\title{
Lernen für das Leben
}

\section{ERSTE ERGEBNISSE DER INTERNATIONALEN SCHULLEISTUNGSSTUDIE}

PISA 2000

OECD

ORGANISATION FÜR WITSCHAFTLICHE ZUSAMMENARBEIT UND ENTWICKLUNG 


\section{ORGANISATION FÜR WIRTSCHAFTLICHE ZUSAMMENARBEIT UND ENTWICKLUNG}

Gemäß Artikel 1 des am 14. Dezember 1960 in Paris unterzeichneten und am 30. September 1961 in Kraft getretenen Übereinkommens fördert die Organisation für wirtschaftliche Zusammenarbeit und Entwicklung (OECD) eine Politik, die darauf gerichtet ist:

- in den Mitgliedstaaten unter Wahrung der finanziellen Stabilität eine optimale Wirtschaftsentwicklung und Beschäftigung sowie einen steigenden Lebensstandard $\mathrm{zu}$ erreichen und dadurch zur Entwicklung der Weltwirtschaft beizutragen;

- in den Mitglied- und Nichtmitgliedstaaten, die in wirtschaftlicher Entwicklung begriffen sind, zu einem gesunden wirtschaftlichen Wachstum beizutragen, und

- im Einklang mit internationalen Verpflichtungen auf multilateraler und nichtdiskriminierender Grundlage zur Ausweitung des Welthandels beizutragen.

Die Gründungsmitglieder der OECD sind: Belgien, Dänemark, Deutschland, Frankreich, Griechenland, Irland, Island, Italien, Kanada, Luxemburg, Niederlande, Norwegen, Österreich, Portugal, Schweden, Schweiz, Spanien, Türkei, Vereinigtes Königreich und Vereinigte Staaten. Folgende Staaten wurden zu den nachstehend genannten Daten Mitglieder der OECD: Japan (28. April 1964), Finnland (28. Januar 1969), Australien (7. Juni 1971), Neuseeland (29. Mai 1973), Mexiko (18. Mai 1994), die Tschechische Republik (21. Dezember 1995), Ungarn (7. Mai 1996), Polen (22. November 1996), Korea (12. Dezember 1996) und die Slowakische Republik (14. Dezember 2000). Die Kommission der Europäischen Gemeinschaften nimmt an den Tätigkeiten der OECD teil (Artikel 13 des Übereinkommens über die OECD).

Die Deutsche Übersetzung wurde im Auftrag des Bundesministeriums für Bildung und Forschung erstellt.

Die englische und die französische Originalfassung wurde veröffentlicht unter dem Titel :

Knowledge and skills for life

FIRST RESULTS FROM PISA 2000

Connaissances et compétences : des atouts pour la vie

PREMIERS RÉSULTATS DE PISA 2000

(C) OECD 2001

Genehmigungen zum Nachdruck von Teilen dieses Werks für nichtkommerzielle Zwecke oder zur Verwendung im Unterricht sind einzuholen beim Centre français d'exploitation du droit de copie (CFC), 20, rue des Grands-Augustins, 75006 Paris, Frankreich, tel: (33-1) 440747 70, fax: (33-1) 463467 19. Dies gilt für alle Länder mit Ausnahme der Vereinigten Staaten, wo das Copyright Clearance Center Inc. (CCC), Customer Service, tel: (508)750-8400, 222 Rosewood Drive, Danvers, MA 01923, USA oder CCC online: www.copyright.com die entsprechenden Genehmigungen erteilt. Alle sonstigen Anträge auf Überlassung von Nachdruck- oder Übersetzungsrechten für das gesamte Dokument oder Teile davon sind zu richten an: OECD Publications, 2, rue André-Pascal, 75775 Paris Cedex 16, Frankreich. 


\section{VORWORT}

Sind die Schülerinnen und Schüler gut vorbereitet für die Herausforderungen der Zukunft? Sind sie in der Lage, ihre Ideen und Vorstellungen effektiv zu analysieren, zu begründen und zu kommunizieren? Verfügen sie über die notwendigen Kompetenzen für lebensbegleitendes Lernen? Eltern und Schüler, die Öffentlichkeit und die Bildungsverantwortlichen brauchen Antworten auf diese Fragen.

In vielen Bildungssystemen wird das Lernverhalten der Schülerinnen und Schüler analysiert, um Antworten auf diese Fragen zu finden. Vergleichende internationale Analysen können die nationale Sichtweise ergänzen und vertiefen, indem sie die nationalen Ergebnisse zur besseren Interpretation in einen größeren Zusammenhang stellen. Sie können den Ländern zeigen, in welchen Bereichen ihre relativen Stärken und Schwächen liegen, und sie dabei unterstützen, Fortschritte zu messen und bessere Leistungen anzustreben. Ferner können sie Orientierungshilfen geben für die nationale Bildungspolitik, die Lehrplangestaltung und die Unterrichtspraxis in den Schulen sowie schließlich für die Lernenden selbst. In Verbindung mit geeigneten Anreizen können sie die Schülerinnen und Schüler zu besserem Lernen, die Lehrkräfte zu besserem Unterrichten und die Schulen zu größerer Effizienz anregen.

Um dem Bedarf an international vergleichbaren Daten über Schulleistungen besser gerecht zu werden, hat die OECD die Internationale Schulleistungsstudie PISA initiiert. Im Rahmen von PISA arbeiten die Regierungen der OECDLänder daran, die an den Schülerleistungen gemessenen Ergebnisse ihrer Bildungssysteme in regelmäßigen Abständen und innerhalb eines gemeinsamen, international vereinbarten Rahmens zu bewerten. Mit PISA soll eine neue Basis für den bildungspolitischen Dialog und die Zusammenarbeit bei der Definition und Operationalisierung von Bildungszielen geschaffen werden, wobei die für das spätere Leben relevanten Kompetenzen im Vordergrund stehen. Die Studie liefert Basismaterial für die Definition von Standards und den Evaluationsprozess. Ferner gibt sie Aufschluss über die Faktoren, die zur Entwicklung wichtiger Kompetenzen beitragen, sowie über die Art und Weise, wie diese in den einzelnen Ländern wirksam werden. Damit dürfte sie zu einem besseren Verständnis von Ursachen und Folgen beobachteter Kompetenzdefizite beitragen. PISA befürwortet eine Verlagerung des Schwerpunkts in der Bildungspolitik von den Inputs auf die Lernergebnisse und kann so die Länder bei ihren Bemühungen um Verbesserung ihrer Schulsysteme unterstützen und dazu beitragen, dass junge Menschen bei ihrem Eintritt in das Erwachsenenleben besser vorbereitet sind auf eine Welt des raschen Wandels und der immer stärker werdenden globalen Interdependenz.

PISA ist ein Kooperationsprojekt, in das wissenschaftliche Fachkenntnisse aus allen Teilnehmerländern einfließen und bei dem übergreifende Entscheidungen von den Teilnehmerstaaten gemeinschaftlich auf der Basis gemeinsamer politischer Interessen getroffen werden. Über einen „Ausschuss der Teilnehmerländer“ übernehmen die beteiligten Staaten die Verantwortung für das Projekt auf der politischen Ebene. Es wurden Arbeitsgruppen mit Experten aus den Teilnehmerstaaten gebildet, die gewährleisten sollen, dass sich die bildungspolitischen Zielsetzungen von PISA auf die besten verfügbaren fachwissenschaftlichen und verfahrenstechnischen Kompetenzen im Bereich des internationalen Leistungsvergleichs stützen. Durch ihre Beteiligung an diesen Expertengruppen stellen die Länder sicher, dass die im Rahmen von PISA eingesetzten Instrumente zur Leistungsmessung international valide sind, dem kulturellen und curricularen Kontext der OECD-Mitgliedsländer Rechnung tragen, eine realistische Basis für die Messungen darstellen und das Schwergewicht auf Authentizität und bildungspolitische Relevanz legen. Die Rahmenkonzeption wie auch die Instrumente zur Leistungsmessung im Rahmen von PISA 2000 sind das Produkt eines mehrjährigen Entwicklungsprozesses und wurden von den OECD-Mitgliedsländern im Dezember 1999 angenommen. 
Der vorliegende Bericht Lernen für das Leben stellt die ersten Ergebnisse von PISA 2000 vor. Er enthält Daten zu den Leistungen von Schülerinnen und Schülern, Schulen und Ländern in den Bereichen Lesekompetenz sowie mathematische und naturwissenschaftliche Grundbildung und bietet Einblick in jene Faktoren, die die Entwicklung dieser Kompetenzen im familiären und schulischen Kontext beeinflussen. Ferner wird untersucht, welche Wechselbeziehungen zwischen diesen Faktoren bestehen und welche Konsequenzen sich hieraus für die Politikgestaltung ergeben.

PISA deckt erhebliche Unterschiede im Leistungsniveau zwischen Schülerinnen und Schülern, Schulen und Ländern auf. Die Studie zeigt, dass der sozioökonomische Hintergrund der Schülerinnen und Schüler, aber auch der Schulen einen bedeutenden Einfluss auf die Schulleistungen hat, der allerdings in einigen Ländern sehr viel weniger ausgeprägt ist als in anderen. Wichtiger ist jedoch die Feststellung, dass einige der Länder, die besonders erfolgreich in ihren Bemühungen waren, die Folgen sozialer Benachteiligung zu mildern, zugleich zu den Ländern mit dem insgesamt gesehen höchsten Schülerleistungsniveau zählen. Diese Länder sind ein konkretes Beispiel dafür, dass ein hohes Leistungsniveau bei gleichzeitiger Minimierung der Ungleichheiten erreicht werden kann. Indem sie verdeutlichen, was in Bezug auf die Verbesserung der Schülerleistungen realisierbar ist, definieren sie eine wichtige Herausforderung auch für andere Länder.

PISA legt den Schluss nahe, dass die Schulen einen maßgeblichen Einfluss ausüben können. Allerdings bedarf es weiterer Analysen, um genau zu ermitteln, welche Wechselwirkungen zwischen Ressourcen, Politik und Praxis der Schulen auf der einen und dem familiären Hintergrund sowie dem gesamtgesellschaftlichen Klima in Bezug auf Lernen und Leistung auf der anderen Seite bestehen und wie sie die Schülerleistungen beeinflussen. Für 2002 und 2003 ist die Veröffentlichung einer Reihe detaillierterer thematischer Berichte vorgesehen, die verstärkten Aufschluss darüber geben sollen, wie sich die Länder und Schulen diesen Herausforderungen stellen können. Vorerst stimmt schon die Tatsache an sich, dass in einigen Ländern ausgezeichnete Lernergebnisse für die Mehrzahl der Schülerinnen und Schüler bereits Realität sind, ermutigend, da dies darauf hindeutet, dass die vor uns liegenden Herausforderungen durchaus zu meistern sind.

Der vorliegende Bericht ist das Produkt eines Kooperationsprojekts zwischen den PISA-Teilnehmerländern, den im Rahmen des PISA-Konsortiums tätigen Experten und Institutionen sowie der OECD. Er wurde erstellt von der OECD-Direktion Bildungswesen, Beschäftigung, Arbeitskräfte und Sozialfragen unter der Leitung von Andreas Schleicher in Zusammenarbeit mit Aletta Grisay, Barry McGaw, Claudia Tamassia, Richard J. Tobin und J. Douglas Willms (der bei der Ausarbeitung von Kapitel 8 eine führende Rolle spielte). Das Datenmaterial für den Bericht wurde vom PISA-Konsortium unter Leitung von Raymond Adams und Christian Monseur vom Australian Council of Educational Research aufbereitet. Die Orientierungen für die Gestaltung des Berichts insgesamt kamen vom Ausschuss der Mitgliedsländer unter dem Vorsitz von Eugene Owen vom National Center for Education Statistics, Vereinigte Staaten. Anhang C des Berichts enthält eine Liste der Mitglieder der verschiedenen PISA-Organe wie auch der einzelnen Fachleute und Consultants, die an diesem Bericht und an PISA allgemein mitgewirkt haben. Die Übersetzung der Publikation ins Deutsche wurde durch die Unterstützung des Bundesministeriums für Bildung, Wissenschaft, Forschung und Technologie der Bundesrepublik Deutschland ermöglicht.

Für diesen Bericht zeichnet der Generalsekretär der OECD verantwortlich.

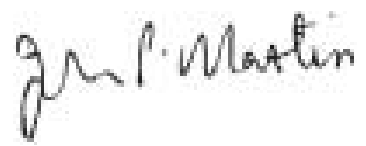

John P. Martin

Leiter der Direktion Bildungswesen, Beschäftigung, Arbeitskräfte und Sozialfragen, OECD

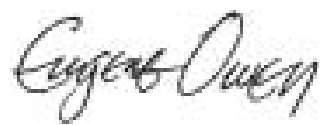

Eugene Owen

Vorsitzender des PISA-

Ausschusses der

Teilnehmerländer 


\section{INHALTSVERZEICHNIS}

Kapitel 1 Einführung in die Internationale Schulleistungsstudie PISA …............... 13

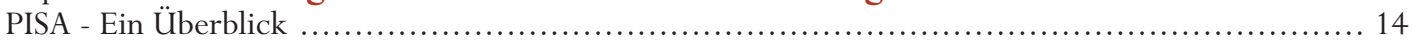

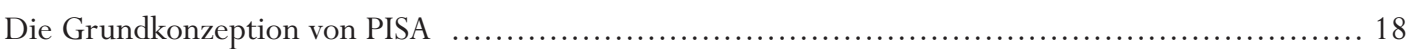

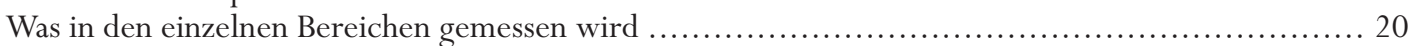

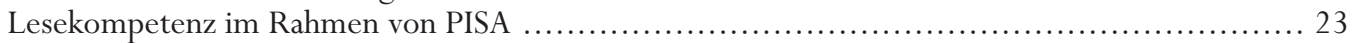

Mathematische Grundbildung im Rahmen von PISA ......................................... 24

Naturwissenschaftliche Grundbildung im Rahmen von PISA …............................... 26

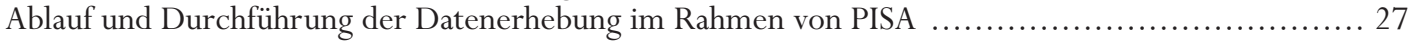

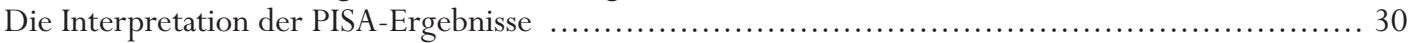

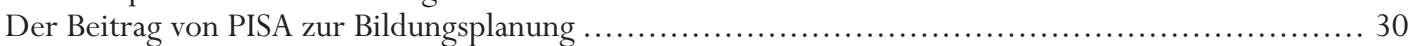

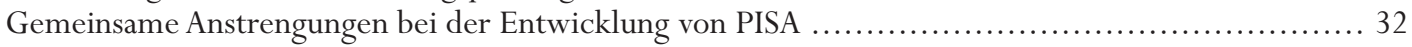

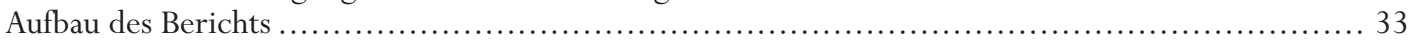

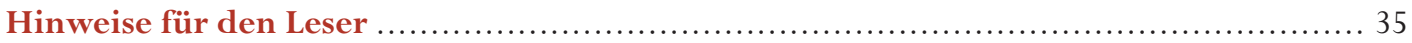

Kapitel 2 Was PISA über die Fähigkeiten 15-Jähriger aussagt:

ein Profil der Schülerleistungen im Bereich Lesekompetenz .............................. 37

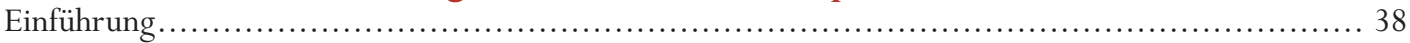

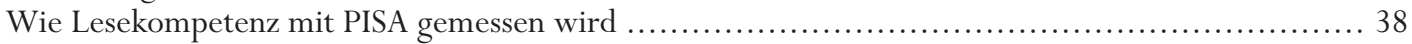

Prozentuale Anteile der Schülerinnen und Schüler auf den verschiedenen Lesekompetenzstufen ....... 42

Kompetenzstufe 5 (über 625 Punkte) .......................................................... 50

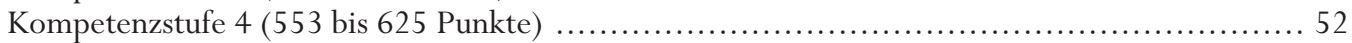

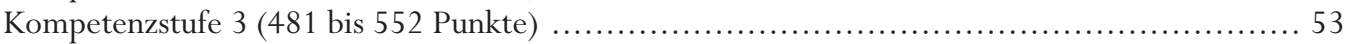

Kompetenzstufe 2 (408 bis 480 Punkte) ................................................. 54

Leistungsniveau 1 (335 bis 407 Punkte) oder darunter (weniger als 335 Punkte) ................. 54

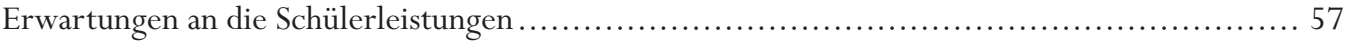

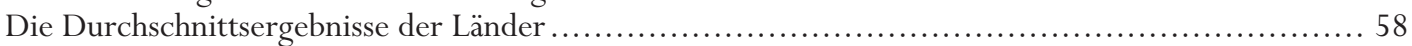

Die Verteilung der Lesekompetenz innerhalb der Länder ......................................... 62

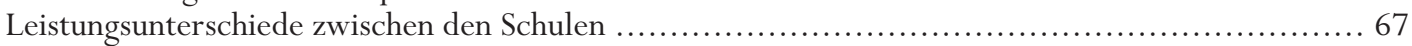

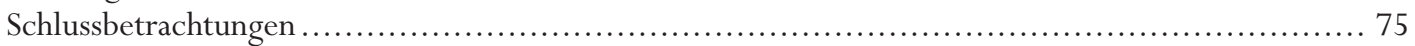

Kapitel 3 Was PISA über die Fähigkeiten 15-Jähriger aussagt: ein Profil der Schülerleistungen in mathematischer und naturwissenschaftlichen Grundbildung ..... 81

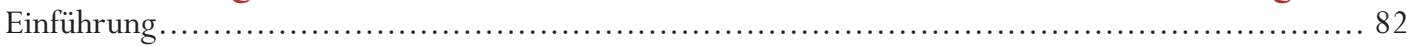

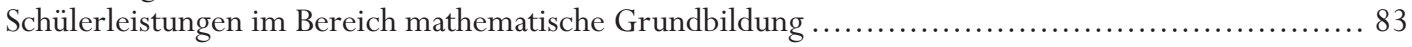

Wie die mathematische Grundbildung in PISA gemessen wird ................................. 83

Die Durchschnittsergebnisse der Länder in mathematischer Grundbildung ........................ 91

Die Verteilung der mathematischen Grundbildung innerhalb der Länder ......................... 93

Leistungen in mathematischer Grundbildung und Lesekompetenz .............................. 96

Schülerleistungen im Bereich naturwissenschaftliche Grundbildung ................................ 97

Wie die naturwissenschaftliche Grundbildung in PISA gemessen wird ........................ 97

Die Durchschnittsergebnisse der Länder in naturwissenschaftlicher Grundbildung ................ 103

Die Verteilung der naturwissenschaftlichen Grundbildung innerhalb der Länder .................. 103

Leistungen in naturwissenschaftlicher Grundbildung und Lesekompetenz ......................... 103

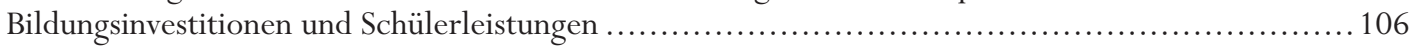

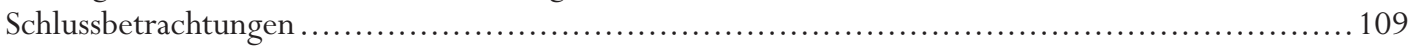




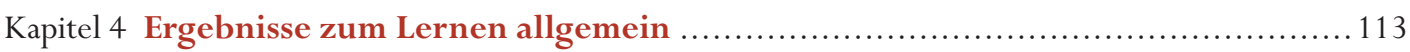

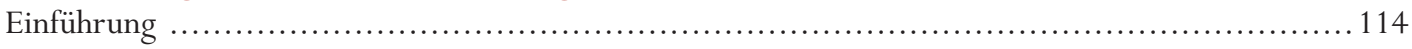

Motivation und Engagement in Bezug auf die Schule .......................................... 116

Fachbezogenes Interesse an Lesen und Mathematik ................................... 116

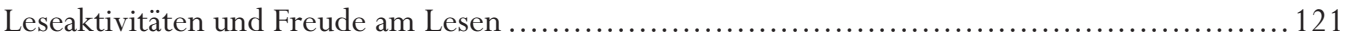

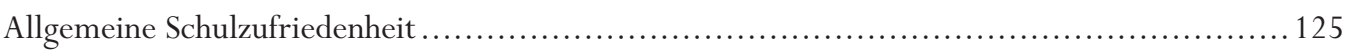

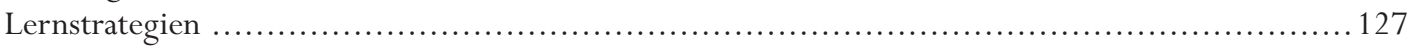

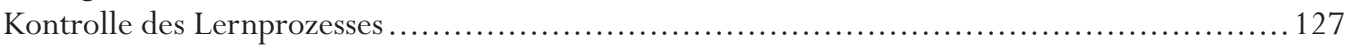

Memorieren und Elaborieren......................................................... 130

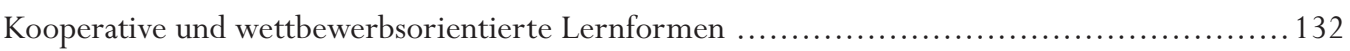

Computer als Hilfsmittel zum Lernen ................................................... 134

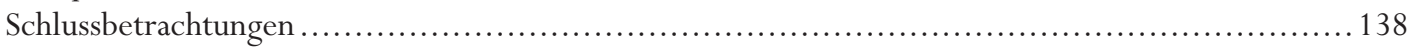

Kapitel 5 Geschlechtsspezifische Leistungsunterschiede ............................ 143

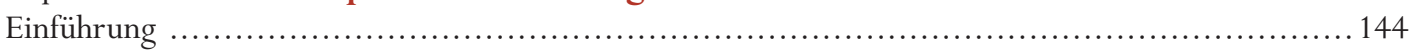

Geschlechtsspezifische Unterschiede in der Lesekompetenz sowie der mathematischen und

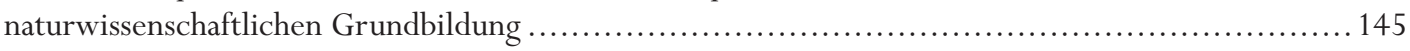

Geschlechtsspezifisch unterschiedliches Interesse an einzelnen Fachgebieten ...................... 151

Geschlechtsspezifische Unterschiede in der Freude am Lesen.................................. 153

Geschlechtsspezifische Unterschiede bei Lernstrategien und Selbstkonzept ....................... 157

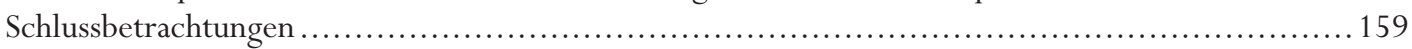

Kapitel 6 Familiärer Hintergrund und Schülerleistungen ........................... 161

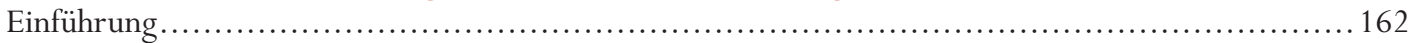

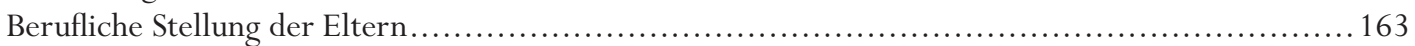

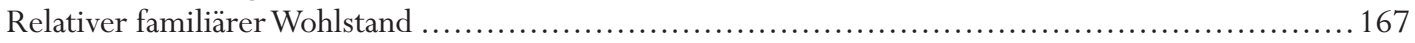

Mit „klassischer“ Kultur verbundene Besitztümer und Aktivitäten................................... 169

Kommunikation über soziale Fragen und kulturelle Aspekte ................................. 172

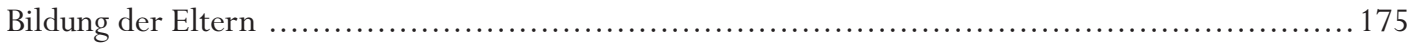

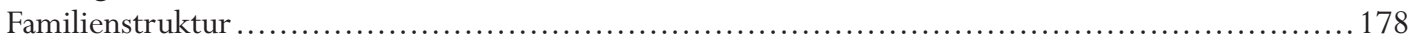

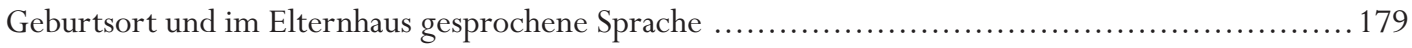

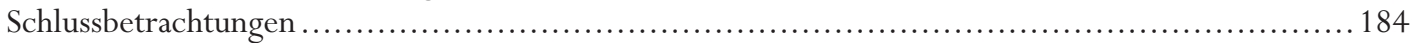

Kapitel 7 Das Lernumfeld und die Schulorganisation ................................ 187

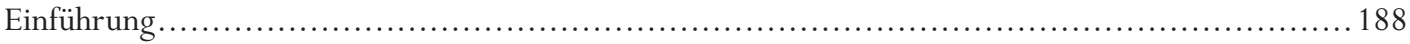

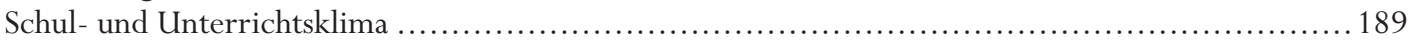

Unterstützung durch die Lehrkräfte............................................... 189

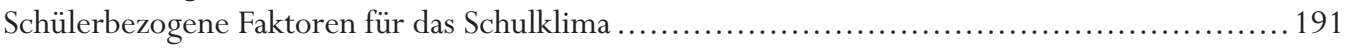

Lehrkräftebezogene Faktoren für das Schulklima ...................................... 197

Das Lernen außerhalb der Schule....................................................... 199

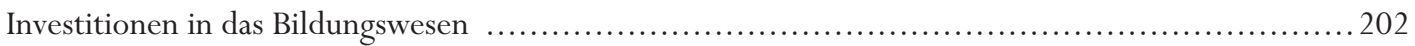

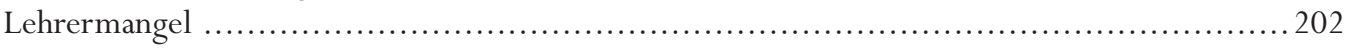

Qualität der räumlichen Bedingungen der Schulen und der Ausstattung mit Lehr- und

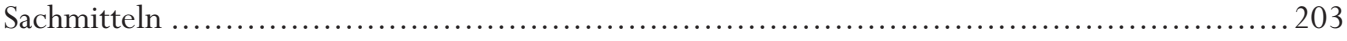

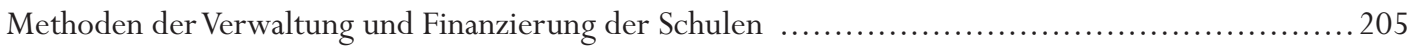


Autonomie der Schulen und Einbeziehung der Lehrkräfte ................................ 205

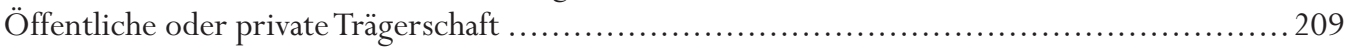

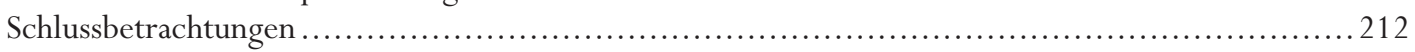

Kapitel 8 Entscheidende Erkenntnisse aus den PISA-Resultaten - Hinweise für die

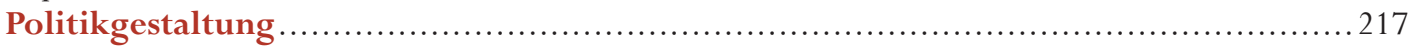

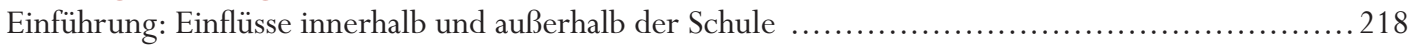

Zusammenhänge zwischen dem sozioökonomischen Hintergrund der Schülerinnen und Schüler und

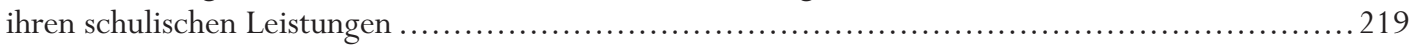

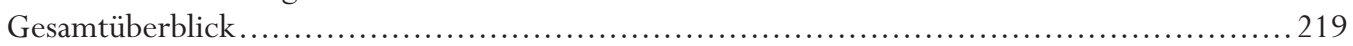

Die Stärke des Einflusses sozioökonomischer Faktoren in den verschiedenen Ländern ............ 222

Komponenten des sozioökonomischen Hintergrunds ..................................... 229

Möglichkeiten der Schulen zur Minderung der Effekte sozioökonomischer Benachteiligung .........2232

Sozioökonomischer Hintergrund und Varianz der Leistungen zwischen den Schulen ............ 234

Die Verstärkung der vom familiären Hintergrund der einzelnen Schülerinnen und Schüler ausgehenden Effekte durch die soziale Struktur der Schulen.............................. 235

Schulische Faktoren, mit denen die Leistungsniveaus angehoben und die Auswirkungen des

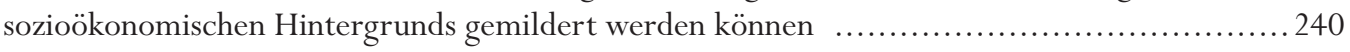

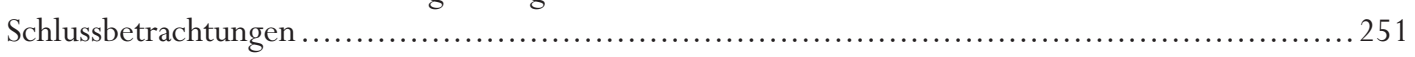

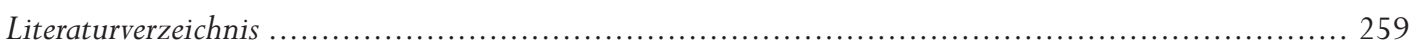

\section{Anhang A:Technische Hinweise}

Anhang A1 Konstruktion der Indizes und anderer von den Kontextfragebogen für

Schülerinnen und Schüler und für Schulen abgeleiteten Messgrößen ................ 262

Anhang A2 Erklärte Varianz der Schülerleistungen ...................................... 272

Anhang A3 PISA-Zielpopulation und PISA-Stichproben .................................... 274

Anhang A4 Standardfehler, Signifikanztests und Ländervergleich ............................. 281

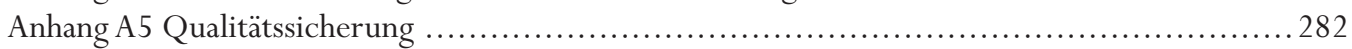

Anhang A6 Entwicklung der PISA-Erhebungsinstrumente .................................. 283

Anhang A7 Reliabilität der Kodierung offener Items .................................... 287

\section{Anhang B: Datentabellen}

Anhang B1 Datentabellen zu den Kapiteln 290

Anhang B2 Leistungsunterschiede zwischen den flämischen und französischen Gemeinschaften in Belgien sowie den Sprachgemeinschaften in der Schweiz.... 361

Anhang C: Entwicklung und Umsetzung von PISA - ein Kooperationsprojekt 363 


\section{VERZEICHNIS DER ABBILDUNGEN}

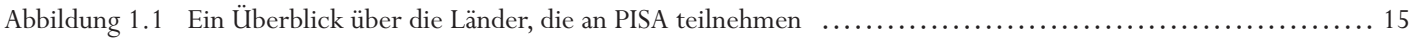

Abbildung 1.2 Schulabschluss, Lesekompetenz und die Wahrscheinlichkeit für die Ausübung einer anspruchsvollen

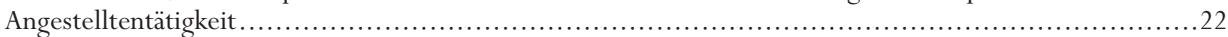

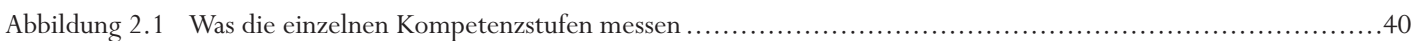

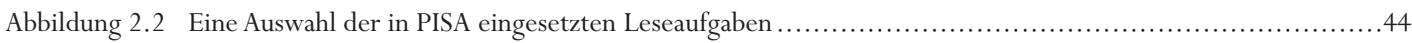

Abbildung 2.3 Leistungsverteilung der Schülerinnen und Schüler auf den verschiedenen Kompetenzstufen

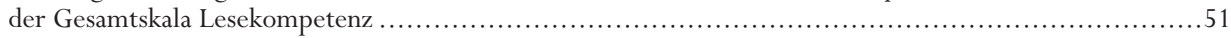

Abbildung 2.4 Vergleich der Durchschnittsergebnisse der Länder auf der Gesamtskala Lesekompetenz ....................60

Abbildung 2.5 Verteilung der Schülerleistungen auf der Gesamtskala Lesekompetenz innerhalb der Länder................63

Abbildung 2.6 Leistungsunterschiede zwischen den Schulen und innerhalb der Schulen auf der

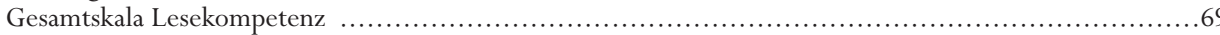

Abbildung 3.1 Eine Auswahl der in PISA eingesetzten Aufgaben im Bereich mathematische Grundbildung ..................88 Abbildung 3.2 Vergleich der Durchschnittsergebnisse der Länder auf der Skala für mathematische Grundbildung ..........92 Abbildung 3.3 Verteilung der Schülerleistungen auf der Skala für mathematische Grundbildung innerhalb der Länder ......94 Abbildung 3.4 Eine Auswahl der in PISA eingesetzten Aufgaben im Bereich naturwissenschaftlicher Grundbildung ..........99 Abbildung 3.5 Vergleich der Durchschnittsergebnisse der Länder auf der Skala für naturwissenschaftliche Grundbildung 104 Abbildung 3.6 Verteilung der Schülerleistungen auf der Skala für naturwissenschaftliche Grundbildung

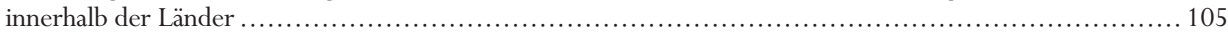

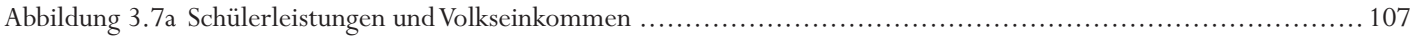

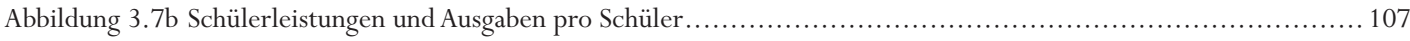

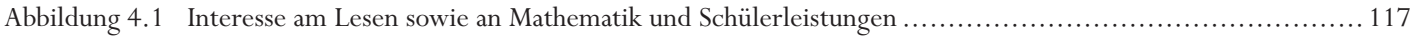

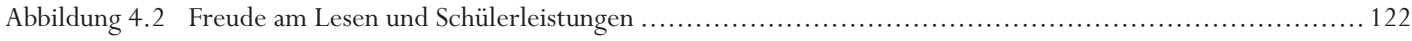

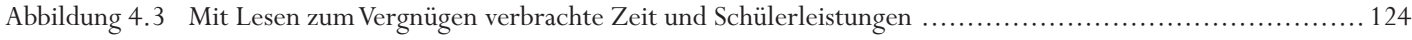

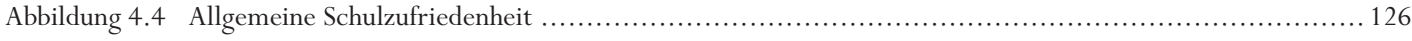

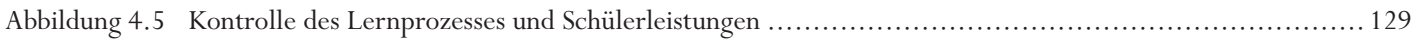

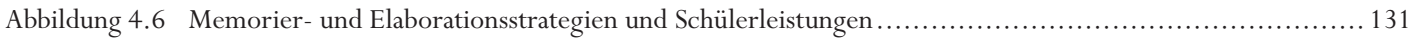

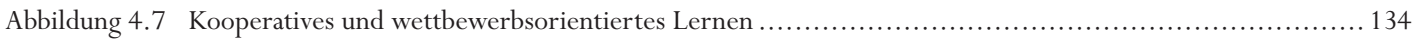

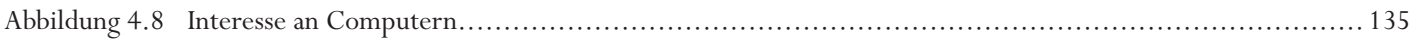

Abbildung 4.9 Vertrautheit und Selbsteinschätzung der Fähigkeit im Umgang mit Computern......................... 137

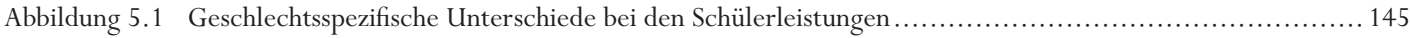

Abbildung 5.2 Der Anteil der Jungen und Mädchen bei den leistungsschwächsten Schülern auf der Gesamtskala Lesekompetenz ......

Abbildung 5.3a Zusammenhang zwischen Leseinteresse und Leistungen auf der Gesamtskala Lesekompetenz bei Jungen und Mädchen.

Abbildung 5.3b Zusammenhang zwischen Mathematikinteresse und Leistungen auf der Skala für mathematische Grundbildung bei Jungen und Mädchen

Abbildung 5.4 Geschlechtsspezifische Unterschiede in Bezug auf die Freude am Lesen - Einstellungen dem

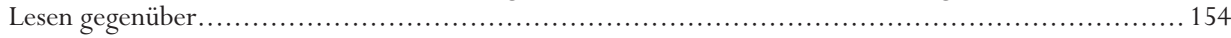

Abbildung 5.5 Geschlechtsspezifische Unterschiede in Bezug auf die Freude am Lesen - mit „Lesen zum Vergnügen“

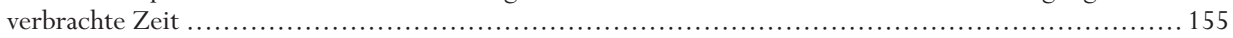

Abbildung 5.6 Geschlechtsspezifische Unterschiede in Bezug auf die Freude am Lesen - Vielfalt des Lesestoffs ............ 156 Abbildung 5.7a Zusammenhang zwischen dem Selbstkonzept Lesen und den Leistungen auf der Gesamtskala Lesekompetenz bei Jungen und Mädchen...... 
Abbildung 5.7b Zusammenhang zwischen dem Selbstkonzept Mathematik und den Leistungen auf der Skala für mathematische Grundbildung bei Jungen und Mädchen

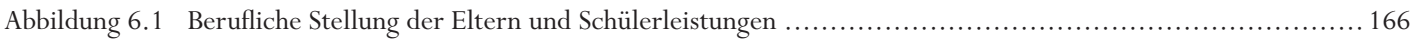

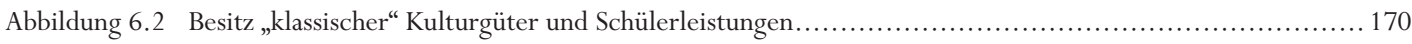

Abbildung 6.3 Soziale und kulturelle Kommunikation mit den Eltern und Schülerleistungen.......................... 173

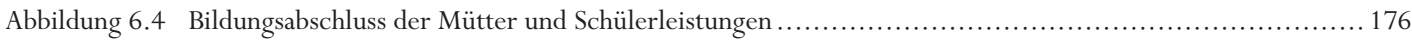

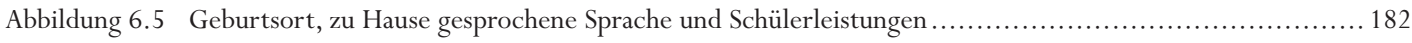

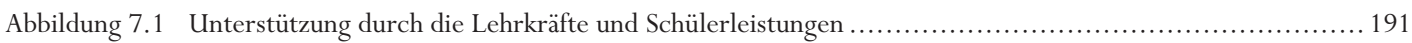

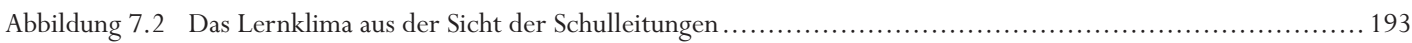

Abbildung 7.3 Das Lernklima aus der Sicht der Schülerinnen und Schüler .......................................... 194

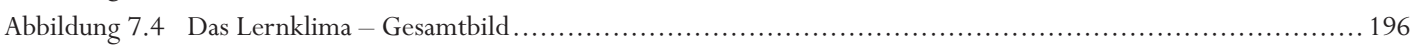

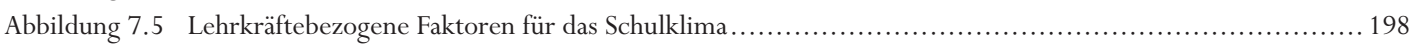

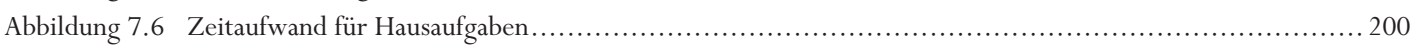

Abbildung 7.7 Qualität der schulischen Ressourcen aus der Sicht der Schulleitungen .................................. 205

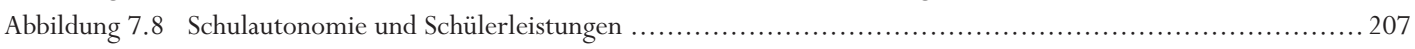

Abbildung 7.9 Schülerleistungen und staatliche oder private Trägerschaft der Schulen ............................. 212

Abbildung 8.1 Zusammenhang zwischen Schülerleistungen und sozioökonomischem Hintergrund, OECD-Raum insgesamt

Abbildungen 8.2 und $8.3 \mathrm{a}-\mathrm{c}$

Zusammenhang zwischen Schülerleistungen und sozioökonomischem Hintergrund in den einzelnen Ländern

Abbildung 8.4 Effekte des sozioökonomischen Hintergrundes Status der Schüler und der Schulen auf die Schülerleistungen auf der Gesamtskala Lesekompetenz.

Abbildung 8.5 Durchschnittliche Auswirkung der Schüler/Lehrer-Quote auf die Schülerleistungen auf der Gesamtskala Lesekompetenz im gesamten OECD-Raum.

\section{VERZEICHNIS DER KÄSTEN}

Kasten 1.1 PISA 2000 - eine international standardisierte Leistungsbeurteilung 15 -jähriger Schülerinnen und Schüler.

Kasten 1.2 Verbessert höhere Lesekompetenz die Beschäftigungsaussichten? ........................................22

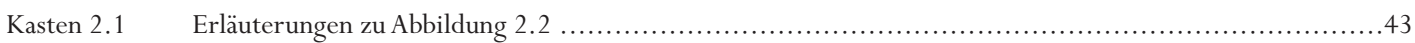

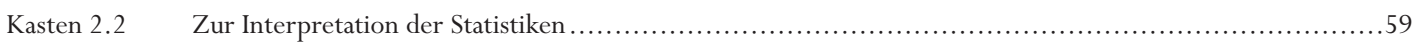

Kasten 2.3 Interpretation der Unterschiede in den PISA-Ergebnissen: Wie groß sind die Abstände? .....................65

Kasten 2.4 Mit Leistungsunterschieden zwischen den Schulen zusammenhängende Faktoren .........................72

Kasten 4.1 Interpretation der Selbstaussagen der Schülerinnen und Schüler...................................... 115

Kasten 5.1 Veränderung geschlechtsspezifischer Leistungsunterschiede in Mathematik und Naturwissenschaften mit zunehmendem Schüleralter ............................................... 146

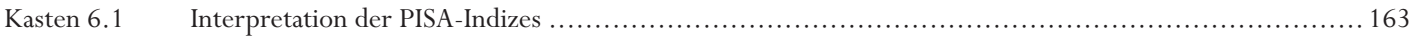

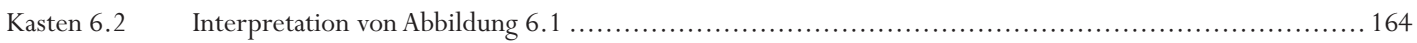

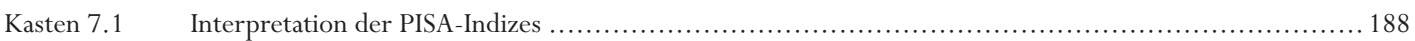

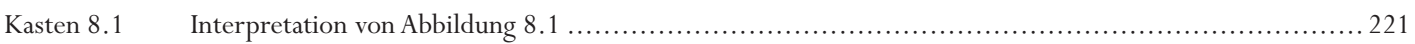

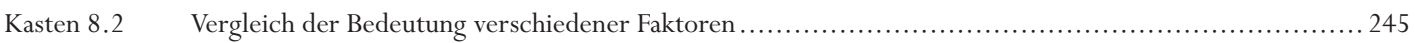

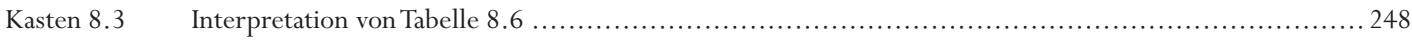




\section{VERZEICHNIS DER TABELLEN}

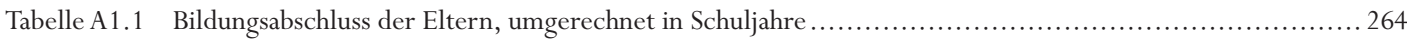

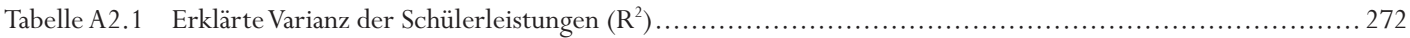

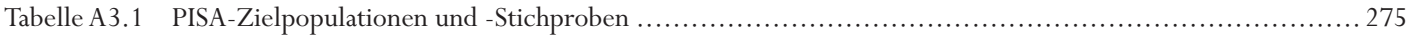

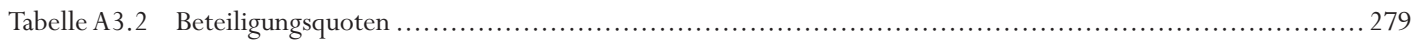

Tabelle A6.1 Itemverteilung nach den Dimensionen der PISA-Rahmenkonzeption auf der Gesamtskala Lesekompetenz . 283

Tabelle A6.2 Itemverteilung nach den Dimensionen der PISA-Rahmenkonzeption auf der Skala für

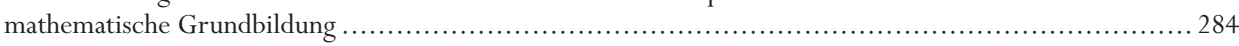

Tabelle A6.3 Itemverteilung nach den Dimensionen der PISA-Rahmenkonzeption auf der Skala für

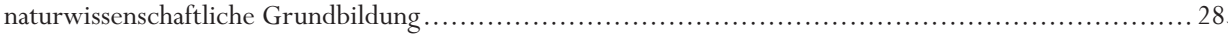

Tabelle 2.1a Prozentsatz der Schülerinnen und Schüler auf den jeweiligen Kompetenzstufen der Gesamtskala Lesekompetenz

Tabelle 2.1b Prozentsatz der Schülerinnen und Schüler auf den jeweiligen Kompetenzstufen der Lesekompetenz-Subskala Informationen Ermitteln.....

Tabelle 2.1c Prozentsatz der Schülerinnen und Schüler auf den jeweiligen Kompetenzstufen der

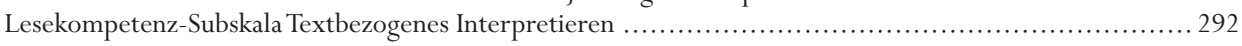

Tabelle 2.1d Prozentsatz der Schülerinnen und Schüler auf den jeweiligen Kompetenzstufen der

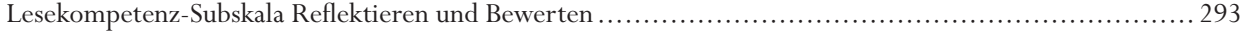

Tabelle 2.2a Vergleich der Durchschnittsergebnisse der Länder auf der Subskala Informationen Ermitteln ............... 294

Tabelle 2.2b Vergleich der Durchschnittsergebnisse der Länder auf der Subskala Textbezogenes Interpretieren ........... 295

Tabelle 2.2c Vergleich der Durchschnittsergebnisse der Länder auf der Subskala Reflektieren und Bewerten ............. 296

Tabelle 2.3a Verteilung der Schülerleistungen auf der Gesamtskala Lesekompetenz ............................... 297

Tabelle 2.3b Verteilung der Schülerleistungen auf der Lesekompetenz-Subskala Informationen Ermitteln ................ 298

Tabelle 2.3c Verteilung der Schülerleistungen auf der Lesekompetenz-Subskala Textbezogenes Interpretieren............ 299

Tabelle 2.3d Verteilung der Schülerleistungen auf der Lesekompetenz-Subskala Reflektieren und Bewerten .............. 300

Tabelle 2.4 Varianz der Schülerleistungen zwischen Schulen und innerhalb von Schulen auf der Gesamtskala

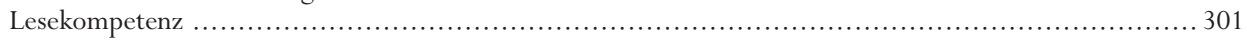

Tabelle 2.5 Festgelegte Mindestnote für die Versetzung und Leistungen auf der Gesamtskala Lesekompetenz............ 302

Tabelle 3.1 Verteilung der Schülerleistungen auf der Skala für mathematische Grundbildung ......................... 303

Tabelle 3.2 Festgelegte Mindestnote für die Versetzung und Leistungen auf der Skala für mathematische

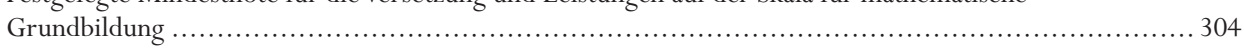

Tabelle 3.3 Verteilung der Schülerleistungen auf der Skala für naturwissenschaftliche Grundbildung.................... 305

Tabelle 3.4 Festgelegte Mindestnote für die Versetzung und Leistungen auf der Skala für

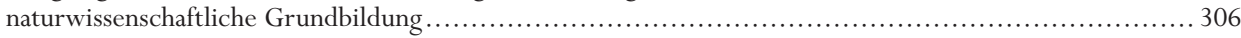

Tabelle 3.5 Varianz der Schülerleistungen zwischen Schulen und innerhalb von Schulen auf den Skalen für mathematische und für naturwissenschaftliche Grundbildung

Tabelle 3.6 Schülerleistungen auf der Gesamtskala Lesekompetenz, der Skala für naturwissenschaftliche Grundbildung und der Skala für mathematische Grundbildung und Volkseinkommen .....

Tabelle 4.1 Index des Leseinteresses und Leistungen auf der Gesamtskala Lesekompetenz nach Indexquartilen für das jeweilige Land

Tabelle 4.2 Index des Mathematikinteresses und Leistungen auf der Skala für mathematische Grundbildung, nach Indexquartilen für das jeweilige Land .....

Tabelle 4.3 Index Freude am Lesen und Leistungen auf der Gesamtskala Lesekompetenz, nach Indexquartilen

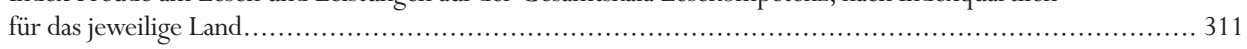

Tabelle 4.4 Täglicher Zeitaufwand für Lesen zum Vergnügen und Leistungen auf der Gesamtskala Lesekompetenz ........... 312

Tabelle 4.5 Index der Kontrollstrategien und Leistungen auf der Gesamtskala Lesekompetenz, nach Indexquartilen für das jeweilige Land ........ 
Tabelle 4.6 Index der Memorierstrategien und Leistungen auf der Gesamtskala Lesekompetenz, nach Indexquartilen für das jeweilige Land .....

Tabelle 4.7 Index der Elaborationsstrategien und Leistungen auf der Gesamtskala Lesekompetenz, nach Indexquartilen für das jeweilige Land .....

Tabelle 4.8 Index des kooperativen Lernens und Leistungen auf der Gesamtskala Lesekompetenz, nach Indexquartilen für das jeweilige Land .....

Tabelle 4.9 Index des wettbewerbsorientierten Lernens und Leistungen auf der Gesamtskala Lesekompetenz, nach Indexquartilen für das jeweilige Land .....

Tabelle 4.10 Index des Interesses an Computern und Leistungen auf der Gesamtskala Lesekompetenz, nach Indexquartilen für das jeweilige Land ....

Tabelle 4.11 Index der Vertrautheit und Selbsteinschätzung der Fähigkeit im Umgang mit Computern und Leistungen auf der Gesamtskala Lesekompetenz, nach Indexquartilen für das jeweilige Land .....

Tabelle 5.1a Schülerleistungen auf der Gesamtskala Lesekompetenz sowie den Skalen für mathematische und für naturwissenschaftliche Grundbildung, nach Geschlecht.

Tabelle 5.1b Schülerleistungen auf den Subskalen Informationen Ermitteln, Textbezogenes Interpretieren, Reflektieren und Bewerten, nach Geschlecht.

Tabelle 5.2a Prozentsatz der Schülerinnen und Schüler auf den jeweiligen Kompetenzstufen der Gesamtskala Lesekompetenz, nach Geschlecht

Tabelle 5.2b Prozentsatz der Schülerinnen und Schüler mit weniger als 400 und mehr als 600 Punkten auf der Skala für mathematische Grundbildung ......

Tabelle 5.3 Täglicher Zeitaufwand für Lesen zum Vergnügen

Tabelle 5.4a Index des Selbstkonzepts Lesen, nach Geschlecht und Leistungen auf der Gesamtskala Lesekompetenz, nach Indexquartilen

Tabelle 5.4b Index des Selbstkonzepts Mathematik, nach Geschlecht und Leistungen auf der Skala für mathematische Grundbildung, nach Indexquartilen

Tabelle 6.1a Internationaler sozioökonomischer Index der beruflichen Stellung der Eltern (ISEI) und Leistungen auf der Gesamtskala Lesekompetenz, nach Indexquartilen für das jeweilige Land ....

Tabelle 6.1b Internationaler sozioökonomischer Index der beruflichen Stellung der Eltern (ISEI) und Leistungen auf der Skala für mathematische Grundbildung, nach Indexquartilen für das jeweilige Land .................... 328

Tabelle 6.1c Internationaler sozioökonomischer Index der beruflichen Stellung der Eltern (ISEI) und Leistungen auf der Skala für naturwissenschaftliche Grundbildung, nach Indexquartilen für das jeweilige Land ............ 329

Tabelle 6.2 Index des relativen Wohlstands der Familie und Leistungen auf der Gesamtskala Lesekompetenz, nach Indexquartilen für das jeweilige Land

Tabelle 6.3 Index des Besitzes von „klassischen“ Kulturgütern im Elternhaus und Leistungen auf der Gesamtskala Lesekompetenz, nach Indexquartilen für das jeweilige Land .....

Tabelle 6.4 Index der „klassischen“ kulturellen Aktivitäten und Leistungen auf der Gesamtskala Lesekompetenz, nach Indexquartilen für das jeweilige Land .....

Tabelle 6.5 Index der sozialen Kommunikation mit den Eltern und Leistungen auf der Gesamtskala Lesekompetenz, nach Indexquartilen für das jeweilige Land

Tabelle 6.6 Index der kulturellen Kommunikation mit den Eltern und Leistungen auf der Gesamtskala Lesekompetenz, nach Indexquartilen für das jeweilige Land ....

Tabelle 6.7 Prozentsatz der Schüler und Leistungen auf der Gesamtskala Lesekompetenz sowie den Skalen für mathematische und für naturwissenschaftliche Grundbildung, nach dem Bildungsabschluss der Mutter ... 335

Tabelle 6.8 Prozentualer Anteil der Hochschulabsolventen an der erwachsenen Bevölkerung und Wahrscheinlichkeit, einen Hochschulabschluss zu erhalten, nach Bildungsstand der Eltern

Tabelle 6.9 Familienstruktur und Leistungen auf der Gesamtskala Lesekompetenz, nach Familientyp.....

Tabelle 6.10 Prozentsatz der Schüler und Leistungen auf der Gesamtskala Lesekompetenz sowie den Skalen für mathematische und für naturwissenschaftliche Grundbildung, nach Staatsangehörigkeit der Schüler und deren Eltern 
Tabelle 6.11 Schülerleistungen auf der Gesamtskala Lesekompetenz sowie den Skalen für mathematische und für naturwissenschaftliche Grundbildung, nach der zu Hause gesprochenen Sprache.....

Tabelle 7.1 Index der Unterstützung der Schüler durch die Lehrkräfte und Leistungen auf der Gesamtskala Lesekompetenz, nach Indexquartilen für das jeweilige Land.

Tabelle 7.2 Index schülerbezogener Faktoren für das Schulklima und Leistungen auf der Gesamtskala Lesekompetenz, nach Indexquartilen für das jeweilige Land.....

Tabelle 7.3 Index der Schuldisziplin und Leistungen auf der Gesamtskala Lesekompetenz, nach Indexquartilen für das jeweilige Land.

Tabelle 7.4 Index lehrkräftebezogener Faktoren für das Schulklima und Leistungen auf der Gesamtskala Lesekompetenz, nach Indexquartilen für das jeweilige Land.

Tabelle 7.5 Index der Stimmung und Arbeitshaltung der Lehrkräfte aus der Sicht der Schulleitung und Leistungen auf der Gesamtskala Lesekompetenz, nach Indexquartilen für das jeweilige Land .....

Tabelle 7.6 Index des wöchentlichen Zeitaufwands für Hausaufgaben in der Testsprache, in Mathematik und den Naturwissenschaften und Leistungen auf der Gesamtskala Lesekompetenz, nach Indexquartilen für das jeweilige Land.

Tabelle 7.7 Teilnahme der Schülerinnen und Schüler an Kursen ausserhalb des Unterrichts ..... 345

Tabelle 7.8 Index des Mangels an Lehrkräften und Leistungen auf der Gesamtskala Lesekompetenz, nach Indexquartilen für das jeweilige Land ....

Tabelle 7.9 Index der Qualität der räumlichen Bedingungen der Schulen und Leistungen auf der Gesamtskala Lesekompetenz, nach Indexquartilen für das jeweilige Land......

Tabelle 7.10 Index der Qualität der Ausstattung mit Lehr- und Sachmitteln und Leistungen auf der Gesamtskala Lesekompetenz, nach Indexquartilen für das jeweilige Land.

Tabelle 7.11 Prozentsatz der Schülerinnen und Schüler in Schulen, in denen die Schulen bei folgenden Aspekten der Schulpolitik und -verwaltung eine gewisse Verantwortung tragen .....

Tabelle 7.12 Prozentsatz der Schülerinnen und Schüler in Schulen, in denen die Lehrkräfte bei folgenden Aspekten der Schulpolitik und -verwaltung die Hauptverantwortung tragen....

Tabelle 7.13 Prozentsatz der Schülerinnen und Schüler und Leistungen auf der Gesamtskala Lesekompetenz, nach Schultyp

Tabelle 8.1 Zusammenhang zwischen Schülerleistungen und sozioökonomischem Hintergrund.....

Tabelle 8.2 Effekte ausgewählter familiärer und demographischer Faktoren auf die Schülerleistungen auf der Gesamtskala Lesekompetenz

Tabelle 8.3 Durch den sozioökonomischen Hintergrund erklärte Varianz der Leistungen auf der Gesamtskala Lesekompetenz

Tabelle 8.4 Effekte des sozioökonomischen Hintergrunds von Schülern und von Schulen auf die Schülerleistungen auf der Gesamtskala Lesekompetenz ....

Tabelle 8.5 Effekte schul- und schülerbezogener Faktoren auf die Leistungen auf der Gesamtskala Lesekompetenz sowie den Skalen für mathematische und für naturwissenschaftliche

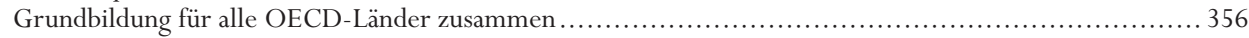

Tabelle 8.5a Effekte schüler- und schulbezogener Faktoren auf die Leistungen auf der Gesamtskala Lesekompetenz ..... 358

Tabelle 8.6 Ländermittel der Indizes ausgewählter schulischer Faktoren und Korrelation mit dem durchschnittlichen wirtschaftlichen, sozialen und kulturellen Status der Schulen (ESCS).....

Tabelle B2.1 Leistungsunterschiede zwischen den flämischen und französischen Gemeinschaften Belgiens sowie Sprachgemeinschaften der Schweiz ..... 


\section{KAPITEL}

1

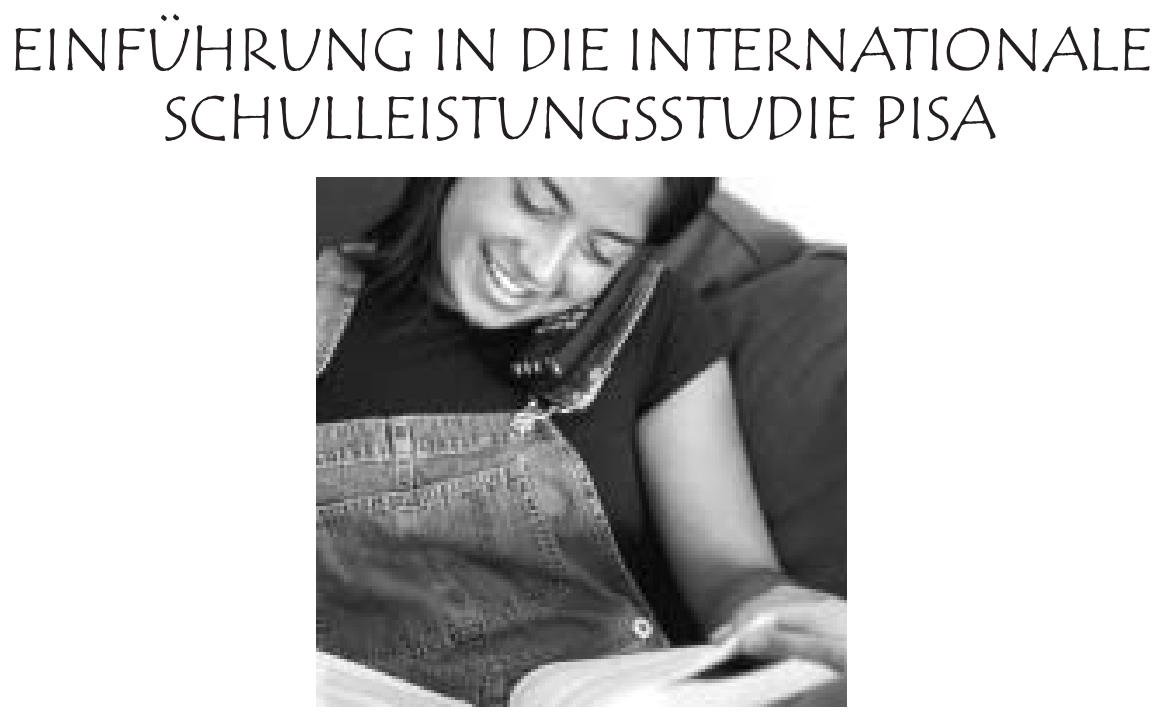


KAPITEL 1 Einführung in die Internationale Schulleistungsstudie PISA

\section{PISA - Ein Überblick}

PISA soll beurteilen, wie gut 15-Jährige für die Herausforderungen des Lebens vorbereitet sind.

Zusammen mit den weltweit führenden Experten haben die teilnehmenden Länder und die OECD länderübergreifend valide Instrumente geschaffen, ...

... die erfassen, was die

Schülerinnen und

Schüler mit dem in

Lesen, Mathematik und

Naturwissenschaften

Gelernten anfangen

können.
Die im Rahmen der OECD durchgeführte internationale Schulleistungsstudie PISA ist ein Kooperationsprojekt der OECD-Mitgliedstaaten, mit dessen Hilfe festgestellt werden soll, wie gut Jugendliche im Alter von 15 Jahren, die somit nahezu das Ende ihrer Pflichtschulzeit erreicht haben, auf die Herausforderungen der heutigen Wissensgesellschaft vorbereitet $\operatorname{sind}^{1}$. Die Erhebung ist zukunftsgerichtet, d.h. sie konzentriert sich weniger auf die Frage, inwieweit die Jugendlichen bestimmte schulische Curricula beherrschen, als vielmehr auf deren Fähigkeit, ihre Kenntnisse und Fertigkeiten zur Bewältigung realitätsnaher Herausforderungen einzusetzen. Diese Orientierung spiegelt auch eine Veränderung in den Zwecken und Zielen von Lehrplänen wider, denn dort geht es zunehmend darum, was die Schülerinnen und Schüler mit ihrem Schulwissen anfangen können, und nicht nur um den Erwerb dieses Wissens im engeren Sinne.

PISA ist das bisher umfassendste und weitreichendste internationale Projekt zur Erfassung von Schülerleistungen und Daten über schülerspezifische, familiäre und institutionelle Faktoren, die zur Erklärung von Leistungsunterschieden herangezogen werden können. Die Entscheidungen über Umfang und Art der Leistungsmessung und der Hintergrundbefragung wurden von führenden Experten in den Teilnehmerstaaten getroffen und von deren Regierungen gemeinschaftlich auf der Basis der ihnen gemeinsamen bildungspolitischen Interessen gesteuert. Es wurden erhebliche Anstrengungen und Ressourcen darauf verwendet, bei dem Erhebungsmaterial kulturelle und sprachliche Breite zu gewährleisten. Für Übersetzungsverfahren, Stichprobenauswahl und Datenerhebung wurden strenge Mechanismen der Qualitätssicherung eingesetzt. Folglich weisen die Ergebnisse der PISA-Studie einen hohen Grad an Validität und Reliabilität auf und können so zu einem wesentlich besseren Verständnis der Bildungserträge in den am meisten entwickelten Ländern der Welt beitragen.

PISA basiert auf einem dynamischen Modell des lebensbegleitenden Lernens, demzufolge die für eine erfolgreiche Anpassung an eine sich verändernde Welt notwendigen neuen Kenntnisse und Fähigkeiten kontinuierlich über die gesamte Lebensspanne hinweg erworben werden. PISA legt den Schwerpunkt auf Aspekte, die 15-Jährige in ihrem späteren Leben brauchen werden, und erhebt, was sie mit dem Gelernten anfangen können. Die Erhebung stützt sich auch, jedoch nicht ausschließlich, auf den gemeinsamen Nenner der Lehrplaninhalte der Teilnehmerstaaten. PISA erhebt das Wissen von Schülerinnen und Schülern, aber ebenso deren Fähigkeit, über dieses Wissen und ihre Erfahrungen zu reflektieren und beides auf realitätsnahe Fragen anzuwenden. Um z.B. bestimmte wissenschaftliche Ratschläge über Nahrungsmittelsicherheit zu verstehen und einzuschätzen, muss ein Erwachsener nicht nur gewisse Grundkenntnisse über die Zusammensetzung von Nährstoffen besitzen, sondern auch in der Lage sein, diese Informationen praktisch anzuwenden. Der Begriff 
Abbildung 1.1

Ein Überblick über die Länder, die an PISA teilnehmen

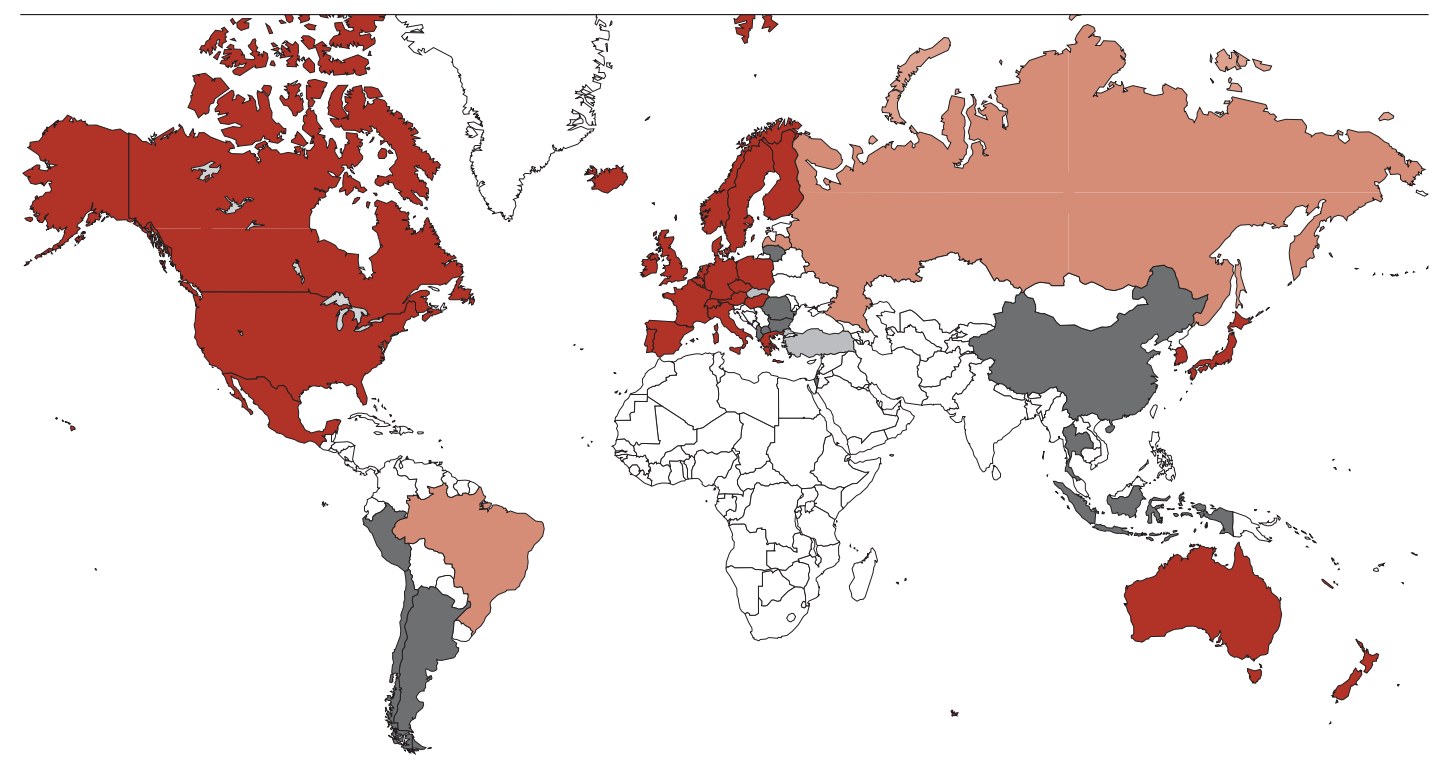

OECD-Länder, die an PISA 2000 teilnahmen

Australien

Belgien

Dänemark

Deutschland

Finnland

Frankreich

Griechenland

Irland

Island

Italien

Japan

Kanada

Korea

Luxemburg

Mexiko

Neuseeland

Niederlande

Norwegen

Österreich

Polen

Portugal

Schweden

Schweiz

Spanien

Tschechische Republik

Ungarn

Vereinigtes Königreich

Vereinigte Staaten
Länder, in denen die Erhebung 2002 abgeschlossen sein wird

Albanien

Argentinien

Bulgarien

Chile

China

Sonderverwaltungs-

region Hong-Kong

Indonesien

Israel

Litauen

Mazedonien

Peru

Rumänien

Thailand 
KAPITEL 1 Einführung in die Internationale Schulleistungsstudie PISA

\section{Kasten 1.1 PISA 2000 - eine international standardisierte Leistungsbeurteilung 15-jähriger Schülerinnen und Schüler}

\section{Stichprobenumfang}

- Im Jahr 2000 wurden mehr als eine Viertel Million Schülerinnen und Schüler getestet, stellvertretend für nahezu 17 Millionen 15-Jährige, die in den 32 Teilnehmerländern zur Schule gehen. Weitere 13 Länder werden die gleiche Erhebung 2002 durchführen.

\section{Inhalt}

- PISA 2000 erfasst drei Bereiche: Lesekompetenz, mathematische Grundbildung und naturwissenschaftliche Grundbildung.

- Im Mittelpunkt von PISA 2000 steht weniger die Frage, wie gut Jugendliche einen bestimmten schulischen Lehrstoff beherrschen, als vielmehr deren Fähigkeit, ihre Kenntnisse und Fertigkeiten zur Bewältigung realitätsnaher Herausforderungen einzusetzen.

- Das Hauptaugenmerk gilt der Beherrschung von Prozessen, dem Verständnis von Konzepten sowie der Fähigkeit, innerhalb des jeweiligen Bereichs mit verschiedenen Situationen umzugehen.

- 25 Länder erhoben im Rahmen einer internationalen Option Informationen über die Einstellungen der Schülerinnen und Schüler zum Lernen.

\section{Methoden}

- Im Rahmen von PISA 2000 wurden Papier-und-Bleistift-Tests durchgeführt, wobei die Testzeit für jeden Schüler zwei Stunden betrug.

- PISA 2000 verwendete sowohl Multiple-Choice-Aufgaben als auch Fragen, bei denen die Schülerinnen und Schüler eigene Antworten formulieren mussten. Die Aufgaben waren in der Regel in Testeinheiten zusammengefasst, die sich jeweils auf eine Beschreibung einer realitätsnahen Situation bezogen.

- Insgesamt wurden Aufgaben für eine Testdauer von rund sieben Stunden eingesetzt, wobei die einzelnen Schülerinnen und Schüler jeweils unterschiedliche Aufgabe-Kombinationen bearbeiteten.

- Die Schülerinnen und Schüler beantworteten einen Hintergrundfragebogen, dessen Bearbeitung etwa 30 Minuten in Anspruch nahm, und - soweit von dieser internationalen Option Gebrauch gemacht wurde - füllten sie auch Fragebogen mit Fragen zu Lernstrategien und zum Umgang mit dem Computer aus.

- Die Schulleitungen beantworteten einen Fragebogen mit Fragen zu ihrer Schule.

\section{Ergebnisse}

- Ein Profil der Kenntnisse und Fähigkeiten von 15-Jährigen.

- Kontextbezogene Indikatoren, mit denen ein Zusammenhang zwischen den Ergebnissen und den Merkmalen von Schülerinnen und Schülern und von Schulen hergestellt wird.

- Eine Wissensbasis für Analysen und Forschung im Bildungsbereich. 
- Trendindikatoren, die Veränderungen in den Ergebnissen über die Zeit hinweg andeuten, sobald Daten aus den folgenden PISA-Zyklen zur Verfügung stehen.

\section{Künftige Erhebungen}

- PISA wird in Dreijahreszyklen fortgesetzt. Im Jahr 2003 wird der Schwerpunkt auf Mathematik und im Jahr 2006 auf Naturwissenschaften liegen. Die Erhebung fächerübergreifender Kompetenzen wird Schritt für Schritt in PISA integriert, wobei 2003 zunächst Problemlösefähigkeiten getestet werden.

„Grundbildung“ (literacy) wird verwendet, um dieses breitere Konzept von Kenntnissen und Fertigkeiten abzudecken.

Die erste PISA-Erhebung wurde im Jahr 2000 in 32 Ländern (darunter 28 OECDLändern) durchgeführt, wobei schriftliche Aufgaben gestellt wurden, die in den Schulen unter Testbedingungen mit unabhängiger Aufsicht zu bearbeiten waren. Weitere 13 Länder werden die gleiche Erhebung 2002 abschließen (vgl. Abb. 1.1). PISA 2000 erfasst die Bereiche Lesekompetenz, mathematische Grundbildung und naturwissenschaftliche Grundbildung, wobei die Lesekompetenz im Mittelpunkt steht. In 25 Ländern wurden im Rahmen einer internationalen Option auch Daten über die Einstellung zum Lernen sowie Informationen über die Art und Weise ihres eigenständigen Lernens erhoben. Die Untersuchung wird alle drei Jahre wiederholt, wobei sich der Schwerpunkt 2003 auf mathematische Grundbildung, 2006 auf naturwissenschaftliche Grundbildung und 2009 dann wieder auf Lesekompetenz verlagert.

Dieser Bericht gibt einen Überblick über die Schülerleistungen und benutzt PISA für eine Analyse der für den schulischen Erfolg förderlichen Aspekte. Er beschreibt die jeweilige Leistungsverteilung in den einzelnen Ländern, beschränkt sich also nicht auf die Wiedergabe bloßer Durchschnittswerte. Darüber hinaus verwendet der Bericht Hintergrundinformationen über Schüler, Schulen und Bildungssysteme, um eine Reihe von Faktoren im Zusammenhang mit den unterschiedlichen Kompetenzstufen zu untersuchen. Indem sie Muster der Schülerleistungen in den jeweiligen Ländern in Verbindung mit Informationen über die Charakteristiken und Erfahrungen der Schülerinnen und Schüler aufzeigt, liefert die PISA-Studie ein effektives Instrument für ein besseres Verständnis dessen, was für den schulischen Erfolg förderlich ist. Im Weiteren befasst sich dieses Kapitel mit folgenden Punkten:

- von welcher Grundkonzeption PISA ausgeht,

- was mit PISA insgesamt und in jedem der drei Grundbildungsbereiche gemessen und nach welchen Methoden dabei vorgegangen wird,
PISA 2000 befasst sich besonders detailliert mit der Lesekompetenz. Im Rahmen eines langfristigen Zyklus verlagert sich der Schwerpunkt in PISA 2003 dann auf die mathematische Grundbildung, in PISA 2006 auf die naturwissenschaftliche Grundbildung usw.

Dieser Bericht gibt einen Überblick über die in PISA 2000 erfassten Schülerleistungen. 
KAPITEL 1 Einführung in die Internationale Schulleistungsstudie PISA

PISA ist ein wichtiges Kooperationsprojekt der Länder, das die Bildungspolitik verbessern kann,...
... indem es die Arbeiten der OECD über internationale Bildungsindikatoren dadurch ergänzt, dass fortan auch die Bildungserträge nachdrücklich und dauerhaft in den Mittelpunkt gerückt werden.
- wie die Ergebnisse interpretiert werden können und wie PISA zu einem besseren Verständnis von Bildung und lebensbegleitendem Lernen in einer Weise beitragen kann, die für die bildungspolitischen Entscheidungsträger in den jeweiligen Ländern relevant ist,

- wie PISA entwickelt wurde und

- wie der Bericht aufgebaut ist.

\section{Die Grundkonzeption von PISA}

PISA erfasst, welches Niveau 15-Jährige in den großen Industriestaaten in einem breiten Spektrum von Kenntnissen und Fähigkeiten erreichen. Bei der Entwicklung von PISA standen folgende Gesichtspunkte im Vordergrund:

- Politikorientierung mit dem Erfordernis, Design und Berichterstattungsmethoden an der Notwendigkeit auszurichten, den Regierungen Informationen an die Hand zu geben, aus denen Lehren für die Politik gezogen werden können;

- innovative Ausrichtung bei der Erfassung von grundlegenden Kompetenzen (literacy), nicht nur im Bereich der Lesekompetenz, sondern auch in naturwissenschaftlicher und in mathematischer Grundbildung;

- Konzentration auf den Nachweis von Kenntnissen und Fähigkeiten in einer Form, die für das tägliche Leben relevant ist;

- große geographische Reichweite mit 45 Teilnehmerländern, die ein Drittel der Weltbevölkerung repräsentieren;

- Regelmäßigkeit, die durch die Verpflichtung gewährleistet ist, die Erhebung alle drei Jahre zu wiederholen;

- kooperativer Charakter, mit gemeinsamer Steuerung des Projekts durch die Regierungen der Teilnehmerländer und einem Konsortium aus den auf diesem Gebiet weltweit führenden Forschungseinrichtungen, die mit den modernsten wissenschaftlichen Erkenntnissen arbeiten.

Die OECD-Mitgliedstaaten arbeiten im Rahmen von PISA gemeinsam daran, qualitativ bessere vergleichende Indikatoren für die Leistung von Bildungssystemen zu entwickeln. Die OECD veröffentlicht in ihrem jährlich erscheinenden Bericht Bildung auf einen Blick (z.B. OECD, 2001) eine Reihe von Indikatoren. Sie vermitteln Informationen über die für Bildung aufgewendeten Human- und Finanzressourcen, über die Funktionsweise undWeiterentwicklung von Bildungssystemen und Lernmethoden sowie über den individuellen, gesellschaftlichen und ökonomischen Nutzen von Bildungsinvestitionen. In der Vergangenheit fehlte es an regelmäßigen und verlässlichen Indikatoren für die Ergebnisse des Bildungswesens in den verschiedenen Ländern, insbesondere 
Indikatoren für Wissen und Fähigkeiten, was ein beträchtliches Manko bei den zur Verfügung stehenden Daten darstellte. Ohne solche Indikatoren haben Politiker, Steuerzahler, Pädagogen und Eltern kein Instrument an der Hand, um die Effektivität ihrer Bildungssysteme vergleichen und beurteilen zu können.

Deshalb hat die OECD gemeinsam mit ihren Mitgliedstaaten nach Möglichkeiten gesucht, die Kenntnisse und Fähigkeiten mit Hilfe international vergleichbarer Erhebungen direkt zu messen. Die zwischen 1994 und 1998 von Statistics Canada und der OECD gemeinsam durchgeführte Internationale Erhebung über Grad und Verteilung elementarer Grundqualifikationen Erwachsener (IALS) lieferte solche Leistungsmessungen für die erwachsene Bevölkerung. Durch die PISA-Studie kommen nun Leistungsmessungen für Schülerinnen und Schüler im Hinblick auf eine eigenverantwortliche Lebensgestaltung hinzu.

Um die Vergleichbarkeit der Ergebnisse zu gewährleisten, muss PISA in vergleichbaren Zielpopulationen erhoben werden. Auf Grund länderspezifischer Unterschiede bei Art und Verbreitung von Einrichtungen des Elementarbereichs, dem regulären Einschulungsalter sowie der Struktur des Bildungssystems sind Vergleiche, die sich auf bestimmte Klassenstufen beschränken, für internationale Vergleiche ungeeignet. Für valide internationale Vergleiche von Schulleistungen müssen vielmehr die Populationen in Bezug auf ein Zielalter definiert werden. PISA bezieht sich auf alle Schülerinnen und Schüler, die zum Zeitpunkt der Erhebung im Alter von 15 Jahren und 3 Monaten bis zu 16 Jahren und 2 Monaten waren, gleichgültig welche Klasse oder Art von Bildungseinrichtung sie besuchten und unabhängig davon, ob es sich um eine Ganztags- oder Halbtagsschule handelte. Bei PISA ausgeklammert sind 15-Jährige, die nicht mehr zur Schule gehen. In diesem Bericht wird diese Population der Einfachheit halber mit dem Begriff „15-Jährige“ bezeichnet. Mit Ausnahme Brasiliens, Luxemburgs und Polens wurden mindestens 95\% dieser Zielpopulation von den aktuellen PISAStichproben abgedeckt, und für die Mehrzahl der Länder beträgt dieser Anteil über 97\% (für weitere Informationen zur Definition der PISA-Population und zum Stichprobenumfang vgl. Anhang A.3). Dieser hohe Erfassungsgrad trägt erheblich mit zur Vergleichbarkeit der Ergebnisse bei.

Daher ist dieser Bericht in der Lage, Aussagen über die Kenntnisse und Fähigkeiten von Personen zu machen, die im gleichen Jahr geboren sind und im Alter von 15 Jahren noch zur Schule gehen, die aber über unterschiedliche inner- wie außerschulische Bildungserfahrungen verfügen. Die Zahl der unterschiedlichen Klassenstufen, in denen sich diese Schülerinnen und Schüler befinden, wird durch die jeweilige nationale Politik in Bezug auf Einschulung und Versetzung bestimmt. Zudem sind die Schülerinnen und Schüler der PISA-Zielpopulation in einigen Ländern unterschiedlichen Bildungssystemen, Bildungsgängen oder -zweigen zugeordnet.
Bei der untersuchten Population handelt es sich um 15-jährige Schülerinnen und Schüler in allen schulformen,...

... so dass

länderübergreifende

vergleiche der

Auswirkungen

unterschiedlicher

Bildungserfahrungen

möglich sind. 
KAPITEL 1 Einführung in die Internationale Schulleistungsstudie PISA

\section{Was in den einzelnen Bereichen gemessen wird}

PISA misst die Lesekompetenz wie auch die mathematische und naturwissenschaftliche

Grundbildung auf kontinuierlichen Skalen, statt einfach eine Zweiteilung vorzunehmen in Personen mit und ohne Grundqualifikationen.

Der Erwerb von Grundqualifikationen ist ein lebenslanger Prozess. PISA untersucht deshalb, ob die Schülerinnen und Schüler zur Fortsetzung dieses Lernprozesses in der Lage sind, ...

... und ob sie mit dem gelernten Wissen in der realen Welt etwas anfangen können.
Internationale Experten aus OECD-Ländern haben zunächst jeden der drei in PISA 2000 untersuchten Grundbildungsbereiche - Leseverständnis, Naturwissenschaften und Mathematik - definiert und sodann eine Rahmenkonzeption für die Erfassung der Leistungen in diesen Bereichen erstellt (OECD, 1999a). Das PISA-Konzept der Grundbildungsbereiche (literacy) ist viel weiter gefasst als die herkömmliche Auffassung, wonach hierunter die Fähigkeit des Lesens und Schreibens zu verstehen ist. Grundkompetenzen werden in Form eines Kontinuums gemessen, nicht als etwas, das man hat oder nicht hat. Für manche Zwecke mag es notwendig oder wünschenswert sein, einen Grenzwert auf einem solchen Kontinuum zu bestimmen, unterhalb dessen ein Kompetenzniveau als unzulänglich anzusehen ist, doch die zugrunde liegende Variationsbreite ist wichtig. Eine mit Grundqualifikationen ausgestattete Person verfügt über eine Reihe von Fähigkeiten. Es ist nicht möglich, eine genau definierbare Trennlinie zwischen solchen Personen zu ziehen, die über voll ausgebildete Grundkompetenzen verfügen, und anderen, die derartige Kompetenzen nicht besitzen.

Der Erwerb von Grundqualifikationen ist ein lebenslanger Prozess, der nicht nur in der Schule oder innerhalb formaler Lernstrukturen stattfindet, sondern auch durch Interaktionen mit Peers, Kollegen und größeren Gemeinschaften bestimmt wird. Von 15-Jährigen kann nicht erwartet werden, dass sie bereits alles gelernt haben, was sie in ihrem späteren Leben als Erwachsene brauchen. Sie benötigen aber ein solides Fundament an Kenntnissen in Bereichen wie Lesen, Mathematik und Naturwissenschaften. Um in diesen Bereichen mehr hinzuzulernen und mit dem gelernten Wissen in der realen Welt etwas anfangen zu können, müssen sie auch gewisse elementare Prozesse und Grundsätze verstehen und fähig sein, diese flexibel in unterschiedlichen Situationen anzuwenden. Deshalb zielt PISA nicht darauf ab, spezifisches Wissen abzufragen, sondern die Fähigkeit zu bewerten, mit Hilfe eines allgemeinen Verständnisses von bestimmten Schlüsselkonzepten Aufgaben in realitätsnahen Situationen zu bewältigen.

Neben der Erhebung der Kompetenzen in den drei Grundbildungsbereichen wird PISA nach und nach auch fächerübergreifende Kompetenzen untersuchen. Im Rahmen von PISA 2000 wurden die Motivation der Schülerinnen und Schüler, andere Aspekte ihrer Einstellung zum Lernen, ihr Umgang mit dem Computer sowie Aspekte der von den Schülerinnen und Schülern angewandten Strategien zur Steuerung und Überwachung ihres eigenen Lernprozesses erfasst. In den folgenden PISA-Erhebungen werden weitere „fächerübergreifende Kompetenzen“, wie Problemlösefähigkeit und Fertigkeiten in Informationstechnologien, eine zunehmend große Rolle spielen.

Inwieweit kann PISA die „Fähigkeiten für das Leben“ wirklich messen? Bei der Beantwortung dieser Frage wird nicht allein von subjektiven Beurteilungen 
dessen, was im Leben wichtig ist, ausgegangen, sondern es wird auch berücksichtigt, ob Personen, die über eine größere Kompetenz in den von PISA gemessenen Bereichen verfügen, tatsächlich größere Erfolgschancen im Leben haben. Wenn sich auch heute über das künftige Abschneiden der an PISA teilnehmenden Schülerinnen und Schüler noch nichts sagen lässt, so zeigt die Internationale Erhebung über Grad und Verteilung elementarer Grundqualifikationen Erwachsener (IALS) doch, dass bei den Erwachsenen unabhängig vom Einfluss des Bildungsabschlusses auch ein enger Zusammenhang zwischen Grundkompetenzen in den Bereichen Leseverständnis und Mathematik und den Arbeitsmarkt- undVerdienstchancen der Betreffenden besteht (vgl. Kasten 1.2).

Die von PISA untersuchten Bereiche werden definiert nach:

- dem Inhalt oder der Struktur des Wissens, das die Schülerinnen und Schüler im jeweiligen Bereich erwerben müssen (z.B. Vertrautheit mit naturwissenschaftlichen Konzepten oder verschiedenen Texttypen);

- den Prozessen, die ausgeführt werden müssen (z.B. Auffinden schriftlicher Informationen in einem Text); und

- dem Kontext, in dem Kenntnisse und Fähigkeiten angewendet werden (z.B. zwecks Entscheidungen im persönlichen Leben oder zum Verständnis des Weltgeschehen).

Mit Hilfe der für PISA entwickelten Instrumente sollen das Wissen und die Fähigkeiten der Schülerinnen und Schüler in jedem der drei Grundbildungsbereiche erfasst werden. Um nach und nach das Verständnis für die einzelnen Bereiche zu vertiefen, wird jedoch in jedem Zyklus einer der Bereiche besonders betont. Bei PISA 2000 stand die Lesekompetenz im Mittelpunkt, worauf dann auch zwei Drittel der Testzeit verwendet wurden. Daher werden in diesem Bericht größtenteils die Ergebnisse von PISA 2000 im Bereich Lesekompetenz erörtert. Für die beiden anderen Bereiche werden zusammenfassende Leistungsprofile erstellt. Im Jahr 2003 wird sich PISA eingehender mit der mathematischen Grundbildung befassen und im Jahr 2006 mit der naturwissenschaftlichen Grundbildung.
Die Testaufgaben sind nach Inhalten, Prozessen und Kontexten definiert. 
KAPITEL 1 Einführung in die Internationale Schulleistungsstudie PISA

\section{Kasten 1.2 Verbessert höhere Lesekompetenz die Beschäftigungsaussichten?}

Die Internationale Erhebung über Grad und Verteilung elementarer Grundqualifikationen Erwachsener (IALS) stellte fest, dass Personen mit höherer Lesekompetenz größere Chancen haben, eine Beschäftigung zu finden und ein höheres Durchschnittsgehalt zu erzielen, als solche mit geringeren Fähigkeiten in diesem Bereich (OECD und Statistics Canada, 2000). Liegt dies allein daran, dass sie ein höheres Bildungsniveau besitzen? Wenn dem so wäre, würde IALS (und PISA) höchstens Kompetenzen messen, die den Einzelnen zu einem besseren Bildungsabschluss und dadurch zu einem besseren Arbeitsplatz verhelfen. Bei IALS erreichten Erwachsene mit einem wie immer gearteten tertiären Bildungsabschluss im Bereich Lesekompetenz im Durchschnitt eine Punktzahl, die um ein bis zwei Kompetenzstufen höher lag als die von Personen ohne Sekundarschulabschluss, es gab aber in den 22 Teilnehmerstaaten eine beträchtliche Zahl von Erwachsenen mit hoher Lesekompetenz und niedrigem Bildungsabschluss, oder umgekehrt. Die wichtigste Erkenntnis lautet, dass die Zukunftsaussichten einer Person auf dem Arbeitsmarkt über den Bildungsabschluss hinaus auch durch das Niveau der Lesekompetenz bestimmt werden.

\section{Abbildung 1.2}

Schulabschluss, Lesekompetenz und die Wahrscheinlichkeit für die Ausübung einer anspruchsvollen Angestelltentätigkeit

Wahrscheinlichkeit einer Anstellung in einer anspruchsvollen Angestelltentätigkeit nach Schulabschluss und steigender Lesekompetenz für alle Länder insgesamt, IALS Prosaskala, Altersgruppe 26-35 Jahre, 1994-1998

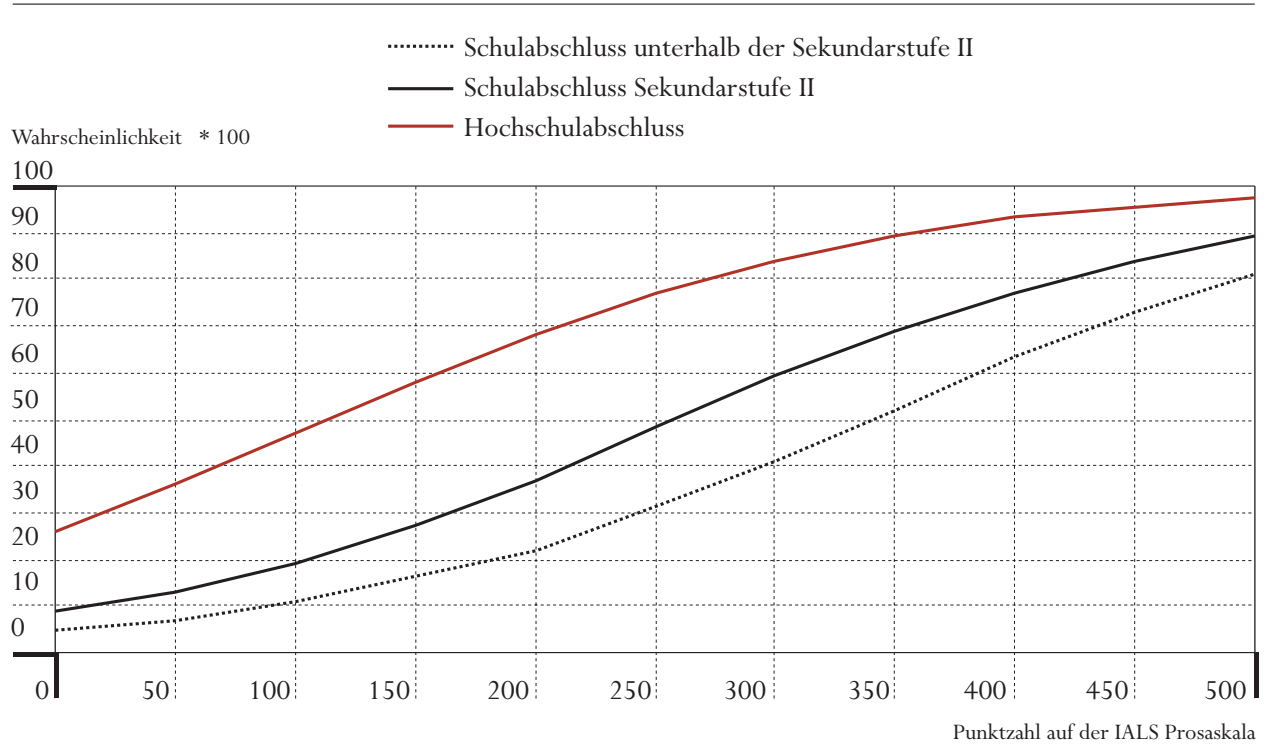

Quelle: OECD und Statistics Canada (2000). 
Abbildung 1.2 verdeutlicht dies, indem sie aufzeigt, wie groß die Wahrscheinlichkeit für Jugendliche mit unterschiedlichen Kombinationen von Lesekompetenz und Bildungsabschlüssen ist, einen Arbeitsplatz als hochqualifizierte Angestellte zu erhalten. Die Abstände zwischen den Kurven veranschaulichen die Effekte eines zunehmenden Bildungsniveaus; der Verlauf der Kurven zeigt den Effekt höherer Lesekompetenz bei einem gegebenen Bildungsniveau. Bei Personen im Alter von 26 bis 35 Jahren mit einer Beschäftigung im Unternehmenssektor nimmt die Wahrscheinlichkeit, eine anspruchsvolle Angestelltentätigkeit auszuüben, mit steigender Lesekompetenz rasch zu. Der unabhängige Effekt der Lesekompetenz auf die Arbeitsmarktergebnisse ist vergleichbar mit dem unabhängigen Effekt des Bildungsabschlusses. Für jemand mit mittlerem Bildungsabschluss (nicht über Sekundarstufe II hinausgehend) stehen die Chancen, einen anspruchsvollen Arbeitsplatz zu finden, bei einem Niveau der Lesekompetenz von 200 (unteres Ende der Skala) bei zwei zu fünf und bei einem Niveau von 400 (hoher Wert) bei vier zu fünf. Umgekehrt stehen die Chancen für eine Person mit mittlerem Niveau im Bereich Lesekompetenz (ein Wert von 300), eine solche Stelle zu erhalten, bei zwei zu fünf, wenn sie einen niedrigen Schulabschluss (nicht mehr als Sekundarstufe I) besitzt, und bei mehr als vier zu fünf, wenn sie über einen höheren Bildungsabschluss (Hochschulniveau) verfügt.

Quelle: OECD und Statistics Canada (2000).

\section{Lesekompetenz im Rahmen von PISA}

Lesekompetenz (reading literacy) ist im Rahmen von PISA definiert als die Fähigkeit, geschriebene Texte zu verstehen, zu nutzen und über sie zu reflektieren, um eigene Ziele zu erreichen, das eigene Wissen und Potential weiterzuentwickeln und aktiv am gesellschaftlichen Leben teilzunehmen. Diese Definition geht über die Vorstellung hinaus, wonach Lesekompetenz in dem Entschlüsseln und wörtlichen Verständnis vonTexten besteht. Zum Lesen gehört auch das Verstehen von Texten und das Nachdenken darüber. Lesekompetenz beinhaltet die Fähigkeit des Einzelnen, schriftliche Informationen so zu nutzen, dass diese seinen jeweiligen Zielen dienen, sowie die entsprechende Fähigkeit komplexer moderner Gesellschaften, schriftliche Informationen so zu nutzen, dass ihre gute Funktionsweise gewährleistet ist. In PISA 2000 wurden rund 140 Aufgaben eingesetzt, die der Art von Lesekompetenz entsprechen, die 15-Jährige wahrscheinlich in ihrem künftigen Leben benötigen werden. Beispiele für die in PISA verwendeten Aufgaben zur Messung der Lesekompetenz sind in Kapitel 2 sowie unter www.pisa.oecd.org zu finden.

Leser gehen auf ganz unterschiedliche Weise an einen gegebenen Text heran, wenn sie das Gelesene zu nutzen und zu verstehen suchen. Dieser dynamische Prozess hat viele Dimensionen, von denen drei herangezogen wurden, um die Erhebungsinstrumente für PISA zu konstruieren. Bereich Lesekompetenz...

PISA definiert Lesekompetenz als die Fähigkeit, geschriebene Texte zu verstehen, zu nutzen und zu bewerten. 
KAPITEL 1 Einführung in die Internationale Schulleistungsstudie PISA

... basieren auf einer Vielzahl von Textformen,

zu denen nicht nur

prosatexte zählen.

Sie verlangen von den

Schülerinnen und

Schülern, Informationen

in einem Text zu

ermitteln, zu verstehen

und darüber zu

reflektieren, ...

.. und sie auf eine

Vielzahl von Situationen

zu beziehen, in denen

der Einzelne Texten

begegnet.

PISA definiert die mathematische

Grundbildung als die Fähigkeit, in bestimmten

Lebenssituationen mathematische Probleme

zu erkennen und zu

lösen.
- Die Art des Lesestoffs oderTextes. Viele bisherige Studien zur Lesekompetenz haben sich auf Prosatexte mit Sätzen und Absätzen bzw. „fortlaufende Texttypen“ konzentriert. Die PISA-Tests enthalten ebenfalls zusammenhängende Prosatexte, wobei zwischen einer Reihe von Prosaformen wie Erzählung, Darlegung und Argumentation unterschieden wird. Zusätzlich bezieht die PISA-Studie jedoch auch „nicht-kontinuierliche Texte“ ein, in denen die Informationen auf eine andere Art und Weise dargestellt sind, z.B. Listen, Formulare, Graphiken und Diagramme. Diese Vielfalt basiert auf der Prämisse, dass jeder Einzelne in der Schule und im Erwachsenenleben verschiedensten Formen von Texten begegnet, die unterschiedliche Techniken der Informationsverarbeitung verlangen. Ein effizienter Leser zeichnet sich durch Flexibilität bzw. die Fähigkeit aus, die für den jeweiligen Texttyp geeignete Technik zur Lokalisierung relevanter Informationen zu wählen.

- Die Art der Leseaufgabe. Sie wird einerseits bestimmt durch die kognitiven Fähigkeiten, die gute Leser benötigen, und andererseits durch die Merkmale der gestellten Fragen. Bei PISA liegt das Gewicht mehr auf „Lesen, um zu Lernen“ als auf „Lesenlernen“. Daher werden grundlegende Lesefertigkeiten bei den Schülern nicht erfasst; es wird davon ausgegangen, dass die meisten 15-Jährigen lesen können. Es wird vielmehr untersucht, inwieweit sie in der Lage sind, Informationen aus Texten zu ermitteln, ein allgemeines Textverständnis zu entwickeln, die Texte zu interpretieren, über Inhalt und Form von Texten unter Bezugnahme auf ihr eigenes Weltwissen kritisch zu reflektieren und ihren eigenen Standpunkt darzulegen und zu begründen.

- Die Art des Gebrauchs, für den der Text geschrieben wurde, d.h. der Kontext oder die Situation, in der er typischerweise gelesen wird. So werden z.B. Romane, persönliche Briefe oder Biographien für den „privaten“ Gebrauch geschrieben. Amtliche Dokumente oder Bekanntmachungen sind für den „öffentlichen“ Gebrauch bestimmt. Handbücher oder Berichte sind für den „beruflichen“ Gebrauch und Lehrbücher oder Arbeitsblätter für den „bildungsbezogenen“ Gebrauch gedacht.

\section{Mathematische Grundbildung im Rahmen von PISA}

Mathematische Grundbildung (mathematical literacy) im Rahmen von PISA ist definiert als die Fähigkeit, mathematische Probleme zu identifizieren, $\mathrm{zu}$ verstehen und sich mit ihnen $\mathrm{zu}$ befassen und fundierte Urteile über die Rolle abzugeben, die die Mathematik im gegenwärtigen und künftigen Privatleben der Betreffenden, im Berufsleben, im sozialen Kontakt mit Peers und Verwandten und im Leben dieser Person als konstruktivem, engagiertem und reflektierendem Bürger spielt. Ebenso wie bei der Lesekompetenz erstreckt sich die Definition nicht allein auf mechanische Operationen, sondern auf allgemeine Anwendungen im Leben des Einzelnen. Der Begriff „mathematische Grundbildung" bezeichnet in dem hier verwendeten Sinn die Fähigkeit, mathematische Kenntnisse und Kompetenzen funktionell zu nutzen, und weniger die Beherrschung des im Curriculum vorgesehenen Lehrstoffs. Der Ausdruck, 
sich mit Mathematik „befassen“, beinhaltet nicht nur soziale Handlungen im engeren Sinne (wie z.B. die Entscheidung, wie viel Wechselgeld in einem Geschäft herauszugeben ist), sondern auch allgemeinere Anwendungen, wie die Vertretung eines Standpunkts oder die Einschätzung eines mathematisch ausgedrückten Sachverhalts (z.B. Stellungnahme zu staatlichen Ausgabenplänen). Zur mathematischen Grundbildung gehört auch die Fähigkeit, mathematische Probleme in ganz unterschiedlichen Situationen zu stellen und zu lösen, sowie die Neigung, dies zu tun, was häufig von persönlichen Charakterzügen wie Selbstvertrauen und Neugierde abhängt.

Um diese Definition in Erhebungsinstrumente mathematischer Grundkenntnisse umzusetzen, wurden drei Dimensionen für PISA 2000 bestimmt:

- Mathematische Inhalte. Diese werden vorwiegend definiert als Cluster relevanter, miteinander vernetzter mathematischer Leitideen, wie sie in realen Situationen und Kontexten anzutreffen sind. Dazu zählen quantitatives Denken, Raum und Form, Veränderung und funktionale Abhängigkeiten sowie Zufall und Wahrscheinlichkeit. Die Wahl dieser Bereiche bedeutet nicht, dass die curricular definierten Stoffgebiete der Mathematik, wie Arithmetik, Algebra und Geometrie, außer Acht gelassen wurden. PISA 2000 setzte Aufgaben ein, die voraussetzen, dass die Schülerinnen und Schüler einen ausgewogenen mathematischen Lehrplan absolviert haben. Da Mathematik bei PISA 2000 nur eine Nebenkomponente darstellt, wurde dieser Bereich in der Erhebung noch einmal reduziert auf die Leitideen Veränderung und funktionale Abhängigkeiten sowie Raum und Form. Diese Bereiche wurden ausgewählt, um eine möglichst breite Berücksichtigung curricularer Stoffgebiete zu gewährleisten und eine Überbetonung von Rechenfertigkeiten zu vermeiden.

- Mathematische Prozesse. Die in der PISA-Erhebung gestellten Fragen sind durch verschiedene Denkfähigkeiten bestimmt, die für Mathematik benötigt werden. Solche Fähigkeiten lassen sich drei „Kompetenzklassen“ zuordnen: Zur ersten Kompetenzklasse Wiedergabe von Fakten und Routineverfahren gehören einfache Berechnungen oder Definitionen, wie sie in herkömmlichen Mathematiktests häufig vorkommen, die zweite Kompetenzklasse Herstellen von Zusammenhängen verlangt, mathematische Ideen und Verfahren zu integrieren, um einfache und mehr oder weniger vertraute Probleme zu lösen, und die dritte Kompetenzklasse mathematisches Denken erfordert mathematische Überlegungen, Verallgemeinerung und Verstehen der Zusammenhänge, wobei die Schülerinnen und Schüler analysieren, die mathematischen Aspekte einer Situation erkennen und Problemstellungen eigenständig formulieren müssen.

- Situationen, in denen Mathematik angewendet wird. Zur Erfassung mathematischer Grundbildung werden den Schülerinnen und Schülern „authentische“ Aufgaben gestellt, die von Situationen ausgehen, die zwar gelegentlich fiktiv sein mögen, aber doch die Art von Problemen repräsentieren,
Die PISA-Aufgaben zur mathematischen Grundbildung verlangen, dass die Schülerinnen und schüler mit den mathematischen schlüsselkonzepten vertraut sind,...
... und zwar in verschiedenen Situationen des täglichen Lebens.

\section{... mathematische Routineverfahren reproduzieren, Zusammenhänge herstellen können und zu mathematischem Denken im weiteren sinne fähig sind, ....}


KAPITEL 1 Einführung in die Internationale Schulleistungsstudie PISA

\section{PISA definiert die naturwissenschaftliche Grundbildung als die Fähigkeit zu naturwissenschaftlichem Denken,... \\ ... wobei davon ausgegangen wird, dass nicht nur eine \\ Minderheit, sondern vielmehr die großse Mehrheit über diese Fähigkeit verfügen muss.}

Die PISA-Aufgaben zur naturwissenschaftlichen

Grundbildung setzen voraus, dass die Schülerinnen und schüler bestimmte naturwissenschaftliche schlüsselkonzepte verstehen ... mit denen sie im wirklichen Leben konfrontiert werden. Die Situationen unterscheiden sich hinsichtlich der „Distanz“ zu den Schülerinnen und Schülern, und sie reichen von Kontexten, die die Einzelnen direkt betreffen (wenn z.B. zu entscheiden ist, ob bei einem Kauf das Preis-Leistungs-Verhältnis stimmt), bis hin zu wissenschaftlichen Problemen von allgemeinerem Interesse. Im Hinblick auf ihre Nähe zu den Schülerinnen und Schülern wurden die Situationen in folgende Kategorien eingeteilt: Privatleben/persönliches Leben, Schulleben, Arbeit und Sport, lokale Gemeinschaft und Gesellschaft undWissenschaft

\section{Naturwissenschaftliche Grundbildung im Rahmen von PISA}

Naturwissenschaftliche Grundbildung (scientific literacy) beinhaltet die Fähigkeit zu naturwissenschaftlichem Denken in einer Welt, in der Naturwissenschaft und Technologie das Leben prägen. Solche Grundkenntnisse setzen ein Verständnis naturwissenschaftlicher Konzepte sowie die Fähigkeit voraus, eine naturwissenschaftliche Perspektive anzuwenden. Im Rahmen von PISA wird naturwissenschaftliche Grundbildung als die Fähigkeit definiert, naturwissenschaftliches Wissen anzuwenden, naturwissenschaftliche Fragen zu erkennen und aus Belegen Schlussfolgerungen zu ziehen, um Entscheidungen zu verstehen und zu treffen, die die natürliche Welt und die durch menschliches Handeln an ihr vorgenommenen Veränderungen betreffen.

Das Erlangen naturwissenschaftlicher Grundbildung wird für alle 15-jährigen Schüler als ein ganz wesentliches Bildungsziel angesehen, unabhängig davon, ob sie ein naturwissenschaftliches Studium anstreben oder nicht. Naturwissenschaftliches Denken wird von allen Bürgern und nicht nur von Naturwissenschaftlern verlangt. Die Einbeziehung naturwissenschaftlicher Grundbildung als eine allgemeine für das Leben wichtige Kompetenz reflektiert die immer zentralere Bedeutung naturwissenschaftlicher und technologischer Fragen. Die für PISA verwendete Definition bedeutet nicht, dass die Erwachsenen von morgen über eine breite Basis an naturwissenschaftlichen Kenntnissen verfügen müssen. Worauf es ankommt, ist die Fähigkeit, naturwissenschaftliche Überlegungen über Befunde anzustellen, auf die sie stoßen werden. PISA 2000 umfasst drei Dimensionen naturwissenschaftlicher Grundbildung:

- Naturwissenschaftliche Konzepte. Die Schülerinnen und Schüler müssen eine Reihe von Schlüsselkonzepten begreifen, um bestimmte Phänomene der natürlichen Umwelt und der durch menschliches Handeln an ihr vorgenommenen Veränderungen zu verstehen. Das sind die übergreifenden Ideen, mit deren Hilfe gewisse Aspekte der physikalischen Umwelt erklärt werden können. Bei den im Rahmen von PISA gestellten Fragen werden Konzepte aus Physik, Chemie, Biologie und Geowissenschaften miteinander kombiniert. Genauer gesagt stammen die Konzepte aus einer Reihe von thematischen Bereichen, darunter Artenvielfalt, Kräfte und Bewegung sowie physiologische Veränderungen. 
- Naturwissenschaftliche Prozesse. PISA erhebt die Fähigkeit zur konkreten Anwendung naturwissenschaftlicher Kenntnisse und deren Verständnis, namentlich die Fähigkeit der Schülerinnen und Schüler, Nachweise zu erbringen, $\mathrm{zu}$ interpretieren und den Implikationen entsprechend $\mathrm{zu}$ handeln. PISA untersucht fünf solcher Prozesse: naturwissenschaftliche Fragestellungen erkennen, Nachweise identifizieren, Schlussfolgerungen ziehen, Schlussfolgerungen kommunizieren und Verständnis für naturwissenschaftliche Konzepte zeigen.

- Naturwissenschaftliche Anwendungssituationen und Anwendungsbereiche. Im PISA-Kontext beinhaltet naturwissenschaftliche Grundbildung weniger die Anwendung der Naturwissenschaften in einem Klassenzimmer oder Labor als vielmehr im täglichen Leben. Ebenso wie in den anderen Grundbildungsbereichen umfasst der Kontext daher Situationen aus dem Alltagsleben und Angelegenheiten von direktem persönlichen Interesse. Die Fragen für PISA 2000 wurden in drei naturwissenschaftliche Anwendungsbereiche untergliedert: Leben und Gesundheit, Erde und Umwelt sowie Technologie.

\section{Ablauf und Durchführung der Datenerhebung im Rahmen von PISA}

PISA 2000 wurde von einem internationalen Netzwerk führender Institutionen und Experten sorgfältig unter dem Gesichtspunkt konzipiert, dass die Studie den oben dargelegten Zwecken gerecht wurde. Alle ausgewählten Schülerinnen und Schüler nahmen in ihrer eigenen Schule an einem schriftlichen zweistündigen Test teil, und sie verbrachten etwa 30 Minuten damit, einen Fragebogen mit Angaben zu ihrer Person auszufüllen. Die Schulleitungen wurden in einem anderen 30-minütigen Fragebogen um weitere Informationen zu Schulmerkmalen gebeten.

Die Erfassung der Schülerleistungen erfolgte in allen drei Bereichen nach den gleichen Grundsätzen, und das wird auch bei den späteren Erhebungen der Fall sein, obwohl der Umfang des Erhebungsmaterials in den jeweiligen Gegenstandsbereichen in jedem Dreijahreszyklus verschieden ausfallen wird. Bei PISA 2000, wo Lesekompetenz den Hauptbereich bildet, wurden die Tests auf folgende Weise durchgeführt (wegen Einzelheiten siehe den PISA 2000 Technical Report):

- Ein breites Spektrum von Testaufgaben: Die Tests im Rahmen von PISA 2000 wurden in schriftlicher Form durchgeführt, die Aufgaben hatten unterschiedliche Formate. Die Schülerinnen und Schüler mussten Überlegungen über Texte und Diagramme anstellen und eine Reihe von Fragen hierzu beantworten. Die meisten Testaufgaben waren so konzipiert, dass sie Aufschluss darüber geben konnten, ob die Schülerinnen und Schüler zu Reflektion und zu aktivem Denken in dem betreffenden Bereich fähig waren. Beispiele für diese Aufgaben sind in den Kapiteln 2 und 3 zu finden.

- Eine weite Erfassung des jeweiligen Grundbildungsbereichs. Für jeden Schüler betrug die Testzeit zwei Stunden, aber nicht allen Schülern wurden

\author{
... und zeigen, dass sie \\ in der Lage sind, \\ Nachweise zu erbringen, \\ zu interpretieren und \\ den Implikationen \\ entsprechend zu \\ handeln,....
}

Im Rahmen von PISA

wurden die Schülerinnen

und Schüler einem

zweistündigen Test

unterzogen und hatten

ebenso wie die

Schulleitungen einen

Fragebogen auszufüllen.

Die Tests umfassen viele unterschiedliche

Aufgabenarten ...

... mit breit gefächertem Inhalt. 
KAPITEL 1 Einführung in die Internationale Schulleistungsstudie PISA

\section{Durch besonders gründliche \\ verfahrensweisen wurde sichergestellt, dass die Testaufgaben länderübergreifend valide sind, ... \\ ... und es wurden strenge Vorkehrungen getroffen, um die Tests in allen Ländern auf die gleiche Weise durchzuführen.}

Bei ungefähr der Hälfte der Fragen zur

Lesekompetenz mussten die Schülerinnen und Schüler eigene Antworten ausarbeiten, ... dieselben Testaufgaben vorgelegt. Um alle Bereiche abzudecken, wurde eine Reihe von Aufgaben ausgewählt, die einerTestzeit von sieben Stunden entsprachen. Diese wurden in unterschiedlichen Kombinationen in neun verschiedenen Testheften aufgeteilt. Jede Aufgabe tauchte in mehreren Testheften auf, womit sichergestellt wurde, dass jede einzelne Aufgabe von einer repräsentativen Schülerstichprobe beantwortet wurde. Jeder Schüler erhielt ein Testheft.

\section{- Zusammenarbeit aller Teilnehmerländer bei der Entwicklung international valider Tests. Auf der Basis der international vereinbarten Rahmenkonzeption und der Testspezifikationen entwickelten die Länder Testaufgaben, die von Spezialisten für die jeweiligen Fächer und von Experten auf dem Gebiet der Schulleistungsmessung überprüft wurden. Um zu gewährleisten, dass alle in der Rahmenkonzeption aufgeführten Bereiche angemessen abgedeckt sind, wurden zusätzliche Testaufgaben erstellt. Die Aufgaben wurden Pilottests unterzogen, die Ergebnisse wurden überprüft und die überarbeitete Aufgabensammlung wurde dann in einem Feldtest erprobt. Um schließlich die Validität sämtlicher Aufgaben in allen Ländern, Sprachen und Kulturen sicherzustellen, wurden die Aufgaben von den Teilnehmerländern ihrer kulturellen Eignung, ihrer curricularen und außercurricularen Relevanz sowie der Angemessenheit ihres Schwierigkeitsgrads nach eingestuft.}

- Standardisierte Verfahren für dieVorbereitung und Durchführung der Tests. Im Rahmen von PISA wurden beispiellose Anstrengungen unternommen, um die Vergleichbarkeit der Ergebnisse für die verschiedenen Länder, Kulturen und Sprachen sicherzustellen. Neben der Gewährleistung einer breiten Erfassung der 15-jährigen Schülerinnen und Schüler in allen Ländern erstreckten sich diese Anstrengungen auch auf die Zusammenarbeit mit zahlreichen Experten in sämtlichen Teilnehmerstaaten, auf die Entwicklung standardisierter Verfahren für die Vorbereitung und Durchführung der Erhebung sowie auf eine strenge Qualitätskontrolle über den gesamten Untersuchungszeitraum. Die Instrumente wurden auf Englisch und Französisch vorbereitet und dann mittels Verfahren, die die linguistische Integrität und Äquivalenz der Instrumente sicherstellen, in die Sprachen der Teilnehmerländer übersetzt. Für nicht englisch- bzw. französischsprachige Länder wurden zwei voneinander unabhängige Übersetzungen der Instrumente angefertigt, die anschließend - in den meisten Fällen unter Heranziehung beider Ausgangsversionen - zu einem Text zusammengefasst wurden. Weitere Informationen über die PISA-Standards und -Verfahren enthält Anhang A.3-A.7.

Die Lesekompetenz wurde anhand einer Reihe von Texten getestet, wobei die Schülerinnen und Schüler für jeden Text eine Reihe von Aufgaben auszuführen hatten. Bei 45\% der Aufgaben mussten die Schülerinnen und Schüler eigene Antworten ausarbeiten, wobei sie entweder eine kurze freie Antwort aus einer ganzen Reihe von möglichen Antworten geben oder eine ausführlichere freie Antwort formulieren konnten, um der Möglichkeit divergierender individueller Antworten und eventuell gegensätzlichen Auffassungen Rechnung zu tragen. Bei 
Aufgaben mit ausführlichen Antworten wurde von den Schülerinnen und Schülern gewöhnlich verlangt, die in dem betreffenden Text enthaltenen Informationen und Ideen zu ihren eigenen Erfahrungen oder Meinungen in Beziehung zu setzen, wobei die Bewertung ihrer Antwort weniger von dem jeweils vertretenen Standpunkt abhing als vielmehr von der Fähigkeit, diesen Standpunkt unter Hinweis auf das Gelesene zu begründen und zu erläutern. Für teilweise richtige oder weniger ausgefeilte Antworten waren abgestufte Punktwerte vorgesehen und alle derartigen Aufgaben wurden per Hand kodiert. Bei weiteren 45\% der Aufgaben handelte es sich um Multiple-Choice-Aufgaben, bei denen die Schülerinnen und Schüler entweder aus vier oder fünf vorgegebenen Alternativen eine auswählen oder zu jeder Frage ein Wort oder einen kurzen Satz (z.B. „ja“ oder „nein“) einkreisen mussten. Die verbleibenden 10\% der Aufgaben erforderten von den Schülerinnen und Schülern, dass sie ihre eigene Antwort formulierten, wobei allerdings nur eine begrenzte Anzahl von Antworten als richtig eingestuft wurde.

Die mathematische Grundbildung wurde anhand einer Kombination verschiedener Arten von Fragen getestet. Wie bei der Lesekompetenz gab es eine Reihe von Testeinheiten, die sich jeweils auf eine Situation oder ein Problem bezogen, zu denen den Schülerinnen und Schülern dann verschiedene Aufgaben gestellt wurden. Einleitend zu jeder Einheit wurden verschiedene Kombinationen von Diagrammen und schriftlichen Informationen gegeben. Etwa zwei Drittel der Aufgaben waren so gestellt, dass die Lösung eindeutig als richtig oder falsch kodiert werden konnte. Die Schülerinnen und Schüler stellten ihr Leistungsniveau unter Beweis, indem sie eine korrekte Antwort auf eine Frage gaben, und zeigten, ob sie die der Aufgabe zu Grunde liegenden mathematischen Grundsätze verstanden hatten. Bei komplexeren Aufgaben konnten die Schülerinnen und Schüler die vollständige oder eine abgestufte Punktzahl für teilweise gelöste Aufgaben erhalten.

Die naturwissenschaftliche Grundbildung wurde in ähnlicher Weise getestet wie die mathematische Grundbildung, d.h. es wurden Testeinheiten verwendet, die jeweils eine reale naturwissenschaftliche Anwendungssituation darstellten, und dann Fragen hierzu gestellt. Rund zwei Drittel der Aufgaben waren so konstruiert, dass die Antworten darauf eindeutig als richtig oder falsch kodiert werden konnten. Bei komplexeren Aufgaben konnten die Schülerinnen und Schüler die vollständige oder eine abgestufte Punktzahl für teilweise gelöste Aufgaben erhalten.

Mit den PISA-Kontextfragebogen wurden Informationen gesammelt, die für die Interpretation und Analyse der Ergebnisse wichtig sind. Die Fragebogen enthielten Fragen zu Schülermerkmalen, wie z.B. Geschlecht, wirtschaftlicher und sozialer Hintergrund, sowie Aktivitäten im häuslichen und schulischen Umfeld. Im Rahmen einer internationalen Option berichteten die Schülerinnen und Schüler auch über ihre Einstellung zum Lernen, über ihren Umgang mit Computern und unter dem Oberbegriff „selbstreguliertes Lernen“ über Strategien zur Steuerung und Überwachung ihres eigenen Lernprozesses. Die Schulleitungen der Schulen, in denen die Erhebungen durchgeführt wurden, wurden über Schulmerkmale (wie z.B. Größe und Ressourcen) sowie über die Organisation des Unterrichts befragt.

\author{
... während die Mehrzahl \\ der Aufgaben in \\ mathematischer \\ Grundbildung ...
}

.. und

naturwissenschaftlicher

Grundbildung so

konstruiert waren, dass

die Antworten eindeutig

als richtig oder falsch

kodiert werden konnten.

Mit den Schülerfrage-

bogen wurden Informa-

tionen über Hintergrund

und Aktivitäten der

schülerinnen und

schüler gesammelt. In

vielen Ländern wurden

die Schülerinnen und

schüler auch danach

gefragt, auf welche Art

und Weise sie lernen. 
KAPITEL 1 Einführung in die Internationale Schulleistungsstudie PISA

Die PISA-Ergebnisse
geben nicht nur
Aufschluss über das
schulische Lernen,
sondern auch über den
Lernprozess generell, ...

... und führen somit in manchen Punkten zu anderen Resultaten als stärker auf die Lehrpläne ausgerichtete Studien.

\section{Die Interpretation der PISA-Ergebnisse}

Wenn ein Land bei den PISA-Messungen besser abschneidet als ein anderes, so lässt sich daraus nicht automatisch schließen, dass die Schulen hier effektiver sind, denn Lernen beginnt bereits vor Schuleintritt und findet in unterschiedlichen institutionellen und außerschulischen Kontexten statt. Gleichwohl lässt sich bei einer höheren Punktzahl eines Landes bei PISA durchaus die Schlussfolgerung ableiten, dass der kumulative Effekt der Lernerfahrungen in diesem Land von der frühen Kindheit bis zum Alter von 15 Jahren, und zwar sowohl im schulischen als auch im außerschulischen Umfeld, zu positiveren Ergebnissen in den von PISA getesteten Bereichen geführt hat.

Wie der Leser dieses Berichts feststellen wird, bestätigen und ergänzen die Ergebnisse von PISA 2000 häufig die Resultate früherer internationaler Erhebungen wie der 3. Internationalen Mathematik- und Naturwissenschaftlichen Studie (TIMSS), die 1995 von der International Association for the Evaluation of Educational Achievement (IEA) bei Schülerinnen und Schülern der dritten/vierten sowie der siebten/achten Klasse und im letzten Sekundarschuljahr durchgeführt und 1999 bei Schülerinnen und Schülern der achten Klasse wiederholt wurde. Trotzdem weichen die PISA-Ergebnisse z.T. von denen in TIMSS ab. Solche Differenzen waren auf Grund der Unterschiede bei den beiden Studien zu erwarten. Die Erhebungsinstrumente für TIMSS wurden auf der Basis einer Analyse des indentierten Curriculums in jedem Teilnehmerland konstruiert, um den wichtigsten und der Mehrzahl der Teilnehmerländer gemeinsamen Lehrstoff abzudecken. Die zuvor beschriebenen Erhebungsinstrumente für PISA erstrecken sich auf eine Reihe von Fertigkeiten und Kompetenzen, die in den jeweils getesteten Bereichen als entscheidende Voraussetzung für die Fähigkeit des Einzelnen angesehen werden, in der modernen Gesellschaft eine aktive Rolle zu spielen und einen erfolgreichen Beitrag dazu zu leisten. Schließlich ist $\mathrm{zu}$ bedenken, dass sich die am Alter orientierte PISA-Zielpopulation der 15-Jährigen von der an Klassenstufen orientierten TIMSS-Population unterscheidet.

\section{Der Beitrag von PISA zur Bildungsplanung}

PISA vermittelt Aufschluss über die Faktoren, die für die

Bildungserträge mitbestimmend sind.

Es soll verglichen werden, wie gut die verschiedenen

Schulsysteme die Schülerinnen und Schüler auf das Leben vorbereiten.
PISA liefert eine umfassende Messung vergleichbarer Lernstände gegen Ende der Pflichtschulzeit, die sowohl als Orientierungshilfe für bildungspolitische Entscheidungen und die Ressourcenzuweisung dienen als auch Aufschlüsse darüber vermitteln kann, welche Faktoren zur Entwicklung von Kenntnissen und Fähigkeiten beitragen und inwieweit diese Faktoren in den verschiedenen Ländern die gleiche Rolle spielen.

PISA liefert internationale Vergleiche der Leistung von Bildungssystemen mit aussagekräftigen, kulturübergreifend validen Messungen von Kompetenzen, die für Erwachsene im täglichen Leben relevant sind. Erhebungen, bei denen lediglich die Beherrschung des in den schulischen Lehrplänen vorgesehenen 
Stoffs geprüft wird, können über die interne Effizienz eines Schulsystems Aufschluss geben. Anhand solcher Evaluierungen kann aber nicht festgestellt werden, wie effektiv die Schülerinnen und Schüler von den Schulen auf ihr späteres Leben nach Abschluss der formalen Schulausbildung vorbereitet werden.

Die von PISA zur Verfügung gestellten Informationen ermöglichen es Bildungspolitikern, sich eingehend mit den Faktoren zu befassen, die mit schulischem Erfolg in Zusammenhang stehen, und nicht nur Vergleiche vereinzelter Ergebnisse anzustellen. PISA kann z.B. Auskunft darüber geben, wie groß der Leistungsabstand zwischen Schülerinnen und Schülern aus sozial besser bzw. schlechter gestellten Familien in ihrem Land im Vergleich zu anderen Ländern ist. PISA liefert auch Aufschlüsse über die spezifischen Schulmerkmale - z.B. die Art der Unterrichtsorganisation - sowie über den Zusammenhang, der zwischen diesen Schulmerkmalen und dem Leistungsniveau der Schülerinnen und Schüler besteht. Die PISA-Daten können herangezogen werden, um festzustellen, welche Aspekte der Einstellung der Schülerinnen und Schüler für das Lernen offenbar von größter Bedeutung sind. In dieser wie auch in vielerlei anderer Hinsicht bietet die PISA-Studie einen neuen Ansatz zur Betrachtung schulischer Ergebnisse, dem die Erfahrungen von Schülerinnen und Schülern in vielen Ländern der Welt und nicht der spezifische kulturelle Kontext eines einzigen Landes zu Grunde gelegt werden. Dieser internationale Kontext erlaubt es den Bildungsplanern, Annahmen hinsichtlich der Qualität der Bildungsergebnisse ihres eigenen Landes in Frage zu stellen.

Die internationale Perspektive von PISA stellt für die Bildungspolitiker eine gute Möglichkeit dar, die Stärken und Schwächen ihrer eigenen Bildungssysteme zu erkennen. Die Tatsache, dass einige Länder bei den Schülerleistungen ein hohes Durchschnittsniveau mit nur geringem Abstand zwischen den besten und den schlechtesten Ergebnissen erreichen können (vgl. Kapitel 2), lässt darauf schließen, dass große Leistungsheterogenität nicht der Preis für ein hohes durchschnittliches Leistungsniveau sein muss. Eine weitere Feststellung, nämlich die ganz unterschiedliche Ausprägung der Zusammenhänge zwischen sozialem Hintergrund und Lernergebnissen in den einzelnen Ländern (vgl. Kapitel 8), zeigt auch, dass es für Schulen und Schulsysteme möglich ist, diese Zusammenhänge zu mildern. Schülerinnen und Schüler aus sozial schwächeren Verhältnissen müssen nicht zwangsläufig ein geringeres Leistungsniveau aufweisen. Schulen - und Bildungspolitiker - können etwas gegen niedrige Leistungen tun.

Schließlich gibt PISA durch die Berichterstattung über die Schülerkompetenzen nach einem festgelegten Zeitplan den Regierungen ein Instrument an die Hand, die Fortschritte ihrer Bildungssysteme unter dem Gesichtspunkt der Schülerleistungen laufend zu beobachten und ihre nationale Politik im Licht der Ergebnisse anderer Länder einzuschätzen. Die hier wiedergegebenen Ergebnisse von PISA 2000 stellen einen Ausgangspunkt dar. In den Jahren 2003, 2006, 2009
PISA identifiziert und vergleicht Zusammenhänge, die zwischen persönlichen, familiären und schulischen Merkmalen einerseits und den schülerleistungen andererseits bestehen ...

... und ermöglicht so den Ländern, das eigene Bildungssystem im Licht der Ergebnisse anderer Länder zu betrachten.
In der Zukunft wird es für die Länder auch möglich sein, Veränderungen der Leistungsergebnisse im Zeitablauf zu beobachten. 
KAPITEL 1 Einführung in die Internationale Schulleistungsstudie PISA

usw. werden die Länder in der Lage sein, sich über die von ihnen erzielten Fortschritte Klarheit zu verschaffen.

Parallel zu diesem ersten internationalen Bericht veröffentlichen die meisten Teilnehmerländer nationale Berichte, die die Ergebnisse von PISA und ihre politischen Implikationen im jeweiligen wirtschaftlichen, sozialen und bildungsbezogenen Kontext untersuchen. Außerdem werden detailliertere internationale thematische Berichte erstellt, um anhand der Ergebnisse von PISA 2000 spezifische Themenkomplexe und deren politischen Implikationen genauer herauszuarbeiten. Besondere Aufmerksamkeit gilt bei diesen thematischen Berichten Gleichstellungsfragen, geschlechtsspezifischen Unterschieden bei Schülerleistungen, Einstellung und Motivation, den Bedürfnissen besonders benachteiligter wie auch außergewöhnlich guter Schüler, der Bedeutung von Engagement und Motivation als wichtige Voraussetzung für Leistung und späteres Weiterkommen, der Natur, der Entwicklung und den Effekten von Grundkompetenzen sowie Aspekten der Lernstrategien und des Selbstkonzepts.

\section{Gemeinsame Anstrengungen bei der Entwicklung von PISA}

PISA ist das Ergebnis einer effektiven

Zusammenarbeit

zwischen nationalen

Organisationen,

Fachleuten und

Schulbehörden ...

... und wird von den

Teilnehmerländern gemeinschaftlich auf der

Basis gemeinsamer politischer Interessen geleitet,...
PISA ist ein weitreichendes Kooperationsprojekt der OECD-Mitgliedstaaten, das auf einem neuen Konzept der Erfassung von Schülerleistungen auf einer regelmäßigen Basis beruht. Die Tests wurden in kooperativer Weise entwickelt, von den Teilnehmerländern akzeptiert und von den nationalen Organisationen durchgeführt. Die konstruktive Mitarbeit von Lehrkräften und Schulleitungen der teilnehmenden Schulen war ein entscheidender Faktor für den Erfolg von PISA in allen Phasen der Entwicklung und Durchführung.

In einem Leitungsgremium, dem Board of Participating Countries (BPC), in dem alle Teilnehmerländer auf hoher Ebene vertreten sind, wurden die politischen Prioritäten und die Standards für die Erarbeitung von Indikatoren, die Entwicklung von Erhebungsinstrumenten und die Berichterstattung über die Ergebnisse festgelegt. Experten aus den Teilnehmerländern wirkten in Arbeitsgruppen mit, die eine Verknüpfung der bildungspolitischen Zielsetzungen des Programms mit der besten auf internationaler Ebene verfügbaren fachwissenschaftlichen und verfahrenstechnischen Kompetenz in den drei Erhebungsbereichen gewährleisten. Durch ihre Beteiligung an diesen Expertengruppen stellten die Länder sicher, dass die Instrumente international valide sind und dem Kontext der Kulturen und Bildungssysteme der verschiedenen OECD-Mitgliedstaaten Rechnung tragen, dass dieTestmaterialien über sehr gute messtechnische Eigenschaften verfügen und dass die Instrumente sowohl Zuverlässigkeit wie auch bildungsbezogene Gültigkeit aufweisen.

Die Teilnehmerstaaten implementierten PISA auf nationaler Ebene durch nationale Projektmanager, und zwar nach einheitlichen technischen und administrativen Verfahren. Diese Projektmanager spielten eine entscheidende Rolle bei der Entwicklung und Validierung der internationalen Erhebungs- 
instrumente und sorgten für eine hohe Qualität bei der Implementierung von PISA. Sie wirkten auch an der Verifizierung und Evaluierung der Ergebnisse, Analysen und Berichte mit.

Verantwortlich für die Konzipierung und die Umsetzung von PISA 2000 innerhalb der vom BPC entwickelten Rahmenkonzeption war ein internationales Konsortium unter Federführung des Australian Council for Educational Research (ACER). Die anderen Partner in diesem Konsortium sind das National Institute for Educational Measurement (CITO) in den Niederlanden, Westat sowie Educational Testing Service (ETS) in den USA und das National Institute for Educational Policy Research (NIER) in Japan.

Die übergreifende Managementverantwortung für die Studie liegt beim OECDSekretariat, das seine Implementierung laufend überwacht, dem BPC als Sekretariat dient, Konsensbildung zwischen den beteiligten Ländern fördert und als Mittler zwischen dem BPC und dem internationalen Konsortium fungiert.

PISA wird gemeinsam von allen beteiligten Ländern finanziert.

\section{Aufbau des Berichts}

Die Kapitel 2 und 3 beschreiben die Schülerleistungen in den drei Grundbildungsbereichen von PISA, und Kapitel 4 ergänzt diese Informationen mit einem Profil 15-jähriger Schülerinnen und Schüler als Lernende im Hinblick auf Motivation, Engagement, Lernstrategien und Vertrauen in ihre eigenen Fähigkeiten. Es enthält auch Informationen über den Umgang von Schülerinnen und Schülern mit dem Computer.

Kapitel 5 untersucht geschlechtsspezifische Unterschiede bei den Schülerleistungen in den drei Grundbildungsbereichen sowohl für die Gesamtstichprobe als auch für bestimmte Untergruppen.

Die Kapitel 6 und 7 analysieren die Leistungen der Schülerinnen und Schüler im Kontext ihres jeweiligen häuslichen Hintergrunds und des generellen Lernumfelds. Das Kapitel 6 konzentriert sich auf eine Beschreibung des familiären Hintergrunds der Schülerinnen und Schüler, einschließlich des wirtschaftlichen, kulturellen und sozialen Hintergrunds zu Hause, und Kapitel 7 untersucht anschließend, inwieweit sich das Lernumfeld und die schulische Organisation in den einzelnen Ländern voneinander unterscheiden. Kapitel 7 befasst sich zusätzlich mit den Human- und Finanzressourcen, die die Länder für das Bildungswesen aufwenden, sowie mit ausgewählten Merkmalen der nationalen Bildungssysteme. ...und zwar durch die

OECD.

Der vorliegende Bericht

befasst sich mit den

Schülerleistungen,...

... geschlechtsspezifischen

Unterschieden,...

... den Leistungen der

schülerinnen und

schüler mit

unterschiedlichem

Hintergrund und

unterschiedlichen

Lernerfahrungen ... 
KAPITEL 1 Einführung in die Internationale Schulleistungsstudie PISA

... sowie mit der Frage, welche Schlüsse die Bildungspolitiker hieraus

hinsichtlich der

Bedeutung einzelner Faktoren ziehen können.
In Kapitel 8 schließlich werden, ausgehend von diesen Ergebnissen, Fragen über die Art der Zusammenhänge zwischen schulischen Leistungen und sozialem Hintergrund behandelt. Anhand einer Analyse der gleichzeitig bestehenden Zusammenhänge zwischen mehreren unterschiedlichen Variablen innerhalb eines breiten Spektrums von Situationen und einer Reihe von Ländern, bei der sowohl familiäre als auch schulische Faktoren berücksichtigt werden, ist es möglich, den individuellen oder kombinierten Einfluss dieser Faktoren abzuschätzen und die relative Bedeutung von schulischen Ressourcen sowie Schulpolitik und -praktiken in verschiedenen Arten von Schulsystemen zu evaluieren. Das kann Hinweise darauf geben, was die Bildungspolitik zu tun vermag, um zum einen die Durchschnittsleistung zu verbessern und zum anderen den Einfluss des familiären Hintergrunds auf die Schülerleistung abzuschwächen.

\section{Anmerkungen}

1. In den meisten OECD-Ländern endet die Schulpflicht mit 15 oder 16 Jahren, in den Vereinigten Staaten jedoch erst mit 17 und in Belgien, Deutschland und den Niederlanden erst mit 18 Jahren (OECD, 2001). 


\section{HINWEISE FÜR DEN LESER}

\section{Die den Abbildungen zugrunde liegenden Daten}

Die Daten, auf die sich die Kapitel 2 bis 8 dieses Berichts beziehen, sind dem Anhang B1 zu entnehmen, zusätliche Informationen finden sich unter www.pisa.oecd.org. Fehlende Daten werden durch vier Symbole ausgedrückt:

a Die Kategorie ist für das betreffende Land nicht anwendbar. Es gibt diesbezüglich keine Daten.

c Die Anzahl der beobachteten Werte reicht nicht aus, um verlässliche Schätzungen zu gewährleisten (z.B. weniger als 5 Schulen oder weniger als 30 Schülerinnen und Schüler mit gültigen Daten für das betreffende Feld).

$m$ Daten sind nicht verfügbar. Soweit nicht anders vermerkt, wurden entsprechende Daten zwar erhoben, später jedoch aus technischen oder anderen Gründen auf Ersuchen des betreffenden Landes aus der Publikation herausgenommen.

$x$ Daten sind in einer anderen Kategorie oder einer anderen Spalte der Tabelle enthalten.

\section{Berechnung der internationalen Durchschnittswerte}

Für die meisten Indikatoren in diesem Bericht wurde der OECD-Durchschnitt errechnet. Für einige Indikatoren wurde auch ein Gesamtwert für den OECD-Raum errechnet.

- Der OECD-Durchschnitt, der zuweilen auch als Ländermittel bezeichnet wird, ist der Mittelwert aus den Daten von allen OECD-Ländern, für die Daten vorliegen oder geschätzt werden konnten. Der OECD-Durchschnitt kann herangezogen werden, um festzustellen, wie ein Land bei einem bestimmten Indikator im Vergleich zu einem typischen OECD-Land abschneidet. Im OECD-Durchschnitt ist die absolute Größe der Schülerpopulation der einzelnen Länder nicht berücksichtigt, d.h. jedes Land trägt in gleicher Weise zum errechneten Mittel bei.

- Der Wert $\boldsymbol{O E C D}$ insgesamt erfasst die OECD-Länder als Einheit, zu der jedes Land proportional zur Anzahl der 15-Jährigen in den Schulen des Landes beiträgt (vgl. die Daten in Anhang A3). Der Gesamtwert veranschaulicht, wie ein Land im Vergleich zum OECD-Raum insgesamt abschneidet.

Drei OECD-Länder wurden aus der Berechnung von Durchschnittswerten und anderen aggregierten Schätzungen ausgeklammert: die Niederlande, die Slowakische Republik (die 2000 OECDMitgliedsland wurde) und die Türkei. Die Niederlande wurden ausgeklammert, da auf Grund der geringen Bearbeitungsquoten keine verlässlichen Schätzungen der Mittelwerte möglich waren (vgl. 
Anhang A3). Die Slowakische Republik und die Türkei werden ab dem Erhebungszyklus 2003 in PISA einbezogen.

Bei anderen Ländern sind für bestimmte Indikatoren möglicherweise keine Daten verfügbar oder einzelne Datenkategorien treffen u.U. nicht zu. Beim Lesen sollte daher beachtet werden, dass sich die Begriffe OECD-Durchschnitt und OECD insgesamt auf die in die jeweiligen Vergleiche einbezogenen OECD-Länder erstrecken.

\section{Darstellung der Schülerdaten}

Der Bericht verwendet generell den Begriff „15-Jährige“ als Kurzform für die Zielpopulation von PISA. In der Praxis bezieht sich dies auf Schülerinnen und Schüler, die zu Beginn der Testperiode im Alter von 15 Jahren und 3 (abgeschlossenen) Monaten bis zu 16 Jahren und 2 (abgeschlossenen) Monaten waren und eine Bildungseinrichtung besuchten, ungeachtet der Klassenstufe und der Art der von ihnen besuchten Bildungseinrichtung und unabhängig davon, ob es sich um eine Ganztagsoder Halbtagsschule handelte (wegen näherer Einzelheiten vgl. Anhang A3).

\section{Darstellung der Schuldaten}

Die Leiterinnen und Leiter der Schulen, in denen Schülerinnen und Schüler am Test teilnahmen, haben durch Ausfüllen eines Schulfragebogens Informationen über Merkmale ihrer Schule geliefert. Bei Darstellung der Antworten der Schulleitungen in dieser Publikation wurde eine Gewichtung vorgenommen, so dass sie im Verhältnis zur Anzahl der 15-Jährigen in der betreffenden Schule stehen.

\section{Runden der Zahlen}

Durch Auf- und Abrunden einiger Zahlen in den Tabellen stimmt die Summe der Zahlen mit der Gesamtsumme möglicherweise nicht immer überein. Summen, Differenzen und Durchschnittswerte werden stets auf Grundlage der exakten Zahlenwerte berechnet und erst nach der Berechnung aufbzw. abgerundet.

\section{Im Bericht benutzte Abkürzungen}

BIP Bruttoinlandsprodukt

ISCED Internationalen Standardklassifikation des Bildungswesens

KKP Kaufkraftparitäten

S.D. Standardabweichung

S.E. Standardfehler

\section{Weterführende Dokumenationen}

Für weitere Informationen zu den angewandten Instrumenten und Methoden in PISA vgl. PISA 2000 Technical Report (verfügbar ab Februar 2002) und die PISA Web site (www.pisa.oecd.org). 


\section{Kapitel}

2

WAS PISA ÜBER DIE FÄHIGKEITEN 15-JÄHRIGER AUSSAGT -

EIN PROFIL DER SCHÜLERLEISTUNGEN IM BEREICH LESEKOMPETENZ

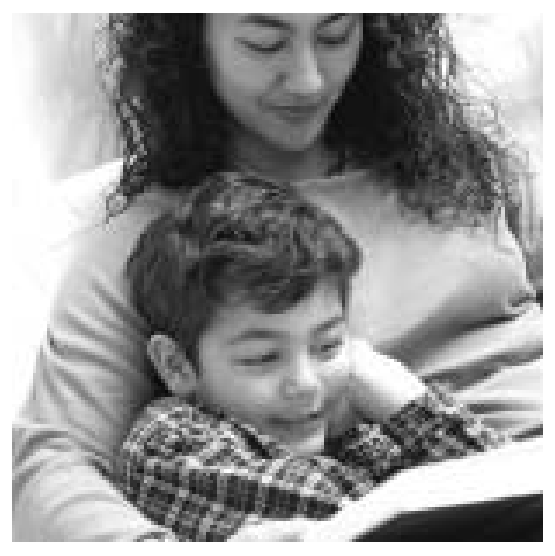


KAPITEL 2 Was PISA über die Fähigkeiten 15-Jähriger aussagt - ein Profil der Schülerleistungen im Bereich Lesekompetenz

\section{Einführung}

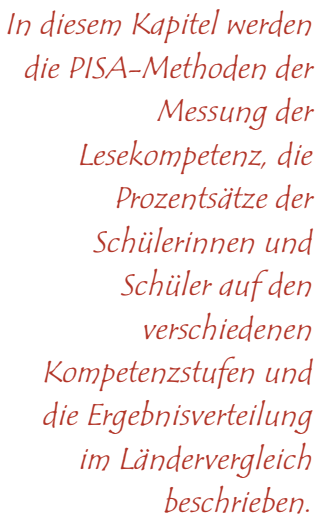

Kapitel 3 und 4 befassen

sich mit der

mathematischen und naturwissenschaftlichen Grundbildung und dem Lernverhalten.

In PISA 2000 wurden

die Schülerinnen und schüler mit einem

breiten Spektrum von

Leseaufgaben mit

unterschiedlichen

Texttypen und Kontexten

konfrontiert,...
Das vorliegende Kapitel liefert ein Profil der Schülerleistungen im Bereich der Lesekompetenz:

- Es wird darin zunächst beschrieben, wie die Leistungen im Bereich der Lesekompetenz in PISA gemessen werden. Dabei werden drei Skalen benutzt, auf denen den Schülerinnen und Schülern Punktwerte zugeteilt werden, die ihren Leistungen bei der Beantwortung von Fragen mit unterschiedlichem Schwierigkeitsgrad entsprechen.

- An zweiter Stelle werden die in den einzelnen Ländern erzielten Leistungen anhand der Verteilungen der Schülerleistungen beschrieben. Um die Darstellung zu erleichtern, ist jede Skala in fünf Kompetenzstufen unterteilt, wobei die Verteilung der in einzelnen Ländern erzielten Ergebnisse durch die Aufschlüsselung der Prozentsätze der Schülerinnen und Schüler dargestellt wird, die jeweils die verschiedenen Stufen erreichen. Die Kompetenzstufen werden anhand von Beispielaufgaben beschrieben, die von den Schülerinnen und Schülern in zufriedenstellender Weise gelöst werden müssen, um die entsprechenden Stufen zu erreichen.

- An dritter Stelle werden die in den einzelnen Ländern erzielten Leistungen im Hinblick auf die durchschnittlichen Werte der Schülerinnen und Schüler und auf die Verteilung der Werte innerhalb der Schülerstichproben untersucht.

Kapitel 3 ergänzt dies durch die Analyse der Schülerleistungen in den Bereichen mathematische und naturwissenschaftliche Grundbildung, wobei auch die Unterschiede zwischen den Schülerleistungen in diesen Bereichen und im Bereich ihrer Lesekompetenz untersucht werden. In Kapitel 4 wird das Profil der PISA-Ergebnisse nochmals erweitert, und zwar durch eine Analyse der Angaben der Schülerinnen und Schüler bezüglich ihres Umgangs mit Computern, ihrer Lernstrategien sowie der nicht kognitiven Ergebnisse der Schulbildung, die für ein lebensbegleitendes Lernen von Bedeutung sind: Motivation, Engagement und Vertrauen in die eigenen Fähigkeiten.

\section{Wie Lesekompetenz mit PISA gemessen wird}

Bei dem in PISA verwendeten Konzept der Lesekompetenz kamen drei verschiedene Dimensionen ins Spiel, die für die Ausarbeitung der Tests maßgeblich waren: die Aufgabenart, die Form und Struktur des Lesestoffs und der Zweck, für den der Text geschrieben wurde. Die Lesekompetenz der einzelnen Schüler lässt sich am besten anhand des ersten dieser Aspekte untersuchen. Bei den beiden anderen handelt es sich um Merkmale der Testmaterialien, die bei der Gewährleistung einer breiten Palette bei den in die Tests aufgenommenen Aufgaben nützlich waren. 
Die Dimension „Art der Leseaufgabe“ wird anhand von drei Subskalen gemessen. Die Skala „Informationen ermitteln“ gibt die Fähigkeit der Schülerinnen und Schüler wieder, Informationen in einem Text zu lokalisieren. Die Skala „Textbezogenes Interpretieren“ umfasst die Fähigkeit, der schriftlichen Information Bedeutung zu verleihen und Schlüsse daraus zu ziehen. Die Skala „Reflektieren und Bewerten“ bezieht sich auf die Fähigkeit der Schülerinnen und Schüler, Verbindungen zwischen dem Text und eigenen Kenntnissen, Vorstellungen und Erfahrungen herzustellen. Die Ergebnisse dieser drei Skalen sind zusätzlich in einer „Gesamtskala Lesekompetenz“ zusammengefasst.

Um die Interpretation der von den Schülerinnen und Schülern erzielten Punktwerte zu erleichtern, wurde die Gesamtskala Lesekompetenz so konstruiert, dass der Mittelwert bei 500 Punkten liegt und rund zwei Drittel der Schülerinnen und Schüler in den OECD-Ländern im Bereich zwischen 400 und 600 Punkten liegen ${ }^{1}$. Diese Bezugspunkte bilden gewissermaßen den „Anker“ der im Rahmen von PISA durchgeführten Messung der Schülerleistungen. Die Mittelwerte auf den drei Subskalen der Gesamtskala Lesekompetenz weichen geringfügig vom Wert $500 \mathrm{ab}$.

Die Werte auf jeder Skala entsprechen unterschiedlichen Leistungsniveaus für jeweils bestimmte Aspekte der Lesekompetenz. Aus einer geringen Punktzahl auf der Skala „Textbezogenes Interpretieren“ lässt sich beispielsweise schließen, dass der jeweilige Schüler nur begrenzt fähig ist, Zusammenhänge zu begreifen, aus einem oder mehreren Teilen eines Textes Bedeutung zu konstruieren oder Schlüsse zu ziehen. Eine hohe Punktzahl auf der Skala „Textbezogenes Interpretieren" deutet hingegen darauf hin, dass der Schüler in diesem Bereich über ausgeprägte Fähigkeiten verfügt.

Für jede der drei Lesekompetenzskalen gibt es leichtere und schwerere Aufgaben, aber es besteht keine hierarchische Beziehung zwischen den drei Subskalen. Jede der drei Lesekompetenzskalen ist entsprechend der Art der Kenntnisse und Fähigkeiten in fünf Kompetenzstufen eingeteilt. Stufe 5 entspricht einer Punktzahl von über 625, Stufe 4 einer Punktzahl zwischen 553 und 625, Stufe 3 einer Punktzahl zwischen 481 und 552, Stufe 2 einer Punktzahl von 408 bis 480 und Stufe 1 einer Punktzahl von 335 bis 407 .

Auf einer gegebenen Stufe können die Schülerinnen und Schüler nicht nur die dieser Stufe zugeordneten Kenntnisse und Fähigkeiten vorweisen, sondern sie verfügen auch über die in den darunter liegenden Stufen geforderten Fähigkeiten. Alle Schülerinnen und Schüler, die den Anforderungen von Stufe 3 genügen, werden demzufolge in der Regel auch den Anforderungen von Stufe 1 und 2 gerecht. Von allen Schülerinnen und Schülern auf einer bestimmten Stufe wird erwartet, dass sie mindestens die Hälfte der Aufgaben dieser Stufe richtig beantworten.

\author{
... wobei ihre \\ Kompetenzen beim \\ Ermitteln von \\ Informationen, \\ textbezogenen \\ Interpretieren und \\ Reflektieren und \\ Bewerten ...
}

\begin{abstract}
... anhand von Skalen
gemessen werden,

auf denen zwei Drittel

der Schüler innerhalb

von plus/minus 100

Punkten vom Mittelwert

500 liegen
\end{abstract}

Die Ergebnisse werden

in fünf Kompetenzstufen

zusammengefasst, ...
... wobei die
Schülerinnen und
Schüler der jeweils
höchsten Stufe
zugeordnet werden, auf
der sie erwartungsgemäfs
die meisten Aufgaben
beantworten können. 
KAPITEL 2 Was PISA über die Fähigkeiten 15-Jähriger aussagt - ein Profil der Schülerleistungen im Bereich Lesekompetenz

Jede Kompetenzstufe

kann anhand der

Fähigkeiten beschrieben

werden, über die die

Schülerinnen und schüler auf dieser Stufe verfügen.
Schülerinnen und Schüler, die weniger als 335 Punkte erzielen, d.h. Stufe 1 nicht erreichen, sind nicht in der Lage, routinemäßig die elementarsten Fähigkeiten nachzuweisen, die in PISA gemessen werden. Das heißt zwar nicht, dass die fraglichen Schülerinnen und Schüler über keinerlei Grundqualifikationen verfügen ${ }^{2}$, aber Leistungen unterhalb der Stufe 1 lassen auf gravierende Defizite in der Fähigkeit von Schülerinnen und Schülern schließen, Lesekompetenzen als Mittel zum Erwerb von Wissen und Fähigkeiten in anderen Bereichen einzusetzen.

Die gleichzeitige Verortung von Aufgabenschwierigkeiten einerseits und der Schülerleistungen in Form von Kompetenzstufen andererseits auf den PISALeistungsskalen ermöglichen nicht nur eine Einstufung der Ergebnisse der Schülerleistungen, sondern auch eine Beschreibung ihrer Fähigkeiten (vgl. Abb. 2.1). Jeder der aufeinander aufbauenden Lesekompetenzstufen entsprechen Aufgaben mit steigendem Schwierigkeitsgrad. Die Aufgaben einer jeden Stufe wurden von einem Expertenteam daraufhin beurteilt, ob sie durch viele gemeinsame Aufgabenmerkmale und -anforderungen gekennzeichnet sind und sich zugleich systematisch von den Aufgaben der nächst höheren oder niedrigeren Stufe abheben. Der Schwierigkeitsgrad der Aufgaben wurde dann empirisch auf der Grundlage der Schülerleistungen in den Teilnehmerländern validiert.

Abbildung 2.1

Was die einzelnen Kompetenzstufen messen

\begin{tabular}{l|c|c} 
Informationen Ermitteln & Textbezogenes Interpretieren & Reflektieren und Bewerten
\end{tabular}

Auf den verschiedenen Lesekompetenzskalen gemessene Fähigkeiten:

\begin{tabular}{|c|c|c|}
\hline $\begin{array}{l}\text { Ermitteln von Informationen bedeutet, einzelne } \\
\text { oder mehrere Informationsteile in einem Text } \\
\text { aufzufinden. }\end{array}$ & $\begin{array}{l}\text { Textbezogenes Interpretieren bedeutet, einem } \\
\text { oder mehreren Teilen eines Textes einen Sinn } \\
\text { zuzuordnen und Schlüsse daraus zu ziehen. }\end{array}$ & $\begin{array}{l}\text { Reflektieren und bewerten bedeutet, einen Text } \\
\text { zu eigenen Erfahrungen, Kenntnissen und Ideen } \\
\text { in Beziehung zu setzen. }\end{array}$ \\
\hline \multicolumn{3}{|c|}{ Aufgabenmerkmale entsprechend den steigenden Schwierigkeitsstufen auf jeder der drei Lesekompetenzskalen: } \\
\hline $\begin{array}{l}\text { Der Schwierigkeitsgrad der Aufgabe hängt davon } \\
\text { ab, wie viele Informationsteile aufzufinden sind. } \\
\text { Ausschlaggebend ist zudem die Zahl der } \\
\text { Bedingungen, die zur Lokalisierung der gefragten } \\
\text { Information erfüllt sein müssen, sowie die Frage, } \\
\text { ob die zu suchende Information in einer } \\
\text { bestimmten Weise geordnet werden muss. Wie } \\
\text { schwierig eine Aufgabe ist, hängt auch von der } \\
\text { „Sichtbarkeit“ der Information und der } \\
\text { Vertrautheit mit dem Kontext ab. Weitere } \\
\text { entscheidende Merkmale sind die Komplexität } \\
\text { desTextes sowie das Vorhandensein und die } \\
\text { Intensität konkurrierender Informationen. }\end{array}$ & $\begin{array}{l}\text { Der Schwierigkeitsgrad der Aufgabe hängt von der } \\
\text { Art des erforderlichen Interpretationsvorgangs ab, } \\
\text { wobei für die einfachsten Aufgaben nur das } \\
\text { Hauptthema einesTextes identifiziert werden muss, } \\
\text { bei schwereren Aufgaben Zusammenhänge erkannt } \\
\text { werden müssen, dieTeil desTextes sind, und bei den } \\
\text { schwierigsten Aufgaben entweder ein Verständnis } \\
\text { für die Bedeutung von Sprache entwickelt oder } \\
\text { analoge Schlussfolgerungen gezogen werden müssen. } \\
\text { Der Schwierigkeitsgrad ergibt sich auch daraus, wie } \\
\text { explizit die Ideen oder Informationen, die der Leser } \\
\text { zur Lösung derAufgabe benötigt, imText ausgedrückt } \\
\text { sind, wie, „sichtbar“die erforderlichen Informationen } \\
\text { sind und wie viele ablenkende Informationen der } \\
\text { Text enthält. Einfluss auf den Schwierigkeitsgrad } \\
\text { haben ferner die Länge und die Komplexität des } \\
\text { Textes sowie die Vertrautheit mit dem Inhalt. }\end{array}$ & $\begin{array}{l}\text { Der Schwierigkeitsgrad der Aufgabe hängt von } \\
\text { der Art der anzustellenden Überlegungen ab, } \\
\text { wobei für die einfachsten Aufgaben nur simple } \\
\text { Verbindungen hergestellt oder Erklärungen } \\
\text { geliefert werden müssen, die einen } \\
\text { Zusammenhang zwischen dem Text und } \\
\text { externen Erfahrungen schaffen, während für } \\
\text { die schwierigeren Aufgaben Hypothesen } \\
\text { aufgestellt oder Beurteilungen abgegeben } \\
\text { werden müssen. Ausschlaggebend für den } \\
\text { Schwierigkeitsgrad ist auch dieVertrautheit mit } \\
\text { dem Wissen, das unabhängig vom Text } \\
\text { herangezogen werden muss, die Komplexität } \\
\text { des Textes selbst, das erforderliche Niveau an } \\
\text { Textverständnis sowie die Frage, wie explizit } \\
\text { der Leser sowohl in der Aufgabenstellung als } \\
\text { auch im Text selbst auf relevante Faktoren } \\
\text { hingewiesen wird. }\end{array}$ \\
\hline
\end{tabular}




\section{Kompetenzstufe}

\begin{tabular}{|c|c|c|}
\hline $\begin{array}{l}\text { Mehrere Teile einer tief eingebetteten } \\
\text { Information, die z.T. auch außerhalb des } \\
\text { Hauptteils des Textes liegen können, } \\
\text { lokalisieren und u.U. ordnen oder } \\
\text { zusammenfügen. Feststellen, welche der } \\
\text { im Text enthaltenen Informationen für } \\
\text { die Aufgabe wichtig sind. Mit sehr } \\
\text { plausiblen und/oder stark } \\
\text { konkurrierenden Informationen umgehen } \\
\text { können. }\end{array}$ & $\begin{array}{l}\text { Die Bedeutung einer nuancierten } \\
\text { Ausdrucksweise analysieren oder ein } \\
\text { volles und detailliertes Verständnis eines } \\
\text { Textes unter Beweis stellen. }\end{array}$ & $\begin{array}{l}\text { Kritisch bewerten oder ausgehend von } \\
\text { Fachwissen Hypothesen aufstellen. Mit } \\
\text { Konzepten umgehen können, die im } \\
\text { Gegensatz zu eigenen Erwartungen } \\
\text { stehen, und gestützt auf eine tiefgreifende } \\
\text { Analyse langer oder komplexer Texte } \\
\text { Rückschlüsse ziehen. }\end{array}$ \\
\hline $\begin{array}{l}\text { Mehrere Teile einer eingebetteten } \\
\text { Information in einem Text, dessen } \\
\text { Kontext oder Form ungewohnt ist, } \\
\text { lokalisieren und u.U. ordnen oder } \\
\text { zusammenfügen, wobei jedes } \\
\text { Informationsteil möglicherweise } \\
\text { mehreren Kriterien entsprechen muss. } \\
\text { Feststellen, welche im Text enthaltenen } \\
\text { Informationen für die Aufgabe wichtig } \\
\text { sind. }\end{array}$ & $\begin{array}{l}\text { Anspruchsvolle, aus dem Text gezogene } \\
\text { Schlüsse zum Verständnis und zur } \\
\text { Anlegung von Kategorien in einem } \\
\text { ungewohnten Kontext einsetzen und die } \\
\text { Bedeutung eines Textteils unter } \\
\text { Berücksichtigung des Textes als Ganzem } \\
\text { analysieren. Mit Mehrdeutigkeiten, den } \\
\text { eigenen Erwartungen zuwiderlaufenden } \\
\text { Vorstellungen und in der Negativform } \\
\text { ausgedrückten Gedanken umgehen } \\
\text { können. }\end{array}$ & $\begin{array}{l}\text { Unter Rückgriff auf schulisches oder } \\
\text { Allgemeinwissen Hypothesen über einen } \\
\text { Text aufstellen oder ihn kritisch bewerten. } \\
\text { Ein genaues Verständnis langer oder } \\
\text { komplexer Texte unter Beweis stellen. }\end{array}$ \\
\hline $\begin{array}{l}\text { Mehrere Informationsteile, die u.U. } \\
\text { jeweils mehreren Kriterien entsprechen } \\
\text { müssen, lokalisieren und manchmal auch } \\
\text { die zwischen ihnen bestehenden } \\
\text { Zusammenhänge erkennen. Mit gut } \\
\text { sichtbaren konkurrierenden } \\
\text { Informationen umgehen können. }\end{array}$ & $\begin{array}{l}\text { MehrereTextteile gedanklich verbinden, um } \\
\text { eine Hauptidee zu identifizieren, einen } \\
\text { Zusammenhang zu begreifen oder die } \\
\text { Bedeutung eines Wortes oder Satzes zu } \\
\text { analysieren. Vergleichen, gegenüberstellen } \\
\text { oder kategorisieren mit Berücksichtigung } \\
\text { zahlreicher Kriterien. Mit konkurrierenden } \\
\text { Informationen umgehen können. }\end{array}$ & $\begin{array}{l}\text { Verbindungen herstellen, Vergleiche } \\
\text { anstellen, Erklärungen liefern oder einen } \\
\text { Textbestandteil beurteilen. Ein } \\
\text { detailliertes Verständnis des Textes unter } \\
\text { Bezugnahme auf vertraute } \\
\text { Alltagskenntnisse unter Beweis stellen } \\
\text { oder dafür auf weniger bekanntes Wissen } \\
\text { zurückgreifen. }\end{array}$ \\
\hline $\begin{array}{l}\text { Ein oder mehrere Informationsteile } \\
\text { lokalisieren, wobei jedes u.U. mehreren } \\
\text { Kriterien entsprechen muss. Mit } \\
\text { konkurrierenden Informationen umgehen } \\
\text { können. }\end{array}$ & $\begin{array}{l}\text { Die Hauptidee eines Textes identifizieren, } \\
\text { Zusammenhänge begreifen, einfache } \\
\text { Kategorien entwickeln und anlegen oder } \\
\text { die Bedeutung eines begrenzten Textteils } \\
\text { analysieren, wenn die Information nicht } \\
\text { leicht sichtbar ist und wenig } \\
\text { anspruchsvolle Schlüsse gezogen werden } \\
\text { müssen. }\end{array}$ & $\begin{array}{l}\text { Vergleiche anstellen oder } \\
\text { Zusammenhänge zwischen dem Text und } \\
\text { außertextlichen Kenntnissen erkennen } \\
\text { oder einen Bestandteil des Textes } \\
\text { ausgehend von eigenen Erfahrungen oder } \\
\text { Standpunkten erklären. }\end{array}$ \\
\hline $\begin{array}{l}\text { Ein oder mehrere unabhängige Teile einer } \\
\text { explizit ausgedrückten Information unter } \\
\text { Berücksichtigung eines einzigen } \\
\text { Kriteriums lokalisieren. }\end{array}$ & $\begin{array}{l}\text { Das Hauptthema oder die Absicht des } \\
\text { Autors in einem Text über ein vertrautes } \\
\text { Thema erkennen, wenn die erforderliche } \\
\text { Information im Text gut sichtbar ist. }\end{array}$ & $\begin{array}{l}\text { Eine einfache Verbindung zwischen der } \\
\text { im Text enthaltenen Information und } \\
\text { allgemeinem Alltagswissen herstellen. }\end{array}$ \\
\hline
\end{tabular}

Quelle: OECD PISA, 2001.

Die in PISA 2000 zur Erfassung der Lesekompetenz eingesetzten Aufgaben unterscheiden sich deutlich sowohl in Bezug auf die Art der Texte, die Situation und die Art der Leseaufgabe (vgl. Kapitel 1) als auch auf ihren Schwierigkeitsgrad. Abbildung 2.2 zeigt Beispiel-Aufgaben aus drei der 36 in PISA 2000 verwendeten Testeinheiten und beschreibt die ihnen zugeordneten Lesekompetenzen, die die Schülerinnen und Schüler auf den verschiedenen Stufen der drei Lesekompetenzskalen unter Beweis stellen müssen. Dabei werden die Fähigkeiten erläutert, die mit der jeweiligen Aufgabe beurteilt werden sollen. Diese Beschreibungen gewähren eine gewisse Vorstellung 
KAPITEL 2 Was PISA über die Fähigkeiten 15-Jähriger aussagt - ein Profil der Schülerleistungen im Bereich Lesekompetenz

Für die Aufgaben am unteren Ende jeder der drei Leseskalen muss vom Text direkt Gebrauch gemacht werden.

Die schwierigeren Aufgaben am oberen Ende erfordern hingegen

einen komplexeren

Umgang mit dem Text und den darin ausgedrückten Gedanken. von der Bandbreite der von den Schülerinnen und Schülern erwarteten Denkprozesse und der auf den verschiedenen Stufen der Lesekompetenzskalen zu erbringenden Leistungen. Ein etwas vollständigeres Aufgabenset kann unter www.pisa.oecd.org abgerufen werden.

Schon ein flüchtiger Blick auf Abbildung 2.2 genügt, um festzustellen, dass zur Lösung der Aufgaben am unteren Ende jeder Skala erwartungsgemäß ganz andere Fähigkeiten erforderlich sind als zur Beantwortung der Fragen am oberen Ende. Bei einer genaueren Analyse des Spektrums der entlang jeder Lesekompetenzskala zu erbringenden Anforderungen lässt sich eine Art geordneter Abfolge von Fähigkeiten und Strategien des Wissensaufbaus erkennen. Alle Aufgaben der Subskala „Informationen ermitteln“ verlangen von den Schülerinnen und Schülern beispielsweise, dass sie Informationen, die in Prosa oder in einer anderen schriftlichen Form ausgedrückt sind, auffinden können. Zur Lösung der einfachsten Aufgaben auf dieser Skala muss eine explizit ausgedrückte Information anhand eines einzigen Kriteriums in einem Text gefunden werden, der nur wenige bzw. keine konkurrierenden Informationen enthält.

Im Gegensatz dazu müssen die Schülerinnen und Schüler, um die Aufgaben am oberen Ende der Skala zu lösen, in der Lage sein, mehrere Teile einer tief eingebetteten Information $\mathrm{zu}$ finden und in die richtige Reihenfolge zu bringen, teilweise unter Berücksichtigung mehrerer Kriterien. Der Text enthält dabei häufig konkurrierende Informationen mit Merkmalen, die z.T. auch die Information kennzeichnen, auf die sich die Frage bezieht. Auf der Subskala „Textbezogenes Interpretieren“ und auf der Subskala „Reflektieren und Bewerten" bestehen ebenfalls große Unterschiede zwischen den Aufgaben am unteren und jenen am oberen Ende, sei es in Bezug auf die Art der zur richtigen Beantwortung notwendigen Denkprozesse, den Umfang der in der Frage oder der Anleitung enthaltenen Hinweise auf die zur Erzielung einer korrekten Antwort erforderlichen Lesestrategien, die Komplexität und den Vertrautheitsgrad des Textes und die in ihm enthaltene Menge an konkurrierenden oder ablenkenden Informationen.

Eine Beschreibung des Rahmenkonzepts, das der Erhebung der Lesekompetenz im Rahmen von PISA zu Grunde liegt, findet sich in Schülerleistungen im internationalen Vergleich - Eine neue Rahmenkonzeption für die Erfassung von Wissen und Fähigkeiten (Deutsches PISA-Konsortium, 2000).

\section{Prozentuale Anteile der Schülerinnen und Schüler auf den verschiedenen Lesekompetenzstufen}

Bei der Beschreibung der Schülerleistungen bezogen auf die fünf Stufen der Lesekompetenz kann entweder angegeben werden, welcher Prozentsatz von ihnen die erforderliche Leistung für eine bestimmte Stufe erbringt oder aber welcher Prozentsatz genau diese Stufe erreicht (wie in den Tabellen 2.1a, b, 
c und d), in dem Sinne, dass diese Stufe dem maximalen Leistungsniveau dieser Schülerinnen und Schüler entspricht. Die erste Vorgehensweise ist zum Vergleich des Leistungsprofils der verschiedenen Länder am geeignetsten und wird daher in der folgenden Beschreibung verwendet. Die Information, dass 10\% aller Schülerinnen und Schüler eines Landes und 20\% aller Schülerinnen und Schüler eines anderen Landes genau auf beispielsweise Stufe 3 liegen, ist nicht sehr aussagekräftig, wenn nicht zugleich angegeben ist, welcher Prozentsatz der Schülerinnen und Schüler die anderen Stufen erreicht. Aussagekräftiger ist die Information, wie viel Prozent der Schülerinnen und Schüler mindestens die für eine bestimmte Stufe erforderlichen Leistungen erbringen, da sich aus dieser Information ableiten lässt, welcher Prozentsatz von ihnen in der Lage ist, bestimmten Anforderungen des Alltags- und Berufslebens gerecht zu werden. Für die Analyse zu einem späteren Zeitpunkt in diesem Bericht und anderenorts, ist es jedoch ebenfalls interessant, die besonderen Merkmale der Schülergruppen zu kennen, die die auf einer bestimmten Stufe gefragte Leistung erbringen, um so untersuchen zu können, wo deren Leistungsgrenzen liegen.

\author{
Die Ergebnisse einer \\ Population können \\ anhand des \\ Prozentsatzes der \\ Schülerinnen und \\ schüler beschrieben \\ werden, die eine \\ bestimmte \\ Kompetenzstufe \\ erreichen, oder \\ derjenigen, die \\ mindestens den \\ Anforderungen dieser \\ Stufe entsprechen (also \\ auch darüber liegen \\ können).
}

\section{Kasten 2.1 Erläuterungen zu Abbildung 2.2}

Ebenso wie den Schülerinnen und Schüler entsprechend ihrer Leistungen eine Punktzahl auf den verschiedenen PISA-Skalen zugeordnet werden kann, so lässt sich auch der Schwierigkeitsgrad jeder Aufgabe auf diesen Skalen einordnen. Während die Schülerinnen und Schüler Skalenpunktwerte erhalten, die ihrer Leistung bei den Testaufgaben entsprechen, wird der Schwierigkeitsgrad der Aufgaben aus den durchschnittlichen Lösungshäufigkeiten in allen Ländern abgeleitet.

Frage 13 aus der in Abbildung 2.2 wiedergegebenen Leseeinheit Graffiti, bei der die Schülerinnen und Schüler die in zwei kurzen Texten enthaltenen Ansichten mit ihren eigenen Anschauungen und Vorstellungen vergleichen sollen, hat beispielsweise einen Schwierigkeitsgrad von 471 Punkten. Ein Schüler mit einem Leistungsniveau von 471 Punkten ist gewöhnlich in der Lage, Aufgaben bis zu diesem Schwierigkeitsgrad zu lösen. Das heißt jedoch nicht, dass jeder Schüler mit einem Ergebnis von 471 Punkten oder darüber dieser Aufgabe richtig beantwortet oder dass alle Schüler mit einem Testergebnis von unter 471 Punkten hier eine falsche Antwort liefern. Es heißt auch nicht, dass alle Schülerinnen und Schüler mit einem Testergebnis von 471 Punkten alle Aufgaben mit einem darunter liegenden Schwierigkeitsgrad richtig und alle mit einem höheren falsch beantworten. Der Schwierigkeitsgrad einer Aufgabe ist so definiert, dass Schüler, deren Punktzahl dem Schwierigkeitsgrad einer gegebenen Aufgabe entspricht, diese mit einer bestimmten Wahrscheinlichkeit ${ }^{3}$ richtig beantworten. Für Schüler, die mehr/weniger als 471 Punkte erzielt haben, ist die Wahrscheinlichkeit, dass sie die Beispiel-Aufgabe (oder andere Aufgaben dieses Typs) richtig beantworten, jeweils entsprechend größer/kleiner.

Bei einigen der offenen Fragen konnten die Schülerinnen und Schüler auch teilweise richtige Antworten geben, so dass für solche Antworten den Schülerinnen und Schülern dann ein Teil der Gesamtpunktzahl zuerkannt wurde, der auf der Leistungsskala einem niedrigeren Wert entspricht als eine vollständig richtige Antwort. 
KAPITEL 2 Was PISA über die Fähigkeiten 15-Jähriger aussagt - ein Profil der Schülerleistungen im Bereich Lesekompetenz

Abbildung 2.2

Eine Auswahl der in PISA eingesetzten Leseaufgaben

\section{GRAFFITI}

Ich koche vor Wut, die Schulwand wird nämlich gerade zum vierten Mal gereinigt und frisch gestrichen, um Graffiti wegzubekommen. Kreativität ist bewundernswert, aber die Leute sollten Ausdrucksformen finden, die der Gesellschaft keine zusätzlichen Kosten aufbürden.

Warum schädigt ihr den Ruf junger Leute, indem ihr Graffiti malt, wo es verboten ist? Professionelle Künstler hängen ihre Bilder doch auch nicht in den Straßen auf, oder? Stattdessen suchen sie sich Geldgeber und kommen durch legale Ausstellungen zu Ruhm.

Meiner Meinung nach sind Gebäude, Zäune und Parkbänke an sich schon Kunstwerke. Es ist wirklich armselig, diese Architektur mit Graffiti zu verschandeln, und außerdem zerstört die Methode die Ozonschicht. Wirklich, ich kann nicht begreifen, warum diese kriminellen Künstler sich so viel Mühe machen, wo ihre „Kunstwerke“ doch bloß immer wieder beseitigt werden und keiner sie mehr sieht.

Helga
Über Geschmack lässt sich streiten. Die Gesellschaft ist voll von Kommunikation und Werbung. Firmenlogos, Ladennamen. Große, aufdringliche Plakate in den Straßen. Sind sie akzeptabel? Ja, meistens. Sind Graffiti akzeptabel? Manche Leute sagen ja, manche nein.

Wer zahlt den Preis für die Graffiti? Wer zahlt letzten Endes den Preis für die Werbung? Richtig! Der Verbraucher.

Haben die Leute, die Reklametafeln aufstellen, dich um Erlaubnis gebeten? Nein. Sollten also die Graffiti-Maler dies tun? Ist das nicht alles nur eine Frage der Kommunikation - der eigene Name, die Namen von Banden und die großen Kunstwerke auf offener Straße?

Denk mal an die gestreiften und karierten Kleider, die vor ein paar Jahren in den Läden auftauchten. Und an die Skibekleidung. Die Muster und die Farben waren direkt von den bunten Betonwänden geklaut. Es ist schon komisch, dass die Leute diese Muster und Farben akzeptieren und bewundern, während sie Graffiti in demselben Stil scheußlich finden.

Harte Zeiten für die Kunst.

Die beiden Briefe kommen aus dem Internet, und es geht in ihnen um Graffiti. Graffiti sind verbotene Malereien und Schrift an Wänden und anderswo. Beziehe dich auf die Briefe, um die anschließenden Fragen zu beantworten. 


\author{
Informationen \\ Ermitteln \\ KOMPETENZSTUFE
}

\section{FRAGE 12}

\section{GRAFFITI}

Warum verweist Sophia auf die Werbung?

Punktekategorie 1 (542*)

- Antworten, die erkennen lassen, dass ein Vergleich zwischen Graffiti und Werbung angestellt wird, und die dem Gedanken entsprechen, dass Werbung eine legale Form von Graffiti ist.

3 oder

- Antworten, die erkennen lassen, dass der Verweis auf die Werbung eine Strategie zur Verteidigung von Graffiti ist.

Zur Beantwortung dieser Frage müssen die

2 Schülerinnen und Schüler eine beabsichtigte Querverbindung in einem Text erkennen.

1

\section{FRAGE 11}

GRAFFITI

Die Absicht der beiden Briefe ist:

- A. zu erklären, was Graffiti sind;

B. Meinungen zu Graffiti zu äußern;

C. die Popularität von Graffiti zu beweisen;

D. den Leuten mitzuteilen, wie viel ausgegeben wird, um Graffiti zu entfernen.

Punktekategorie 1 (421*)

- B: Meinungen zu Graffiti zu äußern.

Zur Beantwortung dieser Aufgabe müssen die Schülerinnen und Schüler erkennen, welche Absicht zwei kurzen Briefen gemeinsam ist, indem sie ihr Hauptthema vergleichen.

\section{Textbezogenes \\ Interpretieren \\ KOMPETENZSTUFE}

\section{GRAFFITI}

Man kann darüber sprechen, was in einem Brief steht (seinen Inhalt).

Man kann über die Art und Weise sprechen, wie ein Brief geschrieben ist (seinen Stil).

Unabhängig davon, welchem Brief du

zustimmst:Welcher Brief ist deiner Meinung nach besser? Erkläre deine Antwort, indem du dich auf die Art und Weise beziehst, wie einer oder beide Briefe geschrieben sind.

Punktekategorie 1 (581*)

- Antworten, mit denen die eigene Meinung unter Bezugnahme auf den Stil oder die Form eines oder beider Briefe erklärt wird. Sie sollten sich auf Kriterien wie Schreibstil, Struktur der Argumentation, Stichhaltigkeit der Argumentation, Tonlage, Strategien zur Überzeugung der Leser beziehen. Ausdrücke wie „bessere Argumente“ müssen belegt werden.

Zur Beantwortung dieser Frage müssen die Schülerinnen und Schüler die Fähigkeit eines Autors beurteilen, indem sie zwei kurze Briefe zum Thema Graffiti vergleichen. Sie müssen sich dabei auf das 2 stützen, was sie selbst unter gutem Stil verstehen.

Reflektieren

und Bewerten KOMPETENZSTUFE

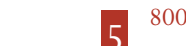

\section{FRAGE 13}

1

GRAFFITI

Welchem der beiden Briefe stimmst du zu? Begründe deine Antwort, indem du mit deinen eigenen Worten wiedergibst, was in einem

oder in beiden Briefen steht.

Punktekategorie 1 (471*)

- Antworten, mit denen der eigene Standpunkt unter Bezugnahme auf den Inhalt eines oder beider Briefe erklärt wird. Sie können sich auf die allgemeine Position der Autorin (also dafür oder dagegen) oder auf ein Detail ihres Arguments beziehen. Die Interpretation des Arguments der Autorin muss plausibel sein. Die Erklärung kann in Form einer Paraphrase eines Teils des Textes erfolgen, darf aber nicht ganz oder weitgehend ohne Veränderungen oder Zusätze abgeschrieben sein.

Zur Beantwortung dieser Frage müssen die Schülerinnen und Schüler die in zwei kurzen Texten ausgedrückten Meinungen mit ihren eigenen Ansichten und

Einstellungen vergleichen. Sie müssen auch unter Beweis stellen, dass sie zumindest einen der beiden Texte prinzipiell verstanden haben. 
KAPITEL 2 Was PISA über die Fähigkeiten 15-Jähriger aussagt - ein Profil der Schülerleistungen im Bereich Lesekompetenz

Abbildung 2.2 (Fortsetzung)

Eine Auswahl der in PISA eingesetzten Leseaufgaben

\section{ERWERBSTÄTIGE BEVÖLKERUNG}

Das folgende Baumdiagramm zeigt die Struktur der erwerbstätigen Bevölkerung bzw. der „Bevölkerung im Erwerbsalter“ eines Landes. Die Gesamtbevölkerung des Landes betrug 1995 etwa 3,4 Millionen.

Die Struktur der erwerbstätigen Bevölkerung, Stand: 31. März 1995 (in Tsd.) ${ }^{1}$

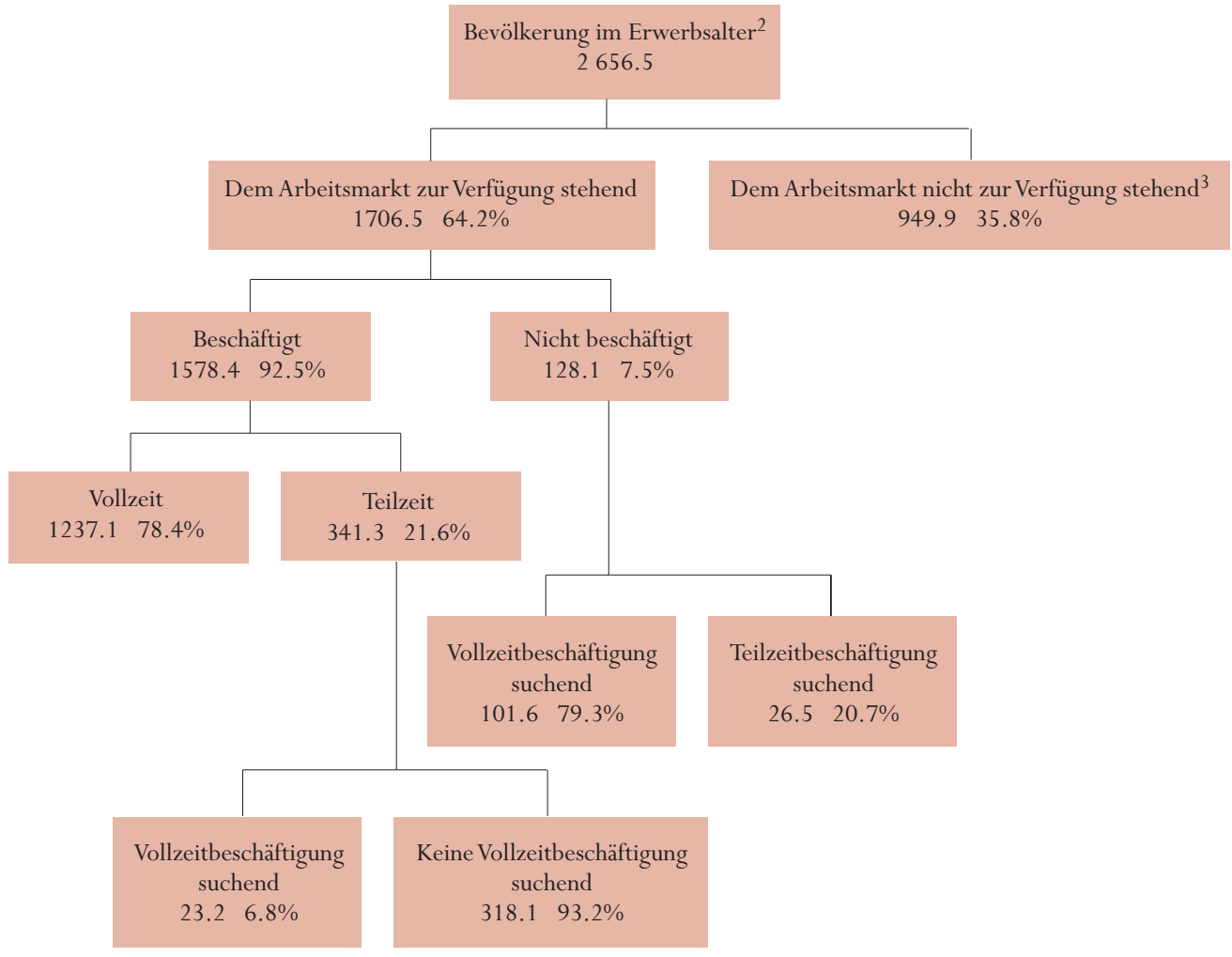

1. Anzahl der Personen in Tausend (Tsd.).

2. Die Bevölkerung im Erwerbsalter ist definiert als Menschen zwischen 15 und 65 Jahren.

3. Bezieht sich auf Personen, die nicht aktiv Arbeit suchen und/oder für Arbeit nicht zur Verfügung stehen.

Quelle: D. Miller, Form 6Eoonomics, ESA Publications, Box 9453, Newmarket,Auckland, NZ, S. 64.

Benutze die Informationen über die erwerbstätige Bevölkerung eines Landes, um die anschließenden Fragen zu beantworten. 
Was PISA über die Fähigkeiten 15-Jähriger aussagt - ein Profil der Schülerleistungen im Bereich Lesekompetenz KAPITEL 2

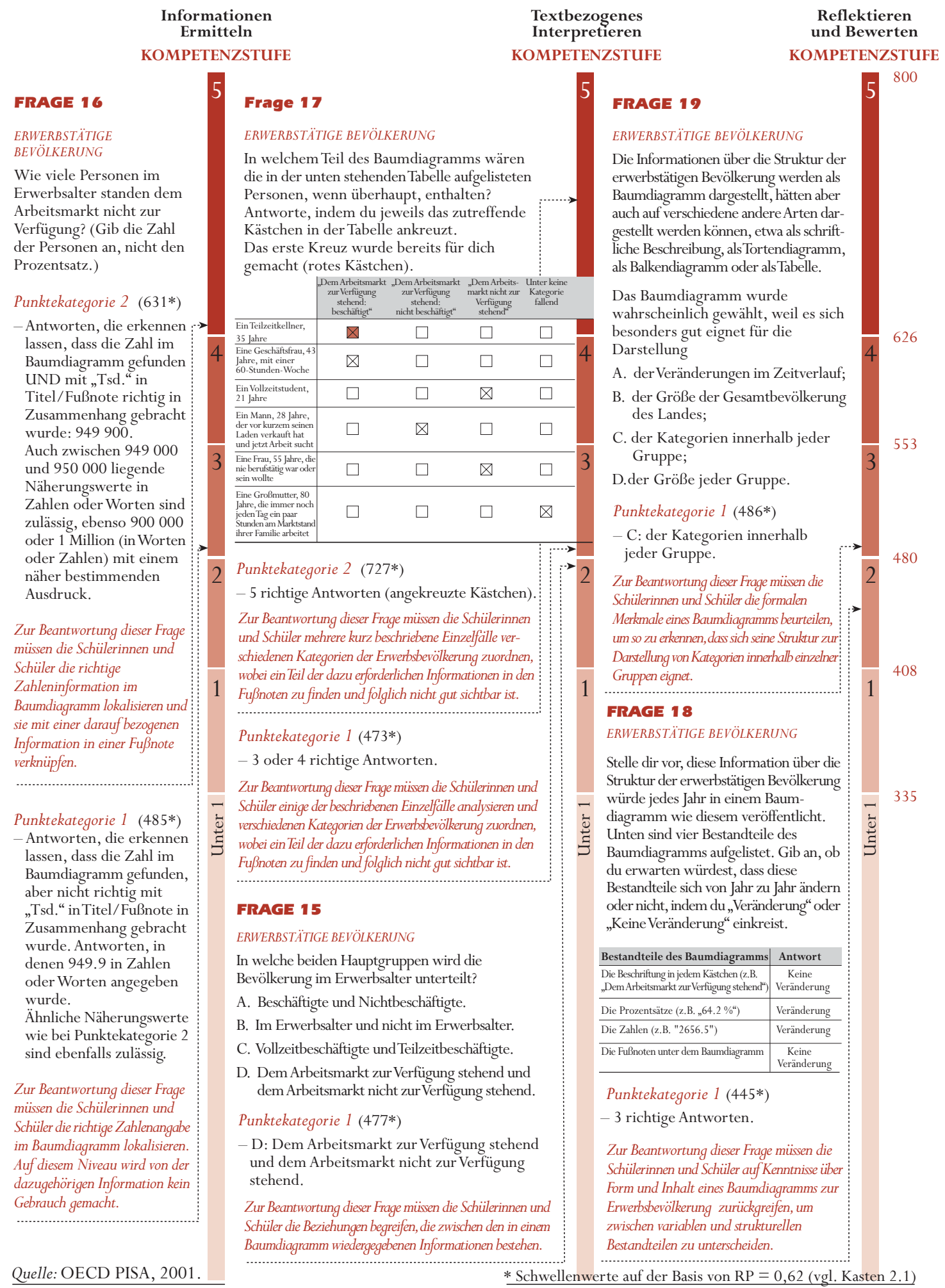


KAPITEL 2 Was PISA über die Fähigkeiten 15-Jähriger aussagt - ein Profil der Schülerleistungen im Bereich Lesekompetenz

Abbildung 2.2 (Fortsetzung)

Eine Auswahl der in PISA eingesetzten Leseaufgaben

Ein Mord wurde
begangen, aber der
Verdächtige streitet
alles ab. Er
behauptet, das Opfer
nicht zu kennen. Er
sagt, er habe ihn nie
gekannt, sei nie in
seiner Nähe gewesen,
hätte ihn nie
angerührt... Polizei
und Justiz sind
überzeugt, dass er
nicht die Wahrheit
sagt. Aber wie ist es
zu beweisen?

Am Tatort haben die

Ermittlungsbeamten jede noch so kleine denkbare Spur und mögliche Beweisstücke zusammengetragen: Gewebefasern, Haare, Fingerabdrücke, Zigarettenstummel ... Die wenigen auf dem Jackett des Opfers gefundenen Haare sind rot. Und sie sehen denen des Verdächtigen merkwürdig ähnlich. Wenn es bewiesen werden könnte, dass diese Haare tatsächlich von ihm stammen, wäre das ein Beweis, dass er dem Opfer doch begegnet war.

\section{Jedes Individuum ist einzigartig}

Die Spezialisten gehen an die Arbeit. Sie untersuchen einige Zellen an der Haarwurzel und ein paar Blutzellen des Verdächtigen. Im Kern jeder Zelle unseres Körpers befindet sich DNS. Was ist das? Die DNS ist wie eine Kette aus zwei

$$
\begin{gathered}
\text { Mikroskop in } \\
\text { einem Polizeilabor }
\end{gathered}
$$

\section{Wissenschaftliche Waffen der Polizei}

umeinander gedrehten Perlenreihen. Stelle dir vor, dass diese Perlen in vier verschiedenen Farben vorkommen und Tausende von Perlen (aus denen ein Gen besteht) in einer ganz bestimmten Reihenfolge aufgezogen sind. Bei jedem einzelnen Individuum ist diese Reihenfolge in allen Zellen des Körpers gleich: die von den Haarwurzeln genauso wie die vom großen Zeh, von der Leber sowie des Magens oder des Blutes. Aber die Reihenfolge der Perlen ist bei jedem Menschen anders. Die Wahrscheinlichkeit, dass zwei Menschen die gleiche DNS haben, ist angesichts der Anzahl derart aufgezogener Perlen sehr gering, mit Ausnahme von eineiigen Zwillingen. Einzigartig für jedes Individuum, ist die DNS damit eine Art genetischer Personalausweis.

Die Genetiker können deshalb den (in seinem Blut festgelegten) genetischen Personalausweis des Verdächtigen mit dem der rothaarigen Person vergleichen. Wenn der genetische Personalausweis derselbe ist, wissen sie, dass der Verdächtige doch in der Nähe des Opfers war, dem er angeblich nie begegnet ist.
Nur ein Beweisstück

Immer häufiger lässt die Polizei bei sexuellen Vergehen, Mord, Diebstahl oder anderen Verbrechen genetische Analysen durchführen. Warum? Um zu versuchen, Beweise dafür zu finden, dass zwei Menschen, zwei

Gegenstände oder ein Mensch und ein Gegenstand miteinander in Berührung gekommen sind. Der Nachweis eines solchen Kontakts ist für die Ermittlungen oft sehr nützlich. Er liefert aber nicht unbedingt den Beweis für ein Verbrechen. Er ist nur ein Beweisstück unter vielen anderen.

Anne Versailles

\section{Wir bestehen aus Milliarden von Zellen}

Jedes Lebewesen besteht aus sehr vielen Zellen. Eine Zelle ist unendlich klein. Man kann sogar sagen „mikroskopisch klein“, da man sie nur mit Hilfe eines Mikroskops sehen kann, das sie um ein Vielfaches vergrößert. Jede Zelle hat eine äußere Hülle und einen Kern, in dem sich die DNS befindet.

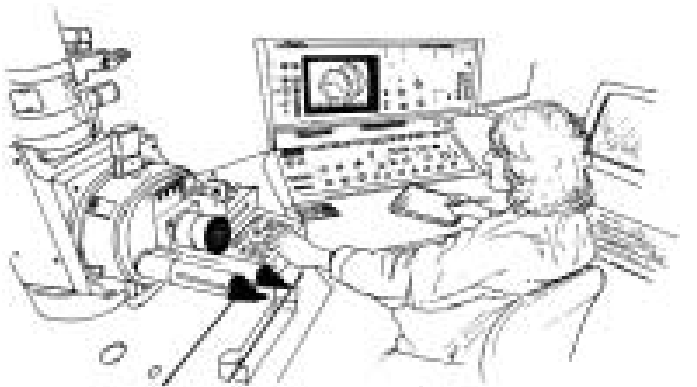

\section{Genetischer WAS?}

Die DNS besteht aus mehreren Genen, von denen jedes aus Tausenden von „Perlen“ gebildet wird. Zusammen bilden diese Gene den genetischen Personalausweis eines Menschen.

Wie findet man den genetischen Personalausweis?

Der Genetiker nimmt die wenigen Zellen von den Wurzeln der Haare, die bei dem Opfer gefunden wurden, oder aus dem Speichel, der an einem Zigarettenstummel haftet. Er taucht sie in eine Substanz, die alles zerstört, was sich um die DNS dieser Zellen herum befindet. Dasselbe macht er dann mit einigen Zellen aus dem Blut des Verdächtigen. Die DNS wird dann speziell für die Analyse vorbereitet. Danach kommt sie in ein spezielles Gel, und durch das Gel wird elektrischer Strom geleitet. Nach ein paar Stunden entstehen dadurch Streifen, ähnlich wie bei einem Strichcode (wie aufWaren, die wir kaufen), die unter einer speziellen Lampe sichtbar werden. Den Strichcode der DNS des Verdächtigen vergleicht man dann mit dem der Haare, die bei dem Opfer gefunden wurden.

Beziehe dich auf den Zeitschriftenartikel, um die anschließenden Fragen zu beantworten. 


Infor
KOMPE
FRAGE 22
WISSENSCHAFTLICHE WAFFEN DER
POLIZEI
Um die Struktur der DNS zu
erklären, spricht der Autor von
einer Perlenkette. Wodurch
unterscheiden sich diese
Perlenketten bei verschiedenen
Menschen?
A. Sie sind von unterschied-
licher Länge.
B. Die Reihenfolge der Perlen
ist unterschiedlich.
C. Die Anzahl der Ketten ist
unterschiedlich.
D. Die Farbe der Perlen ist
unterschiedlich.
Punktekategorie 1 (515*)
- B: Die Reihenfolge der Perlen
ist unterschiedlich.
Zur Beantwortung dieser Frage müssen
die Schülerinnen und Schüler eine
Information lokalisieren, die in einem
Artikel einer wissenschaftlichen
Zeitschriff für junge Leser enthalten
ist, indem sie zwischen konkurrierenden
Informationen die richtige, synonym
ausgedrückte Antwort identifizieren.
-

Quelle: OECD PISA, 2001. formationen

Ermitteln

\section{KOMPETENZSTUFE}

5
4
4

\section{FRAGE 23}

WISSENSCHAFTLICHE WAFFEN DER POLIZEI

Welchen Zweck hat der Kasten mit der Überschrift: „Wie findet man den genetischen Personalausweis?"

Er soll erklären,

A. was die DNS ist;

B. was ein Strichcode ist;

C. wie Zellen analysiert werden, um das Muster der DNS zu finden;

D. wie bewiesen werden kann, dass ein Verbrechen begangen worden ist.

Punktekategorie 1 (518*)

- C: wie Zellen analysiert werden, um das Muster der DNS zu finden.

Zur Beantwortung dieser Frage müssen die Schülerinnen und Schüler die passende Zusammenfassung eines klar definierten Abschnitts eines Artikels identifizieren, der in einer wissenschaftlichen Zeitschrift für junge Leser erschienen ist, indem sie die in mehreren Sätzen enthaltenen Informationen zusammenfügen. Einige konkurrierende Informationen sind im Text enthalten.

\section{FRAGE 24}

WISSENSCHAFTLICHE WAFFEN DER POLIZEI

Was ist das Hauptziel des Autors?

A. Zu warnen.

B. Zu unterhalten

C. Zu informieren.

D. Zu überzeugen

Punktekategorie 1 (406*)

- C: Zu informieren.

Zur Beantwortung dieser Frage müssen die Schülerinnen und Schüler die allgemeine Absicht des Autors eines Artikels aus einer wissenschaftlichen Zeitschrift für junge Leser erkennen.

\section{FRAGE 25}

WISSENSCHAFTLICHE WAFFEN DER POLIZEI

Am Schluss der Einführung (erster grau unterlegter Kasten) heißt es: „Aber wie ist es zu beweisen?"

Im Text steht, dass die Ermittlungsbeamten eine Antwort auf diese Frage zu finden suchen, indem sie

A. Zeugen befragen;

B. Gentests durchführen;

C. den Verdächtigen gründlich verhören;

D. sämtliche Ermittlungsergebnisse noch einmal durchgehen.

Punktekategorie 1 (402*)

- B: Gentests durchführen.

Zur Beantwortung dieser Frage müssen die Schülerinnen und Schüler die in verschiedenen Absätzen enthaltene Information zusammenfügen, um das Hauptthema zu identifizieren, das sich durch den ganzen Artikel einer wissenschaftlichen Zeitschrift für junge Leser zieht.
Reflektieren

$\begin{array}{lc}\text { Interpretieren } & \text { und Bewerten } \\ \text { MPETENZSTUFE } & \text { KOMPETENZSTUFE }\end{array}$

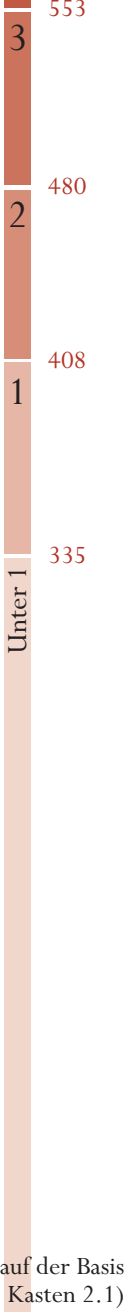

* Schwellenwerte auf der Basis von $\mathrm{RP}=0,62(\operatorname{vgl}$. Kasten 2.1$)$ 
KAPITEL 2 Was PISA über die Fähigkeiten 15-Jähriger aussagt - ein Profil der Schülerleistungen im Bereich Lesekompetenz

Abbildung 2.3 zeigt einen Überblick über die Leistungen auf der Gesamtskala Lesekompetenz (vgl. auch Tabelle 2.1a), wobei die Länge der Balken den prozentualen Anteil der Schülerinnen und Schüler angibt, die die Anforderungen der jeweiligen Kompetenzstufen erfüllen.

\section{Kompetenzstufe 5 (über 625 Punkte)}

Auf Kompetenzstufe 5 sind die Schülerinnen und Schüler in der Lage, anspruchsvolle Leseaufgaben zu lösen, ...

... die Fähigkeiten erfordern, die in einer wissensbasierten Wirtschaft unerlässlich

sind.

In einigen Ländern erreichen über $15 \%$, in

andern jedoch nur

weniger als 5\% der

Schülerinnen und

schüler Stufe 5.

Die Länder, in denen viele Schülerinnen und

schüler Stufe 5

erreichen, sind nicht

immer jene mit dem

geringsten Anteil an

leistungsschwachen

schülerinnen und

schülern.
Schülerinnen und Schüler, die die Anforderungen von Stufe 5 der Gesamtskala Lesekompetenz erfüllen, sind in der Lage, anspruchsvolle Leseaufgaben zu lösen, d.h. mit Informationen umzugehen, die in ungewohnten Texten nur schwer zu finden sind, ein genaues Verständnis dieser Texte nachzuweisen und herauszufinden, welche der im Text enthaltenen Informationen für die Aufgabe von Belang sind, sowie einen Text kritisch zu bewerten und Hypothesen aufzustellen, dabei Fachwissen heranzuziehen und Konzepte zu begreifen, die u.U. im Widerspruch zu den eigenen Erwartungen stehen. Vgl. Abbildung 2.1 wegen einer genaueren Beschreibung.

Schülerinnen und Schüler, die den Anforderungen der höchsten PISAKompetenzstufe genügen, werden die Begabungsreserven ihres Landes vergrößern. Der Prozentsatz der Schülerinnen und Schüler, die heute dieses Leistungsniveau erreichen, dürfte auch Einfluss darauf haben, welchen Beitrag das jeweilige Land zum künftigen Gesamtangebot von Wissenschaftlern der Spitzenklasse in der Weltwirtschaft wird leisten können. Ein Vergleich der Prozentsätze der Schülerinnen und Schüler, die die höchste Lesekompetenzstufe erreichen, ist daher bereits für sich genommen von Interesse.

Im OECD-Raum erfüllen 10\% der in PISA 2000 erfassten Schülerinnen und Schüler die Anforderungen von Stufe 5. In Australien, Kanada, Finnland, Neuseeland und dem Vereinigten Königreich sind es über 15\% und in Belgien, Irland und den Vereinigten Staaten mindestens 12\%; in Brasilien, Griechenland, Lettland, Luxemburg, Mexiko, Portugal, der Russischen Föderation und Spanien erreichen hingegen nur 5\% oder weniger diese Stufe (Tabelle 2.1a).

Bei der Betrachtung dieser Ergebnisse darf nicht vergessen werden, dass nicht nur die Gesamtleistung des Landes im Bereich der Lesekompetenz ausschlaggebend ist für den Prozentsatz der Schülerinnen und Schüler, die Stufe 5 erreichen, sondern auch die innerhalb dieses Landes bestehenden Leistungsdifferenzen zwischen den Schülerinnen und Schülern mit den höchsten und den niedrigsten Ergebnissen. Auch wenn in Ländern mit einem höheren Schüleranteil auf Stufe 5 die generelle Tendenz besteht, einen geringeren Anteil an Schülerinnen und Schülern auf Stufe 1 oder darunter aufzuweisen, ist das aber keineswegs immer der Fall. Es trifft beispielsweise für Finnland zu, wo 18\% der Schülerinnen und Schüler Stufe 5 erreichen, während nur 2\% unter Stufe 1 liegen. Dagegen haben Belgien und die Vereinigten Staaten z.B., die ebenfalls einen überdurchschnittlich hohen Prozentsatz an Schülerinnen und Schülern auf Stufe 5 vorweisen können, zugleich einen relativ hohen Prozentsatz unter Stufe 1 (8\% bzw. 6\%). In Korea hingegen, das zu den Ländern gehört, die in 
Was PISA über die Fähigkeiten 15-Jähriger aussagt - ein Profil der Schülerleistungen im Bereich Lesekompetenz KAPITEL 2

\section{Abbildung 2.3}

Leistungsverteilung der Schülerinnen und Schüler auf den verschiedenen Kompetenzstufen der Gesamtskala Lesekompetenz

Prozentsatz der Schülerinnen und Schüler:

\% Unter Stufe 1 Auf Stufe 1 Auf Stufe 2 Auf Stufe 3 Auf Stufe 4 Auf Stufe 5

100

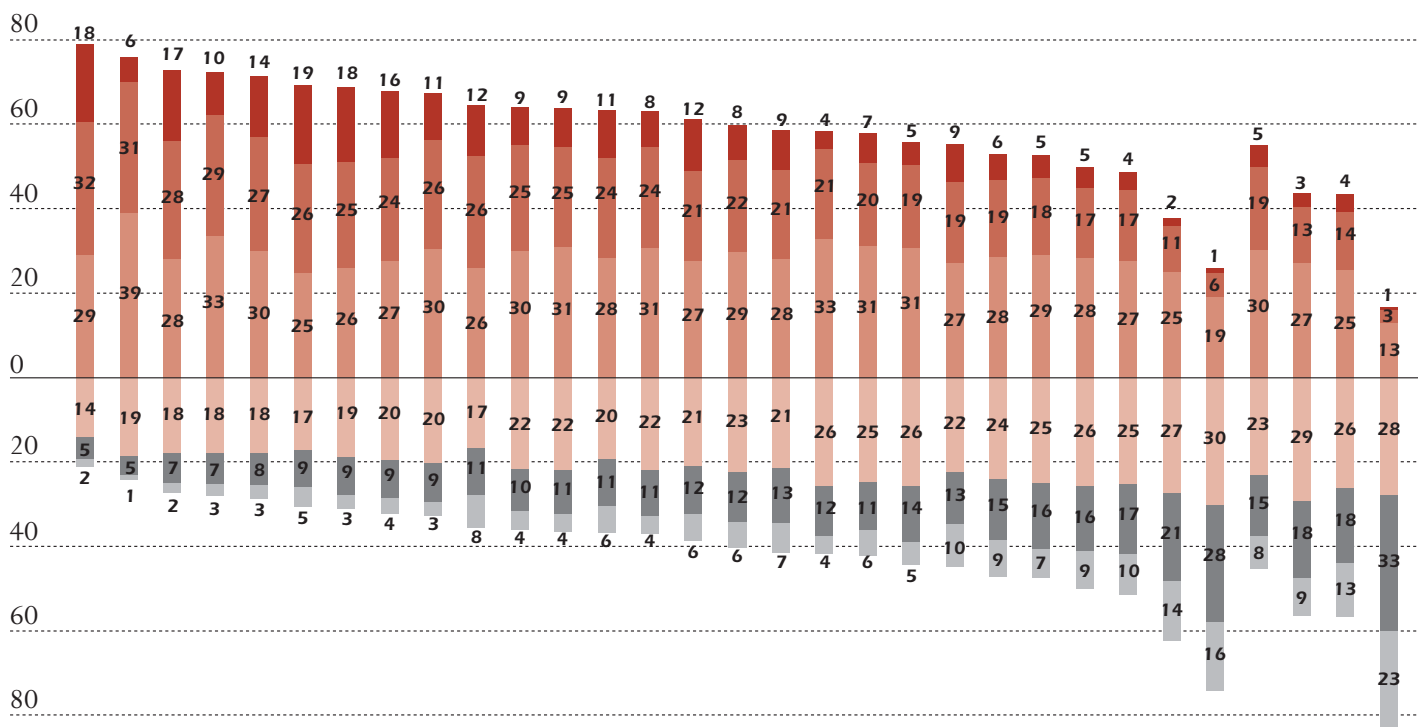

100

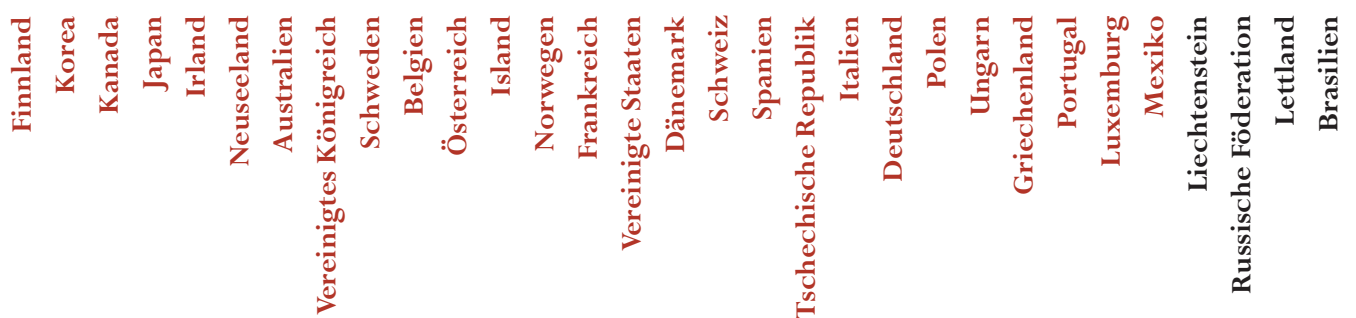

PISA in allen drei Bereichen sehr gut abschneiden, erreichen zwar weniger als $6 \%$ der Schülerinnen und Schüler Stufe 5, dafür liegen dort aber nicht einmal $1 \%$ unter Stufe 1.

Die Betrachtung der drei Komponenten der Gesamtskala Lesekompetenz liefert ein noch differenzierteres Bild, insbesondere was die Länder mit einem überdurchschnittlich hohen Schüleranteil auf Stufe 5 anbelangt. In Finnland erreichen z.B. 26\% der Schülerinnen und Schüler Stufe 5 auf der Skala „Informationen ermitteln“ (Tabelle 2.1b), jedoch nur 14\% auf der Skala „Reflektieren und Bewerten“ (OECD-Durchschnitt: 11\%) (Tabelle 2.1d). Ein

Der Prozentsatz der Schülerinnen und schüler auf Stufe 5 schwankt zwischen den verschiedenen Aspekten der Lesekompetenz. 
KAPITEL 2 Was PISA über die Fähigkeiten 15-Jähriger aussagt - ein Profil der Schülerleistungen im Bereich Lesekompetenz

ähnliches Bild ergibt sich, wenngleich weniger stark ausgeprägt, auch für Australien, Belgien und Schweden. In Kanada und dem Vereinigten Königreich werden hingegen höhere Leistungen auf der Skala „Reflektieren und Bewerten“ verzeichnet als auf den Skalen „Informationen ermitteln“ und „Textbezogenes Interpretieren“. Dies legt den Schluss nahe, dass die in diesen Ländern erzielte hohe Gesamtleistung z.T. auf die guten Schülerergebnisse bei Aufgaben zurückzuführen ist, bei denen eine kritische Auseinandersetzung, der Einsatz von Hypothesen und die Verbindung der Texte mit eigenen Erfahrungen, Kenntnissen und Vorstellungen erforderlich ist (Tabelle 2.1b, c und d) ${ }^{4}$.

Bei den Ländern mit dem geringsten Prozentsatz an Schülerinnen und Schülern, die auf der Gesamtskala Lesekompetenz Stufe 5 erreichen, lässt sich für Griechenland beispielsweise feststellen, dass zwar nur 4\% den Anforderungen von Stufe 5 der Skala „Informationen ermitteln“ genügen, jedoch dreimal so viele, d.h. 12\% (OECD-Durchschnitt: 11\%), diese Stufe auf der Skala „Reflektieren und Bewerten“ erreichen (Tabelle 2.1b und d).

Kompetenzstufe 4 (553 bis 625 Punkte)

Die Aufgaben von Stufe 4 sind noch immer komplex und schwierig,

können aber von rund einem Drittel aller

Schülerinnen und schüler beantwortet werden, ...

Unterschiede zwischen den drei Aspekten der Lesekompetenz weniger stark ausgeprägt sind.
Schülerinnen und Schüler, deren Leistungen Stufe 4 auf der Gesamtskala Lesekompetenz entsprechen, sind in der Lage, schwierige Leseaufgaben zu lösen, beispielsweise eingebettete Informationen zu finden, den Sinn sprachlicher Nuancen zu verstehen und einen Text kritisch zu bewerten (vgl. Abb. 2.1 wegen einer genaueren Beschreibung). Im OECD-Raum erreichen 31\% der Schülerinnen und Schüler mindestens Stufe 4 (d.h. Schülerinnen und Schüler auf Stufe 4 und 5 zusammengenommen) (Tabelle 2.1a). In Finnland erreichen über die Hälfte, in Australien, Kanada, Irland, Neuseeland und dem Vereinigten Königreich 40\% oder mehr mindestens Stufe 4. Außer in Luxemburg und Mexiko erfüllt wenigstens einer von fünf Schülern in den OECD-Ländern mindestens die Leistungsanforderungen von Stufe 4. In Brasilien, wo im Bereich der Lesekompetenz insgesamt die niedrigste Leistung gemessen wurde, erreichen nur 4\% der Schülerinnen und Schüler Stufe 4 oder 5.

Auf Stufe 4 sind die Leistungsunterschiede zwischen den drei Subskalen der Skala Lesekompetenz in der Regel weniger stark ausgeprägt als auf Stufe 5. Allerdings ist in Brasilien der Prozentsatz der Schülerinnen und Schüler auf mindestens Stufe 4 der Skala „Reflektieren und Bewerten“ mit 7\% mehr als doppelt so hoch wie derjenige der Schülerinnen und Schüler, die diese Stufe auf der Skala „Informationen ermitteln“ erreichen (3\%) (Tabelle 2.1b, c und d). In Griechenland, Mexiko, Portugal und Spanien beträgt der Unterschied 6 Prozentpunkte oder mehr. In Belgien, Finnland, Frankreich und Liechtenstein verhält sich es sich umgekehrt. 
Kompetenzstufe 3 (481 bis 552 Punkte)

Auf Stufe 3 der Gesamtskala Lesekompetenz sind die Schülerinnen und Schüler in der Lage, Leseaufgaben mittleren Komplexitätsgrads zu lösen, z.B. mehrere Informationen aufzufinden, Verbindungen zwischen verschiedenen Abschnitten eines Textes herzustellen und den Text zu Alltagswissen in Beziehung zu setzen (vgl.Abb. 2.1 wegen einer genaueren Beschreibung). Im OECD-Raum erreichen 60\% der Schülerinnen und Schüler mindestens Stufe 3 (d.h. Stufe 3, 4 oder 5) auf der Gesamtskala Lesekompetenz (Tabelle 2.1a). In 9 von 27 OECD-Ländern erbringen zwischen zwei Dritteln und 80\% der 15-jährigen Schülerinnen und Schüler mindestens die für Stufe 3 erforderlichen Leistungen.

Zur Beantwortung der Frage, inwieweit sich die Leistungsstrukturen in den verschiedenen Ländern ähneln, empfiehlt es sich, einen Blick auf die neun Länder zu werfen, in denen zwei Drittel bis etwas über drei Viertel der Schülerinnen und Schüler Stufe 3 oder darüber erreichen. Es handelt sich hierbei der Reihenfolge nach um Finnland, Korea, Kanada, Japan, Irland, Neuseeland, Australien, das Vereinigte Königreich und Schweden. Wie schneiden diese Länder auf den anderen Kompetenzstufen ab? In einem von ihnen, Finnland, verfügt ein relativ großer Prozentsatz der Schülerinnen und Schüler über hohe Lesekompetenzen (18\% erreichen Stufe 5, gegenüber 10\% im OECDDurchschnitt) und gleichzeitig liegen vergleichsweise viele über der untersten Stufe (in Finnland liegen nur 7\% auf Stufe 1 oder darunter). Finnland erzielt folglich auf der Gesamtskala Lesekompetenz insgesamt sehr hohe Ergebnisse.

In fünf weiteren Ländern, Australien, Kanada, Irland, Neuseeland und dem Vereinigten Königreich, erreichen zwar viele Schülerinnen und Schüler die höchste Stufe (zwischen 14\% und 19\%), doch dafür ist der Prozentsatz derjenigen, deren Leistungen nur Stufe 1 oder darunter entsprechen, höher als in Finnland: Er liegt zwischen 10\% und 14\% (der OECD-Durchschnitt beträgt 18\%). Diesen Ländern gelingt es also, einerseits bei vielen Schülerinnen und Schülern ein hohes Leistungsniveau zu erzielen, sie haben andererseits jedoch weniger Erfolg bei der Reduzierung des Anteils der leistungsschwachen Schülerinnen und Schüler. In Neuseeland erreichen zwar mehr Schülerinnen und Schüler als in jedem anderen Land Stufe 5 (19\%), doch dafür gehen die Leistungen eines relativ hohen Prozentsatzes (14\%) nicht über die Anforderungen von Stufe 1 hinaus.

Die Ergebnisse Koreas beweisen, dass niedrige Leistungsdifferenzen in der Verteilung der Grundqualifikationen auf relativ hohem Niveau ein erreichbares Ziel darstellen: Drei Viertel der Schülerinnen und Schüler erfüllen in Korea zumindest die Anforderungen von Stufe 3, und nur 6\% liegen auf Stufe 1 oder darunter. Ebenso wie in Korea gibt es auch in Japan viele Schülerinnen und Schüler, die mindestens Stufe 3 erreichen, hingegen nur vergleichsweise wenige auf der höchsten oder niedrigsten Stufe. In Schweden schließlich erreichen zwei Drittel der Schülerinnen und Schüler mindestens die Anforderungen
Drei von fünfSchülern

können Leseaufgaben

mit mittlerem

Schwierigkeitsgrad lösen;

in Finnland sind dazu

bis zu vier von fünf

schülern in der Lage,...

... doch unterscheiden

sich die Länder mit dem

größsten Anteil an

Schülerinnen und

schülern, die mindestens

Stufe 3 erreichen, in

anderer Hinsicht.

In einigen Ländern mit vielen leistungsstarken

schülerinnen und

schülern im Bereich

Lesekompetenz gibt es

jeweils auch einen nicht

geringen Anteil an

leistungsschwachen

schülerinnen und

schülern,...

... während in anderen

Ländern die großse

Mehrheit eher

leistungshomogener ist. 
KAPITEL 2 Was PISA über die Fähigkeiten 15-Jähriger aussagt - ein Profil der Schülerleistungen im Bereich Lesekompetenz

von Stufe 3, die Zahl der besonders leistungsstarken bzw. leistungsschwachen Schülerinnen und Schüler liegt dort aber näher an den Durchschnittswerten.

Kompetenzstufe 2 (408 bis 480 Punkte)

Insgesamt können vier von fünf Schülerinnen und Schülern elementare Leseaufgaben lösen, und

es gibt kein Land, in

dem nicht mindestens die Hälfte dazu fähig ist.

Auch für die einfachsten PISA-Aufgaben müssen die Schülerinnen und schüler mehr leisten, als nur fließsend lesen zu können, ...
Auf Stufe 2 sind die Schülerinnen und Schüler in der Lage, grundlegende Leseaufgaben zu lösen, z.B. eindeutige Informationen zu finden, wenig anspruchsvolle Schlussfolgerungen verschiedener Art zu ziehen, die Bedeutung eines genau definierten Textteils zu erkennen und gewisse externe Kenntnisse zu dessen Verständnis heranzuziehen (vgl.Abb. 2.1 eine für genauere Beschreibung). Im OECD-Raum erfüllen oder übertreffen $82 \%$ der Schülerinnen und Schüler die Anforderungen von Stufe 2 der Gesamtskala Lesekompetenz. In allen OECD-Ländern entsprechen die Leistungen von mindestens einem von zwei Schülern Stufe 2 oder darüber (Tabelle 2.1a).

In Spanien erreichen nur 4\% der Schülerinnen und Schüler Stufe 5, überdurchschnittlich viele (84\%) jedoch mindestens Stufe 2 (Tabelle 2.1a). Interessant ist die Gegenüberstellung der spanischen Ergebnisse mit denen Neuseelands: Während der Prozentsatz der Schülerinnen und Schüler, deren Leistung in beiden Ländern mindestens Stufe 2 entspricht, fast gleich ist (84\% und $86 \%$ ), ist der Schüleranteil mit Stufe 5 in Neuseeland fast fünfmal so hoch wie in Spanien. Im Gegensatz dazu liegt in Spanien bei einem besonders großen Teil der Schülerinnen und Schüler (42\%) das maximale Leistungsniveau bei Stufe 2.

Kompetenzstufe 1 (335 bis 407 Punkte) oder darunter (weniger als 335 Punkte)

Beim PISA-Konzept der Lesekompetenz liegt der Schwerpunkt auf den Kenntnissen und Fähigkeiten, die für das „Lesen, um zu lernen“ notwendig sind im Gegensatz zu den technischen Fertigkeiten des „Lesenlernens“. Da in den OECD-Ländern nur vergleichsweise wenige junge Erwachsene die rein „technischen“ Lesefertigkeiten nicht erworben haben, soll mit PISA daher nicht gemessen werden, inwieweit 15-Jährige fließend lesen können, oder Wörter richtig erkennen können. In Einklang mit den meisten gegenwärtig anerkannten Definitionen der Lesekompetenz zielt PISA darauf ab festzustellen, inwieweit die Schülerinnen und Schüler in der Lage sind, die Bedeutung eines breiten Spektrums von Texten aus allgemeinen schulischen wie außerschulischen Kontexten zu erfassen, zu erweitern und darüber zu reflektieren. Die einfachsten Aufgaben, die diesem Konzept der Lesekompetenz gerecht werden, sind jene auf Stufe 1. Schülerinnen und Schüler, deren Leistungen nur den Anforderungen dieser Stufe entsprechen, können nur die einfachsten der für PISA ausgearbeiteten Leseaufgaben lösen, z.B. eine Einzelinformation finden, das Hauptthema eines Textes erkennen oder eine einfache Verbindung zu Alltagskenntnissen ziehen (vgl. Abb. 2.1 wegen einer genaueren Beschreibung). 
Schülerinnen und Schüler, die weniger als 335 Punkte erziehlen, Stufe 1 also nicht erreichen, sind nicht in der Lage, die elementarsten Lesekompetenzen nachzuweisen, die in PISA gemessen werden. Dies soll nicht heißen, dass die fraglichen Schülerinnen und Schüler über keinerlei Grundqualifikationen verfügen. Die meisten dieser Schülerinnen und Schüler dürften wohl im „technischen“ Sinne durchaus lesen können, und die Mehrzahl von ihnen (54\% im OECD-Durchschnitt ${ }^{5}$ ) sind in der Lage, mindestens 10\% der in PISA 2000 gestellten offenen Fragen, d.h. jenen, bei denen keine Multiplechoice-Antworten vorgegeben sind ${ }^{6}$, erfolgreich zu lösen $(6 \%$ gelang es, ein Viertel dieser Aufgaben richtig zu lösen). Das Muster ihrer Antworten bei den Testaufgaben lässt jedoch darauf schließen, dass sie weniger als die Hälfte der Fragen eines Tests mit ausschließlich Stufe 1 entsprechenden Aufgaben beantworten können und den Anforderungen von Stufe 1 folglich nicht gerecht werden. Solchen Schülerinnen und Schülern bereitet es große Schwierigkeiten, Lesekompetenzen als ein effizientes Mittel zur Förderung und Erweiterung ihrer Kenntnisse und Fähigkeiten in anderen Bereichen einzusetzen. Für Schülerinnen und Schüler, deren Grundqualifikationen unter Stufe 1 liegen, besteht daher nicht nur die Gefahr, dass sie beim Übergang vom Schul- ins Arbeitsleben großen Problemen gegenüberstehen, sondern auch, dass sie in ihrem weiteren Leben Möglichkeiten zur Fort- und Weiterbildung nicht nutzen können.

Wenn in einem Bildungssystem die Leistung eines hohen Prozentsatzes der Schülerinnen und Schüler unter oder gerade noch bei Stufe 1 liegt, steht zu befürchten, dass ein großer Teil der Schülerinnen und Schüler u.U. nicht die erforderlichen Grundkenntnisse und -fähigkeiten erwirbt, um aus den ihnen gebotenen Bildungsmöglichkeiten Nutzen zu ziehen. Diese Situation ist umso besorgniserregender, als zahlreiche Anzeichen dafür sprechen, dass es äußerst schwierig ist, in der Schulzeit entstandene Bildungslücken im späteren Leben zu schließen. Aus OECD-Daten geht im Gegenteil hervor, dass die zwischen den einzelnen Schulabgängern bestehenden Qualifikationsunterschiede im Rahmen der berufsbezogenen Aus- und Fortbildung noch verschärft werden (OECD, 2001). Zwischen dem Grundqualifikationsniveau der Erwachsenen und deren Beteiligung an Fort- und Weiterbildung bestehen enge Zusammenhänge, selbst wenn man auch andere Merkmale kontrolliert, die Einfluss auf die Teilnahme an derartigen Maßnahmen haben. Grundqualifikationen und Ausund Fortbildung scheinen sich gegenseitig zu verstärken, was einer der Gründe dafür ist, dass gerade jene, die sie am nötigsten haben, am seltensten an Weiterbildungsmaßnahmen im Erwachsenenalter teilnehmen.

Im OECD-Raum liegen die Leistungen von insgesamt 12\% der Schülerinnen und Schüler bei Stufe 1 und von 6\% unter Stufe 1, es bestehen jedoch große Unterschiede zwischen den einzelnen Ländern. In Finnland und Korea liegt die Leistung von nur ca. 5\% der Schülerinnen und Schüler auf Stufe 1, und weniger als 2\% liegen darunter. Diese beiden Länder bilden jedoch Ausnahmen. In allen anderen OECD-Ländern liegen zwischen 10\% und 44\% der Schülerinnen und Schüler auf Stufe 1 oder darunter (Tabelle 2.1a). Von ihnen erfüllen über
... so dass Schülerinnen und Schüler, die Stufe

1 nicht erreichen, zwar technisch gesehen durchaus des Lesens mächtig sein können, jedoch im späteren Leben wahrscheinlich großse schwierigkeiten haben werden ...
Der Prozentsatz der Schülerinnen und schüler auf oder unter Stufe 1 variiert stark von Land zu Land, von wenigen Prozent bis zu nahezu der Hälfte, ... 
KAPITEL 2 Was PISA über die Fähigkeiten 15-Jähriger aussagt - ein Profil der Schülerleistungen im Bereich Lesekompetenz

... und in einigen

Ländern erreicht eine

nicht unerhebliche

Minderheit nicht einmal

Stufe 1.

Die Mehrzahl der Schüler auf Stufe 1 ist männlich, stammt aus sozial benachteiligten

Milieus oder hat im

Ausland geborene

Eltern, was vor allem für einige der Länder mit

besonders vielen

leistungsschwachen schülern gilt.
$2 \%$ - in der Hälfte der OECD-Länder sogar über 5\% - nicht einmal die Anforderungen von Stufe 1.

Die Länder, in denen über 20\% der Schülerinnen und Schüler auf Stufe 1 oder darunter liegen, sind der Reihenfolge nach Brasilien, Mexiko, Luxemburg, Lettland, die Russische Föderation, Portugal, Griechenland, Polen, Ungarn, Deutschland, Liechtenstein und die Schweiz. In Brasilien, Mexiko, Luxemburg, Portugal und Deutschland erreichen zwischen 10\% und 23\% nicht einmal Stufe 1, was bedeutet, dass sie nicht in der Lage sind, die elementarsten der in PISA gemessenen Fähigkeiten routinemäßig nachzuweisen. Besonders auffällig ist dies für Deutschland, das den relativ hohen Anteil von 9\% an Schülerinnen und Schüler auf Stufe 5 aufweist.

Bei den Schülerinnen und Schülern, die auf Stufe 1 bzw. darunter liegen, handelt es sich nicht um eine zufällig zusammengesetzte Gruppe. Obwohl sich die besonderen Merkmale dieser Schülerinnen und Schüler am besten im nationalen Kontext untersuchen lassen, stechen doch einige Gemeinsamkeiten ins Auge: In fast allen Ländern ist die Mehrzahl dieser Schüler männlich (vgl. Tabelle 5.2a), und viele kommen aus einem sozial benachteiligten Milieu. In zahlreichen Ländern stammt ein relativ großerTeil der Schüler auf Stufe 1 oder darunter zudem aus dem Ausland bzw. hat ausländische Eltern. In Deutschland und Luxemburg, zwei der vier Länder mit dem höchsten Schüleranteil auf bzw. unter Stufe 1, sind über 26\% bzw. 34\% dieser Schüler ausländischer Herkunft, während dies nur bei 8\% bzw. 11\% derjenigen der Fall ist, die über Stufe 1 liegen. ${ }^{7}$ In Kapitel 5 werden die geschlechtsspezifischen Unterschiede bei den leistungsschwächsten Schülern näher untersucht; die Hintergrundmerkmale der Schülerinnen und Schüler mit hohem und mit niedrigem Leistungsniveau werden in Kapitel 6 und 7 beleuchtet.

Ebenso wie am oberen Ende der Leistungsskala sind auch bei Leistungen auf Stufe 1 und darunter große Unterschiede zwischen den drei Lesekompetenzskalen zu beobachten. In Griechenland, Mexiko, Portugal und Spanien werden die größten Defizite auf der Subskala „Informationen ermitteln“ verzeichnet, wobei der Anteil der Schülerinnen und Schüler bei oder unter Stufe 1 zwischen 5 und 15 Prozentpunkte höher ist als auf der Subskala „Reflektieren und Bewerten“. In Frankreich, Deutschland und der Schweiz hingegen ist der Schüleranteil auf oder unter Stufe 1 auf der Skala „Informationen ermitteln“ mindestens 2 Prozentpunkte niedriger als auf der Skala „Reflektieren und Bewerten“. In Brasilien erreicht über die Hälfte der Schülerinnen und Schüler nicht mehr als Stufe 1 auf der Gesamtskala Lesekompetenz. Auf der Skala „Informationen ermitteln“ kommen über zwei Drittel der Schülerinnen und Schüler in Brasilien nicht über Stufe 1 hinaus, während dieser Anteil auf der Skala „Reflektieren und Bewerten“ nur bei 46\% liegt (Tabellen 2.1b, c und d). 


\section{Erwartungen an die Schülerleistungen}

Beim Vergleich solcher Daten zwischen einzelnen Ländern muss berücksichtigt werden, dass die Bildungssysteme unterschiedlichen wirtschaftlichen Bedingungen unterworfen sind und dass Lehrkräfte, Schulen und die Gesellschaft im weitesten Sinne u.U. ganz unterschiedliche Erwartungen an die Leistungen der Schülerinnen und Schüler stellen. Zeugnisse dienen üblicherweise dazu, Schüler und Eltern darüber zu informieren, inwieweit einzelne Schülerinnen und Schüler den Erwartungen ihrer Lehrkräfte und Schulen gerecht werden. Es bestehen zwar große Unterschiede zwischen den in den verschiedenen Ländern angewandten Beurteilungsmethoden, jedoch enthalten die von den Lehrkräften angelegten Maßstäbe häufig eine Mindestnote für Versetzung bzw. Nichtversetzung, an der abzulesen ist, ob die in verschiedenen Fächern erbrachten Leistungen als ausreichend angesehen werden oder nicht. Wie sich dieser Grenzwert als Note ausdrückt, kann entweder auf Länderebene für alle Schulen oder auf Schulebene bzw. von den einzelnen Lehrkräften für ihre jeweilige Klasse festgelegt werden. Desgleichen können auch Art und Schwierigkeitsgrad der von den 15-Jährigen für eine bestimmte Note zu lösenden Aufgaben unterschiedlich sein, je nachdem, wie anspruchsvoll die jeweilige Schule, das Klassenniveau oder das Lehrprogramm ist.

Im Rahmen von PISA 2000 wurden die Schülerinnen und Schüler gefragt, welche Zeugnisnote sie zuletzt in den Fächern erhalten hatten, die sich auf die Sprache bezogen, in der die Leseaufgaben der PISA-Tests abgefasst waren (wegen Einzelheiten vgl. PISA 2000 Technical Report). Diese Noten wurden dann in drei Kategorien umgerechnet, je nachdem, ob sie die in der jeweiligen Schule/Klasse geltende Mindestnote für die Versetzung übertreffen, erreichen oder unterschreiten. Zwar hat die Tatsache, dass ein Schüler die theoretisch zur Versetzung erforderliche Mindestnote nicht erreicht hat - insbesondere in Ländern, in denen die Versetzung mehr oder minder automatisch erfolgt -, u.U. keine Auswirkungen auf den weiteren Ablauf seiner schulischen Laufbahn. Sie zeigt jedoch, dass der Schüler die in einem bestimmten Fach in seiner Klasse oder seiner Schule gestellten Erwartungen nicht erfüllt hat. Aus dem Vergleich dieser Information mit den Schülerleistungen auf der PISA-Lesekompetenzskala lässt sich ein zusätzlicher Bezugsrahmen für die Interpretation der PISA-Ergebnisse innerhalb eines nationalen Kontextes ableiten, wenngleich mit den Noten in den sprachbezogenen Fächern natürlich ein wesentlich breiteres Spektrum von Kenntnissen und Fähigkeiten bewertet wird als nur die Lesekompetenz.

Die Ergebnisse zeigen, dass vergleichsweise gute Leistungen bei den PISATests nicht zwangsläufig einen vergleichsweise geringen Schüleranteil bedeuten, der den Erwartungen ihrer Lehrkräfte nicht entspricht. Umgekehrt kann in Ländern, die im Durchschnitt relativ schlecht abschnitten, die große Mehrheit der Schülerinnen und Schüler u.U. noch immer den in sie gesetzten Erwartungen gerecht werden.

Frankreich beispielsweise gehört zu den Ländern, in denen vergleichsweise viele Schülerinnen und Schüler angeben, dass sie die theoretisch zur Verset-
Die verschiedenen

Bildungssysteme setzen

unterschiedliche

Erwartungen in ihre

schülerschaft,...

... was sich an den

Schülerangaben

bezüglich ihrer

Zensuren zeigt.
In Ländern, mit hoher PISA Durchschnitts-

werten, erteilen die Lehrkräfte nicht unbedingt allen Schülern die erforderliche Mindestnote,... 
KAPITEL 2 Was PISA über die Fähigkeiten 15-Jähriger aussagt - ein Profil der Schülerleistungen im Bereich Lesekompetenz

... während in einigen

Ländern, die bei

PISA niedrige

Durchschnittsergebnisse

erzielt haben, die

meisten Schülerinnen

und Schüler den

Erwartungen ihrer

Lehrkräfte gerecht

werden.

Durchschnittsergebnisse können nützlich sein, um ein Gesamtbild der Leistung eines Landes zu vermitteln,...

... sie können

jedoch über die

Leistungsunterschiede

hinwegtäuschen, die

gerade innerhalb der

Länder zu beobachten

sind.

In Abbildung 2.4 sind die Ländermittel

dargestellt, ... zung erforderliche Mindestnote nicht erreicht haben (31\%). Gleichwohl erreichen diese Schülerinnen und Schüler im Durchschnitt 492 Punkte, womit sie klar innerhalb Stufe 3 (Tabelle 2.5) und somit nahe am OECD-Durchschnitt liegen.

In Mexiko beläuft sich der Anteil der Schülerinnen und Schüler auf Stufe 1 und darunter auf 44\%. Ungeachtet der Tatsache, dass dort 96\% laut eigener Angabe in den Fächern, die mit der Testsprache in Zusammenhang standen, mindestens die zur Versetzung erforderliche Note erhalten haben, liegt das durchschnittliche Testergebnis dieser Schülerinnen und Schüler nur bei 424 Punkten (Tabelle 2.5). Obwohl diese Schülerinnen und Schüler also nach internationalen Standards schlecht abschneiden dürften, geben trotzdem 96\% von ihnen an, dass sie die Leistungsanforderungen ihrer Lehrkräfte erfüllen bzw. übertreffen. Länder, in denen die Schülerinnen und Schüler, die laut eigener Angabe die theoretisch zur Versetzung erforderliche Mindestnote nicht erreichen, relativ niedrige Testergebnisse erzielen (rund 400 Punkte), sind u.a. Griechenland, Polen, Schweden und das Vereinigte Königreich. Der Prozentsatz dieser Schülerinnen und Schüler lag in diesen Ländern jedoch nur bei 3\% bzw. darunter.

\section{Die Durchschnittsergebnisse der Länder}

Im Mittelpunkt der obigen Analyse stand der Vergleich der Verteilung der Schülerleistungen in den verschiedenen Ländern. Eine weitere Möglichkeit, die Schülerleistungen im Bereich Lesekompetenz zusammenfassend darzustellen und die jeweilige Position der Länder zueinander $\mathrm{zu}$ vergleichen, ist die Darstellung der Ländermittelwerte. Insofern eine hohe Durchschnittsleistung der 15-Jährigen eine für die Zukunft hoch qualifizierte Erwerbsbevölkerung andeutet, haben Länder mit hohen Durchschnittsergebnissen einen erheblichen wirtschaftlichen und sozialen Vorteil.

Es muss jedoch berücksichtigt werden, dass sich hinter Durchschnittsergebnissen erhebliche Unterschiede innerhalb ein und desselben Landes verbergen können, in denen sich die unterschiedliche Leistung zahlreicher verschiedener Schülergruppen widerspiegelt. Wie bereits bei früheren internationalen Schulleistungsstudien, wie der Dritten Internationalen Mathematik- und Naturwissenschaftsstudie (TIMSS) der IEA, entfällt auch bei PISA nur etwa ein Zehntel der erfassten Gesamtvarianz auf Leistungsunterschiede zwischen den einzelnen Ländern, so dass auch nur ein Zehntel davon in einem Vergleich der Durchschnittswerte der einzelnen Länder dargestellt werden kann (vgl. Tabelle 8.3). Die übrigen Leistungsunterschiede sind innerhalb der einzelnen Länder zu beobachten, d.h. zwischen verschiedenen Bildungssystemen und Lehrplänen, zwischen verschiedenen Schulen und zwischen Schülern innerhalb derselben Schulen.

In Abbildung 2.4 sind die Leistungen der Teilnehmerländer auf der Gesamtskala Lesekompetenz dargestellt. Aus Tabelle 2.2a, b und c sind die entsprechenden Informationen für die drei Subskalen ersichtlich. Abbildung 2.4 zeigt auch, 


\section{Kasten 2.2 Zur Interpretation der Statistiken}

Standardfehler und Konfidenzintervalle. Die in diesem Bericht enthaltenen Statistiken stellen Schätzwerte der nationalen Leistung auf der Basis von Schülerstichproben dar, und nicht etwa aus den Antworten sämtlicher Schülerinnen und Schüler eines Landes auf sämtliche Fragen errechnete Werte. Daher ist es wichtig, die mögliche Höhe des Messfehlers dieser Schätzungen zu kennen. In PISA 2000 wird bei jeder Schätzung ein Messfehler angegeben, der durch den Standardfehler (S.E.) ausgedrückt ist. Die Verwendung von Konfidenzintervallen ermöglicht es, Schlüsse in Bezug auf die Populationsdurchschnittswerte und -prozentsätze zu ziehen und dabei den an die Stichprobenschätzungen geknüpften Messfehler zu berücksichtigen. Es kann davon ausgegangen werden, dass das tatsächlich beobachtete statistische Ergebnis einer gegebenen Population in 95 von 100 Wiederholungsmessungen mit unterschiedlichen Stichproben derselben Population innerhalb des Konfidenzintervalls liegen würde.

Überprüfung der Unterschiedshypothesen. Für diesen Bericht wurden die Unterschiede zwischen den für die Länderstichproben ermittelten Prozentsätzen und Durchschnittsergebnissen auf ihre statistische Signifikanz hin getestet, um beurteilen zu können, ob diese Unterschiede auch tatsächlich zwischen den Populationen bestehen, denen die Stichproben entstammen. Bei jedem Test wurde davon ausgegangen, dass die Irrtumswahrscheinlichkeit, mit der aus einem zwischen zwei Stichproben beobachteten Unterschied infolge eines Stichproben- und Messfehlers auf einen Unterschied zwischen den entsprechenden Populationen geschlossen wird, obwohl in Wirklichkeit zwischen den beiden Populationen kein echter Unterschied besteht, 5\% nicht übersteigt. In den Abbildungen und Tabellen zum Vergleich von Ländermittelwerten wird mit der Signifikanzprüfung eine Methode angewendet, bei der festgelegt wird, dass die Wahrscheinlichkeit, mit der es sich bei den in den Stichproben gefundenen Unterschiede um zufällige Unterschiede handelt, die in der Grundgesamtheit nicht existieren, 5\% nicht übersteigt (wegen Einzelheiten hierzu vgl. Anhang A4).

welche Länder unter oder über dem OECD-Durchschnitt liegen und welche nicht wesentlich davon abweichen.

Die Ergebnisse Finnlands auf der Gesamtskala Lesekompetenz sind höher als die aller anderen OECD-Länder (Tabelle 2.4). Sein Landesdurchschnitt liegt mit 546 Punkten fast zwei Drittel einer Kompetenzstufe über dem OECDMittelwert von 500 Punkten (bzw. übertrifft den Mittelwert statistisch gesehen um mehr als eine halbe internationale Standardabweichung). Länder mit einem Mittelwert der Schülerleistungen signifikant über dem OECD-Mittelwert sind Australien, Österreich, Belgien, Kanada, Finnland, Island, Irland, Japan, Korea, Neuseeland, Schweden und Vereinigtes Königreich, in fünf Ländern entspricht er in etwa dem OECD-Durchschnitt (Dänemark, Frankreich, Norwegen, Schweiz und Vereinigte Staaten) und in 14 Ländern liegt er signifikant unter dem OECD-Durchschnitt (Brasilien, Tschechische Republik, Deutschland, Griechenland, Ungarn, Italien, Lettland, Liechtenstein, Luxemburg, Mexiko, Polen, Portugal, Russische Föderation und Spanien) ${ }^{8}$.

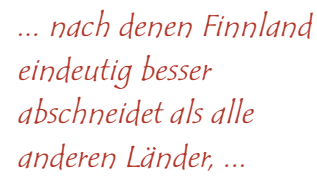

anderen Länder,... 
KAPITEL 2 Was PISA über die Fähigkeiten 15-Jähriger aussagt - ein Profil der Schülerleistungen im Bereich Lesekompetenz

\section{Abbildung 2.4}

Vergleich der Durchschnittsergebnisse der Länder auf der Gesamtskala Lesekompetenz

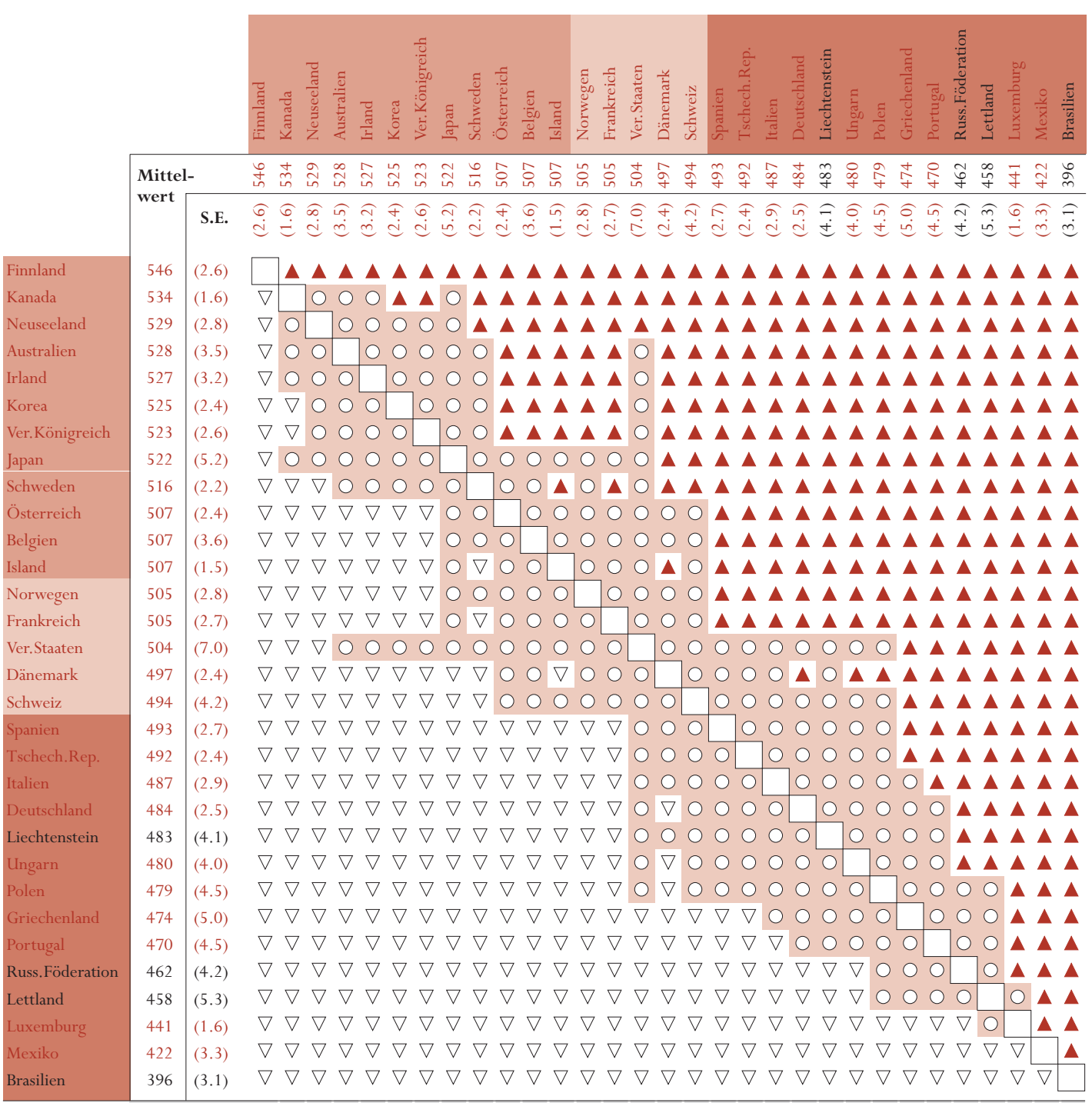

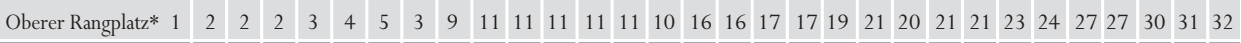

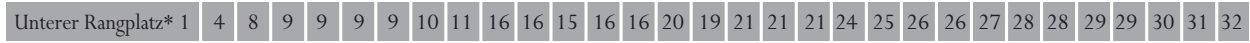

* Anmerkung: Da die Daten auf Stichprobenziehungen beruhen, ist es nicht möglich, den genauen Rangplatz eines Landes zu bestimmen. Es können jedoch der jeweils obere und untere Rangplatz angegeben werden, zwischen denen das Land mit 95\%iger Wahrscheinlichkeit liegt.

Erläuterungen

Zum Vergleich der Ergebnisse eines Landes mit denen der Länder im Tabellenkopf ist die Zeile des betreffenden Landes zu lesen. Die Symbole zeigen, ob die Durchschnittsergebnisse des Landes in der jeweiligen Zeile statistisch signifikant über oder unter denen des Vergleichslandes liegen oder ob zwischen den Durchschnittsergebnissen beider Länder kein signifikanter Unterschied besteht.

Quelle: OECD PISA Datenbank, 2001.
A Durchschnittsergebnisse statistisch signifikant höher als im Vergleichsland Kein statistisch signifikanter Unterschied gegenüber dem Vergleichsland $\nabla$ Durchschnittsergebnisse statistisch signifikant niedriger als im Vergleichsland

Statistisch signifikant über dem OECD-Durchschnitt

Kein statistisch signifikanter Unterschied zum OECD-Durchschnitt Statistisch signifikant unter dem OECD-Durchschnitt 
Wie in Kasten 2.2 beschrieben, ist es bei der Interpretation der Durchschnittsleistungen wichtig, nur die statistisch signifikanten Unterschiede zu berücksichtigen. Die Position eines Landes gemäß Abbildung 2.4 sollte daher nicht beurteilt werden, ohne gleichzeitig zu untersuchen, ob die Ergebnisse der Länder, die auf den nächst höheren oder nächst niedrigeren Plätzen liegen, wirklich signifikante Unterschiede aufweisen. Aus Abbildung 2.4 geht hervor, bei welchen Länderpaaren die Mittelwertsunterschiede so groß sind, um mit ziemlicher Sicherheit davon auszugehen, dass die höhere Leistung der Schülerstichprobe eines Landes als repräsentativ für dessen gesamte Population der 15-Jährigen angesehen werden kann. In den Zeilen der links aufgelisteten Länder ist angegeben, wie sich die Ergebnisse eines jeweiligen Landes im Vergleich zu denen der Länder verhalten, die in der Kopfzeile der Abbildung aufgeführt sind. Aus den Symbolen ist ersichtlich, ob die durchschnittlichen Ergebnisse des jeweiligen Landes signifikant unter denen des Vergleichslandes liegen, nicht in statistisch signifikanter Weise von diesen abweichen oder signifikant höher sind. Neuseeland schneidet beispielsweise laut Abbildung 2.4 deutlich niedriger ab als Finnland, nicht signifikant anders als Australien, Kanada, Irland, Japan, Korea und das Vereinigte Königreich, und signifikant höher als alle übrigen Länder. Die Leistungen Finnlands liegen signifikant höher als die aller anderen Ländern.

Die Durchschnittsergebnisse von Brasilien und Mexiko liegen signifikant niedriger als die aller anderen Länder, beide liegen mehr als eine ganze Kompetenzstufe unter dem OECD-Durchschnitt. Bei der Interpretation ihres jeweiligen Durchschnittsergebnisses muss jedoch beachtet werden, dass sich die 15-Jährigen in beiden Ländern in zahlreichen verschiedenen Klassenstufen befinden. Die 15-Jährigen, die in Brasilien und Mexiko die 10. Klasse besuchen (d.h. die typische Klassenstufe der 15-Jährigen in den OECD-Ländern), erzielten im Durchschnitt 463 bzw. 466 Punkte, womit ihre Durchschnittsergebnisse zwischen denen der Russischen Föderation und Portugals liegen (Daten abrufbar unter www.pisa.oecd.org).

Die Tabellen 2.2a, b und c liefern Informationen $\mathrm{zu}$ den durchschnittlichen Leistungen auf den drei Subskalen nach dem gleichen Muster wie Abbildung 2.4 für die Gesamtskala Lesekompetenz. Allerdings sollten die Werte der drei Subskalen zu den Aspekten der Lesekompetenz nicht direkt miteinander verglichen werden. Es ist jedoch möglich, die relative Stärke zweier Länder in diesen Aspekten der Lesekompetenz anhand ihrer jeweiligen relativen Position auf den drei Subsksalen zu vergleichen ${ }^{9}$ (Die Werte in Klammern geben die mittlere Punktzahl auf den Subskalen "Informationen ermitteln" bzw. "Reflektieren und Bewerten” an).

- Der Vergleich ergibt, dass Österreich (502, 512), Kanada (530, 542), Irland (524, 533), Portugal $(455,480)$, Spanien $(483,506)$ und das Vereinigte
... allerdings sind nicht

alle dargestellten

Unterschiede auch

statistisch signifikant.
In Ländern mit niedrigen Durchschnittsergebnissen, in denen leistungsschwache schüler häufig wiederholen, erzielen die sich in der ihrem Alter entsprechenden Klassenstufe befindenden Schüler im internationalen Vergleich wesentlich bessere Ergebnisse.

In einigen Ländern schneiden die schülerinnen und Schüler auf einer der subskalen besser ab als aufden anderen - diese Unterschiede fallen jedoch nur in wenigen Ländern ins Gewicht. 
KAPITEL 2 Was PISA über die Fähigkeiten 15-Jähriger aussagt - ein Profil der Schülerleistungen im Bereich Lesekompetenz

Königreich $(523,539)$ auf der Skala „Reflektieren und Bewerten“ vergleichsweise besser abschneiden als auf der Skala „Informationen ermitteln“.

- Australien (536, 526), Belgien (515, 497), Finnland $(556,533)$, Frankreich $(515,496)$, Deutschland $(483,478)$ und die Schweiz $(498,488)$ erzielen auf der Skala „Informationen ermitteln“ vergleichsweise bessere Ergebnisse als auf der Skala „Reflektieren und Bewerten“.

- In Bezug auf die übrigen Länder bestehen keine statistisch signifikanten Unterschiede zwischen den Schülerleistungen auf den drei Subskalen.

\section{Die Verteilung der Lesekompetenz innerhalb der Länder}

Hohe Durchschnittsergebnisse allein sind nicht ausreichend: Die

Länder sind auch bestrebt, das Niveau der leistungsschwachen schüler anzuheben.

Am Ausmaßs der Leistungsunterschiede zeigt sich, wie schwer die Aufgabe ist, vor der sie dabei stehen,...

... was aus Abbildung 2.5 hervorgeht, ...
Zur Beurteilung der Qualität von Schulen und Bildungssystemen werden in der Regel Durchschnittsergebnisse herangezogen. Durchschnittswerte liefern jedoch, wie bereits erwähnt, kein vollständiges Bild der Schülerleistungen und können zudem über erhebliche Unterschiede zwischen den Leistungen der einzelnen Schüler einer Klasse, einer Schule oder eines Bildungssystems hinwegtäuschen. Zudem sind manche Länder nicht nur bestrebt, hohe Leistungen zu fördern, sondern auch, die Leistungsdifferenzen innerhalb ihrer Schülerschaft gering zu halten. Sowohl die Eltern als auch die breite Öffentlichkeit sind sich über die Folgen niedriger schulischer Leistungen im Klaren und wissen, dass Schulabgänger, denen es an grundlegenden Kompetenzen mangelt, auf dem Arbeitsmarkt wenig Chancen haben. Ein hoher Prozentsatz von Schülerinnen und Schülern am unteren Ende der Lesekompetenzskala könnte zu Befürchtungen Anlass geben, dass es einem großen Teil der Arbeitskräfte und Wähler von morgen an den nötigen Fähigkeiten fehlen wird, um die ihnen abverlangten fundierten Entscheidungen treffen zu können.

Die in diesem Abschnitt enthaltene Analyse muss von der obigen Untersuchung der Verteilung der Schülerleistungen über die verschiedenen PISAKompetenzstufen unterschieden werden. Während aus der Verteilung der Schülerinnen und Schüler auf die einzelnen Kompetenzstufen hervorgeht, welcher Prozentsatz der Schülerinnen und Schüler eines Landes ein bestimmtes Niveau an Kenntnissen und Fähigkeiten nachweisen kann, was einen Ländervergleich auf der Basis von absoluten Normwertern (benchmarking) der Schülerleistung ermöglicht, liegt der Schwerpunkt der nun folgenden Analyse auf der relativen Streuung der Ergebnisse, d.h. dem Abstand zwischen den Schülerinnen und Schülern mit den höchsten und den niedrigsten Leistungen innerhalb eines Landes. Dies ist ein wichtiger Indikator für eine ausgewogene Verteilung der Bildungserträge im Bereich Lesekompetenz (vgl. Kasten 2.3).

Abbildung 2.5 stellt die Verteilung der Punktwerte auf der Gesamtskala Lesekompetenz dar (Tabelle 2.3a). Da die Ergebnisse für alle drei Subskalen relativ ähnlich ausfallen, werden sie in diesem Abschnitt nicht einzeln untersucht. Angaben über die Verteilung der Ergebnisse auf den verschiedenen Subskalen sind den Tabellen 2.3b, c und d zu entnehmen. 
Was PISA über die Fähigkeiten 15-Jähriger aussagt - ein Profil der Schülerleistungen im Bereich Lesekompetenz KAPITEL 2

\section{Abbildung 2.5}

Verteilung der Schülerleistungen auf der Gesamtskala Lesekompetenz innerhalb der Länder
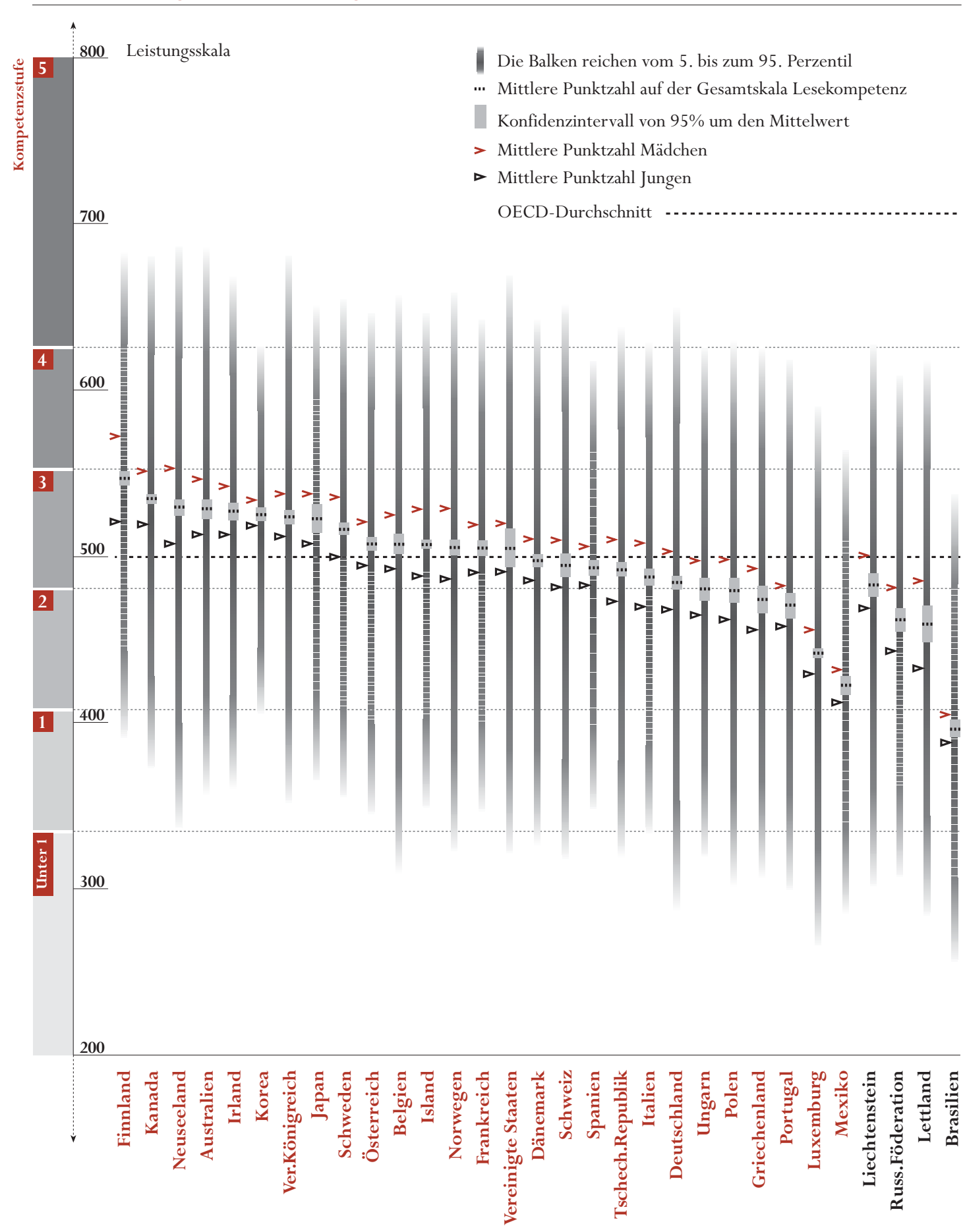

Quelle: OECD PISA Datenbank, 2001. Tabelle 2.3a. 
KAPITEL 2 Was PISA über die Fähigkeiten 15-Jähriger aussagt - ein Profil der Schülerleistungen im Bereich Lesekompetenz

... in der die Die schattierten Balken in Abbildung 2.5 zeigen die Leistungsverteilung eines Leistungsverteilung der einzelnen Länder

dargestellt ist. jeden Landes zwischen dem 5. Perzentil (dem Punkt, unterhalb dessen die leistungsschwächsten 5\% der Schülerinnen und Schüler liegen) und dem 95. Perzentil (dem Punkt, unter dem 95\% bzw. über dem die leistungsstärksten 5\% der Schülerinnen und Schüler eines Landes liegen). Der Schattierungsgrad der Balken gibt den Anteil der Schülerinnen und Schüler wieder, der die den jeweiligen Skalenpunkten entsprechende Leistung erbringt. Die Tabellen 2.3a, b, c und d enthalten zusätzlich Angaben über das 25. und das 75. Perzentil, d.h. die Skalenpunkte, die das leistungsschwächste und das leistungsstärkste Quartil der Schülerinnen und Schüler des jeweiligen Landes kennzeichnen. Die drei schwarzen Punkte nahe der Balkenmitte geben die mittlere Punktzahl der einzelnen Länder an (die im vorigen Abschnitt analysiert wurde), der kleine schattierte Kasten, der sie umgibt, bezeichnet das entsprechende Konfidenzintervall.

Abbildung 2.5 verdeutlicht, dass das Ausmaß der Leistungsunterschiede innerhalb der einzelnen Länder größer ist als das der Mittelwertsunterschiede zwischen den Ländern, ...

... und dass es in jedem Land jeweils solche Schülerinnen und Schüler gibt, deren Leistungen über dem Mittelwert des leistungsstärksten Landes bzw. unter dem des leistungsschwächsten Landes liegen.
Aus Abbildung 2.5 ist ersichtlich, dass auf der Gesamtskala Lesekompetenz innerhalb der einzelnen Länder große Leistungsdifferenzen bestehen. Die Länge der Balken, die die mittleren 90\% der Population darstellt, übersteigt bei weitem den Abstand, der zwischen dem Mittelwert des leistungsschwächsten und dem des leistungsstärksten Landes besteht. Für die mittleren 90\% der Population, die jeweils durch die Balken dargestellt sind, gehen die innerhalb der einzelnen Länder festgestellten Leistungsunterschiede weit über die Streuungsbreite der in den einzelnen Ländern erreichten Mittelwerte hinaus. In fast allen OECDLändern gehört zu der dargestellten mittleren Gruppe von 90\% sowohl ein Teil der Schülerinnen und Schüler, die Stufe 5 erreichen, als auch ein Teil derjenigen, deren Leistung höchstens Stufe 1 entspricht. Mit Ausnahme von fünf Ländern ist in allen OECD-Ländern die Varianz der Schülerleistungen auch noch innerhalb der mittleren 50\% der Population (d.h. zwischen dem 25. und dem 75. Perzentil) größer als der Unterschied zwischen dem Mittelwert des leistungsschwächsten und dem des leistungsstärksten Landes. In allen Ländern ist die Streuung der Ergebnisse der mittleren 50\% der Schülerpopulation höher als eine Kompetenzstufe und in Australien, Belgien, Deutschland und Neuseeland übersteigt sie sogar mehr als zwei Kompetenzstufen (der OECD-Durchschnitt beträgt 1,8 Kompetenzstufen). Daraus lässt sich schließen, dass Lehrpläne, Schulen und Lehrkräfte mit einer großen Bandbreite an unterschiedlichen Schülerkenntnissen und -fähigkeiten zurechtkommen müssen.

In allen Ländern erreichen mindestens 5\% der Schülerinnen und Schüler nicht das durchschnittliche Leistungsniveau von Mexiko, dem OECD-Land mit den niedrigsten Ergebnissen (Tabelle 2.3a). In Deutschland, Ungarn und Polen erreicht ein Viertel nicht einmal den niedrigsten Ländermittelwert, während zugleich ein Viertel den höchsten von einem Land erzielten Mittelwert übertrifft. Am oberen Ende der Skala liegen in allen Ländern mindestens 5\% der Schülerinnen und Schüler über dem durchschnittlichen Leistungsniveau Finnlands, dem Land mit dem höchsten Durchschnittsergebnis. 
In einigen der Länder mit hohen Durchschnittsergebnissen, z.B. in Australien, Neuseeland und dem Vereinigten Königreich, liegt das 25. Perzentil auf der Gesamtskala Lesekompetenz klar innerhalb von Stufe 2 (rund 458 Punkte), was darauf schließen lässt, dass die dortigen Schülerinnen und Schüler beim 25. Perzentil in absoluter Rechnung noch immer recht gut abschneiden. Der Abstand, der in diesen Ländern zwischen den Schülerleistungen beim 25. und beim 75. Perzentil der nationalen Leistungsverteilung besteht, könnte jedoch darauf
Auch in Ländern, in denen das untere Schülerquartil im internationalen Vergleich noch gut abschneidet, können großse Leistungsunterschiede Anlass zur Besorgnis geben.

Kasten 2.3 Interpretation der Unterschiede in den PISA-Ergebnissen: Wie groß sind die Abstände?

Was bedeutet ein Unterschied von beispielsweise 50 Punkten zwischen den Ergebnissen von zwei verschiedenen Schülergruppen? Ein Unterschied von 73 Punkten entspricht der Höhe einer Kompetenzstufe innerhalb der Skala für Lesekompetenz. Ein Unterschied von einer Kompetenzstufe kann konkret als ein vergleichsweise großer Unterschied zwischen der Leistung verschiedener Schüler angesehen werden. Stufe 3 auf der Subskala “Textbezogenes Interpretieren” unterscheidet beispielsweise Schülerinnen und Schüler, die für gewöhnlich in der Lage sind, mehrere Teile eines Texts miteinander zu verknüpfen, Zusammenhänge zu begreifen oder den Sinn eines Ausdrucks oder eines Satzes zu analysieren, konkurrierende Informationen zu vergleichen und einander gegenüberzustellen und anhand mehrerer Kriterien in Kategorien zu unterteilen, von denen auf Stufe 2, die in der Regel nur das Hauptthema eines Texts identifizieren, Zusammenhänge begreifen und einfache Kategorien aufstellen oder anwenden und den Sinn eines nur begrenzten Textteils analysieren können, bei dem keine anspruchsvollen Schlüsse gezogen werden müssen (vgl. Abb. 2.1).

Als weitere Referenzgröße kann der Leistungsunterschied auf der Gesamtskala Lesekompetenz zwischen den OECD-Ländern mit dem dritthöchsten und dem drittniedrigsten durchschnittlichen Ergebnis herangezogen werden: Er beträgt 59 Punkte. Der Unterschied zwischen den OECDLändern mit dem fünfthöchsten und dem fünftniedrigsten beläuft sich auf 48 Punkte.

Ergebnisunterschiede können auch anhand der Leistungsdifferenzen untersucht werden, die zwischen verschiedenen Schülergruppen auf der Gesamtskala Lesekompetenz zu beobachten sind:

- Der Abstand zwischen der Leistung des oberen und des unteren Schülerquartils eines Landes nach dem sozioökonomischen PISA-Index liegt im OECD-Durchschnitt bei 81 Punkten (Tabelle 6.1a). Konkret heißt das, dass zwischen den Schülerinnen und Schülern, deren Eltern z.B. Gymnasiallehrer oder Leiter kleiner Firmen sind, und solchen, deren Eltern Maurer, Zimmermann oder Anstreicher sind, im OECD-Durchschnitt ein Leistungsunterschied von 81 Punkten besteht.

- Der Unterschied zwischen der Leistung der Schülerinnen und Schüler, deren Mütter einen Hochschulabschluss besitzen, und jenen, deren Mütter die Sekundarstufe II nicht abgeschlossen haben, liegt im OECD-Durchschnitt bei 67 Punkten (Tabelle 6.7).

- Der Unterschied zwischen der Leistung der Schülerinnen und Schüler, die zu Hause normalerweise die Sprache sprechen, die in den Tests verwendet wurde, und jenen, für die dies nicht der Fall ist, liegt im OECD-Durchschnitt bei 68 Punkten (Tabelle 6.11). 
KAPITEL 2 Was PISA über die Fähigkeiten 15-Jähriger aussagt - ein Profil der Schülerleistungen im Bereich Lesekompetenz

Sind die beobachteten

Leistungsunterschiede unvermeidlich? Diese

Frage ist schwer zu

beantworten,...

... doch ist in einigen

Ländern die

Streungsbreite der

Ergebnisse wesentlich

geringer als in anderen.

Dies gilt sogar für Länder mit ähnlichen

Mittelwerten.

Am wichtigsten ist jedoch, dass einige Länder sowohl hohe Durchschnittsergebnisse als auch eine recht große Leistungshomogenität erzielen. hindeuten, dass die Schülerinnen und Schüler beim 25. Perzentil deutlich weniger leisten, als von ihnen innerhalb ihres nationalen Bildungssystems erwartet wird.

Inwieweit diese Leistungsunterschiede nun eine Verteilung der Schülerbegabungen widerspiegeln und somit nur schwer mit Änderungen der politischen Weichenstellung zu beeinflussen sind, ist eine Frage, die sich kaum allein anhand der PISA-Daten beantworten lässt - zumal die Differenzen zwischen den Ländern auch auf Unterschiede im sozialen und wirtschaftlichen Bildungs- und Lernumfeld zurückzuführen sind. Einige Ergebnisse deuten jedoch darauf hin, dass die Politik durchaus einen Beitrag leisten kann:

- Erstens ist der Umfang der innerhalb der einzelnen Länder beobachteten Leistungsdifferenzen von Land zu Land sehr unterschiedlich. Die Leistungsabstände zwischen dem 75. und dem 25. Perzentil reichen von 92 Punkten in Korea bis zu über 140 Punkten in Australien, Belgien, Deutschland, Neuseeland, der Schweiz und den Vereinigten Staaten (Tabelle 2.3a). Am größten ist der Abstand in Belgien, wo zwischen dem 75. und dem 25. Perzentil 150 Punkte liegen. Dieser große Abstand lässt sich zumindest teilweise durch die unterschiedlichen Leistungen der flämischen und wallonischen Sprachgruppen in Belgien erklären (vgl. Anhang B2 wegen Einzelheiten).

- Zweitens weisen Länder mit ähnlichen Durchschnittsergebnissen große Unterschiede bei den Leistungsdifferenzen der Schülerinnen und Schüler auf. Korea und das Vereinigte Königreich verzeichnen z.B. beide mit ca. 525 Punkten überdurchschnittlich gute Ergebnisse auf der Gesamtskala Lesekompetenz. Der Abstand zwischen dem 75. und dem 25. Perzentil beträgt in Korea jedoch nur 93 Punkte - er ist also signifikant kleiner als im OECDDurchschnitt - während er im Vereinigten Königreich bei 137 Punkten liegt und damit signifikant größer als der OECD-Durchschnitt ist. Die gleiche Beobachtung kann auch für unterdurchschnittlich abschneidende Länder gemacht werden. Deutschland und Italien, zwei Länder mit Durchschnittswerten von jeweils rund 486 Punkten, was signifikant unter dem OECD-Durchschnitt liegt, unterscheiden sich bezüglich ihrer internen Leistungsdifferenzen. In Italien beträgt der Abstand zwischen dem 75. und dem 25. Perzentil 124 Punkte, während er sich in Deutschland auf 146 Punkte beläuft. Bemühungen zur Anhebung des Niveaus des leistungsschwächsten Schülerquartils in Richtung des derzeitigen Mittelwerts wären eine Möglichkeit, um in Ländern mit großen Leistungsdifferenzen das Gesamtniveau zu steigern. Die Tatsache, dass einige Länder sowohl ein hohes durchschnittliches Leistungsniveau als auch niedrige Leistungsdifferenzen aufweisen, ist von weitreichender Bedeutung.

- Drittens zeigt sich beim Vergleich der Leistungsverteilung in einem Land mit dessen Durchschnittswerten, dass große Leistungsheterogenität keine notwendige Voraussetzung für ein hohes Gesamtniveau darstellt. So gehören z.B. die drei Länder mit dem geringsten Abstand zwischen dem 75. und dem 
25. Perzentil - Finnland, Japan und Korea - auch zu denen, die die höchsten Leistungen bei der Lesekompetenz erzielen. Im Gegensatz dazu liegt eines der drei Länder mit den größten Leistungsdifferenzen - Deutschland - deutlich unter dem OECD-Durchschnitt.

Die Analyse der Streuungsbreite der Ergebnisse zwischen dem 25. und dem 5. Perzentil liefert einen Anhaltspunkt für die Leistung der Schülerinnen und Schüler mit der niedrigsten Punktzahl im Vergleich zur Gesamtleistung des betreffenden Landes. Die Frage lautet hier: Vergrößern sich die Leistungsdifferenzen am unteren Ende der Verteilung? Im Allgemeinen weisen Länder mit einem geringen Abstand zwischen dem 75. und dem 25. Perzentil, z.B. Finnland, Japan, Korea und Spanien, auch geringere Leistungsunterschiede am unteren Ende zwischen dem 25. und dem 5. Perzentil auf. Die drei Länder mit dem größten Abstand zwischen dem 75. und dem 25. Perzentil - Belgien, Deutschland und Neuseeland - weisen auch am unteren Ende die größten Leistungsunterschiede auf: Der Abstand zwischen dem 25. und dem 5. Perzentil liegt dort bei über 122 Punkten.

In den vier OECD-Ländern, die auf der Gesamtskala Lesekompetenz die niedrigsten Ergebnisse erzielen - Griechenland, Luxemburg, Mexiko und Portugal -, liegt weniger als ein Viertel der Schülerinnen und Schüler über dem Durchschnitt des leistungsstärksten Landes. In einem Land - Mexiko - ist dies sogar nur für 10\% der Fall. In Brasilien liegen über 75\% der Schülerinnen und Schüler unter dem OECD-Durchschnitt. Die oberen 10\% liegen dort knapp und die oberen 5\% deutlich über dem OECD-Durchschnitt.

In einigen Ländern mit unterdurchschnittlichen Ergebnissen schneiden die leistungsstärksten Schülerinnen und Schüler dennoch sehr gut ab. So erzielen beispielsweise in Deutschland 5\% über 650 Punkte, während die oberen 5\% in Korea nur über 629 Punkte erzielen - und dies, obwohl der deutsche Mittelwert signifikant unter und der koreanische Mittelwert signifikant über dem OECD-Durchschnitt liegt. Umgekehrt können die Ergebnisse der leistungsschwächsten Schülerinnen und Schüler in Ländern mit hohen Durchschnittsergebnissen vergleichsweise niedrig sein. In einem der Länder mit der höchsten durchschnittlichen Lesekompetenz - Neuseeland - liegen 5\% der Population unter dem vergleichsweise niedrigen Wert von 337 Punkten, d.h. der Anteil der Schülerinnen und Schüler mit niedrigen Werten ist dort höher als in einigen Ländern mit eher durchschnittlichen Mittelwerten.

Diese Ergebnisse machen nochmals deutlich, dass sich Lehrkräfte, Schulen und Schulsysteme unbedingt mit den Leistungsdifferenzen innerhalb von Klassen, Schulen und Ländern auseinandersetzen müssen.

\section{Leistungsunterschiede zwischen den Schulen}

Der bildungspolitische und institutionelle Kontext des Schulbesuchs der 15-Jährigen in den OECD-Ländern kann sehr unterschiedliche Formen
Länder mit der gröjsten

Gesamtvarianz der

Ergebnisse weisen auch

die größsten

Leistungsdifferenzen am

unteren Ende der

Verteilung auf.

In den

leistungsschwächsten

Ländern gibt es eine kleine

Minderheit, deren

Leistungen knapp über den

Durchschnittswerten der

anderen Länder liegen, ...

... in anderen Ländern mit unterdurchschnittlichen

Ergebnissen schneiden die

obersten 5\% hingegen sehr gut ab, selbst verglichen

mit den leistungsstärksten

schülern anderer Länder.

Die in PISA erfassten

15-Jährigen besuchen

zahlreiche verschiedene

schultypen,... 
... auf die sie manchmal entsprechend ihrer Fähigkeiten verteilt sind.

Spielen die Unterschiede zwischen den Schulen innerhalb der in PISA erfassten Differenzen eine Rolle? annehmen. In manchen Ländern befindet sich ein Teil der Schülerinnen und Schüler in berufsorientierten Schulen, während ein anderer Teil Schulen besucht, die in erster Linie auf ein Hochschulstudium vorbereiten sollen. In Ländern, in denen der Übergang von der Sekundarstufe I zur Sekundarstufe II in etwa im Alter von 15 Jahren erfolgt, besuchen einige der in PISA erfassten Schülerinnen und Schüler noch Sekundarstufe I, während andere bereits in Sekundarstufe II sind. Hinzu kommt, dass in allen außer zwei OECD-Ländern die Mehrzahl zwar in öffentlichen Schulen unterrichtet wird, in mehreren OECD-Ländern jedoch eine nicht unerhebliche Minderheit privat verwaltete und in einigen Fällen auch finanzierte Schulen besucht.

Die vorangegangene Analyse hat gezeigt, dass in den meisten Ländern innerhalb der jeweiligen Bildungssysteme erhebliche Leistungsunterschiede bestehen. Diese Unterschiede können durch den sozioökonomischen Hintergrund der Schüler und Schulen, den Umfang der den Schulen zur Verfügung stehenden personellen und finanziellen Mittel, unterschiedliche Curricula, vorhandene Selektionsmechanismen und durch die Art und Weise, wie der Unterricht organisiert und durchgeführt wird, bedingt sein (vgl. auch Kapitel 6 und 8). Einige Länder haben nicht selektive Schulsysteme, die allen Schülerinnen und Schülern die gleichen Lernmöglichkeiten bieten wollen und allen Schulen die Möglichkeit geben, die gesamte Palette der Schülerleistungen abzudecken. Andere Länder versuchen der Verschiedenheit der Schülerinnen und Schüler gerecht zu werden, indem sie sie bewusst (durch Selektion zwischen oder innerhalb der Schulen) in Gruppen mit ähnlichem Leistungsniveau einteilen, mit dem Ziel, sie entsprechend ihrer besonderen Bedürfnisse bestmöglich zu fördern. In wieder anderen Ländern werden diese beiden Ansätze kombiniert. Auch in Gesamtschulsystemen können große Unterschiede zwischen den einzelnen Schulen bestehen, bedingt z.B. durch die sozioökonomischen und kulturellen Merkmale der von ihnen versorgten Gemeinden oder durch geographische Unterschiede (zwischen einzelnen Regionen, Provinzen oder Bundesstaaten in föderativen Regierungsformen oder zwischen ländlichen und städtischen Gebieten). Zudem können zwischen einzelnen Schulen auch Unterschiede bestehen, die sich nicht ohne weiteres quantifizieren oder anderweitig beschreiben lassen und die z.T. auf Differenzen in der Qualität und der Effizienz des von ihnen angebotenen Unterrichts zurückzuführen sein könnten.

Könnten die innerhalb der einzelnen Länder beobachteten Leistungsdifferenzen, außer auf Unterschiede zwischen einzelnen Schulen und zwischen sozialen Gruppen, nicht auch auf andere identifizierbare Faktoren zurückzuführen sein? Welchen Einfluss haben beispielsweise die für die Schulsysteme der einzelnen Länder prägenden politischen und historischen Strukturen auf die Leistungsunterschiede zwischen den Schülerinnen und Schülern und in welchem Zusammenhang stehen sie mit ihnen? Sind in Ländern, deren Bildungssysteme eine explizite Einteilung in Leistungsgruppen vorsehen, insgesamt größere Unterschiede in den Schülerleistungen zu beobachten als in Ländern mit nicht selektiven Bildungssystemen? Diese Fragen stellen sich vor allem für jene 
Was PISA über die Fähigkeiten 15-Jähriger aussagt - ein Profil der Schülerleistungen im Bereich Lesekompetenz KAPITEL 2

\section{Abbildung 2.6}

Leistungsunterschiede zwischen den Schulen und innerhalb der Schulen auf der Gesamtskala Lesekompetenz ausgedrückt in Prozentanteilen der durchschnittlichen Varianz der Schülerleistungen

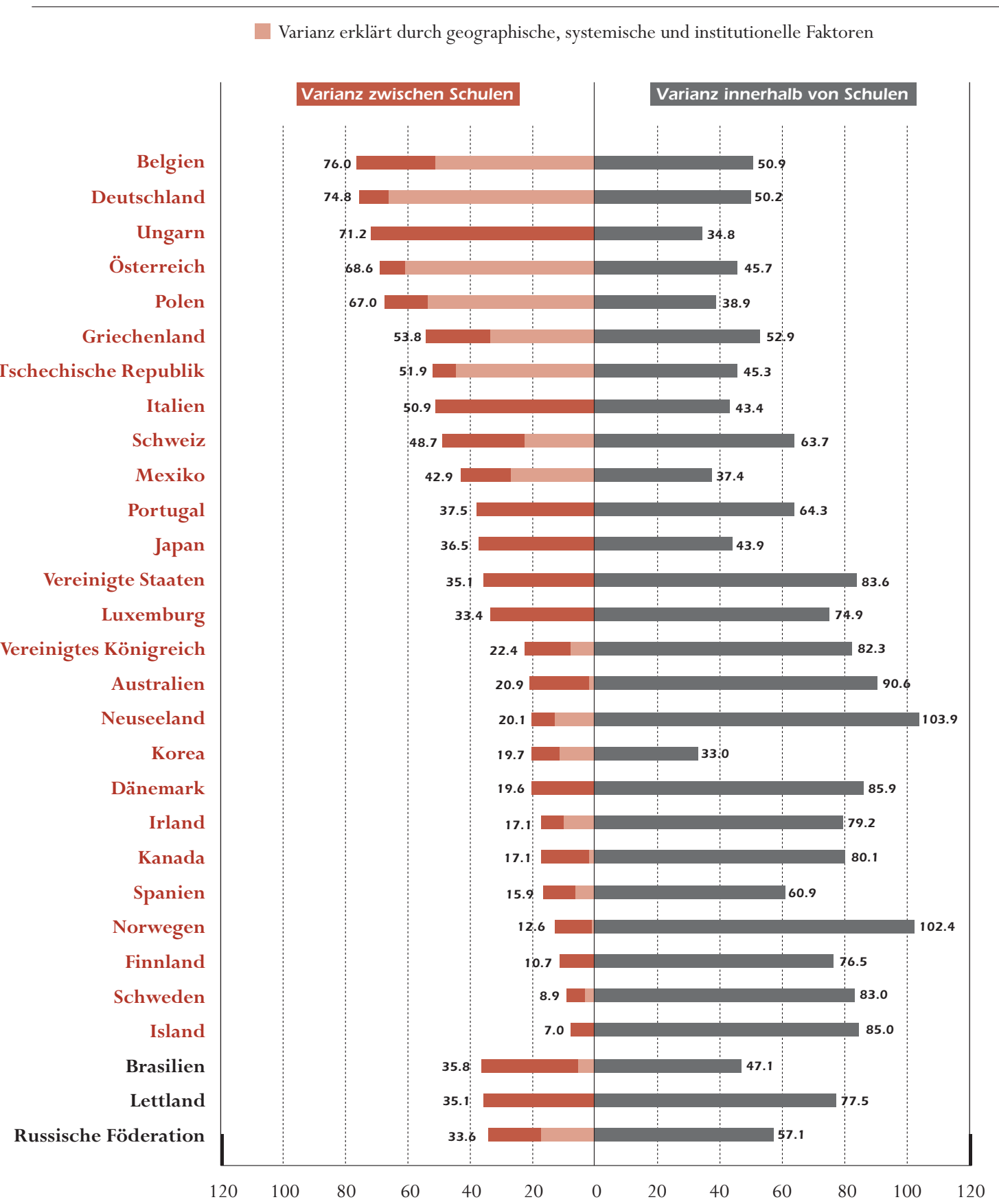

Quelle: OECD PISA Datenbank, 2001. Tabelle 2.4. 
KAPITEL 2 Was PISA über die Fähigkeiten 15-Jähriger aussagt - ein Profil der Schülerleistungen im Bereich Lesekompetenz

Abbildung 2.6 vergleicht die innerhalb der Länder beobachtete Varianz, ...

... aufgegliedert in die Varianz zwischen den Schulen und die Varianz innerhalb von Schulen, ...

wobei sich zeigt, dass in selektiveren Bildungssystem über die Hälfte der

Gesamtvarianz auf Unterschiede zwischen den Schulen entfällt.

In manchen Ländern sind die Leistungsunterschiede sowohl zwischen als auch innerhalb der Schulen gering, ...
Länder, in denen besonders große Leistungsheterogenität zu beobachten ist, z.B. in Belgien, Deutschland, Neuseeland, Norwegen und den Vereinigten Staaten.

Abbildung 2.6 zeigt, in welchem Ausmaß die in den verschiedenen Ländern ermittelten Leistungsunterschiede jeweils auf die verschiedenen Faktoren zurückzuführen sind. Die Länge der Balken verdeutlicht die beobachtete Varianz der Schülerleistungen auf der Gesamtskala Lesekompetenz (Spalte 2 von Tabelle 2.4) ${ }^{10}$. Die Werte in Abbildung 2.6 und Tabelle 2.4 sind als Prozentsätze der Durchschnittsvarianz der Schülerleistungen zwischen den OECD-Ländern nach der Gesamtskala Lesekompetenz ausgedrückt, die 9277 Einheiten entspricht ${ }^{11}$. Werte in Spalte 2, die größer sind als 100, deuten darauf hin, dass die Leistungsdifferenzen zwischen den Schülern des entsprechenden Landes größer sind als in einem typischen OECD-Land. Kleinere Werte als 100 bedeuten dementsprechend, dass die Leistungsdifferenzen kleiner sind als im Durchschnitt.

Die den einzelnen Ländern entsprechenden Balken sind in Abbildung 2.6 so angeordnet, dass die Varianz zwischen den Schulen durch die Länge des Balkenabschnitts links der vertikalen Linie in der Abbildungsmitte und die Varianz innerhalb der Schulen durch die Länge des Balkenabschnitts rechts dieser Linie dargestellt ist. Längere Balkensegmente links der Vertikalen deuten darauf hin, dass die Durchschnittsergebnisse der Schulen weiter auseinander liegen. Längere Balkenabschnitte rechts der Vertikalen weisen auf größere Leistungsunterschiede zwischen den Schülern derselben Schulen hin.

Wie aus Abbildung 2.6 ersichtlich, gehen die Leistungsunterschiede zwischen den Schülern in den meisten Ländern auf Differenzen zwischen den Schulen zurück. In den 26 in diesen Vergleich einbezogenen OECD-Ländern lassen sich 36\% der im OECD-Durchschnitt beobachteten Varianz in den Schülerleistungen auf Unterschiede zwischen den Schulen zurückführen. In Österreich, Belgien, der Tschechischen Republik, Deutschland, Griechenland, Ungarn, Italien und Polen entfallen sogar über 50\% der Gesamtvarianz auf Unterschiede zwischen den Schulen (Spalte 3, Tabelle 2.4). Wo große Leistungsdifferenzen zwischen den Schulen bestehen, die Leistungsunterschiede zwischen den Schülern der einzelnen Schulen aber vergleichsweise gering sind, kann davon ausgegangen werden, dass die Schülerinnen und Schüler Schulen besuchen, in denen das Niveau ihrer Mitschüler in etwa ähnlich ist. Diese Aufteilung kann auf bewusste Entscheidungen der Familien in Bezug auf die Schul- oder Wohnortwahl, die Aufnahmebestimmungen der Schulen oder die von den Bildungsbehörden verfolgte Politik hinsichtlich der Verteilung der Schüler oder der Gestaltung der Lehrpläne zurückzuführen sein.

In Korea beträgt die Varianz der Schülerleistungen auf der Gesamtskala Lesekompetenz insgesamt nur rund die Hälfte des OECD-Durchschnitts, dort sind auch nur 20\% der durchschnittlichen OECD-Varianz in den Schülerleistungen auf Unterschiede zwischen den Schulen zurückzuführen. Korea erzielt somit im Bereich der Lesekompetenz nicht nur hohe Durchschnittsleistungen mit 
geringen Gesamtdifferenzen zwischen den Schülerleistungen, sondern ist auch in der Lage, die Unterschiede zwischen den Durchschnittsleistungen der Schülerinnen und Schüler verschiedener Schulen niedrig zu halten. Auch in Spanien sind sowohl die Gesamtvarianz (die sich auf rund drei Viertel des OECD-Durchschnitts beläuft) als auch die Unterschiede zwischen den Schulen gering (16\% der durchschnittlichen Varianz der Schülerleistungen in den OECD-Ländern), doch liegt der spanische Mittelwert, im Gegensatz zum koreanischen, deutlich unter dem OECD-Durchschnitt (Abb. 2.4).

Im Bereich Lesekompetenz sind die geringsten Leistungsdifferenzen zwischen den Schulen in Finnland, Island und Schweden zu beobachten, wo diese Unterschiede nur zwischen 7\% und 11\% der durchschnittlichen Differenzen in den Schülerleistungen ausmachen. In diesen drei Ländern besteht im Großen und Ganzen kein Zusammenhang zwischen den Leistungen der Schüler und den Schulen, die sie besuchen. Die Schülerinnen und Schüler treffen dort mit großer Wahrscheinlichkeit überall auf ein ähnliches Lernumfeld, was das Spektrum der Fähigkeiten ihrer Mitschüler anbelangt. Dabei ist zu erwähnen, dass in diesen Ländern auch die Gesamtdifferenz zwischen den Schülerleistungen unter dem OECD-Durchschnitt liegt und nur wenige Schülerinnen und Schüler die in ihren Schulen oder von ihren Lehrkräften festgelegte Mindestnote zur Versetzung nicht erreichen. Den Bildungssystemen dieser Länder gelingt es also, sowohl die Unterschiede zwischen den Schulen als auch die Leistungsdifferenzen zwischen den Schülern insgesamt im Hinblick auf die Lesekompetenz gering zu halten.

Australien, Neuseeland und Norwegen gehören (mit 112\%, 126\% bzw. $116 \%$ der durchschnittlichen Varianz der Schülerleistungen in den OECDLändern) zu den Ländern mit den größten Leistungsunterschieden im Bereich Lesekompetenz, doch nur ein geringer Teil dieser Unterschiede (21\%, 20\% bzw. 13\% der OECD-Durchschnittsleistung) geht auf Unterschiede zwischen den Schulen zurück. In diesen Ländern ist die Varianz der Schülerleistungen innerhalb der einzelnen Schulen am größten, weshalb die Schulen dort äußerst unterschiedlichen Zielpersonen gerecht werden müssen.

Weitere Länder mit vergleichsweise hohen Differenzen in den Schülerleistungen sind Belgien, Deutschland und die Schweiz (124\%, 133\% und 112\% der durchschnittlichen Varianz der Schülerleistungen in den OECD-Ländern), doch resultiert in diesen Ländern ein großer Teil der Varianz (76\%, 75\% bzw. 49\% der durchschnittlichen Leistungsvarianz der OECD-Länder) aus Leistungsunterschieden zwischen den Schulen.

Die Vereinigten Staaten, ein weiteres Land mit einer vergleichsweise hohen Varianz in den Schülerleistungen (118\% des OECD-Durchschnitts), liegen in etwa im mittleren Bereich: Im Vergleich zur durchschnittlichen Varianz der Schülerleistungen in den OECD-Ländern entfallen dort 35\% auf Unterschiede zwischen den Schulen

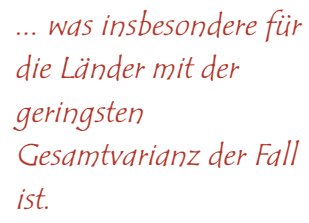

Eine hohe

Gesamtvarianz kann auf

erhebliche Leistungs-

unterschiede innerhalb

von Schulen,...
... oder aufeine Kombination dieser beiden Faktoren zurückzuführen sein. 
KAPITEL 2 Was PISA über die Fähigkeiten 15-Jähriger aussagt - ein Profil der Schülerleistungen im Bereich Lesekompetenz

Kasten 2.4 Mit Leistungsunterschieden zwischen den Schulen zusammenhängende Faktoren

Die Unterschiede zwischen den durchschnittlichen Leistungen der Schülerinnen und Schüler verschiedener Schulen werden durch vielerlei Faktoren beeinflusst. Einige davon sind im Folgenden genannt:

- Unterschiede zwischen verschiedenen Verwaltungseinheiten: In mehreren Ländern sind die Schulsysteme dezentral organisiert und werden beispielsweise auf Ebene der verschiedenen Sprachgemeinschaften wie in Belgien, der Provinzen wie in Kanada, der Bundesländer wie in Deutschland oder der Bundesstaaten wie in Australien oder den Vereinigten Staaten verwaltet. In anderen Ländern unterscheiden sich die Schulsysteme durch die Kombination verschiedener Verwaltungsdistrikte und Sprachgruppen (z.B. in der Schweiz).

- Stadt und Land: Oft sind die Unterrichtsformen und die Lehrinhalte in städtischen und ländlichen Regionen unterschiedlich.

- Staatliche oder private Schulen: In vielen Ländern stehen öffentlich und privat verwaltete Schulen miteinander im Wettbewerb. In manchen Ländern haben Privatschulen gewöhnlich strengere Aufnahmebestimmungen. Zudem können bei privat finanzierten Schulen Zugangsbarrieren für Schülerinnen und Schüler bestehen, die aus in sozioökonomischer Hinsicht benachteiligten Milieus kommen.

-Ausrichtung des schulischen Angebots: In einigen Bildungssystemen wird zwischen verschiedenen Schultypen unterschieden, deren Curricula deutlich voneinander abweichen können (z.B. in der Vorbereitung auf ein Hochschulstudium oder auf den direkten Eintritt ins Berufsleben). Selbst in Systemen, bei denen die Einteilung der Schülerinnen und Schüler in Leistungsgruppen innerhalb der Schulen erfolgt, können zwischen den Schulen Unterschiede hinsichtlich des Prozentsatzes der Schülerinnen und Schüler bestehen, die berufsbildende oder allgemeinbildende Zweige besuchen.

- Klassenstufe: In ein paar Ländern besucht ein Teil der 15-Jährigen bereits Sekundarstufe II, während ein anderer noch in Sekundarstufe I ist, wofür entweder der Geburtsmonat der Schüler oder die geltenden Versetzungsregeln ausschlaggebend sind. In anderen Ländern beherbergt dieselbe Schule wiederum mehrere Bildungsstufen. Daraus ergibt sich, dass die Leistungsdifferenzen zwischen Sekundarstufe I und II im ersten Fall auf Unterschiede zwischen den Schulen, im zweiten Fall auf Unterschiede zwischen den Schülern innerhalb einer Schule zurückzuführen sind.

-Sozioökonomischer Hintergrund der Schülerinnen und Schüler: Die sozioökonomischen Merkmale der Einzugsgebiete der Schulen sind häufig unterschiedlich, wenngleich die Größenordnung dieser Disparitäten zwischen den einzelnen Ländern ebenfalls stark abweichen kann. Der unterschiedliche sozioökonomische Hintergrund der Schülerinnen und Schüler einer Schule kann Einfluss auf die Schülerleistungen haben. 
Die Antwort auf die Frage, wie es zu diesen Unterschieden kommt, kann von Land zu Land anders ausfallen (vgl. auch Kasten 2.4). Die Teilnehmerländer haben Angaben zu den in PISA erfassten geographischen, systemischen oder institutionellen Aspekten ihrer Bildungssysteme gemacht, denen sie im Hinblick auf die Leistungsunterschiede zwischen den Schulen die größte Bedeutung beimessen. Der Varianzanteil, der sich aus diesen Variablen erklären lässt, ist in Abbildung 2.6 im linken Teil der Balken dargestellt.

- In Australien reduzieren sich die Leistungsdifferenzen zwischen den Schulen nach Berücksichtigung der zwischen den verschiedenen Bundesstaaten und Territorien bestehenden Unterschiede von $21 \%$ auf $19 \%$ der durchschnittlichen Varianz der Schülerleistungen (vgl. Tabelle 2.4).

- In Österreich reduzieren sich die Leistungsdifferenzen zwischen den Schulen nach Berücksichtigung der Aufteilung der Schüler auf sechs verschiedene Schultypen von 68\% auf $8 \%$. In Belgien verringern sich die Leistungsdifferenzen zwischen den Schulen nach Abzug der Unterschiede zwischen den Sprachgruppen sowie den verschiedenen Schultypen von 76\% auf 25\%. Nach Berücksichtigung der Unterschiede zwischen den verschiedenen Schultypen und Schulprogrammen reduzieren sich die Leistungsdifferenzen zwischen den Schulen in Deutschland von 75\% auf 10\%, in Ungarn von 71\% auf 19\%, in Polen von 67\% auf 14\% und in Korea von 20\% auf 9\%.

- Die Berücksichtigung der Unterschiede zwischen allgemein- und berufsbildenden Schulen sowie zwischen Sekundarstufe I und Sekundarstufe II hat in der Tschechischen Republik eine Verringerung der Leistungsdifferenzen zwischen den Schulen von 52\% auf 7\% und in Griechenland von 54\% auf $21 \%$ zur Folge.

- In Irland reduzieren sich die Leistungsdifferenzen zwischen den Schulen nach Berücksichtigung der unterschiedlichen Schultypen sowie der Unterschiede zwischen den Schulen in ländlichen bzw. in städtischen Gebieten von 17\% auf $7 \%$.

- In Italien verringern sich die Differenzen zwischen den Schulen nach Berücksichtigung der Unterschiede zwischen den Bildungsstufen und den Schultypen (Licei versus berufsbildende Schulen und istituti tecnichi) von 51\% auf 23\% und in Mexiko von 43\% auf 16\%.

- In Kanada reduziert sich die Leistungsdifferenz zwischen den Schulen nach Abzug der Unterschiede zwischen den Provinzen von 17\% auf 16\%.

- In Island hat die Berücksichtigung der Schulgröße und des Urbanisierungsgrads eine Verringerung der Leistungsdifferenzen zwischen den Schulen von 7\% auf $6 \%$ zur Folge.
Ein Teil der Unterschiede zwischen den Schulen ist durch geographische oder institutionelle Faktoren oder auch die Auswahl der Schülerschaft entsprechend ihrer Fähigkeiten bedingt, ... 
KAPITEL 2 Was PISA über die Fähigkeiten 15-Jähriger aussagt - ein Profil der Schülerleistungen im Bereich Lesekompetenz

- In Neuseeland reduzieren sich die Leistungsdifferenzen zwischen den Schulen nach Berücksichtigung des sozialen Hintergrunds der Schüler (Einstufung nach dem sozioökonomischen Index sowie Anteil der Maori-Schüler und der Schüler aus anderen Pazifikregionen) von 20\% auf $7 \%$.

- In Norwegen schrumpfen die Leistungsunterschiede zwischen den Schulen nach Abzug der auf Migrantenkinder entfallenden Leistungsdifferenz von 13\% auf $12 \%$ und in Schweden von 9\% auf 6\%.

- In Spanien verringern sich die Leistungsdifferenzen zwischen den Schulen nach Berücksichtigung der Unterschiede zwischen öffentlich und privat verwalteten Schulen von 16\% auf 10\%.

- In der Schweiz reduzieren sich die Leistungsunterschiede zwischen den Schulen nach Berücksichtigung der Differenzen zwischen den verschiedenen Schultypen, den Bildungsstufen und den Sprachregionen, in denen die Schulen angesiedelt sind, von $49 \%$ auf $27 \%$.

- Im Vereinigten Königreich schrumpft der Abstand zwischen den Schulen nach Berücksichtigung der Unterschiede zwischen den von nachgeordneten Gebietskörperschaften oder von anderen Organen - beispielsweise eigenständigen Einrichtungen oder kirchlichen Stiftungen - verwalteten Schulen, zwischen den gemischten Schulen und den getrennten Mädchen- und Jungenschulen sowie zwischen den verschiedenen Regionen von 22\% auf $15 \%$.

wobei die Anhäufung sozial besser gestellter Schülerinnen und Schüler, vor allem in

Ländern mit unterschiedlichen

Sekundarschultypen, als verstärkender Faktor hinzukommen kann,...
Die vorliegenden Daten legen insgesamt auch den Schluss nahe, dass in Bildungssystemen, die eine Einteilung in verschiedene Schultypen vorsehen, Schülerinnen und Schüler mit bestimmten sozioökonomischen Merkmalen häufiger in denselben Schulen zusammentreffen als in Systemen, in denen sich die Curricula der verschiedenen Schulen nicht wesentlich unterscheiden. In Österreich, Belgien, der Tschechischen Republik, Deutschland, Italien und den Niederlanden beispielsweise wird der mit der Verteilung der Schüler auf unterschiedliche Schultypen zusammenhängende Leistungsabstand zwischen den Schulen durch Differenzen in Bezug auf den sozialen und familiären Hintergrund zusätzlich verstärkt. Dies könnte die Folge eines Selektionsbzw. Autoselektionsphänomens sein: Wenn der Bildungsmarkt eine gewisse Differenzierung vorsieht, besteht die Tendenz, dass Schülerinnen und Schüler aus unteren sozialen Schichten in weniger anspruchsvolle Lehrprogramme gelenkt werden bzw. sich selbst für solche Programme entscheiden oder beschließen, nicht an den im Bildungssystem vorgesehenen Auswahlverfahren teilzunehmen.

Die ausführlichere Analyse hierzu in Kapitel 8 deutet darauf hin, dass der soziale Hintergrund der Gesamtheit der Schülerinnen und Schüler einer Schule in der Regel größere Auswirkungen hat als der soziale Hintergrund des einzelnen Schülers. Schülerinnen und Schüler aus einer unteren sozialen Schicht erzielen 
in der Regel wesentlich bessere Ergebnisse, wenn sie Schulen besuchen, deren Schülerschaft im Durchschnitt einem gehobenen Milieu entstammt, als wenn sie in Schulen gehen, in denen der sozioökonomische Hintergrund der Schülerschaft unter dem Durchschnitt liegt. Das Gegenteil ist der Fall für Schülerinnen und Schüler aus besser gestellten Milieus in Schulen, deren sozioökonomischer Hintergrund weniger günstig ist. Dies ist zwar an sich nicht überraschend, frappierend ist jedoch das Ausma $\beta$ der Unterschiede. Wie in Kapitel 8 beschrieben, dürfte die Differenz zwischen dem zu erwartenden Ergebnis eines beliebigen Schülers in zwei verschiedenen Schulen, die nach dem durchschnittlichen sozioökonomischen Status ihrer Schülerinnen und Schüler (nach der Definition des PISA-Index) beispielsweise 10 Punkte auseinanderliegen, größer sein als der zu erwartende Unterschied zwischen zwei verschiedenen Schülern ein und derselben Schule, zwischen denen ein Abstand von 10 Punkten besteht. Daraus lässt sich schließen, dass die im Bildungssystem vorgesehene institutionelle Differenzierung - häufig verstärkt durch Unterschiede beim sozialen Hintergrund der Schülerinnen und Schüler sowie die eigenen Entscheidungen der Schülerinnen und Schüler bzw. ihrer Eltern - großen Einfluss auf den schulischen Erfolg der einzelnen Schüler haben kann.

\section{Schlussbetrachtungen}

Die Ergebnisse von PISA 2000 zeigen, dass hinsichtlich der Kenntnisse und Fähigkeiten der 15-Jährigen im Bereich der Lesekompetenz zwischen den Ländern große Unterschiede bestehen. 124 Skalenpunkte, d.h. 1,7 Kompetenzstufen, trennen die OECD-Länder, die gemessen an ihren Durchschnittsergebnissen auf der Gesamtskala Lesekompetenz am besten abschneiden, von denen, die die niedrigsten Ergebnisse erzielen. Die Unterschiede zwischen den Ländern machen jedoch nur einen Bruchteil der Gesamtdifferenzen in den Schülerleistungen aus: Die Leistungsunterschiede innerhalb der Länder sind durchschnittlich ungefähr zehnmal so groß wie die Unterschiede zwischen den Durchschnittsleistungen der einzelnen Länder. Einer derartig unterschiedlich zusammengesetzten Schülerschaft gerecht zu werden und die Leistungsunterschiede der Schülerinnen und Schüler zu verringern, stellt daher für alle Länder eine große Herausforderung dar.

Durchschnittlich 10\% der 15-Jährigen erreichen die höchste in PISA vorgesehene Kompetenzstufe und stellen damit ihre Fähigkeit unter Beweis, komplexe Leseaufgaben zu lösen, ein detailliertes Verständnis der Texte sowie der Bedeutung ihrer einzelnen Bestandteile zum Ausdruck zu bringen, Informationen kritisch zu bewerten und ausgehend von Fachkenntnissen Hypothesen aufzustellen. Am anderen Ende der Leistungsskala erreichen durchschnittlich 6\% der Schülerinnen und Schüler nicht einmal Stufe 1. Diese Schüler sind nicht in der Lage, die elementarsten Kenntnisse und Fähigkeiten, die in PISA 2000 gemessen wurden, routinemäßig nachzuweisen. Sie können zwar durchaus des Lesens im technischen Sinne mächtig sein, haben jedoch ernste Schwierigkeiten dabei, die Lesekompetenz als ein Mittel zur Förderung

\author{
... da die \\ Schülerleistungen nicht \\ nur von ihrem eigenen \\ familiären Hintergrund, \\ sondern auch - und \\ zwar in größserem Maße \\ - vom Hintergrund der \\ Gesam theit der \\ Schülerschaft einer \\ schule beeinflusst \\ werden.
}

Die Leistungsunterschiede zwischen den verschiedenen Ländern im Bereich der Lesekompetenz sind erheblich, die innerhalb der einzelnen Länder bestehenden Unterschiede sind jedoch noch größser.

PISA verweist auf einen gewaltigen Abstand zwischen den $10 \%$ der schülerinnen und schüler, die anspruchsvolle Leseaufgaben bewältigen können, und den $6 \%$, die nicht einmal einfache Aufgaben lösen können, ... 
KAPITEL 2 Was PISA über die Fähigkeiten 15-Jähriger aussagt - ein Profil der Schülerleistungen im Bereich Lesekompetenz

.. bzw. den $12 \%$, die nur zur Lösung einfacher Aufgaben in der Lage sind und die ebenfalls eine Herausforderung darstellen, vor allem in jenen Ländern, in denen ihr Anteil am größten

Selbst dort, wo nur relativ wenige Schülerinnen und schüler im internationalen Vergleich schwach abschneiden, kann ein grofser Teil von ihnen den im eigenen Land geltenden Leistungsnormen nicht gerecht werden. und Erweiterung ihrer Kenntnisse und Fähigkeiten in anderen Bereichen einzusetzen. Obwohl der Prozentsatz dieser Schülerinnen und Schüler in drei Ländern nur 2\% beträgt und in nur zwei OECD-Ländern 14\% übersteigt, muss das Vorhandensein einer solchen kleinen, aber dennoch ins Gewicht fallenden Minderheit von Schülerinnen und Schülern, denen gegen Ende der Pflichtschulzeit die für den weiteren Lernprozess erforderlichen Grundqualifikationen fehlen, für die politischen Entscheidungsträger jedoch ein echter Grund zur Besorgnis sein, wenn sie lebensbegleitendes Lernen für alle anstreben. Anlass zur Sorge besteht in diesem Zusammenhang umso mehr, als sich die Anzeichen dafür häufen, dass die weitere Aus- und Fortbildung nach Abschluss der Schulzeit die Qualifikationsunterschiede, die auf eine unterschiedlich erfolgreiche Absolvierung der schulischen Erstausbildung zurückzuführen sind, eher verschärft als verringert.

Rechnet man zu dem Prozentsatz der Schülerinnen und Schüler, die Stufe 1 nicht erreichen, den Prozentsatz jener hinzu, die nicht über Stufe 1 hinauskommen, derjenigen also, die nur die einfachsten Leseaufgaben bewältigen können (z.B. eine einfache Information finden, das Hauptthema eines Textes identifizieren oder eine simple Verbindung mit Alltagswissen herstellen), erhöht sich der Prozentsatz der leistungsschwachen Schülerinnen und Schüler in den OECDLändern auf durchschnittlich 18\%. In Ländern, in denen ein hoher Prozentsatz der Schüler unter oder auf Stufe 1 liegt, müssen sich Eltern, Pädagogen und politische Entscheidungsträger darüber klar werden, dass ein erheblicher Teil der Schülerinnen und Schüler keinen ausreichenden Nutzen aus den Bildungsmöglichkeiten zieht und möglicherweise auch nicht die notwendigen Kenntnisse und Fähigkeiten erwirbt, um dies während der weiteren Schulzeit oder im späteren Leben effizient zu tun. In Ländern wie Brasilien oder Mexiko, deren Volkseinkommen vergleichsweise niedrig ist und deren Bildungsaufwendungen pro Schüler bis zum Alter von 15 Jahren nur rund ein Viertel des OECD-Durchschnitts betragen (Tabelle 3.6), stellt die Förderung der Schülerinnen und Schüler mit den größten Defiziten eine gewaltige Herausforderung dar. Zudem wurden die besonderen staatlichen Maßnahmen, mit denen diese Aufgabe bewältigt werden soll, in vielen Fällen erst in letzter Zeit eingeleitet.

Große Unterschiede in den Schülerleistungen bedeuten indessen nicht immer auch gleichzeitig, dass ein großer Prozentsatz der Schülerinnen und Schüler ein niedriges Lesekompetenzniveau besitzt. In einigen Ländern, die sowohl hohe Durchschnittswerte als auch eine hohe Varianz in den Schülerleistungen aufweisen, wie Australien, Neuseeland und das Vereinigte Königreich, liegt das 25. Perzentil auf der Gesamtskala Lesekompetenz noch immer klar im Bereich von Stufe 2 (ca. 458 Punkte), woraus sich schließen lässt, dass die Schülerinnen und Schüler beim 25. Perzentil im internationalen Vergleich noch immer recht gut abschneiden. Die große Streuung bei den Schülerleistungen in diesen Ländern deutet jedoch darauf hin, dass die Schülerinnen und Schüler 
beim 25. Perzentil möglicherweise weit unter den Leistungsnormen des eigenen Landes liegen.

Nun stellt sich die Frage, inwieweit die Leistungsheterogenität zwischen den Schülern eine Verteilung der Schülerbegabungen widerspiegelt und die Bildungssysteme somit vor eine Herausforderung stellt, der nicht unmittelbar mit bildungspolitischen Maßnahmen begegnet werden kann. Die in diesem Kapitel enthaltene Analyse hat gezeigt, dass es nicht nur große Unterschiede in Bezug auf die Differenzen in den Schülerleistungen innerhalb eines Landes gibt, sondern auch, dass eine breite Streuung der Leistungen nicht zwangsläufig Voraussetzung für ein hohes Gesamtniveau ist. Obwohl beim Vergleich solcher Unterschiede zwischen den Ländern auch andere - allgemeinere Umfeldfaktoren berücksichtig werden müssen, kann man davon ausgehen, dass die staatliche Politik durchaus zur Gewährleistung von Chancengleichheit und ausgewogenen Lernständen beitragen kann. PISA zeigt, dass zwischen den Ländern nicht nur Unterschiede in Bezug auf die durchschnittlichen Schülerleistungen bestehen, sondern auch im Hinblick auf die Fähigkeit, den Abstand zwischen den leistungsschwächsten und den leistungsstärksten Schülern zu verringern und einige der Barrieren abzubauen, die einer ausgewogenen Verteilung der Lernergebnisse entgegenstehen. PISA liefert somit Erkenntnisse von unmittelbarer bildungspolitischer Relevanz.

Die Leistungsunterschiede zwischen den Schülern werden durch viele Faktoren bedingt. Sie können auf den sozioökonomischen Hintergrund der Schülerinnen und Schüler und der Schulen, auf den Umfang der den Schulen zur Verfügung stehenden personellen und finanziellen Ressourcen, auf unterschiedliche Curricula und auf die Art und Weise, wie der Unterricht organisiert und durchgeführt wird, zurückzuführen sein. Da die Ursachen der Leistungsunterschiede zwischen den Schülern sehr unterschiedlich sind, suchen die verschiedenen Länder diesem Problem auch mit unterschiedlichen Methoden zu begegnen. Einige Länder haben nicht-selektive Schulsysteme aufgebaut, die allen Schülerinnen und Schülern dieselben Lernmöglichkeiten geben sollen und in denen die einzelnen Schulen auf das gesamte Leistungsspektrum der Schülerinnen und Schüler ausgerichtet sind. Andere Länder antworten bewusst auf die Verschiedenartigkeit der Schülerinnen und Schüler, indem sie sie durch Selektion zwischen oder innerhalb der Schulen in Gruppen mit ähnlichen Leistungsniveaus einteilen, mit dem Ziel, die Schülerinnen und Schüler ihrer besonderen Bedürfnisse entsprechend zu fördern.

Wie wirken sich diese Vorgehensweisen und Praktiken nun auf die Schülerleistungen aus? Die Frage lässt sich nicht eindeutig beantworten, da diese innerhalb der Schulen oft auf informeller Basis umgesetzt werden und zwischen den Ländern nur schwer vergleichbar sind. Gleichwohl legen die Daten aus PISA 2000 den Schluss nahe, dass sowohl die Gesamtvarianz zwischen den Schülerleistungen generell als auch der auf Unterschiede zwischen den Schulen entfallende Anteil dieser Varianz in solchen Ländern größer ist, in denen die Schülerinnen und Schüler bereits zu einem frühen
Jene Länder, die hohe Durchschnittsleistungen und zugleich eine relativ geringe Leistungsheterogenität erzielen, können den anderen als Ansporn dienen, ...

... vor allem solchen Ländern, deren Schulsysteme große Unterschiede zwischen den Schulen zulassen,... 
KAPITEL 2 Was PISA über die Fähigkeiten 15-Jähriger aussagt - ein Profil der Schülerleistungen im Bereich Lesekompetenz

In einigen Ländern

müssen die

Leistungsunterschiede

zwischen den Schulen,

in anderen die

Unterschiede innerhalb

von Schulen und in

wieder anderen beide

Arten von

Unterschieden reduziert

werden.
Zeitpunkt auf verschiedene Lehrprogramme und Schultypen verteilt werden. Die PISA-Ergebnisse lassen auch darauf schließen, dass in Bildungssystemen mit unterschiedlich gegliederten Schulsystemen die Wahrscheinlichkeit einer sozialen Clusterbildung größer ist als in solchen, in denen zwischen den Curricula der verschiedenen Schulen kaum nennenswerte Unterschiede bestehen. Diese Ergebnisse werden in Kapitel 8 ausführlicher untersucht. Dort wird auch auf politische Instrumente eingegangen, mit denen es einigen Ländern zu gelingen scheint, auf die zwischen dem sozialen Hintergrund und der schulischen Leistung bestehenden Beziehungen Einfluss zu nehmen und so zu einer größeren Chancengleichheit der Schüler im Bildungswesen beizutragen.

In Ländern, in denen auf der Gesamtskala Lesekompetenz zwischen den Schülerleistungen in denselben Schulen große Differenzen bestehen, wie dies beispielsweise für Neuseeland, Norwegen und Australien der Fall ist, könnten sich schulinterne Reformen, die darauf abzielen, die Leistungen der schwächeren Schülerinnen und Schüler anzuheben, im Hinblick auf die Steigerung des Leistungsniveaus insgesamt als sinnvoller erweisen als Reformen, die auf eine Verbesserung des Niveaus bestimmter Schulen ausgerichtet sind. In Ländern hingegen, in denen große Leistungsunterschiede zwischen den Schulen bestehen, z.B. in Österreich, Belgien, Deutschland, Ungarn und Polen, könnten die Gesamtleistungen wahrscheinlich durch Reformen gesteigert werden, die auf eine Anhebung der Leistungen der Schulen mit den niedrigsten Ergebnissen abzielen. 


\section{Anmerkungen}

1. Formal wurden der Mittelwert für die Schülerleistungen in den OECD-Ländern auf 500 und die Standardabweichung auf 100 festgelegt und die Daten so gewichtet, dass alle OECD-Länder gleichermaßen berücksichtigt werden.

2. Das in PISA zugrunde liegende Konzept der Lesekompetenz ist wesentlich weiter gefasst als die traditionelle Definition des Lesen- und Schreibenkönnens. Nach der in PISA verwendeten Definition bedeutet Lesekompetenz mehr als nur schriftliches Material dekodieren zu können und Texte wörtlich zu verstehen, so dass diese Art rein technischer Lesefertigkeiten in PISA nicht gemessen wurde. Schülerinnen und Schüler, die Kompetenzstufe 1 nicht erreichen, können folglich durchaus des Lesens und Schreibens im technischen Sinne mächtig sein.

3. In Anbetracht der Tatsache, dass alle Schülerinnen und Schüler auf einer gegebenen Kompetenzstufe - auch jene, die im untersten Bereich dieser Stufe liegen - in der Regel mindestens die Hälfte der dieser Stufe zugeordneten Aufgaben richtig beantworten, sowie auf Grund des festgelegten Umfanges einer Kompetenzstufe kann angenommen werden, dass ein Schüler mit einer bestimmten erreichten Punktzahl eine Aufgabe, deren Schwierigkeitsgrad der betreffenden Punktzahl entspricht, mit einer Wahrscheinlichkeit von $62 \%$ richtig beantwortet. Hierauf bezieht sich der in einigen Tabellen und Abbildungen vorkommende Wert RP (Lösungswahrscheinlichkeit) von 0,62.

4. Zur Bestätigung der statistischen Signifikanz der Unterschiede wurde die relative Wahrscheinlichkeit eines jeden Landes, eine entsprechende Position in der Rangordnung der einzelnen Lesekompetenzskalen einzunehmen über den Mittelwert des Landes, den Standardfehler und die Kovarianz zwischen den zwei Skalen bestimmt. Dies gibt mit 95\%iger Wahrscheinlichkeit an, ob ein Land auf einer Subskala signifikant höher, signifikant niedriger oder auf der relativ gleichen Position liegt wie auf der anderen Subskala. Wegen genauerer Angaben hierzu vgl. PISA 2000 Technical Report.

5. Wegen genauerer Angaben vgl. PISA 2000 Technical Report.

6. Multiple-Choice-Aufgaben wurden in diesen Vergleich nicht einbezogen, da die Schülerinnen und Schüler sie durch einfaches Raten zufällig richtig beantworten konnten.

7. In Deutschland sind 11,3\% der Schülerinnen und Schüler im Ausland geboren (Standardfehler 0,59); 5, 1\% der Schülerinnen und Schüler sind im Ausland geboren und liegen auf Stufe 1 oder darunter (Standardfehler 0,51); 88,7\% der Schülerinnen und Schüler sind in Deutschland geboren (Standardfehler 0,59); 14,4\% der Schülerinnen und Schüler sind in Deutschland geboren und liegen auf Stufe 1 oder darunter (Standardfehler 0,82). In Luxemburg sind 18,6\% der Schülerinnen und Schüler im Ausland geboren (Standardfehler 0,64); 11,5\% der Schülerinnen und Schüler sind im Ausland geboren und liegen auf oder unter Stufe 1 (Standardfehler 0,55); 81,5\% der Schülerinnen und Schüler sind in Luxemburg geboren (Standardfehler 0,64); 22,3\% der Schülerinnen und Schüler sind in Luxemburg geboren und liegen auf Stufe 1 oder darunter (Standardfehler 0,62).

8. Die Leistungen der polnischen Schülerinnen und Schüler dürften etwas zu hoch geschätzt sein, da die 6,7\% der 15-Jährigen, die noch in einer Grundschule eingeschriebenen sind, nicht berücksichtigt wurden. Daher dürfte die Position Polens in der Rangordnung bezüglich der Gesamtskala Lesekompetenz um zwei Rangplätze überschätzt sein. Die Leistungen der niederländischen Schülerinnen und Schüler können nicht genau geschätzt werden, da die Beteiligungsquote der Schulen zu niedrig war. Es kann jedoch mit ziemlicher Sicherheit gesagt werden, dass die Niederlande auf der Gesamtskala für Lesekompetenz unter den OECD-Ländern zwischen dem 2. und 14. Rangplatz liegen würden (wegen Einzelheiten vgl. Anhang A3).

9. Auf der Grundlage der mittleren Punktzahl der Länder, der entsprechenden Standardfehler und der Kovarianz zwischen den Leistungsskalen zweier Bereiche wurde die relative Wahrscheinlichkeit bestimmt, mit der ein Land jede der Rangpositionen auf jeder der Skalen erreichen kann. Auf diese Weise kann gefolgert werden, ob ein Land mit einer Wahrscheinlichkeit von $95 \%$ in einem Teilbereich statistisch signifikant über dem im anderen Teilbereich erzielten Niveau, auf diesem Niveau oder statistisch signifikant darunter liegen würde. Wegen Einzelheiten zur angewandten Methode vgl. PISA 2000 Technical Report. 
KAPITEL 2 Was PISA über die Fähigkeiten 15-Jähriger aussagt - ein Profil der Schülerleistungen im Bereich Lesekompetenz

10. Auf Grund der in Japan angewandten Methode der Stichprobenziehung sind in den Leistungsdifferenzen zwischen den Schulen auch die Leistungsunterschiede zwischen verschiedenen Klassen derselben Schule inbegriffen.

11. Die Höhe der Unterschiede in Abbildung 2.6 und Tabelle 2.4 ist durch die statistische Varianz ausgedrückt, die sich aus der Quadrierung der an anderer Stelle in diesem Kapitel erwähnten Standardabweichung ergibt. Für die Zwecke dieses Vergleichs wurde statt auf die weiter oben in diesem Kapitel erwähnte Standardabweichung auf die statistische Varianz zurückgegriffen, um die Anteile der Varianz in den Schülerleistungen aufschlüsseln zu können. Aus Gründen, die im PISA 2000 Technical Report näher erläutert werden, kann die Summe der Komponenten der Leistungsvarianz zwischen den Schulen und der Leistungsvarianz innerhalb der Schulen für einige Länder u.U. geringfügig vom Quadrat der in Tabellen 2.3a wiedergegebenen Standardabweichung differieren. Der Durchschnittswert wurde für die in Tabelle 2.4 angegebenen OECD-Länder errechnet. 


\section{Kapitel}

3

WAS PISA ÜBER DIE FÄHIGKEITEN 15-JÄHRIGER AUSSAGT - EIN PROFIL DER SCHÜLERLEISTUNGEN IN MATHEMATISCHER UND NATURWISSENSCHAFTLICHER GRUNDBILDUNG

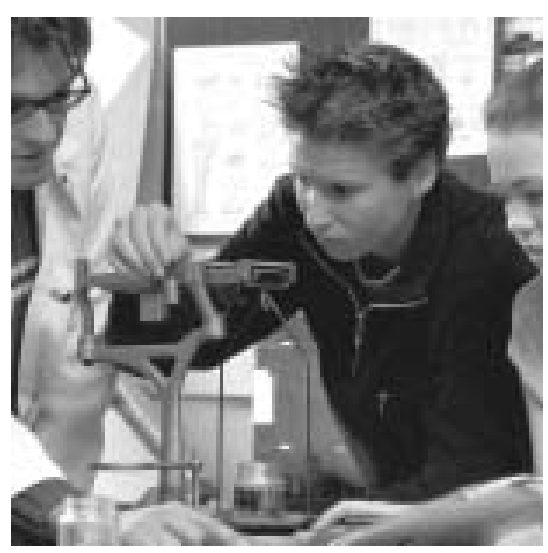


KAPITEL 3 Was PISA über die Fähigkeiten 15-jähriger aussagt

\begin{abstract}
Zur Anwendung
mathematischer und

naturwissenschaftlicher

Kenntnisse sollte heutzutage

nicht nur eine Minderheit,

sondern die großse Mehrheit

der Bevölkerung in der Lage

sein, ...
\end{abstract}

... um die moderne Welt zu verstehen und an ihr teilzuhaben.

PISA untersucht die

mathematischen und naturwissenschaftlichen

Kenntnisse und

Fähigkeiten der

Schülerinnen und Schüler, wobei die für ihr

späteres Leben

relevanten Kompetenzen im Vordergrund stehen.

\section{Einführung}

Im vergangenen Jahrhundert standen die Curricula der Schulmathematik und der naturwissenschaftlichen Fächer meist im Zeichen der Notwendigkeit, eine solide Grundlage für die berufliche Ausbildung einer kleinen Zahl von Mathematikern, Wissenschaftlern und Ingenieuren zu liefern. Mit der zunehmenden Bedeutung von Naturwissenschaften, Mathematik und Technologie für das moderne Leben erfordern Ziele wie Selbstverwirklichung, Berufstätigkeit und aktive Teilnahme am gesellschaftlichen Leben allerdings, dass alle Erwachsenen, und nicht nur diejenigen, die eine naturwissenschaftliche Karriere anstreben, über mathematische, naturwissenschaftliche und technische Grundkompetenzen verfügen.

Mathematische und naturwissenschaftliche Grundbildung sind für dasVerständnis ökologischer, medizinischer, ökonomischer und sonstiger Probleme von Bedeutung, denen sich die modernen Gesellschaften heute gegenübersehen, die sehr stark vom technologischen und naturwissenschaftlichen Fortschritt abhängen. Außerdem haben die Ergebnisse der leistungsstärksten Schülerinnen und Schüler eines Landes in Mathematik und naturwissenschaftlichen Fächern möglicherweise Auswirkungen auf die Rolle, die dieses Land in den Spitzentechnologiesektoren von morgen einnehmen wird und auf seine allgemeine internationale Wettbewerbsfähigkeit. Defizite in der mathematischen und naturwissenschaftlichen Grundbildung können hingegen durchaus negative Konsequenzen für die Arbeitsmarkt- und Einkommensaussichten des Einzelnen sowie für dessen aktive Teilnahme am gesellschaftlichen Leben haben.

Infolgedessen messen politische Entscheidungsträger und Pädagogen gleichermaßen der mathematischen und naturwissenschaftlichen Bildung große Bedeutung bei. Um der wachsenden Nachfrage nach mathematischen und naturwissenschaftlichen Kompetenzen gerecht zu werden, bedarf es eines in jeder Hinsicht hervorragenden Bildungssystems, und es ist hilfreich zu verfolgen, wie gut die Länder junge Erwachsene mit den Grundkompetenzen in diesen Bereichen ausstatten. So sind die mathematischen und naturwissenschaftlichen Kenntnisse und Fähigkeiten fester Bestandteil des PISA-Konzepts der Grundbildung (literacy). Ferner haben die Ergebnisse wegen der PISAspezifischen Definition der mathematischen und naturwissenschaftlichen Grundbildung, die in Kapitel 1 beschrieben wurde, für die fortgeschrittenen Industrieländer eine größere Bedeutung als Untersuchungen, die sich ausschließlich auf den gemeinsamen Nenner der nationalen Lehrpläne beziehen.

Das vorliegende Kapitel bietet einen Überblick über die PISA-Ergebnisse für die mathematische und naturwissenschaftliche Grundbildung. Ferner wird untersucht, bis zu welchem Grad sie den in Kapitel 2 in Bezug auf die Lesekompetenz dargelegten Ergebnissen entsprechen oder von ihnen abweichen. Das vorliegende Kapitel enthält: 
- eine Beschreibung der Kriterien für die Leistungsmessung in mathematischer und naturwissenschaftlicher Grundbildung sowie Beispielaufgaben mit einfachem, mittlerem und hohem Schwierigkeitsgrad aus PISA 2000;

- eine Zusammenfassung der Schülerleistungen in den einzelnen Ländern anhand der von den Schülerinnen und Schülern erreichten mittleren Punktzahl sowie der Punkteverteilung in den einzelnen Schülerpopulationen;

- eine Untersuchung der Leistungsdifferenzen zwischen Lesekompetenz, mathematischer und naturwissenschaftlicher Grundbildung.

In Kapitel 4 wird die Diskussion dann um die Analyse der nicht kognitiven Aspekte der Lernstände erweitert, wie beispielsweise die Motivation 15-Jähriger, ihr Engagement, ihre Lernstrategien wie auch ihr Vertrauen in die eigenen Fähigkeiten.

In PISA 2000 stand die Erfassung der Lesekompetenz im Mittelpunkt des Interesses. Aus diesem Grund wurden mathematische und naturwissenschaftliche Grundbildung nur eingeschränkt erhoben, und daher ist auch die Analyse auf diesen Gebieten nicht so detailliert wie im Bereich Lesekompetenz. Diese Bereiche werden in den nächsten PISA-Erhebungen vertieft, wobei 2003 das Hauptaugenmerk auf der mathematischen Grundbildung liegt und 2006 die naturwissenschaftliche Grundbildung im Mittelpunkt steht. Eine Beschreibung der Rahmenkonzeption, die den Leistungserhebungen von PISA in den Bereichen mathematische und naturwissenschaftliche Grundbildung als Basis dient, findet sich in Schülerleistungen im internationalen Vergleich - Eine neue Rahmenkonzeption für die Erfassung von Wissen und Fähigkeiten (Deutsches PISAKonsortium, 2000).

\section{Schülerleistungen im Bereich mathematische Grundbildung}

\section{Wie die mathematische Grundbildung in PISA gemessen wird}

In PISA 2000 werden die Leistungen in mathematischer Grundbildung anhand einer Gesamtskala gemessen, die, wie im Fall der Lesekompetenz, so konstruiert wurde, dass der Mittelwert bei 500 Punkten liegt, die Standardabweichung 100 Punkte beträgt und rund zwei Drittel der Schülerinnen und Schüler in den OECD-Ländern Ergebnisse zwischen 400 und 600 Punkten aufweisen ${ }^{1}$. Die Skala misst die Fähigkeit der Schülerinnen und Schüler, mathematische Probleme zu erkennen und zu interpretieren, denen sie in ihrem Umfeld begegnen, diese Probleme in mathematische Strukturen umzusetzen, mathematische Kenntnisse und Verfahren zur Lösung von Problemen innerhalb dieses mathematischen Kontexts anzuwenden, die Lösung im Hinblick auf das Ausgangsproblem zu interpretieren, über die angewandte Methode zu reflektieren und die Ergebnisse $\mathrm{zu}$ formulieren und zu kommunizieren.
Die Leistungen in mathematischer Grundbildung wurden anhand einer Gesamtskala gemessen, ... 
KAPITEL 3 Was PISA über die Fähigkeiten 15-jähriger aussagt

... wobei der Schwierigkeitsgrad der Aufgaben bestimmt wird durch die Komplexität der notwendigen Prozessschritte, ...

... durch die Anforderungen hinsichtlich der Herstellung von Querverbindungen zwischen verschiedenen

Informationen ...

...und durch die Anforderungen, die die

Formulierung und Lösung von Problemen an das mathematische

Denken der

Schülerinnen und

schüler stellen.

ie schwierigsten

Aufgaben setzen

kreatives

mathematisches Denken

und ein tieferes

mathematisches

Verständnis voraus, ...
Der wachsende Schwierigkeitsgrad der Aufgaben auf der Skala wird anhand folgender Kriterien definiert:

- Anzahl und Komplexität der für die Bewältigung einer Aufgabe notwendigen Prozessbzw. Rechenschritte. Die Aufgaben reichen von einschrittigen Problemen, bei denen von den Schülerinnen und Schülern verlangt wird, grundlegendes mathematisches Faktenwissen abzurufen oder einfache Rechenschritte durchzuführen, bis hin zu mehrschrittigen Problemen, die fortgeschrittenes mathematischesWissen und komplexe Entscheidungsprozesse, dieVerarbeitung von Informationen sowie Fähigkeiten zur mathematischen Problemlösung und Modellierung voraussetzen.

- Anforderungen in Bezug auf die Herstellung von Querverbindungen und Zusammenhängen. Bei den einfachsten Aufgaben wird von den Schülerinnen und Schülern generell verlangt, eine einzige mathematische Darstellung oder Technik auf eine Einzelinformation anzuwenden. Bei den komplizierteren Aufgaben wird von ihnen erwartet, Querverbindungen zwischen verschiedenen Einzelinformationen herzustellen und diese unter Verwendung verschiedener mathematischer Darstellungen, Instrumente oder Kenntnisse in einem mehrschrittigen Prozess zu verknüpfen.

- Anforderungen an Darstellung und Interpretation von Materialien sowie Reflexion über Situationen und Methoden. Die Aufgaben reichen von der Erkennung und Anwendung bekannter Formeln bis hin zur Formulierung, Übertragung oder Konzipierung eines geeigneten Modells in einem ungewohnten Kontext sowie derVerwendung eines tieferen mathematischen Verständnisses, Beweisführens, Argumentierens und Verallgemeinerns.

Da das Schwergewicht in PISA 2000 weniger auf der Erhebung mathematischer und naturwissenschaftlicher Grundbildung und stärker auf dem Bereich Lesekompetenz lag, wurde auch kein Versuch unternommen, Kompetenzstufen für mathematische und naturwissenschaftliche Grundbildung zu definieren. Dennoch ist es möglich, die Leistungen in mathematischer und naturwissenschaftlicher Grundbildung anhand der Kenntnisse und Kompetenzen ausführlich zu beschreiben, über die Schülerinnen und Schüler auf bestimmten Punkten der Skalen verfügen müssen.

Im Fall der Skala für mathematische Grundbildung bedeutet dies konkret:

- Am oberen Ende der Skala bei rund 750 Punkten nehmen die Schülerinnen und Schüler bei der Lösung mathematischer Probleme eine kreative und aktive Rolle ein. Sie interpretieren und formulieren Probleme anhand mathematischer Ausdrücke, können mit komplexeren Informationen umgehen und wägen zwischen einer Reihe von Prozessschritten ab. Schülerinnen und Schüler auf dieser Stufe wissen, welche Instrumente und Kenntnisse relevant sind, und wenden diese (häufig auch in einem ungewohnten Problemzusammenhang) an, zeigen tieferes Verständnis in das Wesen 
der Probleme, um geeignete Lösungsstrategien zu identifizieren, und greifen zur Erläuterung bzw. Kommunikation von Ergebnissen auf andere, höhere kognitive Prozesse zurück, wie Verallgemeinern, Beweisführung und mathematisches Argumentieren.

- Bei rund 570 Punkten auf der Skala sind die Schülerinnen und Schüler generell in der Lage, verschiedene Darstellungen eines mathematischen Problems oder Informationen aus verschiedenen Quellen zu interpretieren, zu verknüpfen und $\mathrm{zu}$ integrieren und/oder ein bestimmtes Modell anzuwenden oder zu manipulieren, das in vielen Fällen algebraische Formeln oder sonstige symbolische Darstellungen enthält, und/oder gegebene Lösungsvorschläge bzw. Modelle zu überprüfen oder zu testen. Die Schülerinnen und Schüler arbeiten in der Regel mit bestimmten Strategien, Modellen oder Thesen (z.B. indem sie ein Muster erkennen und fortführen), und sie wählen das mathematische Wissen aus und wenden es an, das in einer bestimmten Problemsituation, in der der Lösungsweg einige wenige Prozessschritte umfasst, relevant ist.

- Am unteren Ende der Skala bei rund 380 Punkten sind die Schülerinnen und Schüler in der Regel nur in der Lage, einen einzigen Rechenschritt auszuführen, der darin besteht, grundlegende mathematische Fakten oder Prozesse wiederzugeben bzw. einfache Rechenfertigkeiten anzuwenden. Die Schülerinnen und Schüler erkennen generell Informationen, die in ihnen vertrauten und einfachen Diagrammen und Textformen enthalten sind und in denen mathematische Formeln mitgeliefert werden oder leicht zu erkennen sind. Jede Interpretation oder Argumentation erfordert normalerweise zumindest die Erfassung eines vertrauten Elements des Problems. Der Lösungsprozess sieht auch die Anwendung eines Routineverfahrens mit einem einzigen Rechenschritt vor.

Im Durchschnitt aller OECD-Länder erreichen 5\% der leistungsstärksten Schülerinnen und Schüler 655 Punkte, 10\% 625 Punkte und 25\% 571 Punkte. Am unteren Ende der Skala erreichen über drei Viertel der Schülerinnen und Schüler zumindest 435 Punkte, über 90\% 367 und über 95\% 326 Punkte (Tabelle 3.1).

Die für die Beurteilung der mathematischen Grundbildung in PISA eingesetzten Aufgaben weisen höchst unterschiedliche Schwierigkeitsgrade auf. Abbildung 3.1 enthält Aufgaben, die in zwei der 16 Testeinheiten zur Erfassung der mathematischen Grundbildung gestellt wurden, sowie eine Beschreibung der Kriterien zur Kodierung der Schülerantworten (ein etwas vollständigeres Aufgabenset findet sich unter www.pisa.oecd.org).
... weniger schwierige

Aufgaben verlangen von

den Schülerinnen und

Schülern, Informationen

aus verschiedenen

Quellen miteinander zu

verknüpfen und zu

verarbeiten, ...

... während die

einfachsten Aufgaben

nur einen einzigen

Rechenschritt in einem

vertrauten Kontext

erfordern.

Die Mathematikaufgaben weisen höchst

unterschiedliche

Schwierigkeits-

grade auf... 
KAPITEL 3 Was PISA über die Fähigkeiten 15-jähriger aussagt

Nur eine kleine Zahl von Schülerinnen und schülern ist in der Lage, Aufgaben zu lösen, die ein tieferes Verständnis

für mathematische

Funktionen voraussetzten; ...

... ein Viertel der

Schülerinnen und Schülern ist in der Lage,

weniger schwierige Aufgaben zulösen, die von ihnen verlangten,

Ausdrücke zu

interpretieren,

verschiedene

Darstellungsformen miteinander zu verknüpfen und

Lösungen zu vergleichen; ...

... die Hälfte ist in der Lage, eine Beschreibung in eine Tabelle umzusetzen, die sie vervollständigen müssen;...
Frage 3 in der Testeinheit Äpfel ist die schwierigste der Beispielfragen in Abbildung 3.1. Den Schülerinnen und Schülern wird eine hypothetische Situation präsentiert, in der es darum geht, Apfelbäume in einem quadratischen Muster anzupflanzen, mit einer „Reihe“ schützender Nadelbäume um den Obstgarten herum. In dieser Situation wird von den Schülerinnen und Schülern erwartet, dass sie ein tieferes Verständnis für mathematische Funktionen unter Beweis stellen, indem sie die Zunahme einer linearen mit der einer quadratischen Funktion vergleichen. Es wird verlangt, eine Verallgemeinerung des Modells verbal zu formulieren und mit Hilfe algebraischer Formeln zu belegen. Für die richtige Antwort müssen die Schülerinnen und Schüler sowohl die in der Beschreibung des Musters verwendeten algebraischen Formeln als auch die zu Grunde liegenden funktionalen Beziehungen so weit verstehen, dass sie die Verallgemeinerung dieses Zusammenhangs auch in einem ungewohnten Kontext erkennen und erklären können. Um die volle Punktzahl für Frage 3 zu bekommen, d.h. 723 Punkte auf der Skala für mathematische Grundbildung, müssen die Schülerinnen und Schüler sowohl die korrekte Antwort als auch eine zutreffende Erklärung geben. Schülerinnen und Schüler mit einem Leistungsniveau von 723 Punkten werden Fragen dieses Schwierigkeitsgrads wahrscheinlich in 62 von 100 Fällen richtig beantworten (vgl. Kasten 2.1). Im Durchschnitt der OECD-Länder erhielten 8\% der Schülerinnen und Schüler die volle Punktzahl für diese offene Frage. Weitere 10\% erhielten eine abgestufte Punktzahl für teilweise gelöste Aufgaben (Daten abrufbar unter www.pisa.oecd.org).

In Frage 2 dieser Testeinheit - eine etwas weniger schwierige Frage mit einem Schwierigkeitsgrad von 655 auf der Skala für mathematische Grundbildung - werden die Schülerinnen und Schüler mit zwei algebraischen Formeln konfrontiert, die beschreiben, wie die Anzahl der Bäume bei Vergrößerung des Obstgartens steigt. Die Schülerinnen und Schüler sollen einen Wert bestimmen, bei dem die Anzahl der Apfel- und Nadelbäume gleich groß ist. Zur Beantwortung dieser Frage müssen die Schülerinnen und Schüler Ausdrücke interpretieren, die Worte und Symbole enthalten, und jeweils für zwei Funktionen (eine quadratische und eine lineare) verschiedene Darstellungsformen (Zeichnung, Text und Algebra) miteinander verknüpfen. Sie müssen eine Strategie entwickeln, um zu bestimmen, wann die beiden Funktionen die gleiche Lösung haben, und das Ergebnis dann unter Begründung des Lösungswegs und der verschiedenen Rechenschritte kommunizieren. In den OECD-Ländern bekamen durchschnittlich 25\% der Schülerinnen und Schüler für diese offene Frage die volle Punktzahl.

Bei der einfachsten Frage in der Testeinheit Äpfel werden die Schülerinnen und Schüler aufgefordert, eine Tabelle mit Werten zu vervollständigen, die mit Hilfe der Funktionen generiert wird, die beschreiben, wie die Zahl der Bäume bei Vergrößerung des Obstgartens zunimmt. Zur Beantwortung dieser Frage müssen die Schülerinnen und Schüler eine schriftliche Beschreibung einer Situation interpretieren, diese mit einer tabellarischen Darstellung 
ÄPFEL

Ein Bauer pflanzt Apfelbäume an, die er in einem quadratischen Muster anordnet. Um diese Bäume vor dem Wind zu schützen, pflanzt er Nadelbäume um den Obstgarten herum.

Im folgenden Diagramm siehst du das Muster, nach dem Apfelbäume und Nadelbäume für eine beliebige Anzahl (n) von Apfelbaumreihen gepflanzt werden:

$\mathbf{X}=$ Nadelbaum

- Apfelbaum

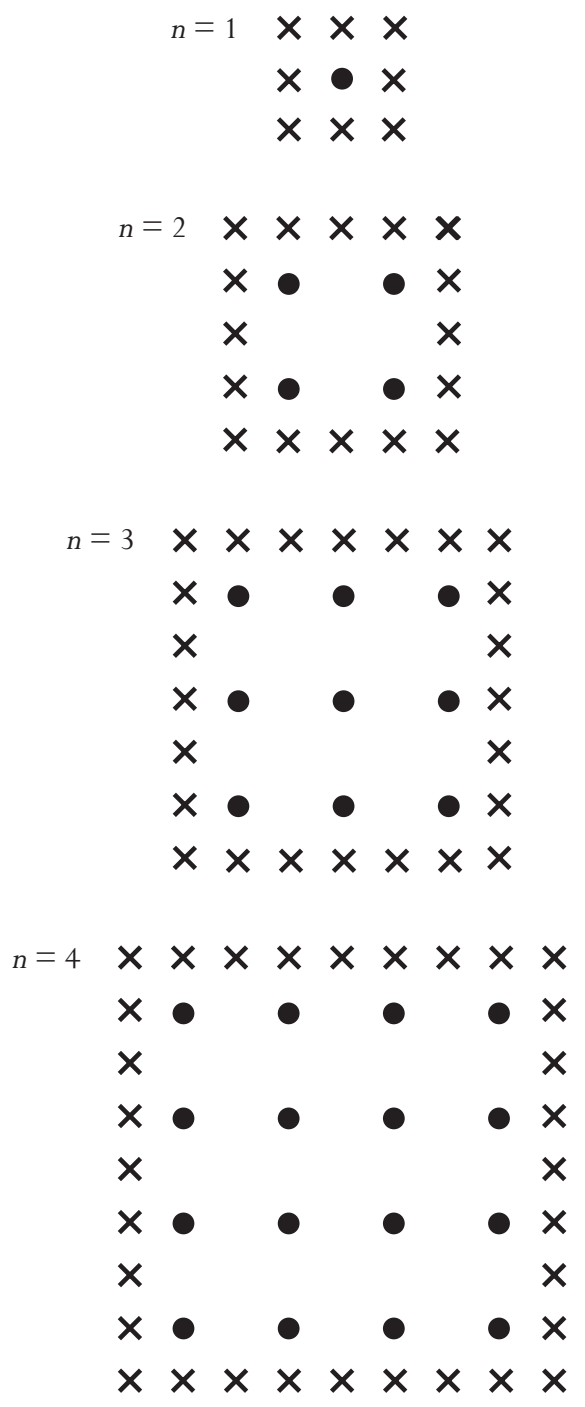


KAPITEL 3 Was PISA über die Fähigkeiten 15-jähriger aussagt

\section{SCHWIERIGKEITSGRAD}

\section{FRAGE 3}

ÄPFEL

Angenommen, der Bauer möchte einen viel größeren Obstgarten mit vielen Reihen von Bäumen anlegen. Was wird schneller zunehmen, wenn de Bauer den Obstgarten vergrößert: die Anzahl der Apfelbäume oder die Anzahl der Nadelbäume?

Erkläre, wie du zu deiner Antwort gekommen bist.
Punktekategorie 2 (723*) algebraische Erklärungen auf der Basis der Formeln $n^{2}$ und $8 n$ gegeben werden.

Punktekategorie 1

- Korrekte Antworten (Apfelbäume) auf der Basis von spezifischen Beispielen oder dem Weiterführen der Tabelle.

- Richtige Antworten (Apfelbäume) zusammen mit Anhaltspunkten, die zeigen, dass die Beziehung zwischen $\mathrm{n}^{2}$ und $8 \mathrm{n}$ verstanden wurde, aber nicht so klar ausgedrückt wie in Punktekategorie 2.
- Korrekte Antworten (Apfelbäume), wobei

Bei dieser Aufgabe wird von den Schülerinnen und Schülern erwartet, dass sie tieferesVerständnis für mathematische Funktionen unter Beweis stellen, indem sie die Zunahme einer linearen mit der einer quadratischen Funktion vergleichen. Es wird von ihnen verlangt, eineVerallgemeinerung des Problems verbal zu formulieren und mit Hilfe algebraischer Formeln zu belegen. Die Schülerinnen und Schüler müssen sowohl die in der Beschreibung des Musters verwendeten algebraischen Formeln als auch die $750 \mathrm{zu}$ Grunde liegenden funktionalen Beziehungen so weit verstehen, dass sie dieVerallgemeinerung dieser Zusammenhänge in einem unbekannten

- Kontext erkennen und erklären können. Eine Argumentationskette ist erforderlich, die in einer schriftlichen Erläuterung kommuniziert werden muss.

Bei dieser Aufgabe wird von den Schülerinnen und Schülern erwartet, dass sie Ausdrücke interpretieren, dieWorte und Symbole enthalten, $\rightarrow$ und dass sie verschiedene Darstellungsformen (Zeichnung, Text und Algebra) jeweils für zwei Funktionen (eine quadratische und eine lineare) miteinander verknüpfen. Die Schülerinnen und Schüler müssen eine Strategie entwickeln, um zu bestimmen, wann die beiden Funktionen die gleiche Lösung haben (z.B. durch Ausprobieren oder mit Hilfe algebraischer Formeln), und das Ergebnis dann unter Erläuterung des Lösungswegs und der verschiedenen Rechenschritte kommunizieren. 570

der Nadelbäume für das oben beschriebene Muster zu berechnen:

- Anzahl der Apfelbäume $=n^{2}$ - Anzahl der Nadelbäume $=8 \mathrm{n}$ - wobei $n$ die Anzahl der Apfelbaumreihen bezeichnet. Es gibt einen Wert für $n$, bei dem die Anzahl der Apfelbäume gleich groß ist wie die Anzahl der Nadelbäume. Bestimme diesen Wert und gib an, wie du ihn berechnet hast. aber nicht klar sichtbar oder keine Berechnungen angegeben.

Punktekategorie 2 (655*)

- Antworten mit $n=8$, algebraische Methode explizit angegeben.

- Antworten mit $\mathrm{n}=8$ mit anderen Lösungswegen, z.B. Fortsetzen des Musters in der Tabelle oder Zeichnung.

\section{FRAGE 1}

ÄPFEL

Vervollständige die Tabelle:

\begin{tabular}{c|cc}
$n$ & $\begin{array}{c}\text { Anzahl der } \\
\text { Apfelbäume }\end{array}$ & $\begin{array}{c}\text { Anzahl der } \\
\text { Nadelbäume }\end{array}$ \\
\hline 1 & 1 & 8 \\
\hline 2 & 4 & 16 \\
\hline 3 & 9 & 24 \\
\hline 4 & 16 & 32 \\
\hline 5 & 25 & 40 \\
\hline
\end{tabular}

Punktekategorie 2 (548*)

-Antworten mit 7 korrekten Einträgen. 
einiger Informationen verknüpfen, ein Muster erkennen und dieses dann weiterführen. Sie müssen mit den gegebenen Modellen arbeiten und zwei unterschiedliche Darstellungsformen (Zeichnung und Tabelle) zweier Funktionen (einer quadratischen und einer linearen) zur Fortsetzung des Musters miteinander verknüpfen. Im Durchschnitt der OECD-Länder erhielten $50 \%$ der Schülerinnen und Schüler die volle Punktzahl für diese offene Frage und weitere 13\% eine abgestufte Punktzahl für teilweise gelöste Aufgaben

Die zweite in Abbildung 3.1 gezeigte Testeinheit Rennwagen enthält Aufgaben, die das Niveau im mittleren Feld und am unteren Ende der Skala für mathematische Grundbildung veranschaulichen. Frage 5, die auf der Skala für mathematische Grundbildung bei 492 Punkten liegt, enthält einen Graphen, der die Geschwindigkeit eines Rennwagens auf einer Rennstrecke anzeigt. Die Aufgabe besteht darin, anhand einer Interpretation des Graphen eine

\author{
... zwei Drittel verfügen \\ über die Fähigkeit, durch \\ verknüpfung von zwei \\ Merkmalen einen \\ Graphen zu \\ interpretieren;...
}

\section{GESCHWINDIGKEIT EINES RENNWAGENS}

Dieser Graph zeigt, wie die Geschwindigkeit eines Rennwagens während seiner zweiten Runde auf einer drei Kilometer langen ebenen Rennstrecke variiert.

\section{Geschwindigkeit eines Rennwagens auf einer Strecke von 3 km (2. Runde)}

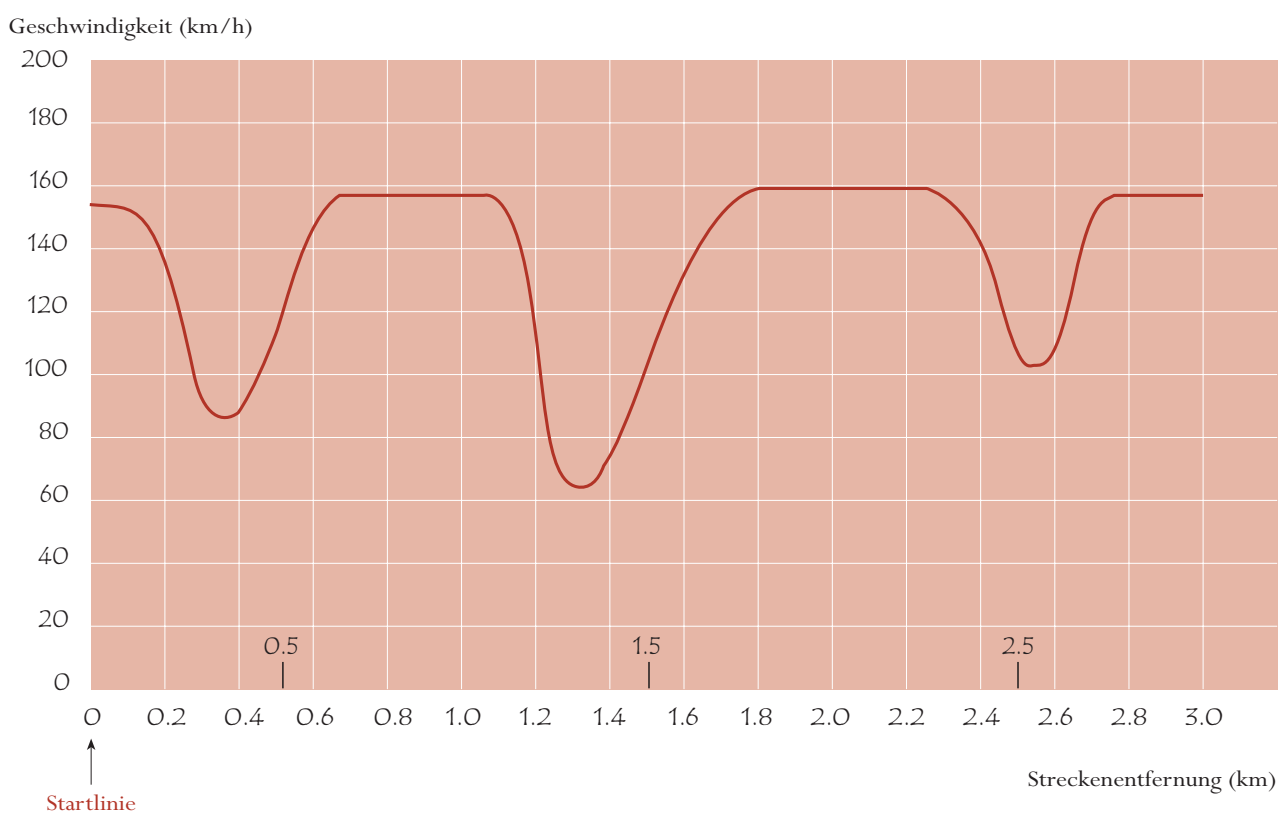


KAPITEL 3 Was PISA über die Fähigkeiten 15-jähriger aussagt

FRAGE 8

GESCHWINDIGKEIT EINES RENNWAGENS

Hier siehst du Abbildungen von fünf Rennstrecken: Auf welcher dieser Rennstrecken fuhr der

Wagen, so dass der am Anfang gezeigte Geschwindigkeitsgraph entstand?

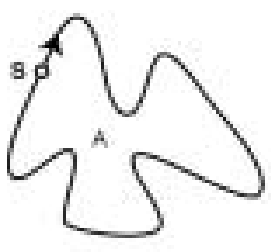

S: Startlinie

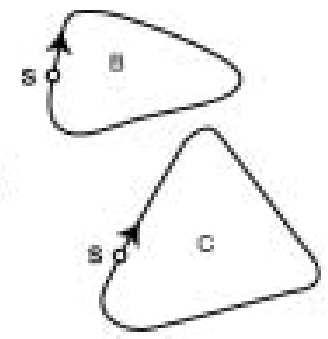

$s p o$

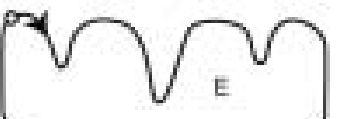

FRAGE 5

GESCHWINDIGKEIT EINES RENNWAGENS

Wie groß ist die ungefähre Entfernung von der Startlinie bis zum Beginn des längsten geradlinigen Abschnitts der Rennstrecke?

A. $0,5 \mathrm{~km}$

B. $1,5 \mathrm{~km}$

C. $2,3 \mathrm{~km}$

D. $2,6 \mathrm{~km}$

\section{FRAGE 7}

GESCHWINDIGKEIT EINES RENNWAGENS

Was kannst du über die Geschwindigkeit des Wagens zwischen den Markierungen 2,6 km und 2,8 km sagen?

A. Die Geschwindigkeit des Wagens bleibt konstant.

B. Die Geschwindigkeit des Wagens nimmt zu.

C. Die Geschwindigkeit des Wagens nimmt ab.

D. Die Geschwindigkeit des Wagens kann anhand des Graphen nicht

bestimmt werden.

FRAGE 6

GESCHWINDIGKEIT EINES RENNWAGENS $\quad$ Punktekategorie 2 (403*)

Wo wurde während der zweiten Runde $\quad$ - C: bei etwa 1,3 km.

die geringste Geschwindigkeit gemessen?

A. An der Startlinie.

B. Bei etwa $0,8 \mathrm{~km}$

C. Bei etwa $1,3 \mathrm{~km}$

D. Nach der halben Runde.

Quelle: OECD PISA, 2001.

Punktekategorie 1 (492*)

- B: 1,5 km.

(413*) nimmt zu.
SCHWIERIGKEITSGRAD

Punktekategorie 1 (655*)

Antwort B.

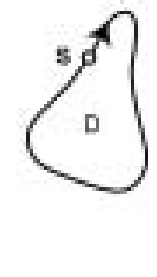

B: Die Geschwindigkeit des Wagens
Bei dieser Aufgabe müssen die Schülerinnen und Schüler eine graphische Darstellung zweier Elemente, zwischen denen ein physikalischer

750 Zusammenhang besteht (Geschwindigkeit und Entfernung einesWagens), verstehen und interpretieren und das Ganze auf eine reale Situation anwenden.. Die Schülerinnen und Schüler müssen zwei ganz unterschiedliche visuelle

Darstellungsformen einer Fahrt auf einer Rennstrecke miteinander verknüpfen und integrieren. Sie müssen

$\rightarrow$ unter den gegebenen schwierigen

Antwortmöglichkeiten die richtige Option wählen.

Bei dieser Aufgabe wird von den Schülerinnen und Schülern erwartet, dass sie eine graphische Darstellung zweier Elemente, zwischen denen ein physikalischer Zusammenhang besteht (Entfernung und Geschwindigkeit eines Rennwagens auf unbekannter Rennstrecke), interpretieren. Sie müssen den Graphen interpretieren, indem sie eine verbale Beschreibung mit zwei besonderen Merkmalen des Graphen verknüpfen (ein einfaches und offensichtliches Merkmal und ein zweites, das 570 ein tieferes Verständnis mehrerer Elemente des Graphen und seiner Bedeutung voraussetzt), und dann die relevanten Informationen identifizieren und dem Graphen entnehmen sowie unter den Antwortmöglichkeiten die beste Option auswählen.

Bei dieser Aufgabe müssen die Schülerinnen und Schüler einem Graphen Informationen entnehmen, zwischen denen ein physikalischer Zusammenhang besteht (Geschwindigkeit und Entfernung eines Wagens). Sie müssen genau den Punkt auf dem Graphen finden, auf den in der verbalen Beschreibung Bezug genommen wird, erkennen, wie sich die Geschwindigkeit desWagens an diesem Punkt verhält, und dann die passendste Option unter den Antwortmöglichkeiten auswählen.

Bei dieser Aufgabe müssen die Schülerinnen und Schüler dem Graphen einen einzigenWert entnehmen, der eine einfache Bedingung erfüllt. Die Fragestellung verlangt von ihnen, einem

- Graphen Informationen zu entnehmen, zwischen 380 denen ein physikalischer Zusammenhang besteht (Geschwindigkeit und Entfernung eines Wagens). Die Schülerinnen und Schüler müssen ein spezifisches Merkmal des Graphen identifizieren (die Anzeige der Geschwindigkeit), aus dem Graphen direkt denWert ablesen, an dem die Geschwindigkeit am niedrigsten war, und dann unter den Antwortmöglichkeiten die passendste Option auswählen.

* Schwellenwerte auf der Basis von RP $=0,62$, (vgl. Kasten 2.1). 
Entfernung zu ermitteln, die eine bestimmte Bedingung erfüllt. Dabei müssen die Schülerinnen und Schüler eine verbale Beschreibung mit zwei besonderen Merkmalen des Graphen verknüpfen (ein einfaches und offensichtliches Merkmal und ein zweites, das ein tieferes Verständnis mehrerer Elemente der Graphik und seiner Bedeutung voraussetzt) und dann die relevanten Informationen identifizieren und dem Graphen entnehmen sowie unter den Antwortmöglichkeiten die beste Option auswählen. Im Durchschnitt der OECD-Länder beantworteten 67\% der Schülerinnen und Schüler diese Multiple-Choice-Aufgabe richtig.

Am unteren Ende der Skala für mathematische Grundbildung erfordert Frage 7 mit einem Schwierigkeitsgrad von 413 Punkten von den Schülerinnen und Schülern, das Tempo des Autos an einem bestimmten Punkt des Graphen abzulesen. Zur Beantwortung der Frage müssen die Schülerinnen und Schüler einem Graphen Informationen entnehmen, zwischen denen ein physikalischer Zusammenhang besteht (Geschwindigkeit und Entfernung eines Wagens). Sie müssen genau den Punkt des Graphen finden, auf den in der verbalen Beschreibung Bezug genommen wird, erkennen, wie sich die Geschwindigkeit des Wagens an diesem Punkt verhält, und dann die passendste Option unter den Antwortmöglichkeiten auswählen. Im Durchschnitt der OECD-Länder beantworteten 83\% der Schülerinnen und Schüler diese Multiple-ChoiceAufgabe richtig (Daten abrufbar unter www.pisa.oecd.org).

\section{Die Durchschnittsergebnisse der Länder in mathematischer Grundbildung}

Für die politischen Entscheidungsträger in den OECD-Ländern stellen die internationalen Vergleiche der Schülerleistungen mittlerweile ein wesentliches Instrument zur Einschätzung der Leistungsfähigkeit der Bildungssysteme ihrer Länder dar. Diese Vergleiche bieten einen externen Referenzpunkt für die objektive Evaluierung der Effizienz von Bildungssystemen. Die erste Frage, die in diesem Zusammenhang häufig gestellt wird, bezieht sich auf das Abschneiden der Länder im Vergleich ihrer Durchschnittsergebnisse. Wie bei der Lesekompetenz lassen sich die Leistungen in der mathematischen Grundbildung anhand der von den Ländern erreichten mittleren Punktzahl zusammenfassend wiedergeben.

In Abbildung 3.2 sind die Länder in der Reihenfolge ihrer mittleren Punktzahl auf der Skala für mathematische Grundbildung aufgelistet. Ferner zeigt die Abbildung, welche Länder über, unter oder in etwa auf dem Niveau des OECDDurchschnitts liegen.

Wie bei der Lesekompetenz, so ist es auch hier wichtig, nur jene Differenzen zwischen den Ländern zu berücksichtigen, die statistisch signifikant sind. Aus Abbildung 3.2 geht hervor, für welche Länderpaare die Mittelwertsunterschiede so groß sind, um mit ziemlicher Sicherheit sagen zu können, dass die höheren Leistungen der Schülerinnen und Schüler eines Landes auf die
... und die überwiegende Mehrzahl der

Schülerinnen und

schüler ist in der Lage,

einfache Informationen

aufeinem Graphen

abzulesen und zu

verstehen.
Die Länder

unterscheiden sich stark voneinander hinsichtlich ihrer durchschnittlichen Leistung in

mathematischer

Grundbildung,... 
KAPITEL 3 Was PISA über die Fähigkeiten 15-jähriger aussagt

\section{Abbildung 3.2}

Vergleich der Durchschnittsergebnisse der Länder auf der Skala für mathematische Grundbildung

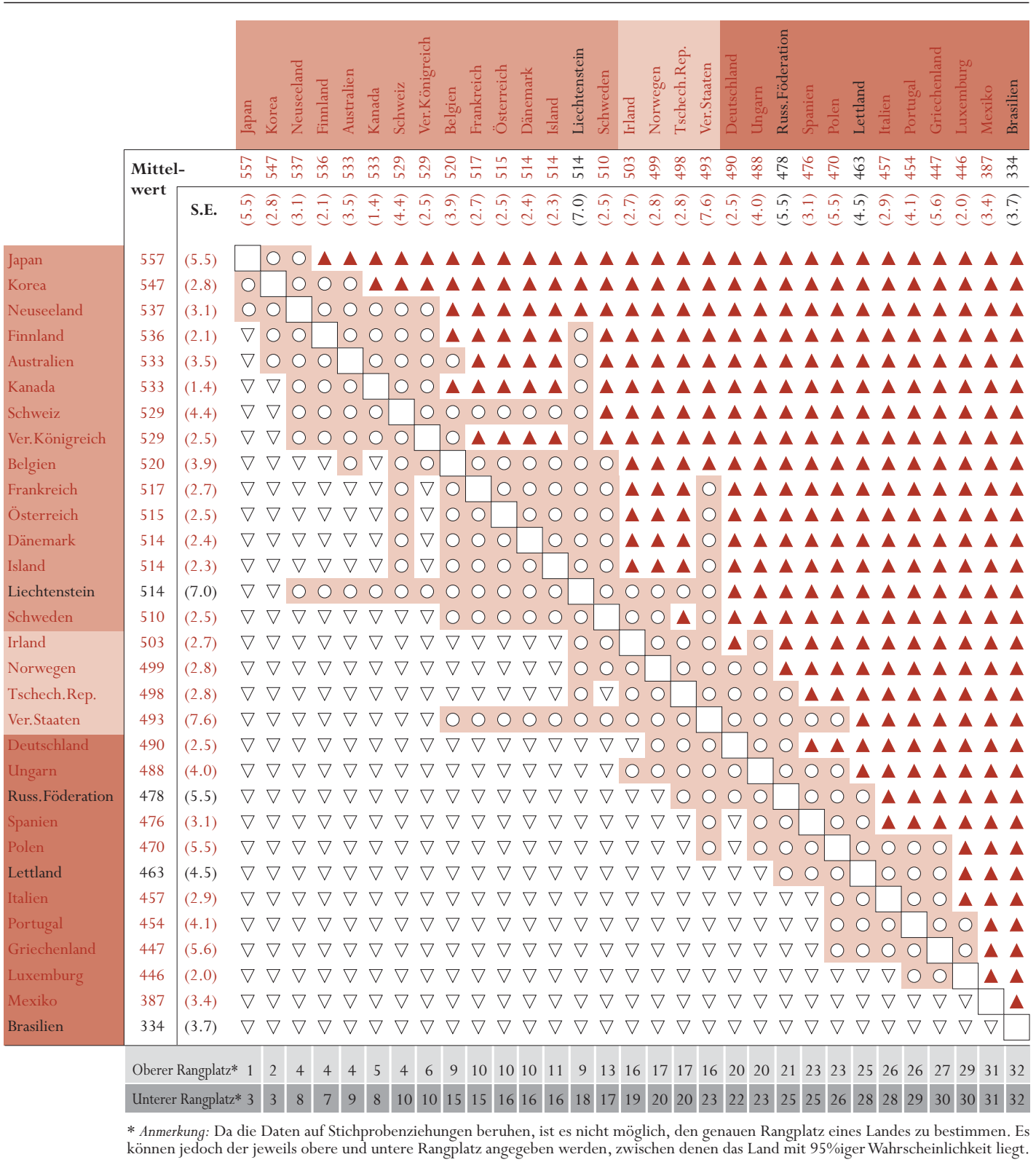

Erläuterungen

Zum Vergleich der Ergebnisse eines Landes mit denen der Länder im Tabellenkopf ist die Zeile des betreffenden Landes zu lesen. Die Symbole zeigen, ob die Durchschnittsergebnisse des Landes in der jeweiligen Zeile statistisch signifikant über oder unter denen des Vergleichslandes liegen oder ob zwischen den Durchschnittsergebnissen beider Länder kein signifikanter Unterschied besteht.

Quelle: OECD PISA Datenbank, 2001.
A Durchschnittsergebnisse statistisch signifikant höher als im Vergleichsland

Kein statistisch signifikanter Unterschied gegenüber dem Vergleichsland

$\nabla$ Durchschnittsergebnisse statistisch signifikant niedriger als im Vergleichsland

Statistisch signifikant über dem OECD-Durchschnitt

Kein statistisch signifikanter Unterschied zum OECD-Durchschnitt Statistisch signifikant unter dem OECD-Durchschnitt 
gesamte Schülerschaft des Landes übertragen werden können. Zum Vergleich der Ergebnisse eines Landes mit denen der Länder im Tabellenkopf ist die Zeile des betreffenden Landes zu lesen. Die Symbole zeigen, ob die Durchschnittsergebnisse des Landes in der jeweiligen Zeile signifikant über denen des Vergleichslands oder darunter liegen oder ob zwischen den Durchschnittsergebnissen beider Länder kein signifikanter Unterschied besteht ${ }^{2}$.

Die Schülerinnen und Schüler in Japan erreichen in der mathematischen Grundbildung die höchste mittlere Punktzahl, allerdings bestehen zwischen dem Durchschnittsergebnis von Japan und denen von Korea und Neuseeland keine statistisch signifikanten Unterschiede. Die anderen Länder, deren Punktzahlen ebenfalls über dem OECD-Durchschnitt liegen, sind Australien, Österreich, Belgien, Kanada, Dänemark, Finnland, Frankreich, Island, Liechtenstein, die Niederlande ${ }^{3}$, Schweden, die Schweiz und das Vereinigte Königreich.

Obwohl die in PISA im Bereich mathematische Grundbildung eingesetzten Aufgaben so entwickelt wurden, dass Schülerinnen und Schüler ohne Taschenrechner keinen Nachteil bei der Bearbeitung haben, hatten die Schülerinnen und Schüler die Erlaubnis, ihren eigenen oder einen von der Testleitung gestellten Taschenrechner zu benutzen. Es gibt keinen Hinweis darauf, dass die Schülerinnen und Schüler, die einen Taschenrechner benutzten, einen Vorteil gegenüber den anderen Schülerinnen und Schülern hatten ${ }^{4}$.

\section{Die Verteilung der mathematischen Grundbildung innerhalb der Länder}

Auch wenn die Durchschnittsergebnisse der einzelnen Länder stark voneinander abweichen, sind die Leistungsunterschiede zwischen den Schülern in den einzelnen Ländern ebenso wie im Bereich der Lesekompetenz um ein Vielfaches größer. So zeichnen die Durchschnittsergebnisse kein vollständiges Bild der Schülerleistungen und können signifikante Unterschiede innerhalb einer einzigen Klasse, einer Schule oder eines Bildungssystems verschleiern. Für die Bildungssysteme besteht eine der größten Herausforderungen darin, ein hohes Leistungsniveau zu fördern und gleichzeitig interne Unterschiede auf ein Mindestmaß zu reduzieren.

Abbildung 3.3 stellt die Verteilung der Ergebnisse auf der Skala für mathematische Grundbildung dar ${ }^{5}$. Die schattierten Balken zeigen das Ergebnisspektrum eines jeden Landes zwischen dem 5. und 95. Perzentil. Der Schattierungsgrad der Balken gibt den Anteil der Schülerinnen und Schüler wieder, der die den jeweiligen Skalenpunkten entsprechenden Leistungen erbringt. Tabelle 3.1 enthält zusätzlich Angaben über das 25. und das 75. Perzentil, d.h. die Skalenpunkte, die das untere und obere Quartil der Schülerinnen und Schüler des jeweiligen Landes abgrenzen. Die drei schwarzen Punkte Linie in der Mitte geben die mittlere Punktzahl eines Landes an, die im vorangegangenen Abschnitt erörtert wurde, ergänzt um das entsprechende Konfidenzintervall.
... doch die Leistungsunterschiede innerhalb der Länder sind um ein Vielfaches größser, ... 
KAPITEL 3 Was PISA über die Fähigkeiten 15-jähriger aussagt

\section{Abbildung 3.3}

Verteilung der Schülerleistungen auf der Skala für mathematische Grundbildung innerhalb der Länder

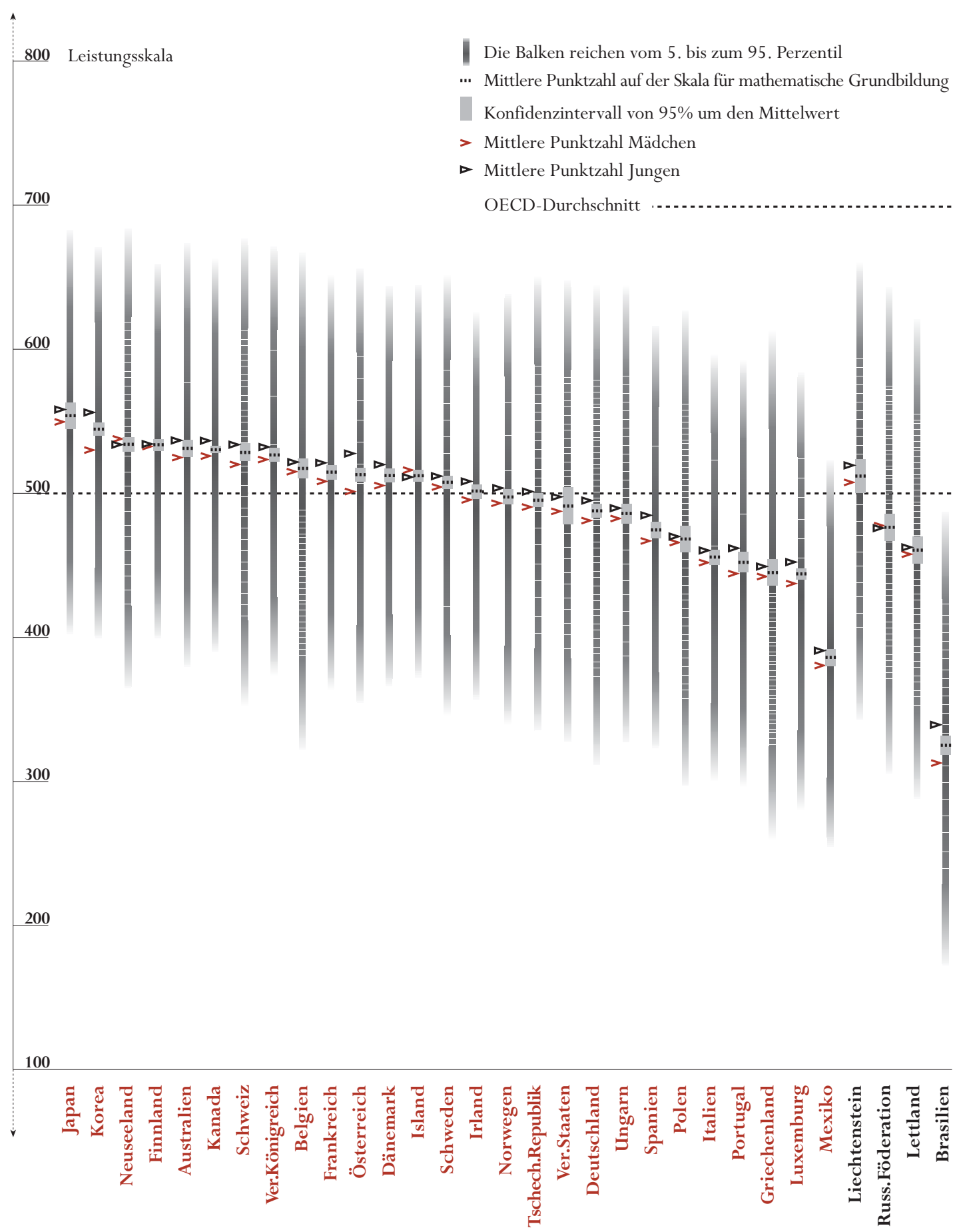

Quelle: OECD PISA Datenbank, 2001. Tabelle 3.1. 
In allen Ländern müssen die Bildungssysteme und -programme wie auch die Schulen und die Lehrkräfte einem breiten Spektrum an Schülerwissen und -fähigkeiten gerecht werden und effizient fördern. In ungefähr der Hälfte der Länder erreichen über 10\% der Schülerinnen und Schüler noch nicht einmal die Durchschnittspunktzahl des leistungsschwächsten OECD-Lands (Tabelle 3.1). Aus Abbildung 3.1 geht hervor, dass diese Schülerinnen und Schüler generell Schwierigkeiten haben, einfache Aufgaben zu lösen, die in der Wiedergabe von grundlegenden mathematischen Fakten oder Verfahren bzw. Anwendung einfacher Rechenfertigkeiten bestehen. Aufgaben, die über die Erkennung eines einzelnen bekannten Elements des Problems hinaus Interpretations- bzw. Argumentationsfähigkeiten erfordern, und Lösungsprozesse, die komplexer sind als die Anwendung eines Routineverfahrens in einem einzigen Prozessschritt, würden normalerweise die Kenntnisse und Fähigkeiten dieser Schülerinnen und Schüler übersteigen. So liegen die in Abbildung 3.1 aufgeführten Beispielfragen generell über dem Kompetenzniveau der Schülerinnen und Schüler, deren Leistungen hinter dem Durchschnittsergebnis Mexikos (387 Punkte) zurückbleiben.

Am anderen Ende der Skala liegen in allen Ländern mit zwei Ausnahmen zumindest $10 \%$ der Schülerinnen und Schüler über dem Mittelwert des leistungsstärksten Landes, d.h. Japan (557 Punktwerte).

Diese Ergebnisse deuten darauf hin, dass die Bildungssysteme in vielen Ländern einem breiten Spektrum an Schülerbedürfnissen angemessen gerecht werden müssen, insbesondere denen der Schülerinnen und Schüler mit den größten Defiziten wie auch jenen der außergewöhnlich leistungsstarken Schülerinnen und Schüler.

Aus dem Vergleich der Streuung der Leistungen innerhalb eines Landes mit dem von diesem erzielten Durchschnittsergebnis geht hervor, dass große Leistungsheterogenität nicht unbedingt eine Voraussetzung dafür darstellt, dass ein Land ein insgesamt hohes Leistungsniveau erreicht. Augenfällig ist ganz im Gegenteil, dass die Durchschnittsergebnisse in sechs der acht Länder mit der geringsten Varianz zwischen dem 75. und 25. Perzentil (das die mittleren 50\% der nationalen Leistungsverteilung abdeckt), d.h. Kanada, Dänemark, Finnland, Island, Japan, und Korea, alle signifikant über dem OECD-Durchschnitt liegen (Tabelle 3.1). Außerdem gehören vier dieser Länder, nämlich Kanada, Finnland, Japan, und Korea zu den sechs leistungsstärksten Ländern des OECD-Raums in mathematischer Grundbildung.

Hingegen liegen in vier der fünf Länder mit der höchsten Leistungsheterogenität im Bereich mathematische Grundbildung (gemessen anhand der Differenz zwischen dem 75. und 25. Perzentil), d.h. Deutschland, Griechenland, Ungarn und Polen, die Ergebnisse statistisch signifikant unter dem OECD-Durchschnitt (Belgien stellt mit einer sehr heterogenen Punkteverteilung, aber einem Mittelwert über dem OECD-Durchschnitt eine Ausnahme dar).

\author{
... wobei eine nicht \\ unbedeutende \\ Minderheit von \\ Schülerinnen und \\ schülern in vielen \\ Ländern Schwierigkeiten \\ bei der Anwendung \\ einfacher \\ Rechenfertigkeiten \\ hat,...
}

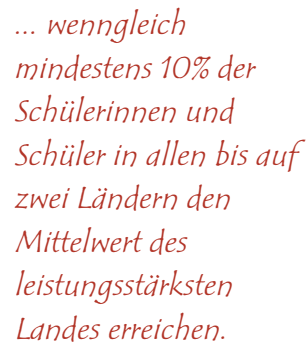

Auffällig ist, dass die Unterschiede in leistungsstarken Ländern tendenziell am geringsten sind, ...

\author{
... während in einigen \\ Ländern mit niedrigen \\ Durchschnittsergebnissen \\ großse Differenzen zu \\ beobachten sind.
}


Für Schülerinnen und Schüler aus Ländern mit niedrigen

Durchschnittsergebnissen in mathematischer Grundbildung ist die Wahrscheinlichkeit, dass

sie den Erwartungen ihrer Lehrkräfte gerecht werden, nicht unbedingt geringer.

Die Länder haben in jeweils unterschiedlichen Bereichen Stärken und Schwächen,...
Diese Struktur der Leistungsverteilung auf der Skala für mathematische Grundbildung ähnelt in der Tendenz der Leistungsverteilung im Bereich der Lesekompetenz, wobei Belgien, Deutschland, Griechenland, Ungarn, Neuseeland, Polen, die Schweiz, und die Vereinigten Staaten zwischen dem 75. und dem 25. Perzentil einen recht großen Abstand aufweisen - zwischen 135 und 149 Punkten auf der Skala für mathematische Grundbildung (Tabelle 3.1). Demgegenüber bestehen in Finnland, Island, Irland, Japan und Korea mit weniger als 113 Punkten zwischen dem 75. und dem 25. Perzentil verhältnismäßig geringe Leistungsunterschiede. Es gibt aber Ausnahmen: Australien z.B. weist auf der Gesamtskala Lesekompetenz verhältnismäßig große Leistungsunterschiede auf, während die Unterschiede zwischen dem 75. und 25. Perzentil bei den Ergebnissen für mathematische Grundbildung mit jeweils 121 Punktwerten unter dem Durchschnittswert des Interquartilbereichs im OECD-Raum liegt.

Wie in Kapitel 2 erläutert, wurden die am PISA-Test teilnehmenden Schülerinnen und Schüler gebeten, die letzte Zeugnisnote in Mathematik mitzuteilen und anzugeben, wie ihre Noten von der Schule interpretiert wurden. Auf der Grundlage dieser Information kann festgestellt werden, ob die Noten der Schülerinnen und Schüler über, an oder unter der von der Schule oder der jeweiligen Lehrkraft festgelegten Mindestnote liegen. Ein Vergleich dieser Information mit den Schülerleistungen auf der PISA-Skala für mathematische Grundbildung liefert einen Bezugsrahmen für die Interpretation der PISA-Ergebnisse im nationalen Kontext (Tabelle 3.2). Die Daten zeigen, dass in den Ländern, die in PISA vergleichsweise hohe Leistungen erzielen, der Anteil der Schülerinnen und Schüler, die die Erwartungen ihrer Lehrkräfte erfüllen, nicht automatisch gering ist. In Ländern mit recht niedrigen Durchschnittsergebnissen gibt die überwiegende Mehrzahl der Schülerinnen und Schüler nach wie vor an, dass sie den Erwartungen ihrer Lehrkräfte gerecht werden. Die internationalen Belege für die erheblichen Unterschiede bei den Durchschnittsleistungen zwischen den Ländern lassen vermuten, dass Länder, in denen sowohl die Anforderungen als auch die durchschnittlichen Leistungsniveaus niedrig sind, beide anheben könnten.

\section{Leistungen in mathematischer Grundbildung und Lesekompetenz}

Ein direkter Vergleich der auf den Skalen für mathematische Grundbildung und Lesekompetenz erzielten Werte ist nicht angemessen (die in Klammern aufgeführten Mittelwerte der Skala für Lesekompetenz und der Skala für mathematische Grundbildung sind nur als Hinweis gedacht.). Dennoch lässt sich die relative Leistungsstärke der Länder in diesen beiden Bereichen anhand ihres Platzes in der Rangordnung auf der jeweiligen Skala bestimmen ${ }^{6}$. Zu beachten ist dabei, dass dieser Vergleich nicht auf einen Leistungsvergleich zwischen den Ländern abzielt, sondern vielmehr auf eine Gegenüberstellung der Ergebnisse in den beiden Bereichen innerhalb eines Landes. 
- Unter Zugrundelegung dieses Vergleichs weisen Dänemark (497, 514), Ungarn (480, 488), Lettland (458, 463), Liechtenstein (483, 514), Japan $(522,557)$, Korea $(525,547)$, die Russische Föderation $(462,478)$ und die Schweiz $(494,529)$ im Bereich mathematische Grundbildung verhältnismäßig höhere Leistungen auf als im Bereich Lesekompetenz.

- Kanada (534, 533), Finnland (546, 536), Griechenland (474, 447), Irland (527, 503), Italien (487, 457), Norwegen (505, 499), Spanien (493, 476), Schweden $(516,510)$ und die Vereinigten Staaten $(504,493)$ schneiden im Bereich der Lesekompetenz vergleichsweise besser ab.

- Die übrigen Länder zeigen auf beiden Skalen im wesentlichen die gleiche relative Leistungsstärke.

\section{Schülerleistungen im Bereich naturwissenschaftliche Grundbildung}

\section{Wie die naturwissenschaftliche Grundbildung in PISA gemessen wird}

Ebenso wie in der mathematischen Grundbildung werden auch die Leistungen in der naturwissenschaftlichen Grundbildung in PISA 2000 anhand einer einzigen Skala gemessen, die so aufgebaut wurde, dass das Durchschnittsergebnis bei 500 Punkten liegt, die Standardabweichung 100 Punkte beträgt und rund zwei Drittel der Schülerinnen und Schüler in den OECD-Ländern Ergebnisse zwischen 400 und 600 Punkten aufweisen. Die Skala misst die Fähigkeit der Schülerinnen und Schüler, naturwissenschaftliches Wissen anzuwenden (Verständnis naturwissenschaftlicher Konzepte), naturwissenschaftliche Fragen zu erkennen bzw. zu identifizieren, worum es bei naturwissenschaftlichen Untersuchungen geht (Verständnis für das Wesen einer naturwissenschaftlichen Untersuchung), Behauptungen und Schlussfolgerungen mit naturwissenschaftlichen Daten zu verbinden (Einsatz naturwissenschaftlicher Beweise) und diese Aspekte der Naturwissenschaften zu kommunizieren.

Zu den Kriterien, die den wachsenden Schwierigkeitsgrad der Aufgaben auf der Skala bestimmen, gehören: die Komplexität der verwendeten Konzepte, der Umfang des gelieferten Datenmaterials, die notwendige Argumentationskette und die in der Kommunikation geforderte Genauigkeit. Außerdem wird der Schwierigkeitsgrad durch den Kontext der Information, das Format und die Präsentation der Frage beeinflusst. Die PISA-Aufgaben setzen folgende naturwissenschaftlichen Kenntnisse voraus (mit aufsteigendem Schwierigkeitsgrad): Abrufen einfacher naturwissenschaftlicher Kenntnisse oder von naturwissenschaftlichem Allgemeinwissen bzw. allgemein bekannten Daten, die Anwendung naturwissenschaftliche Konzepte oder Fragestellungen und Grundlagenkenntnisse über Untersuchungen, die Anwendung entwickelter Wissenschaftskonzepte bzw. Argumentationsketten sowie Kenntnis einfacher konzeptueller Modelle oder Analysen von Belegen für alternative Perspektiven.
... wobei einige Länder im Bereich mathematische Grundbildung besser abschneiden als im Bereich Lesekompetenz, ...

... während auf andere das Gegenteil zutrifft.
Bei der naturwissenschaftlichen Grundbildung wird die Fähigkeit der Schülerinnen und schüler gemessen, naturwissenschaftliche Kenntnisse anzuwenden und von einem naturwissenschaftlichen Ansatz aus an Probleme heranzugehen,...

... wobei der schwierigkeitsgrad durch die Komplexität der Konzepte, den Umfang des gelieferten

Datenmaterials sowie die notwendige Argumentationskette bestimmt wird. 
KAPITEL 3 Was PISA über die Fähigkeiten 15-jähriger aussagt

Die schwierigsten

Aufgaben setzen komplexe begriffliche Fähigkeiten voraus, ...

... weniger schwierige Aufgaben erfordern immerhin noch ein gewisses Maßs an wissenschaftlichem Denken,...

... und die leichteren Aufgaben verlangen die

Fähigkeit, einfaches naturwissenschaftliches Faktenwissen abzurufen und anzuwenden.

Den Schülerinnen und schülern wurde

beispielsweise ein experimentelles Problem vorgelegt, mit dem ein Wissenschaftler des 19.

Jahrhunderts konfrontiert war, ...
- Am oberen Ende der Skala für naturwissenschaftliche Grundbildung (bei rund 690 Punkten) sind die Schülerinnen und Schüler generell in der Lage, mit Hilfe konzeptueller Modelle Vorhersagen zu machen und Erklärungen zu geben; naturwissenschaftliche Untersuchungen z.B. in Verbindung mit der Versuchsanordnung oder Erkennung einer Idee, die in einer bestimmten Untersuchung geprüft wurde, zu analysieren; auf der Basis des Vergleichs von Daten alternative Standpunkte oder unterschiedliche Perspektiven zu evaluieren; und wissenschaftliche Argumente und/oder Darlegungen in allen Einzelheiten und mit Präzision zu kommunizieren.

- Bei rund 550 Punkten sind die Schülerinnen und Schüler generell in der Lage, naturwissenschaftliche Konzepte zu benutzen, um Vorhersagen zu machen oder Erklärungen zu geben; Fragen zu erkennen, die durch naturwissenschaftliche Untersuchungen beantwortet werden können, und/oder Details über den Gegenstand einer naturwissenschaftlichen Untersuchung zu identifizieren; ferner können sie beim Ziehen bzw. bei der kritischen Bewertung von Schlussfolgerungen relevante Informationen aus konkurrierenden Daten oder Argumentationsketten auswählen.

- Am unteren Ende der Skala (bei rund 400 Punkten) sind die Schülerinnen und Schüler in der Lage, einfaches naturwissenschaftliches Faktenwissen abzurufen (d.h. Namen, Fakten, Terminologien, einfache Regeln und Gesetze) und naturwissenschaftliches Allgemeinwissen beim Ziehen oder Bewerten von Schlussfolgerungen zu verwenden.

Eine Beschreibung der Rahmenkonzeption, die der PISA-Erhebung der naturwissenschaftlichen Grundbildung zu Grunde liegt, findet sich in Schülerleistungen im internationalen Vergleich - Eine neue Rahmenkonzeption für die Erfassung vonWissen und Fähigkeiten (Deutsches PISA-Konsortium, 2000).

In PISA erreichten im Durchschnitt aller OECD-Länder die 5\% der leistungsstärksten Schülerinnen und Schüler 657 Punkte, 10\% 627 Punkte und 25\% 572 Punkte. Am unteren Ende der Skala erreichten über drei Viertel der Schülerinnen und Schüler zumindest 431 Punkte, über 90\% mindestens 368 und über 95\% 332 Punkte (Tabelle 3.3).

Zur Beurteilung der naturwissenschaftlichen Grundbildung wurden in der PISA-Erhebung ganz unterschiedliche Aufgaben gestellt. Abbildung 3.4 sind die Aufgaben einer der 13 in PISA 2000 verwendeten Testeinheiten in Verbindung mit einer Beschreibung der zur Kodierung der Schülerleistungen verwendeten Kriterien zu entnehmen (ein vollständigeres Set von Beispielaufgaben findet sich unter www.pisa.oecd.org). Die genannte Testeinheit bezieht sich auf die Forschungen von Semmelweis über die Ursachen des Kindbettfiebers. Semmelweis fiel die enorm hohe Sterberate in einer Entbindungsstation auf, die auf das Kindbettfieber zurückgeführt wurde. Den Schülerinnen und Schülern wird dieser Sachverhalt anhand eines Diagramms präsentiert, und sie werden dann mit der Vermutung konfrontiert, das Kindbettfieber 


\section{SEMMELWEIS'TAGEBUCH-TEXT 1}

„Juli 1846. NächsteWoche trete ich meine Stelle als, Herr Doktor ' auf der ersten Station der Entbindungsklinik im Allgemeinen Krankenhaus vonWien an. Ich war entsetzt, als ich vom Prozentsatz der Patienten hörte, die in dieser Klinik sterben. In diesem Monat starben dort sage und schreibe 36 von 208 Müttern, alle an Kindbettfieber. Ein Kind zurWelt zu bringen ist genauso gefährlich wie eine Lungenentzündung ersten Grades."

\section{Anzahl der Todesfälle je 100 Geburten wegen Kindbettfieber}

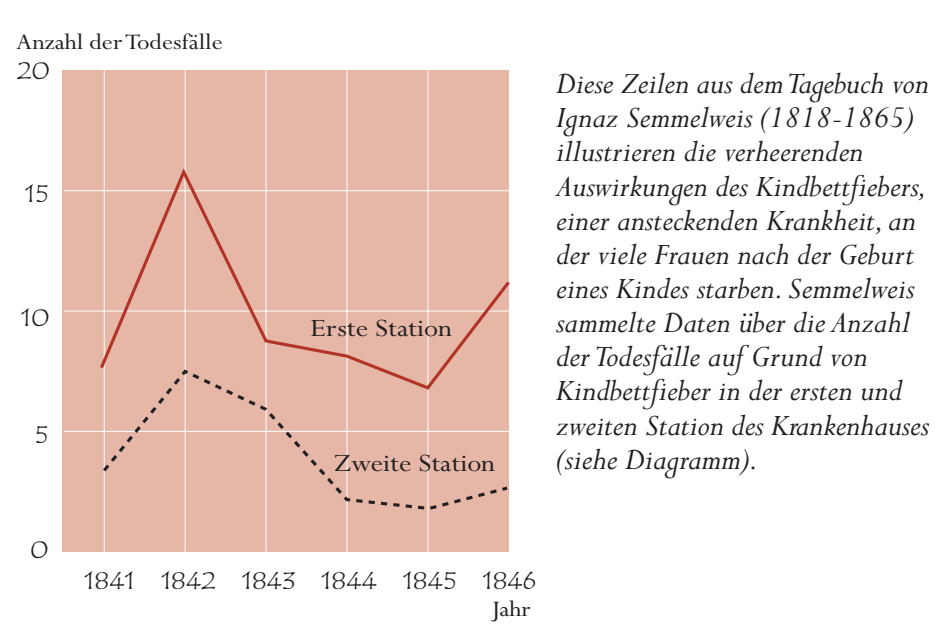

Die Ärzte, darunter auch Semmelweis, tappten in Bezug auf die Ursache des Kindbettfiebers völlig im Dunkeln. Semmelweis schrieb in sein Tagebuch:

„Dezember 1846. Warum sterben so viele Frauen nach einer völlig problemlosen Geburt an diesem Fieber? Seit Jahrhunderten lehrt uns dieWissenschaft, es handle sich um eine unsichtbare Epidemie, die Mütter tötet. Als mögliche Ursachen gelten Veränderungen in der Luft, irgendwelche außerirdischen Einflüsse oder eine Bewegung der Erde selbst, ein Erdbeben."

Heutzutage würde kaum jemand außerirdische Einflüsse oder ein Erdbeben als mögliche Ursachen für Fieber in Erwägung ziehen. Zu Lebzeiten von Semmelweis taten dies allerdings viele, auch Wissenschaftler! Wir wissen heute, dass es etwas mit hygienischen Bedingungen zu tun hat. Semmelweis wusste jedoch, dass außerirdische Einflüsse oder ein Erdbeben als Ursachen für Fieber eher unwahrscheinlich waren. Er machte auf die Daten, die er gesammelt hatte, aufmerksam (siehe Diagramm) und versuchte, damit seine Kollegen zu überzeugen.

\section{SEMMELWEIS' TAGEBUCH - TEXT 2}

Zur Forschung in den Krankenhäusern gehörte das Sezieren. Der Körper einer verstorbenen Person wurde aufgeschnitten, um eine Todesursache zu finden. Semmelweis schrieb, dass auf der Ersten Station tätige Studenten üblicherweise am Sezieren von Frauen teilnahmen, die am Vortag gestorben waren. Direkt anschließend untersuchten sie Frauen, die gerade ein Kind geboren hatten. Sie achteten nicht besonders darauf, sich nach dem Sezieren zu waschen. Manche waren sogar stolz darauf, dass man roch, dass sie vorher in der Leichenhalle gearbeitet hatten, weil man daran ihren Fleiß erkennen konnte!

Ein Freund von Semmelweis starb, nachdem er sich beim Sezieren geschnitten hatte. Beim Sezieren seines Leichnams zeigte sich, dass er dieselben Symptome aufwies wie Mütter, die an Kindbettfieber gestorben waren. Dadurch bekam Semmelweis eine neue Idee. 
KAPITEL 3 Was PISA über die Fähigkeiten 15-jähriger aussagt

\section{SCHWIERIGKEITSGRAD}

\section{FRAGE 1}

SEMMELWEIS 'TAGEBUCH

Nimm an, du wärst

Semmelweis. Nenne einen Grund dafür (ausgehend von den Daten, die Semmelweis gesammelt hat), dass Erdbeben als Ursache für Kindbettfieber unwahrscheinlich sind
Punktekategorie 2 (666*)

- Bezieht sich auf die unterschiedliche Anzahl der Todesfälle (je 100 Geburten) in den beiden Stationen.
Punktekategorie 1 (638*)

- Bezieht sich darauf, dass Erdbeben nicht oft vorkommen.

- Bezieht sich darauf, dass Erdbeben auch die Leute außerhalb der Stationen beeinflussen würden.

- Bezieht sich auf den Gedanken, dass Männer bei Erdbeben kein Kindbettfieber bekommen.

\section{FRACE 4}

- Multiple-choices
SEMMELWEIS 'TAGEBUCH
Viele Krankheiten können
durch den Einsatz von
Antibiotika geheilt werden. In
den letzten Jahren hat jedoch
die Wirksamkeit einiger
Antibiotika gegen
Kindbettfieber nachgelassen.
Worauf ist das zurückzuführen?1

\section{FRAGE 2}

- Multiple-choices

SEMMELWEIS 'TAGEBUCH

Semmelweis' neue Idee hängt mit dem hohen Prozentsatz

verstorbener Frauen auf den

Entbindungsstationen und dem

Verhalten der Studenten zusammen.

Was war seine Idee? ${ }^{1}$

\section{FRAGE 3}

\section{SEMMELWEIS 'TAGEBUCH}

Semmelweis' Versuche, die Anzahl der Todesfälle auf Grund von Kindbettfieber zu senken, zeigten Erfolg. Aber selbst heute bleibt Kindbettfieber eine Krankheit, die sich schwer bekämpfen lässt.

Schwer zu heilende Arten von Fieber sind in den Krankenhäusern immer noch ein Problem. Zahlreiche Routinemaßnahmen dienen dazu, das Problem unter Kontrolle zu halten. Zu diesen Maßnahmen zählt das Waschen der Bettwäsche bei hoher Temperatur.

Erkläre, warum eine hohe Temperatur (beim Waschen der Bettwäsche) dazu beiträgt, das

Risiko, dass Patienten Fieber bekommen, zu senken.

1. Wegen der vollständigen Antwortalternativen siehe unter www.pisa.oecd.org. Quelle: OECD PISA, 2001.
Punktekategorie 1 (493*)

- A: Wenn man die Studenten veranlasst, sich nach dem Sezieren zu waschen, sollten weniger Fälle von Kindbettfieber auftreten.

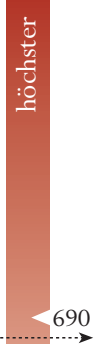

Bei dieser Aufgabe wird von den Schülerinnen und Schülern erwartet, dass sie ausgehend von den vorgegebenen Daten Schlüsse ziehen und bewerten.

Bei dieser Aufgabe wird von den Schülerinnen und Schülern erwartet, dass sie auf der Basis wissenschaftlicher Belege in einer eigenen, nicht vorgegebenen, Argumentationskette Daten systematisch mit möglichen Schlussfolgerungen verbinden.

Bei dieser Frage werden die Schülerinnen und Schüler aufgefordert, über das historische Beispiel hinauszugehen und naturwissenschaftlichesWissen herzuleiten, das zur Erläuterung eines naturwissenschaftlichen Phänomens notwendig ist. Sie sollen für ihre Erklärungen naturwissenschaftliche Konzepte (im Gegensatz zu naturwissenschaftlichem Allgemeinwissen)

$\rightarrow$ heranziehen.

$\rightarrow$ Bei dieser Aufgabe sollen die Schülerinnen und Schüler sich auf bestimmte Daten und Informationen beziehen und eine Schlussfolgerung daraus ziehen.

$\rightarrow$ Bei dieser Aufgabe wird von den Schülerinnen und Schülern erwartet, dass sie den naturwissenschaftlichen Grundsatz anwenden, dass Hitze Bakterien tötet, um zu erklären, warum diesesVerfahren wirksam ist. 
werde möglicherweise durch außerirdische Einflüsse oder Naturkatastrophen ausgelöst, zu Semmelweis‘ Zeiten kein ungewöhnlicher Gedanke. Semmelweis versuchte, seine Kollegen davon zu überzeugen, nach rationaleren Erklärungen zu suchen. Die Schülerinnen und Schüler werden aufgefordert, sich in die Lage von Semmelweis zu versetzen und auf der Basis der von Semmelweis gesammelten Daten die Idee zu verteidigen, dass Erdbeben als Ursache für diese Krankheit unwahrscheinlich sind. Die zeitlichen Verlaufskurven des Diagramms zeigen einen ähnlichen Verlauf bei den Sterberaten, wobei die erste Station konstant höhere Sterberaten aufweist als die zweite. Wenn Erdbeben die Ursache wären, dann müssten die Sterberaten in beiden Entbindungsstationen mehr oder minder gleich hoch sein. Die Kurven legen den Schluss nahe, dass die Ursache für die abweichenden Beobachtungen in den Entbindungsstationen selbst liegt. Abbildung 3.4 enthält einen Auszug aus den zur Kodierung der Schülerantworten verwendeten Kriterien.

Um in dieser Testeinheit für Frage 1 die volle Punktzahl zu erhalten, müssen die Schülerinnen und Schüler auf den Gedanken kommen, dass der Verlauf der Sterberaten in beiden Entbindungsstationen ähnlich hätte sein müssen, wenn Erdbeben die Ursache gewesen wären. Die volle Punktzahl für diese Frage entspricht einem Wert von 666 Punkten auf der Skala für naturwissenschaftliche Grundbildung. Schülerinnen und Schüler mit einer erreichten Punktzahl von 666 beantworten Fragen dieses Schwierigkeitsgrads wahrscheinlich in 62 von 100 Fällen richtig (vgl. auch Kasten 2.2). Im Durchschnitt aller OECD-Länder beantworteten 22\% der Schülerinnen und Schüler diese Frage korrekt (Daten abrufbar unter www.pisa.oecd.org). Einige Schülerinnen und Schüler lieferten Antworten, die nicht auf die Forschungsergebnisse von Semmelweis Bezug nehmen, sondern auf eine Eigenschaft von Erdbeben, die diese als Ursache gleichermaßen unwahrscheinlich macht: die Unregelmäßigkeit der Beben, während das Fieber ständig auftrat. Andere Schülerinnen und Schüler geben originelle und vertretbare Stellungnahmen ab, so z.B. „wenn Erdbeben die Ursache sind, warum sind dann nur Frauen und nicht auch Männer von der Krankheit betroffen?“ oder „wenn das zutrifft, dann müssten auch Frauen außerhalb der Entbindungsstation das Fieber bekommen“. Auch wenn argumentiert werden kann, dass sich diese Schülerinnen und Schüler nicht, wie in der Aufgabenstellung verlangt, auf die von Semmelweis gesammelten Daten stützten, erhalten sie dennoch einen Teilwert der Höchstpunktzahl, da ihre Antworten zeigen, dass sie in der Lage sind, wissenschaftliche Fakten zu verarbeiten, um daraus Schlussfolgerungen zu ziehen. Im Durchschnitt der OECD-Länder erhielten 28\% der Schülerinnen und Schüler für diese Frage zumindest Teilpunkte (Daten abrufbar unter www.pisa.oecd.org).
... wobei die schwierigste

Aufgabe, die nur von wenigen Schülerinnen und Schülern richtig beantwortet wird, verlangt, Fakten zu beurteilen und schlussfolgerungen zu ziehen,... 
KAPITEL 3 Was PISA über die Fähigkeiten 15-jähriger aussagt

... während diese bei den einfacheren Fragen gefordert sind, Informationen aus dem

Text miteinander zu verknüpfen,...

... auf wissenschaftliches Allgemeinwissen zurückzugreifen, ...

... und Erklärungen mit wissenschaftlichen

Konzepten zu untermauern.
In Frage 2 der Testeinheit werden die Schülerinnen und Schüler aufgefordert, unter den Ideen von Semmelweis diejenige zu identifizieren, die für die Reduzierung des Kindbettfiebers am relevantesten war. Hier müssen die Schülerinnen und Schüler zwei wichtige Informationselemente des Textes miteinander verknüpfen: das Verhalten eines Medizinstudenten und den Tod einer Freundin von Semmelweis an Kindbettfieber, nachdem der Student einen Leichnam seziert hatte. Diese Aufgabe, der 493 Punkte auf der Leistungsskala entsprechen, ist ein Beispiel für den mittleren Schwierigkeitsgrad. Bei dieser Frage wird von den Schülerinnen und Schülern erwartet, dass sie zur Ziehung von Schlussfolgerungen auf bestimmte Daten oder Informationen zurückgreifen, und es wird ihrVerständnis für das Wesen naturwissenschaftlicher Untersuchungen erfasst. Im Durchschnitt der OECD-Länder beantworteten 64\% der Schülerinnen und Schüler diese Frage richtig, indem sie die Antwort wählten, der zufolge weniger Fälle von Kindbettfieber auftreten sollten, wenn man die Studenten veranlasst, sich nach dem Sezieren zu waschen.

Die meisten Menschen sind sich mittlerweile der Tatsache bewusst, dass Bakterien viele Krankheiten auslösen und Hitze diese Bakterien töten kann. Viele Menschen erkennen u.U. jedoch nicht, dass diese Beobachtung bei Routinemaßnahmen in Krankenhäusern genutzt wird, um die Gefahr von fieberhaften und sonstigen Erkrankungen zu reduzieren. In Frage 3 der Testeinheit wird von den Schülern erwartet, die allgemein bekannte wissenschaftliche Erkenntnis anzuwenden, dass Hitze Bakterien tötet, um zu erklären, warum diese Verfahren effizient sind. Es handelt sich hierbei um ein weiteres Beispiel für eine Frage mit niedrigem bis mittlerem Schwierigkeitsgrad, mit einem Wert von 467 auf der Skala für naturwissenschaftliche Grundbildung. Im Durchschnitt aller OECD-Länder bekamen 68\% der Schülerinnen und Schüler für die Beantwortung dieser offenen Frage die volle Punktzahl.

Frage 4 geht schließlich über das historische Beispiel hinaus und erwartet von den Schülerinnen und Schülern, naturwissenschaftliches Grundwissen herzuleiten, das zur Erklärung eines naturwissenschaftlichen Phänomens notwendig ist. Die Schülerinnen und Schüler sollen erläutern, warum die Wirksamkeit von Antibiotika nachlässt. Für die richtige Antwort müssen sie wissen, dass die häufige und langfristige Einnahme von Antibiotika Bakterienstämme hervorbringt, die gegenüber den zuvor tödlichen Effekten resistent sind. Auf der Skala für naturwissenschaftliche Grundbildung liegt diese Frage mit 508 Punktwerten im mittleren Bereich, da von den Schülerinnen und Schülern (im Gegensatz zum Rückgriff auf wissenschaftliches Allgemeinwissen, dem ein niedrigeres Niveau zugeordnet ist) erwartet wird, dass sie ihre Erklärungen mit wissenschaftlichen Konzepten untermauern. Im Durchschnitt der OECDLänder beantworteten 60\% der Schülerinnen und Schüler diese Frage richtig, indem sie die Antwortmöglichkeit wählten, der zufolge Bakterien gegen Antibiotika widerstandsfähig werden. 


\section{Die Durchschnittsergebnisse der Länder in naturwissenschaftlicher Grundbildung}

Wie bei der mathematischen Grundbildung lassen sich die Leistungen in der naturwissenschaftlichen Grundbildung anhand der mittleren Punktzahl der Länder zusammenfassend darstellen (Abb. 3.5). Japan und Korea weisen auf der Skala für naturwissenschaftliche Grundbildung die höchsten Ergebnisse auf. Die anderen Länder, deren Ergebnisse statistisch signifikant über dem OECD-Durchschnitt liegen, sind Australien, Österreich, Kanada, die Tschechische Republik, Finnland, Irland, Neuseeland, Schweden und das Vereinigte Königreich. Die Mittelwerte in Belgien, Frankreich, Ungarn, Island, Norwegen, der Schweiz und den Vereinigten Staaten unterscheiden sich nicht signifikant vom OECD-Durchschnitt? .

Einige Länder haben in allen drei Bereichen Werte, die signifikant über dem OECD-Durchschnitt liegen: Australien, Österreich, Kanada, Finnland, Japan, Korea, Neuseeland, Schweden und das Vereinigte Königreich.

\section{Die Verteilung der naturwissenschaftlichen Grundbildung innerhalb der Länder}

In Abbildung 3.6 wird die Verteilung der Leistungspunkte auf der Skala für naturwissenschaftliche Grundbildung in einer ähnlichen Form dargestellt wie in Abbildung 3.3. Zusätzlich liefert Tabelle 3.5 den auf Unterschiede zwischen den Schulen entfallenden Varianzanteil. Zur Interpretation dieser Daten sei auf Kapitel 2 verwiesen. Wie bei der Lesekompetenz und der mathematischen Grundbildung lassen sich auch hier drei Hauptschlussfolgerungen ziehen: Die Varianz der Schülerleistungen innerhalb der Länder ist wesentlich größer als die zwischen den Ländern, die Leistungsdifferenzen sind in den einzelnen Ländern sehr unterschiedlich und zwischen der Höhe der Leistungsunterschiede innerhalb der Länder und deren durchschnittlichem Gesamtergebnis besteht kein Zusammenhang (Tabelle 3.3).

\section{Leistungen in naturwissenschaftlicher Grundbildung und Lesekompetenz}

Die meisten Länder nehmen im Bereich naturwissenschaftlicher Grundbildung einen ähnlichen Platz in der Rangordnung ein wie im Bereich Lesekompetenz, aber es gibt Ausnahmen. Ein Vergleich der relativen Position eines Landes in der Rangordnung ergibt folgende Erkenntnisse in Bezug auf die Schülerleistungen in Lesekompetenz und naturwissenschaftlicher Grundbildung (in Klammern sind die jeweilige mittlere Punktzahl im Bereich Lesekompetenz bzw. im Bereich naturwissenschaftlicher Grundbildung angegeben):

- Österreich (507, 519), die Tschechische Republik (492, 511), Ungarn (480, $496)$, Japan $(522,550)$ und Korea $(525,552)$ sowie das Vereinigte Königreich (523, 532) verzeichnen im Bereich naturwissenschaftliche Grundbildung vergleichsweise höhere Ergebnisse als im Bereich Lesekompetenz.
Die mittleren

Punktzahlen der Länder

im Bereich der

naturwissenschaftlichen

Grundbildung sind in

Abbildung 3.5

zusammenfassend

dargestellt.

Einige Länder haben

sowohl hohe

Durchschnittsleistungen

als auch geringe

Leistungsunterschiede in

naturwissenschaftlicher

Grundbildung.

Viele Länder nehmen in

Bezug auf die

Schülerleistungen in

Lesekompetenz sowie in

mathematischer und

naturwissenschaftlicher

Grundbildung einen

ähnlichen Platz in der

Rangordnung ein, aber

es gibt Ausnahmen. 
KAPITEL 3 Was PISA über die Fähigkeiten 15-jähriger aussagt

\section{Abbildung 3.5}

Vergleich der Durchschnittsergebnisse der Länder auf der Skala für naturwissenschaftliche Grundbildung

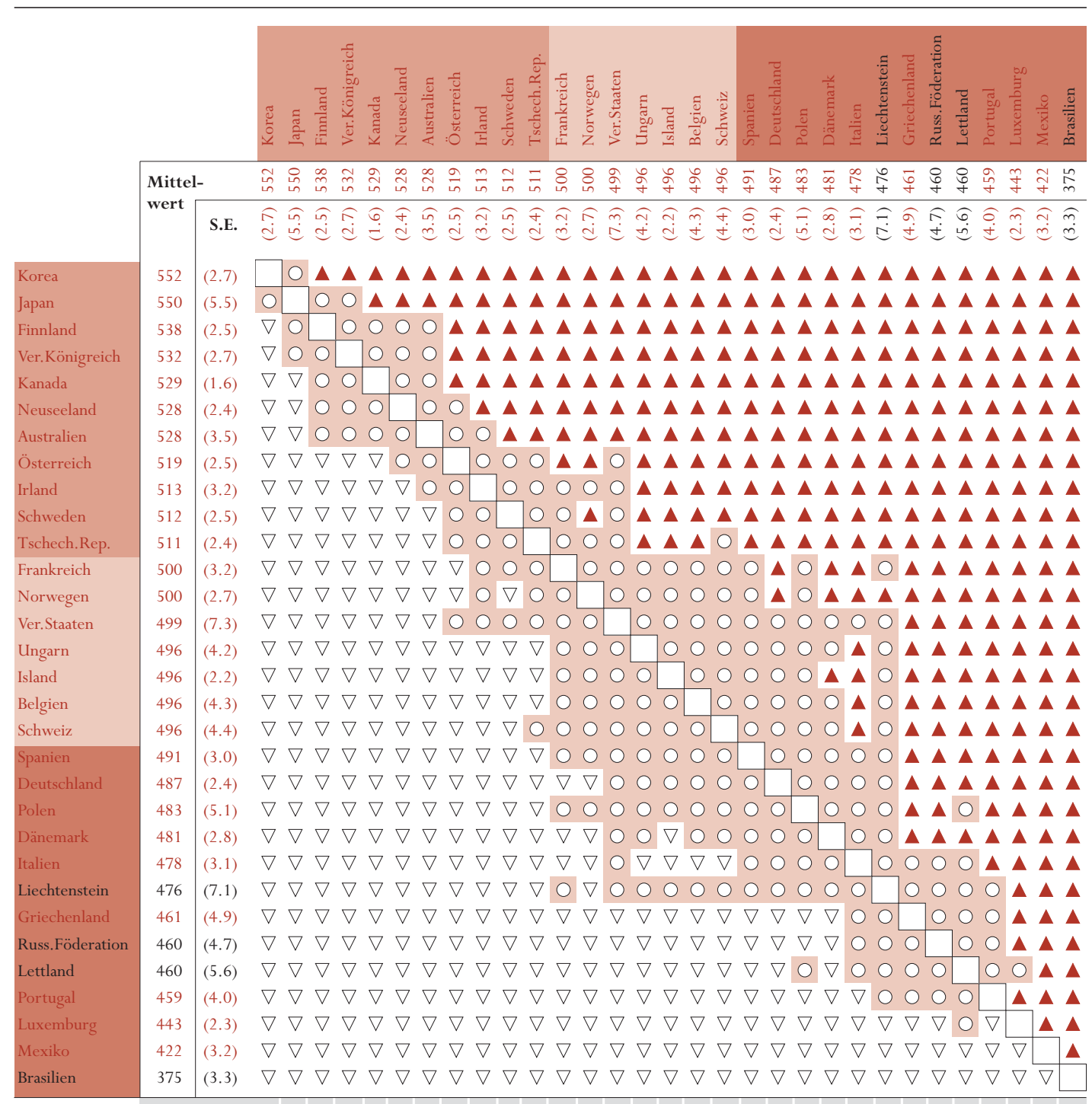

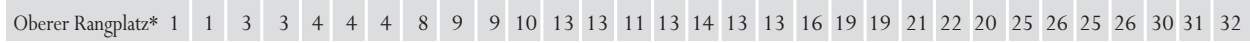

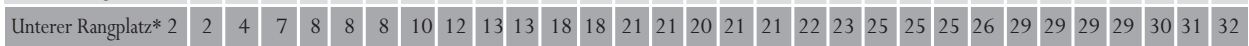

* Anmerkung: Da die Daten auf Stichprobenziehungen beruhen, ist es nicht möglich, den genauen Rangplatz eines Landes zu bestimmen. Es können jedoch der jeweils obere und untere Rangplatz angegeben werden, zwischen denen das Land mit 95\%iger Wahrscheinlichkeit liegt.

\section{Erläuterungen}

Zum Vergleich der Ergebnisse eines Landes mit denen der Länder im Tabellenkopf ist die Zeile des betreffenden Landes zu lesen. Die Symbole zeigen, ob die Durchschnittsergebnisse des Landes in der jeweiligen Zeile statistisch signifikant über oder unter denen des Vergleichslandes liegen oder ob zwischen den Durchschnittsergebnissen beider Länder kein signifikanter Unterschied besteht.

Quelle: OECD PISA Datenbank, 2001.
D Durchschnittsergebnisse statistisch signifikant höher als im Vergleichsland

Kein statistisch signifikanter Unterschied gegenüber dem Vergleichsland

$\nabla$ Durchschnittsergebnisse statistisch signifikant niedriger als im Vergleichsland

Statistisch signifikant über dem OECD-Durchschnitt

Kein statistisch signifikanter Unterschied zum OECD-Durchschnitt Statistisch signifikant unter dem OECD-Durchschnitt 


\section{Abbildung 3.6}

Verteilung der Schülerleistungen auf der Skala für naturwissenschaftliche Grundbildung innerhalb der Länder

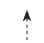

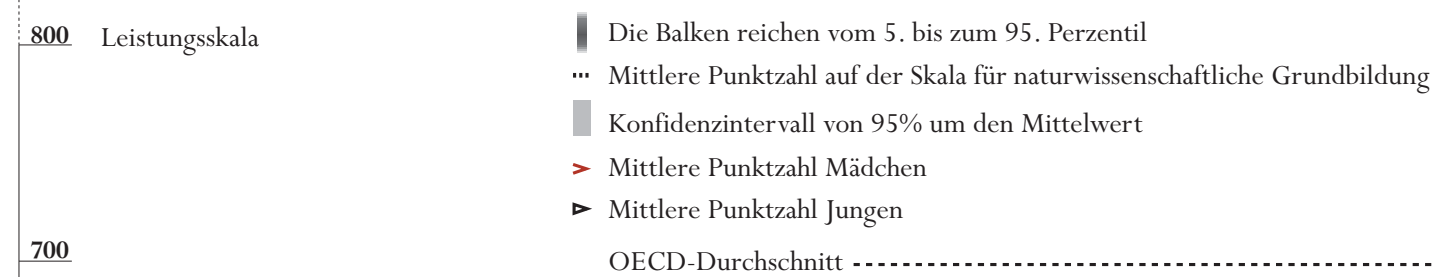

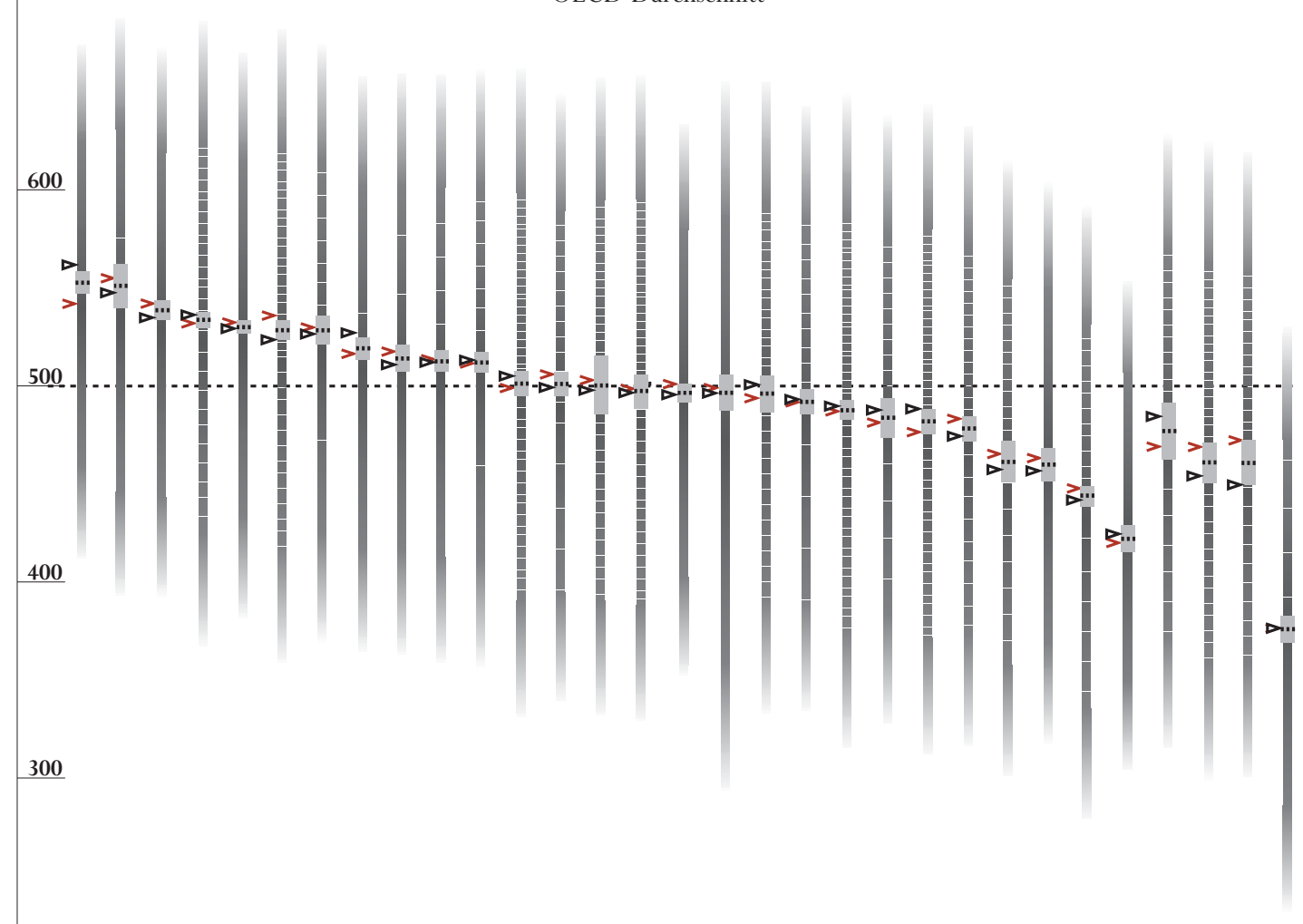

200

100

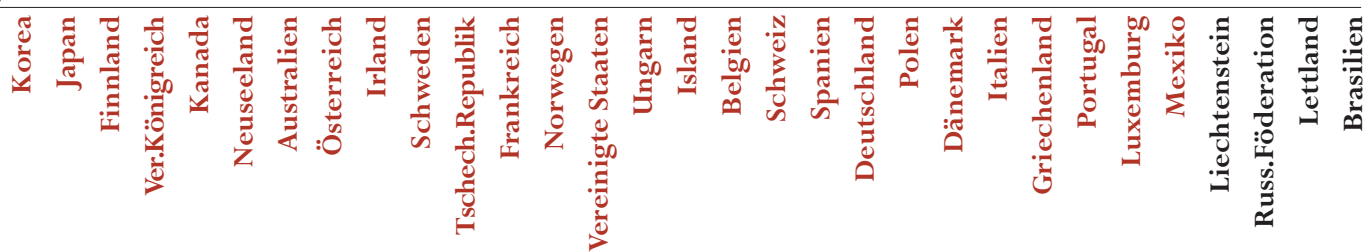

Quelle: OECD PISA Datenbank, 2001. Tabelle 3.3. 
KAPITEL 3 Was PISA über die Fähigkeiten 15-jähriger aussagt

Bei der mathematischen Grundbildung sind die

Unterschiede zwischen den Punktzahlen der Länder größser als bei der Lesekompetenz, da sie wahrscheinlich enger mit der Schule verknüpft

Die Länderergebnisse müssen unter Berücksichtigung des wirtschaftlichen Kontextes interpretiert werden.
- Belgien (507, 496), Kanada (534, 529), Dänemark (497, 481), Finnland (546, 538), Island (507, 496), Irland $(527,513)$ und Italien $(487,478)$ schneiden im Bereich der Lesekompetenz besser ab als in der naturwissenschaftlichen Grundbildung.

- Die übrigen Länder zeigen auf beiden Skalen im Wesentlichen die gleiche relative Leistungsstärke.

Das Leistungsniveau der einzelnen Länder weist große Unterschiede auf, besonders auf der Skala für mathematische Grundbildung. 169 Punkte (mehr als ein und eine halbe internationale Standardabweichung) trennt die beiden Länder mit der höchsten und niedrigsten mittleren Punktzahl auf der Skala für mathematische Grundbildung, und 101 Punkte trennen immer noch die beiden Länder mit der Zweithöchsten und zweitniedrigsten Punktzahl (Tabelle 3.1). Die Abweichungen zwischen den Durchschnittsergebnissen der Länder sind im Bereich naturwissenschaftliche Grundbildung geringer; am geringsten sind sie im Bereich Lesekompetenz ${ }^{8}$. Ein Grund hierfür ist möglicherweise die Tatsache, dass der Erwerb mathematischer und naturwissenschaftlicher Kenntnisse sehr viel enger mit der Schule verknüpft ist, so dass die Unterschiede zwischen den Bildungssystemen in diesen Bereichen sehr viel größer sind als im Bereich der Lesekompetenz.

\section{Bildungsinvestitionen und Schülerleistungen}

Bei einer Gegenüberstellung der Ergebnisse der verschiedenen Bildungssysteme ist es unumgänglich, dem wirtschaftlichen Kontext in den einzelnen Ländern wie auch den Ressourcen Rechnung zu tragen, die sie für Bildungszwecke zur Verfügung stellen. So erleichtert der relative Wohlstand es einigen Ländern, Bildungsinvestitionen zu tätigen, während andere durch ihr relativ geringes Volkseinkommen hier Einschränkungen unterliegen.

In Abbildung 3.7a wird zwischen dem Kaufkraftbereinisch Pro-KopfVolkseinkommen (BIP) und den in den einzelnen Ländern in PISA erzielten durchschnittlichen Schülerergebnissen eine Beziehung hergestellt. Für diesen Vergleich wurde ein Mittelwert der Durchschnittsleistungen der einzelnen Länder in den Bereichen Lesekompetenz sowie mathematische und naturwissenschaftliche Grundbildung errechnet. Bei den BIP-Werten handelt es sich um das Pro-Kopf-BIP zu jeweiligen Preisen von 2000, das um Kaufkraftunterschiede zwischen den OECD-Ländern bereinigt wurde (Tabelle 3.6). Ferner zeigt die Abbildung für die 23 OECD-Länder, für die vergleichbare Daten für alle Spalten in Tabelle 3.6 verfügbar sind ${ }^{9}$, eine Regressionsgerade, die den Zusammenhang zwischen Pro-Kopf-BIP und den durchschnittlichen Schülerleistungen in den drei Grundbildungsbereichen zusammenfassend darstellt. Dabei muss unbedingt berücksichtigt werden, dass die Anzahl der in den Vergleich einbezogenen Länder klein ist und die Regressionsgerade daher sehr stark von den einbezogenen Ländern abhängt. 


\section{Abbildung 3.7a}

Schülerleistungen und Volkseinkommen

Durchschnittsergebnisse über die Gesamtskala Lesekompetenz sowie die Skalen für mathematische und naturwissenschaftliche Grundbildung im Verhältnis zum Pro-Kopf-BIP, in US-Dollar, umgerechnet auf KKP-Basis (Kaufkraftparität)

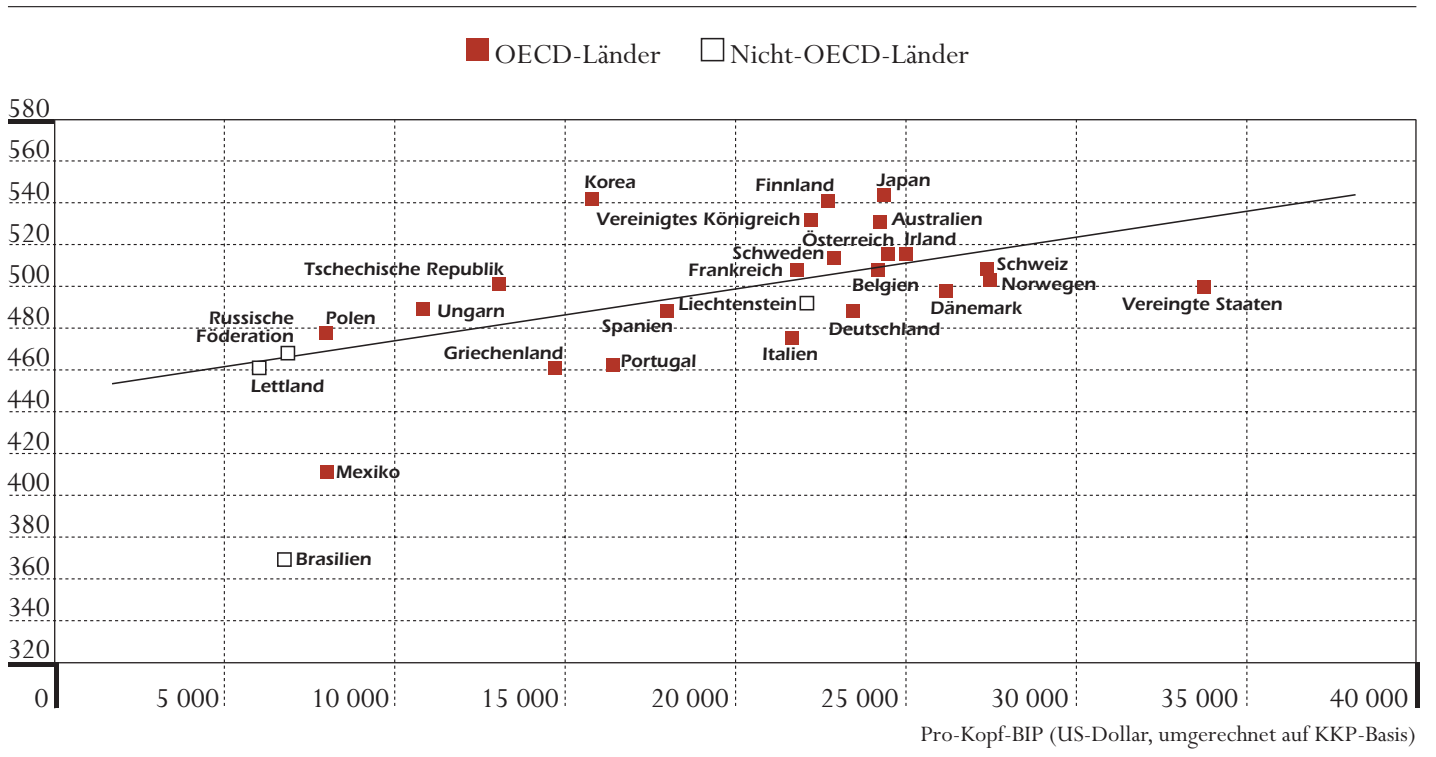

\section{Abbildung 3.7b}

Schülerleistungen und Ausgaben pro Schüler

Durchschnittsergebnisse über die Gesamtskala Lesekompetenz sowie die Skalen für mathematische und naturwissenschaftiche Grundbildung im Verhältnis zu den kumulativen Ausgaben für Bildungseinrichtungen für Schülerinnen und Schüler bis zu 15 Jahren, in US-Dollar, umgerechnet auf KKP-Basis

\begin{tabular}{|c|c|c|c|c|c|c|c|c|}
\hline \multicolumn{9}{|c|}{ OECD-Länder } \\
\hline \multicolumn{9}{|l|}{580} \\
\hline \multicolumn{9}{|l|}{560} \\
\hline 540 & & & & Korea & Finnland & Japan & & \\
\hline 520 & & & \multicolumn{4}{|c|}{ Vereinigtes Königreich $\square$ Australiẹn } & & \\
\hline 500 & & \multicolumn{2}{|c|}{$\begin{array}{l}\text { Tschechische Republik } \\
\text { Ungarn }\end{array}$} & Irland & \multicolumn{4}{|c|}{ Belgien } \\
\hline 480 & & Ungarn & ㄱoma- & Spanien & Deutschland & & Dänemark Ver & \\
\hline 460 & & Polen & & & & & Italien & \\
\hline 440 & & & Griechenlan & Portugal & & & & \\
\hline 420 & & & & & & & & \\
\hline 400 & & Mexiko & & & & & & \\
\hline 380 & & & & & & & & \\
\hline 360 & Brasilien $\square$ & & & & & & & \\
\hline 340 & & & & & & & & \\
\hline 320 & & & & & & & & \\
\hline 0 & 10000 & 20000 & 30000 & 40000 & 50000 & 60000 & 70000 & 80000 \\
\hline
\end{tabular}

Anmerkung: Bei der Regressionsgeraden bleiben Nicht-OECD-Länder unberücksichtigt.

Quelle: OECD PISA Datenbank, 2001. Tabelle 3.6. 
KAPITEL 3 Was PISA über die Fähigkeiten 15-jähriger aussagt

Länder mit höherem

Pro-Kopf-Einkommen

schneiden im

Durchschnitt tendenziell

besser $a b, \ldots$

doch weisen einige

Länder höhere oder

niedrigere

Durchschnittsergebnisse auf, als bei ihrem

Volkseinkommen

eigentlich zu erwarten

wäre, ...

Volkseinkommen hängt zwar mit den Leistungen zusammen, determiniert sie jedoch nicht.

Ein weiterer Faktor sind die Ausgaben je schüler,...

... die ein Sechstel der zwischen den Ländern bestehenden Varianz erklären.
Die Graphik deutet darauf hin, dass Länder mit höherem Volkseinkommen auf der Gesamtskala Lesekompetenz, der Skala für mathematische Grundbildung und der Skala für naturwissenschaftliche Grundbildung tendenziell besser abschneiden als Länder mit niedrigerem Volkseinkommen. Bis zu 27\% der Varianz zwischen den mittleren Punktzahlen der Länder können auf der Basis ihres Pro-Kopf-BIP vorhergesagt werden ${ }^{10}$.

Die Länder dicht an der Geraden befinden sich genau dort, wo sie laut Pro-Kopf-BIP als Prädiktor liegen sollten; Beispiele hierfür sind Österreich, Belgien, Frankreich, Irland und Spanien. So übertrifft z.B. Irland Spanien in allen drei geprüften Bereichen, und zwar in einem Ausmaß, das auch - wie Abbildung 3.7a zeigt - anhand des Abstands zwischen dem Pro-Kopf-BIP dieser Länder hätte prognostiziert werden können. Die Länder über der Geraden verzeichnen bessere Durchschnittsergebnisse bei den PISA-Messungen, als auf der Grundlage ihres Pro-Kopf-BIP (und der für die Schätzung des Zusammenhangs zu Grunde gelegten spezifischen Ländergruppe) zu erwarten war. Länder unterhalb der Geraden zeigen niedrigere Leistungen als auf der Basis des BIP zu erwarten wäre.

Das Vorhandensein einer Korrelation bedeutet nicht zwangsläufig, dass zwischen den beiden Variablen ein Kausalzusammenhang besteht; wahrscheinlich spielen auch viele andere Faktoren eine Rolle. Abbildung 3.7a legt indessen den Schluss nahe, dass Länder mit höherem Volkseinkommen verhältnismäßig im Vorteil sind. Dies sollte insbesondere bei der Interpretation des Leistungsniveaus von Ländern mit vergleichsweise niedrigem Volkseinkommen berücksichtigt werden.

Das Pro-Kopf-BIP liefert einen Anhaltspunkt für die Mittel, die ein Land in Bildung investieren könnte, ohne jedoch direkt zu messen, welche Summen konkret für das Bildungswesen aufgewendet werden. In Abbildung 3.7b werden die Beträge, die die Länder von Beginn der Primarschule bis zum 15. Lebensjahr pro Schüler durchschnittlich ausgeben, mit den Leistungen 15-jähriger Schülerinnen und Schüler anhand der Durchschnittspunktzahl in den drei geprüften Bereichen verglichen. Es wird ein Näherungswert für die Ausgaben je Schüler ermittelt, indem die öffentlichen und privaten Ausgaben für Bildungseinrichtungen je Schüler im Jahr 1998 auf jeder Bildungsstufe mit der theoretischen Dauer der Bildungsmaßnahme auf dem jeweiligen Niveau bis zum Alter von 15 Jahren multipliziert werden ${ }^{11}$. Die Ergebnisse sind unter Verwendung von Kaufkraftparitäten (KKP) in US-Dollar ausgedrückt (OECD, 2001).

Die Abbildung deutet auf einen positiven Zusammenhang zwischen den Ausgaben je Schüler und den Durchschnittsergebnissen eines Landes hin, wenn alle drei Erhebungsbereiche im Mittel betrachtet werden (Tabelle 3.6). Mit wachsenden Ausgaben je Schüler für Bildungseinrichtungen steigen auch die Durchschnittsergebnisse der Schülerinnen und Schüler in den 
einzelnen Ländern, wobei die Ausgaben je Schüler 17\% der Varianz in den Durchschnittsleistungen zwischen den Ländern erklären ${ }^{12}$.

Die Abweichungen von der Regressionsgeraden legen den Schluss nahe, dass moderate Ausgaben je Schüler nicht automatisch mit einer mangelhaften Leistungsfähigkeit der Bildungsstätten gleichgesetzt werden können. Korea und Japan erzielen im Durchschnitt in den drei geprüften Bereichen ähnliche Ergebnisse, wenngleich Korea nur 30844 US-\$ auf KKP-Basis pro Schüler für die Bildung ausgibt, Japan hingegen 53255 US-\$ auf KKP-Basis. Analog hierzu schneidet Irland in allen drei Bereichen statistisch signifikant besser ab als Deutschland, wendet aber pro Schüler rund ein Viertel weniger auf als Deutschland.

Die Abbildungen deuten ferner darauf hin, dass die Ausgaben für Bildungseinrichtungen, die natürlich eine unabdingbare Voraussetzung für eine qualitativ hochwertige Bildung sind, allein nicht ausreichen, um ein hohes Leistungsniveau zu erzielen. Es gibt eine Reihe von Ländern, für die aufgrund der Geraden bessere Ergebnisse zu erwarten wären, als in Wirklichkeit erzielt werden. Hierzu zählen Dänemark, Griechenland, Italien, Mexiko, Portugal und die Vereinigten Staaten.

\section{Schlussbetrachtungen}

In einer zunehmend technologischen Welt müssen alle Erwachsenen, und nicht nur jene, die eine wissenschaftliche Karriere anstreben, über eine solide mathematische und naturwissenschaftliche Grundbildung verfügen. Die aus der Analyse des vorliegenden Kapitels hervorgehenden großen Unterschiede bei den Schülerleistungen auf den Skalen für mathematische und naturwissenschaftliche Grundbildung deuten indessen darauf hin, dass dieses Ziel noch in weiter Ferne liegt, und dass die Länder einem breiten Spektrum von Schülerfähigkeiten gerecht werden müssen, und dies gilt nicht nur für die Schülerinnen und Schüler mit den größten Defiziten, sondern auch für die außergewöhnlich Leistungsstarken.

Gleichzeitig geht aus den Analysen wie auch bei der Lesekompetenz hervor, dass eine hohe Leistungsheterogenität keine unabdingbare Voraussetzung für ein insgesamt hohes Leistungsniveau eines Landes ist. Augenfällig ist, dass die Ergebnisse von sechs der acht Länder mit der geringsten internen Varianz auf der Skala für mathematische Grundbildung alle statistisch signifikant über dem OECD-Durchschnitt liegen.
Geld spielt zwar eine Rolle, ...

... ist aber nicht der einzige bedeutsame Faktor.

Das Ziel einer mathematischen und naturwissenschaftlichen Grundbildung für alle liegt noch in weiter Ferne, ...

... und doch gibt es Länder, die sowohl von ihrem insgesamt hohen durchschnittlichen Leistungsniveau als auch von den nur geringen

Differenzen her gut abschneiden. 
KAPITEL 3 Was PISA über die Fähigkeiten 15-jähriger aussagt

Die Leistungsunterschiede zwischen den

Ländern könnten Auswirkungen auf deren künftige Wettbewerbsfähigkeit haben.

Here Ausgaben sind mit besseren Leistungen verbunden, jedoch kein

Garant dafür.
Obwohl die Varianz der Schülerleistungen innerhalb der Länder um ein Vielfaches größer ist als die zwischen den Ländern, darf nicht übersehen werden, dass zwischen den Ländern in Bezug auf die durchschnittlichen Ergebnisse der Schülerinnen und Schüler erhebliche Unterschiede bestehen. In dem Maße, wie diese für die spätere Karriere der Schülerinnen und Schüler mit vorherbestimmend sind, können sie insbesondere in Fächern wie Mathematik und Naturwissenschaften Fragen in Bezug auf die künftige Wettbewerbsfähigkeit von OECD-Ländern aufwerfen. Darüber hinaus können die Leistungsunterschiede zwischen den Ländern in den drei Grundbildungsbereichen auf wichtige systemische Faktoren hinweisen, die die Leistungsfähigkeit der Schülerinnen und Schüler beeinflussen.

Die Gegenüberstellung der Ausgaben je Schüler mit den durchschnittlichen Schülerleistungen in den Ländern ist nicht kausal zu verstehen. Dennoch weisen die Daten darauf hin, dass zwischen beiden Komponenten ein eindeutig positiver Zusammenhang besteht. Der Vergleich legt zudem nicht nur den Schluss nahe, dass die Ausgaben für Bildungseinrichtungen eine unabdingbare Voraussetzung für ein qualitativ hochwertiges Bildungsangebot sind, sondern deutet auch darauf hin, dass diese Ausgaben allein nicht ausreichen, um ein hohes Leistungsniveau zu erzielen und dass andere Faktoren, wie z.B. die Effizienz der Bildungsinvestitionen, ebenfalls eine entscheidende Rolle spielen. 


\section{Anmerkungen}

1. Formal wurden der Mittelwert für die Schülerleistungen in den OECD-Ländern auf 500 und die Standardabweichung auf 100 festgelegt und die Daten gewichtet, so dass alle OECD-Länder gleichermaßen berücksichtigt werden.

2. Die Leistungen der polnischen Schülerinnen und Schüler dürften etwas zu hoch geschätzt sein, da 6,7\% der 15 -jährigen, noch in einer Grundschule eingeschriebenen Schüler nicht berücksichtigt wurden. Diese Ausklammerung wird den Platz Polens auf der Skala für mathematische Grundbildung aber wahrscheinlich nicht beeinflussen (wegen näherer Einzelheiten vgl. Anhang A3).

3. Die Leistungen der niederländischen Schülerinnen und Schüler können nicht genau geschätzt werden, da die Beteiligungsquote der Schulen so niedrig war. Gleichwohl kann mit Gewissheit gesagt werden, dass die Niederlande auf der Skala für mathematische Grundbildung unter den OECD-Ländern einen der ersten vier Plätze einnehmen würden. Die Niederlande sind deshalb in Abbildung 3.2 nicht mit aufgeführt (wegen näherer Einzelheiten vgl. Anhang A3).

4. In Australien, Österreich, Kanada, der Tschechischen Republik, Dänemark, Finnland, Deutschland, Griechenland, Island, Liechtenstein, Mexiko, den Niederlanden, Neuseeland, Norwegen, Portugal, Schweden, der Schweiz, dem Vereinigten Königreich und den Vereinigten Staaten benutzten zwischen der Hälfte und Drei Viertel der Schülerinnen und Schüler einen Taschenrechner, in Belgien, Frankreich, Ungarn, Italien, Lettland, der Russischen Föderation und Spanien zwischen einem Drittel und der Hälfte. Ein geringerer Anteil der Schülerinnen und Schüler, die einen Taschenrechner benutzten, gab es in Polen (31\%), Irland (27\%), Luxemburg (7\%) und Brasilien (6\%). In Japan benutzten die Schülerinnen und Schüler keinen Taschenrechner. Für Korea fehlen die Angaben hierzu. Mit der Ausnahme von Brasilien und Griechenland waren die Punktzahlen der Schülerinnen und Schüler, die einen Taschenrechner benutzten, auf der Skala für mathematische Grundbildung tendenziell etwas höher als die der Schülerinnen und Schüler ohne Taschenrechnerbenutzung. Allerdings spiegeln die Leistungsunterschiede zwischen den Schülerinnen und Schülern mit Taschenrechnerbenutzung und denen ohne auf der Skala für mathematische Grundbildung die gleichen Unterschiede wider, die zwischen diesen beiden Schülergruppen in den Leistungen auf der Skala für Lesekompetenz bestehen (die kein Rechnen erforderte). Es gibt demnach keinen Hinweis darauf, dass die Benutzung eines Taschenrechners einen Vorteil für die betreffenden Schülerinnen und Schüler hinsichtlich ihrer Leistungen in PISA darstellt.

5. Außerdem liefert Tabelle 3.5 Anhaltspunkte dafür, bis zu welchem Grad die Varianz auf Unterschiede zwischen den Schulen zurückzuführen ist. Wegen einer Interpretation dieser Daten vgl. Kapitel 2.

6. Auf der Grundlage der mittleren Punktzahl der Länder, der Standardfehler und der Kovarianz zwischen den Leistungsskalen zweier Bereiche kann die relative Wahrscheinlichkeit bestimmt werden, mit der ein Land seine Stelle in der Rangordnung auf jeder Skala auch wirklich einnehmen wird. Hieraus lassen sich Schlussfolgerungen darüber ziehen, ob ein Land in einem Bereich mit 95\%iger Wahrscheinlichkeit statistisch signifikant über dem im anderen Bereich erzielten Niveau, auf diesem Niveau oder statistisch signifikant darunter liegen würde. Wegen Einzelheiten zur verwendeten Methode vgl. PISA 2000 Technical Report.

7. Die Leistungen der polnischen Schülerinnen und Schüler dürften etwas zu hoch geschätzt sein, da 6,7\% der 15 -jährigen, noch in einer Grundschule eingeschriebenen Schüler nicht berücksichtigt wurden. Daher dürfte die Position Polens in der Rangordnung bezüglich der Skala für naturwissenschaftliche Grundbildung um zwei Rangplätze überschätzt sein. Die Leistungen der niederländischen Schülerinnen und Schüler können nicht genau geschätzt werden, da die Beteiligungsquote der Schulen so niedrig war. Gleichwohl kann mit Gewissheit gesagt werden, dass die Niederlande auf der Skala für naturwissenschaftliche Grundbildung unter den OECD-Ländern einen Rang zwischen der dritten und vierzehnten Position einnehmen würden (wegen näherer Einzelheiten vgl. Anhang A3).

8. Leistungsdifferenzen zwischen den Ländern lassen sich auch als Anteil der Gesamtvarianz der Schülerleistungen in der gesamten Schülerpopulation der OECD-Länder zusammenfassen, die auf Unterschiede zwischen den Ländern zurückzuführen ist. Dieser Anteil macht 14\% auf der Skala für mathematische Grundbildung, $8 \%$ auf der Gesamtskala Lesekompetenz und 9\% auf der Skala für naturwissenschaftliche Grundbildung aus. 
KAPITEL 3 Was PISA über die Fähigkeiten 15-jähriger aussagt

9. Kanada, Island, Luxemburg und Neuseeland bleiben in diesem Vergleich unberücksichtigt, da die Ausgaben je Schüler in diesen Ländern nicht auf Vergleichsbasis geschätzt werden können.

10. Für die 23 Vergleichsländer beträgt der Korrelationskoeffizient zwischen der durchschnittlichen Schülerleistung in den drei geprüften Bereichen insgesamt und dem BIP 0,52. Die erklärte Varianz ergibt sich aus dem Quadrat des Koeffizienten. Die Korrelation zwischen Schülerleistung und Pro-Kopf-BIP kann für die drei Grundbildungsbereiche auch getrennt berechnet werden. Im Bereich Lesekompetenz beträgt sie 0,59, in der mathematischen Grundbildung 0,55 und in der naturwissenschaftlichen Grundbildung 0,39.

11. Die Gesamtausgaben für ein gegebenes Land werden wie folgt näherungsweise errechnet: Stehen $n(0), n(1), n(2), n(3)$ für die Anzahl der Jahre, die ein Schüler zwischen 6 und 15 Jahren in der Grundschule, Sekundarstufe I und Sekundarstufe II verbringt und E(0), E(1), E(2), E(3) für die jährlichen Ausgaben je Schüler in US-Dollar in der Grundschule, Sekundarstufe I und Sekundarstufe II, dann lassen sich die Gesamtausgaben durch Multiplikation der jeweiligen Jahresausgaben (E) mit der Regeldauer (n) auf jeder Bildungsstufe (i) unter Verwendung der nachstehenden Formel berechnen:

$C E=\sum_{i=0}^{2} n(i) * E(i)$

Die Schätzungen der Bildungsdauer n(i) basieren auf der Internationalen Standardklassifikation des Bildungswesens (ISCED) (OECD, 1997).

12. Die Korrelation für das Gesamtverhältnis beträgt 0,42. Bei getrennter Betrachtung ergibt sich eine Korrelation von 0,44 für die Gesamtskala Lesekompetenz, 0,47 für die Skala für mathematische Grundbildung und 0,29 für die Skala für naturwissenschaftliche Grundbildung. 


\section{Kapitel}

4

\section{ERGEBNISSE ZUM LERNEN ALLGEMEIN}

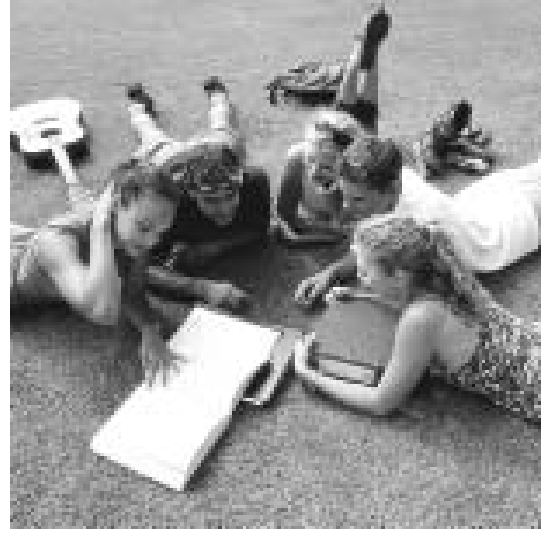


KAPITEL 4 Ergebnisse zum Lernen allgemein

\begin{abstract}
Bei Abschluss der Schule müssen die Schülerinnen und Schüler nicht nur über solide Kenntnisse in den einzelnen Fächern verfügen, sondern auch bereit sein, den Lernprozess

fortzusetzen,...
\end{abstract}

... und sie müssen zur Steuerung ihres eigenen Lernens in der Lage sein.

Im Rahmen von PISA wurden das Interesse und die Freude der Schülerinnen und Schüler am Lernen sowie ihre Fähigkeit zur

Organisation von Lernprozessen ermittelt, ...

... und es wurden die Zusammenhänge zwischen diesen Merkmalen und den

Leistungen in den von PISA untersuchten Grundbildungsbereichen geprüft.

\section{Einführung}

Die meisten Kinder sind bei ihrer Einschulung bereit und willig, etwas zu lernen. Wie können die Schulen diese Neigung fördern und stärken, und wie können sie sicherstellen, dass die jungen Erwachsenen die Schule mit der Fähigkeit verlassen, den Lernprozess ein ganzes Leben lang fortzusetzen? Die Schülerinnen und Schüler müssen in der Lage sein, die in Lesen, Mathematik und Naturwissenschaften erworbenen Kenntnisse und Fertigkeiten in ihrem späteren Leben anzuwenden. Ohne diese Kenntnisse und Kompetenzen und ohne die Fähigkeit, diese weiter auszubauen, ist der Einzelne nicht hinreichend gerüstet für den Erwerb neuer Kenntnisse und Kompetenzen, die für eine erfolgreiche Anpassung an die sich wandelnden äußeren Umstände erforderlich sind.

In den Schulen wird ein Großteil des Lernprozesses der Schülerinnen und Schüler von den Lehrkräften gesteuert. Für den Prozess des Lernens ist es jedoch förderlicher, wenn die Schülerinnen und Schüler eine positive Einstellung zum Lernen haben und zu selbstreguliertem Lernen fähig sind; und nach Abschluss der Schule muss jeder das Lernen überwiegend selbst in die Hand nehmen. Das setzt wiederum die Fähigkeit voraus, Ziele aufzustellen, Ausdauer zu zeigen, die eigenen Fortschritte zu überwachen, seine Lernstrategie gegebenenfalls anzupassen und Lernschwierigkeiten zu überwinden.

Bei einer umfassenden Einschätzung der Leistungen eines Landes im Bildungsbereich müssen diese allgemeinen Ergebnisse ebenso berücksichtigt werden wie die Leistungen in den traditionellen Unterrichtsfächern. Zu diesem Zweck wurden im Rahmen von PISA 2000 die Einstellung 15-jähriger Schülerinnen und Schüler gegenüber Lesen und Mathematik sowie ihr allgemeineres Engagement für Lernen und Schule überhaupt erfasst. Darüber hinaus entschieden sich 25 der insgesamt 32 an PISA 2000 teilnehmenden Länder für die Option, auch die Lernstrategien der Schülerinnen und Schüler sowie deren Vertrauen in die eigenen Fähigkeiten zu ermitteln ${ }^{1}$. Im vorliegenden Kapitel werden die entsprechenden Ergebnisse vorgestellt, und es wird versucht, das PISA-Lernprofil 15-jähriger Schülerinnen und Schüler auf diese Weise zu erweitern. Das Kapitel befasst sich zunächst mit Motivation und Engagement der Schülerinnen und Schüler, wobei der Akzent darauf liegt, gewisse Muster in Bezug auf das Interesse an Lesen und Mathematik sowie die allgemeine Einstellung zur Schule herauszuarbeiten. Sodann wird untersucht, wie die Schülerinnen und Schüler ihren Lernprozess steuern und welcher Lernstrategien sie sich bedienen.

Wenngleich effektive Strategien für lebensbegleitendes Lernen ein wichtiges Ergebnis der Schulbildung darstellen und mithin eine gesonderte Betrachtung verdienen, stellt sich darüber hinaus natürlich die Frage, inwieweit effektive Lernstrategien auch eine Voraussetzung für Schulerfolge darstellen. Um diesen Fragen nachzugehen, werden im vorliegenden Kapitel nicht nur Art und Verteilung der Einstellung der Schülerinnen und Schüler gegenüber dem 
Kasten 4.1 Interpretation der Selbstaussagen der Schülerinnen und Schüler

Die Daten im vorliegenden Kapitel basieren auf Selbstaussagen der Schülerinnen und Schüler über ihre Verhaltensweisen und Präferenzen sowie darauf, wie sie ihre Fähigkeiten selbst beurteilen. Sie beziehen sich also nicht auf externe Beobachtungen, sondern auf eigene Angaben der Schülerinnen und Schüler, und sie können durch kulturelle Unterschiede beim Antwortverhalten bzw. hinsichtlich der sozialen Erwünschheit bestimmter Antworten beeinflusst sein. Daher ist bei den Vergleichen Vorsicht geboten, auch wenn die Instrumente zur Erfassung der Art und Weise, wie die Schülerinnen und Schüler an das Lernen herangehen und wie sie ihre eigenen Fähigkeiten einschätzen, auf bewährten Untersuchungsmethoden beruhen und vor ihrer Verwendung in PISA 2000 ausgiebig getestet wurden.

Eine Reihe von Messwerten sind in Form von Indizes dargestellt, die die Antworten der Schülerinnen und Schüler auf eine Reihe miteinander zusammenhängender Fragen zusammenfassen. Die Fragen wurden auf der Basis etablierter theoretischer Überlegungen und früherer Forschungsarbeiten aus größeren Konstrukten ausgewählt (vgl. auch Anhang A1). Es wurden Strukturgleichungsmodelle verwendet, um zu ermitteln, ob sich die theoretisch erwarteten Indexergebnisse bestätigen, und um deren Vergleichbarkeit zwischen den einzelnen Ländern zu validieren. Zu diesem Zweck wurden für jedes Land einzeln sowie für alle OECD-Länder zusammengenommen Modellschätzungen durchgeführt.

Die Indizes wurden so konstruiert, dass zwei Drittel der OECD-Schülerpopulation im Bereich zwischen - 1 und 1 liegen und die durchschnittliche Punktzahl 0 beträgt (d.h. der Mittelwert der gesamten Schülerpopulation der OECD-Teilnehmerländer wurde mit 0 und die Standardabweichung mit 1 angesetzt). Dabei ist zu beachten, dass negative Indexwerte nicht zwangsläufig negative Antworten der Schüler auf die betreffenden Fragen bedeuten. Ein negativer Wert sagt lediglich aus, dass die Aussagen einer Gruppe von Schülerinnen und Schülern (oder in einem gegebenen Land aller Schüler zusammengenommen) weniger positiv sind als im Durchschnitt der OECDLänder. Analog dazu bedeutet ein positiver Indexwert, dass die Antworten einer Gruppe von Schülerinnen und Schülern zustimmender bzw. positiver sind als die sämtlicher Schülerinnen und Schüler im Durchschnitt der OECD-Länder. Vgl. Anhang A1 zu ausführlichen Erläuterungen zur Indexbildung.

Lernen sowie deren Rückgriff auf bestimmte Lernstrategien geprüft, sondern es wird auch versucht, den Zusammenhang zwischen diesen Faktoren und den PISA-Testergebnissen zu ermitteln.

Das wirft wiederum Fragen zu Richtung und Ursachen derartiger Zusammenhänge auf. Aber so relevant diese Fragen auch sein mögen, so schwer lassen sie sich doch nach wie vor beantworten. Es kann z.B. sein, dass sich gute Leistungen und eine positive Lernhaltung wechselseitig verstärken oder dass höher begabte Schülerinnen und Schüler sowohl leistungsstark sind als auch bestimmte Lernstrategien anwenden. Es können aber auch Drittfaktoren am Werk sein, wie z.B. Faktoren, die mit dem familiären Hintergrund der
Möglicherweise bestehen zwischen Leistungen, Verhaltensweisen und Lernstrategien nicht einfach einseitige Kausalbeziehungen, sondern sich wechselseitig verstärkende Effekte. 
KAPITEL 4 Ergebnisse zum Lernen allgemein

Schülerinnen und Schüler oder mit Unterschieden ihres jeweiligen schulischen Umfelds zusammenhängen. Der Leser sollte daher bei den nachstehenden Ausführungen bedenken, dass die genaue Art und die Stärke der UrsacheWirkungs-Zusammenhänge ungesichert sind und eine entsprechende Analyse den Rahmen dieses ersten PISA-Berichts sprengen würde. Der Nachweis, dass derartige Beziehungen grundsätzlich existieren, könnte jedoch Anregungen für den politischen Diskurs und künftige Forschungsarbeiten liefern.

Die wichtige Frage nach geschlechtsspezifischen Unterschieden in Einstellung, Motivation und Selbstkonzept wird in Kapitel 5 behandelt.

Der vorliegende Bericht enthält nur eine kleine Auswahl von PISA-Daten über die Art und Weise, wie die Schülerinnen und Schüler grundsätzlich an das Lernen herangehen sowie über die Selbsteinschätzung ihrer Fähigkeiten, wobei insbesondere solche Daten gewählt wurden, bei denen die Vergleichbarkeit zwischen den einzelnen Ländern überprüft wurde (vgl. Kasten 4.1). Ein breiteres Spektrum von Ergebnissen wird Gegenstand eines thematischen Berichts im Jahr 2002 sein.

\section{Motivation und Engagement in Bezug auf die Schule}

Motivation und

Engagement sind

maßgebliche

Voraussetzungen für

lebensbegleitendes

Lernen, ...

... und die Fähigkeit zu eigenständigem Lernen

kann in der Schule gefördert werden.

Fachbezogenes Interesse kann die Freude am Lernen beeinflussen.
Motivation und Engagement sind die „Energiequellen“ des Lernens. Schülerinnen und Schüler, die, wenn sie die Schule verlassen, in der Lage sind, sich selbständig Lernziele zu setzen, und wissen, dass sie diese effektiv erreichen können, sind potentiell Lernende auf Lebenszeit. Motivation und Engagement können sich auch auf die Lebensqualität der Schülerinnen und Schüler während der Adoleszenz auswirken und Einfluss darauf haben, ob der Einzelne später in der Lage ist, die sich ihm bietenden Bildungs- bzw. Arbeitsmarktchancen erfolgreich zu nutzen.

Eigenständiges Lernen setzt die Fähigkeit voraus, sowohl die Schwierigkeit einer Aufgabe kritisch und realistisch zu beurteilen als auch genügend Energie zu ihrer Bewältigung aufzubringen. Diese Fähigkeit ist das Ergebnis von Lerngewohnheiten, die u.a. durch die regelmäßige Beschäftigung mit Schulaufgaben sowie deren Beurteilung durch die Lehrkräfte entwickelt und geprägt werden. Sowohl die Freude am Lernen als auch lernförderliche Aktivitäten erhöhen die Motivation.

\section{Fachbezogenes Interesse an Lesen und Mathematik}

Das Interesse an bestimmten Fächern wirkt sich sowohl auf die Intensität und Kontinuität der Freude am Lernen als auch auf den Grad des Verständnisses aus. Dieser Effekt ist weitgehend unabhängig von der allgemeinen Lernmotivation. So können z.B. Schülerinnen und Schüler, die sich für Mathematik interessieren und daher eher fleißig auf diesem Gebiet arbeiten, auch eine allgemein hohe Lernmotivation aufweisen, doch muss dies nicht unbedingt der Fall sein - und umgekehrt gilt das Gleiche. Daher ist es wichtig, die Struktur des Interesses der 
Interesse am Lesen sowie an Mathematik und Schülerleistungen

A

Index des Leseinteresses: O Oberstes Quartil $\square$ Drittes Quartil $\square$ Zweites Quartil $\Delta$ Unterstes Quartil Index des Mathematikinteresses: • Oberstes Quartil — Drittes Quartil — Zweites Quartil $\Delta$ Unterstes Quartil B

Leistungen auf der Gesamtskala Lesekompetenz: @ Oberstes Quartil - Drittes Quartil - Zweites Quartil ^ Unterstes Quartil Leistungen auf der Skala für math. Grundbildung: $\square$ Oberstes Quartil - Drittes Quartil $\square$ Zweites Quartil $\Delta$ Unterstes Quartil

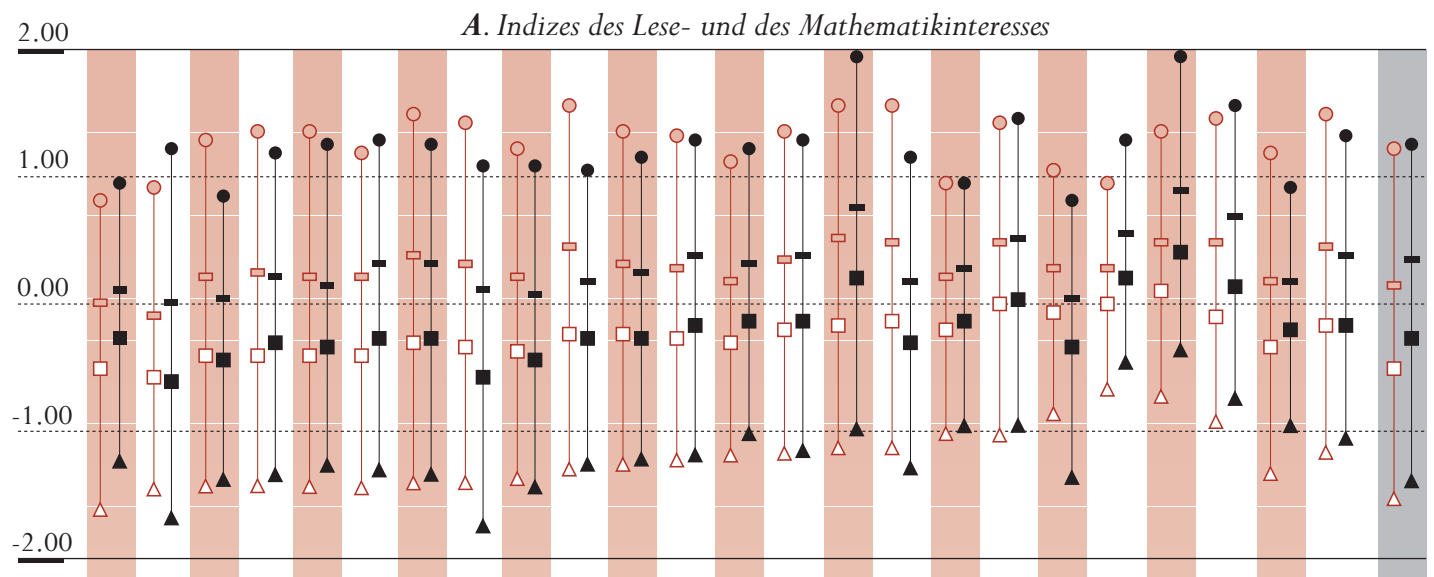

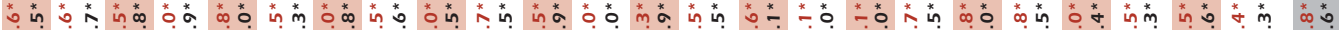

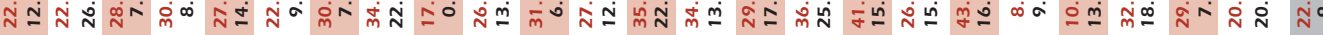

700 B. Leistungen auf der Gesamtskala Lesekompetenz und der Skala für mathematische Grundbildung, nach Indexquartilen

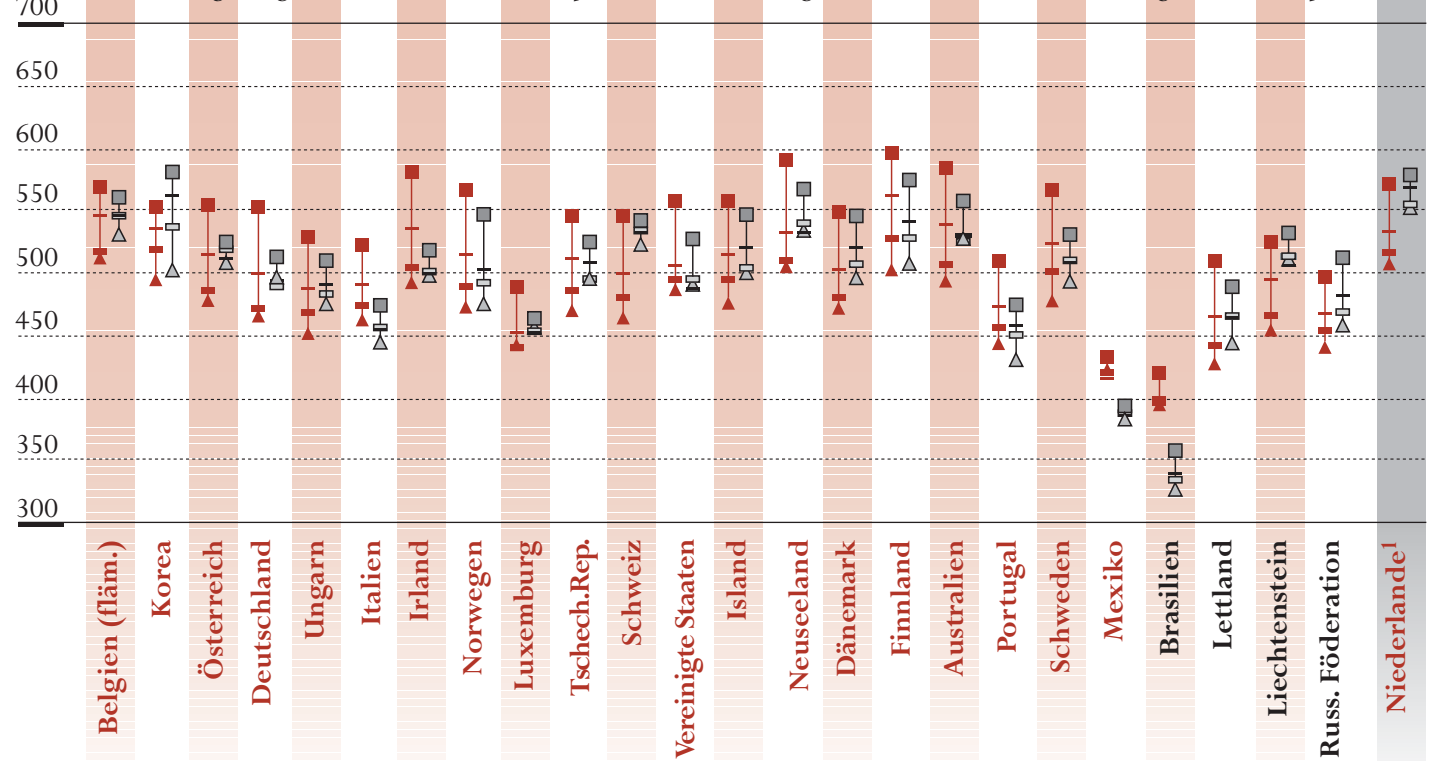

* Punktzahlveränderung auf der Gesamtskala Lesekompetenz je Indexeinheit des Index des Leseinteresses und des Index des Mathematikinteresses.

1. Die Beteiligungsquote ist zu niedrig, um die Vergleichbarkeit zu gewährleisten (vgl. Anhang A3).

Anmerkung: Zur Definition der Indizes siehe Anhang A1.

Quelle: OECD PISA Datenbank, 2001. Tabellen 4.1 und 4.2. 
KAPITEL 4 Ergebnisse zum Lernen allgemein

Die positive Einstellung zum Lesen ist je nach Land sehr unterschiedlich ...

... und eine positive Einstellung ist innerhalb der Länder mit höherer Lesekompetenz verbunden.
Schülerinnen und Schüler an den verschiedenen Fächern zu analysieren. Eine derartige Analyse kann signifikante Stärken und Schwächen der Schulsysteme in Bezug auf die von ihnen verfolgte Strategie zur Förderung der Lernmotivation verschiedener Gruppen von Schülerinnen und Schülern in den einzelnen Fächern aufzeigen.

Etwa die Hälfte der in PISA untersuchten 15-Jährigen steht dem Lesen grundsätzlich positiv gegenüber. Für durchschnittlich 21\% der Schülerinnen und Schüler in den OECD-Ländern insgesamt trifft die Aussage zu: „Weil mir

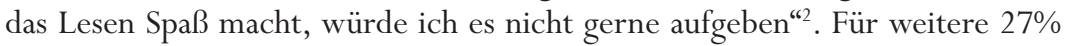
trifft diese Feststellung „eher“ zu. 28\% der Schülerinnen und Schüler geben ferner an, dass sie beim Lesen alles um sich herum vergessen, und für weitere 29\% trifft dies „eher“ zu. Bei der Beantwortung jeder dieser Fragen gibt es erhebliche Unterschiede zwischen den einzelnen Ländern. Während z.B. in Belgien (flämische Gemeinschaft) und Korea für weniger als ein Drittel der Schülerinnen und Schüler die Aussage „weil mir das Lesen Spaß macht, würde ich es nicht gerne aufgeben“ zutrifft bzw. eher zutrifft, teilen in Dänemark, Mexiko und Portugal mindestens 60\% der Schülerpopulation diese Einstellung zum Lesen (Daten abrufbar unter www.pisa.oecd.org).

In Abbildung 4.1 werden die Länder anhand eines Index des Leseinteresses verglichen. Der Index ist so konstruiert, dass die durchschnittliche Punktzahl für alle Länder 0 beträgt und zwei Drittel der Werte zwischen 1 und -1 liegen. Ein positiver Indexwert bedeutet, dass die Schülerinnen und Schüler ein höheres Leseinteresse bekunden als im OECD-Durchschnitt, während ein negativer Wert auf ein unter dem OECD-Durchschnitt liegendes Interesse hinweist (Angaben zur Definition des Index und Hinweise auf die begrifflichen Grundlagen finden sich in Anhang A1). Im oberen Teil von Abbildung 4.1 ist die Verteilung auf dem Index des Leseinteresses angegeben. Eine halbe Standardabweichung auf diesem Index trennt Dänemark, Finnland und Portugal, d.h. Länder mit hohem Leseinteresse der Schüler, von Belgien (flämische Gemeinschaft) und Korea, wo das Leseinteresse am niedrigsten ist.

In Abbildung 4.1 wird ferner für die einzelnen Länder die Lesekompetenz des Schülerquartils mit dem größten Leseinteresse mit der des Quartils verglichen, in dem das Leseinteresse am geringsten ist. Die Ergebnisse besagen jedoch nicht, dass Länder mit im Durchschnitt eifrigeren Lesern auch durchschnittlich bessere Werte auf der Skala Lesekompetenz erzielen. In der Tat weisen einige Länder mit überdurchschnittlichen Leseleistungen wie Korea und Österreich ein vergleichsweise niedriges Leseinteresse auf. Was die Ergebnisse hingegen sehr deutlich zeigen, ist, dass innerhalb der einzelnen Länder Schülerinnen und Schüler mit größerem Leseinteresse tendenziell bessere Resultate erzielen als solche mit geringerem Interesse ${ }^{3}$. Im Durchschnitt ist der Leistungsunterschied auf der Gesamtskala Lesekompetenz zwischen Schülern des obersten und des untersten Quartils des Index für Leseinteresse beträchtlich und beträgt 75 Punkte, was in etwa der Größenordnung einer ganzen Kompetenzstufe 
entspricht (Tabelle 4.1). In Finnland, dem Land mit den höchsten Gesamtwerten, erreicht das Quartil der Schülerinnen und Schüler mit dem geringsten Leseinteresse das OECD-Durchschnittsniveau (502 Punkte), während das Quartil mit dem höchsten Leseinteresse 599 Punkte erzielt, womit die Differenz 97 Punkte beträgt.

Die Art der hier bestehenden Kausalbeziehung ist wahrscheinlich komplex und lässt sich schwer ermitteln. Das fächerspezifische Interesse und die Leistungen könnten sich wechselseitig verstärken. Auch dritte Faktoren könnten eine Rolle spielen, wie z.B. der soziale Hintergrund der Schülerinnen und Schüler und ihrer Schulen. Wie diese Zusammenhänge auch beschaffen sein mögen eine positive Grundeinstellung zum Lesen stellt auf jeden Fall ein wichtiges Bildungsziel dar.

Beim Ländervergleich der Leistungen von Schülerinnen und Schülern im obersten und untersten Indexquartil sollte beachtet werden, dass das Gesamtniveau des Leseinteresses als solches je nach Land unterschiedlich sein kann. Außerdem sind diese Differenzen, wie in Kapitel 5 gezeigt wird, z.T. auf geschlechtsspezifische Unterschiede zurückzuführen. So geben wie aus Tabelle $4.1 \mathrm{zu}$ entnehmen die Schülerinnen und Schüler im obersten Indexquartil in der Tschechischen Republik, Dänemark, Finnland und Irland beispielsweise ein sehr viel größeres Leseinteresse an als Schülerinnen und Schüler des entsprechenden Quartils in Belgien (flämische Gemeinschaft) oder Korea. Desgleichen ist auch die Streuungsbreite der Werte auf dem Index des Leseinteresses von Land zu Land sehr unterschiedlich. In Irland bestehen größere Unterschiede beim Leseinteresse der Schülerinnen und Schüler als in Mexiko. Um diesen Differenzen Rechnung zu tragen und den relativen Einfluss des Leseinteresses auf die Schülerleistungen auf der Gesamtskala Lesekompetenz zu erfassen, enthält Abbildung 4.1 auch Angaben darüber, wie groß die Verbesserung der Leseleistung in den einzelnen Ländern bei Zunahme des Leseinteresses um eine Indexeinheit ist ${ }^{4}$. In Australien und Schweden z.B. erhöht sich die Leseleistung auf der Gesamtskala Lesekompetenz bei einem Anstieg des Leseinteresses um eine Indexeinheit um mehr als 40 Punkte (der OECD-Durchschnitt beträgt 28 Punkte).

Rund die Hälfte der 15-Jährigen sieht Mathematik als wichtig an, doch längst nicht alle davon halten dies für einen Grund, mit Mathematik weiter zu machen. In den OECD-Ländern insgesamt erklären durchschnittlich 20\% der Schülerinnen und Schüler, dass Mathematik ihnen persönlich wichtig ist $^{5}$. Weitere 32\% stimmen dieser Aussage „eher“ zu. Hingegen stimmen im OECD-Länderdurchschnitt nur 14\% der Aussage zu, dass sie, weil ihnen „die Beschäftigung mit Mathematik Spaß macht, das nicht gerne aufgeben würden“; weitere 26\% stimmen dieser Aussage „eher“ zu. 26\% stimmen nicht zu, dass Mathematik Spaß macht und weitere 29\% „eher“ nicht (Daten abrufbar unter www.pisa.oecd.org).
Nur eine Minderheit von schülerinnen und Schülern sieht Mathematik als wichtig für ihr späteres Leben an. 
KAPITEL 4 Ergebnisse zum Lernen allgemein

Beim Mathematikinteresse sind die Unterschiede zwischen den einzelnen Ländern größser als beim Leseinteresse

Ebenso wie beim Lesen ist ein durchschnittlich schwaches Interesse nicht immer gleichbedeutend mit niedrigen länderspezifischen Leistungen, ...

wenngleich innerhalb der Länder ein positiver Zusammenhang zwischen Mathematikinteresse und -leistungen besteht.
Abbildung 4.1 enthält neben den Angaben zum Leseinteresse auch einen Überblick über das Mathematikinteresse (Angaben zur Definition des Index und Hinweise auf die begrifflichen Grundlagen finden sich in Anhang A1). Je mehr man sich dem oberen Ende des Index nähert, desto mehr der Schülerinnen und Schüler geben an, dass sie bei Mathematikaufgaben alles um sich herum vergessen, dass Mathematik ihnen persönlich wichtig ist und dass sie, weil ihnen die Beschäftigung mit Mathematik Spaß macht, das nicht gerne aufgeben würden.

Die Mittelwerte der Länder weichen beim Index des Mathematikinteresses stärker voneinander $\mathrm{ab}$ als beim Index des Leseinteresses - obgleich die Unterschiede innerhalb der Länder auch hier wiederum sehr viel ausgeprägter sind als zwischen den Ländern (Tabelle 4.2). Da das Lernen von Mathematik im Vergleich zum Lesen weitaus häufiger in der Schule stattfindet, könnten die zwischen den Ländern bestehenden Unterschiede darauf hindeuten, dass die Bildungssysteme einen Einfluss auf die Einstellung junger Menschen zur Mathematik haben. Dies ist, sofern es durch weitere Untersuchungen bestätigt werden sollte, ein für die Bildungspolitik wichtiges Ergebnis. Angesichts der wachsenden Bedeutung der Mathematik für das spätere Leben der Schülerinnen und Schüler sollten die Bildungssysteme unbedingt sicherstellen, dass bei den Schülerinnen und Schülern sowohl das Interesse als auch die Motivation bestehen, in diesem Bereich auch nach dem Schulabschluss weiter zu lernen.

Ebenso wie beim Lesen ergibt sich auch hinsichtlich der länderspezifischen Beziehung zwischen dem Mathematikinteresse und der Leistung auf der Skala für mathematische Grundbildung ein gemischtes Bild. Das Beispiel von Dänemark und in geringerem Maße auch das Islands und Neuseelands zeigt, dass ein durchschnittlich relativ hohes Interesse der Schülerinnen und Schüler an Mathematik mit hohen Mathematikleistungen des jeweiligen Landes einhergehen kann (Tabelle 4.2). Auf der anderen Seite aber erzielen die Schülerinnen und Schüler in Österreich, Korea und Schweden über dem OECD-Durchschnitt liegende Leistungen auf der Skala für mathematische Grundbildung, obgleich sie nur ein geringes oder durchschnittliches Interesse an Mathematik bekunden.

Wenn auch zwischen den einzelnen Ländern ein unterschiedliches Bild besteht, ist doch innerhalb der Länder der Zusammenhang zwischen dem Mathematikinteresse und den Leistungen im Bereich mathematischer Grundbildung durchgehend positiv, wenngleich weniger stark als im Fall der Lesekompetenz $^{6}$. In den OECD-Ländern insgesamt trennen durchschnittlich 35 Punkte auf der Skala für mathematische Grundbildung das oberste vom untersten Schülerquartil auf dem Index des Mathematikinteresses, im Vergleich zu 75 Punkten bei der Lesekompetenz.

Die Tatsache, dass zumindest in einigen Ländern das Interesse der Schülerinnen und Schüler je nach Gegenstandsbereichen variiert, kann ein weiterer Hinweis darauf sein, dass das Interesse von der Art und Weise des Lernens und 
Unterrichtens abhängt. Am größten ist der Unterschied in Dänemark, wo die Schülerinnen und Schüler ein weitaus höheres Mathematik- als Leseinteresse zeigen, was sich in überdurchschnittlichen Leistungen bei der mathematischen Grundbildung gegenüber nur durchschnittlichen Leistungen bei der Lesekompetenz widerspiegelt. Die drei Länder mit den nächstgroßen Unterschieden sind Finnland, Norwegen und Schweden. In diesen Ländern weisen die Schülerinnen und Schüler ein sehr viel stärkeres Lese- als Mathematikinteresse auf.

\section{Leseaktivitäten und Freude am Lesen}

Neben der thematischen Motivation sind Leseaktivitäten und Freude am Lesen entscheidend an der Aufrechterhaltung und Weiterentwicklung der Lesekompetenz beteiligt. Das Ergebnis der Internationalen Erhebung über Grad und Verteilung elementarer Grundqualifikationen Erwachsener (IALS), wonach sich die Lesekompetenz nach Beendigung der schulischen Erstausbildung verschlechtern kann, wenn sie nicht ständig angewendet wird (OECD und Statistics Canada, 1995), zeigt, welche Bedeutung der Aufrechterhaltung der Lesekompetenz zukommt. Positive Leseaktivitäten und Freude am Lesen sind daher wichtige Ergebnisse der schulischen Erstausbildung und stellen Prädiktoren für Lernerfolge während des gesamten Lebens dar.

Die Angaben der Schülerinnen und Schüler darüber, wie oft sie z.B. zum Vergnügen lesen, gern mit anderen Leuten über Bücher sprechen oder gern in Buchhandlungen oder Büchereien gehen, können ebenso wie die allgemeine Bedeutung, die sie dem Lesen beimessen, Aufschluss darüber geben, welchen Platz sie dem Lesen in ihrem künftigen Leben einräumen werden (Angaben zur Definition des Index und Hinweise auf die begrifflichen Grundlagen finden sich in Anhang A1).

Die PISA-2000-Ergebnisse für die OECD-Länder legen den Schluss nahe, dass noch sehr viel mehr getan werden muss, um die Freude der Schülerinnen und Schüler am Lesen zu fördern. In den OECD-Ländern insgesamt lesen durchschnittlich 44\% der Schülerinnen und Schüler eigenen Angaben zufolge nur, um Informationen zu bekommen, die sie brauchen ${ }^{7}$, über ein Drittel liest demnach nur, wenn es sein muss, und 21\% stimmen ganz oder eher der Aussage zu, dass Lesen Zeitverschwendung ist ${ }^{8}$ (Daten abrufbar unter www.pisa.oecd.org).

Abbildung 4.2 vergleicht die Länder anhand eines Index, der die verschiedenen, in PISA 2000 enthaltenen Fragen über die Einstellung der Schüler zum Lesen zusammenfasst. Bei diesem Index wurde der Index von Abbildung 4.1 durch die Einbeziehung einer größeren Zahl unterschiedlicher Verhaltensweisen gegenüber dem Lesen erweitert. Ein positiver Indexwert bedeutet, dass die Schülerinnen und Schüler des betreffenden Landes häufiger als im OECDDurchschnitt angeben, dass Lesen eines ihrer liebsten Hobbys ist, dass sie gern mit anderen Leuten über Bücher sprechen, dass sie sich freuen, wenn
Die Tatsache, dass das

Interesse je nach

Gegenstandsbereich

unterschiedlich sein kann,

legt den Schluss nahe,

dass es durch die Art

und Weise des Lernens

beeinflusst wird.

Leseaktivitäten und Freude am Lesen spielen eine entscheidende Rolle bei der Weiterentwicklung und Aufrechterhaltung der Lesekompetenz,...

... viele Schülerinnen und schüler lesen jedoch nur, wenn es sein muss, oder betrachten Lesen als Zeitverschwendung. 
KAPITEL 4 Ergebnisse zum Lernen allgemein

Abblidung 4.2

Freude am Lesen und Schülerleistungen
A O Oberstes Quartil $\square$ Drittes Quartil —Zweites Quartil $\Delta$ Unterstes Quartil
B $\square$ Oberstes Quartil - Drittes Quartil $\square$ Zweites Quartil $\Delta$ Unterstes Quartil

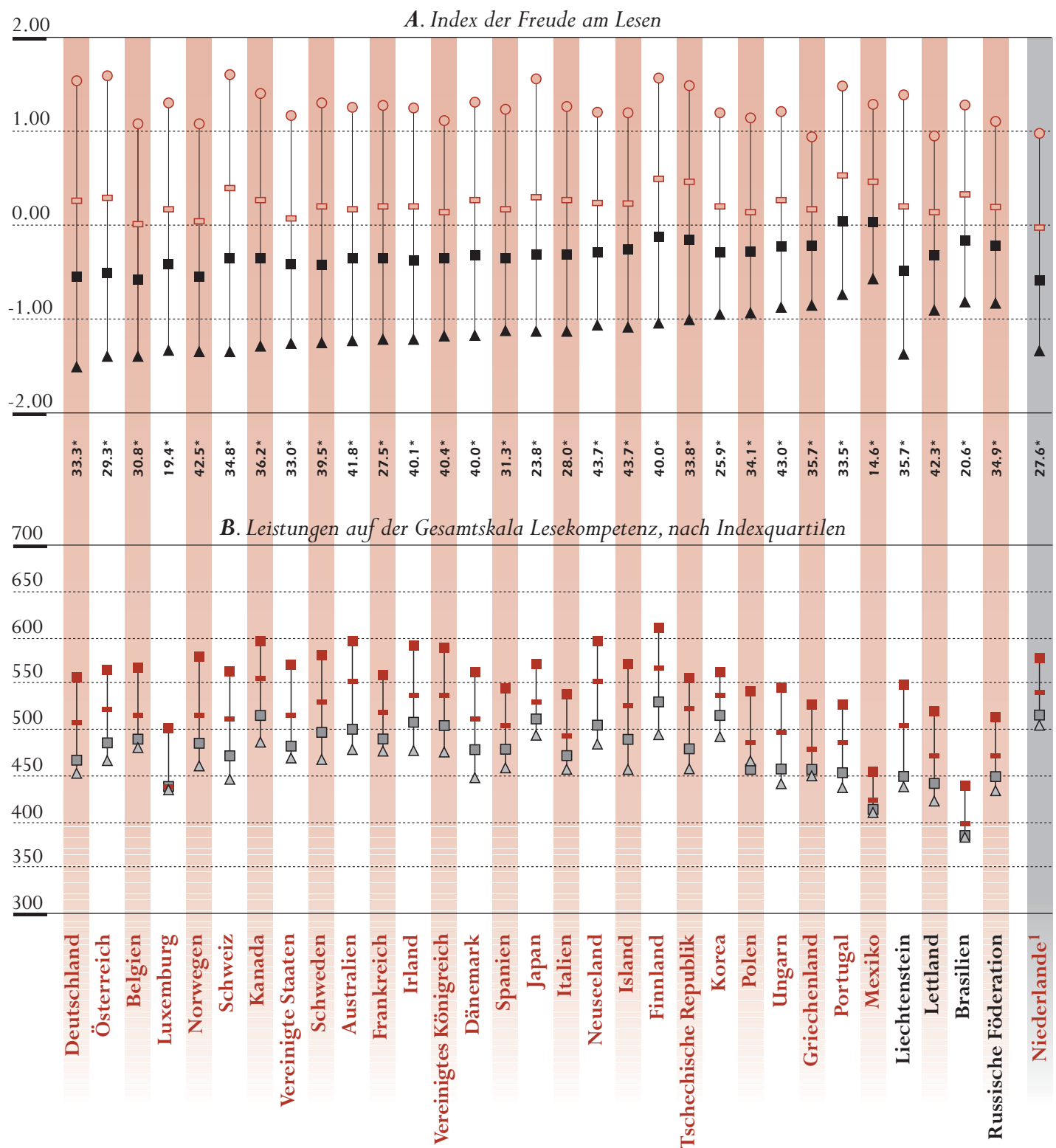

* Punktzahlveränderung auf der Gesamtskala Lesekompetenz je Indexeinheit des Index der Freude am Lesen. 1. Die Beteiligungsquote ist zu niedrig, um die Vergleichbarkeit zu gewährleisten (vgl. Anhang A3). Anmerkung: Zur Definition des Index siehe Anhang A1. Quelle: OECD PISA Datenbank, 2001. Tabelle 4.3. 
sie ein Buch geschenkt bekommen, und dass sie gern in Buchhandlungen oder Büchereien gehen. Ein positiver Wert bedeutet ebenfalls, dass die betreffenden Schüler weniger häufig angeben, dass sie nur lesen, wenn es sein muss, dass es ihnen schwerfällt, Bücher zu Ende zu lesen, dass Lesen Zeitverschwendung ist, dass sie nur lesen, um Informationen zu bekommen, die sie brauchen, bzw. dass sie nicht länger als ein paar Minuten stillsitzen und lesen können.

In Abbildung 4.2 sind die Mittelwerte der Länder auf dem Index der Einstellung zum Lesen dargestellt. Der Abstand zwischen den Ländern, die ein hohes Niveau in Bezug auf die Freude am Lesen aufweisen (Tschechische Republik, Finnland, Mexiko, Portugal) und den Ländern mit diesbezüglich niedrigen Niveaus (Belgien, Niederlande, Norwegen) beträgt ungefähr eine halbe Standardabweichung auf dem Index (Tabelle 4.3).

Abbildung 4.2 vergleicht ebenfalls die auf der Gesamtskala Lesekompetenz erfassten Leistungen von Schülerinnen und Schülern des untersten und obersten Quartils des Index der Freude am Lesen sowie dieVerbesserung der Leseleistung bei Zunahme der Lesefreude um eine Indexeinheit. In praktisch allen Ländern besteht ein enger Zusammenhang zwischen der Freude am Lesen und den Leistungen der Schülerinnen und Schüler, der in 12 von 28 Ländern mehr als 100 Punkte beträgt, was einer internationalen Standardabweichung und in allen bis auf sieben Ländern einer Kompetenzstufe entspricht (Tabelle 4.3) ${ }^{9}$.

Eine weitere wichtige Variable unter den Bestimmungsfaktoren für die Freude der Schülerinnen und Schüler am Lesen ist die Zeit, die diese damit verbringen, zum Vergnügen zu lesen. Bei der Interpretation der hier beobachteten Unterschiede darf natürlich nicht übersehen werden, dass die Zeit, die die Schülerinnen und Schüler mit Lesen tatsächlich verbringen können, von Land zu Land je nach der Länge des Schultags, den geforderten Hausaufgaben oder anderen außerschulischen Aktivitäten unterschiedlich sein kann.

Abbildung 4.3 zeigt, wie viel Zeit die Schülerinnen und Schüler täglich zum Vergnügen lesen, und gibt zugleich die Leistungen auf der Gesamtskala Lesekompetenz an. In den OECD-Ländern insgesamt erklären durchschnittlich 32\% der Schülerinnen und Schüler, und in Österreich, Belgien, Deutschland, Japan und den Vereinigten Staaten sogar über 40\%, dass sie überhaupt nicht zum Vergnügen lesen ${ }^{10}$. Der durchschnittliche Mittelwert dieser Schülerinnen und Schüler auf der Gesamtskala Lesekompetenz liegt mit 474 Punkten weit unter dem OECD-Durchschnitt insgesamt (Tabelle 4.4).

Weitere 31\% der Schülerinnen und Schüler in den OECD-Ländern insgesamt lesen durchschnittlich bis zu 30 Minuten täglich zum Vergnügen. Ihre mittlere Leistung liegt mit 513 Punkten über dem OECD-Durchschnitt. Ein weiteres Drittel der Schülerinnen und Schüler in den OECD-Ländern liest durchschnittlich zwischen 30 Minuten und 2 Stunden pro Tag zum Vergnügen, und ihr Leistungsniveau liegt bei rund 527 Punkten. Schülerinnen und Schüler, die längere Lesezeiten angeben, liegen mit 506 Punkten nahe
Die Freude am Lesen ist in den einzelnen Ländern unterschiedlich stark ausgeprägt, ...

... jedoch schneiden die eifrigen Leser in allen Ländern sehr viel besser ab als Schülerinnen und Schüler mit nur geringer Freude am Lesen.

Eine nicht unbedeutende Minderheit an Schülerinnen und schülern...

... verbringt überhaupt keine Zeit mit Lesen zum eigenen Vergnügen.

Bildungssysteme sollten sich um ein dem auferschulischen Lesen förderliches Lernumfeld bemühen. 
KAPITEL 4 Ergebnisse zum Lernen allgemein

Abbildung 4.3

Mit Lesen zum Vergnügen verbrachte Zeit und Schülerleistungen

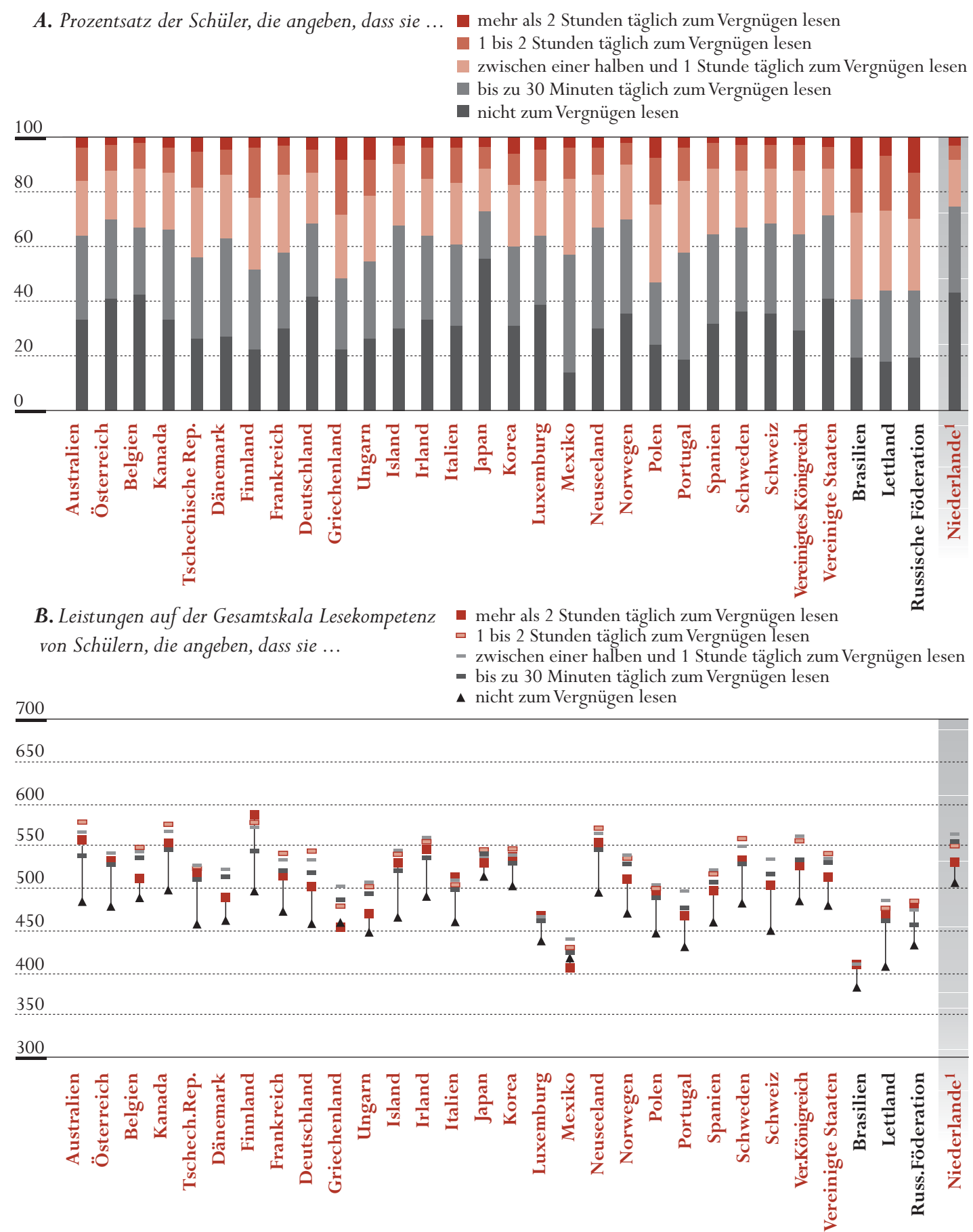

1. Die Beteiligungsquote ist zu niedrig, um die Vergleichbarkeit zu gewährleisten (vgl. Anhang A3).

Quelle: OECD PISA Datenbank, 2001. Tabelle 4.4. 
am OECD-Durchschnitt (Tabelle 4.4), wobei ihre niedrigeren Leistungen darauf zurückzuführen sein könnten, dass sie mehr Zeit zum Lesen ihres Materials brauchen als leistungsstarke Schüler. Das niedrige Leistungsniveau von Schülerinnen und Schülern, die überhaupt nicht zum Vergnügen lesen, zeigt für die Bildungssysteme die Notwendigkeit auf, ein für das außerschulische Lesen förderliches Lernumfeld zu schaffen.

\section{Allgemeine Schulzufriedenheit}

Störendes Verhalten, Absentismus und eine negative Einstellung zur Schule können häufig mit niedrigen schulischen Leistungen und der Entscheidung zum Schulabbruch einhergehen. Auf der anderen Seite haben Untersuchungen gezeigt, dass wenn sich die Schülerinnen und Schüler am Unterricht bzw. an außercurricularen Aktivitäten aktiv beteiligen und enge Beziehungen zu anderen Schülern und Lehrkräften entwickeln, die Wahrscheinlichkeit größer ist, dass sie gute schulische Leistungen erreichen und die Sekundarschulstufe abschließen.

Im Rahmen von PISA 2000 wurden 15-jährige Schülerinnen und Schüler nach ihren Einstellungen der Schule gegenüber befragt. In 20 der 28 OECD-Länder stimmt über ein Viertel der Schülerinnen und Schüler der Aussage, wonach die Schule ein Ort ist, an den sie nicht hingehen möchten, eher bzw. ganz zu. In Belgien, Kanada, Frankreich, Ungarn, Italien und den Vereinigten Staaten reicht dieser Anteil in der genannten Reihenfolge von 35\% bis 42\% (Daten abrufbar unter www.pisa.oecd.org). Selbst in einigen der leistungsstärksten Länder im Rahmen von PISA, wie Australien, Kanada und Korea, stimmen zwischen 30\% und 37\% der Schülerinnen und Schüler eher oder ganz der Feststellung zu, dass die Schule ein Ort ist, an den sie nicht hingehen möchten. Hingegen beträgt dieser Anteil für Dänemark, Mexiko, Portugal und Schweden weniger als 20\%. In nahezu der Hälfte der OECD-Länder stimmen die meisten Schülerinnen und Schüler auch eher oder ganz der Aussage zu, dass die Schule ein Ort ist, an dem sie sich oft langweilen ${ }^{11}$, und in Griechenland, Irland und Spanien gilt dies sogar für mehr als zwei Drittel der Schülerinnen und Schüler. Aber auch hier wiederum sind die Antworten je nach Land sehr unterschiedlich (Daten abrufbar unter www.pisa.oecd.org), was den Schluss nahe legt, dass das Desinteresse an der Schule in diesem Alter zwar weit verbreitet, aber nicht unvermeidlich ist.

Welche Bedeutung kommt dem Befund zu, dass viele Schülerinnen und Schüler nicht gern zur Schule gehen? Vielleicht muss jungen Leuten ja nicht alles gefallen, was gut für sie ist. Die Befunde zeigen allerdings, dass die Leistungen von Schülerinnen und Schülern, die gern zur Schule gehen, besser sind als die von Schülerinnen und Schülern, bei denen dies nicht der Fall ist. Schülerinnen und Schüler, die gerne zur Schule gehen, schneiden in fast allen Ländern auf der Gesamtskala Lesekompetenz besser ab als diejenigen, für die die Schule ein Ort ist, an den sie nicht hingehen möchten (Daten abrufbar unter www.pisa.oecd.org).
Eine positive Einstellung

zur Schule ist wichtig.

In vielen Ländern jedoch ist eine großse Anzahl von schülerinnen und Schülern der Schule gegenüber negativ eingestellt, ...

... und diese schneiden im Durchschnitt schlechter ab als Schülerinnen und Schüler mit einer positiveren Einstellung. 
KAPITEL 4 Ergebnisse zum Lernen allgemein

\section{Abbildung 4.4}

Allgemeine Schulzufriedenheit

Verteilung der mittleren Prozentsätze der Schülerinnen und Schüler, die folgende Aussagen mit „stimmt eher“ und „stimmt ganz genau“ beantworten. „Die Schule ist ein Ort, ...

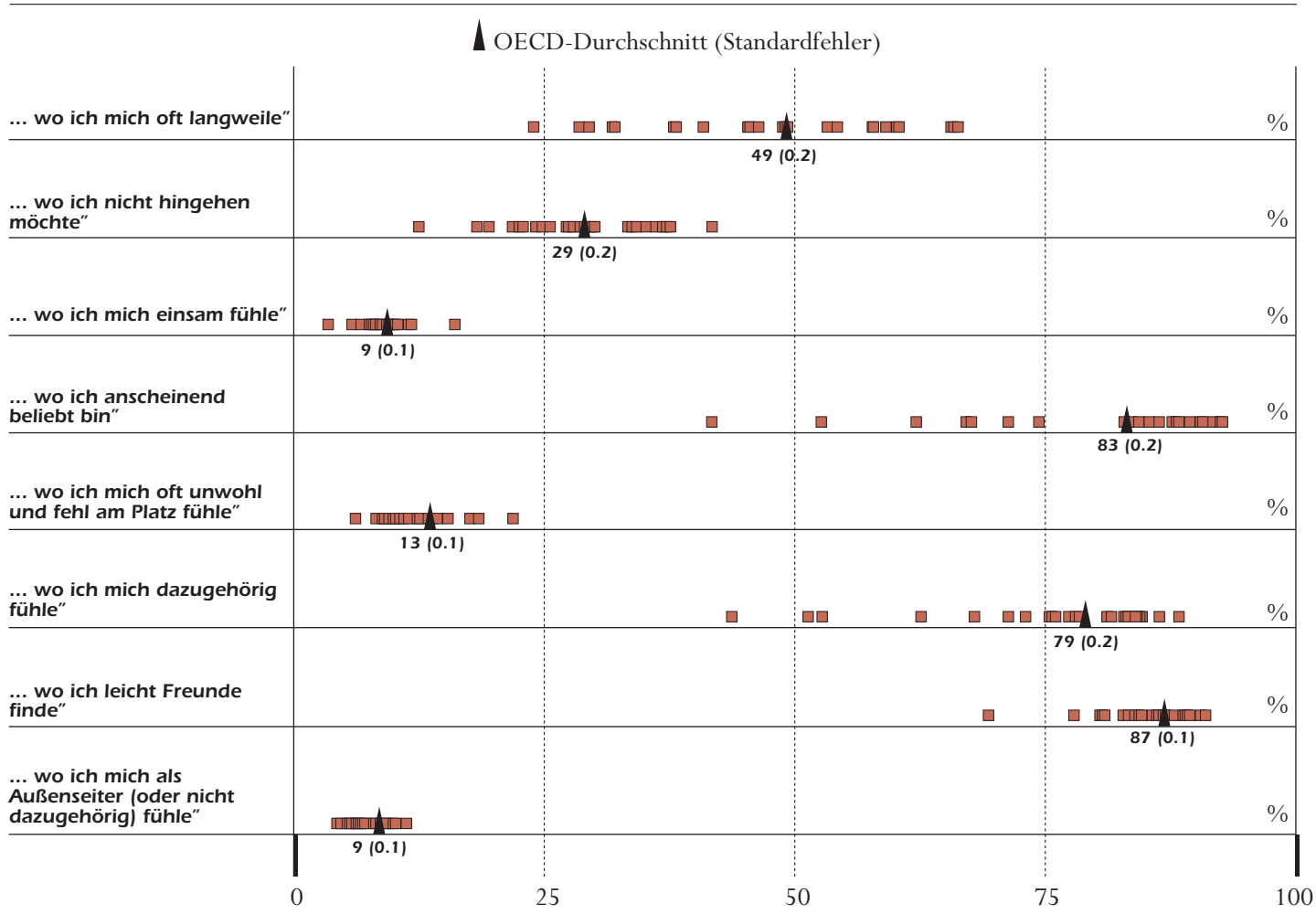

Anmerkung: Die Länder sind dargestellt durch die Quadrate.

Quelle: OECD PISA Datenbank, 2001. Daten abrufbar unter www.pisa.oecd.org.

Abbildung 4.4 zeigt, dass OECD-weit durchschnittlich 86\% der Schülerinnen und Schüler erklären, die Schule sei ein Ort, an dem sie leicht Freunde finden, und drei Viertel aller Schüler bezeichnen die Schule als einen Ort, an dem sie sich dazugehörig fühlen ${ }^{12}$ (wobei die Prozentsätze von höchstens ungefähr der Hälfte aller Schülerinnen und Schüler in Frankreich und Spanien bis hin zu 88\% in Ungarn und Mexiko reichen) (Daten abrufbar unter www.pisa.oecd.org). Hingegen gibt es eine kleine, aber bedeutsame Gruppe von Schülerinnen und Schülern, für die die Schule ein schwieriges soziales Umfeld darstellt. In den OECD-Ländern insgesamt erklären durchschnittlich 13\% aller Schülerinnen und Schüler, dass die Schule ein Ort ist, an dem sie sich oft unwohl und fehl am Platz fühlen, während dieser Prozentsatz in Schweden und im Vereinigten Königreich bei weniger als 9\% liegt. 
Die Befunde lassen keine Kausale Interpretation des Zusammenhangs zwischen diesen Faktoren und den schulischen Leistungen zu. Zudem gibt es andere Faktoren, die sowohl die Leistung als auch die Einstellung zur Schule beeinflussen, bzw. es könnte auch sein, dass gute schulische Leistungen der Grund dafür sind, dass die betreffenden Schülerinnen und Schüler gern zur Schule gehen, statt umgekehrt. Angesichts der in allen Ländern hohen Bildungsinvestitionen ist allerdings die Feststellung unbefriedigend, dass bei einer nicht unbedeutenden Minderheit, und in manchen Fällen sogar bei einer Mehrheit der Schülerinnen und Schüler, keine Schulzufriedenheit, sondern vielmehr eine negative Einstellung der Schule gegenüber festzustellen ist. Es ist schwer vorstellbar, dass Schulen optimale Resultate erzielen können, wenn die Schülerinnen und Schüler der Schule nicht grundsätzlich positiv gegenüberstehen. Wenn eine solche Einstellung für die im Rahmen von PISA getestete Altersgruppe auch gar nicht so einfach zu verwirklichen sein dürfte, führen die Ergebnisse doch zu dem Schluss, dass Schulpolitik und -praxis der Schaffung eines positiven Lernumfelds für alle Schülerinnen und Schüler hinreichende Aufmerksamkeit widmen sollten.

Zudem besteht nicht nur ein negativer Zusammenhang zwischen fehlender Schulzufriedenheit und schulischen Leistungen, sondern bei Schülerinnen und Schülern, die kein Interesse für die Schule aufbringen, dürfte auch die Wahrscheinlichkeit geringer sein, dass sie sich in ihrem späteren Leben an Lernaktivitäten innerhalb oder außerhalb formaler Bildungseinrichtungen beteiligen.

\section{Lernstrategien}

\section{Kontrolle des Lernprozesses}

Informationen werden von Schülerinnen und Schülern nicht passiv aufgenommen und verarbeitet. Vielmehr sind diese aktiv am Lernprozess beteiligt, indem sie sich den Inhalt des zu lernenden Stoffes auf eine Art und Weise erarbeiten, die durch ihr jeweiliges Vorwissen sowie ihre neu erworbenen Erfahrungen geprägt ist. Schülerinnen und Schüler mit gut entwickelten Fähigkeiten zu selbstreguliertem Lernen sind in der Lage, sich geeignete Lernziele zu setzen, sich beim Lernen an bereits vorhandenen Kenntnissen und Fertigkeiten $\mathrm{zu}$ orientieren und Lernstrategien zu wählen, die der jeweiligen Aufgabe angemessen sind. Diese Kompetenzen undVerhaltensweisen, deren Entwicklung nicht immer ausdrücklich im Mittelpunkt des Schulunterrichts gestanden hat, werden mittlerweile immer häufiger explizit als wichtige schulische Ziele artikuliert und sollten dementsprechend auch als maßgebliche Erträge des Lernprozesses betrachtet werden.

Effektiv lernen heißt, Informationen effizient zu verarbeiten. Hierzu ist mehr nötig als die Fähigkeit zum Memorieren neuer Informationen. Es bedarf der Fähigkeit, neue Informationen zu bestehendem Wissen in Beziehung zu setzen und herauszufinden, wie die Kenntnisse im wirklichen Leben angewendet
Negative Einstellungen zur schule müssen nicht notwendigerweise niedrige Leistungen hervorrufen, doch sie sind auf keinen Fall wünschenswert ...

... und können sich negativ aufkünftiges Lernen auswirken.

Die Schülerinnen und Schüler sollten bei der Organisation ihres Lernprozesses aktiv mitwirken ... 
KAPITEL 4 Ergebnisse zum Lernen allgemein

PISA stellte den Schülerinnen und Schülern auch Fragen über den Lernprozess als solchen ...

... und stellte fest, dass zwischen der Anwendung von Strategien zur persönlichen Lernorganisation und den Leistungen ein positiver Zusammenhang besteht. werden können. Wenn ein Schüler effektive Lernstrategien hinreichend beherrscht, wird er auch eher imstande sein, das eigene Lernen zu organisieren. Wer gut lernt, ist in der Lage, ein effizientes Repertoire an Lernstrategien je nach Fall flexibel einzusetzen. Hingegen stehen Schülerinnen und Schülern, denen selbständiges Lernen schwerfällt, häufig keine effektiven Strategien zur Verfügung, die ihnen das Lernen und dessen Selbstkontrolle erleichtern könnten, bzw. sie sind nicht in der Lage, eine zur Lösung der jeweiligen Aufgabe geeignete Strategie zu wählen.

Im Rahmen von PISA wurden den Schülerinnen und Schülern auch Fragen über den Lernprozess als solchen vorgelegt. Ausgehend von den Antworten auf Fragen danach, wie oft sich die Schülerinnen und Schüler beim Lernen zuerst überlegen, was sie lernen müssen, beim Lernen nach und nach herauszufinden suchen, welche Begriffe sie nicht verstanden haben, nach zusätzlichen Informationen suchen, wenn sie etwas nicht verstehen, selbst kontrollieren, ob sie sich an das Gelernte erinnern, und sich vergewissern, dass sie die wichtigsten Dinge behalten haben, wurde ein Index für Kontrollstrategien entwickelt. Der Index wurde so konstruiert, dass der Durchschnittswert der Länder bei 0 liegt und die Standardabweichung 1 beträgt. (Angaben zur Definition des Index und Hinweise auf die begrifflichen Grundlagen finden sich in Anhang A1.) Ein positiver Indexwert bedeutet, dass diese Kontrollstrategien häufiger als im OECD-Durchschnitt angewendet werden.

Abbildung 4.5 vergleicht die Länder anhand der Angaben der 15-Jährigen über ihre Anwendung von Kontrollstrategien beim Lernen. Die mittlere Punktzahl auf dem Index der Kontrollstrategien variiert, wobei die Schülerinnen und Schüler aus Österreich, der Tschechischen Republik, Deutschland, Ungarn, Italien und Portugal den häufigsten Rückgriff auf Selbstregulierungsstrategien melden. Am wenigsten wenden Schülerinnen und Schüler aus Finnland, Island, Korea und Norwegen ihren eigenen Angaben zufolge derartige Strategien an (Tabelle 4.5).

Zwischen der effektiven Anwendung von Kontrollstrategien und den Schülerleistungen besteht ein positiver Zusammenhang. Innerhalb der jeweiligen Länder erzielen Schülerinnen und Schüler, die häufiger auf derartige Strategien zurückgreifen, tendenziell höhere Punktzahlen auf der PISA-Gesamtskala für Lesekompetenz als Schülerinnen und Schüler, die dies nicht tun (wenngleich nicht festzustellen ist, ob die Lernstrategien wirklich die Ursache für die besseren Ergebnisse sind). Am deutlichsten tritt dieser Zusammenhang in Australien, Neuseeland und Portugal zu Tage, wo das Quartil der Schülerinnen und Schüler, die diese Lernstrategien am meisten anwenden, dem Schülerquartil, das am wenigsten davon Gebrauch macht, um durchschnittlich eine ganze Kompetenzstufe voraus ist. Im OECD-Durchschnitt beträgt der Abstand zwischen dem obersten und dem untersten Quartil 52 Punkte. Relativ gesehen entspricht eine Differenz von einer Indexeinheit 16 Punkten im Durchschnitt der OECD-Länder insgesamt ${ }^{13}$. Derartige Strategien sind von 
Kontrolle des Lernprozesses und Schülerleistungen
A O Oberstes Quartil $\square$ Drittes Quartil
- Zweites Quartil
$\Delta$ Unterstes Quartil
B @ Oberstes Quartil - Drittes Quartil $\square$ Zweites Quartil $\Delta$ Unterstes Quartil
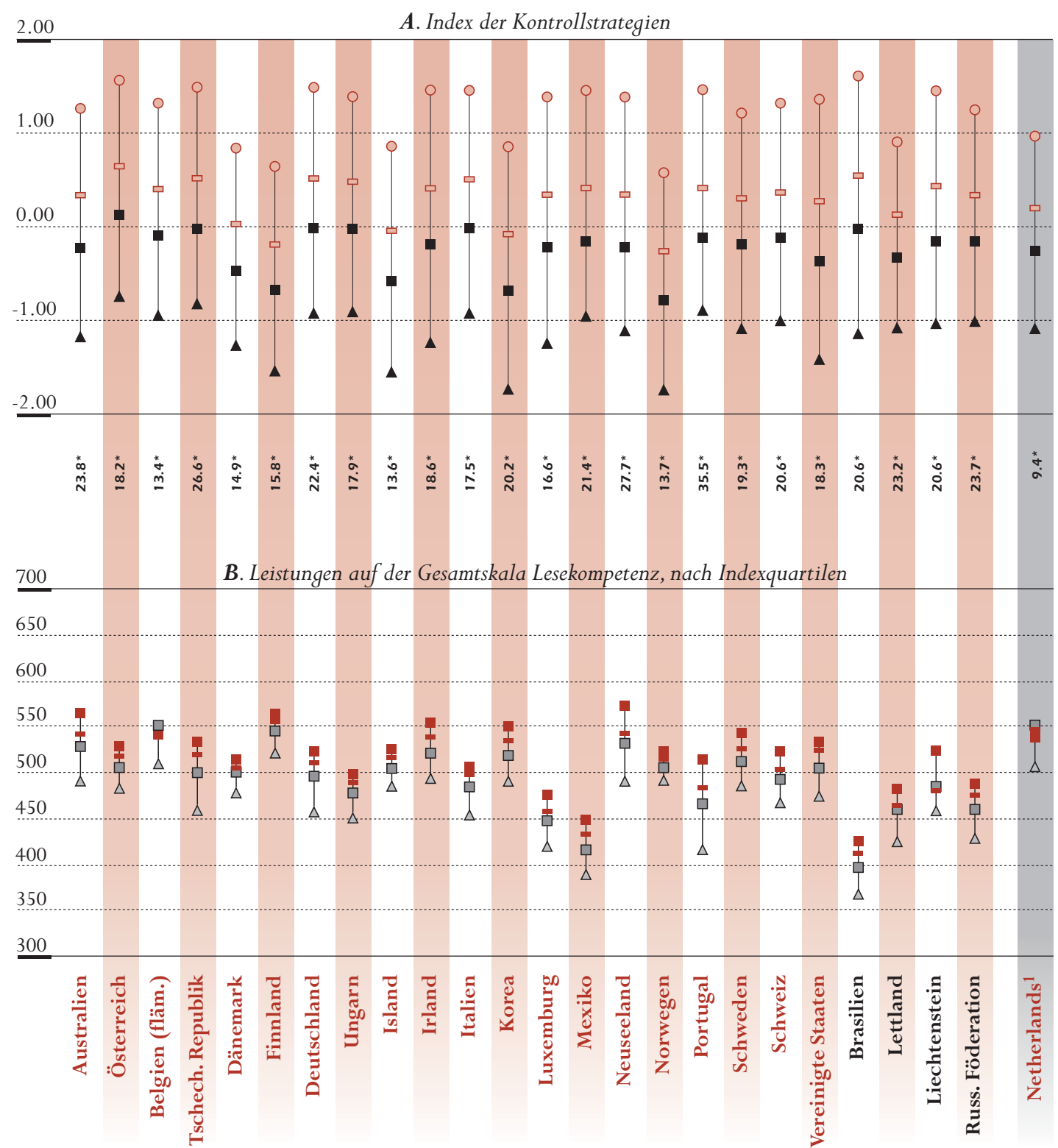

* Punktzahlveränderung auf der Gesamtskala Lesekompetenz je Indexeinheit des Index der Kontrollstrategien.

1. Die Beteiligungsquote ist zu niedrig, um die Vergleichbarkeit zu gewährleisten (vgl. Anhang A3).

Anmerkung: Zur Definition des Index siehe Anhang A1.

Quelle: OECD PISA Datenbank, 2001. Tabelle 4.5. 
KAPITEL 4 Ergebnisse zum Lernen allgemein

wesentlicher Bedeutung für effizientes selbstreguliertes Lernen, weil sie den Schülerinnen und Schülern dabei helfen, ihren Lernprozess den spezifischen Anforderungen der jeweiligen Aufgabe anzupassen. Die Schulen sollten sich vielleicht ausdrücklicher darum bemühen, den Schülerinnen und Schülern die Möglichkeit zu geben, ihren Lernprozess selbst zu steuern und zu kontrollieren, um auf diese Weise allen Schülern bei der Entwicklung effektiver Strategien zu helfen, die diese nicht nur bei ihrem schulischen Lernen unterstützen, sondern ihnen auch die praktischen Mittel für die Selbstregulierung ihrer Lernprozesse im späteren Leben an die Hand geben.

\section{Memorieren und Elaborieren}

Die Schülerinnen und Schüler müssen sowohl neue Informationen memorieren, als auch in der Lage sein, diese zu ihrem jeweiligen Vorwissen in Beziehung zu setzen.

Memorier- und Elaborationsstrategien ergänzen einander.
Memorierstrategien (z.B. wiederholtes lautes Lesen der Texte und Auswendiglernen von Schlüsselbegriffen) sind bei vielen Aufgaben wichtig, führen aber normalerweise nur zu einer wörtlichen Repräsentation des Wissensstoffes, was bedeutet, dass neue Informationen mehr oder minder unverarbeitet im Gedächtnis gespeichert werden. Wenn sich der Lernende zum Ziel setzt, die gespeicherten Informationen in derselben Form abrufen zu können, wie sie eingegeben wurden, dann ist das Memorieren eine geeignete Strategie, aber ein derartiges „mechanisches Lernen“ führt nur selten zu wirklichem Textverständnis. Letzteres setzt vielmehr die Integration der neuen Informationen in die bereits bestehende Wissensbasis des Lernenden voraus. $\mathrm{Zu}$ diesem Zweck können Elaborationsstrategien eingesetzt werden (indem z.B. untersucht wird, wie die Informationen mit bereits in einem anderen Kontext gelernten Dingen zusammenhängen, bzw. indem gefragt wird, wie die betreffenden Informationen in einem anderen Kontext angewendet werden könnten).

Den Schülerinnen und Schülern wurden gesonderte Fragen über die Anwendung von Memorier- oder Elaborationsstrategien vorgelegt. Ausgehend von ihren Antworten hierauf wurde für jede dieser beiden Strategien ein Index konstruiert. Der Memorierindex wurde von den Antworten der Schülerinnen und Schüler auf die Frage abgeleitet, wie oft die Schüler beim Lernen versuchen, alles auswendig zu lernen, was drankommen könnte, sich alles Neue so einzuprägen suchen, dass sie es aufsagen können, und üben, indem sie den Stoff immer wieder aufsagen. Der Elaborationsindex wurde von den Antworten auf Fragen danach abgeleitet, wie oft der Lernende versucht, den Stoff besser zu verstehen, indem erVerbindungen zu Dingen herstellt, die er schon kennt, oder neuen Stoff mit Dingen zu verbinden, die er in anderen Fächern gelernt hat, bzw. überlegt, inwiefern die Information im wirklichen Leben nützlich sein könnte (Angaben zur Definition der Indizes und Hinweise auf die begrifflichen Grundlagen finden sich in Anhang A.1). Die Indizes wurden so konstruiert, dass die durchschnittliche Punktzahl in allen Ländern 0 und die Standardabweichung 1 beträgt. 
Memorier- und Elaborationsstrategien und Schülerleistungen

A Index der Memorierstrategien: OOberstes Quartil $\square$ Drittes Quartil $\square$ Zweites Quartil $\triangle$ Unterstes Quartil Index der Elaborationsstrategien: • Oberstes Quartil - Drittes Quartil — Zweites Quartil \ Unterstes Quartil

Leistungen auf der Gesamtskala Lesekompetenz

B nach Quartilen des Index der Memorierstrategien: - Oberstes Quartil - Drittes Quartil • Zweites Quartil $\Delta$ Unterstes Quartil

Leistungen auf der Gesamtskala Lesekompetenz $\quad$ OOberstes Quartil - Drittes Quartil $\square$ Zweites Quartil $\Delta$ Unterstes Quartil nach Quartilen des Index der Elaborationsstragtegien:

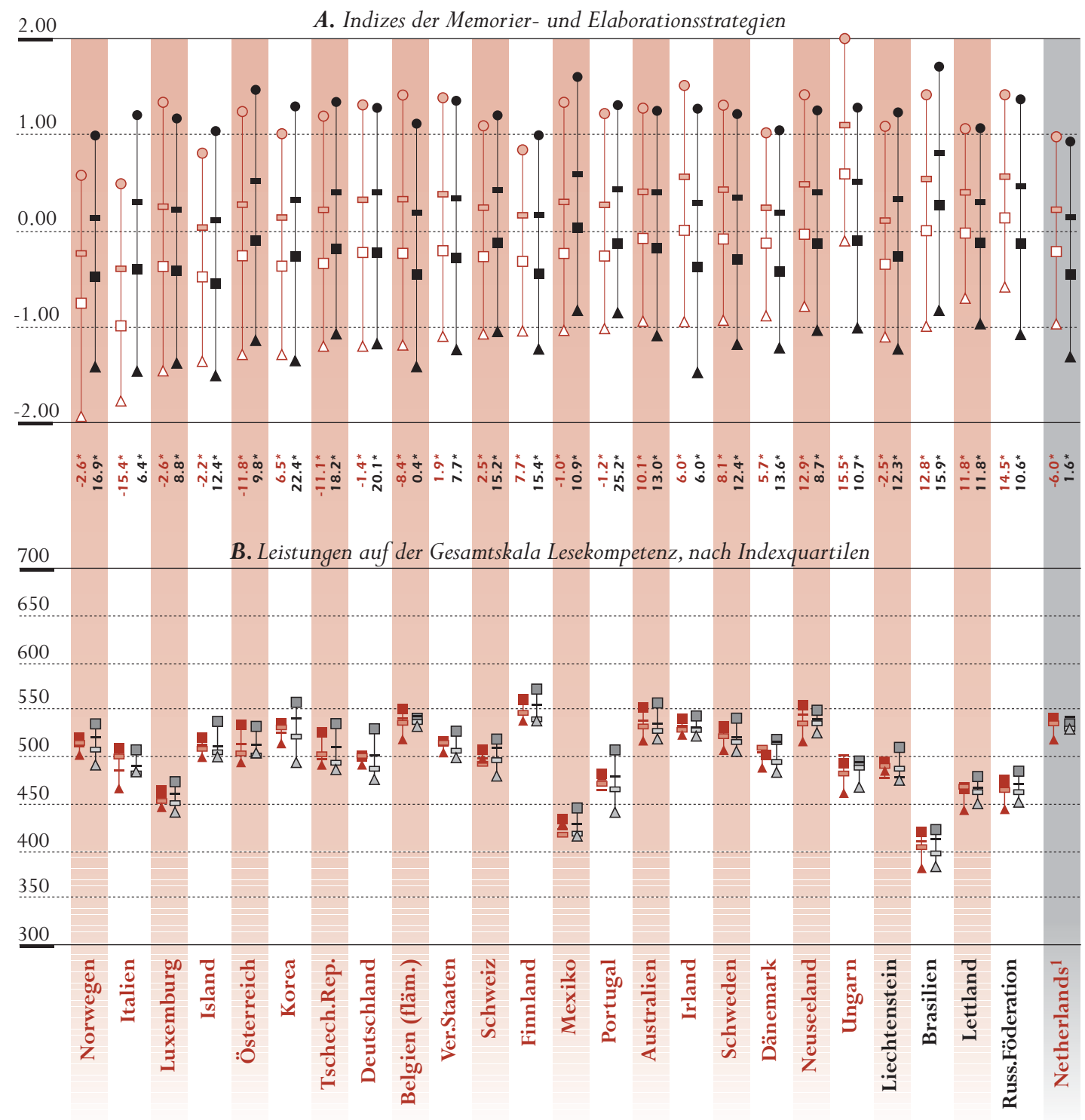

* Punktzahlveränderung auf der Gesamtskala Lesekompetenz je Indexeinheit der Indizes der Memorier- und Elaborationsstrategien. 1. Die Beteiligungsquote ist zu niedrig, um die Vergleichbarkeit zu gewährleisten (vgl. Anhang A3).

Anmerkung: Zur Definition der Indizes siehe Anhang A1.

Quelle: OECD PISA Datenbank, 2001. Tabellen 4.6 und 4.7. 
KAPITEL 4 Ergebnisse zum Lernen allgemein

Die Anwendung von Elaborationsstrategien ist mit guten Leistungen verbunden,...

Memorierstrategien anscheinend keine so bedeutende Rolle spielen.

Die Schulen sollten die schülerinnen und Schüler beim Verständnis für und der Entwicklung von Strategien für selbstreguliertes Lernen unterstützen. bewerbsorientiertes Lernen können einander ergänzen.
In Abbildung 4.6 werden mit Hilfe der Memorier- und Elaborationsindizes Ländervergleiche anhand der Angaben der 15-Jährigen über den Gebrauch angestellt, den sie von diesen beiden Strategien machen.

Die häufige Anwendung von Elaborationsstrategien weist tendenziell in allen Ländern einen positiven Bezug zu den Leistungen auf der Gesamtskala Lesekompetenz auf, wobei der Unterschied zwischen den länderspezifischen obersten und untersten Indexquartilen für alle OECD-Länder insgesamt durchschnittlich 32 Punkte beträgt. Die Höhe des offensichtlichen Vorteils des Gebrauchs dieser Strategien ist jedoch sehr unterschiedlich: So beträgt der Abstand zwischen dem obersten und dem untersten Schülerquartil in Portugal 61 Punkte (was nahezu einer ganzen Kompetenzstufe entspricht), in Korea 60 Punkte und in Deutschland 51 Punkte, in Belgien (flämische Gemeinschaft), Irland, Italien, den Niederlanden, Neuseeland und den Vereinigten Staaten hingegen weniger als 25 Punkte (Tabelle 4.7) ${ }^{14}$.

Bei der Anwendung von Memorierstrategien ergibt sich hinsichtlich des Zusammenhangs mit den Punktzahlen auf der Gesamtskala Lesekompetenz ein gemischtes Bild. So sind die Schülerleistungen im obersten Indexquartil in 11 Ländern höher als im untersten Quartil, während das Verhältnis in 5 Ländern umgekehrt ist und in den übrigen Ländern keine statistisch signifikanten Unterschiede festzustellen sind (Tabelle 4.6).

Alles in allem legen die Befunde den Schluss nahe, dass sich Elaborationsstrategien stärker auf die Schülerleistungen auswirken. Mithin könnte ihnen größere Bedeutung zukommen als einfachen Memorierstrategien, auf die die Schüler vermutlich intuitiv eher zurückgreifen. Auf jeden Fall sollten die Schulen ebenso wie bei den Kontrollstrategien den Schülerinnen und Schülern bei der Aufklärung über und der Entwicklung von Strategien helfen, die dem Lernen besonders förderlich sind. Diese Strategien werden den Schülerinnen und Schülern nicht nur während ihrer Schulausbildung zugute kommen, sondern wahrscheinlich mehr noch in ihrem späteren Leben, wenn sie beim Lernen auf weniger Hilfe von außen zählen können. Aber auch hier wiederum sollten etwaige Schlussfolgerungen nur in enger Verbindung mit dem kulturellen und bildungsspezifischen Kontext der jeweiligen Länder gezogen werden.

\section{Kooperative und wettbewerbsorientierte Lernformen}

Lernen im Erwachsenenalter findet überwiegend in Situationen statt, in denen Menschen zusammenarbeiten und aufeinander angewiesen sind. Im formalen Bildungswesen hingegen, insbesondere auf der Sekundar- und Tertiärstufe, findet das Lernen oft isoliert im Rahmen der Vorbereitung auf wettbewerbsorientierte Prüfungssituationen statt. Wenn zwischen kooperativem und wettbewerbsorientiertem Lernen auch zuweilen ein Konflikt bestehen kann, kann doch die eine wie die andere Form zu hohen Leistungen führen. Die Ergebnisse von PISA 2000 lassen vermuten, dass beide Lernformen zu höherer 
Lerneffizienz beitragen können, wenn sie als einander ergänzende Instrumente begriffen werden.

Ausgehend von den Angaben der Schülerinnen und Schüler wurden getrennte PISA-Indizes für das kooperative und das wettbewerbsorientierte Lernen konstruiert. Der Index des kooperativen Lernens wurde von den Antworten auf Fragen danach abgeleitet, ob die Schülerinnen und Schüler gern mit anderen Schülern zusammenarbeiten, ob sie anderen gern dabei helfen, in einer Gruppe gute Arbeit zu leisten, ob sie am meisten lernen, wenn sie mit anderen Schülern zusammenarbeiten, und ob sie die beste Arbeit leisten, wenn sie mit anderen Schülern zusammenarbeiten. Der Index des wettbewerbsorientierten Lernens wurde von den Antworten der Schülerinnen und Schüler auf Fragen danach abgeleitet, ob sie gerne versuchen, besser zu sein als andere Schülerinnen und Schüler, gerne in irgendeinem Bereich der/die Beste wären, gute Arbeit leisten, wenn sie versuchen, besser als andere zu sein, bzw. schneller lernen, wenn sie versuchen, besser zu sein als die anderen. Dabei ist zu beachten, dass die Schülerinnen und Schüler die Möglichkeit hatten, auf jeden der beiden Fragenkomplexe unabhängig voneinander positiv oder negativ zu antworten. Die Indizes wurden so konstruiert, dass die durchschnittliche Punktzahl in allen Ländern 0 und die Standardabweichung 1 beträgt (Angaben zur Definition der Indizes und Hinweise auf die begrifflichen Grundlagen finden sich in Anhang A1).

Die Mittelwerte auf diesen Indizes sind in Abbildung 4.7 dargestellt, weitere Informationen hierzu befinden sich in den Tabellen 4.8 und 4.9. Die Schülerinnen und Schüler in Dänemark, Neuseeland, Portugal und den Vereinigten Staaten erzielen hohe Werte auf dem Index für kooperatives Lernen. Hingegen stehen die koreanischen Schülerinnen und Schüler sowohl dem kooperativen als auch dem wettbewerbsorientierten Lernen deutlich negativ gegenüber, wenn diese negative Einstellung auch im letzteren Fall weniger ausgeprägt ist.

Sowohl das wettbewerbsorientierte als auch das kooperative Lernen beeinflussen die Punktzahl auf der Gesamtskala Lesekompetenz tendenziell positiv, wenngleich diese Relation im ersteren Fall ausgeprägter ist als im letzteren. In den OECD-Ländern insgesamt trennen durchschnittlich 23 Punkte auf der Gesamtskala Lesekompetenz das oberste vom untersten Quartil auf dem Index des kooperativen Lernens, wobei die Differenz in 20 von 25 Ländern statistisch signifikant ist. Beim wettbewerbsorientierten Lernen beträgt die durchschnittliche Differenz 33 Punkte und ist in 20 Ländern statistisch signifikant.

Diejenigen Schülerinnen und Schüler, die kooperatives Lernen stärker betonen, erzielen vergleichsweise höhere Leistungen als die, bei denen dies nicht der Fall ist. Aber auch diejenigen, denen eher wettbewerbsorientiertes Lernen zusagt, sind tendenziell leistungsstärker. Dahinter verbirgt sich eine allgemeine Tendenz, nämlich die, dass sich Schülerinnen und Schüler, die für kooperatives
In mehreren Ländern geben die Schülerinnen und Schüler an, dass sie sich sowohl kooperativer als auch wettbewerbsorientierten Lernformen bedienen, ...

... wobei in beiden Fällen ein positiver Zusammenhang mit den Leistungen besteht, ...

... was den Schluss nahe legt, dass aktiv Lernende sich je nach Bedarf der einen oder der anderen Strategie bedienen. 
KAPITEL 4 Ergebnisse zum Lernen allgemein

Abbildung 4.7

Kooperatives und wettbewerbsorientiertes Lernen

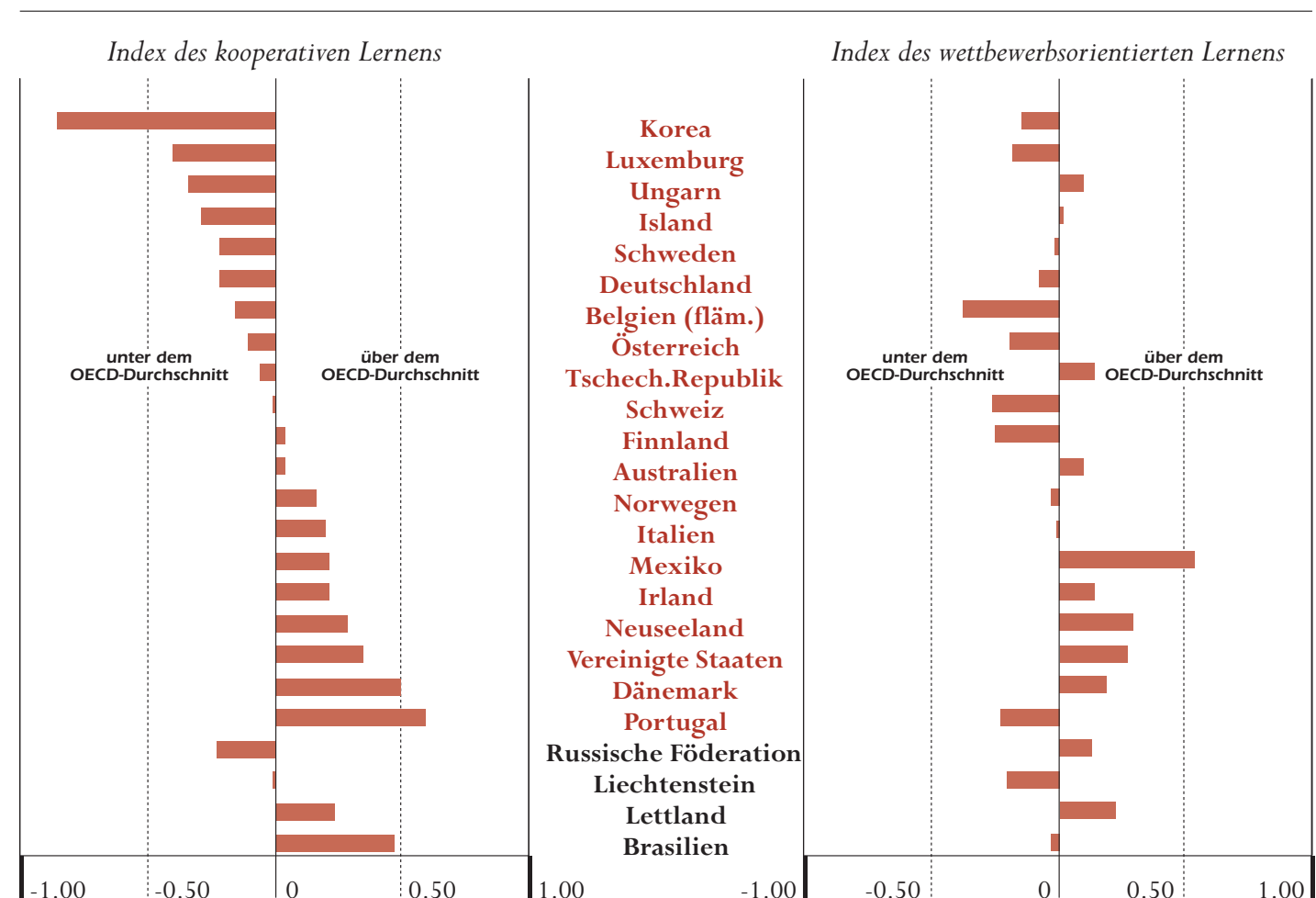

Anmerkung: Zur Definition der Indizes siehe Anhang A1

Quelle: OECD PISA Datenbank, 2001. Tabellen 4.8 und 4.9.

Lernen sind, auch bei wettbewerbsorientiertem Lernen wohl fühlen, und möglicherweise haben sie prinzipiell eine positive Einstellung dem Lernen gegenüber. Dieser Befund lässt vermuten, dass sich aktiv Lernende je nach Kontext beider Strategien bedienen, statt sich auf eine einzige zu beschränken, die in einer gegebenen Situation vielleicht nicht die beste ist. Es bedarf weiterer Forschungsarbeiten, um diese Aspekte eingehender zu untersuchen.

\section{Computer als Hilfsmittel zum Lernen}

Computerfertigkeiten sind heutzutage unentbehrlich.
Die Volkswirtschaften der OECD-Länder hängen zunehmend von den technologischen Kenntnissen und Fertigkeiten der Erwerbsbevölkerung ab. Schülerinnen und Schülern, die nur wenig oder gar keinen Zugang zu Computern und Informationstechnologie haben, dürfte es schwer fallen, sich problemlos in den modernen Arbeitsmarkt einzugliedern.

Im Rahmen von PISA 2000 wurden drei Aspekte im Hinblick auf den Umgang 15-jähriger Schülerinnen und Schüler mit Computern untersucht, nämlich 
ihr Interesse an Computern, die Selbsteinschätzung ihrer Einstellung zu und ihrer Fähigkeit zum Umgang mit Computern sowie die Benutzung von und die Erfahrungen mit Computern. Diese Erhebung stellte eine internationale Option dar, von der 20 der insgesamt 32 Teilnehmerländer Gebrauch machten ${ }^{15}$.

Im Durchschnitt der 16 befragten OECD-Länder erklären 65\% der Schülerinnen und Schüler, dass sie einen Computer benutzen, weil sie sehr daran interessiert sind, und dieser Prozentsatz liegt in keinem Land unter 50\% (Daten abrufbar unter www.pisa.oecd.org). Durchschnittlich 60\% der Schülerinnen und Schüler bezeichnen es als sehr wichtig, mit dem Computer zu arbeiten.

In Abbildung 4.8 werden die Länder anhand eines Index verglichen, der die verschiedenen Fragen nach dem Interesse an Computern zusammenfasst. Der Index wurde so konstruiert, dass die mittlere Punktzahl in allen Ländern 0 und die Standardabweichung 1 beträgt (Angaben zur Definition des Index und
Die Mehrheit der

Schülerinnen und Schüler

ist an der Benutzung von

Computern interessiert, ...
Abbildung 4.8

Interesse an Computern

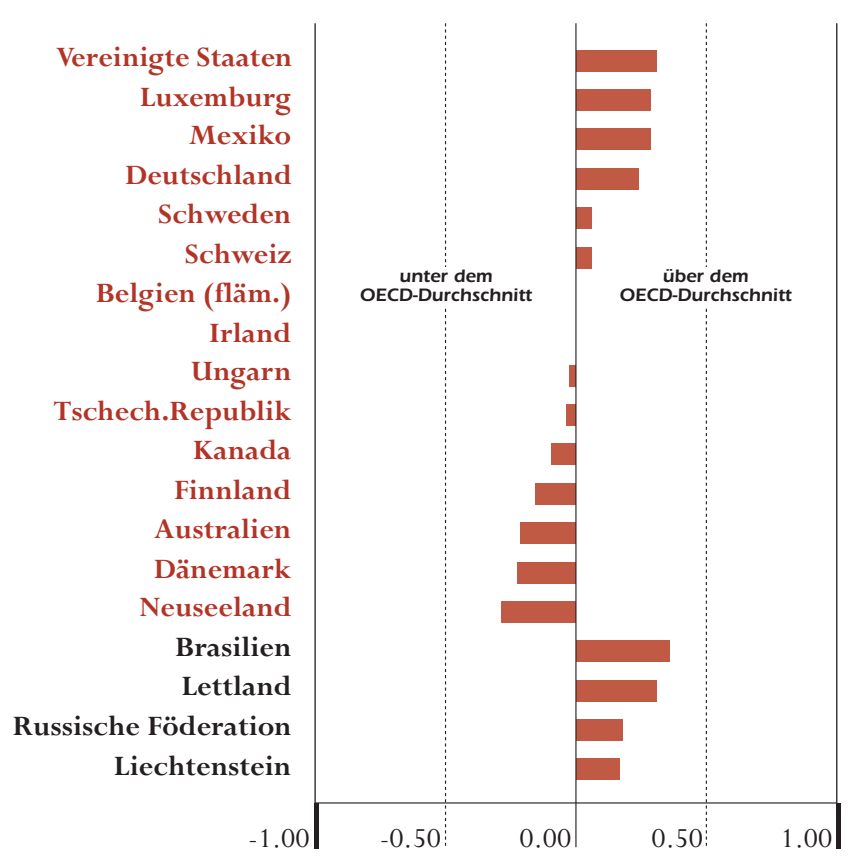

Anmerkung: Zur Definition des Index siehe Anhang A1.

Quelle: OECD PISA Datenbank, 2001. Tabelle 4.10. 
KAPITEL 4 Ergebnisse zum Lernen allgemein

... jedoch bestehen sowohl

Unterschiede zwischen den Ländern ...

wie auch zwischen den Geschlechtern.

Die Mehrheit der Schülerinnen und Schüler sagt von sich, dass sie gut im Umgang mit Computern

sind.
Hinweise auf die begrifflichen Grundlagen finden sich in Anhang A1). Ein positiver Indexwert bedeutet, dass Schülerinnen und Schüler häufig angeben, dass es sehr wichtig für sie ist, mit dem Computer zu arbeiten, dass es ihnen richtig Spaß macht, am Computer zu spielen oder zu arbeiten, dass sie den Computer benutzen, weil sie das sehr interessiert, und dass sie, wenn sie am Computer arbeiten, nicht merken, wie die Zeit vergeht. Der Abbildung sind die Mittelwerte auf dem Index des Interesses an Computern zu entnehmen.

Das größte Interesse an Computern bekunden die Schülerinnen und Schüler in Deutschland, Luxemburg, Mexiko und den Vereinigten Staaten; ihre Werte liegen um weit mehr als eine halbe Standardabweichung über denen der Schülerinnen und Schüler in Dänemark und Neuseeland, den Ländern mit dem niedrigsten Computerinteresse (Tabelle 4.10).

In den meisten Ländern zeigen Jungen ein sehr viel stärkeres Interesse an Computern als Mädchen, wobei die Vereinigten Staaten jedoch insofern eine Ausnahme bilden, als dort das Interesse an Computern bei Jungen und Mädchen gleich stark ist (Tabelle 4.10).

Das Interesse an Computern dürfte eine der Voraussetzungen für deren effektive Benutzung sein, darüber hinaus ist aber auch die Vertrautheit im Umgang mit Computern notwendig. In den OECD-Ländern insgesamt erklären durchschnittlich 69\% der Schülerinnen und Schüler, dass sie beim Schreiben eines Aufsatzes auf dem Computer gut oder sehr gut sind, und 55\% geben an, dass sie gut oder sehr gut wären, wenn sie eine Klassenarbeit am Computer schreiben müssten (Daten abrufbar unter www.pisa.oecd.org).

In Abbildung 4.9 werden die Länder anhand eines Index verglichen, der die verschiedenen Fragen hinsichtlich der Vertrautheit mit Computern und der Selbsteinschätzung der Fähigkeit im Umgang mit Computern zusammenfasst. Der Index wurde so konstruiert, dass die mittlere Punktzahl in allen Ländern 0 und die Standardabweichung 1 beträgt (Angaben zur Definition des Index und Hinweise auf die begrifflichen Grundlagen finden sich in Anhang A1). Ein positiver Indexwert bedeutet, dass die Schülerinnen und Schüler häufig angeben, dass es für sie sehr wichtig ist, mit dem Computer zu arbeiten, dass sie gut im Umgang mit dem Computer sind, dass sie beim Schreiben eines Aufsatzes auf dem Computer gut sind, dass sie gut wären, wenn sie eine Klassenarbeit am Computer schreiben müssten, und dass sie ihre Fähigkeiten im Umgang mit Computern im Vergleich zu anderen 15-Jährigen als besser beurteilen (Tabelle 4.11). 


\section{Abbildung 4.9}

Vertrautheit und Selbsteinschätzung der Fähigkeit im Umgang mit Computern

Index derVertrautheit und Selbsteinschätzung der Fähigkeit im Umgang mit Computern

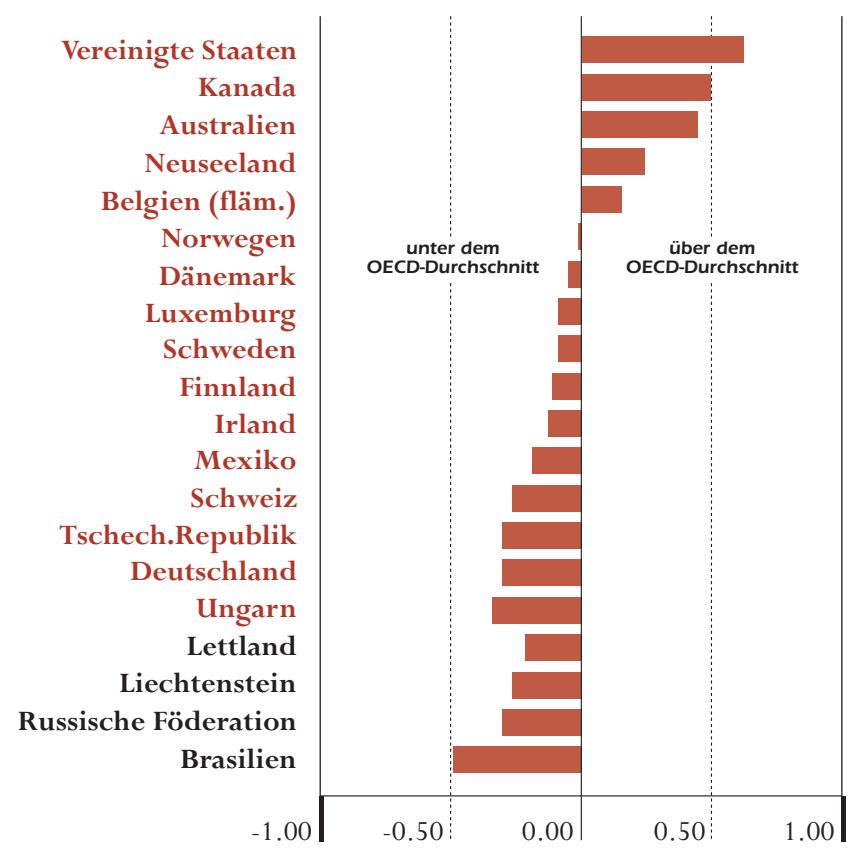

Anmerkung: Zur Definition des Index siehe Anhang A1.

Quelle: OECD PISA Datenbank, 2001. Tabelle 4.11.

Schülerinnen und Schüler mit höheren Werten auf dem Index des Computerinteresses erzielen tendenziell höhere Punktzahlen auf der Gesamtskala Lesekompetenz. Bei dieser Relation ist jedoch große Vorsicht geboten, da es nicht nur an Informationen über deren Richtung mangelt, sondern auch Drittfaktoren am Werk sein könnten wie beispielsweise die Möglichkeit, dass Schülerinnen und Schüler aus wohlhabenderen Familien oder solche, die zu Hause oder in der Schule über ein größeres Bildungskapital verfügen, auch besseren Zugang zu und mithin größeres Interesse an Computern haben.

Inwieweit besteht ein Zusammenhang zwischen dem Arbeiten am Computer und der Schule? In den OECD-Ländern insgesamt benutzen durchschnittlich $60 \%$ der Schülerinnen und Schüler fast jeden Tag oder zumindest mehrmals pro Woche einen Computer zu Hause, wobei dieser Prozentsatz allerdings von 21\% in Mexiko und 42\% bzw. 45\% in Ungarn und in der Tschechischen Republik bis zu über $70 \%$ in Australien, Kanada, Norwegen und Schweden reicht (Daten abrufbar unter www.pisa.oecd.org). Der Prozentsatz der Schülerinnen und Schüler, die fast jeden Tag oder zumindest mehrmals pro Woche einen Computer in der Schule benutzen, ist sehr viel niedriger und liegt im
Das Interesse an Computern geht mit höherer Lesekompetenz einher, doch ist dieser Zusammenhang kompliziert.

Die meisten Schülerinnen und Schüler arbeiten zu Hause und in der Schule regelmäfig am Computer... 
KAPITEL 4 Ergebnisse zum Lernen allgemein

... und in einigen Ländern benutzt fast die Hälfte jeden Tag das Internet.

Der Computer findet nur allmählich Eingang in das Lehren und Lernen, doch ist er für die 15-Jährigen heutzutage bereits ein sehr wichtiges Hilfsmittel.

Schülerinnen und Schüler, die nach Abschluss der Schule in der Lage sind, sich eigene Lernziele zu setzen in dem Bewusstsein, diese Ziele auch erreichen zu können, sind potentiell lebenslang Lernende,...

... und PISA zeigt einige der Lernstrategien auf, die den Schülerinnen und Schülern dabei helfen können, diese ziele zu erreichen.
OECD-Durchschnitt bei 36\%, wobei sich die Spannweite von 15\% in Deutschland bis zu 55\% oder mehr in Dänemark, Ungarn und dem Vereinigten Königreich erstreckt.

In den OECD-Ländern insgesamt benutzt durchschnittlich ein Viertel der Schülerinnen und Schüler jeden Tag und ein weiteres Viertel mindestens mehrmals pro Woche das Internet ${ }^{16}$. Am häufigsten wird das Internet in Kanada und Schweden benutzt, wo $46 \%$ bzw. $48 \%$ der Schülerinnen und Schüler sich fast jeden Tag über einen Computer Zugang zum Internet verschaffen. Durchschnittlich 42\% der Schülerinnen und Schüler benutzen einen Computer nahezu jeden Tag oder zumindest mehrmals pro Woche zur elektronischen Kommunikation, und 30\% benutzen ihn als Hilfsmittel zum Lernen für die Schule (Daten abrufbar unter www.pisa.oecd.org).

Wenn Computer auch nur schrittweise in den Lehr- und Lernprozess Eingang finden (Pelgrum und Anderson, 1999), legen die Ergebnisse von PISA 2000 doch den Schluss nahe, dass Computer die 15-Jährigen als Hilfsmittel bereits interessieren und dass viele von ihnen mit der Benutzung eines Computers für alltägliche Anwendungszwecke vertraut sind.

\section{Schlussbetrachtungen}

Es besteht auf breiter Basis Einigkeit darüber, dass lebensbegleitendes Lernen eine Notwendigkeit für jeden Einzelnen darstellt, und dieser Notwendigkeit sucht die heutige Bildungspolitik zunehmend Rechnung zu tragen. In diesem Zusammenhang ergeben sich wichtige Fragen hinsichtlich der Möglichkeiten und des Zugangs zu lebensbegleitendem Lernen jenseits des formalen Bildungswesens, aber auch danach, wie die Fähigkeiten des Einzelnen zur Nutzung dieser Möglichkeiten entwickelt werden können.

Die Entwicklung der Bereitschaft der Schülerinnen und Schüler zum Lernen wie auch der Fähigkeit zur Umsetzung dieser Lernbereitschaft in die Praxis, stellen wichtige schulische Ziele dar, die auch zunehmend in den nationalen Bildungspolitiken, insbesondere im Blick auf die Förderung lebensbegleitenden Lernens, artikuliert werden. Junge Menschen, die nach Abschluss der Schule in der Lage sind, sich selbständig Lernziele zu setzen in dem Bewusstsein, diese Ziele auch erreichen zu können, sind potentiell lebenslang Lernende. Motivation und Engagement können auch für die Lebensqualität der Schülerinnen und Schüler in der Adoleszenz wichtig sein und einen Einfluss darauf haben, ob sie später imstande sein werden, Möglichkeiten der weiterführenden Bildung bzw. Arbeitsmarktchancen erfolgreich zu nutzen.

Die PISA-Ergebnisse zeigen, dass diejenigen, die am meisten dazu neigen, Informationen zu memorieren, nicht immer die besten Ergebnisse erzielen, sondern dass diejenigen gut abschneiden, die die von ihnen zu lernenden Informationen verarbeiten bzw. elaborieren. Schließlich lässt sich mit Hilfe von PISA weder belegen, dass die kooperative der wettbewerbsorientierten 
Lernform überlegen ist, noch dass das Gegenteil der Fall ist. Die Befunde lassen vielmehr darauf schließen, dass die beiden Strategien zur Leistungsverbesserung als einander ergänzende Methoden angewendet werden können. Da insbesondere der Einsatz kooperativer Lernformen stark von der Art und Weise abhängt, wie die Lerngelegenheiten in den Schulen organisiert sind, kommt dieser Schlussfolgerung sowohl für die Bildungspolitik wie auch für die Bildungspraxis Bedeutung zu.

Angesichts der erheblichen Investitionen aller Länder in das Bildungswesen ist die Feststellung unbefriedigend, dass eine nicht unbedeutende Minderheit von Schülerinnen und Schülern in all diesen Ländern eine negative Haltung gegenüber dem Lernen an den Tag legt und Schulzufriedenheit vermissen lässt, auch wenn dies bis zu einem gewissen Grad auf das Alter der erfassten Population zurückzuführen sein dürfte. Eine negative Einstellung wirkt sich allem Anschein nach nicht nur negativ auf die schulischen Leistungen aus, sondern darüber hinaus ist bei Schülerinnen und Schülern, die Desinteresse am schulischen Lernen bekunden, auch die Wahrscheinlichkeit geringer, dass sie sich in ihrem späteren Leben auf inner- oder außerschulische Lernaktivitäten einlassen.

Die Zusammenhänge zwischen Einstellung, Motivation und Leistungen sind naturgemäß komplex und die in diesem Kapitel enthaltene Analyse kann nicht für sich in Anspruch nehmen, Kausalbeziehungen aufgedeckt zu haben. In der Tat könnte zwischen Leistung und Einstellung durchaus eine reziproke Beziehung bestehen, d.h. Schüler haben das gern, worin sie gut sind, und sie sind gut in dem, was ihnen gefällt. Schulen und Bildungssysteme sollten ihr Augenmerk sowohl auf die Leistung als auch auf die Schulzufriedenheit richten, und sie sollten nicht das Risiko eingehen, sich auf eine der beiden Komponenten zu konzentrieren in der Hoffnung, die andere werde sich dann von selbst ergeben. Wenn jedoch beide gegeben sind, ist eine verlässlichere Grundlage für einen produktiven und engagierten Prozess des lebensbegleitenden Lernens vorhanden.
Es muss mehr getan

werden, um eine positive

Lernhaltung der

schülerinnen und Schüler zu fördern, ...
... so dass sich hohe
Leistungen und
Schulzufriedenheit
gegenseitig verstärken. 
KAPITEL 4 Ergebnisse zum Lernen allgemein

\section{Anmerkungen}

1. Zu den 25 Ländern, die sich für die internationale Option entschieden, Zusatzfragen über die Art und Weise zu stellen, wie die Schülerinnen und Schüler an das Lernen herangehen und wie sie ihre Fähigkeiten selbst beurteilen, gehörten 20 OECDLänder. Der OECD-Durchschnitt bezieht sich allein auf diese Länder. Die Daten für Belgien gelten nur für die flämische Gemeinschaft.

2. Die Skala hat die Antwortkategorien „stimmt überhaupt nicht“, „stimmt eher nicht“, „stimmt eher“, „stimmt ganz genau“.

3. In allen Ländern außer Mexiko ist die Differenz bei der Lesekompetenz zwischen den Schülerinnen und Schülern im obersten und untersten Quartil des Index für das Leseinteresse statistisch signifikant. Weniger eindeutig ist jedoch der Wirkungszusammenhang zwischen zwei aufeinander folgenden Quartilen, wobei sich dieser in einigen Ländern von einem Quartil zum anderen sogar umkehrt.

4. Es handelt sich hierbei um eine Schätzung mit Hilfe der unstandardisierten Regressionskoeffizienten der Ergebnisse auf der Gesamtskala Lesekompetenz des PISA-Index für das Leseinteresse. Diese Koeffizienten geben an, welchen Unterschied bei der Lesekompetenz ein Anstieg um eine Einheit (d.h. eine Standardabweichung) auf dem PISA-Index für das Leseinteresse verursacht. Der Zusammenhang ist in allen Ländern statistisch signifikant. In den OECD-Ländern insgesamt ist dieser Zusammenhang für durchschnittlich 10\% der Gesamtvariation der Schülerleistungen verantwortlich.

5. Diese und die weiteren Skalen in diesem Abschnitt haben die Antwortkategorien „trifft nicht zu“, „trifft eher nicht zu“, „trifft eher zu“, „trifft zu“.

6. In allen Ländern außer Liechtenstein, Luxemburg, Mexiko und der Schweiz ist die Leistungsdifferenz auf der Skala für mathematische Grundbildung zwischen dem obersten und dem untersten Schülerquartil des Index für das Mathematikinteresse statistisch signifikant. Weniger eindeutig ist jedoch der Wirkungszusammenhang zwischen zwei aufeinander folgenden Quartilen, wobei sich dieser in einigen Ländern von einem zum anderen Quartil sogar umkehrt.

7. Diese Schülerinnen und Schüler stimmen eher bzw. ganz der Aussage zu ,ich lese nur, um die Informationen zu bekommen, die ich brauche“, wobei auf der entsprechenden Skala die Antwortkategorien „stimmt überhaupt nicht“, „stimmt eher nicht“, „stimmt eher“ und „stimmt ganz genau“ vorgesehen waren.

8. Die Skala hat die Antwortkategorien „stimmt überhaupt nicht“, „stimmt eher nicht“, „stimmt eher“ und „stimmt ganz genau“.

9. In allen Ländern ist die Leistungsdifferenz auf der Gesamtskala Lesekompetenz zwischen dem obersten und dem untersten Schülerquartil des Index der Freude am Lesen statistisch signifikant. Weniger eindeutig ist jedoch der Zusammenhang zwischen zwei aufeinander folgenden Quartilen, wobei sich dieser in einigen Ländern von einem zum anderen Quartil sogar umkehrt. Die unstandardisierten Regressionskoeffizienten sind ebenfalls in allen Ländern statistisch signifikant.

10. Die Antwortkategorien der Skala lauten: „ich lese nicht zum Vergnügen“, „bis zu 30 Minuten täglich“, „zwischen einer halben und 1 Stunde täglich“, „1 bis 2 Stunden täglich“ und „mehr als 2 Stunden täglich“.

11. Die Skala hat die Antwortkategorien: „stimmt überhaupt nicht“, „stimmt eher nicht“, „stimmt eher“ und „stimmt ganz genau“.

12. Die Skala hat die Antwortkategorien: „stimmt überhaupt nicht“, „stimmt eher nicht“, „stimmt eher“ und „stimmt ganz genau“.

13. In den OECD-Ländern insgesamt erklärt dieser Zusammenhang durchschnittlich $4,6 \%$ der Gesamtvarianz der Schülerleistungen. 
14. In Belgien (flämische Gemeinschaft), den Niederlanden und den Vereinigten Staaten sind die Differenzen statistisch nicht signifikant.

15. Zu den 20 Ländern, die sich für die internationale Option entschieden, Zusatzfragen nach der Vertrautheit mit Computern zu stellen, zählten 16 OECD-Länder. Der OECD-Durchschnitt bezieht sich allein auf diese Länder.

16. Die Antwortkategorien der Skalen in diesem Abschnitt lauten: „fast jeden Tag“, „mehrmals pro Woche“, „zwischen einmal pro Woche und einmal im Monat“, „weniger als einmal pro Monat“ und „nie“. 



\section{Kapitel}

5

GESCHLECHTSSPEZIFISCHE LEISTUNGSUNTERSCHIEDE

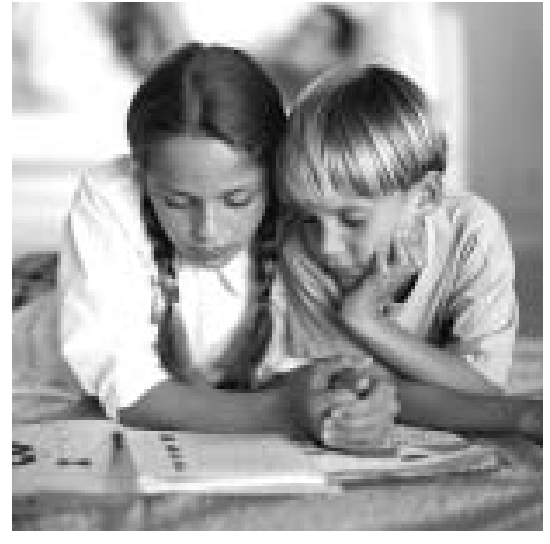


KAPITEL 5 Geschlechtsspezifische Leistungsunterschiede

\section{Einführung}

Alle Länder streben einen Abbau geschlechtsspezifischer Leistungsunterschiede an ...

... und waren diesbezüglich im Bereich der Bildungsabschlüsse generell erfolgreich, ...

... obwohl Frauen in einigen Studienfächern noch immer unterrepräsentiert sind.

Mädchen schneiden in einigen Bereichen mittlerweile so gut ab, dass die schwachen Leistungen der Jungen nun zunehmend Anlass zur Besorgnis geben.

Dieses Kapitel befasst sich mit geschlechtsspezifischen Unterschieden bei den Leistungen wie auch bei allgemeineren Aspekten des Lernens.
Da Bildung Auswirkungen auf die Arbeitsmarktbeteiligung, die berufliche Mobilität und die Lebensqualität hat, sind alle Länder nachdrücklich daran interessiert, Bildungsunterschiede zwischen Frauen und Männern abzubauen.

Die geschlechtsspezifischen Unterschiede in den Bildungsabschlüssen von Frauen und Männern konnten erheblich verringert werden. So weisen jüngere Frauen heute mit sehr viel höherer Wahrscheinlichkeit als die Frauen vor 30 Jahren einen Hochschulabschluss vor: In 18 der 29 OECD-Länder, für die Vergleichsdaten vorhanden sind, ist die Zahl der Frauen mit tertiärem Bildungsabschluss in der Altersgruppe der 25- bis 34-jährigen Frauen mehr als doppelt so hoch wie in der Altersgruppe der 55- bis 64-jährigen. Zudem ist bei den Hochschulabschlüssen der Frauenanteil in 17 der 25 OECD-Länder mit vergleichbaren Daten heute gleich hoch oder höher als der Anteil der Männer (OECD, 2001).

Dessen ungeachtet bestehen in gewissen Studiengängen nach wie vor starke geschlechtsspezifische Unterschiede bei den tertiären Abschlüssen. Der Frauenanteil unter den Hochschulabsolventen der Studienrichtungen Mathematik und Informatik liegt im Durchschnitt der OECD-Länder bei unter 31\%. In Österreich, der Tschechischen Republik, Ungarn, Island, den Niederlanden, Norwegen und der Schweiz liegt der Anteil zwischen 12\% und 19\%. Auch bei den Hochschulabschlussquoten in den Biowissenschaften und Physik besteht ein, wenn auch weniger stark ausgeprägter, Unterschied zwischen Frauen und Männern (OECD, 2001). In diesem Zusammenhang sei darauf hingewiesen, dass den Ergebnissen früherer internationaler Studien zufolge die auf unteren Bildungsstufen relativ geringen geschlechtsspezifischen Unterschiede zu Gunsten der männlichen Schüler bei den Bildungsergebnissen in Mathematik und Naturwissenschaften sich auf den höheren Bildungsstufen in vielen Ländern oft verstärken (vgl. Kasten 5.1).

Bislang galt die Besorgnis über geschlechtsspezifische Unterschiede überwiegend den schwächeren Leistungen von Mädchen. Da die Mädchen den Rückstand aber zunächst wettgemacht und die Jungen in vielen Bildungsbereichen sodann überflügelt haben, fällt die Aufmerksamkeit zunehmend auf Leistungsdefizite bei Jungen. Die politischen Entscheidungsträger sollten sich daher eingehend mit den geschlechtsspezifischen Leistungsunterschieden auseinandersetzen.

Dieses Kapitel rundet das Schülerleistungsprofil ab, mit dessen Erstellung in den vorangegangenen Kapiteln begonnen wurde, und zwar durch eine Untersuchung der geschlechtsspezifischen Leistungsunterschiede in den drei Grundbildungsbereichen sowie durch eine Beschreibung der geschlechtsspezifischen Unterschiede in Bezug auf das Interesse der Schülerinnen und Schüler bezüglich verschiedener Fächer, in Bezug auf ihre Motivation und ihr „Selbstkonzept“. In den weiteren Kapiteln dieses Berichts werden 
die Schülerleistungen im Hinblick auf den Hintergrund und das weitere Lernumfeld der Schülerinnen und Schüler, die personellen und finanziellen Bildungsinvestitionen der einzelnen Länder, ausgewählte Merkmale der nationalen Bildungssysteme sowie die grundlegende Beziehung zwischen den Ergebnissen auf Schulebene und dem familiären Hintergrund untersucht.

\section{Geschlechtsspezifische Unterschiede in der Lesekompetenz sowie} der mathematischen und naturwissenschaftlichen Grundbildung

Abbildung 5.1 zeigt die Unterschiede bei den Durchschnittsleistungen in den drei PISA-Untersuchungsbereichen. Für den Leistungsvergleich zwischen

\section{Abbildung 5.1}

Geschlechtsspezifische Unterschiede bei den Schülerleistungen

Unterschiede bei den Ergebnissen auf den PISA-Skalen

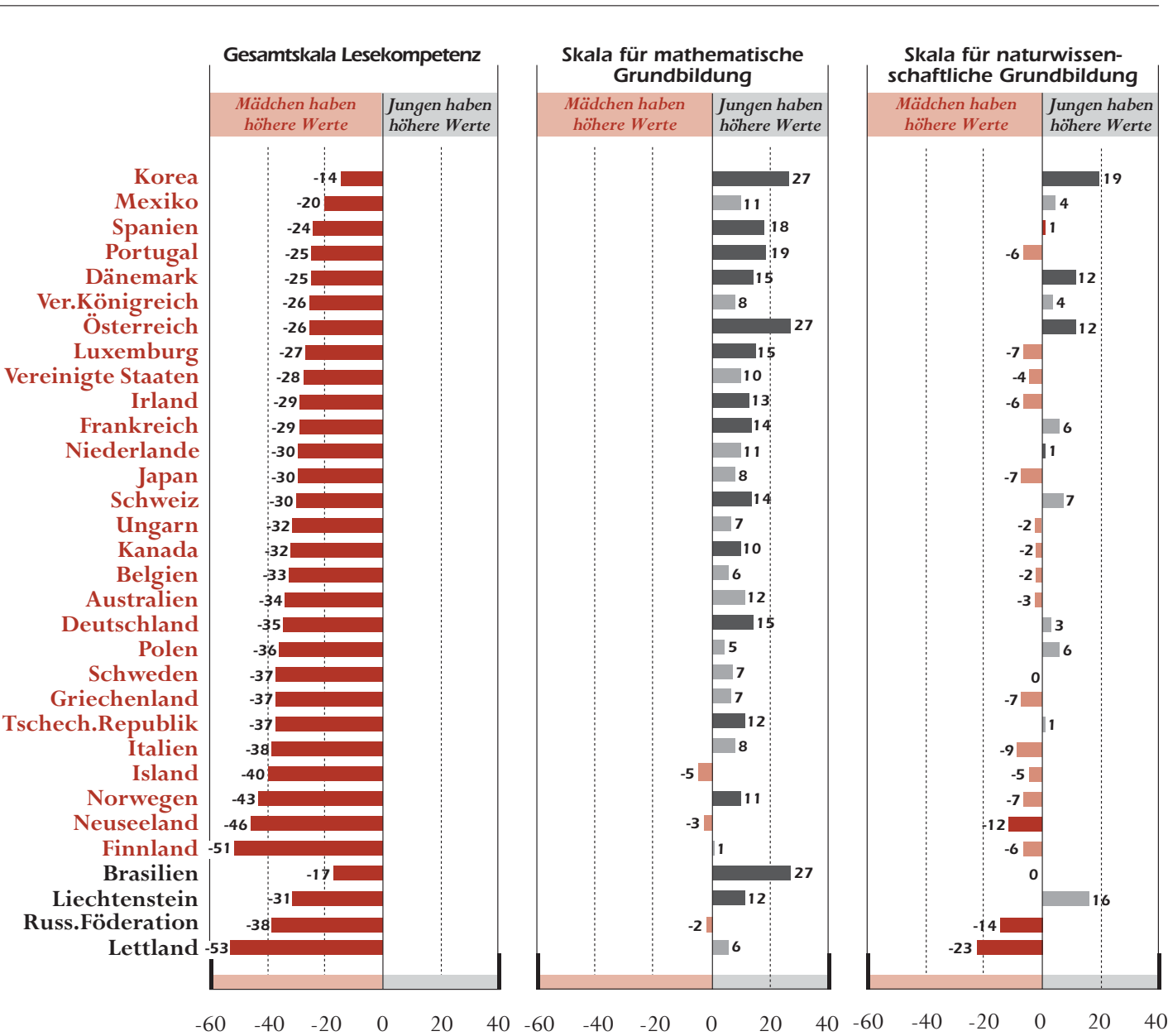

Anmerkung: Statistisch signifikante Unterschiede sind in schwarz und rot gekennzeichnet.

Quelle: OECD PISA Datenbank, 2001. Tabelle 5.1a. 
KAPITEL 5 Geschlechtsspezifische Leistungsunterschiede

Jungen und Mädchen wird die gleiche Skala wie die für den Leistungsvergleich zwischen den einzelnen Ländern in Kapitel 2 und 3 herangezogen. Auf dieser Skala liegen etwa zwei Drittel der 15-Jährigen im OECD-Raum in einem Bereich von plus/minus 100 Punkten von der mittleren Punktzahl, rund 50 Punkte trennen die Länder mit dem fünfthöchsten und fünftniedrigsten Ergebnis auf der Gesamtskala Lesekompetenz, und eine Kompetenzstufe entspricht knapp über 70 Punkten. Wie in Kapitel 2 und 3 ist auch hier zu berücksichtigen, dass sich hinter der unterschiedlichen mittleren Punktzahl von Jungen und Mädchen in diesem Kapitel eine erhebliche Varianz der geschlechtsspezifischen Unterschiede zwischen den verschiedenen Bildungsprogrammen, Schulen oder Schülertypen verbergen kann.

Kasten 5.1 Veränderung geschlechtsspezifischer Leistungsunterschiede in Mathematik und Naturwissenschaften mit zunehmendem Schüleralter

1994/1995 ergab die 3. Internationale Mathematik- und Naturwissenschaftliche Studie der IEA (TIMSS) in nur 3 von 16 teilnehmenden Ländern (Japan, Korea und Niederlande) bei Schülerinnen und Schülern der 4. Klasse statistisch signifikante geschlechtsbezogene Unterschiede in Mathematik, wobei durchgehend Jungen die besseren Ergebnisse aufwiesen. Hingegen zeigte dieselbe Studie für die 8. Klasse in 6 derselben 16 OECD-Länder im Fach Mathematik statistisch signifikante Unterschiede zwischen Jungen und Mädchen, in allen Fällen zu Gunsten der Jungen. Schließlich wurden in derTIMSS-Studie für das letzte Schuljahr der Sekundarstufe II bei den Leistungen im Fach Mathematik in allen teilnehmenden OECD-Ländern, mit Ausnahme Ungarns und der Vereinigten Staaten, zwischen Schülerinnen und Schülern große und statistisch signifikante Unterschiede (wiederum alle zu Gunsten der männlichen Schüler) nachgewiesen. Ein ähnliches und sogar noch deutlicheres Bild ergab sich in den Naturwissenschaften (Beaton et al., 1996; Mullis et al., 1998).

Obwohl sich die auf beiden Klassenstufen untersuchten Schülergruppen nicht aus den gleichen Schülerinnen und Schülern zusammensetzten, lassen die Ergebnisse darauf schließen, dass die Unterschiede zwischen Jungen und Mädchen in Mathematik und Naturwissenschaften in vielen OECD-Ländern in den höheren Klassen ausgeprägter und weiter verbreitet sind.

Trotz dieses allgemeinen Trends hat TIMSS auch gezeigt, dass es einigen Ländern gelungen ist, die wachsenden geschlechtsspezifischen Unterschiede auf die höheren Klassenstufen zu begrenzen (OECD, 1996; OECD, 1997).

Mädchen verfügen über eine höhere

Lesekompetenz; Jungen

verzeichnen in der

Hälfte der Länder höhere

Mathematikergebnisse.
PISA 2000 zeigt ein mehr oder weniger konsistentes Muster der geschlechtsspezifischen Unterschiede zwischen den einzelnen Ländern: In allen Ländern erreichen Mädchen in der Lesekompetenz im Durchschnitt ein höheres Leistungsniveau als Jungen. Im Bereich der mathematischen Grundbildung bestehen in etwa der Hälfte der Länder statistisch signifikante Unterschiede, wobei durchgehend die Jungen die besseren Ergebnisse aufweisen. Im Bereich der naturwissenschaftlichen Grundbildung sind die Differenzen zwischen Jungen 
und Mädchen geringer, und das Muster der Unterschiede ist uneinheitlich. In 24 OECD-Ländern zeigen sich in den Naturwissenschaften keine statistisch signifikanten Leistungsunterschiede zwischen den Geschlechtern (Tabelle 5.1a).

Der Leistungsvorsprung der Mädchen beim Leseverständnis ist nicht nur überall feststellbar, sondern auch groß. Im Durchschnitt sind es 32 Punkte, d.h. fast eine halbe Kompetenzstufe. Der Wert ist generell höher, als die typische Differenz in der mittleren Punktzahl zwischen den einzelnen Ländern. In Mathematik ist der Durchschnittsabstand nur etwa ein Drittel so groß (11 Punkte zu Gunsten der Jungen). In den naturwissenschaftlichen Fächern halten sich die geschlechtsspezifischen Unterschiede zwischen den Ländern die Waage (Tabelle 5.1a).

Der in allen Ländern beobachtete erhebliche Vorsprung der Mädchen in der Lesekompetenz sowie die in vielen Ländern besseren Ergebnisse der Jungen in der mathematischen Grundbildung sind zum Teil vielleicht auch auf den allgemeinen gesellschaftlichen und kulturellen Hintergrund bzw. auf pädagogische Maßnahmen und Praktiken zurückzuführen. Die PISA-Ergebnisse legen jedoch auch die Vermutung nahe, dass die Länder bei der Beseitigung geschlechtsspezifischer Unterschiede unterschiedlich erfolgreich waren und dass Mädchen nach wie vor im Bereich Lesekompetenz und Jungen in Mathematik besser abschneiden.

Indessen ergibt sich aus den großen Differenzen bezüglich geschlechtsspezifischer Unterschiede zwischen den Ländern (der bei der Lesekompetenz von 25 Punkten oder weniger in Dänemark, Korea, Mexiko, Portugal und Spanien bis $\mathrm{zu}$ mehr als dem Doppelten in Finnland und Lettland, und in der mathematischen Grundbildung von statistisch nicht signifikanten Unterschieden in 15 OECD-Ländern bis zu 27 Punkten in Österreich, Brasilien und Korea reicht), dass die derzeit bestehenden Unterschiede nicht zwangsläufig aus Differenzen im Lernstil von Jungen und Mädchen resultieren. Derartige Unterschiede können abgebaut werden.

Einige Länder bieten ganz offenbar ein Lernumfeld, das beiden Geschlechtern gleichermaßen zugute kommt, was entweder unmittelbar auf bildungspolitische Maßnahmen oder auf einen günstigeren gesellschaftlichen Kontext zurückgeführt werden kann. Korea, und in geringerem Ausmaß auch Irland, Japan und dem Vereinigten Königreich gelingt es, bei der Lesekompetenz sowohl höhere durchschnittliche Punktwerte zu erreichen als auch die geschlechtsspezifischen Unterschiede in Grenzen zu halten. In der mathematischen Grundbildung erzielen Belgien, Finnland, Japan, Neuseeland und das Vereinigte Königreich einerseits hohe Durchschnittsleistungen und andererseits auch geringe geschlechtsspezifische Unterschiede (Tabelle 5.1a).
Der Vorsprung der Mädchen im Bereich Lesekompetenz ist grofs,...

... in einigen Ländern aber sehr viel größser als in anderen, ...

... was darauf hindeutet, dass geschlechtsspezifische Unterschiede nicht unvermeidlich sind.

Mehrere Länder vereinen geringe geschlechtsspezifische Ungleichheiten mit hohem Leistungsnivedu, ... 
KAPITEL 5 Geschlechtsspezifische Leistungsunterschiede

... einige leistungsstarke

Länder weisen indessen

auch mit die größten

geschlechtsspezifischen

unterschiede auf.

Mädchen liegen auf der Subskala "Reflektieren und Bewerten" der

Lesekompetenz am weitesten vorn

Bei den Erwachsenen scheinen die Frauen die

Männer beim

Leseverständnis deutlich überholt zu haben.

In Mathematik und Naturwissenschaften liegen Mädchen nicht so stark zurück wie es in TIMSS der Fall war,...
Gleichzeitig verzeichnet ein Teil der Länder mit den größten geschlechtsspezifischen Unterschieden hohe Gesamtergebnisse. In Finnland z.B. schneiden die Jungen bei der Lesekompetenz keineswegs schlecht ab - ihre Ergebnisse liegen weit über dem Durchschnitt aller von der PISAStudie erfassten Schüler, und in keinem anderen Land erreichen Jungen bessere Resultate -, vielmehr sind es die Mädchen, die außergewöhnlich gute Ergebnisse aufweisen: sie haben 18 Punkte Vorsprung vor dem Land mit der nächsthöchsten Punktzahl für Mädchen (Neuseeland).

Die Unterschiede in der Lesekompetenz zwischen Jungen und Mädchen sind zumeist ausgeprägter auf der Skala „Reflektieren und Bewerten“, also bei Aufgaben, die eine kritische Einschätzung erfordern und bei denen es darum geht, Texte mit eigenen Erfahrungen, Kenntnissen und Vorstellungen zu verknüpfen. Im Durchschnitt machen die geschlechtsspezifischen Unterschiede auf der Skala „Reflektieren und Bewerten“ 45 Punkte zu Gunsten der Mädchen aus, im Vergleich zu 29 Punkten auf der Skala „Textbezogenes Interpretieren“ und 24 Punkten auf der Skala „Informationen ermitteln“ (Tabelle 5.1b). In Finnland, dem Land mit den größten Abständen zwischen Jungen und Mädchen, zeigt sich bei den Mädchen mit 564 Punkten ein außergewöhnlich hoher Durchschnittswert auf der Skala "Reflektieren und Bewerten", während die Jungen mit lediglich 501 Punkten im OECD-Durchschnitt liegen. Diese Ergebnisse könnten mit der Art des Lesestoffs zusammenhängen, mit dem Jungen und Mädchen jeweils in Berührung kommen bzw. der von ihnen gewöhnlich bevorzugt wird (vgl. weiter unten).

Künftige PISA-Erhebungen werden auch der Frage nachgehen, inwieweit geschlechtsspezifische Leistungsunterschiede sich im Zeitablauf verändern. Einstweilen ergibt ein Vergleich der in der Internationalen Erhebung über Grad und Verteilung elementarer Grundqualifikationen Erwachsener (IALS) beobachteten geschlechtsspezifischen Unterschiede zwischen verschiedenen Altersstufen, dass Frauen sich immer mehr an die Spitze setzen. So erreichten beispielsweise bei den 56- bis 64-Jährigen Männer gegenüber Frauen auf der IALS-Dokumentenskala in 17 von 21 Ländern bessere Ergebnisse. Diese Diskrepanz wurde aber in der Gruppe der 16- bis 25-Jährigen ausgeglichen. Desgleichen lagen auf der IALS-Skala für Prosatexte in der Altersgruppe der 56- bis 64-Jährigen in der Mehrzahl der Länder die Männer im Vorderfeld, in der Altersgruppe der 16- bis 25-Jährigen lagen jedoch in allen außer zwei der IALS-Länder die Frauen vorn (OECD und Statistics Canada, 2000).

In naturwissenschaftlicher und mathematischer Grundbildung, wo Jungen in der Vergangenheit häufig besser abschnitten, sind die geschlechtsspezifischen Unterschiede meist sehr viel geringer ausgeprägt als die Unterschiede zu Gunsten der Mädchen bei der Lesekompetenz. In den Naturwissenschaften lässt sich kein eindeutiges Muster der geschlechtsspezifischen Unterschiede feststellen: Mädchen schneiden in Lettland, Neuseeland und der Russischen Föderation besser ab als Jungen, während in Österreich, Dänemark und 
Korea die Jungen vor den Mädchen liegen. In den übrigen Ländern sind die Differenzen statistisch nicht signifikant (Tabelle 5.1a). Hier unterscheiden sich die Ergebnisse recht deutlich von den Resultaten der 3. Internationalen Mathematik- und Naturwissenschaftlichen Studie (TIMSS) der IEA, wo in den Naturwissenschaften bei Schülern der 8. Klasse sehr viel größere geschlechtsspezifische Unterschiede festgestellt wurden und die Jungen fast durchweg die besseren Resultate aufwiesen.

Die unterschiedlichen Ergebnisse von PISA und TIMSS lassen sich zum Teil dadurch erklären, dass in PISA im Bereich naturwissenschaftliche Grundbildung den Biowissenschaften stärkeres Gewicht beigemessen wird als in TIMSS. Hierbei handelt es sich um einen Sektor, in dem Mädchen auch in der TIMSS-Studie zumeist gut abgeschnitten haben, in der im Vergleich zu PISA die Physik mehr im Vordergrund stand, in der Jungen in der Regel gute Ergebnisse erreichen. Zudem wurden in PISA naturwissenschaftliche Prozesse und die Anwendung von Wissen stärker betont. Schließlich hat vielleicht auch die Tatsache, dass PISA einen höheren Anteil offener und kontextbezogener Aufgaben enthält, bei denen Mädchen in der Regel besser abschneiden, und weniger Multiple-Choice-Aufgaben, bei denen Jungen zumeist besser abschneiden, zu der höheren Leistung der Mädchen beigetragen.

In allen Ländern zeigen sich in den drei inhaltlichen Bereichen Lesekompetenz, mathematische und naturwissenschaftliche Grundbildung in der Regel ähnliche geschlechtsspezifische Unterschiede, was den Schluss nahe legt, dass einige grundlegende Merkmale der Bildungssysteme und/oder Gesellschaften und Kulturen die gesamte schulische Laufbahn hindurch die geschlechtsspezifischen Leistungsunterschiede beeinflussen. Gleichwohl bestehen aber auch einige wesentliche Differenzen. Finnland z.B. weist die höchsten geschlechtsspezifischen Unterschiede auf der Gesamtskala Lesekompetenz auf (51 Punkte zu Gunsten der Mädchen), hingegen sind dort die Unterschiede zwischen Jungen und Mädchen auf der Skala für mathematische und naturwissenschaftliche Grundbildung gering. Umgekehrt ergeben sich für Korea die geringsten geschlechtsspezifischen Unterschiede bei der Lesekompetenz (14 Punkte zu Gunsten der Mädchen), während die Abstände zwischen Jungen und Mädchen in der mathematischen Grundbildung (27 Punkte zu Gunsten der Jungen) und in der naturwissenschaftlichen Grundbildung (19 Punkte zu Gunsten der Jungen) zu den größten im gesamten OECD-Raum gehören. Solche Abweichungen zwischen den verschiedenen Bildungsbereichen zeigen, dass diese Disparitäten durch die unterschiedlichen Lernerfahrungen der Schülerinnen und Schüler bedingt sind und somit durch Veränderungen der politischen Rahmenbedingungen abgebaut werden können.

\author{
... was sich u. U. durch \\ die Betonung der \\ Anwendung von Wissen \\ in PISA und der im \\ Zentrum stehenden \\ Inhalte erklären lässt.
}

In einigen Ländern sind die Leistungsunterschiede zwischen Jungen und Mädchen in den einzelnen Fächern unterschiedlich stark ausgeprägt, was darauf hindeutet, dass sich diese Differenzen aus den Lernerfahrungen erklären. 
KAPITEL 5 Geschlechtsspezifische Leistungsunterschiede

Abbildung 5.2

Der Anteil der Jungen und Mädchen bei den leistungsschwächsten Schülern auf der Gesamtskala Lesekompetenz

Prozentsätze der Jungen und Mädchen auf und unter Kompetenzstufe 1

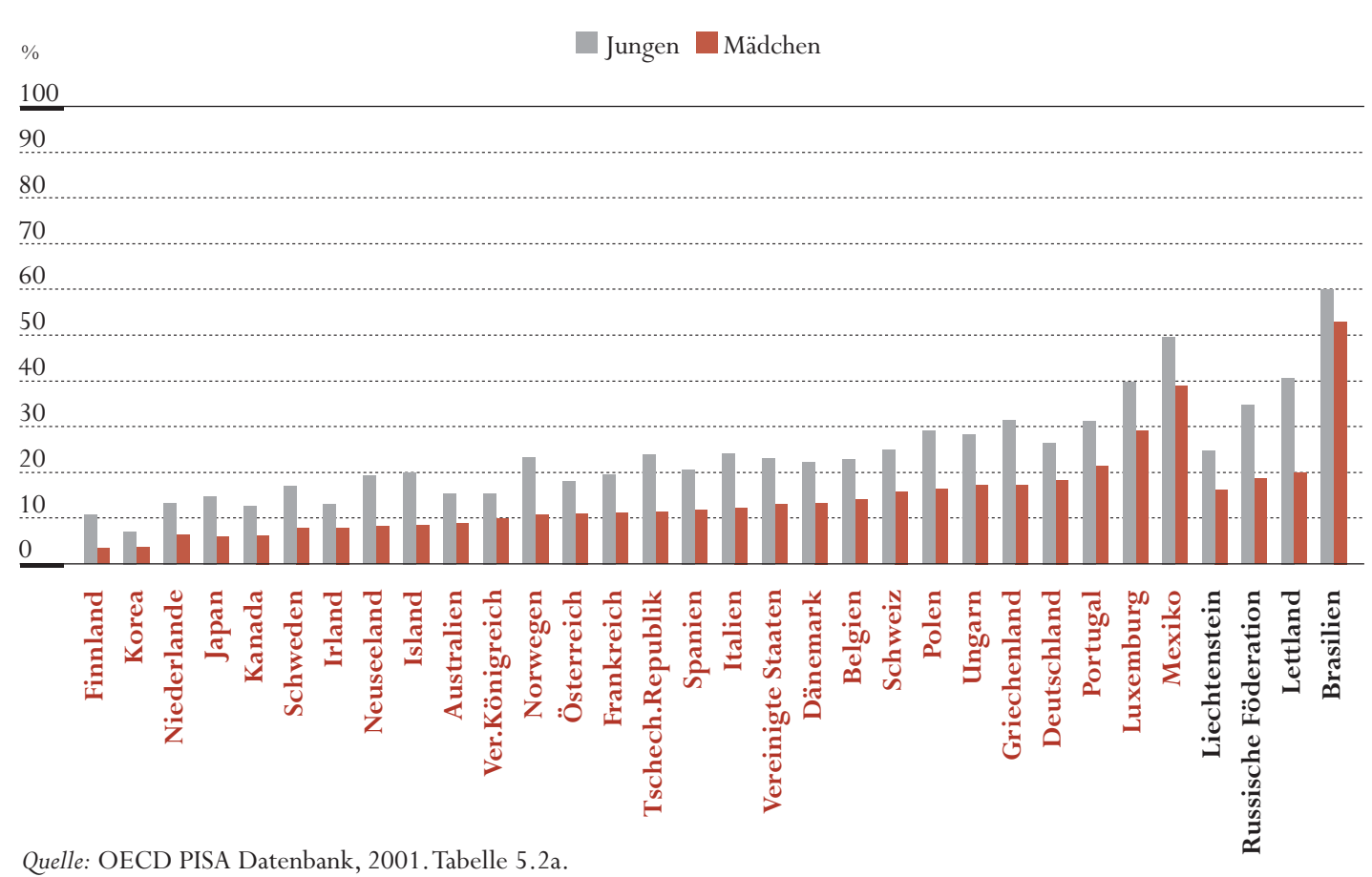

In allen Ländern gibt es

unter den

leistungsschwächsten

Lesern mehr Jungen als

Mädchen, ...
Die beträchtlichen geschlechtsspezifischen Unterschiede unter den leistungsschwächsten Schülern sollten den politischen Entscheidungsträgern Anlass zur Besorgnis geben (Abb. 5.2). In allen OECD-Ländern ist für Jungen die Wahrscheinlichkeit, zu den leistungsschwächsten Schülern zu zählen, also auf der Gesamtskala Lesekompetenz auf Leistungsstufe 1 und darunter zu liegen, höher als für Mädchen, wobei das Verhältnis zwischen Jungen und Mädchen auf dieser Stufe in den OECD-Ländern von 1,3 bis 3,5 reicht. So liegen in Kanada, Finnland, Japan und Korea 6\% oder weniger der Mädchen auf Leistungsstufe 1 oder darunter, gegenüber einem Anteil von 7 bis $14 \%$ bei den Jungen (Tabelle 5.2a). Selbst in Finnland, dem Land mit den besten Ergebnissen, liegen lediglich 3\% der Mädchen auf Leistungsstufe 1 oder darunter, im Vergleich zu 11\% der Jungen. 
Auf der Skala für mathematische Grundbildung schneiden Jungen im Allgemeinen besser ab als Mädchen. Ein Großteil dieser Unterschiede ist indessen auf die größeren Differenzen zu Gunsten der Jungen bei den leistungsstärkeren Schülern und nicht auf einen relativen Mangel an Jungen unter den leistungsschwächsten zurückzuführen. Bei den Schülerinnen und Schülern, deren Leistungen um mindestens 100 Punkte unter dem internationalen Durchschnitt der Skala für mathematische Grundbildung liegen (also Schülerinnen und Schüler, die meist nur einen einzelnen Verarbeitungsschritt vornehmen können, wie z.B. grundlegende mathematische Fakten oder Prozesse nachvollziehen oder simple Rechentechniken anwenden), ist das Verhältnis zwischen Jungen und Mädchen mehr oder minder ausgewogen (Tabelle 5.2b). In 15 der teilnehmenden OECD-Länder hingegen ist die Wahrscheinlichkeit bei Jungen grösser, zu den leistungsstärksten Schülern zu gehören, deren Punktzahlen mehr als eine Standardabweichung über dem OECD-Durchschnitt liegen, während in keinem Land das Gegenteil der Fall ist.

Diese Ergebnisse zeigen, dass die schwachen Leistungen der Jungen eine ernste bildungspolitische Herausforderung darstellen, der besondere Aufmerksamkeit gewidmet werden sollte, um geschlechtsspezifische Unterschiede abzubauen und den Anteil der Schülerinnen und Schüler auf dem untersten Leistungsniveau zu verringern.

Die Selektions- und Autoselektionsmechanismen in stark gegliederten Bildungssystemen sind möglicherweise ein Faktor, der für die geschlechtsspezifischen Unterschiede mit verantwortlich ist. Mit Ausnahme Koreas sind Mädchen in den anspruchsvolleren Sekundarstufe-II-Programmen, die zur Hochschulreife hinführen, überrepräsentiert ${ }^{1}$. In den 16 Ländern, deren Bildungssysteme unterschiedliche Schulzweige aufweisen und die Angaben zur Schülerbeteiligung an den einzelnen Programmen machen, liegt der Anteil der Mädchen in hochschulorientierten Programmen um 8\% über dem der Jungen, in Polen beträgt der Abstand mehr als 20\% (Daten abrufbar unter www.pisa.oecd.org). Unter den Schülerinnen und Schülern von Zweigen, die zur Hochschulreife hinführen, sind die geschlechtsspezifischen Unterschiede im Bereich Lesekompetenz generell geringer (zu Gunsten der Mädchen), während sie tendenziell in der mathematischen Grundbildung (zu Gunsten der Jungen) im Vergleich zur Gesamtpopulation der 15-jährigen Schüler doppelt so groß sind.

\section{Geschlechtsspezifisch unterschiedliches Interesse an einzelnen Fachgebieten}

Abbildung 5.3a vergleicht das Leseinteresse von Schülerinnen und Schülern (Abszisse) mit den jeweiligen Leistungen auf der Gesamtskala Lesekompetenz (Ordinate). Der Index des Leseinteresses wird in Kapitel 4 beschrieben. Jedes Land ist in dieser Abbildung durch zwei Symbole repräsentiert: Die Symbole in grau zeigen den durchschnittlichen Index des Leseinteresses für Jungen und ihr mittleres Ergebnis auf der Gesamtskala Lesekompetenz. Die entsprechenden

\author{
... und in der \\ mathematischen \\ Grundbildung ist der \\ durchschnittliche \\ vorsprung der Jungen \\ offenbar auf die \\ herausragenden \\ Leistungen einiger \\ zurückzuführen.
}

Die schwachen Leistungen

bei 15-jährigen Jungen

sind deshalb eine wichtige

Angelegenheit.

Die geschlechts-

spezifischen

Unterschiede sind

möglicherweise auch

eine Folge der

Selektions- und

Autoselektionsmechanismen

in stark gegliederten

Bildungssystemen.

Jungen und Mädchen

unterscheiden sich auch hinsichtlich ihres

Interesses an einzelnen

Fachgebieten. 
KAPITEL 5 Geschlechtsspezifische Leistungsunterschiede

Abbildung 5.3a

Zusammenhang zwischen Leseinteresse und Leistungen auf der Gesamtskala Lesekompetenz bei Jungen und Mädchen

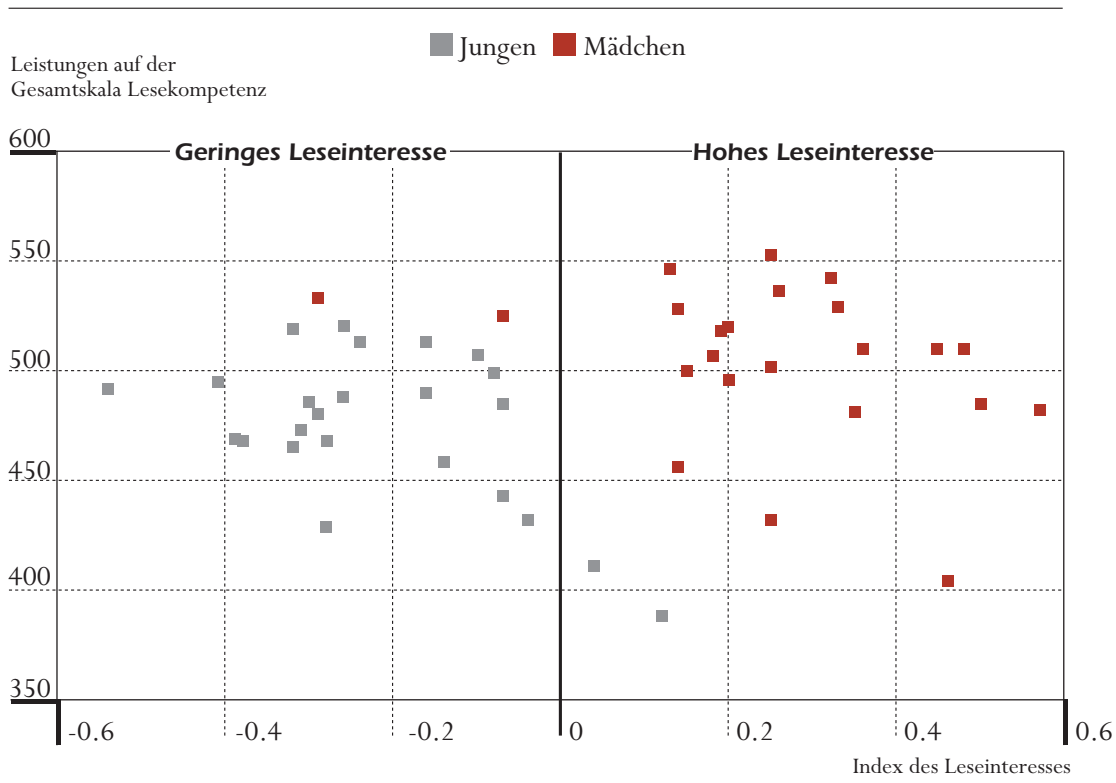

Zur Definition des Index siehe Anhang A1.

Anmerkung: Die Länder sind durch die Kästchen dargestellt.

Quelle: OECD PISA Datenbank, 2001. Tabelle 4.1.

Länderwerte für Mädchen sind rot angegeben. Das obere Ende der Ordinate stellt hohe Durchschnittsleistungen in der Lesekompetenz dar. Am rechten Ende der Abszisse geben Schülerinnen und Schüler im Durchschnitt häufiger an, in ihrer Freizeit zu lesen, dass Lesen für sie persönlich wichtig ist, dass sie das Lesen nicht gerne aufgeben würden, weil es Spaß macht, und dass sie beim Lesen manchmal alles um sich herum vergessen. Abbildung $5.3 \mathrm{~b}$ zeigt die Struktur des Zusammenhangs zwischen dem Mathematikinteresse und den Leistungen auf der Skala für mathematische Grundbildung.

Die geschlechtsspezifischen

Unterschiede in den

fachbezogenen Interessen spiegeln deutlich die in den Leistungen wider, ...
Die Abbildungen 5.3a und b zeigen deutlich, dass Mädchen in der Regel ein stärkeres Interesse am Lesen bekunden als Jungen, während bei Mathematik das Gegenteil der Fall ist. Die geschlechtsspezifischen Leistungsunterschiede in Lesekompetenz und mathematischer Grundbildung spiegeln sich also deutlich in dem unterschiedlichen Interesse, das Schülerinnen und Schüler den jeweiligen Fachgebieten entgegenbringen, wider. So kann gesagt werden, dass dieser Gesamtzusammenhang für alle Länder in Bezug auf das Lesen und für die Mehrzahl der Länder im Bereich Mathematik nachweisbar ist (Tabellen 4.1 und 4.2), wobei nur in Portugal Mädchen ein höheres Interesse an Mathematik angeben als Jungen. 
Abbildung 5.3b

Zusammenhang zwischen Mathematikinteresse und Leistungen auf der Skala für mathematische Grundbildung bei Jungen und Mädchen

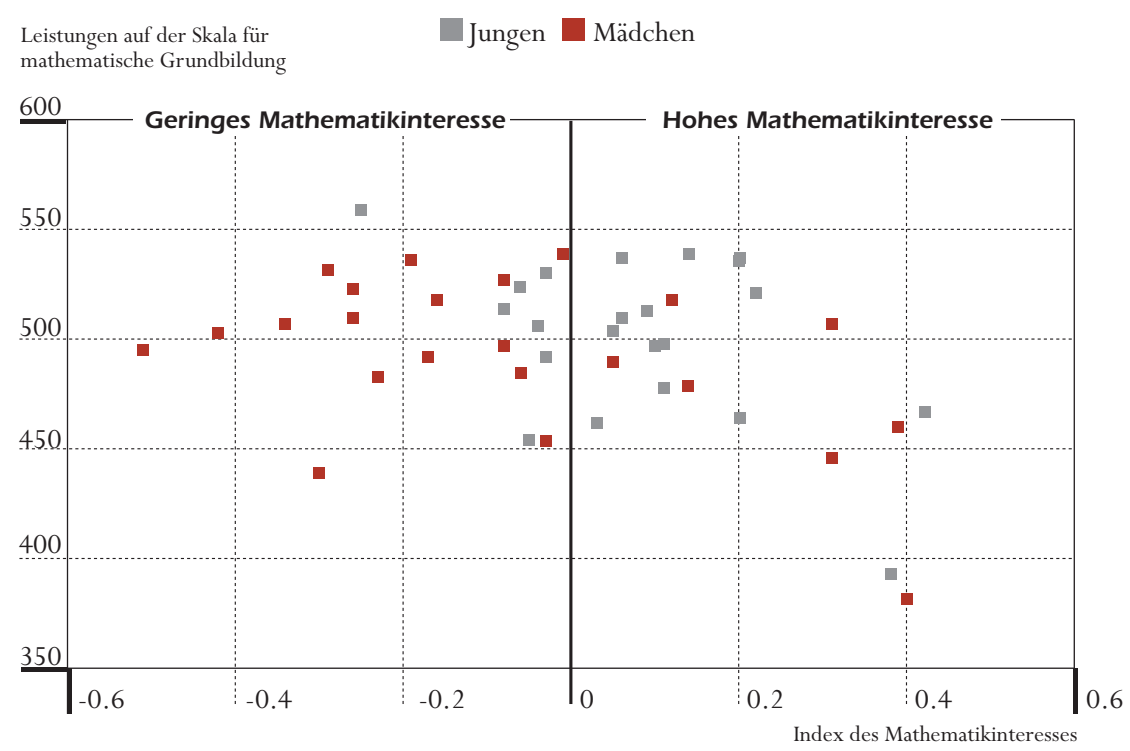

Zur Definition des Index siehe Anhang A1.

Anmerkung: Die Länder sind durch die Kästchen dargestellt.

Quelle: OECD PISA Datenbank, 2001. Tabelle 4.2.

Aus dem vorliegenden Datenmaterial lässt sich kein Kausalzusammenhang dieser Beziehungen ableiten, die auch insofern komplexer sein dürften, als sich Interesse und Leistungen vermutlich gegenseitig verstärken. Dennoch ist die Tatsache, dass fachspezifische Interessen zwischen Jungen und Mädchen sich durchgängig unterscheiden und ein so enger Zusammenhang mit dem Lernerfolg in den jeweiligen Fächern besteht, an sich schon für die Konzipierung bildungspolitischer Maßnahmen von Bedeutung. Dies belegt die Ungleichheiten zwischen den Geschlechtern in Bezug auf die Effektivität von Schule und Gesellschaft bei der Förderung von Motivation und Interesse an den verschiedenen Fachgebieten. Die Ergebnisse deuten ferner auf mögliche Konsequenzen hin, die diese Ungleichheiten für die Bildungserträge haben.

\section{Geschlechtsspezifische Unterschiede in der Freude am Lesen}

Geschlechtsspezifische Unterschiede zu Gunsten der Mädchen zeigen sich auch bei der Freude am Lesen von Schülerinnen und Schülern, die von PISA daran gemessen wurde, wie oft Schülerinnen und Schüler nach ihren eigenen Angaben zum eigenen Vergnügen lesen, gerne mit anderen Leuten über Bücher sprechen, Buchhandlungen und Bibliotheken aufsuchen und welche allgemeine Bedeutung sie der Lektüre beimessen.
... was darauf hindeutet, dass Jungen und Mädchen in Schule und Gesellschaft nicht gleichermafsen motiviert werden.

Mädchen haben mehr Freude am Lesen,... 
KAPITEL 5 Geschlechtsspezifische Leistungsunterschiede

Abbildung 5.4

Geschlechtsspezifische Unterschiede in Bezug auf die Freude am Lesen - Einstellungen dem Lesen gegenüber Verteilung der mittleren Prozentsätze der Jungen und Mädchen, die die folgenden Aussagen mit „stimmt eher“ oder „stimmt ganz genau“ beantworten

Ländermittelwerte Ländermittelwerte \OECD-Durchschnitt Prozentsatz \ OECD-Durchschnitt Prozentsatz Prozentsatz der Jungen Prozentsatz der Mädchen der Jungen (Standardfehler) der Mädchen (Standardfehler)

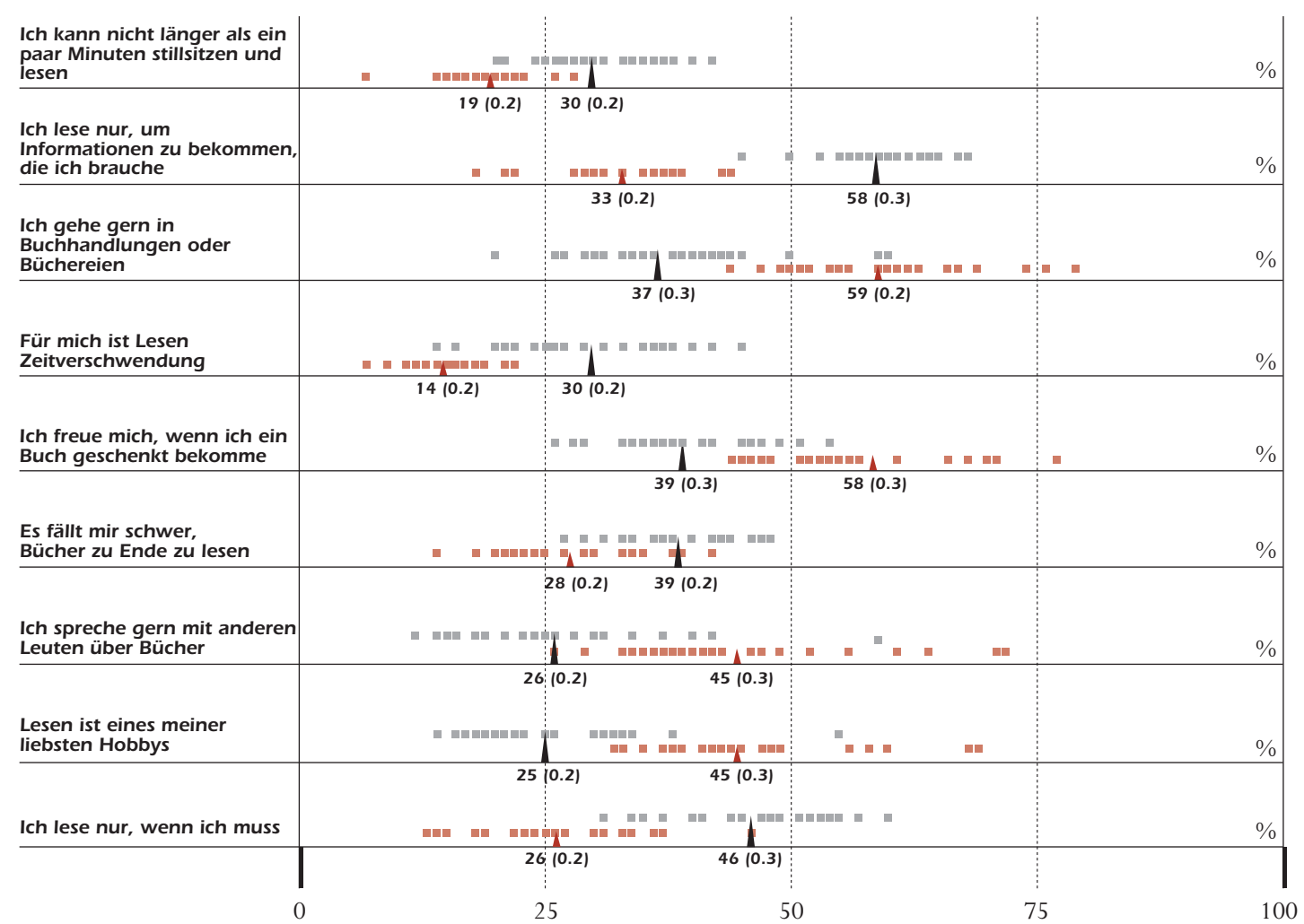

Anmerkung: Die Länder sind dargestellt durch die Quadrate.

Quelle: OECD PISA Datenbank, 2001. Daten abrufbar unter www.pisa.oecd.org.

... wobei Jungen 15-jährige Jungen scheinen nur in begrenztem Maß eine über das verlangte tendenziell nurlesen, Ausmaß hinausgehende Lesebereitschaft aufzubringen (Abb. 5.4). Im wenn sie müssen ... D Durchschnitt der OECD-Länder lesen 46\% der Jungen nur, wenn sie müssen, was bei Mädchen nur für 26\% gilt (Daten für die einzelnen Länder abrufbar unter www.pisa.oecd.org). Außerdem geben 58\% der Jungen (gegenüber 33\% der Mädchen) an, dass sie nur lesen, um die von ihnen benötigten Informationen zu bekommen, und dieserWert steigt in derTschechischen Republik, Deutschland, Irland und Mexiko sogar auf über zwei Drittel der Jungen. Analog hierzu geben 45\% der Mädchen an, Lesen sei eines ihrer liebsten Hobbys, und 45\% erklären, gerne mit anderen Leuten über Bücher zu sprechen, während dies bei den Jungen lediglich für 25\% der Fall ist (Daten für die einzelnen Länder abrufbar unter www.pisa.oecd.org). 
Abbildung 5.5

Geschlechtsspezifische Unterschiede in Bezug auf die Freude am Lesen - mit „Lesen zum Vergnügen“ verbrachte Zeit

Prozentsätze der Jungen und Mädchen, die täglich mehr als 30 Minuten zum Vergnügen lesen

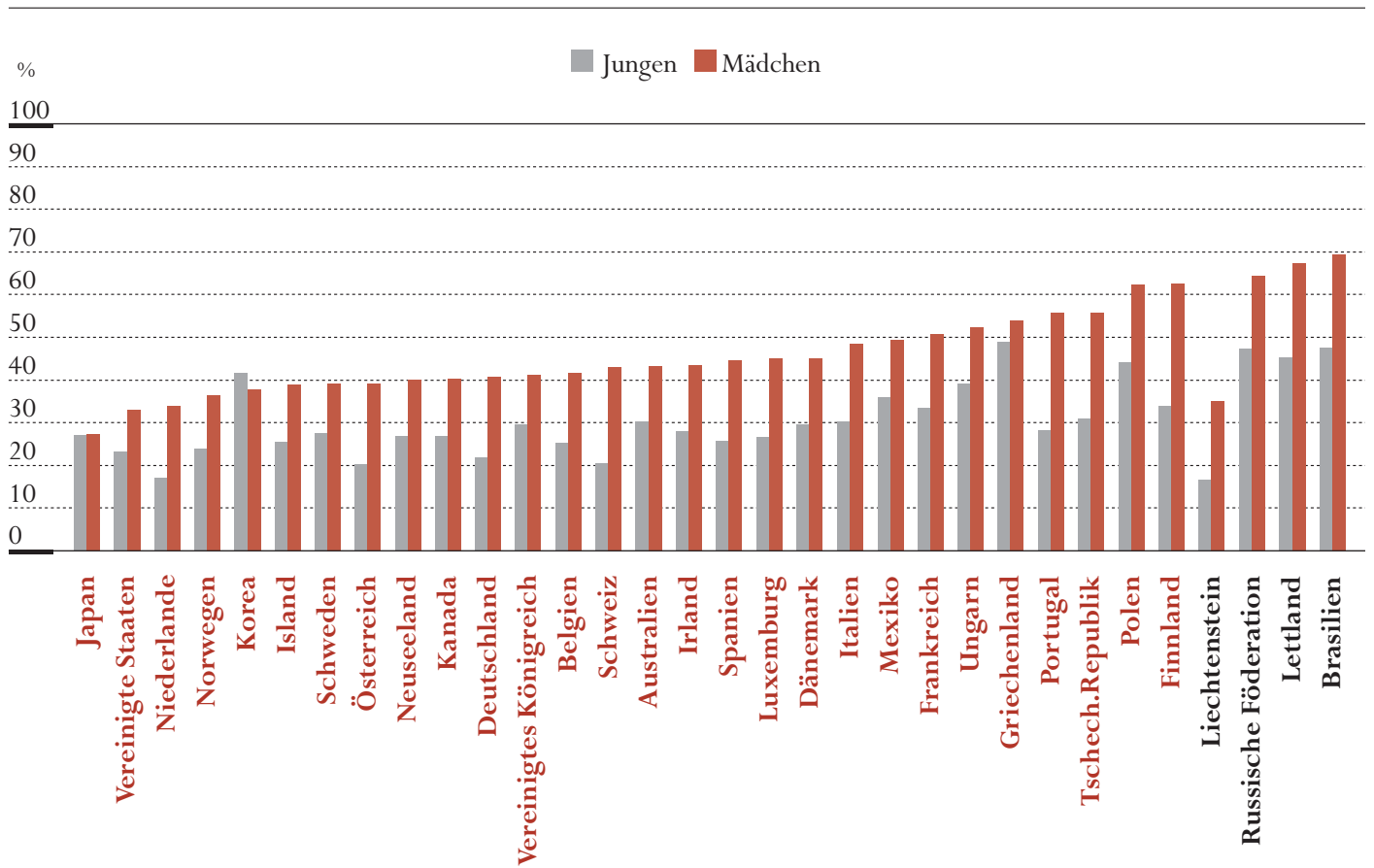

Quelle: OECD PISA Datenbank, 2001. Tabelle 5.3.

Jungen wenden auch wesentlich weniger Zeit für das Lesen zu ihrem Vergnügen auf als Mädchen. Im Durchschnitt der OECD-Länder geben 45\% der Mädchen an, jeden Tag länger als 30 Minuten zum Vergnügen zu lesen (die Anteile reichen von 27\% der Mädchen in Japan bis zu mehr als dem doppelten Prozentsatz in derTschechischen Republik, Finnland, Polen und Portugal). Der entsprechende Wert liegt für Jungen bei 30\%, wobei sich der Anteil in einer Bandbreite von 20\% oder weniger in Österreich, den Niederlanden und der Schweiz bis zu über 40\% in Griechenland, Korea und Polen bewegt (Abb. 5.5 und Tabelle 5.3).

Obwohl diese Ergebnisse keine Herstellung von Kausalzusammenhängen erlauben, zeigen sie doch, dass die unterschiedlichen Lesegewohnheiten von Mädchen und Jungen weitreichende Konsequenzen für den Lernprozess haben können, auf die näher eingegangen werden muss, um innerhalb der Schulsysteme gleiche Bedingungen für Jungen und Mädchen zu erreichen.
... und die Mädchen

lesen eher zum

Vergnügen.

Die unterschiedlichen

Lesegewohnheiten von

Jungen und Mädchen

können weitreichende

Konsequenzen haben. 
KAPITEL 5 Geschlechtsspezifische Leistungsunterschiede

Abbildung 5.6

Geschlechtsspezifische Unterschiede in Bezug auf die Freude am Lesen - Vielfalt des Lesestoffs Mittlere Prozentsätze der Jungen und Mädchen, die die nachstehenden Arten von Lesestoff mehrmals pro Monat oder proWoche lesen

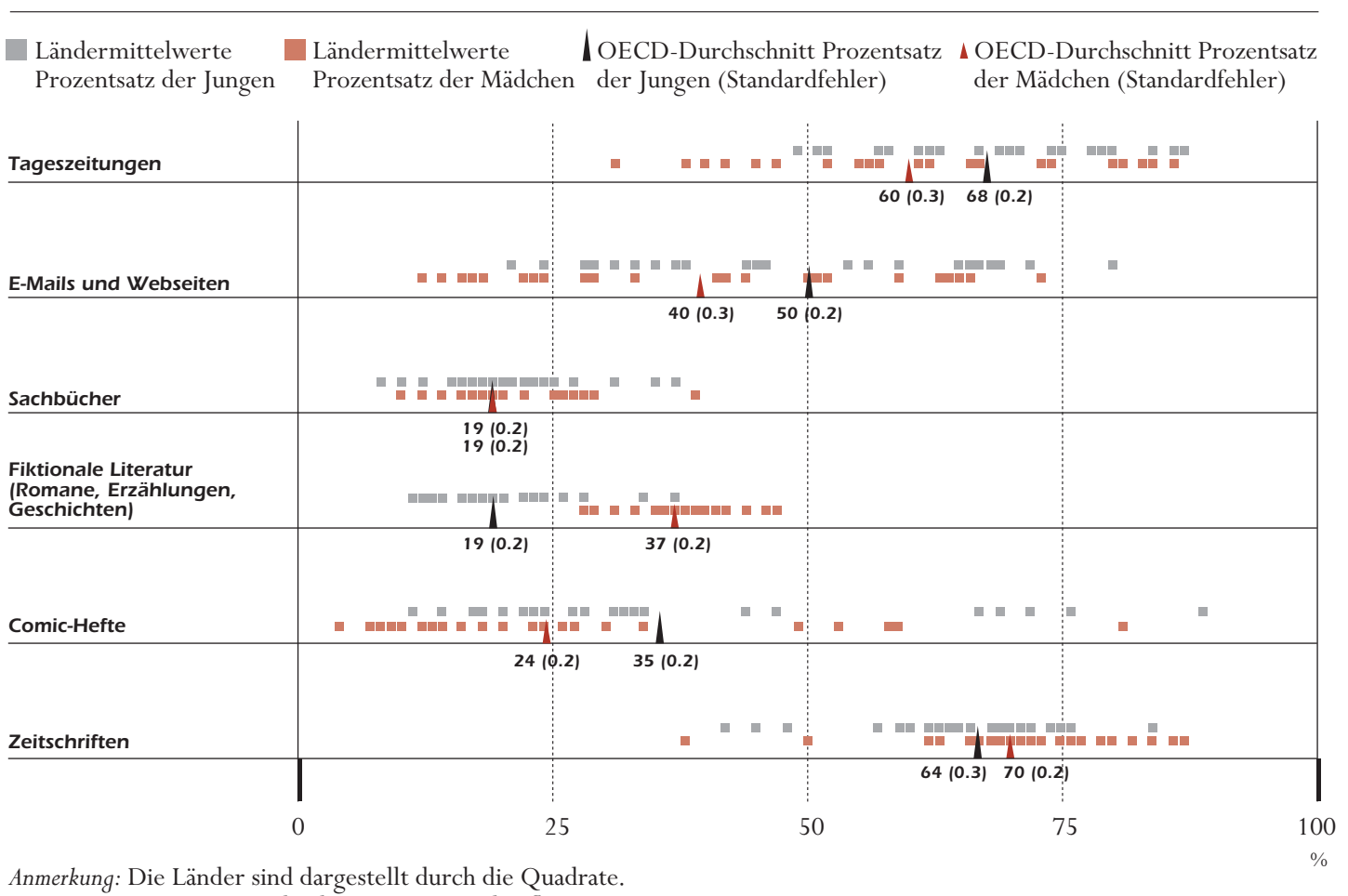

Quelle: OECD PISA Datenbank, 2001. Daten abrufbar unter www.pisa.oecd.org.

Jungen und Mädchen haben auch Präferenzen

für unterschiedliche Arten von Lesestoffen, ...

... wobei Mädchen mehr fiktionale Literatur und Jungen mehr Tageszeitungen, Comic-

Hefte, E-Mails und Webseiten lesen.
Schließlich unterscheiden sich 15-jährige Jungen und Mädchen nicht nur hinsichtlich ihrer Freude am Lesen, sondern auch bei der Wahl des bevorzugten Lesestoffs (Abb. 5.6). Im Allgemeinen zeigen Mädchen eine stärkere Neigung, Lektüre mit anspruchsvollerem Inhalt zu lesen (Daten für die einzelnen Länder abrufbar unter www.pisa.oecd.org).

Im Durchschnitt aller Länder weisen Mädchen eine größere Bereitschaft zum Lesen von Romanen, Erzählungen und Geschichten auf (37\% der Mädchen lesen solche Lesestoffe mehrmals im Monat oder in der Woche gegenüber $19 \%$ der Jungen). Jungen zeigen eine stärkere Neigung zum Lesen von Tageszeitungen $(68 \%$ mehrmals in der Woche oder im Monat, verglichen mit 60\% der Mädchen), Comic-Heften (35\% der Jungen mehrmals im Monat oder in der Woche, gegenüber 24\% der Mädchen) sowie E-Mails undWebseiten (50\% der Jungen mehrmals im Monat oder in der Woche verglichen mit 40\% der Mädchen). Daten zu einzelnen Ländern sind abrufbar unter www.pisa.oecd.org.

Die Bereitschaft von Mädchen und Jungen zum Lesen von Zeitschriften (rund ein Drittel der Jungen und Mädchen geben an, Zeitschriften mehrmals wöchentlich 
zu lesen) und Sachbüchern (19\% der Mädchen und Jungen geben an Sachbücher mehrmals im Monat oder in der Woche zu lesen) ist im Durchschnitt aller Länder gleich stark ausgeprägt.

\section{Geschlechtsspezifische Unterschiede bei Lernstrategien und Selbstkonzept}

Kapitel 4 zeichnet ein Profil der Schülerlernstrategien nach den Eigenangaben der Schülerinnen und Schüler in PISA 2000. Dabei ist es bemerkenswert, dass bei den Lernstrategien zwischen Jungen und Mädchen ihren eigenen Angaben zufolge durchgängig Unterschiede bestehen.

In der Mehrzahl der Länder geben die 15-jährigen Mädchen an, dass sie größeres Schwergewicht auf Memorierstrategien legen als Jungen, und nur drei Länder weisen eine statistisch signifikante Differenz in die gegenteilige Richtung auf (Tabelle 4.6). Hingegen geben Jungen in der Regel häufiger als Mädchen den Einsatz von Elaborationsstrategien an, und nur in einem Land geben die Mädchen statistisch signifikant häufiger den Einsatz von Elaborationsstrategien an (Tabelle 4.7).

In fast allen Ländern mit statistisch signifikanten geschlechtsspezifischen Unterschieden verwenden Mädchen indessen häufiger als Jungen Kontrollstrategien (Tabelle 4.5). Dies bedeutet, dass Mädchen zwar eher ihren Lernprozess von sich aus beurteilen, aber das Einüben von Elaborationsstrategien könnte ihnen in den meisten Ländern dennoch zugute kommen. Andererseits wäre für Jungen eine allgemeinere Unterstützung bei der Planung, Organisation und Strukturierung ihrer Lernaktivitäten vorteilhaft.

Schließlich gibt es zahlreiche Hinweise darauf, dass zwischen der Selbsteinschätzung des Einzelnen und seinem Lernerfolg ein enger Zusammenhang besteht. Erfolgreich Lernende vertrauen auf ihre Fähigkeiten und glauben, dass es nutzbringend sein kann, in Lernen zu investieren. Schülerinnen und Schüler hingegen, die kein Zutrauen zu ihrer Fähigkeit haben, sich den nach eigenem Urteil wesentlichen Lernstoff anzueignen, laufen Gefahr, nicht nur in der Schule, sondern auch als Erwachsene im Leben zu versagen. Aus diesem Grund wurde im Rahmen von PISA 2000 das „Selbstkonzept“ von Schülerinnen und Schülern in Lesen und Mathematik untersucht. Dargestellt wird dies mit Hilfe von zwei Indizes, die die Schülerantworten auf eine Reihe von Fragen im Zusammenhang mit dem Selbstkonzept zusammenfassen, die ihrerseits aus den Konstrukten früherer Forschungsarbeiten ausgewählt wurden (vgl. auch Anhang A1). Auf der in den Indizes verwendeten Skala weisen zwei Drittel der OECD-Schülerpopulation Werte zwischen -1 und +1 mit einer durchschnittlichen Punktzahl von 0 auf.
Die Lernstrategien von Jungen und Mädchen unterscheiden sich ebenfalls,...

... wobei Mädchen den Akzent eher auf Memorierstrategien und Jungen eher auf Elaborationsstrategien legen.

Mädchen überprüfen ihr Lernen häufiger.

Schülerinnen und schüler mit gröfserem Selbstvertrauen schneiden generell besser $a b, \ldots$ 
KAPITEL 5 Geschlechtsspezifische Leistungsunterschiede

... und das Abbildung 5.7a zeigt den Zusammenhang zwischen dem Selbstkonzept im Lesen Selbstvertrauen der und den Leistungen auf der Gesamtskala Lesekompetenz. Die Punkte stehen Jungen und Mädchen in für die durchschnittliche Position der Jungen und der Mädchen im jeweiligen Lesen und Mathematik Teilnehmerland. In allen Ländern, mit Ausnahme Koreas, geben Mädchen

entsprichtihren häufiger an, in sprachlichen Unterrichtsfächern gute Noten zu bekommen Leistungen in diesen und rasch zu lernen. Besonders ausgeprägt sind die Unterschiede in Finnland,

Fächern,... Deutschland, Italien und den Vereinigten Staaten (Tabelle 5.4a). In Mathematik (Abbildung 5.7b) weisen Jungen im Allgemeinen ein höheres Selbstkonzept auf als Mädchen, insbesondere in Deutschland, Norwegen und der Schweiz (Tabelle 5.4b). Diese geschlechtsspezifischen Unterschiede hängen eng mit den unterschiedlichen Lese- und Mathematikleistungen von Jungen und Mädchen zusammen. Zwischen Selbstkonzept und Schülerleistungen besteht ein positiver Zusammenhang, der in Mathematik noch stärker als beim Lesen zutage tritt.

... was neue Fragen für weitere Analysen aufwirt.
Viele dieser Fragen bedürfen der weiteren Untersuchung, und dies wird der Inhalt eines thematischen Berichts sein, der derzeit in Vorbereitung ist. Es zeigt sich jedoch jetzt schon deutlich, dass geschlechtsspezifische Unterschiede bei den Schülerleistungen in engem Zusammenhang mit Gewohnheiten, Einstellungen und Selbstkonzept von Mädchen und Jungen überprüft und analysiert werden müssen.

\section{Abbildung 5.7a}

Zusammenhang zwischen dem Selbstkonzept Lesen und den Leistungen auf der Gesamtskala Lesekompetenz bei Jungen und Mädchen

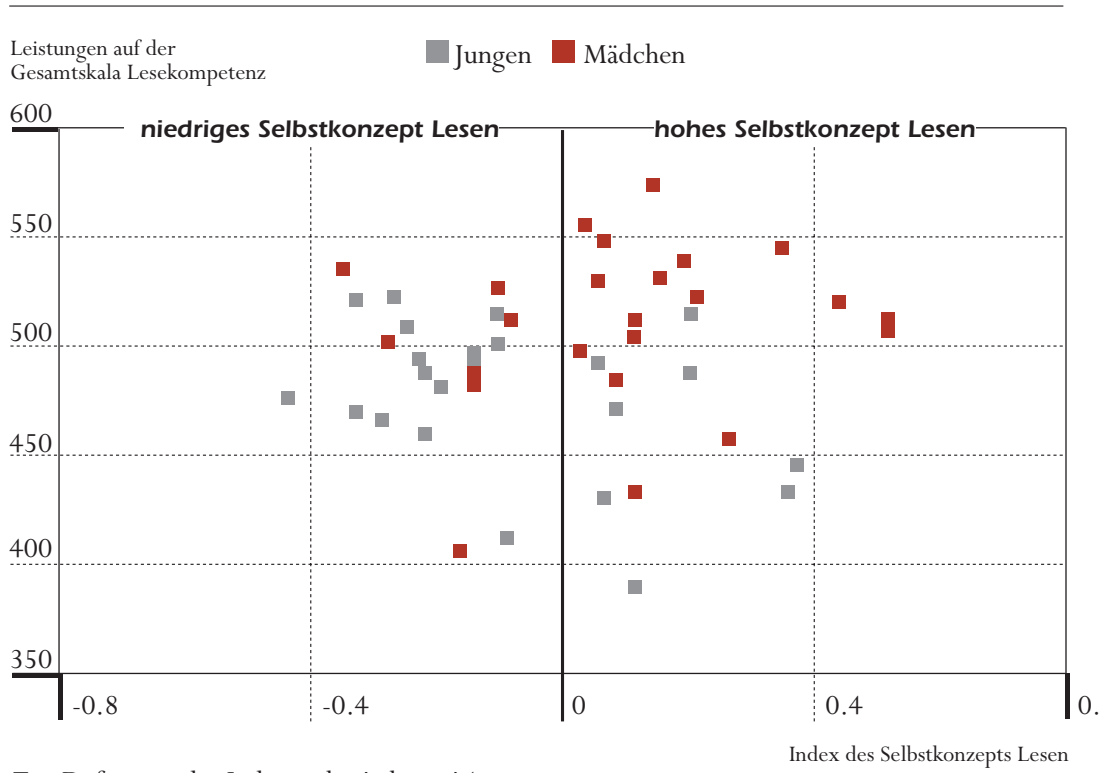

Zur Definition des Index siehe Anhang A1. Anmerkung: Die Länder sind durch die Kästchen dargestellt. Quelle: OECD PISA Datenbank, 2001. Tabelle 5.4a. 


\section{Abbildung 5.7b}

Zusammenhang zwischen dem Selbstkonzept Mathematik und den Leistungen auf der Skala für mathematische Grundbildung bei Jungen und Mädchen

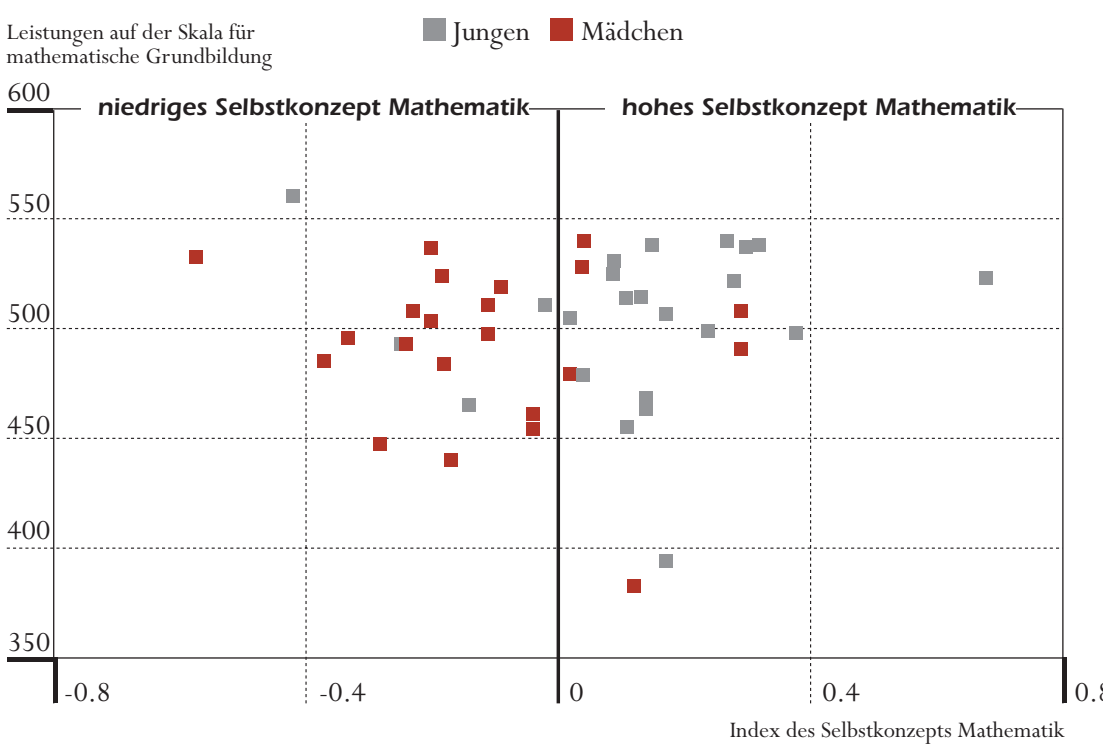

Zur Definition des Index siehe Anhang A1.

Anmerkung: Die Länder sind durch die Kästchen dargestellt.

Quelle: OECD PISA Datenbank, 2001. Tabelle 5.4b.

\section{Schlussbetrachtungen}

Die politischen Entscheidungsträger haben Fragen der Gleichstellung von Mann und Frau eine erhebliche Priorität eingeräumt, wobei den Benachteiligungen von Frauen besondere Aufmerksamkeit geschenkt wurde. Die Ergebnisse von PISA weisen einerseits darauf hin, dass viele Länder in ihren Bemühungen erfolgreich waren, andererseits aber auch auf zunehmende Probleme männlicher Schüler vor allem im Bereich Lesekompetenz und am unteren Ende des Leistungsspektrums. In Mathematik bleibt in vielen Ländern im Durchschnitt ein Rückstand bei den Schülerinnen bestehen, doch ist der Vorsprung der Jungen in den Ländern, in denen er nach wie vor existiert, vor allem auf das hohe Leistungsniveau einer vergleichsweise kleinen Zahl von männlichen Schülern zurückzuführen.

Gleichzeitig zeigen sich beim Ausmaß der geschlechtsspezifischen Differenzen zwischen den einzelnen Ländern erhebliche Unterschiede. Die Ergebnisse der Länder, in denen heute keine Nachteile für Schülerinnen mehr vorhanden sind, zeigen an, dass mit Hilfe wirksamer Maßnahmen und Praktiken ein Zustand überwunden werden kann, der lange Zeit als das unvermeidliche Ergebnis von unterschiedlichen Lernstilen und sogar grundlegenden Begabungen der Jungen und Mädchen betrachtet wurde. Tatsächlich wird durch die Ergebnisse von PISA 2000 deutlich, dass einige Länder ein Lernumfeld bzw. einen weiteren
Mädchen sind in einiger Hinsicht zwar noch immer benachteiligt, doch stellt die Leistungsschwäche der Jungen ein wachsendes Problem dar,...

... wenngleich einige Länder gezeigt haben, dass geschlechtsspezifische Unterschiede erfolgreich überwunden werden können. 
KAPITEL 5 Geschlechtsspezifische Leistungsunterschiede

In der Förderung von

Motivation und des

Interesses in

verschiedenen Fächern

bestehen

geschlechtsspezifische

ungleichheiten fort.

Eine Erhöhung der

Freude am Lesen bei

Jungen und der

Förderung des

Selbstkonzepts in

Mathematik bei

Mädchen bleiben

unerlässlich, wenn

gewährleistet werden

soll, dass alle

Schülerinnen und

Schüler ihr Potential voll

ausschöpfen
Kontext zur Verfügung stellen, der beiden Geschlechtern gleichermaßen zugute kommt. Die fortbestehenden Unterschiede in anderen Ländern und der weit verbreitete Rückstand der Jungen in der Lesekompetenz zeigen, dass hier erhöhte politische Aufmerksamkeit gefordert ist.

Die Analyse zeigt auch, dass die Motivation und das Interesse von Jungen und Mädchen an den jeweiligen Fachgebieten von Schule und Gesellschaft unterschiedlich wirksam gefördert werden. Die enge Wechselbeziehung zwischen dem Interesse an einem bestimmten Fach und den Lernerfolgen weist auch darauf hin, dass die unterschiedlichen Gewohnheiten und Interessen von Jungen und Mädchen weitreichende Konsequenzen für das Lernen haben und dass die Bildungspolitik hierauf eingehen muss.

Obgleich die Bildungssysteme in den vergangenen Jahrzehnten bei der Überbrückung der Differenzen zwischen Jungen und Mädchen im Hinblick auf die Bildungsabschlüsse einen großen Schritt weiter gekommen sind (OECD, 2001), bleibt noch viel zu tun. Mit 15 Jahren stehen viele Schülerinnen und Schüler gerade an der Schwelle von der Schule ins Arbeitsleben. Ihre schulischen Leistungen wie auch ihre Motivation und ihre Einstellungen in verschiedenen Fachgebieten können ihren künftigen Bildungs- und Berufsweg stark beeinflussen. Und diese Bildungs- und Berufswege werden nicht nur die jeweiligen Karriere- und Gehaltsaussichten, sondern auch die allgemeine Effektivität beeinflussen, mit der in den OECD-Ländern und -Gesellschaften Humankapital entwickelt und eingesetzt wird. Eine Steigerung der Freude am Lesen bei den Jungen sowie die Förderung des Interesses und des Selbstkonzepts der Mädchen in Mathematik sollten vorrangige bildungspolitische Ziele sein, wenn zwischen Jungen und Mädchen größere Gleichheit im Hinblick auf den Bildungserfolg erreicht werden soll.

\section{Anmerkungen}

1. Hierbei handelt es sich um Programme, die in der Internationalen Standardklassifikation des Bildungswesens (ISCED) auf Stufe $3 \mathrm{~A}$ angeordnet sind. 


\section{Kapitel}

6

\section{FAMILIÄRER HINTERGRUND UND SCHÜLERLEISTUNGEN}

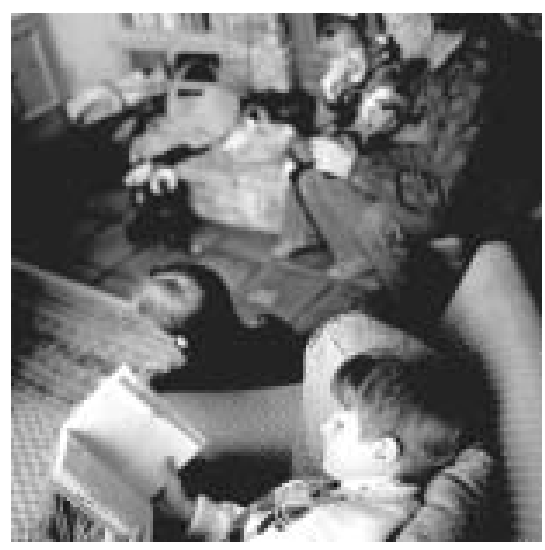


KAPITEL 6 Familiärer Hintergrund und Schülerleistungen

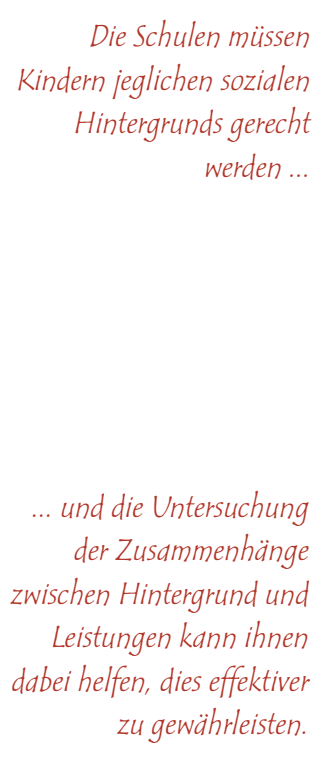

und die Untersuchung

Zusammenhange

zu gewährleisten.

Die berufliche Stellung der

Eltern ist ein Maß für den

sozioökonomischen Status

und kann die Ziele und

Einstellungen der

Schülerinnen und Schüler

beeinflussen.

\section{Einführung}

Schülerinnen und Schüler kommen aus ganz unterschiedlichen familiären, sozioökonomischen und kulturellen Verhältnissen. Daher müssen die Schulen sich bemühen, dieser vielfältig zusammengesetzten Schülerschaft adäquate und gleiche Bildungschancen zu geben. Das Lernumfeld der Schulen kann durch den unterschiedlichen Hintergrund und die vielfältigen Interessen der Schülerinnen und Schüler zwar angeregt werden, gleichzeitig vergrößern die Heterogenität der Fähigkeiten sowie die unterschiedliche Art der Schulvorbereitung allerdings die Schwierigkeiten, vor die sich die Schulen gestellt sehen, wenn sie den Bedürfnissen von Schülerinnen und Schülern mit ganz unterschiedlichem sozioökonomischen Hintergrund gerecht werden wollen.

Die Bestimmung der spezifischen Merkmale von Schülerinnen und Schülern, die niedrige Leistungen erzielen, kann Pädagogen und politischen Entscheidungsträgern helfen, die Bereiche zu identifizieren, in denen Handlungsbedarf besteht. Desgleichen kann die Identifizierung der spezifischen Merkmale von Schülerinnen und Schülern mit hervorragenden schulischen Leistungen den politischen Entscheidungsträgern dabei helfen, die Bedingungen für hohe Leistungsniveaus zu fördern. Wenn gezeigt werden kann, dass einige Länder weniger Probleme dabei haben, beiden Gruppen gerecht zu werden als andere, könnte dies den Schluss nahe legen, dass die Förderung von Gleichheit und Qualität gleichermaßen möglich ist.

Im vorliegenden Kapitel wird der Zusammenhang zwischen den Schülerleistungen im Bereich Lesekompetenz, mathematische und naturwissenschaftliche Grundbildung sowie den verschiedenen Aspekten des familiären Hintergrunds wie Bildungsstand und Berufstätigkeit der Eltern, das jeweilige wirtschaftliche und kulturelle Umfeld, das Geburtsland und die im Elternhaus gesprochene Sprache untersucht (vgl. Kasten 6.1).

\section{Berufliche Stellung}

Eine höhere berufliche Stellung der Eltern kann den Berufswunsch sowie die beruflichen Zielsetzungen und Erwartungen der Schülerinnen und Schüler und damit auch ihren Lerneifer als Mittel zur Erreichung dieser Ziele beeinflussen. Eine hohe berufliche Stellung der Eltern kann auch das Spektrum der im Bewusstsein der Kinder existierenden Optionen erweitern.

PISA erfasst diesen Aspekt des familiären Hintergrunds der Schülerinnen und Schüler durch international vergleichbare Informationen über die berufliche Tätigkeit der Eltern sowie die damit in Zusammenhang stehenden Aktivitäten. Der entsprechende sozioökonomische Index der beruflichen Stellung (Ganzeboom et al., 1992), der Werte zwischen 0 und 90 aufweist, misst diejenigen Berufsattribute, die den Niederschlag der Bildung einer Person in deren Einkommen aufzeigen. Je höher der Indexwert, umso höher die berufliche Stellung der Schülereltern. Im Durchschnitt der OECD-Länder beträgt der Indexwert 49 und seine Standardabweichung 16 (vgl. Kasten 6.2). 


\section{Kasten 6.1 Interpretation von PISA-Indizes}

Die in PISA 2000 enthaltenen Indizes basieren auf Angaben von Schülerinnen und Schülern sowie von Schulleitungen über das Lernumfeld und die Schulorganisation sowie den sozialen und ökonomischen Kontext, in dem das Lernen stattfindet. Die Indizes fußen nicht auf externen Beobachtungen, sondern auf Selbstangaben und können von zwischen den Kulturen bestehenden Unterschieden im Antwortverhalten sowie der sozialen Erwünschtheit bestimmter Antworten beeinflusst sein.

Mehrere Indizes fassen die Antworten der Schülerinnen und Schüler bzw. der Schulleitungen auf eine Reihe von miteinander verbundenen Fragen zusammen. Die Fragen entstammen größeren Konstrukten, die auf theoretischen Überlegungen und früheren Forschungsarbeiten basieren ${ }^{1}$.

Falls nicht anders angegeben, beziehen sich die Vergleiche der Schülerleistungen im vorliegenden Kapitel auf die Ergebnisse der Schülerinnen und Schüler auf der Gesamtskala Lesekompetenz.

Strukturgleichungsmodelle wurden als Methode eingesetzt, um die theoretisch erwarteten Dimensionen der Indizes zu bestätigen und um ihre länderübergreifende Vergleichbarkeit zu validieren. Zu diesem Zweck wurden die Modelle für jedes Land einzeln sowie für alle OECDLänder zusammengenommen geschätzt.

Wie aus Abbildung 6.1 ersichtlich wird, gehen Unterschiede im sozioökonomischen Index der beruflichen Stellung mit großen Differenzen zwischen den Schülerleistungen innerhalb der Länder einher (Tabelle 6.1a). Unter den Schülerinnen und Schülern, die nach dem sozioökonomischen Index dem oberen Quartil angehören, liegt die mittlere Punktzahl auf der Gesamtskala Lesekompetenz in den OECD-Ländern bei 545 Punkten, also 45 Punkte über dem OECD-Durchschnitt. Hingegen beträgt das Durchschnittsergebnis für das jeweils untere Quartil der Schülerinnen und Schüler nach dem sozioökonomischen Index lediglich 463 Punkte. Die Durchschnittsdifferenz zwischen den beiden Gruppen im Bereich Lesekompetenz beträgt mehr als eine ganze Kompetenzstufe ${ }^{2}$. Im Durchschnitt der OECD-Länder erklärt der Index 11\% der Gesamtvarianz der Schülerleistungen auf der Gesamtskala Lesekompetenz (vgl. Anhang A2). Für mathematische und naturwissenschaftliche Grundbildung gelten ähnliche Ergebnisse (Tabellen 6.1b und c).

Abbildung 6.1 zeigt ebenfalls, dass die Höhe der Leistungsunterschiede auf der Gesamtskala Lesekompetenz zwischen Schülern, die sich nach dem sozioökonomischen Index im oberen und unteren Quartil eines jeden Landes befinden, von Land zu Land erheblich variiert. Die geringste Differenz ist in Korea festzustellen (33 Punkte). In diesem Land erzielen die Schülerinnen und Schüler, deren Eltern einen niedrigen beruflichen Status aufweisen, gute Leistungen sowohl relativ, d.h. im Vergleich zu koreanischen Schülerinnen und Schülern, deren Eltern einen hohen beruflichen Status aufweisen, als auch absolut, d.h. im Vergleich zu Schülerinnen und Schülern aus anderen Ländern, deren Eltern einen ähnlichen beruflichen Status haben. Finnland und Island

Schülerinnen und Schüler, deren Eltern angesehene Berufe ausüben, erreichen im Durchschnitt höhere Leistungen, ...
... doch ist der Vorteil in einigen Ländern wesentlich größser als in anderen ... 
KAPITEL 6 Familiärer Hintergrund und Schülerleistungen

\section{Kasten 6.2 Interpretation der Abbildung 6.1}

Der in PISA eingesetzte sozioökonomische Index der beruflichen Stellung teilt die Schülerinnen und Schüler nach der beruflichen Stellung ihrer Eltern in Gruppen ein, wobei die direkte Rolle der beruflichen Tätigkeit bei der Einkommensmaximierung die Rangfolge bestimmt. In jedem Land wird die Bevölkerung in Quartile unterteilt und die Rangordnung nach den nationalen Werten auf dem Index festgesetzt. Die Fähigkeiten, die nötig sind, um den Anforderungen einer beruflichen Tätigkeit zu genügen, sind das primäre Kriterium, nach dem die verschiedenen Niveaus beruflicher Stellungen unterschieden werden.

In Abbildung 6.1 werden die Durchschnittsleistungen der Schülerinnen und Schüler im oberen Indexquartil mit denen der Schülerinnen und Schüler im unteren Quartil desselben Landes verglichen. Anhand der Länge der verschiedenen Linien wird dargestellt, welcher Abstand in jedem Land zwischen Personen der höchsten und niedrigsten nationalen Berufsgruppen besteht. Anzumerken ist, dass der in jedem Quartil erfasste durchschnittliche berufliche Status der einzelnen OECD-Länder unterschiedlich ist, wenngleich der Mittelwert des unteren Quartils, mit Ausnahme von vier Ländern, in allen OECD-Ländern auf der Basis eines 90-Punkte-Index, um höchstens 5 Punkte variiert. Für das obere Quartil ist die Variationsbreite etwas größer.

Typische Berufstätigkeiten der Eltern von 15-Jährigen mit 16 bis 35 Indexpunkten sind z.B. Kleinbauern, Werkzeugmacher, Kfz-Mechaniker,Taxi- und Lkw-Fahrer sowie Kellner. Die häufigsten beruflichen Tätigkeiten im Bereich von 35 bis 53 Indexpunkten sind Buchhalter, Verkaufspersonal, Manager von Kleinbetrieben oder Pflegepersonal. Je höher die für eine Tätigkeit geforderten Qualifikationen sind, umso höher ist auch der damit verbundene berufliche Status. Zwischen 54 und 70 Indexpunkten liegen z.B. Tätigkeiten wie Marketingmanager, Lehrer, Hoch- und Tiefbauingenieur und Wirtschaftsprüfer. Im oberen internationalen Indexquartil liegen mit Punkten zwischen 71 und 90 berufliche Tätigkeiten wie Arzt, Hochschullehrer und Juristen.

sind die beiden europäischen OECD-Länder mit den geringsten Differenzen zwischen den zwei extremen Quartilen (52 bzw. 53 Punkte). In diesen drei Ländern bestehen auch zwischen Schülern im unteren und oberen Quartil des Index die geringsten Leistungsdifferenzen auf der Skala für mathematische und naturwissenschaftliche Grundbildung.

Die größten Unterschiede, nämlich 100 Punkte und mehr in allen drei Grundbildungsbereichen weisen Belgien, Deutschland und die Schweiz auf. In Deutschland ist der Unterschied besonders auffallend. Schülerinnen und Schüler, deren Eltern die höchsten beruflichen Stellungen haben (das obere Quartil auf dem berufsbezogenen Index), erzielen durchschnittlich in etwa die gleichen Leistungen wie der Durchschnitt aller Schülerinnen und Schüler in Finnland, dem nach PISA leistungsstärksten Land; die Schülerinnen und Schüler, deren Eltern den niedrigsten beruflichen Status haben, erzielen in etwa die gleichen Leistungen wie der Durchschnitt aller Schülerinnen und Schüler in Mexiko, dem leistungsschwächsten OECD-Land. 
Auch in der Tschechischen Republik, in Ungarn, dem Vereinigten Königreich und den Vereinigten Staaten betragen die Unterschiede für Schülerinnen und Schüler in den jeweiligen oberen und unteren nationalen Quartilen des sozioökonomischen Index in allen drei Grundbildungsbereichen über 90 Punkte, womit sie weit über das Äquivalent einer Kompetenzstufe hinausgehen. Wie auch in Belgien, Deutschland und der Schweiz, so ist auch in diesen Ländern für die Schülerinnen und Schüler im unteren Quartil des berufsbezogenen Index im Vergleich zu anderen Schülerinnen und Schülern die Wahrscheinlichkeit, ebenfalls zu den unteren 25\% ihres Landes auf den PISA-Leistungsskalen zu gehören, mehr als doppelt so groß (Tabelle 6.1a).

Es darf jedoch nicht unterstellt werden, dass alle diese Differenzen ein unmittelbares Ergebnis der auf dem familiären Hintergrund beruhenden Vorteile und der durch Eltern mit angesehener beruflicher Stellung geweckten Erwartungen sind. Die Leistungen der Schülerinnen und Schüler in den drei Grundbildungsbereichen werden von vielen Faktoren beeinflusst. Der sozioökonomische Status kann z.B. mit dem Ort zusammenhängen, an dem die Schülerinnen und Schüler leben, und mit der Qualität der Schulen, zu denen sie Zugang haben (dies wäre in Schulsystemen wichtig, die von lokalen Steuern abhängen), mit der Wahrscheinlichkeit des Besuchs von Privatschulen, dem Grad der Unterstützung durch die Eltern und deren Engagement usw.

Es ist wichtig festzuhalten, dass die Schülerinnen und Schüler im unteren nationalen Quartil zwar niedrigere Mittelwerte erzielen als ihre Mitschüler im jeweiligen Land, sie absolut gesehen aber dennoch nicht unbedingt schlecht abschneiden. So erzielen z.B. die Schülerinnen und Schüler im unteren Quartil des sozioökonomischen Index in Finnland und Korea Mittelwerte, die in jedem der Testbereiche statistisch signifikant über dem OECD-Durchschnittswert von 500 Punkten liegen, sowie gleichzeitig höhere Durchschnittspunktzahlen als die Schülerinnen und Schüler des oberen Quartils in zwei anderen Ländern. Mit anderen Worten: Wenngleich ein höherer sozioökonomischer Status mit einem Leistungs-„Vorteil“ innerhalb der Länder verbunden ist, besteht dieser Vorteil nicht systematisch in allen Ländern. Gleicherweise bedeutet die Platzierung im oberen Quartil des sozioökonomischen Index nicht automatisch in jedem Land den gleichen relativen Vorteil.

Es sollte ebenfalls berücksichtigt werden, dass die Platzierung im oberen (oder unteren) nationalen Quartil des sozioökonomischen Index in einem Land nicht notwendigerweise gleichwertig mit der Platzierung im oberen (oder unteren) Quartil eines anderen Landes ist. Gleicherweise ist der „Abstand“ bzw. die Differenz zwischen den im unteren und oberen Quartil erfassten Schülerinnen und Schülern von Land zu Land sehr unterschiedlich. Die Tschechische Republik und Frankreich verzeichnen zwar z.B. identische Mittelwerte (48 Indexpunkte) auf dem sozioökonomischen Index der beruflichen Stellung (vgl. Tabelle 6.1a), doch beträgt die durchschnittliche Differenz zwischen Schülern im unteren und oberen Quartil in der Tschechischen Republik

\author{
... und viele Faktoren \\ beeinflussen diesen \\ Zusammenhang
}

In einigen Ländern

erreichen Schülerinnen und

Schüler, deren Eltern einen

niedrigeren beruflichen

Status haben, höhere

Leistungen als der

Durchschnittsschüler des

OECD-Raums. 
KAPITEL 6 Familiärer Hintergrund und Schülerleistungen

\section{Abbildung 6.1}

Berufliche Stellung der Eltern und Schülerleistungen

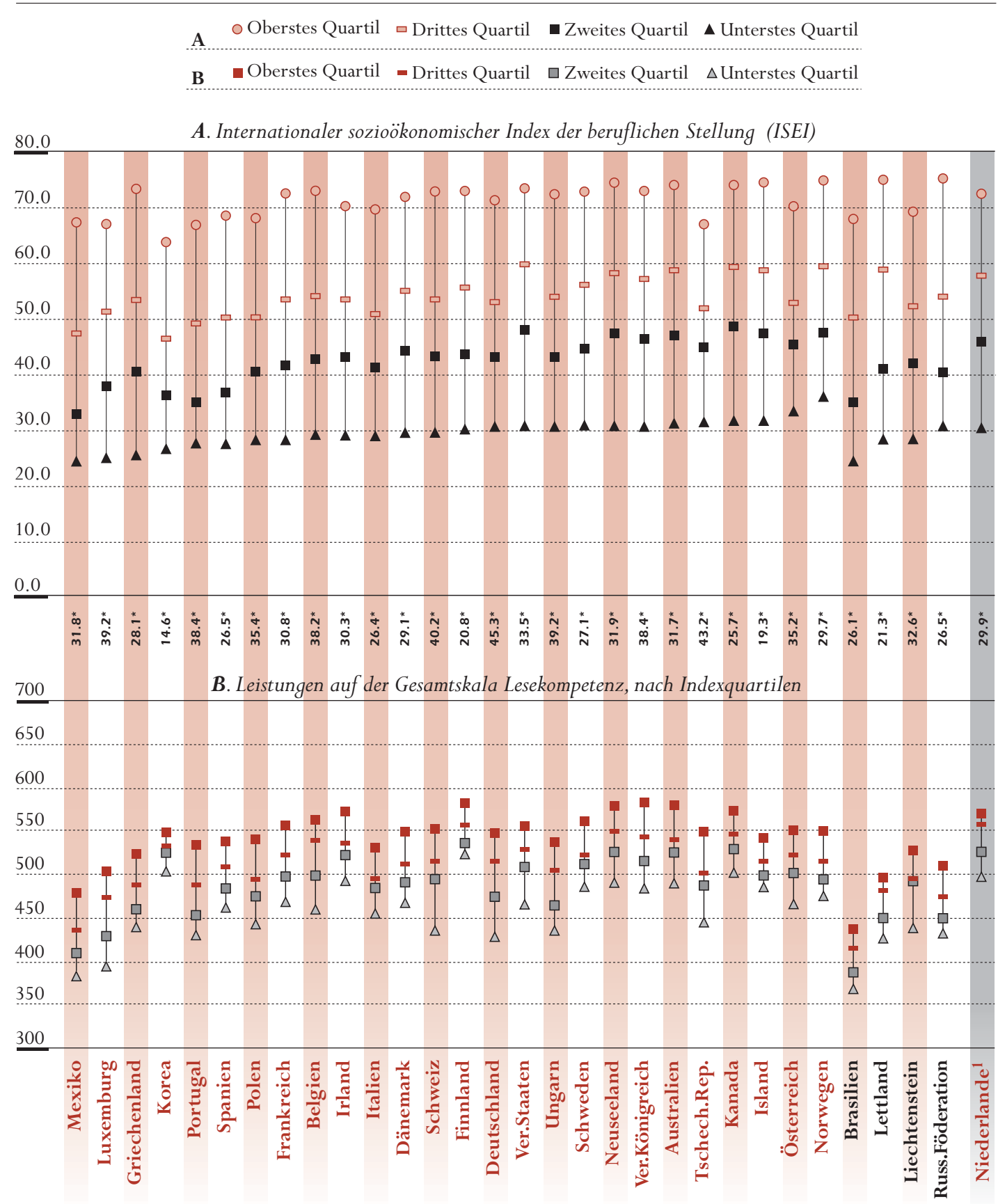

* Punktzahlveränderung auf der Gesamtskala Lesekompetenz je 16,3 Indexeinheiten (1 Standardabweichung) des Internationalen sozioökonomischen Index der beruflichen Stellung.

1. Die Beteiligungsquote ist zu niedrig, um die Vergleichbarkeit zu gewährleisten (vgl. Anhang A3).

Anmerkung: Zur Definition des Index siehe Anhang A1.

Quelle: OECD PISA Datenbank, 2001. Tabelle 6.1a. 
35 Punkte und in Frankreich 44, woraus hervorgeht, dass in Frankreich größere Differenzen im sozioökonomischen Status bestehen als in der Tschechischen Republik. Um diesen Unterschieden Rechnung zu tragen, können auch die Differenzen bei den Durchschnittspunktzahlen von Schülerinnen und Schülern im oberen und unteren Quartil des Index Lesekompetenz verglichen werden, die mit einem Unterschied von einer internationalen Standardabweichung (16 Einheiten) auf dem sozioökonomischen Index der beruflichen Stellung (Tabelle 6.1a, b und c) verbunden sind. Diese Analyse bestätigt die relativ hohe Bedeutung des sozioökonomischen Status in einigen Ländern sowie dessen relativ geringe Bedeutung in anderen.

Diese Ergebnisse sind für die politisch Verantwortlichen potentiell von großer Bedeutung. Die Internationale Erhebung über Grad und Verteilung elementarer Grundqualifikationen Erwachsener (IALS) hat gezeigt, dass die Lesekompetenz eine Fähigkeit ist, die Beschäftigungschancen und Verdiensthöhe verbessert (OECD und Statistics Canada, 2000). Darüber hinaus ist die Lesekompetenz ebenfalls eine wichtige Grundlage für lebensbegleitendes Lernen. Daher könnte die generationsübergreifende Mobilität zwischen niedrigem und höherem sozioökonomischen Status in denjenigen Ländern begrenzt sein, in denen ein hoher Prozentsatz der Menschen schwächere oder marginale Lesekompetenzen aufweist. Die schwächeren Leser (sowie die Personen mit nur geringer mathematischer und naturwissenschaftlicher Grundbildung) dürften nur die geringsten Chancen haben, Beschäftigungsmöglichkeiten mit wirtschaftlichen Aufstiegschancen zu finden. Wie die Ausführungen über die zwischen den einzelnen Ländern bestehenden Unterschiede in der Relation zwischen Schülerleistungen und sozioökonomischem Status jedoch zeigen, variieren diese Unterschiede in den verschiedenen Ländern erheblich, was möglicherweise bedeutet, dass sie nicht unvermeidlich sind.

\section{Relativer familiärer Wohlstand}

Relativer Wohlstand, der in der Regel in engem Zusammenhang mit dem ausgeübten Beruf steht, ist im Allgemeinen ein Vorteil. Per Definitionem haben wohlhabendere Personen Zugang zu mehr Ressourcen. Sie haben in der Regel auch ein höheres frei verfügbares Einkommen und können daher die von ihnen gewünschten Dinge problemloser erwerben, darunter auch Güter und Dienstleistungen hoher Qualität. Allerdings sollte der familiäre Wohlstand von Schülerinnen und Schülern in öffentlich geförderten, der Chancengleichheit verpflichteten Bildungssystemen nicht den Zugang zu Bildungsqualität beeinflussen.

Zur Messung des Zusammenhangs zwischen Wohlstand und Bildungserträgen wurde auf Schülerangaben zu den verschiedenen, in ihrem Elternhaus vorhandenen Gütern zurückgegriffen und anhand der Antworten ein kombinierter Index des relativen Wohlstands der Familie erstellt. Für die OECD-Länder ist bei diesem Index der Durchschnittswert mit 0 und die Standardabweichung mit 1 angesetzt.
Die Leistungsunterschiede bremsen generationsübergreifend die wirtschaftliche und soziale Mobilität.

Der relative Wohlstand der Familie sollte für die Bildungschancen in öffentlichen

Bildungssystemen keine Rolle spielen,... 
KAPITEL 6 Familiärer Hintergrund und Schülerleistungen

... doch schneiden Schülerinnen und Schüler

aus wohlhabenderen Familien in der Regel besser ab, auch wenn das Muster

hier weniger stark ausgeprägt ist als beim beruflichen Status der

Eltern.

In einigen Ländern ist der Zusammenhang wesentlich ausgeprägter als in anderen, ...

in denen ein relativer Mangel an Wohlstand kein Hindernis für Leistungen über dem internationalen Durchschnitt ist.
Wie aus Tabelle 6.2 hervorgeht, ist der Zusammenhang zwischen Wohlstand und Leistung in der PISA-Studie uneinheitlich, generell jedoch positiv. Schülerinnen und Schüler aus wohlhabenderen Familien schneiden in der Regel in allen getesteten Bereichen besser $a b$ als Schülerinnen und Schüler aus den am wenigsten begüterten Familien. Innerhalb der Länder erreichen Schülerinnen und Schüler aus wohlhabenderen Familien in der Regel höhere Punktzahlen als Schülerinnen und Schüler aus weniger wohlhabenden Familien. Im Durchschnitt liegen die Ergebnisse von Schülerinnen und Schülern in den OECD-Ländern, die auf dem Index des relativen Wohlstands dem obersten Quartil angehören, auf der Gesamtskala Lesekompetenz um 34 Punkte höher als die der Schülerinnen und Schüler des untersten Quartils (die Differenzen sind in allen außer drei OECD-Ländern statistisch signifikant $)^{3}$.

Unter den an PISA beteiligten Ländern bestehen in den Vereinigten Staaten die größten Unterschiede; der Abstand bei der Leseleistung beträgt zwischen den Schülern im oberen und unteren Quartil beim Index des familiären Wohlstands 85 Punkte (OECD-Durchschnitt: 34 Punkte). Anders ausgedrückt besteht für die Schülerinnen und Schüler in den Vereinigten Staaten, wenn sie dem unteren Quartil des PISA-Index des relativen Wohlstands der Familie angehören, eine zumindest doppelt so großeWahrscheinlichkeit, hinsichtlich der Lesekompetenz zu den leistungsschwächsten 25\% zu gehören, als wenn sie sich im oberen Quartil befinden. Brasilien, Mexiko und Portugal weisen die nächstgrößeren Differenzen auf, nämlich durchschnittlich rund 70 Punkte für die drei Grundbildungsbereiche (siehe Tabelle 6.2 für Lesekompetenz sowie unter www.pisa.oecd.org für mathematische und naturwissenschaftliche Grundbildung).

In den nordischen Ländern, aber auch in Österreich, Belgien, Italien, Japan, Lettland und Polen ist der Zusammenhang zwischen familiärem Wohlstand und Schülerleistung verhältnismäßig schwach ${ }^{4}$. Ferner ist in mehreren OECDLändern ein relativer Mangel an Wohlstand kein Hindernis für Leistungen über dem internationalen Durchschnitt. Tatsächlich erzielen in rund einem Drittel der Länder Schülerinnen und Schüler, die dem unteren nationalen Wohlstandsquartil angehören, mittlere Punktzahlen, die den Durchschnittspunktzahlen für den OECD-Raum entsprechen oder diese übertreffen. Bei den Schülerinnen und Schülern im unteren Wohlstandsquartil sind hohe Punktzahlen in Finnland (Lesekompetenz) und in Japan (alle drei Grundbildungsbereiche) zu beobachten (siehe Tabelle 6.2 für Lesekompetenz sowie unter www.pisa.oecd.org für mathematische und naturwissenschaftliche Grundbildung). Die Schülerinnen und Schüler des untersten Wohlstandsquartils erreichen in diesen Ländern höhere mittlere Punktzahlen als die Schülerinnen und Schüler des obersten Wohlstandsquartils in vielen anderen OECD-Ländern. 


\section{Mit „klassischer“ Kultur verbundene Besitztümer und Aktivitäten}

Neben dem relativen Wohlstand der Familie haben Schülerinnen und Schüler zudem möglicherweise Zugang zu Besitztümern, die zur klassischen Kultur gerechnet werden und deren Einfluss auf Bildungserfolge von der Forschung häufig nachgewiesen wurde. Im Unterschied zum familiären Wohlstand, der in der Regel auf das erfolgreiche Abschneiden der Eltern auf dem Arbeitsmarkt zurückzuführen ist, sind Kulturgüter zumindest grundsätzlich für den, der sich darum bemüht, leichter verfügbar. Zur Messung des Zusammenhangs zwischen Kulturgütern und Leistungen in den drei Grundbildungsbereichen wurden die Schüler in PISA aufgefordert anzugeben, ob es in ihrem Elternhaus klassische Literatur, Bücher mit Gedichten und Kunstgegenstände (z.B. Bilder) gibt. Anhand der kombinierten Antworten wurde ein Index des Besitzes von „klassischen” Kulturgütern im Elternhaus entwickelt; für die OECD-Länder wurde der Durchschnitt mit 0 und die Standardabweichung mit 1 angesetzt.

Die höchsten Werte hinsichtlich des Besitzes von klassischen Kulturgütern sind in Island, Lettland und der Russischen Föderation zu beobachten. In Island erklären z.B. 75\% oder mehr der Schülerinnen und Schüler, dass ihre Familie Werke der klassischen Literatur, der Dichtkunst oder Kunstgegenstände besitzt (Daten abrufbar unter www.pisa.oecd.org). In der Russischen Föderation geben nahezu 90\% der Schülerinnen und Schüler an, dass es in ihrem Elternhaus Werke der klassischen Literatur und der Dichtkunst gibt. Diese Prozentsätze stehen im Kontrast zu Ländern wie Neuseeland und Mexiko, wo weniger als ein Drittel der Schülerinnen und Schüler angibt, dass im Elternhaus klassische Literatur vorhanden ist, sowie Brasilien, Frankreich und Mexiko, wo ein Drittel oder weniger der Schüler erklärt, dass es in ihrem Elternhaus Kunstgegenstände gibt.

Wie Abbildung 6.2 $2^{5}$ entnehmen ist, steht der Besitz von „klassischen” Kulturgütern in engem Zusammenhang mit Leistungsunterschieden, und dieser Zusammenhang ist generell stärker als beim familiären Wohlstand. Zudem sind die Leistungsunterschiede zwischen Schülern im unteren und oberen nationalen Quartil des Index des Besitzes von Kulturgütern im Elternhaus im Durchschnitt der OECD-Länder durchgängig hoch; sie reichen von 55 Punkten in der mathematischen Grundbildung bis zu 68 Punkten in der Lesekompetenz (vgl. Tabelle 6.3 für Lesekompetenz sowie www.pisa.oecd.org für mathematische und naturwissenschaftliche Grundbildung) ${ }^{6}$. Dieser Zusammenhang ist natürlich eng mit anderen Hintergrundfaktoren wie sozioökonomischer Status und Wohlstand verknüpft.

Das Fehlen von „klassischen” Kulturgütern hat selbstverständlich relativen Charakter. In manchen Ländern mögen Schülerinnen und Schüler nur über wenige derartige Güter verfügen und, gemessen an ihren Mitschülerinnen und Mitschülern, schwache Leistungen erbringen. Gleichzeitig können solche Schülerinnen und Schüler jedoch im Vergleich zu Schülern im unteren Quartil des Index in anderen Ländern gut abschneiden. Ein relativer Mangel an
In PISA wurden die Schüler auch danach gefragt, ob es in ihrem Elternhaus klassische Kulturgüter, wie z.B. Literatur und Kunstgegenstände, gibt.

Innerhalb der einzelnen Länder ist der Besitz von Kulturgütern enger mit den Leistungen verbunden als der relative familiäre Wohlstand,...

... obgleich Schülerinnen und Schüler, die diese Güter entbehren, im internationalen Vergleich nicht überall schwach abschneiden. 
KAPITEL 6 Familiärer Hintergrund und Schülerleistungen

\section{Abbildung 6.2}

Besitz „klassischer” Kulturgüter und Schülerleistungen
A O Oberstes Quartil $\square$ Drittes Quartil
- Zweites Quartil $\Delta$ Unterstes Quartil
B \Oberstes Quartil - Drittes Quartil $\square$ Zweites Quartil $\Delta$ Unterstes Quartil
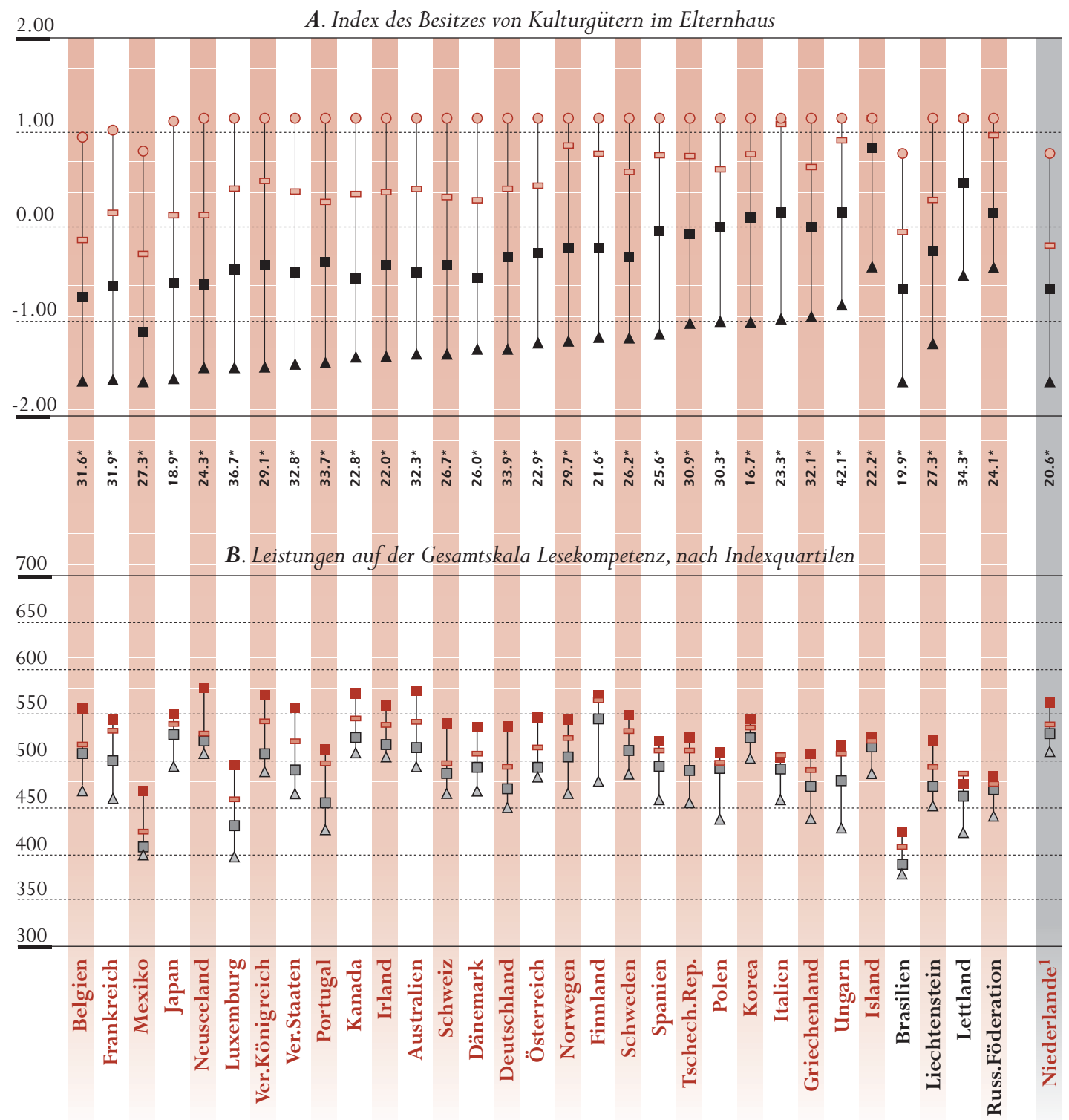

* Punktzahlveränderung auf der Gesamtskala Lesekompetenz je Indexeinheit.

1. Die Beteiligungsquote ist zu niedrig, um die Vergleichbarkeit zu gewährleisten (vgl. Anhang A3).

Anmerkung: Zur Definition des Index siehe Anhang A1 .

Quelle: OECD PISA Datenbank, 2001. Tabelle 6.3. 
„klassischen” Kulturgütern hindert Schülerinnen und Schüler in mehreren Ländern daher offensichtlich nicht daran, in jedem Grundbildungsbereich Leistungen über dem OECD-Durchschnitt zu erzielen.

Schülerinnen und Schüler mit den höchsten Werten auf dem Index des Besitzes von „klassischen” Kulturgütern im Elternhaus erbringen in der Regel außergewöhnlich hohe Leistungen. In acht OECD-Ländern erzielen diese Schülerinnen und Schüler in jedem Bereich Durchschnittspunktzahlen von 550 oder mehr. Im Bereich Lesekompetenz wären diese Schülerinnen und Schüler im Grenzbereich zwischen Kompetenzstufe 3 und 4 platziert. In Australien und dem Vereinigten Königreich gehen die durchschnittlichen Ergebnisse für Schülerinnen und Schüler im obersten Quartil in der Lesekompetenz sowie in mathematischer und naturwissenschaftlicher Grundbildung über 560 Punkte hinaus und liegen bei der Lesekompetenz innerhalb von Kompetenzstufe 4. Im Durchschnitt der OECD-Länder nimmt die Leseleistung für jeden Anstieg um eine Einheit auf dem Index des Besitzes von „klassischen” Kulturgütern im Elternhaus um 27 Punkte zu (Tabelle 6.3).

Es kann ebenfalls untersucht werden, wie die PISA-Punktzahlen für Grundbildung bei Schülerinnen und Schülern mit sukzessiv höherem Kulturgüterniveau schrittweise steigen. Noch deutlicher als im Fall des familiären Wohlstands zeigt sich, dass hier durchschnittlich 40\% dieser Differenz zwischen dem unteren und dem zweiten Quartil des Kulturgüterindex liegen. In Island, Italien, Japan, Lettland, Polen, der Russischen Föderation und Spanien sind es sogar über $60 \%$.

Wenngleich Kulturgüter eng mit anderen Faktoren verbunden sind, so macht die Art des kulturellen Kapitals, auf der schulische Lehrprogramme häufig aufbauen und das in Prüfungen und Tests bewertet wird, doch einen Unterschied. Dass die Effekte bei der Lesekompetenz sogar noch ausgeprägter sind als bei mathematischer und naturwissenschaftlicher Grundbildung (Daten abrufbar unter www.pisa.oecd.org), unterstreicht die Tatsache, dass ein Zugang zu Literatur und anderen Kulturgütern über das Elternhaus im Bildungsbereich von Nutzen ist.

In PISA 2000 wurden die Schülerinnen und Schüler ferner aufgefordert, die Häufigkeit anzugeben, mit der sie an Aktivitäten im Bereich der klassischen Kultur teilnehmen, wie z.B. Besuche von Museen oder Kunstausstellungen, von Theateraufführungen oder Oper, Ballett und klassischen Konzerten. Anhand der Antworten wurde ein kombinierter Index der „klassischen“ kulturellen Aktivitäten entwickelt. Indexwerte über 0 bedeuten, dass die Schülerinnen und Schüler häufiger als der Schülerdurchschnitt in allen OECD-Ländern an kulturellen Aktivitäten teilnehmen; Werte unter 0 drücken aus, dass die Schülerinnen und Schüler weniger häufig als der typische oder durchschnittliche 15-Jährige in den OECD-Ländern an derartigen Aktivitäten teilnehmen.
Das Schülerquartil mit den meisten Kulturgütern erzielt in vielen Ländern sehr hohe Punktzahlen,...

... und ein Großsteil der Differenz besteht zwischen dem untersten Quartil und den übrigen.

In PISA wurde auch nach "klassischen" kulturellen Aktivitäten gefragt, ... 
KAPITEL 6 Familiärer Hintergrund und Schülerleistungen

... die von den Schülern aus verschiedenen Ländern

mit unterschiedlicher Häufigkeit wahrgenommen werden ...

... und das Ergebnis lautet, dass praktisch überall diejenigen, die solche Aktivitäten am häufigsten wahrnehmen, im Durchschnitt eine höhere Grundbildung aufwiesen.

wird weithin als
grundlegend für den
Schulerfolg betrachtet,...

... und in PISA wurden die Schülerinnen und Schüler daher gefragt, wie häufig sie mit ihren Eltern ...

... in kulturellen und sozialen Kontexten kommunizieren.
Die Länder mit der höchsten Häufigkeit derTeilnahme an kulturellen Aktivitäten sind dieTschechische Republik, Ungarn und Lettland (Tabelle 6.4). Nahezu 30\% der Schülerinnen und Schüler in der Tschechischen Republik und in Ungarn geben drei oder mehr Theaterbesuche für das vergangene Jahr an (gegenüber dem internationalen Durchschnitt von lediglich 12\%) (Daten abrufbar unter www.pisa.oecd.org). Desgleichen hatte ein Drittel der ungarischen Schülerinnen und Schüler im vergangenen Jahr dreimal oder noch häufiger ein Museum oder eine Kunstgalerie besucht (gegenüber dem internationalen Durchschnitt von 14\%). Andererseits geben durchschnittlich 43\% der Schülerinnen und Schüler an, noch nie ein Museum oder eine Kunstgalerie besucht zu haben.

Aus der Häufigkeit der Teilnahme an „klassischen” kulturellen Aktivitäten, wie sie dieser Index misst, erklären sich im OECD-Länderdurchschnitt 5,7\% der Varianz der Schülerleistungen auf der Gesamtskala Lesekompetenz (vgl. Anhang A2). Mit Ausnahme von Brasilien besteht in jedem Land ein statistisch signifikanter Unterschied zwischen den durchschnittlichen Schülerleistungen im unteren nationalen Quartil des Index der „klassischen” kulturellen Aktivitäten und denen im oberen Quartil, wobei diese Unterschiede in Belgien, Deutschland, Spanien, dem Vereinigten Königreich und den Vereinigten Staaten mit 70 Skalenpunkten oder mehr ganz besonders groß sind (Tabelle 6.4).

\section{Kommunikation über soziale Fragen und kulturelle Aspekte}

Die Lernförderung der Kinder im Elternhaus wird weithin als ein grundlegendes Element des schulischen Erfolgs angesehen. Wenn Interaktion und Kommunikation zwischen Eltern und Kindern gut funktionieren, können die Eltern ihre Kinder ermutigen, ihr Interesse an den Fortschritten der Kinder zeigen und auf andere Weise kundtun, wie wichtig die Entwicklung ihrer Kinder innerhalb und außerhalb der Schule für sie ist. In umfangreichen Forschungsarbeiten der Vergangenheit wurde deutlich nachgewiesen, dass zwischen elterlichem Engagement und schulischen Erfolgen der Kinder ein starker Zusammenhang besteht.

In PISA wurden die Schülerinnen und Schüler aufgefordert anzugeben, wie häufig es in den folgenden sechs Bereichen zu Interaktion und Kommunikation mit ihren Eltern kommt: Diskussion über politische oder soziale Fragen, Diskussion über Bücher, Filme oder Fernsehsendungen, gemeinsames Hören klassischer Musik, Gespräche über die Schulleistungen, gemeinsame Einnahme der Hauptmahlzeit sowie Zeit, um miteinander zu reden. Die Forschung bestätigt, dass derartige Fragen gute Indikatoren für soziale und kulturelle Kommunikation darstellen.

Anhand der kombinierten Antworten auf die drei ersten Fragen wurde ein Index der kulturellen Kommunikation entwickelt; mittels der kombinierten Antworten auf die drei letzten Fragen wurde ein Index der sozialen Kommunikation konstruiert. Für beide Indizes bedeuten Werte oberhalb 


\section{Abbildung 6.3}

Soziale und kulturelle Kommunikation mit den Eltern und Schülerleistungen Index der sozialen Kommunikation und Index der kulturellen Kommunikation

Index der sozialen Kommunikation Index der kulturellen Kommunikation

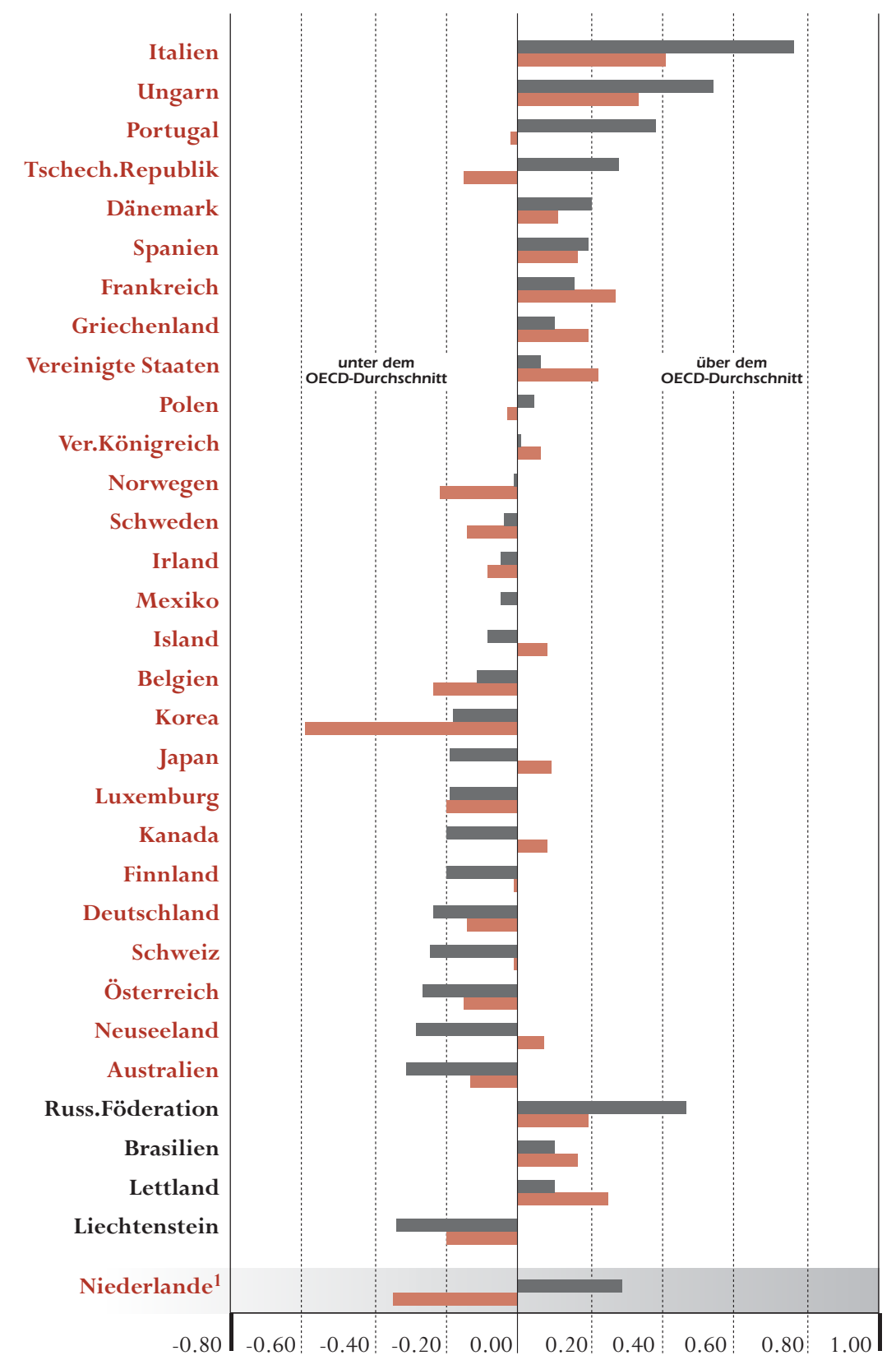

1. Die Beteiligungsquote ist zu niedrig, um die Vergleichbarkeit zu gewährleisten (vgl. Anhang A3). Anmerkung: Zur Definition der Indizes siehe Anhang A1.

Quelle: OECD PISA Datenbank, 2001. Tabellen 6.5 und 6.6. 
KAPITEL 6 Familiärer Hintergrund und Schülerleistungen

Bessere Ergebnisse erzielen diejenigen, die mehr

kommunizieren, insbesondere über kulturelle Themen. des OECD-Durchschnitts, der mit Null angesetzt wurde, relativ hohe Kommunikationsniveaus zwischen Schülern und ihren Eltern. Dabei ist anzumerken, dass unterdurchschnittliche Werte nicht das Fehlen von Kommunikation anzeigen, sondern nach Angaben der Schülerinnen und Schüler lediglich besagen, dass Kommunikation und Interaktion weniger häufig stattfinden als im Durchschnitt der OECD-Länder.

Nach den Aussagen der Schülerinnen und Schüler erzielen italienische Eltern, gefolgt von Eltern in Ungarn und der Russischen Föderation, das höchste Niveau bei der Kommunikation über soziale Fragen mit ihren Kindern (Tabelle 6.5 und Abbildung 6.3). In diesen drei Ländern geben z.B. über 70\% der Schülerinnen und Schüler an, dass ihre Eltern mit ihnen über ihre Schulleistungen sprechen oder sich mehrmals in der Woche Zeit nehmen, "um einfach nur mit ihnen zu reden” (Daten abrufbar unter www.pisa.oecd.org). Die Schülerinnen und Schüler in Italien geben zudem das höchste Kommunikationsniveau bei kulturellen Themen an, gefolgt von Schülerinnen und Schülern in Ungarn, Frankreich und Lettland (Tabelle 6.6 und Abbildung 6.3). In Brasilien, Ungarn, Italien und der Russischen Föderation geben mehr als 40\% der Schülerinnen und Schüler an, dass ihre Eltern mehrmals in der Woche mit ihnen über Bücher, Filme oder Fernsehsendungen sprechen. Dieser Prozentsatz steht im Gegensatz zu der Situation in mehreren anderen Ländern, in denen mindestens 40\% der Schüler nie oder nur einige Male im Jahr mit ihren Eltern über Bücher oder Filme sprechen (Daten abrufbar unter www.pisa.oecd.org).

In jedem Land gilt für beide Indizes, dass mit zunehmender Kommunikation und Interaktion der Durchschnitt der Mittelwerte auf der Gesamtskala Lesekompetenz steigt ${ }^{7}$. Im Durchschnitt steht die Kommunikation über kulturelle Themen in engerer Beziehung zu den Lesekompetenz-Punktzahlen als die soziale Kommunikation. Veranschaulicht wird dies durch die Tatsache, dass die durchschnittliche OECD-Punktzahl für Lesekompetenz von 471 für das untere Quartil des Index der kulturellen Kommunikation auf 530 für das obere Quartil (eine Differenz von nahezu 60 Punkten) und im Fall des Index der sozialen Kommunikation von 481 Punkten auf 511 (eine Differenz von 30 Punkten) steigt (Tabelle 6.5 und 6.6) ${ }^{8}$.

Wiederum ist anzumerken, dass der Effekt der Kommunikation mit den Eltern im Zusammenhang mit anderen Hintergrundfaktoren gesehen werden muss (vgl. auch Kapitel 8). Gleichwohl zeigt diese einfache Analyse, dass der Bildungserfolg mit positiven Synergien zwischen dem Elternhaus und dem schulischen Umfeld in Zusammenhang stehen kann, und dass die Kommunikation zwischen Eltern und Kindern die Leistungen der Kinder positiv beeinflussen kann. Daher könnte es für die staatliche Politik ein wichtiges Ziel sein, mit den Eltern zusammenzuarbeiten, insbesondere mit denjenigen, die niedrigere Bildungsabschlüsse vorweisen, um deren Interaktion mit ihren Kindern und den Schulen ihrer Kinder so zu fördern, dass das Lernen der Kinder begünstigt wird. Dies ist keine leichte Aufgabe, aber es ist wichtig, die Unterstützung 
der Eltern bei ihren erzieherischen Aufgaben als ein Schlüsselelement für die Bildung der Kinder anzusehen.

\section{Bildung der Eltern}

Einschlägigen Forschungsarbeiten ist durchgängig zu entnehmen, dass das Bildungsniveau der Eltern trotz der erheblichen Bemühungen der Länder um Gewährleistung gleicher Bildungschancen für alle weiterhin eine wichtige Ursache von Unterschieden in den Schülerleistungen ist. Ein besonders förderliches Lernumfeld im Elternhaus, zu dem ein hoher Bildungsabschluss des Vaters oder der Mutter beitragen kann, wird sich wahrscheinlich in höheren Bildungsergebnissen der Kinder niederschlagen. Ein solches günstiges Umfeld kann nicht nur in Form der finanziellen Mittel zum Ausdruck kommen, die für die Bildung der Kinder - einschließlich der Hochschulbildung - und deren daraus resultierenden höheren beruflichen Status aufgewendet werden, sondern ebenfalls in Form regelmäßiger Interaktionen zwischen Eltern und Kindern, die für die Art der von den Schulen vermittelten Bildung von größerem Wert ist.

Abbildung 6.4 gibt drei Kategorien von Bildungsabschlüssen (Primar- oder Sekundarstufe I, Sekundarstufe II und Hochschule) für die Mütter der Schülerinnen und Schüler an und führt mittlere Punktzahlen der Lesekompetenz für jedes Land auf. Diese Kategorien sind so definiert, dass ihre internationale Vergleichbarkeit auf der Basis der Internationalen Standardklassifikation des Bildungswesens (ISCED) gegeben ist. Die Bildung der Mutter wurde gewählt, da sie in der Fachliteratur häufig als stärkerer Prädiktor für die Schülerleistungen identifiziert wurde als die Bildung des Vaters.

Schülerinnen und Schüler, deren Mütter die Sekundarstufe II abgeschlossen haben, weisen in allen Ländern höhere Lesekompetenzen auf als andere Schüler, und in den meisten Ländern bedeutet der Hochschulabschluss der Mutter einen zusätzlichen Vorteil (Tabelle 6.7).

Schülerinnen und Schüler, deren Mütter die Sekundarstufe II nicht abgeschlossen haben, sind besonders benachteiligt. In allen OECD-Ländern liegen die mittleren Punktzahlen der Schülerinnen und Schüler, deren Mütter zu dieser Kategorie gehören, hinsichtlich der Lesekompetenz sowie der mathematischen und naturwissenschaftlichen Grundbildung um rund 45 Punkte niedriger als für diejenigen, deren Mütter die Sekundarstufe II abgeschlossen haben

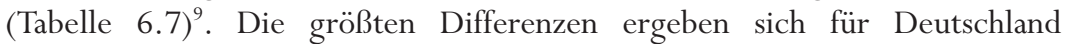
(99 Punkte), Mexiko (75 Punkte), Belgien und die Schweiz (je 73 Punkte) sowie die Tschechische Republik (71 Punkte). In diesen Ländern besteht für die Schülerinnen und Schüler, deren Mütter keinen Abschluss der Sekundarstufe II erreicht haben, eine 2,1 bis 3-mal so hohe Wahrscheinlichkeit, Leistungen im unteren nationalen Quartil der Schülerpopulation zu erzielen, als für Schülerinnen und Schüler, deren Mütter die Sekundarstufe II abgeschlossen haben. Die Größenordnung des Nachteils der Schülerinnen und Schüler, deren Mütter die Sekundarstufe II nicht abgeschlossen haben, wird in Abbildung 6.4
Das Bildungsniveau der Eltern kann ebenfalls zu einem günstigen familiären Umfeld beitragen.

Die Bildung der Mütter steht in positivem Zusammenhang mit den Schülerleistungen...

... mit dem Ergebniss, dass niedrige Leistungen unter den Schülerinnen und schülern auftreten, deren Mütter die Sekundarstufe II nicht abgeschlossen haben,... 
KAPITEL 6 Familiärer Hintergrund und Schülerleistungen

\section{Abbildung 6.4}

Bildungsabschluss der Mütter und Schülerleistungen

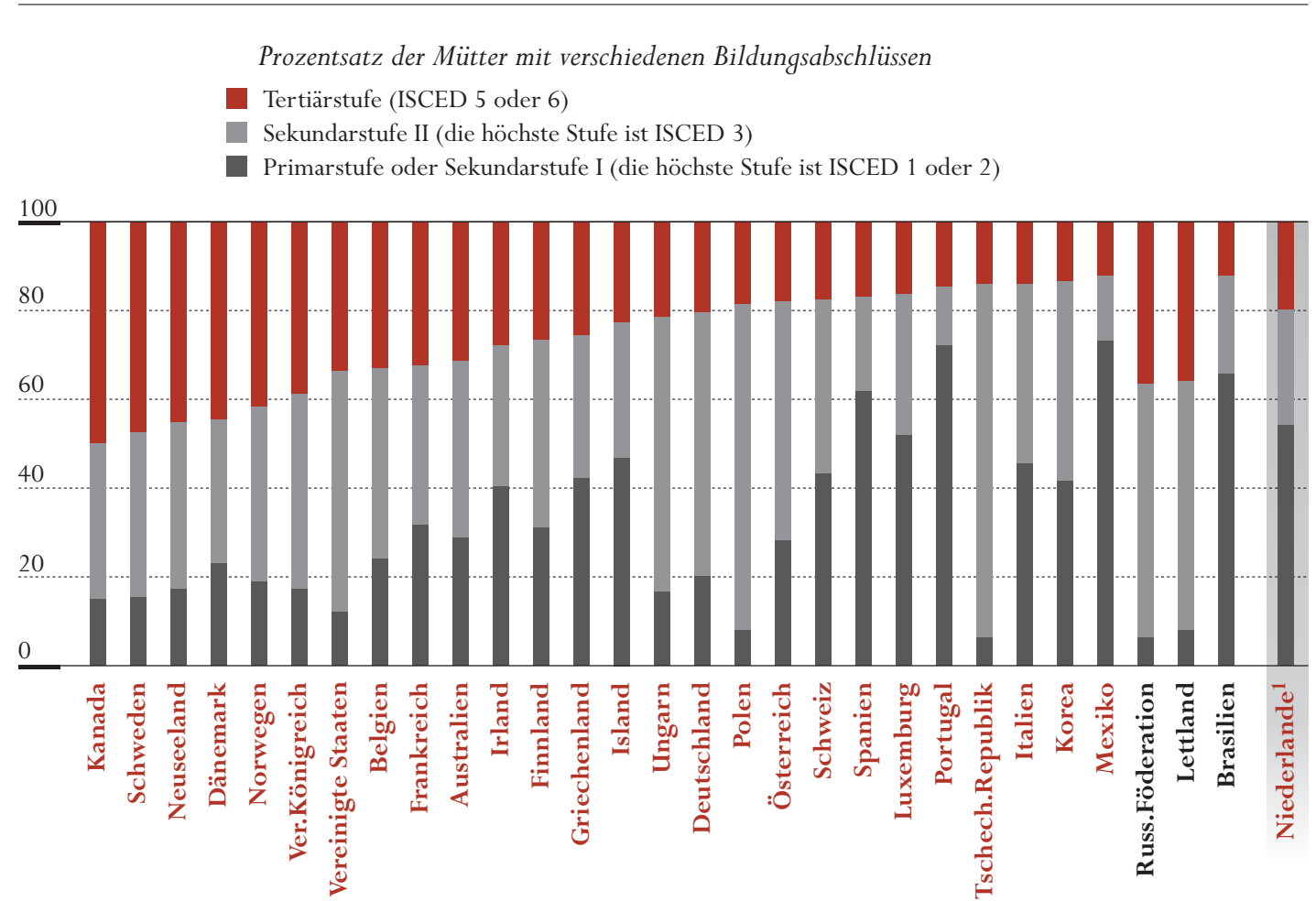

GrößereWahrscheinlichkeit für Schülerinnen und Schüler, deren Mütter die Sekundarstufe II nicht abgeschlossen haben, im untersten nationalen Quartil der Gesamtskala Lesekompetenz zu liegen ${ }^{2}$

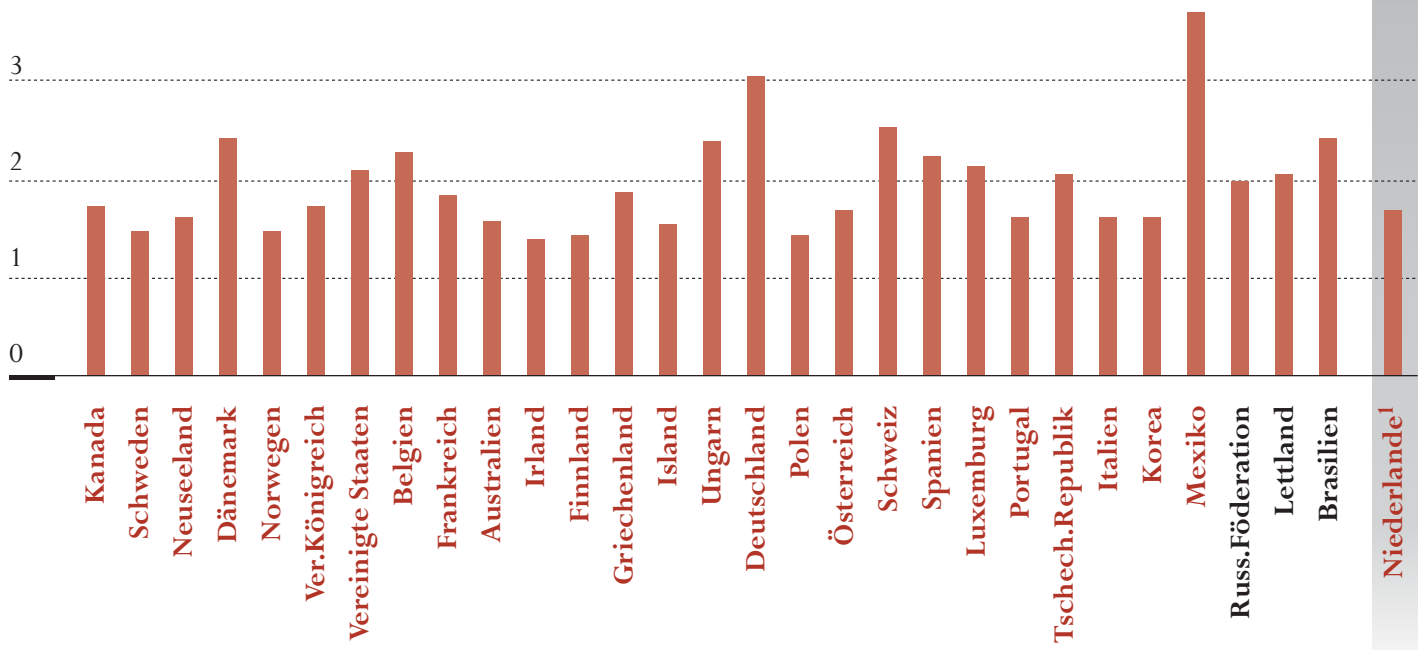

1. Die Beteiligungsquote ist zu niedrig, um die Vergleichbarkeit zu gewährleisten (vgl. Anhang A3).

2. Für alle Länder ist der Quotient statistisch signifikant größer als 1.

Quelle: OECD PISA Datenbank, 2001. Tabelle 6.7. 
(zweiter Teil) zusammengefasst, in der die erhöhte Wahrscheinlichkeit ausgewiesen wird, mit der sich diese Kinder im unteren Quartil der nationalen Verteilung der Leseleistung befinden. Trotz dieser Ergebnisse hat der erwartete Zusammenhang zwischen der Bildung der Mutter und den Schülerleistungen keinen universellen und daher möglicherweise auch nicht automatischen oder unvermeidlichen Charakter.

Ein Hochschulabschluss der Mutter bedeutet in einigen Ländern keinen oder nur einen geringen zusätzlichen Pluspunkt für die mittleren Leistungen ihres Kindes gegenüber dem Effekt, der aus dem Abschluss der Sekundarstufe II resultiert. In anderen Ländern ergeben sich daraus hingegen große Unterschiede.

Wenngleich aus den Daten hervorgeht, dass die Schülerinnen und Schüler, deren Mütter die niedrigsten Bildungsabschlüsse aufweisen, häufig die niedrigsten mittleren Punktzahlen erzielen, sollten solche „niedrigen“ Punktzahlen in ihrem jeweiligen Zusammenhang betrachtet werden. In Australien, Finnland und Korea liegen die mittleren Punktzahlen dieser Schülerinnen und Schüler in allen drei Grundbildungsbereichen über dem OECD-Durchschnitt. Schülerinnen und Schüler, deren Mütter die niedrigsten Bildungsabschlüsse haben, liegen in Irland im Bereich Lesekompetenz und in Kanada, Island und Neuseeland im Bereich mathematische Grundbildung über dem OECD-Durchschnitt (vgl. Kapitel 2 undTabelle 6.7). Dies deutet ebenfalls darauf hin, dass andere bildungsund gesellschaftsbezogene Faktoren elterliche Bildungsdefizite kompensieren können.

In Gesellschaften, die nach Chancengleichheit streben, besteht immer noch eine enge Beziehung zwischen den Bildungsergebnissen der Schülerinnen und Schüler und dem Bildungsabschluss der Mütter. Wenngleich die PISADaten nicht für eine direkte Vorherbestimmung der Leistungsunterschiede im Bildungsverlauf der über 15-Jährigen verwendet werden können, liefern die Daten der Internationalen Erhebung über Grad und Verteilung elementarer Grundqualifikationen Erwachsener (IALS) Erkenntnisse über die Wahrscheinlichkeit, mit der verschiedene Gruppen einen Hochschulabschluss erzielen werden, wenn die Eltern unterschiedliche Bildungsabschlüsse vorweisen. Aus Tabelle 6.8 geht hervor, wie hoch die Chance für einen Hochschulabschluss ist, wenn zumindest ein Elternteil einen entsprechenden Abschluss erreicht hat, sowie die Wahrscheinlichkeit, einen solchen Abschluss zu erhalten, wenn keiner der beiden Elternteile die Sekundarstufe II abgeschlossen hat.

In den 12 der in IALS erfassten Länder reicht die generationsübergreifende Mobilität von 2,0 in Australien bis zu 5,8 in Polen; d.h. dass die Wahrscheinlichkeit, einen Hochschulabschluss zu erlangen, in Australien zweimal so hoch und in Polen fast sechsmal so hoch ist, wenn die Eltern einen hohen Bildungsabschluss haben, als dann, wenn die Eltern ein niedriges Bildungsniveau vorweisen. Diese Ergebnisse lassen darauf schließen, dass in vielen OECDLändern, dabei in einigen mehr als in anderen, die Bildungsmobilität weiterhin
... während ein Hochschulabschluss weniger durchgängig mit den Schülerleistungen assoziiert ist.

In einigen Ländern schneiden auch Schülerinnen und Schüler mit weniger gebildeten Müttern im internationalen vergleich gut ab, ...
... und möglicherweise den Einfluss der verbleibenden und immer noch beträchtlichen Hindernisse für Bildungsmobilität mindern können. 
KAPITEL 6 Familiärer Hintergrund und Schülerleistungen

durch Hemmnisse eingeschränkt ist, die den Bemühungen um Chancengleichheit und um eine Anhebung des allgemeinen Niveaus des vorhandenen Humankapitals zuwiderlaufen.

\section{Familienstruktur}

Alleinerziehende könnten es im vergleich zu

Elternpadren als schwieriger empfinden, Schülerinnen und Schülern ausreichende Unterstützung zu gewähren.

Schülerinnen und Schüler aus Ein-Eltern-Familien erzielen in einigen Ländern niedrigere Leistungen ...

und der Abstand ist dort am größten, wo Ein-Eltern-Familien am häufigsten sind, ...
Auch das familiäre Umfeld kann die schulischen Leistungen fördern. Die Eltern können jungen Lernenden vorlesen, ihnen bei den Hausaufgaben helfen und in einigen Ländern ehrenamtlich Aufgaben in der Schule übernehmen. Für ältere Schülerinnen und Schüler kann ein unterstützendes familiäres Umfeld ebenfalls im Hinblick auf Hausaufgaben, Ermutigung sowie Teilnahme an Zusammenkünften mit Lehrkräften oder Angehörigen der Schulverwaltung von Bedeutung sein. Ein solches Umfeld zu schaffen und auf Dauer beizubehalten, dürfte mitunter schwierig sein, wenn die Schülerinnen und Schüler in EinEltern-Familien leben, in denen die Eltern häufig die Doppelbelastung von Beruf und Kindererziehung zu tragen haben.

In den PISA-Kontextfragebogen wurden die Schülerinnen und Schüler danach gefragt, wer normalerweise mit ihnen zusammen im Elternhaus lebt. Anhand der Antworten können die Leistungen der Schülerinnen und Schüler aus verschiedenen Familientypen miteinander verglichen werden ${ }^{10}$. Unter allen OECD-Ländern erklären rund 15\% der in PISA erfassten Schülerinnen und Schüler, dass sie in Ein-Eltern-Familien leben (Tabelle 6.9). Den höchsten Anteil weisen mit etwas über 20\% Lettland, Neuseeland, das Vereinigte Königreich und die Vereinigten Staaten auf, während Griechenland, Korea und Polen mit weniger als $10 \%$ den niedrigsten Anteil verzeichnen. In den meisten OECDLändern zeigen die PISA-Ergebnisse, dass Schülerinnen und Schüler in EinEltern-Familien weniger gut abschneiden als ihre Mitschüler aus anderen Familientypen ${ }^{11}$.

Global besteht hinsichtlich der Leistung auf der Gesamtskala Lesekompetenz eine Differenz von 12 Punkten zu Ungunsten der Schülerinnen und Schüler aus Ein-Eltern-Familien. Die größten Unterschiede, d.h. mindestens 28 Punkte, sind in den Niederlanden, im Vereinigten Königreich und den Vereinigten Staaten zu beobachten (Tabelle 6.9). Das heißt, zwei der drei Länder mit dem höchsten Anteil an Schülerinnen und Schülern aus Ein-Eltern-Familien weisen auch die höchsten Differenzen bei den mittleren Punktzahlen für Lesekompetenz auf. Mit anderen Worten ist nicht nur die Zugehörigkeit zu einer Ein-Eltern-Familie im Vereinigten Königreich und in den Vereinigten Staaten häufiger, sondern auch die Auswirkungen für die schulischen Leistungen scheinen dort ausgeprägter ${ }^{12}$. Weitere Länder mit vergleichsweise großen Differenzen sind u.a. Belgien, Finnland, Frankreich, Dänemark, Irland, Korea, Neuseeland, Norwegen und Schweden. 
Allerdings wird dieser Zusammenhang durch das Einwirken anderer sozialer Hintergrundfaktoren, wie z. B. Wohlstand, häufig verstärkt (vgl. auch Kapitel 8), wenn auch in unterschiedlichem Ausmaß, da das Sozialprofil Alleinerziehender stark variiert.

Fragen in Bezug auf die Familienstruktur sind nicht unproblematisch, sind zugleich aber auch von großer Bedeutung. Erkenntnisse, wonach Kinder in Familien mit beiden Elternteilen höhere Leistungen erzielen, dürften für Personen, für die ein Ein-Eltern-Familien-Arrangement aus außerschulischen Gründen vorzuziehen und in jedem Fall bereits Realität ist, nicht gerade ein Trost sein. Wenn andererseits nachteilige Auswirkungen erwiesen sind, müssen Abhilfemöglichkeiten gesucht werden. Dabei geht es darum, wie das Lernen der Kinder durch konstruktive Unterstützung im Elternhaus gefördert werden kann, ohne dass von den Eltern ein Zeitaufwand gefordert wird, der die Möglichkeiten Alleinerziehender übersteigt. Eine strategische Zeiteinteilung zu Gunsten von Aktivitäten mit dem höchsten potentiellen Ertrag steigert die Effizienz, wenn nur begrenzte Zeit zur Verfügung steht. Die Grundsatzfrage für die Bildungssysteme und die einzelnen Schulen, die mit den Eltern in Kontakt stehen, lautet, welche Arten elterlichen Engagements gefördert werden können.

\section{Geburtsort und im Elternhaus gesprochene Sprache}

Migration von einem Land in ein anderes wird immer mehr zur Normalität, da die internationalen Handelsbeziehungen sich ausweiten, Beschäftigungsmöglichkeiten die Menschen dorthin ziehen, wo ihnen bessere oder andere Existenzgrundlagen geboten werden, und Staaten Flüchtlingen beistehen, die vor politischen Unruhen oder wirtschaftlicher Not geflohen sind. Unabhängig von den Gründen, die Menschen zur Migration veranlassen, sehen sich ihre schulpflichtigen Kinder häufig einem neuen Umfeld gegenüber, in dem sie in mancher Weise anders sind oder dessen Unterrichtssprache ihnen fremd ist. Wenngleich der Migrationskontext von Land zu Land nur sehr schwer verglichen werden kann, könnte eine länderübergreifende Analyse gewisse Erkenntnisse über die Gegebenheiten liefern, mit deren Hilfe einige Länder diesen Ungleichheiten erfolgreicher begegnen als andere.

Um die mit dem Migrationsstatus und der gesprochenen Sprache verbundenen Effekte auf die Leistungen in den drei Grundbildungsbereichen untersuchen zu können, wurden die Schülerinnen und Schüler in dem PISA-Kontextfragebogen aufgefordert anzugeben, ob sie selbst und jeder ihrer beiden Elternteile in dem Land geboren wurden, in dem sie leben, oder in einem anderen Land. Sie wurden ebenfalls gefragt, welche Sprache sie normalerweise zu Hause sprechen.
... doch handelt es sich um einen komplexen

Zusammenhang, bei dem viele Faktoren eine Rolle spielen.

Migration kann Bildungsprobleme verstärken,...

... und daher wurden die Schülerinnen und Schüler in PISA zu ihrem

Migrationsstatus und der zu Hause gesprochenen sprache befragt. 
KAPITEL 6 Familiärer Hintergrund und Schülerleistungen

Diese Fragen decken allerdings nicht die ganze Bandbreite an Situationen ab, in denen sich Kinder aus Migrantenfamilien befinden.

In der Analyse werden ...

... die Gruppe 1 (im Inland geborene Schülerinnen und

schüler mit mindestens einem im Inland geborenen Elternteil),...

... die Gruppe 2 (im Inland geborene Schülerinnen und

Schüler mit im Ausland geborenen Eltern)...

... und die Gruppe 3 (im Ausland geborene Schülerinnen und Schüler mit im Ausland geborenen Eltern) miteinander verglichen.

Für viele Schülerinnen und Schüler, die im Ausland geboren wurden oder deren Eltern aus dem Ausland stammen, ist die Sprache ein Schlüsselfaktor.
Es ist wichtig, den begrenzten Charakter der vorhandenen Daten zu erkennen. PISA stellte den Schülerinnen und Schülern nicht die Frage, wie lange sie schon in dem Land leben, in dem die Erhebung durchgeführt wurde. Während es sich bei vielen Schülerinnen und Schülern um Kinder von zugewanderten Eltern handelt, die die Unterrichtssprache wahrscheinlich fließend sprechen, waren andere zweifellos erst vor kurzer Zeit zugewandert und besuchten die Schule in ihrem „neuen“ Land lediglich im zweiten Jahr ${ }^{13}$. Es liegen ebenfalls keine Informationen darüber vor, wie ähnlich oder verschieden die Erstsprache eines Schülers im Vergleich zur Unterrichtssprache ist. Es ist anzunehmen, dass die Punktzahl eines Schülers, dessen Eltern z. B. in Neuseeland geboren wurden und der nunmehr in Australien oder den Vereinigten Staaten lebt, weniger von diesen Umständen beeinflusst wird als im Fall eines Schülers, dessen Eltern in einem Land geboren wurden, in dem z.B. eine andere Sprache als Englisch gesprochen wird. Ganz unabhängig davon, wie entgegenkommend sich die Schulen in den „neuen“ Ländern verhalten, dürften die Sprachunterschiede die Leseleistungen in der Zweitsprache (oder die Leistungen in mathematischer bzw. naturwissenschaftlicher Grundbildung) senken, insbesondere bei erst seit kurzer Zeit im Land ansässigen Schülerinnen und Schülern.

Um den Einfluss des Geburtsorts auf die Leistungen zu bewerten, wurden - wie in Abbildung 6.5 dargestellt - drei Schülerkategorien miteinander verglichen:

- Schülerinnen und Schüler, die in dem Erhebungsland geboren wurden und bei denen zumindest ein Elternteil ebenfalls in diesem Land geboren wurde (nachfolgend als „Gruppe 1“ bezeichnet);

- Schülerinnen und Schüler, die in dem Erhebungsland geboren wurden, deren Eltern jedoch in einem anderen Land geboren wurden (nachfolgend als „Gruppe 2“ bezeichnet);

- Schülerinnen und Schüler, die außerhalb des Erhebungslandes geboren wurden und deren Eltern ebenfalls in einem anderen Land geboren wurden (nachfolgend als „Gruppe 3“ bezeichnet).

Für viele Angehörigen der Gruppe 3 ist die Testsprache eine Zweitsprache, und einige von ihnen haben noch keine langjährigen Erfahrungen mit dem Bildungssystem des Landes, in dem der Test stattfand. Die Schülerinnen und Schüler der Gruppe 3 leben zudem in Familien, in denen die Erstsprache bzw. die zu Hause gesprochene Sprache möglicherweise nicht die Unterrichtssprache ist. Die zur zweiten oder dritten Gruppe gehörenden Schülerinnen und Schüler müssen unabhängig von ihrem Geburtsort die gleichen Kenntnisse 
und Fähigkeiten erwerben, die von den Angehörigen der Gruppe 1 erwartet werden, je näher sie dem Abschluss ihrer regulären Bildung kommen.

Bei einem Vergleich der Lesekompetenz von Schülerinnen und Schülern der Gruppe 2 und von Angehörigen der Gruppe 1 in den Ländern, in denen Angehörige der Gruppe 2 mindestens 3\% der in PISA 2000 erfassten Schülerinnen und Schüler ausmachen, treten in zehn Ländern verhältnismäßig große und statistisch signifikante Unterschiede zu Gunsten der Angehörigen der Gruppe 1 zu Tage. Auf der Gesamtskala Lesekompetenz liegen die Leistungsunterschiede in Frankreich, Neuseeland, Schweden und den Vereinigten Staaten zwischen 31 und 41 Punkten, in Österreich, Liechtenstein und der Schweiz bei etwa 53 bis 62 Punkten, in Deutschland, Luxemburg und den Niederlanden bei über 70 Punkten, d.h. ungefähr eine volle Kompetenzstufe, und steigen in Belgien auf 112 Punkte (Tabelle 6.10). Ein ähnliches Muster gilt für die Beziehung zwischen Geburtsort und Leistungen auf den Skalen für mathematische und naturwissenschaftliche Grundbildung.

Diese Unterschiede sind beunruhigend, da beide Schülergruppen im Erhebungsland geboren wurden und anzunehmen ist, dass sie durch die gleichen Lerninhalte geprägt wurden, die im Rahmen des jeweiligen nationalen Bildungssystems allen Schülern vermittelt werden. Trotz aller möglichen Ähnlichkeiten des individuellen schulischen „Werdegangs“ ist die Tatsache, zur Gruppe $2 \mathrm{zu}$ gehören, in diesen Ländern in irgendeiner Form mit einem relativen Nachteil verbunden. Besorgnis über derartige Differenzen ist besonders in den Ländern angebracht, in denen signifikante Leistungsunterschiede und vergleichsweise hohe Prozentsätze an Angehörigen der Gruppe 2 vorhanden sind, insbesondere in Belgien (8\%), Frankreich (10\%), Luxemburg (18\%) und der Schweiz (9\%).

Wie zu erwarten, liegen die Schülerinnen und Schüler der Gruppe 3 in der Regel leistungsmäßig sogar noch deutlicher hinter denen der Gruppe 1 als die der Gruppe 2. Im Durchschnitt übertreffen in 10 der 16 verglichenen Länder die Angehörigen der Gruppe 1 ihre Mitschülerinnen und Mitschüler der Gruppe 3 in der Lesekompetenz um mindestens 71 Punkte bzw. eine volle Kompetenzstufe. Die Differenz liegt in Liechtenstein, Luxemburg und der Schweiz zwischen 103 und 112 Punkten sowie in Österreich, Belgien, Dänemark, Deutschland, Griechenland, den Niederlanden, Norwegen und Schweden zwischen 60 und 100 Punkten. Die kleinsten, aber immer noch statistisch signifikanten Differenzen sind in Kanada (27 Punkte), Neuseeland (30 Punkte) und den Vereinigten Staaten (45 Punkte) festzustellen. Für Neuseeland entspricht der Unterschied in etwa dem zwischen Schülerinnen und Schülern der Gruppen 3 und 2 in diesem Land.
In den meisten Ländern mit einem bedeutsamen Anteil an Immigranten liegen die Schülerinnen und Schüler der Gruppe 2 in der Lesekompetenz weit unter dem Niveau der schülerinnen und Schüler von Gruppe 1,...

... und das frappierenderweise, obwohl sie selbst im Land geboren wurden.

Im Ausland geborene Schülerinnen und Schüler liegen sogar noch weiter zurück, allerdings in höchst unterschiedlichem Ausmaßs in den einzelnen Ländern. 
KAPITEL 6 Familiärer Hintergrund und Schülerleistungen

\section{Abbildung 6.5}

Geburtsort, zu Hause gesprochene Sprache und Schülerleistungen

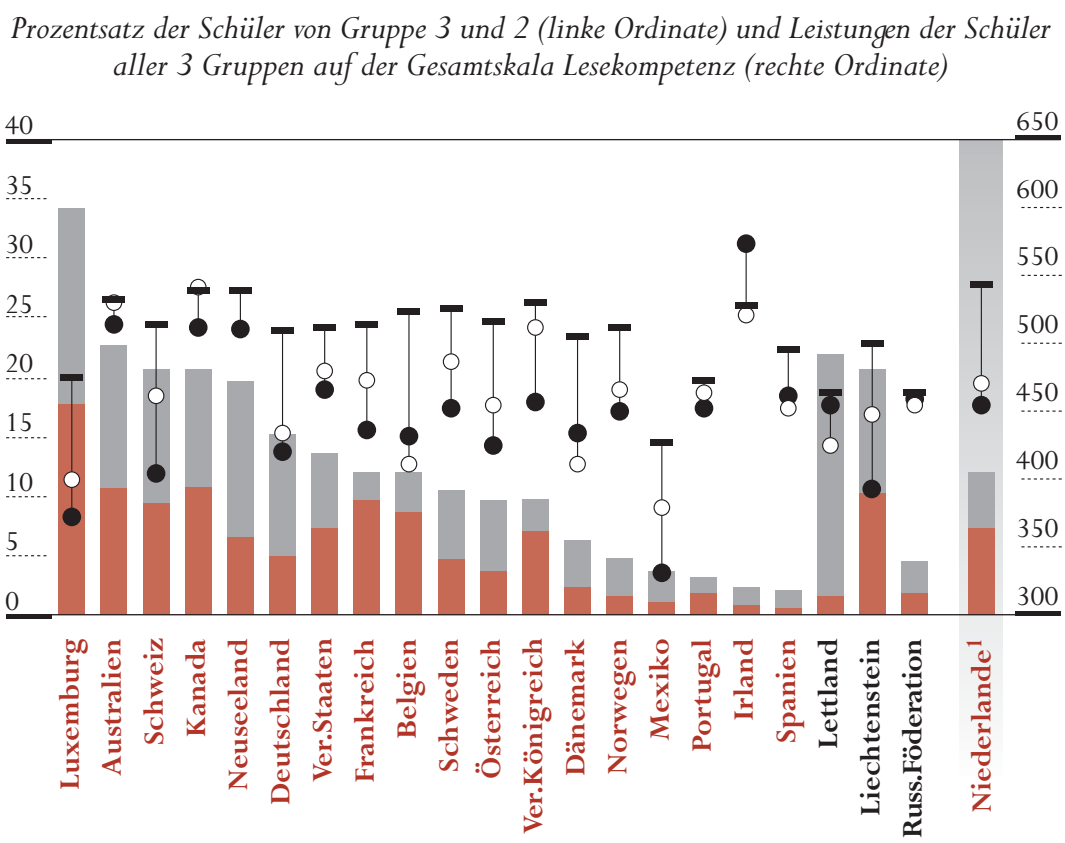

Prozentsatz der Schüler, die zu Hause normalerweise eine andere Sprache sprechen als die Testsprache (linke Ordinate) und Leistungen auf der Gesamtskala Lesekompetenz (rechte Ordinate), nach Sprachgruppen

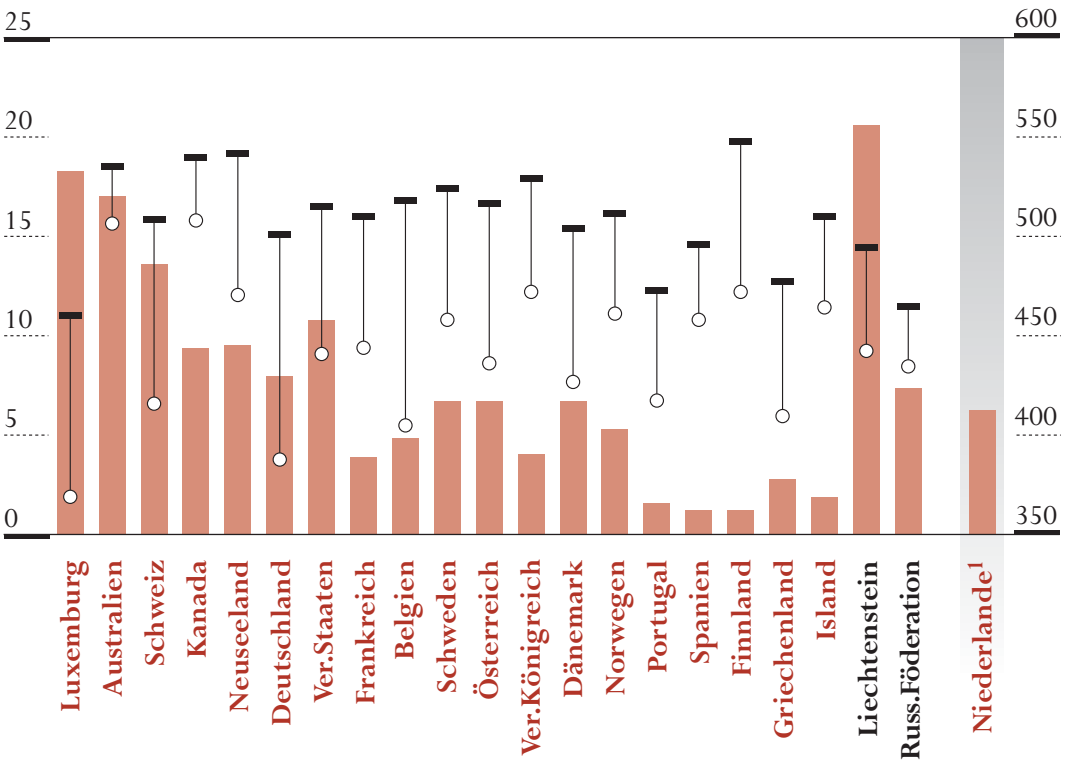

1. Die Beteiligungsquote ist zu niedrig, um die Vergleichbarkeit zu gewährleisten (vgl. Anhang A3). Quelle: OECD PISA Datenbank, 2001. Tabelle 6.10.

\section{Linke Ordinate}

Prozentsatz der im Ausland geborenen Schülerinnen und Schüler mit im Ausland geborenen Eltern (Gruppe 3)

Prozentsatz der im Inland geborenen Schülerinnen und Schüler mit im Ausland geborenen Eltern (Gruppe 2)

\section{Rechte Ordinate}

- Durchschnittsleistungen auf der Gesamtskala

Lesekompetenz von im Inland geborenen

Schülerinnen und Schülern mit mindestens einem im

Inland geborenen Elternteil (Gruppe 1)

Durchschnittsleistungen auf der Gesamtskala

Lesekompetenz von im Inland geborenen

Schülerinnen und Schülern mit im Ausland geborenen

Eltern (Gruppe 2)

- Durchschnittsleistungen auf der Gesamtskala Lesekompetenz von im Ausland geborenen Schülerinnen und Schülern mit im Ausland geborenen Eltern (Gruppe 3)

\section{Linke Ordinate}

Prozentsatz der Schülerinnen und Schüler, die zu Hause normalerweise eine Sprache sprechen, die sich von der Testsprache, anderen offiziellen Landessprachen oder nationalen Dialekten unterscheidet.

Rechte Ordinate

- Leistungen auf der Gesamtskala Lesekompetenz von Schülerinnen und Schülern, die zu Hause normalerweise die gleiche Sprache sprechen wie die Testsprache, eine andere offizielle Landessprache oder andere nationale Dialekte.

$\bigcirc$ Leistungen auf der Gesamtskala Lesekompetenz von Schülerinnen und Schülern, die zu Hause normalerweise eine andere Sprache sprechen als die Testsprache, eine andere offizielle Landessprache oder andere nationale Dialekte. 
Australien und Kanada bilden eine interessante Ausnahme von dem allgemeinen Schema. In Australien scheinen weder der Geburtsort der Schülerinnen und Schüler noch der Geburtsort ihrer Eltern die Leseleistung sowie die Leistungen in mathematischer und naturwissenschaftlicher Grundbildung zu beeinflussen. Australien erzielt diese Ergebnisse trotz hoher Anteile an Angehörigen der Gruppe 3 (12\% aller Schülerinnen und Schüler) und der Gruppe 2 (11\%). Ähnliche Erfolgsmuster werden auch in Kanada beobachtet, da die Leistungen der drei Gruppen der kanadischen Schülerinnen und Schüler in zwei der drei Grundbildungsbereiche statistisch über dem OECD-Durchschnitt liegen.

Es ist anzunehmen, dass Schülerinnen und Schüler, die im Elternhaus normalerweise die Testsprache oder eine andere Landessprache bzw. einen nationalen Dialekt sprechen („Schüler mit majoritätssprachlichem Hintergrund“), in PISA höhere Leistungen erzielen als Schülerinnen und Schüler, die mit ihren Eltern und Geschwistern üblicherweise in einer anderen Sprache kommunizieren („Schüler mit minoritätssprachlichem Hintergrund“) ${ }^{14}$. Global bestätigen die Daten diese Annahme. In den OECD-Ländern beträgt die durchschnittliche Differenz zwischen den zwei Gruppen 66 Punkte in der Lesekompetenz und in der naturwissenschaftlichen Grundbildung sowie 49 Punkte in der mathematischen Grundbildung (Tabelle 6.11).

Aus diesen Unterschieden folgt u.a., dass für die 15-jährigen Schülerinnen und Schüler in Belgien, Dänemark, Deutschland, Luxemburg und der Schweiz, die zu Hause nicht die Testsprache oder eine andere offizielle Landessprache bzw. einen nationalen Dialekt sprechen, die Wahrscheinlichkeit zumindest zweieinhalbmal so hoch ist, zu den leistungsschwächsten 25\% der Schülerinnen und Schüler im Bereich Lesekompetenz zu gehören, wie für Schülerinnen und Schüler, die normalerweise die Testsprache sprechen. In Österreich, Frankreich, Griechenland, den Niederlanden, Neuseeland, Schweden und den Vereinigten Staaten ist für Schülerinnen und Schüler mit minoritätssprachlichem Hintergrund die Wahrscheinlichkeit mehr als zweimal so hoch, in allen Grundbildungsbereichen im unteren Quartil zu liegen, wie für Schülerinnen und Schüler mit majoritätssprachlichem Hintergrund.

Die Unterschiede zwischen Schülerinnen und Schülern mit majoritäts- und minoritätssprachlichem Hintergrund hinsichtlich der mittleren Punktzahl im Bereich Lesekompetenz beeinflussen eindeutig das Gesamtergebnis der Länder in diesem Sektor. Wenn es Deutschland z.B. gelänge, die mittleren Punktzahlen der minoritätssprachlichen Schülerinnen und Schüler auf das Niveau der majoritätssprachlichen anzuheben, würde die mittlere Punktzahl dieses Landes im Bereich mathematische Grundbildung über dem OECD-Durchschnitt von 500 Punkten liegen und nicht 10 Punkte darunter.
Einige Länder stechen hervor, da es ihnen anscheinend gelingt, diese Differenzen zu vermeiden.

Nicht überraschend ist, dass Schülerinnen und Schüler, die zu Hause nicht die Majoritätssprache sprechen, wesentlich schlechter abschneiden als diejenigen, bei denen das der Fall ist, ...

... und eine beträchtlich höhere Wahrscheinlichkeit aufweisen, im unteren Schülerquartil ihres Landes zuliegen, ... 
KAPITEL 6 Familiärer Hintergrund und Schülerleistungen

Die Tatsache, dass die

Auswirkungen des

familiären Hintergrunds

auf die Schülerleistungen

von Land zu Land

unterschiedlich hoch sind,

weckt die Hoffnung, dass

diese Einflüsse gemindert

werden können.

Die berufliche Stellung der

Eltern ist eng mit den

Schülerleistungen

verbunden, ...

... und dasselbe gilt für

Besitz und Aktivitäten im

Zusammenhang mit

"klassischen" Kulturgütern.

Die Bildung der Eltern und die Qualität ihres Kommunikationsverhaltens gegenüber den Kindern wirken sich ebenfalls positiv aus, ...

\section{Schlussbetrachtungen}

Ziel der öffentlichen Bildungspolitik muss es sein, allen Schülerinnen und Schülern gleiche Chancen zu bieten, um ihr Potential voll zu entwickeln. Wenngleich der starke Einfluss familiärer Hintergrundfaktoren auf die Schülerleistungen der Erreichung dieses Ziels im Wege steht, gibt doch die Tatsache, dass diese Effekte in den einzelnen Ländern sehr unterschiedlich zum Tragen kommen, Grund zu der optimistischen Annahme, dass mehr Chancengleichheit im Bildungswesen realisierbar ist.

Die im vorliegenden Kapitel untersuchten Aspekte des familiären Hintergrunds stehen miteinander in Beziehung, und ihre Effekte sind nicht isoliert zu sehen. Gleichwohl enthält jede Komponente Implikationen für die Bildungspolitik:

- Die berufliche Stellung der Eltern steht in ausgesprochen enger Beziehung zu den Schülerleistungen in den Bereichen Lesekompetenz sowie mathematische und naturwissenschaftliche Grundbildung. Üben die Eltern eine Tätigkeit mit höherem Berufsprestige aus, kann dies die beruflichen Erwartungen der Schülerinnen und Schüler beeinflussen und ihren Lerneifer positiv beeinflussen, der dann als Mittel zur Erreichung ehrgeiziger beruflicher Ziele betrachtet wird. Ein hoher beruflicher Status der Eltern kann überdies die im Bewusstsein der Kinder existierende Bandbreite von Optionen ausweiten. Die Bildungssysteme müssen sowohl für eine Erweiterung des Wissens über verschiedene Berufe und der entsprechenden Berufsziele sorgen, als auch Lösungen für nahe liegendere Probleme im Hinblick auf die Bildungsleistungen finden.

- Güter und Aktivitäten, die zur „klassischen“ Kultur zu rechnen sind, spielen eine komplexe Rolle; es kann jedoch gesagt werden, dass der Besitz des Typs von kulturellem Kapital, auf dem die Lehrpläne in der Regel aufbauen und das in Prüfungen und Tests bewertet wird, offenbar mit den schulischen Leistungen in Zusammenhang steht. Wenngleich zwischen Vorteilen dieser Art und den anderen familiären Hintergrundfaktoren eine Verbindung besteht, sind ihre Effekte isoliert gesehen generell hoch. Dass die Effekte im Bereich Lesekompetenz sogar noch ausgeprägter sind als in den Bereichen mathematische und naturwissenschaftliche Grundbildung, weist deutlich darauf hin, wie positiv der häusliche Zugang zu Literatur und anderen kulturellen Gütern in den Bildungsergebnissen zum Ausdruck kommt.

- Die Bildung der Eltern und die sozialen und kulturellen Kommunikationsmuster zwischen Eltern und Kindern, insbesondere die Kommunikation hinsichtlich der Schule und des Lernens, stehen in Wechselbeziehung miteinander und können für die Kinder von bildungsmäßigem Nutzen sein. Die PISA-Ergebnisse zeigen, dass Bildungserfolge möglicherweise mit Kommunikationsmustern zwischen Eltern und Kindern in Beziehung stehen. Daher sollte ein zentrales Ziel der staatlichen Politik darin bestehen, die Eltern zu unterstützen, insbesondere jene, die nur niedrigere Bildungsabschlüsse vorweisen können, um ihre Interaktion mit den Kindern und den Schulen 
ihrer Kinder auf eine Weise zu fördern, die den Lernprozess der Kinder unterstützt.

- Familiärer Wohlstand, der mit der beruflichen Stellung der Eltern verbunden ist, steht ebenfalls mit höheren Leistungen in Zusammenhang, wenngleich diese Verbindung offenbar schwächer ist, als bei den anderen im vorliegenden Kapitel untersuchten Faktoren.

- Schließlich ist in allen OECD-Ländern eine zunehmende Migration zu beobachten, wobei in vielen Fällen die von den Migranten zu Hause gesprochene Sprache nicht die Unterrichtssprache der von ihren Kindern besuchten Schulen ist. Welcher Art die für die ethnischen Minderheiten angehörenden Schülerinnen und Schüler und/oder Migrantenkinder aus dieser Tatsache erwachsenden Bildungsnachteile sind, wird in erheblichem $\mathrm{Maß}$ von dem Kontext bestimmt, aus dem sie kommen. Ein Bildungsnachteil, der bereits in ihrem Herkunftsland vorhanden war, kann sich in ihrem Aufnahmeland noch vergrößern, obgleich sich ihre schulischen Leistungen in absoluter Rechnung möglicherweise verbessert haben. Konzentrierte Hilfe in der Unterrichtssprache könnte eine Politikoption für derartige Schülerinnen und Schüler sein. Diese Schüler sind im Bildungsbereich möglicherweise im Nachteil, weil sie sich als Zuwanderer entweder an ein neues Bildungssystem gewöhnen müssen oder weil sie eine neue Sprache in einem familiären Umfeld lernen müssen. In jedem Fall wird es wohl nötig sein, diesen Schülerinnen und Schülern besondere oder zusätzliche Aufmerksamkeit zu widmen.

Insgesamt können sich aus diesen Erkenntnissen für die politischen Entscheidungsträger bedeutende Konsequenzen ergeben. Grundbildungskompetenzen sind ein wichtiges Fundament für lebensbegleitendes Lernen und für bessere zukünftige Beschäftigungs- und Verdienstchancen. Daher könnte die generationsübergreifende Mobilität von niedrigen zu höheren Niveaus des sozioökonomischen Status in den Ländern begrenzt sein, die einen hohen Anteil an schwachen oder nur marginal leistungsfähigen Lesern aufweisen. Die schwächeren Schülerinnen und Schüler dürften zugleich diejenigen sein, für die die geringste Wahrscheinlichkeit besteht, Beschäftigungsmöglichkeiten mit wirtschaftlichen Aufstiegschancen zu finden. Diese Analyse wird in Kapitel 8 weiter vertieft. Dort wird zunächst die Interaktion zwischen den verschiedenen Hintergrundfaktoren erörtert und anschließend untersucht, über welche politischen Instrumente die verschiedenen Länder verfügen, um die durchschnittlichen Leistungen zu verbessern und parallel die Effekte des familiären Hintergrunds auf die Schülerleistungen zu mildern.
... während familiärer Wohlstand für die Schülerleistungen in der Regel eine geringere Rolle spielt.

Die Nachteile von Migranten sind komplex und vielschichtig, erfordern aber Masnahmen.

Familiäre Nachteile werden sich über die Generationen weiter verstärken, wenn die Bildungssysteme diese Effekte nicht mildern. 
KAPITEL 6 Familiärer Hintergrund und Schülerleistungen

\section{Anmerkungen}

1. Wegen genaueren Informationen zur Konstruktion der Indizes siehe Anhang A1.

2. In allen Ländern sind die Unterschiede statistisch signifikant.

3. Standardisiert entspricht die Erhöhung je Einheit auf dem Index des relativen Wohlstands der Familie 20 Punkten auf der Gesamtskala Lesekompetenz (vgl. Tabelle 6.2), 24 Punkten auf der Skala für mathematische Grundbildung und 18 Punkten auf der Skala für naturwissenschaftliche Grundbildung (Daten abrufbar unter www.pisa.oecd.org).

4. Im Durchschnitt der OECD-Länder erklärt dieser Index 2,6\% der Varianz der Schülerleistungen auf der Gesamtskala Lesekompetenz.

5. Im Durchschnitt der OECD-Länder erklärt dieser Index 8,2\% der Varianz der Schülerleistungen auf der Gesamtskala Lesekompetenz.

6. In allen OECD-Ländern und in allen drei Grundbildungsbereichen sind die Unterschiede statistisch signifikant.

7. Im Bereich der kulturellen Kommunikation sind die Unterschiede in allen Ländern statistisch signifikant. Für die soziale Kommunikation sind sie, mit Ausnahme Italiens, in allen Ländern statistisch signifikant. Der Index der kulturellen Kommunikation erklärt 5,8\% der Varianz der Schülerleistungen auf der Gesamtskala Lesekompetenz, der Index der sozialen Kommunikation 2,2\%.

8. Eine andere Methode zur Bewertung der Bedeutung von sozialer und kultureller Kommunikation besteht darin, ihren relativen Beitrag zu den Leistungsunterschieden auf der Gesamtskala Lesekompetenz zu untersuchen. In allen OECD-Ländern erhöht sich bei einem Anstieg der kulturellen Kommunikation um eine Indexeinheit die durchschnittliche Leseleistung um rund 21 Punkte, während sie sich bei einem Anstieg der sozialen Kommunikation um eine Indexeinheit nur um 10 Punkte erhöht.

9. In allen Ländern und in allen drei Erhebungsbereichen sind diese Differenzen statistisch signifikant. Ausnahmen sind Portugal und Polen im Bereich mathematische Grundbildung.

10. In der nachfolgenden Untersuchung werden Schülerinnen und Schüler aus Ein-Eltern-Familien mit Schülerinnen und Schülern aus allen anderen Familientypen verglichen, d.h. mit Kernfamilien (traditionelle Familien mit einer Mutter und einem Vater), zusammengesetzten Familien (eine Familie mit einer Mutter und einem männlichen Erziehungsberechtigten, einem Vater und einer weiblichen Erziehungsberechtigten oder zwei Erziehungsberechtigten) und sonstigen familiären Umfeldern (z.B. bei Großeltern oder Geschwistern lebend).

11. Die Unterschiede sind in 14 Ländern statistisch signifikant.

12. Wenngleich Schülerinnen und Schüler aus Ein-Eltern-Familien im Ver.Königreich schlechtere Leistungen erzielen als ihre Mitschülerinnen und Mitschüler aus anderen Familientypen, übertreffen die Ergebnisse beider Schülergruppen den OECDDurchschnitt auf der Gesamtskala Lesekompetenz oder kommen diesem nahe. In den Vereinigten Staaten ist dies nicht der Fall.

13. Schülerinnen und Schüler, die sich nicht schriftlich oder mündlich in der Testsprache ausdrücken konnten, da sie weniger als ein Jahr in der Testsprache unterrichtet worden waren, wurden von der Erhebung ausgeschlossen (vgl. Anhang A3).

14. Auf die Frage „welche Sprache sprecht ihr normalerweise zu Hause“ konnten die Schülerinnen und Schüler antworten, dass sie die Sprache, in der der Test vorgenommen wurde, eine „andere offizielle Landessprache“, „andere nationale Dialekte “ oder „andere Sprachen“ sprechen. Die in diesem Zusammenhang aufgeführten Daten vergleichen Schülerinnen und Schüler der letzten Gruppe (d.h. ,andere Sprachen“) mit Schülerinnen und Schülern in den ersten drei Gruppen in den Ländern, in denen mindestens 3\% oder mehr der Schüler angeben, dass die im Elternhaus zumeist gesprochene Sprache eine „andere Sprache“ ist. 


\section{Kapitel}

7

\section{DAS LERNUMFELD UND DIE SCHULORGANISATION}

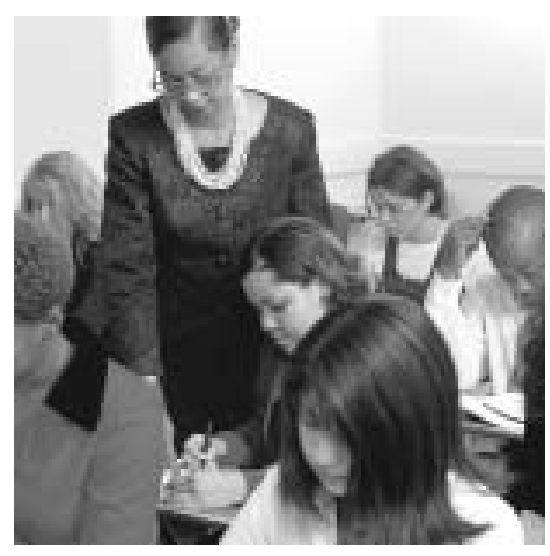


KAPITEL 7 Das Lernumfeld und die Schulorganisation

\section{Einführung}

Im vorliegenden Kapitel Der Umfang der erworbenen Kenntnisse und Fähigkeiten hängt in erheblichem wird untersucht, in Maße davon ab, inwieweit die Schülerinnen und Schüler sowohl zu Hause als welchem Zusammenhang auch in der Schule Zugang zu effektiven Lernmöglichkeiten haben. Dieses das schulische Umfeld mit Kapitel geht bei der Ermittlung der Faktoren, die zum schulischen Erfolg dem Erfolg der beitragen, einen Schritt weiter, indem das Augenmerk auf das Lernumfeld Schülerinnen und Schüler gelenkt wird, das die 15-jährigen Schülerinnen und Schüler in Schule und

steht. Familie vorfinden, und indem einige Aspekte der Schulleitung und -finanzierung miteinander verglichen werden.

\section{Kasten 7.1 Interpretation der PISA-Indizes}

Mehrere der Indizes fassen die Antworten der Schülerinnen und Schüler bzw. der Schulleitungen auf eine Reihe miteinander verbundener Fragen zusammen. Diese Fragen wurden aus größeren Komplexen auf der Grundlage von theoretischen Überlegungen und vorherigen Forschungsarbeiten ausgewählt ${ }^{1}$. Genauere Informationen bezüglich der Konstruktion der Indizes sind Anhang A1 zu entnehmen.

Die PISA-2000-Indizes basieren auf den Angaben der Schülerinnen und Schüler sowie der Schulleitungen zu dem Lernumfeld und der Organisation der Schulen sowie zu dem sozialen und ökonomischen Kontext des Lernprozesses. Den Indizes liegen eher persönliche Einschätzungen als externe Beobachtungen zu Grunde, weshalb sie durch kulturell bedingte Unterschiede im Antwortverhalten der Befragten sowie durch das Bestreben beeinflusst sein können, sozial erwünschte Antworten zu geben.

Bei der Interpretation der Daten müssen gewisse Einschränkungen hinsichtlich der Zuverlässigkeit der bei den Schulleitungen erfragten Informationen berücksichtigt werden. In den meisten Ländern haben nicht mehr als 150 Schulleitungen an der Erhebung teilgenommen. Obwohl die Schulleitungen zwar am besten in der Lage sind, Informationen über ihre Schulen zu liefern, ist es zudem kein leichtes Unterfangen, allgemeine Schlüsse aus Informationen zu ziehen, die für jede Schule jeweils nur aus einer einzigen Quelle stammen (und diese dann mit den von den Schülerinnen und Schülern gelieferten Informationen in Einklang zu bringen). Am schwersten fällt dabei ins Gewicht, dass die Schülerleistungen in jedem der Fachbereiche von zahlreichen Faktoren abhängen, und insbesondere von der gesamten Bildung, die sie in den Vorjahren erhalten haben, und nicht nur während des Zeitraums, in dem sie von ihren derzeitigen Lehrkräften unterrichtet wurden. Gleichwohl können die aus den Schulleiterfragebogen gewonnenen Informationen sehr aufschlussreich sein, da sie einen einzigartigen Einblick in die Wege gewähren, mit denen die zuständigen Stellen auf nationaler und subnationaler Ebene ihre bildungspolitischen Zielvorgaben umsetzen.

In diesem Bericht wiedergegebene Informationen, die sich auf die Aussagen der Schulleitungen stützen, wurden entsprechend der Zahl der in der jeweiligen Schule eingeschriebenen 15-Jährigen gewichtet.

Soweit nicht anders angegeben, beziehen sich die Vergleiche der Schülerleistungen in diesem Kapitel auf die Ergebnisse der Schülerinnen und Schüler auf der Gesamtskala Lesekompetenz. 


\section{Schul- und Unterrichtsklima}

\section{Unterstützung durch die Lehrkräfte}

Die Literatur über die Effizienz von Schulen lässt darauf schließen, dass Schülerinnen und Schüler (insbesondere solche mit niedrigem Leistungsniveau) von Unterrichtsmethoden profitieren, die das Interesse der Lehrkräfte an den Fortschritten ihrer Schülerinnen und Schüler zum Ausdruck bringen, die deutlich machen, dass von sämtlichen Schülerinnen und Schülern ein angemessenes Leistungsniveau erwartet wird, und die von der Bereitschaft zeugen, allen Schülerinnen und Schülern dabei zu helfen, den entsprechenden Maßstäben gerecht zu werden. Um zu untersuchen, inwieweit solche Unterrichtsmethoden in den verschiedenen OECD-Ländern üblich sind und inwieweit sie ein höheres Leistungsniveau fördern, wurden die Schülerinnen und Schüler gebeten anzugeben, wie oft es vorkommt, dass sich die Lehrkräfte der Testsprache für den Lernfortschritt jedes Einzelnen interessieren, den Schülerinnen und Schülern Gelegenheit geben, ihre Meinung zu sagen, ihnen bei der Arbeit helfen und ein Unterrichtsthema so lange erklären, bis sie es wirklich verstanden haben.

Ausgehend von den Antworten auf diese sowie damit zusammenhängende Fragen wurde ein Gesamtindex der Unterstützung durch die Lehrkräfte konstruiert. Über dem OECD-Durchschnitt, der gleich Null gesetzt wurde, liegende Werte zeigen an, dass die Unterstützung durch die Lehrkräfte nach Einschätzung der Schülerinnen und Schüler (zumindest im Unterricht in der Testsprache) überdurchschnittlich gut ist; demgegenüber zeigen negative Werte an, dass die Unterstützung durch die Lehrkräfte nach Einschätzung der Schülerinnen und Schüler unter dem Durchschnitt liegt ${ }^{2}$.

Am positivsten wird die Unterstützung durch die Lehrkräfte von den Schülerinnen und Schülern in Australien, Brasilien, Kanada, Neuseeland, Portugal, dem Vereinigten Königreich und den Vereinigten Staaten eingeschätzt. In Österreich, Belgien, der Tschechischen Republik, Frankreich, Deutschland, Italien, Japan, Korea, Lettland, Luxemburg und Polen hingegen liegt die Unterstützung durch die Lehrkräfte in der Testsprache nach Einschätzung der Schülerinnen und Schüler unter dem Durchschnitt (Tabelle 7.1).

So geben z.B. zwischen zwei Dritteln und drei Vierteln der Schülerinnen und Schüler in Australien, Kanada, Dänemark, Island, Neuseeland, Portugal, Schweden und dem Vereinigten Königreich an, dass ihre Lehrkräfte in der Testsprache in den meisten Stunden oder in jeder Stunde den Unterrichtsstoff so lange erklären, bis die Schülerinnen und Schüler ihn verstanden haben. Demgegenüber geben das in der Tschechischen Republik, Japan, Korea und Polen nur weniger als die Hälfte der Schülerinnen und Schüler an. Ebenso erklären mindestens zwei Drittel der Schülerinnen und Schüler in Australien, Kanada, Dänemark, Ungarn, Island, Neuseeland, Portugal, Schweden und dem Vereinigten Königreich, dass ihre Lehrkräfte in der Testsprache in den
Forschungsarbeiten haben gezeigt, dass Schülerinnen und Schüler von klaren Leistungserwartungen profitieren und höhere Leistungen bringen, wenn die Lehrkräfte Interesse an den Lernfortschritten haben und sie dabei unterstützen, die Erwartungen zu erfüllen.

In einigen Ländern ist die großse Mehrzahl der Schüler mit der Unterstützung durch die Lehrkräfte zufrieden, in anderen Ländern gilt dies jedoch nur für eine Minderheit. 
KAPITEL 7 Das Lernumfeld und die Schulorganisation

Zwischen der

Unterstützung durch die

Lehrkräfte und den

Schülerleistungen besteht

ein komplexer

Zusammenhang, ...

... in den meisten Ländern mit starker Unterstützung durch die Lehrkräfte kann jedoch nur ein geringfügig positiver Zusammenhang mit den Schülerleistungen festgestellt werden.

In Ländern mit einer generell geringeren Unterstützung durch die Lehrkräfte ist das Bild uneinheitlicher,... meisten Stunden oder in jeder Stunde intensiv bemüht sind, ihnen zu helfen, während dies in Frankreich, Korea, Japan, Luxemburg und Polen nicht einmal für die Hälfte der Schülerinnen und Schüler gilt (Daten abrufbar unter www.pisa.oecd.org). Die verfügbaren Daten liefern keine Möglichkeit festzustellen, inwieweit diese Ergebnisse tatsächliche - sowohl innerhalb als auch zwischen den Ländern - bestehende Unterschiede in Bezug auf die Haltung und Methoden der Lehrkräfte widerspiegeln oder lediglich Unterschiede in den subjektiven Einschätzungen der Schülerinnen und Schüler, die in jedem Land jeweils nur ihr persönliches Urteil abgaben. Trotz dieser Einschränkung scheinen einige der zwischen den Ländern bestehenden Differenzen groß genug zu sein, um besondere Aufmerksamkeit zu verdienen.

Insofern die Lehrkräfte in Klassen, die mehrheitlich von leistungsschwächeren Schülerinnen und Schülern besucht werden, in der Regel vergleichsweise häufiger auf „unterstützende“ Praktiken zurückgreifen, wäre zu erwarten, dass die Korrelation zwischen Unterstützung und Leistungen negativ ist. Auf der anderen Seite dürfte, insofern die gebotene Förderung wirksam ist, gleichzeitig zu erwarten sein, dass die Leistungen in Klassen, die mehr Unterstützung als andere erfahren, höher sind.

Wie aus dem Vorstehenden bereits geschlossen werden kann, sind die Zusammenhänge uneinheitlich und im Allgemeinen schwach ${ }^{3}$. In den meisten Ländern, in denen der Wert auf dem Index der Unterstützung durch die Lehrkräfte über dem OECD-Durchschnitt liegt, ist die Korrelation zwischen der Unterstützung durch die Lehrkräfte und den Leistungen im Bereich Lesekompetenz tendenziell jedoch positiv und statistisch signifikant (Abb. 7.1).

In Ländern, in denen die Unterstützung durch die Lehrkräfte nach den Angaben der Schülerinnen und Schüler unter dem OECD-Durchschnitt liegt, ist das Muster der Zusammenhänge uneinheitlich. So liegen z.B. die Werte für Japan, Korea, Lettland und Polen auf dem Index der Unterstützung durch die Lehrkräfte unter dem Durchschnitt, jedoch erzielen jene Schülerinnen und Schüler, denen eine stärkere Unterstützung durch die Lehrkräfte zuteil wird, höhere PISA-Punktzahlen als andere Schülerinnen und Schüler. Demgegenüber handelt es sich bei Belgien, derTschechischen Republik, Deutschland, Italien und Luxemburg um Länder mit unterdurchschnittlicher Unterstützung durch die Lehrkräfte, in denen Schülerinnen und Schüler, die nach eigenen Angaben eine stärkere Unterstützung durch die Lehrkräfte erhalten, tendenziell niedrigere Ergebnisse erzielen. In diesen Ländern geben mindestens 51\% der Schülerinnen und Schüler an, dass ihre Lehrkräfte im Testsprachenunterricht sich niemals für den Lernfortschritt der einzelnen Schüler interessieren oder dies lediglich in einigen Stunden tun; mindestens 27\% der Schülerinnen und Schüler erklären dort ferner, dass ihre Lehrkräfte ihnen nie oder lediglich in einigen Stunden Gelegenheit geben, ihre Meinung zu sagen, und 58\% oder mehr der 
Unterstützung durch die Lehrkräfte und Schülerleistungen

Korrelation zwischen dem Index der Unterstützung durch die Lehrkräfte und den Schülerleistungen auf der Gesamtskala Lesekompetenz

\begin{tabular}{|c|c|c|c|}
\hline & $\begin{array}{l}\text { Positive Korrelation zwischen } \\
\text { dem Index der Unterstützung } \\
\text { durch die Lehrkräfte und den } \\
\text { Leistungen auf der Gesamtskala } \\
\text { Lesekompetenz }\end{array}$ & $\begin{array}{l}\text { Keine } \\
\text { statistisch } \\
\text { signifikante } \\
\text { Korrelation }\end{array}$ & $\begin{array}{l}\text { Negative Korrelation zwischen } \\
\text { dem Index der Unterstützung } \\
\text { durch die Lehrkräfte und den } \\
\text { Leistungen auf der Gesamtskala } \\
\text { Lesekompetenz }\end{array}$ \\
\hline $\begin{array}{l}\text { Das Indexmittel bei der } \\
\text { Unterstützung durch } \\
\text { die Lehrkräfte liegt } \\
\text { über dem OECD- } \\
\text { Mittel }(0,0)\end{array}$ & $\begin{array}{l}\text { Australien, Brasilien, Kanada, } \\
\text { Dänemark, Finnland, } \\
\text { Island, Neuseeland, } \\
\text { Russische Föderation, } \\
\text { Schweden, Ver.Königreich, } \\
\text { Vereinigte Staaten }\end{array}$ & $\begin{array}{l}\text { Griechenland, } \\
\text { Ungarn, Irland, } \\
\text { Mexiko, Portugal, } \\
\text { Spanien }\end{array}$ & Liechtenstein, Schweiz \\
\hline $\begin{array}{l}\text { Das Indexmittel bei der } \\
\text { Unterstützung durch die } \\
\text { Lehrkräfte liegt unter } \\
\text { dem OECD-Mittel }(0,0)\end{array}$ & $\begin{array}{l}\text { Japan, Korea, Lettland, } \\
\text { Norwegen, Polen }\end{array}$ & $\begin{array}{l}\text { Österreich, } \\
\text { Tschech.Republik, } \\
\text { Frankreich, Niederlande }\end{array}$ & $\begin{array}{l}\text { Belgien, Deutschland, Italien, } \\
\text { Luxemburg }\end{array}$ \\
\hline
\end{tabular}

Quelle: OECD PISA Datenbank, 2001.

Schülerinnen und Schüler geben an, dass ihre Lehrkräfte ihnen nie oder nur in einigen Stunden beim Lernen helfen (Daten abrufbar unter www.pisa.oecd.org).

$\mathrm{Zu}$ diesem Muster könnten zahlreiche Faktoren beitragen, für deren Untersuchung weitergehende Studien erforderlich sind. So ist es möglich, dass in Ländern mit einem unterdurchschnittlichen Niveau der Unterstützung durch die Lehrkräfte und einer negativen Korrelation mit der Leistung die Unterstützungsbereitschaft in geringerem Maße als in anderen Ländern in der Berufskultur der Lehrkräfte verankert ist und die Lehrkräfte dazu tendieren, ihre Anstrengungen auf Klassen bzw. einzelne Schülerinnen und Schüler mit besonders großen Schwierigkeiten zu beschränken. Vielleicht verhält es sich auch so, dass im Hinblick auf die in einer Schule gebotene Unterstützung durch die Lehrkräfte erst ein bestimmtes Niveau erreicht sein muss, bevor die Auswirkungen auf die Schülerleistungen groß genug sind, um diese positiv zu beeinflussen.

\section{Schülerbezogene Faktoren für das Schulklima}

Sowohl die an die Schulleiterinnen und Schulleiter als auch die an die Schülerinnen und Schüler gerichteten PISA-Kontextfragebogen enthielten Fragen, die die Identifizierung sowie den Vergleich der Faktoren ermöglichen, die das Lernklima der Schulen beeinträchtigen. So wurden die Schulleitungen danach gefragt, inwieweit das Lernen an ihrer Schule durch Faktoren wie die häufige Abwesenheit von Schülerinnen und Schülern, den Konsum von Alkohol oder illegalen Drogen und Störung des Unterrichts durch Schülerinnen und Schüler beeinträchtigt wird. Die Schülerinnen und Schüler wurden ihrerseits

\author{
... was darauf \\ zurückzuführen sein \\ könnte, dass die Lehrkräfte \\ die Unterstützung auf \\ leistungsschwache Schüler \\ beschränken.
}

In PISA wurde untersucht, inwieweit der Lernprozess aus Sicht der schulleitungen durch unangemessenes schülerverhalten beeinträchtigt wird. 
Fast die Hälfte der Schulleitungen ist über die negativen Auswirkungen der häufigen Abwesenheit der Schülerinnen und Schüler und des Störens im Unterricht besorgt..., Schülerinnen und Schüler den Zeitverlust zu Beginn der Unterrichtsstunden als störendsten Faktor ansehen. gefragt, wie häufig der Unterricht in der Testsprache bei ihnen durch bestimmte Vorfälle gestört wird. So geben die Schülerinnen und Schüler z.B. die Häufigkeit an, mit der folgende Aussagen für ihren Unterricht in der Testsprache zutreffen: „wir Schülerinnen/wir Schüler können nicht ungestört arbeiten“, „es ist laut, und alles geht durcheinander" und es "vergehen zu Beginn der Stunde mehr als fünf Minuten, in denen gar nichts passiert“. Bei der Interpretation dieser Angaben ist jedoch eine gewisse Vorsicht angebracht. Die Schulleitungen legen in den verschiedenen Ländern bei der Beurteilung des Lernklimas nicht zwangsläufig dieselben Kriterien zu Grunde. So können z.B. Schulleitungen in Ländern, in denen die Schülerinnen und Schüler dem Unterricht insgesamt eher selten fernbleiben, eine relativ geringe Abwesenheitsquote in ihren Schulen als eine der Hauptursachen für Disziplinprobleme ansehen, während Schulleitungen in Ländern, in denen die Abwesenheitsquote generell hoch ist, dies vielleicht anders beurteilen.

In sämtlichen OECD-Ländern nennen die Schulleiterinnen und Schulleiter die häufige Abwesenheit von Schülerinnen und Schülern als den Faktor, der den Lernprozess am häufigsten beeinträchtigt, wobei $48 \%$ von ihnen im Durchschnitt angeben, dass dies die 15-Jährigen bis zu einem gewissen Grad oder stark am Lernen hindert (vgl. Abb. 7.2 und www.pisa.oecd.org). Als nächster Faktor wird mit 42\% die Störung des Unterrichts genannt, danach folgen mit $33 \%$ das Schwänzen von Unterrichtsstunden und mit 24\% der fehlende Respekt der Schülerinnen und Schüler vor den Lehrkräften. 14\% der Schulleiterinnen und Schulleiter geben an, dass das Einschüchtern oder Schikanieren von Schülerinnen und Schülern durch Mitschülerinnen und Mitschüler das Lernen bis zu einem gewissen Grad oder sogar stark beeinträchtigt.

Von den Schülerinnen und Schülern wird die Zeitverschwendung zu Unterrichtsbeginn als häufigstes Disziplinproblem genannt. Durchschnittlich $40 \%$ der Schülerinnen und Schüler geben an, dass in den meisten Stunden oder in jeder Stunde des Testsprachenunterrichts zu Beginn der Stunde mehr als fünf Minuten vergehen, in denen gar nichts passiert, und ein Viertel der Schülerinnen und Schüler erklärt, dass sie erst lange Zeit nach Beginn der Stunde zu arbeiten anfangen (vgl. Abb. 7.3 und www.pisa.oecd.org). Im Durchschnitt der OECDLänder gibt ein Drittel der Schülerinnen und Schüler an, dass die Lehrkräfte in den meisten Stunden oder in jeder Stunde lange warten müssen, bis Ruhe eintritt und dass es im Testsprachenunterricht laut ist und alles durcheinander geht. In Dänemark, Island, Japan, Mexiko, Polen, der Schweiz und dem Vereinigten Königreich erklärt nicht einmal einer von fünf Schülern, dass die Schülerinnen und Schüler in der Regel nicht auf das hören, was die Lehrerin oder der Lehrer sagt, wohingegen das für rund ein Drittel der Schülerinnen und Schüler in Korea und Italien der Fall ist. 
Abbildung 7.2

Das Lernklima aus der Sicht der Schulleitungen

Verteilung der mittleren Prozentsätze der Schülerinnen und Schüler in Schulen, wo das Lernen nach Angaben der Schulleitungen ,etwas“ oder „sehr“ durch folgende Faktoren beeinträchtigt wird

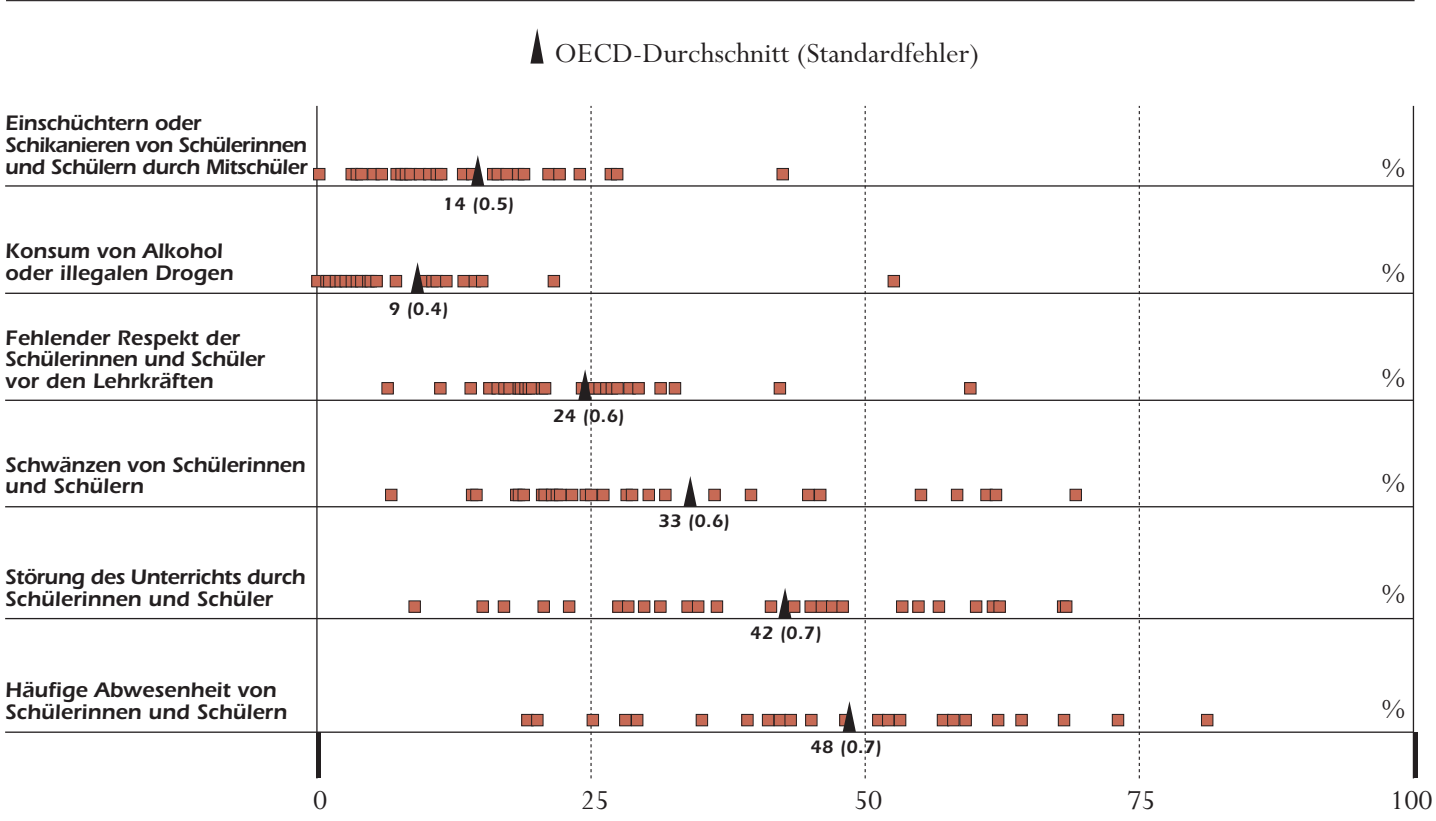

Anmerkung: Die Länder sind dargestellt durch die Quadrate.

Quelle: OECD PISA Datenbank, 2001. Daten abrufbar unter www.pisa.oecd.org.

Diese Durchschnittswerte vermitteln ein Gesamtbild von der Lage im OECDRaum, es darf dabei aber nicht außer Acht gelassen werden, dass sich dahinter erhebliche Unterschiede zwischen den einzelnen OECD-Ländern verbergen. Um diese Unterschiede zu untersuchen, wurden summarische Indizes konstruiert, die zum einen auf den von den Schulleitungen und zum anderen auf den von den Schülerinnen und Schülern gemachten Angaben basieren. Im Fall des Index aus Schülersicht ist das Lernklima des Bildungssystems nach Ansicht der Schülerinnen und Schüler umso positiver, je weiter die Punktzahl über Null liegt. Auf dem Schulleiterindex spiegeln Werte über Null eine positive Einschätzung der Schuldisziplin durch die Schulleiterinnen und Schulleiter wider, was heißt, dass die verschiedenen in den Index einfließenden Faktoren, die den Lernprozess ihrer Ansicht nach beeinträchtigen, im jeweiligen Land weniger stark ausgeprägt sind als im OECD-Durchschnitt. Aus Indexzahlen unter Null lässt sich hingegen ablesen, dass die Beurteilung des Lernklimas (Schulleiterindex) und der Schuldisziplin (Schülerindex) jeweils schlechter ausfällt als im OECD-Durchschnitt.
Doch die Antwortmuster sind von Land zu Land verschieden, wie sich an den beiden Indizes des Lernklimas aus der Sicht der Schulleitungen und aus der Sicht der Schülerinnen und Schüler zeigt. 
KAPITEL 7 Das Lernumfeld und die Schulorganisation

\section{Abbildung 7.3}

Das Lernklima aus der Sicht der Schülerinnen und Schüler

Verteilung der mittleren Prozentsätze der Schülerinnen und Schüler, nach deren Ansicht die folgenden Aussagen für die meisten oder alle Unterrichtsstunden zutreffen

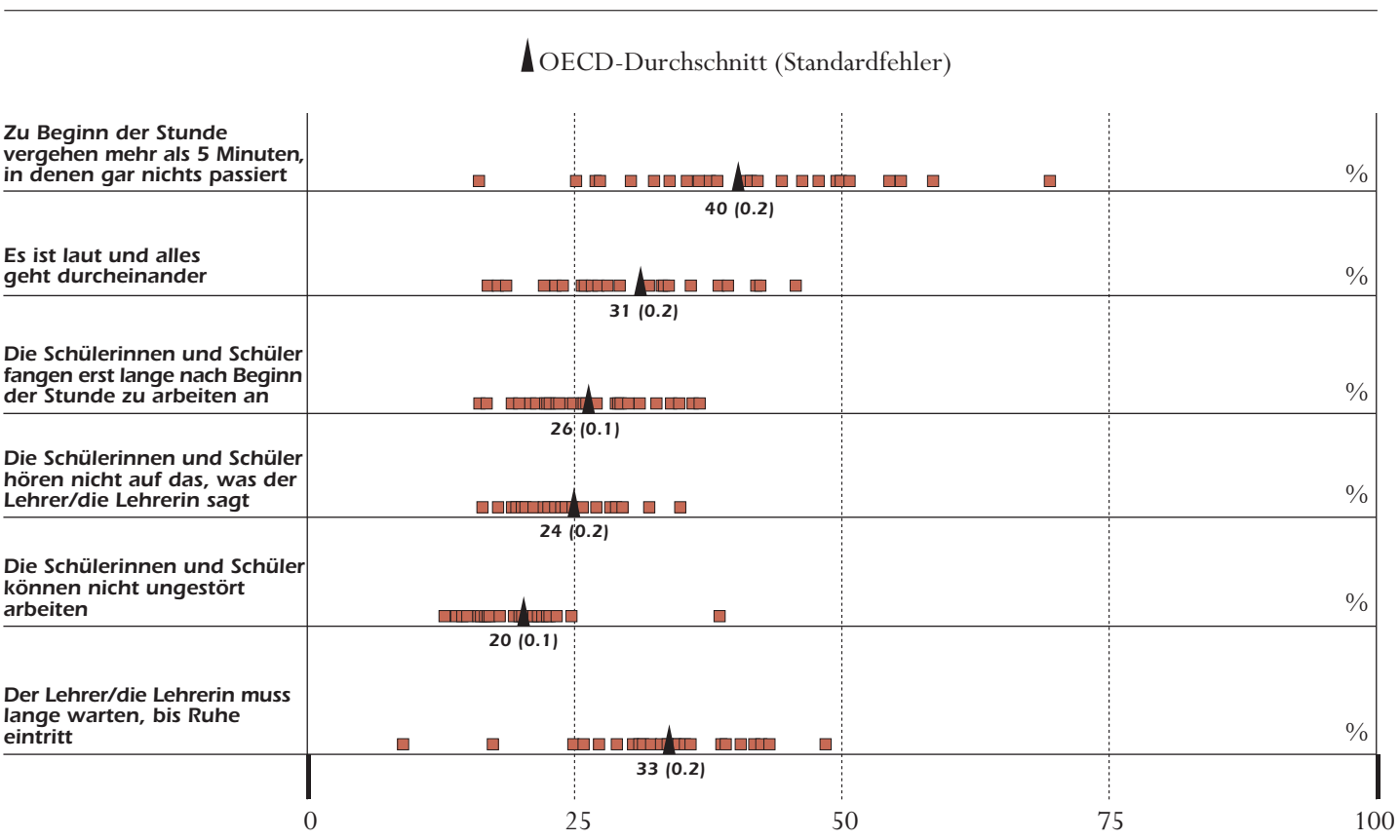

Anmerkung: Die Länder sind dargestellt durch die Quadrate.

Quelle: OECD PISA Datenbank, 2001. Daten abrufbar unter www.pisa.oecd.org.

Am besorgtesten zeigen sich die Schulleitungen in

Griechenland und in der Russischen Föderation, ...

... während die

Schulleitungen in der Tschechischen Republik,

Dänemark, Japan und

Kored am wenigsten

beunruhigt sind, ...
Unter den Schulleitungen sind jene in Griechenland und in der Russischen Föderation am stärksten der Ansicht, dass ihre Schulen unter Disziplinproblemen wie häufige Abwesenheit, Störung des Unterrichts durch Schülerinnen und Schüler und Schwänzen von Unterrichtsstunden zu leiden haben. 85\% der 15-Jährigen besuchen in der Russischen Föderation z.B. Schulen, in denen nach den Aussagen der Schulleitungen das häufige Schwänzen von Unterrichtsstunden den Lernprozess bis zu einem gewissen Grad oder stark beeinträchtigt (Daten abrufbar unter www.pisa.oecd.org).

Zu den Ländern, in denen Disziplinprobleme nach Ansicht der Schulleitungen am seltensten sind, zählen die Tschechische Republik, Dänemark, Japan und Korea (Tabelle 7.2). Jedoch lässt sich selbst in diesen Ländern, in denen das Lernklima im internationalen Vergleich relativ gut zu sein scheint, aus den Antworten der Schulleitungen nicht schließen, dass Disziplinprobleme dort nicht vorhanden sind. 
Betrachten wir z.B. Japan und Korea, zwei der drei Länder mit der höchsten Punktzahl auf dem Index der schülerbezogenen Faktoren für das Schulklima nach Angaben der Schulleitungen. In Japan geben 39\% der Schulleitungen dennoch an, dass die häufige Abwesenheit von Schülerinnen und Schülern das Lernen etwas oder stark beeinträchtigt (OECD-Durchschnitt: 48\%), 18\% sind der Ansicht, dass dies auch auf das Schwänzen von Unterrichtsstunden zutrifft (OECD-Durchschnitt: 33\%), 29\% geben an, dass sich der mangelnde Respekt der Schülerinnen und Schüler vor den Lehrkräften in gleicher Weise nachteilig auswirkt (OECD-Durchschnitt: 24\%), und 5\% sagen, dass Einschüchterungspraktiken und Schikanen von Schülerinnen und Schülern gegenüber ihren Mitschülern das Lernklima etwas oder stark beeinträchtigen (OECD-Durchschnitt: 14\%) (vgl. Abb. 7.2 und www.pisa.oecd.org). Desgleichen nennen 20\% der Schulleitungen in Korea die häufige Abwesenheit der Schülerinnen und Schüler, 17\% das Stören im Unterricht, 14\% das Schwänzen von Unterrichtsstunden und 29\% den fehlenden Respekt der Schülerinnen und Schüler vor den Lehrkräften als Hindernisse, die das Lernen etwas oder stark beeinträchtigen. Das lässt darauf schließen, dass selbst in Ländern mit vergleichsweise geringen Problemen Spielraum für Verbesserungen besteht.

Die Schülerinnen und Schüler in Lettland, Liechtenstein, Japan, Polen, der Russischen Föderation und der Schweiz geben am häufigsten an, dass es bei ihnen selten oder nur gelegentlich zu Störungen im Unterricht kommt (Tabelle 7.3). Demgegenüber sind die Schülerinnen und Schüler in Brasilien, Griechenland und Norwegen der Auffassung, dass ihr Unterricht häufig durch unpassendes Verhalten gestört wird.

Wie stellen sich die Auffassungen der Schülerinnen und Schüler auf der einen und der Schulleiterinnen und Schulleiter auf der anderen Seite nun im Vergleich dar? Zwar befassen sich beide Fragebogen mit dem Lernklima, doch geschieht dies, wie bereits erwähnt, aus unterschiedlichen Perspektiven und anhand verschiedener Fragen. Gleichwohl ist es möglich, die Einschätzungen des Lernklimas durch die beiden Gruppen indirekt miteinander zu vergleichen, und zwar durch die Gegenüberstellung ihrer Antworten auf dem jeweiligen Fragenkomplex. In vielen Ländern besteht ein relativ hoher Grad an Übereinstimmung zwischen den Ansichten der Schulleitungen und denen der Schülerinnen und Schüler über die schülerbezogenen Aspekte der Schuldisziplin, allerdings gibt es - wie aus Abbildung 7.4 ersichtlich ist - einige Ausnahmen.

Am wichtigsten ist jedoch die Frage, welcher Zusammenhang zwischen den Einschätzungen des Lernklimas und den Schülerleistungen besteht. Die Tabellen 7.2 und 7.3 zeigen mittlere Punktzahlen auf der Gesamtskala Lesekompetenz für die oberen und unteren Quartile der Schulklimaindizes der jeweiligen Länder (wobei das obere Quartil einem wünschenswerteren Lernklima entspricht). In vielen Ländern besteht ein enger Zusammenhang zwischen der Beurteilung der Schulleitungen hinsichtlich der schülerbezogenen Faktoren, die das Schulklima beeinträchtigen, und den Schülerleistungen. In Belgien, Deutschland, Ungarn,

\author{
... doch selbst in den \\ Ländern mit den geringsten \\ Problemen besteht noch \\ Raum für Verbesserungen.
}

Die Ansichten der

Schulleitungen und der

schüler über das

Schulklima sind in vielerlei

Hinsicht ähnlich.

\begin{abstract}
Die Schülerleistungen
stehen in engem

Zusammenhang mit dem

schulklima, insbesondere

wenn man die Sicht der

schulleitungen zugrunde-

legt, ...
\end{abstract}


KAPITEL 7 Das Lernumfeld und die Schulorganisation

\section{Abbildung 7.4}

Das Lernklima - Gesamtbild

Index der Schuldisziplin und Index schülerbezogener Faktoren für das Schulklima

Index der Schuldisziplin aus der

Index schülerbezogener Faktoren für da

Sicht der Schülerinnen und Schüler

Schulklima nach Angaben der Schulleitungen

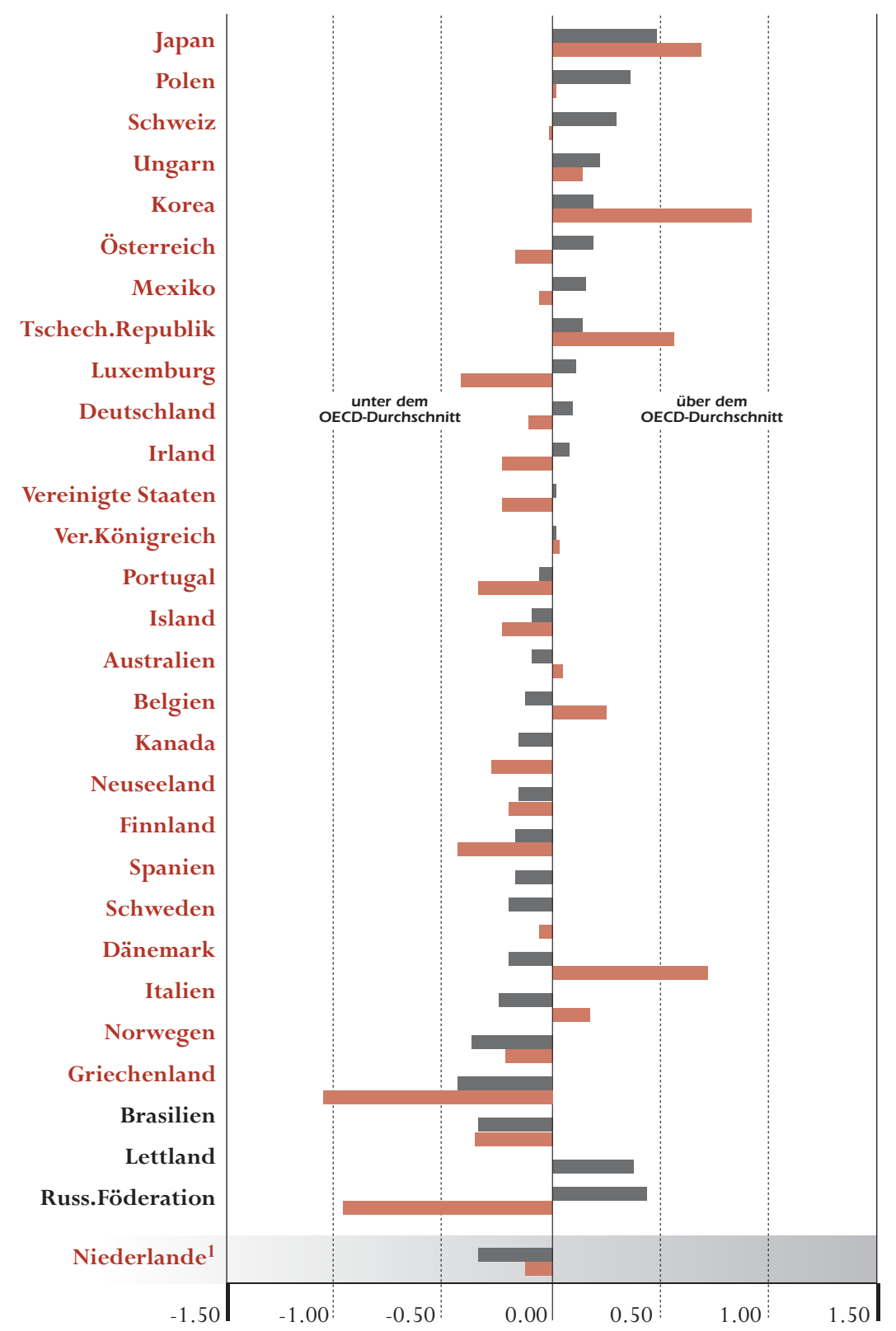

1. Die Beteiligungsquote ist zu niedrig, um die Vergleichbarkeit zu gewährleisten (vgl. Anhang A3). Anmerkung: Zur Definition der Indizes siehe Anhang A1.

Quelle: OECD PISA Datenbank, 2001. Tabellen 7.2 und 7.3. 
Italien, Japan, Polen, den Niederlanden und dem Vereinigten Königreich erklärt der Index der schülerbezogenen Faktoren für das Schulklima zwischen 12\% und $21 \%$ der Leistungsunterschiede im Bereich Lesekompetenz ${ }^{4}$. In diesen Ländern beträgt der Leistungsabstand zwischen Schülerinnen und Schülern von Schulen, die auf Grund der Angaben der Schulleitungen im oberen Quartil des Index der schülerbezogenen Faktoren mit Einfluss auf das Schulklima liegen, und solchen von Schulen im unterem Quartil zwischen 80 und 114 Punkten. Der Zusammenhang zwischen der Einschätzung der Schuldisziplin durch die Schülerinnen und Schüler und der Leseleistung ist in der Tendenz schwächer, aber in vielen Ländern deutlich erkennbar ${ }^{5}$.

Die Untersuchung der Funktionsweise dieser Beziehungen sowie der kontextbezogenen und vermittelnden Faktoren, die sie möglicherweise beeinflussen, würde den Rahmen dieses ersten Berichts sprengen und muss daher Gegenstand weitergehender Studien und Analysen werden.

\section{Lehrkräftebezogene Faktoren für das Schulklima}

Den Schulleiterinnen und Schulleitern wurden im Rahmen von PISA 2000 auch Fragen über ihre Einschätzung der lehrkräftebezogenen Faktoren mit Einfluss auf das Schulklima gestellt. Sie sollten insbesondere angeben, wie sehr der Lernprozess in ihrer Schule z.B. durch folgende Faktoren behindert wird: niedrige Erwartungen der Lehrkräfte, schlechtes Schüler-Lehrer-Verhältnis, häufige Abwesenheit von Lehrkräften, Widerstand des Kollegiums gegen Veränderungen, Nichteingehen der Lehrkräfte auf die individuellen Bedürfnisse der Schülerinnen und Schüler sowie mangelnde Ermutigung der Schülerinnen und Schüler zur vollen Ausschöpfung ihres Potentials. Die Antworten wurden kombiniert, um einen Gesamtindex der lehrkräftebezogenen Faktoren für das Schulklima zu konstruieren. Positive Werte drücken aus, dass sich die lehrkräftebezogenen Faktoren für das Schulklima aus Schulleitersicht weniger nachteilig auf den Lernprozess auswirken als im OECD-Durchschnitt; negative Werte bedeuten, dass das Verhalten der Lehrkräfte den Lernprozess nach Ansicht der Schulleitungen stärker als im OECD-Durchschnitt beeinträchtigt.

Im Vergleich zu denen anderer Länder zeigen sich die Schulleitungen in Griechenland, Luxemburg, Mexiko, den Niederlanden und der Russischen Föderation am stärksten beunruhigt über lehrkräftebezogene Faktoren, die den Lernprozess behindern. Demgegenüber berichten die Schulleitungen in der Tschechischen Republik, Dänemark, Ungarn, Island, Korea und Lettland am wenigsten über Probleme mit lehrkräftebezogenen Faktoren, die das Schulklima beeinträchtigen (Tabelle 7.4).

Wie zu erwarten, besteht in den meisten Ländern in der Tendenz ein positiver Zusammenhang zwischen der Schulleitereinschätzung der lehrkräftebezogenen Faktoren für das Schulklima und der Leseleistung, d.h. je größer die Besorgnis der Schulleitungen über die lehrkräftebezogenen Faktoren für das Schulklima ist, umso geringer sind die Schülerleistungen im Bereich Lesekompetenz.
... obwohl u.U. zahlreiche

kontextbezogene und

vermittelnde Faktoren auf

diesen Zusammenhang mit

einwirken.

Bei PISA wurde auch

untersucht, ob das

Verhalten und die

Einstellung der Lehrkräfte

die Lernergebnisse

beeinflussen, ...
..., wobei sich ein
schwacher positiver
Zusammenhang mit den
Schülerleistungen ergibt. 
KAPITEL 7 Das Lernumfeld und die Schulorganisation

\section{Abbildung 7.5}

Lehrkräftebezogene Faktoren für das Schulklima

Verteilung der mittleren Prozentsätze der Schülerinnen und Schüler in Schulen, wo das Lernen nach Angaben der Schulleitungen ,etwas“ oder „sehr“ durch folgende Faktoren beeinträchtigt wird

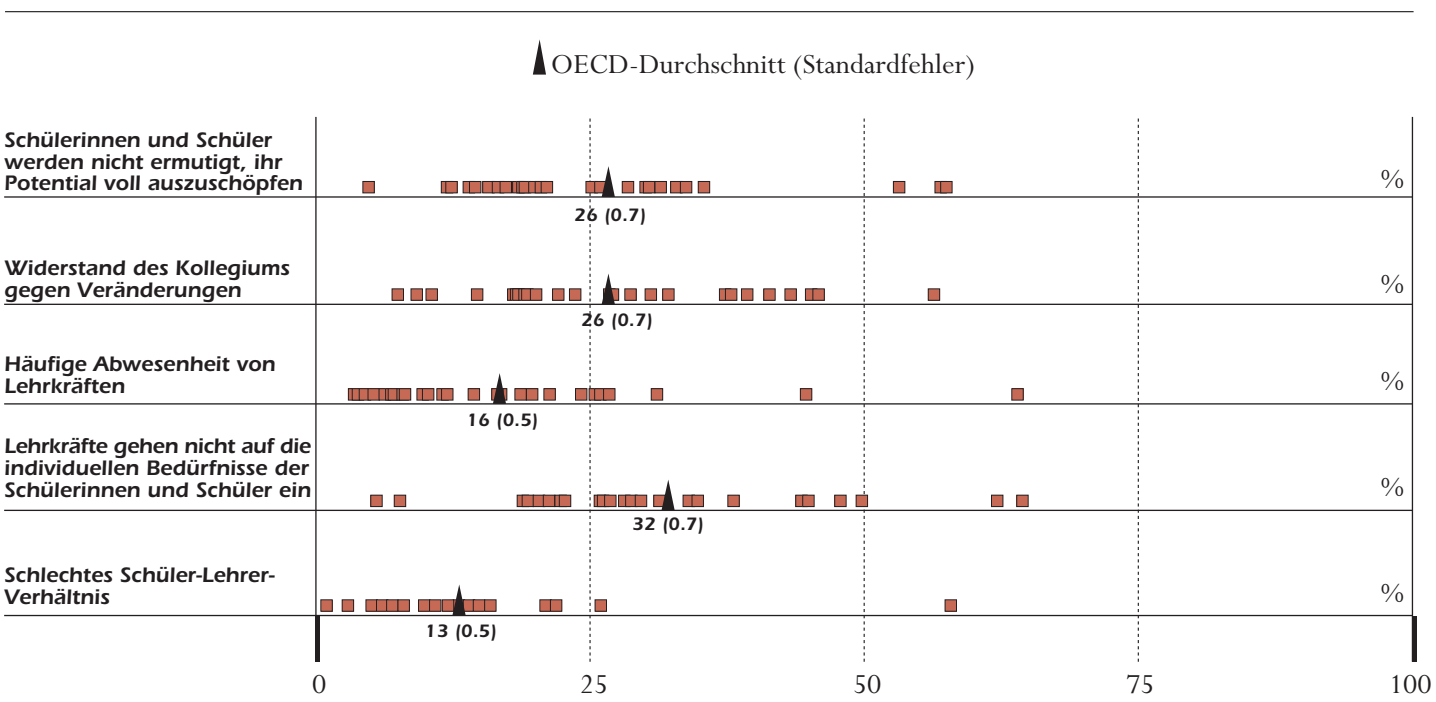

Anmerkung: Die Länder sind dargestellt durch die Quadrate .

Quelle: OECD PISA Datenbank, 2001. Daten abrufbar unter www.pisa.oecd.org.

Dieser Zusammenhang ist jedoch, von einigen wenigen Ausnahmen abgesehen, nicht sehr stark ausgeprägt (vgl. Tabelle 7.4) ${ }^{6}$.

Die Schulleitungen wurden auch zur Stimmung und Arbeitshaltung sowie zum Engagement der Lehrkräfte befragt,...
Ergänzend zu den Fragen über das Schulklima beeinträchtigende lehrkräftebezogene Faktoren wurden die Schulleitungen auch um Aussagen über die Stimmung und Arbeitshaltung der Lehrkräfte sowie deren Engagement gebeten. Zu diesem Zweck sollten sie angeben, in welchem Umfang sie z.B. folgende Aussagen als zutreffend bzw. nicht zutreffend ansahen: „die Lehrkräfte arbeiten mit großem Engagement“, „die Lehrkräfte sind stolz auf diese Schule“ und „die Stimmung und Arbeitshaltung der Lehrkräfte sind in dieser Schule gut“. Ausgehend von ihren Antworten wurde ein Index der Stimmung und Arbeitshaltung der Lehrkräfte konstruiert, wobei der OECDLänderdurchschnitt mit Null angesetzt wurde. Höhere Indexwerte spiegeln eine im Vergleich mit dem OECD-Mittel bessere Einschätzung von Stimmung und Arbeitshaltung wider.

Österreich weist den höchsten positiven Wert auf, was darauf hindeutet, dass die Stimmung und Arbeitshaltung der Lehrkräfte dort nach Ansicht der Schulleitungen besonders gut ist. Im Gegensatz dazu meinen die Schulleitungen in Korea, Italien, Polen und Portugal, dass der Grad der Stimmung und Arbeitshaltung ihrer Lehrkräfte vergleichsweise niedrig ist (Tabelle 7.5). 
Der Zusammenhang zwischen der Stimmung und Arbeitshaltung der Lehrkräfte aus Schulleitersicht und den Ergebnissen ihrer Schülerinnen und Schüler im Bereich Lesekompetenz ist in der Tendenz eher schwach. In einigen Ländern ist er jedoch stärker ausgeprägt. So erklärt der Index der Stimmung und Arbeitshaltung der Lehrkräfte in Belgien, Japan, Luxemburg und Polen zwischen 4\% und 8\% der Varianz der Leseleistung, und auch in Australien, Ungarn, Korea, der Russischen Föderation, Spanien und dem Vereinigten Königreich liegt dieser Anteil immer noch bei über $2 \%{ }^{7}$.

\section{Das Lernen außerhalb der Schule}

Politischen Entscheidungsträgern, die um eine Steigerung der Bildungserträge bemüht sind, muss daran gelegen sein, dass die Schülerinnen und Schüler mehr Zeit auf Lernaktivitäten verwenden bzw. diese Zeit effizienter nutzen. Die Unterrichtsdauer, d.h. die Zahl der Stunden, die die Schülerinnen und Schüler im Schulunterricht zubringen, steht in einem engen Zusammenhang mit Faktoren wie Klassengröße, Arbeitszeit der Lehrkräfte (Unterrichtszeit) sowie Schüler-Lehrer-Quote. Das optimale Gleichgewicht zwischen diesen Faktoren kann je nach Fachbereich oder Klassenstufe unterschiedlich sein.

Die Regeln und Vorgehensweisen in Bezug auf die Hausaufgaben stellen ein anderes Element in dieser Gleichung dar, das einen wesentlichen Einfluss darauf haben kann, wie viel Zeit die Schülerinnen und Schüler auf das Lernen verwenden. In vielen OECD-Ländern entfällt auf die Hausaufgaben ein bedeutender Teil der Zeit, die die Schülerinnen und Schüler mit Lernen verbringen. Bei PISA 2000 wurden die Schülerinnen und Schüler gebeten anzugeben, wie viel Zeit sie pro Woche auf die Erledigung der Hausaufgaben im Testsprachenunterricht, in Mathematik und in den naturwissenschaftlichen Fächern verwenden (Abbildung 7.6).

Aus der Addition dieser Antworten ergibt sich ein Durchschnitt von 4,6 Stunden allein in den drei PISA-Fächern, wobei die Spanne von 3,3 Stunden oder weniger in Japan und Schweden bis $\mathrm{zu} 5,8$ Stunden und mehr in

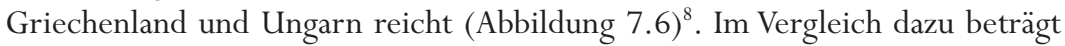
die durchschnittliche wöchentliche Regelunterrichtszeit in diesen drei Fächern zusammen 12 Stunden (OECD, 2001). Darüber hinaus gibt ein großer Teil der Schülerinnen und Schüler an, gelegentlich oder regelmäßig Zusatzunterricht oder Nachhilfestunden außerhalb der Schule zu nehmen, um ihre Leistungen zu verbessern. Im Durchschnitt der OECD-Länder berichteten z.B. 25\% der Schülerinnen und Schüler, in den letzten drei Jahren gelegentlich oder regelmäßig Förderkurse in der Testsprache oder in anderen Fächern oder Zusatzunterricht außerhalb ihrer Schulen besucht zu haben. In Japan und Korea liegt dieser Anteil bei 64\% bzw. 71\% (Tabelle 7.7).

\author{
... die tendenziell in einem \\ schwächeren \\ Zusammenhang mit den \\ schülerleistungen stehen, \\ der in einigen Ländern \\ jedoch stärker ausgeprägt \\ ist.
}

Die auf das Lernen

verwendete Zeit muss

effizient genutzt werden.

Hausaufgaben und sonstige aufserschulische Lernaktivitäten spielen eine wichtige Rolle, ... 
KAPITEL 7 Das Lernumfeld und die Schulorganisation

\section{Abbildung 7.6}

Zeitaufwand für Hausaufgaben

Index des Zeitaufwands für Hausaufgaben in der Testsprache, Mathematik und Naturwissenschaften

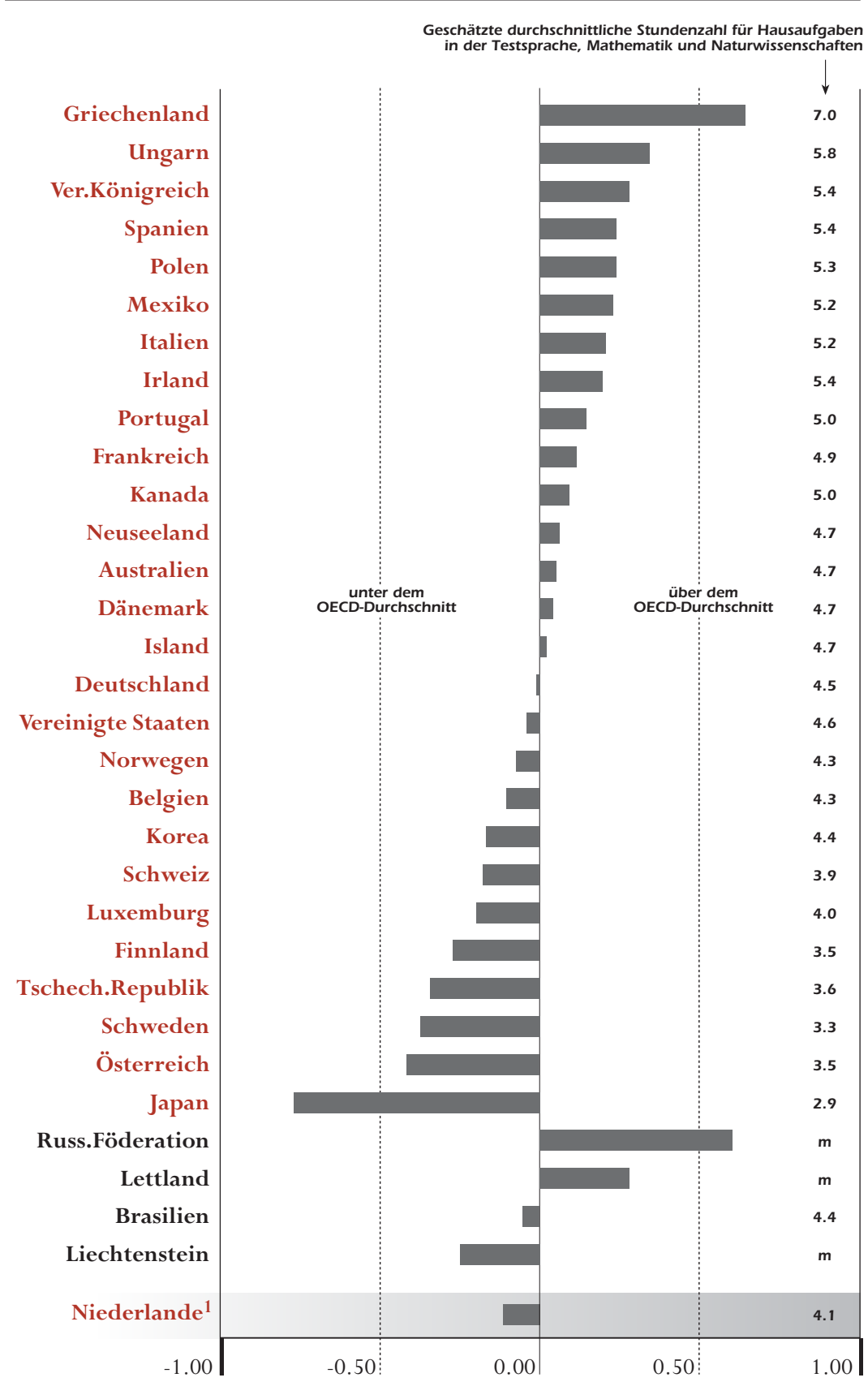

1. Die Beteiligungsquote ist zu niedrig, um die Vergleichbarkeit zu gewährleisten (vgl. Anhang A3). Anmerkung: Zur Definition des Index siehe Anhang A1.

Quelle: OECD PISA Datenbank. 2001. Tabelle 7.6. 
Durch die Hausaufgaben erhalten die Schülerinnen und Schüler mehr Gelegenheit, Zeit mit Lernen zu verbringen, weshalb ein positiver Zusammenhang mit den Lernergebnissen zu erwarten wäre. Auf Grund verschiedener Faktoren ist dieser Zusammenhang jedoch wesentlich komplizierter. So können z.B. die Lehrkräfte dazu neigen, jenen Schülerinnen und Schülern, die ihre Leistungen am deutlichsten verbessern müssen, mehr (oder regelmäßiger) Hausaufgaben aufzutragen. Desgleichen benötigen Schüler, die langsamer lernen, u.U. mehr Zeit als andere, um eine gleiche Menge an Hausaufgaben zu erledigen. Umgekehrt kann es sich bei den Schülerinnen und Schülern, die nach eigener Angabe vergleichsweise wenig Zeit auf die Hausaufgaben verwenden, entweder um besonders begabte Schüler handeln, die ihre Hausaufgaben rasch erledigen können, oder um desinteressierte Schüler, die zu Hause nicht viel Zeit auf schulische Aktivitäten verwenden wollen.

Trotz dieser vielschichtigen Beziehungen ist der Zusammenhang zwischen der Zeit, die auf die Hausaufgaben in den drei Fachbereichen verwendet wird, und den Schülerleistungen in der Tendenz durchweg positiv. Zur Analyse dieses Zusammenhangs ist es aus technischen Gründen am zweckmäßigsten, einen Index nach dem Modell der zuvor in diesem Kapitel herangezogenen Indizes zu konstruieren, in dem die Angaben der Schülerinnen und Schüler über die Zeit, die sie auf die Hausaufgaben in den drei PISA-Testbereichen aufwenden, zusammengefasst werden. Dieser Index wird in Tabelle 7.6 und Abbildung 7.6 dargestellt.

Der Index des wöchentlichen Zeitaufwandes für Hausaufgaben erklärt zwischen 7\% und 15\% der Varianz der Leseleistung in Australien, Belgien, Frankreich, Griechenland, Ungarn, Italien, Polen, Spanien, der Russischen Föderation, dem Vereinigten Königreich und den Vereinigten Staaten ${ }^{9}$. Der Zusammenhang ist in der Tendenz stärker in Ländern, in denen das Indexmittel größer als 0,2 ist, was darauf hindeuten könnte, dass in einem Bildungssystem eine kritische Schwelle bei der Hausaufgabenmenge erreicht sein muss, bevor deren positive Auswirkungen konsistent bzw. deutlich zu Tage treten.

Nun stellt sich u.a. die Frage, ob die Erteilung von Hausaufgaben nicht die Unterschiede zwischen den Schülerleistungen, die sich aus familiären Hintergrundfaktoren ergeben, zusätzlich verstärken könnte. In der Tat erklären sich aus dem sozioökonomischen Index der beruflichen Stellung in einigen Ländern, wie Belgien, Griechenland, Ungarn, Korea und dem Vereinigten Königreich, 2\% und mehr der Varianz des Hausaufgabenindex. In anderen Ländern, darunter Italien und Polen, besteht jedoch ein positiver Zusammenhang zwischen dem PISA-Hausaufgabenindex und der Leseleistung, und es ist praktisch keine Beziehung zum sozioökonomischen PISA-Index der beruflichen Stellung festzustellen. Das lässt darauf schließen, dass die Hausaufgaben in einer Weise erteilt werden können, die auch Schülerinnen und Schüler aus sozioökonomisch benachteiligten Milieus zum Lernen ermutigt (vgl. Tabelle 7.6 und Anhang A2).

\author{
Aus verschiedenen \\ Gründen erzielen \\ schülerinnen und Schüler, \\ die mehr Zeit auf die \\ Hausaufgaben verwenden, \\ nicht immer die besten \\ Ergebnisse,...
}

... aber sie schneiden im
Durchschnitt besser ab, ...

... vor allem in Ländern

mit einer vergleichsweise

hohen durchschnittlichen

Hausaufgabenmenge. 
KAPITEL 7 Das Lernumfeld und die Schulorganisation

Investitionen in das Bildungswesen

\section{Lehrermangel}

Einige Länder beklagen

einen Mangel an

qualifizierten

Lehrkräften, ...

Schulleitungen in PISA

auch gefragt wurden,

inwieweit der Lernprozess

durch den Lehrermangel

behindert wird.
Hoch qualifizierte Lehrkräfte einstellen und halten zu können, ist in den OECDLändern ein wichtiges Anliegen der Politik. Auf Grund der zunehmenden Alterung der Lehrerschaft und steigender Schülerzahlen erhöht sich in vielen Ländern nach wie vor der Druck auf die Nachfrage nach Lehrkräften, während die angehenden Lehrerinnen und Lehrer in einigen Ländern zugleich zu der Auffassung gelangen, dass der Lehrerberuf mit unverhältnismäßig viel Stress verbunden ist, nicht genügend Ansehen genießt und das Gehaltsniveau zudem unter dem anderer Berufe mit ähnlichem Qualifikationsniveau liegt (OECD, 2001).

Der PISA-Fragebogen für Schulleiterinnen und Schulleiter bietet eine Möglichkeit, um zu beurteilen, ob die Schulleitungen das Lehrkräfteangebot für ausreichend halten und inwieweit sich ein von ihnen eventuell festgestellter Lehrermangel auf die Schülerleistungen auswirkt. Ausgehend von den Antworten auf vier Fragen darüber, wie sehr der Lernprozess der 15-Jährigen in ihrer Schule durch fehlende oder unzureichend qualifizierte Lehrkräfte für die Testsprache sowie für Mathematik und Naturwissenschaften beeinträchtigt wird, wurde ein Index des Lehrkräftemangels konstruiert, anhand dessen die Auswirkungen des Lehrermangels auf die Lernerfolge der Schülerinnen und Schüler untersucht wurden. Für alle OECD-Länder wurde bei diesem Index ein Mittelwert von 0 festgesetzt. Je weiter der Wert über dem Indexmittel liegt, umso besser ist das Lehrkräfteangebot, zumindest aus der Sicht der Schulleitungen. Werte unter 0 bedeuten, dass die festgestellte Beeinträchtigung des Lernprozesses der 15-Jährigen durch fehlende oder unzureichend qualifizierte Lehrkräfte höher ist als im OECD-Durchschnitt.

Bei einer Gegenüberstellung aller PISA-Teilnehmerländer ergibt sich, dass die Schulleitungen in Griechenland und in der Russischen Föderation am häufigsten der Ansicht sind, dass fehlende oder unzureichend qualifizierte Lehrkräfte den Lernprozess an ihren Schulen beeinträchtigten. Relativ hoch ist die Besorgnis der Schulleitungen über den Mangel an Lehrkräften auch in Island, Mexiko, den Niederlanden und im Vereinigten Königreich. In Österreich, der Tschechischen Republik und Spanien wird Lehrkräftemangel von den Schulleitungen am seltensten als ein Hindernis für den Lernprozess gesehen (Tabelle 7.8).

Bei der Interpretation dieser Antworten muss berücksichtigt werden, dass der Lehrkräftemangel nicht in Form einer international vergleichbaren Messgröße bestimmt wurde, wie der Zahl der Schülerinnen und Schüler pro Lehrkraft, sondern dass der Schwerpunkt bei PISA auf der Frage lag, wie sehr ein unzureichendes Lehrkräfteangebot den Lernprozess nach Ansicht der Schulleitungen beeinträchtigt. So gehören beispielsweise einige der Länder, in denen sich die Schulleitungen überdurchschnittlich stark über den negativen Effekt eines unzureichenden Lehrkräfteangebots auf die Schülerleistungen 
besorgt zeigen, darunter Griechenland, Italien und Norwegen, zu den OECDLändern mit den niedrigsten Schüler-Lehrer-Quoten (OECD, 2001).

Alles in allem besteht offenbar ein schwach negativer Zusammenhang zwischen einem festgestellten Lehrkräftemangel und den Schülerleistungen im Bereich Lesekompetenz $^{10}$. Je größer der Mangel nach Ansicht der Schulleiterinnen und Schulleiter ist, desto stärker nehmen erwartungsgemäß die Leistungen ab. Die höchsten Punktzahlen im Bereich Lesekompetenz können in der Regel Schulen sowie Schülerinnen und Schüler im oberen Quartil vorweisen (wo höhere Indexwerte anzeigen, dass auf Seiten der Schulleitungen keine oder nur wenig Besorgnis über fehlende oder unzureichend qualifizierte Lehrkräfte besteht).

Im OECD-Durchschnitt besteht im Bereich Lesekompetenz zwischen der mittleren Punktzahl des oberen und des unteren Quartils des Index des Mangels an Lehrkräften ein Abstand von insgesamt 22 Punkten, die Streuungsbreite der Ergebnisse weicht jedoch zwischen den Ländern erheblich ab. In mehreren Ländern sinken die durchschnittlichen Punktzahlen im Bereich Lesekompetenz zwischen dem oberen und dem unteren Quartil des Index des Mangels an Lehrkräften um mindestens 40 Punkte, so z.B. in Österreich, Belgien, der Tschechischen Republik, Polen, dem Vereinigten Königreich und vor allem in Deutschland (98 Punkte) (Tabelle 7.8). Demgegenüber sind die Unterschiede bei der durchschnittlichen Punktzahl zwischen dem oberen und dem unteren Quartil in Griechenland, Irland, Italien, Luxemburg, Mexiko, den Niederlanden, Norwegen und der Russischen Föderation statistisch nicht signifikant, obwohl nach Ansicht der Schulleitungen in diesen Ländern ebenfalls Lehrkräftemangel herrscht.

Obwohl nach Einschätzung der Schulleitungen in Australien, Island, Irland, Japan, Neuseeland, Norwegen, Schweden und dem Vereinigten Königreich durchgängig ein überdurchschnittlicher Lehrkräftemangel vorhanden ist, erzielt in jedem dieser Länder das Quartil der Schülerinnen und Schüler, in deren Schulen nach Angaben der Schulleitungen die größten diesbezüglichen Schwierigkeiten bestehen, im Bereich Lesekompetenz im Schnitt immer noch eine Punktzahl um oder über dem OECD-Durchschnitt.

\section{Qualität der räumlichen Bedingungen der Schulen und der Ausstattung mit Lehr- und Sachmitteln}

Die Bereitstellung einer geeigneten räumlichen Infrastruktur und einer hinreichenden Ausstattung mit Lehr- und Sachmitteln ist zwar keine Garantie für hohe Leistungen, doch kann das Fehlen eines solchen Umfelds den Lernprozess möglicherweise beeinträchtigen. Gebäude in gutem Zustand und ausreichende Unterrichtsräume sind beides Faktoren, die zu einem lernfördernden materiellen Umfeld beitragen. Dasselbe gilt mehr oder minder für die ausreichende Ausstattung der Schulen mit Lehr- und Sachmitteln, wie Computern, Bibliotheken und Unterrichtsmaterial, einschließlich Schulbüchern, sowie Multimediaressourcen für Unterrichtszwecke.
Insgesamt liegen die Schülerleistungen in Schulen mit größserem Lehrkräftemangel etwas niedriger, ...

... der Leistungsabstand ist jedoch in einigen Ländern vergleichsweise größer, während er in anderen überhaupt nicht festzustellen ist, ...
Die Räumlichkeiten sowie das Angebot an Büchern und sonstigen Ressourcen bestimmen das Lernumfeld. 
KAPITEL 7 Das Lernumfeld und die Schulorganisation

$\begin{aligned} \text { Die Schulleitungen wurden } & \text { Ausgehend von den Antworten der Schulleitungen auf eine Reihe von Fragen } \\ \text { danach gefragt, ob } & \text { darüber, inwieweit der Lernprozess der 15-Jährigen nach ihrer Ansicht durch } \\ \text { schlechte räumliche } & \text { den Mangel an geeigneten Räumlichkeiten sowie Lehr- und Sachmitteln } \\ \text { Bedingungen oder } & \text { gehemmt wird, wurden zwei Indizes konstruiert: einer für die Qualität der } \\ \text { mangelnde Lehr-und } & \text { räumlichen Bedingungen der Schulen und ein anderer für die Ausstattung } \\ \text { Sachmittel den Lernprozess } & \text { mit Lehr- und Sachmitteln, beide aus Sicht der Schulleitungen. Wie } \\ \text { behindern. } & \text { bei den vorstehend erörterten Indizes wurde der Mittelwert der OECD- } \\ & \text { Länder auch hier mit Null und die Standardabweichung mit } 1 \text { angesetzt. } \\ & \text { Positive Werte bedeuten, dass die Besorgnis der Schulleitungen über etwaige } \\ & \text { negative Auswirkungen der räumlichen Bedingungen und der Ausstattung mit } \\ & \text { Lehr- und Sachmitteln ihrer Schulen auf den Lernprozess der 15-Jährigen } \\ & \text { unterdurchschnittlich ist. }\end{aligned}$

In der Tschechischen Republik, Ungarn und der Schweiz geben nur wenige Schulleitungen an, dass die Qualität der räumlichen Bedingungen ihrer Schulen den Lernprozess beeinträchtigt (Tabelle 7.9). Zu den anderen Ländern, in denen die Schulleitungen relativ selten erklärten, dass die Qualität der räumlichen Bedingungen ihrer Schule den Lernprozess hemmt, zählen Belgien, Brasilien, Kanada und Island. Demgegenüber zeigen sich die Schulleitungen in Griechenland, Korea, Luxemburg, Mexiko, Norwegen, der Russischen Föderation und dem Vereinigten Königreich zumindest im Vergleich mit dem OECD-Durchschnitt stärker besorgt über die möglichen Auswirkungen der ihrer Ansicht nach unzureichenden Qualität der räumlichen Bedingungen.

Bei den räumlichen Bedingungen besteht, wenn überhaupt, nur ein geringer

Zusammenhang mit den Schülerleistungen, ...

... während der Zusammenhang mit den Lehr-und Sachmitteln etwas stärker ist.
In Belgien, Ungarn, der Schweiz und den Vereinigten Staaten geben die Schulleitungen vergleichsweise selten an, dass die Qualität der Ausstattung mit Lehr- und Sachmitteln den Lernprozess beeinträchtigt (Tabelle 7.10). Im Gegensatz dazu sind die Schulleitungen in Griechenland, Lettland, Mexiko, Norwegen, der Russischen Föderation und dem Vereinigten Königreich im Vergleich zum OECD-Durchschnitt stärker über die unzulängliche Ausstattung ihrer Schulen mit Lehr- und Sachmitteln besorgt.

Die Frage lautet nun, ob sich Unterschiede hinsichtlich der Qualität der räumlichen Bedingungen auch auf die Schülerleistungen auswirken. Zwar sind im Bereich der Lesekompetenz zwischen dem oberen und dem unteren Quartil des Index der Qualität der räumlichen Bedingungen der Schulen gewisse Unterschiede bei den Punktzahlen der Schülerinnen und Schüler festzustellen, doch sie sind meistens gering und statistisch nicht signifikant ${ }^{11}$.

Die Ausstattung mit Lehr- und Sachmitteln scheint in engerem Zusammenhang mit den Schülerleistungen zu stehen als die Qualität der Räumlichkeiten ${ }^{12}$. In 13 OECD-Ländern sowie in Brasilien können Unterschiede hinsichtlich der Ausstattung mit Lehr- und Sachmitteln mit Differenzen von mehr als 22 Punkten bei der Leseleistung in Verbindung gebracht werden, wobei beim Vergleich des untersten mit dem obersten Quartil auf dem Index der Ausstattung mit Lehrund Sachmitteln die größten Unterschiede in Mexiko (81 Punkte), Luxemburg 


\section{Abbildung 7.7}

Qualität der schulischen Ressourcen aus der Sicht der Schulleitungen

Verteilung der mittleren Prozentsätze der Schülerinnen und Schüler in Schulen, wo das Lernen nach Angaben der Schulleitungen „etwas" oder „sehr" durch folgende Faktoren beeinträchtigt wird

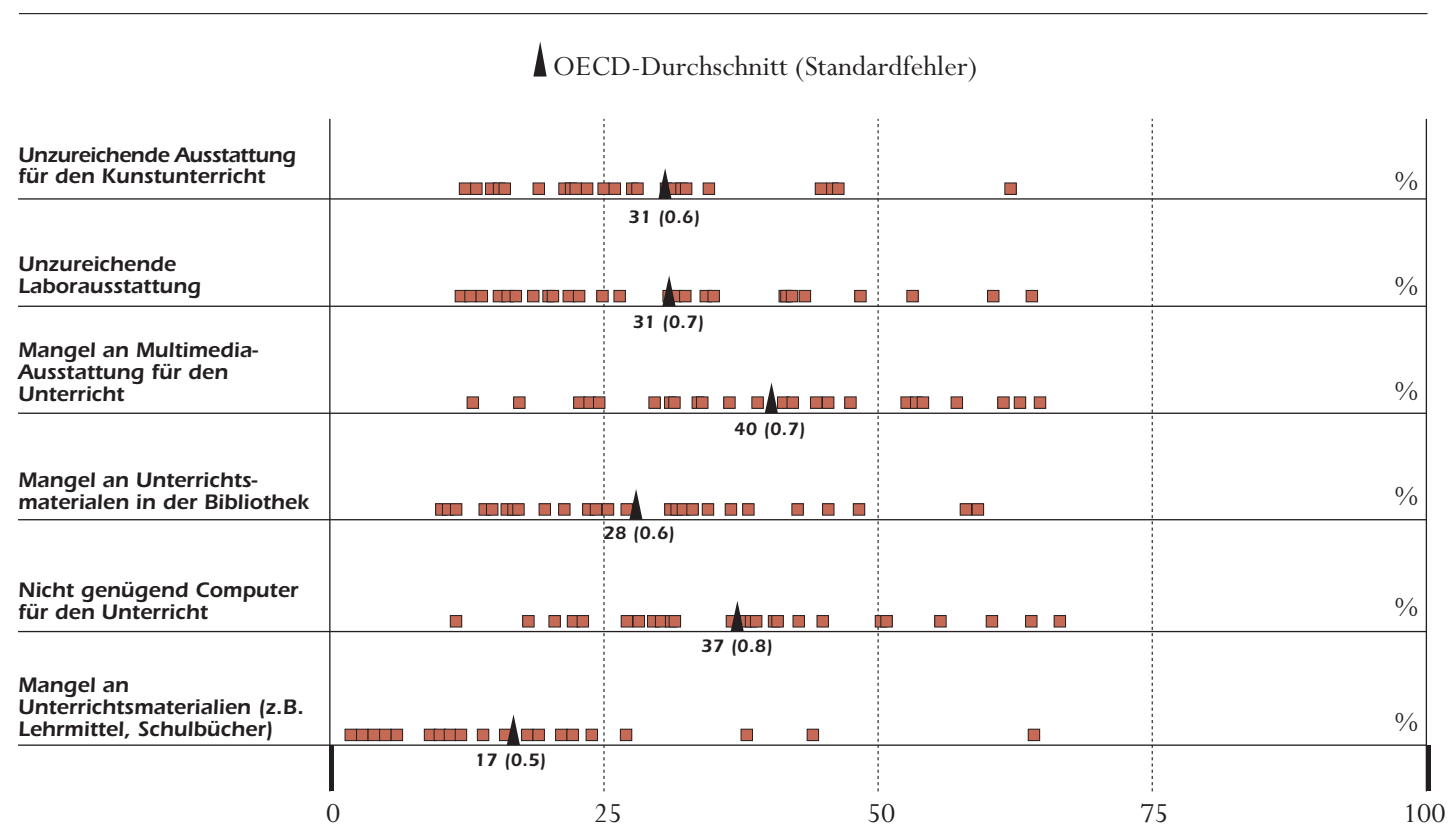

Anmerkung: Die Länder sind dargestellt durch die Quadrate.

Quelle: OECD PISA Datenbank, 2001. Daten abrufbar unter www.pisa.oecd.org.

(63 Punkte), Deutschland (55 Punkte), Brasilien (41 Punkte) und dem Vereinigten Königreich (39 Punkte) festzustellen sind. Die durchschnittliche Differenz für die OECD-Länder insgesamt beträgt rund 23 Punkte $^{13}$.

\section{Methoden der Verwaltung und Finanzierung der Schulen}

\section{Autonomie der Schulen und Einbeziehung der Lehrkräfte}

Eines der Hauptziele der Struktur- und Systemreformen des Bildungswesens, die ab Anfang der achtziger Jahre in vielen Ländern eingeleitet wurden, war es, die unteren Ebenen mit mehr Entscheidungsbefugnissen auszustatten. Durch die Verlagerung derVerwaltung auf die Ebene der Schulen soll deren Kreativität und Anpassungsfähigkeit an die Bedürfnisse der jeweiligen Einzugsgebiete gefördert werden. Dies beinhaltet eine Stärkung der Entscheidungsverantwortung und Rechenschaftspflicht der Schulleiterinnen und Schulleiter sowie in manchen

Die Schulleitungen liefern in PISA Angaben dazu, wer für ihre Schule die Verantwortung in verschiedenen Bereichen der schulischen Politik und verwaltung trägt. Fällen auch der Verantwortung der Lehrkräfte und Fachbereichsleiter in Verwaltungsangelegenheiten. Eine größere Autonomie ermöglicht es den Schulen zwar, den Anforderungen des jeweiligen lokalen Umfelds besser gerecht zu werden, sie wird jedoch gelegentlich auch als ein Faktor angesehen, der 
KAPITEL 7 Das Lernumfeld und die Schulorganisation

In der Mehrzahl der Länder haben die meisten Schulen kein Mitspracherecht bei der Festsetzung der Anfangsgehälter ihrer Lehrkräfte,...

wohingegen viele Einfluss auf die Einstellung und Entlassung von Lehrkräften haben.
Auswahlmechanismen in Gang setzt, die hauptsächlich bereits privilegierten gesellschaftlichen Gruppen zugute kommen.

Um den Umfang des Mitspracherechts des Lehrerkollegiums und der Schulleitung in Fragen der schulischen Politik und Verwaltung beurteilen zu können, wurden die Schulleitungen gebeten, anzugeben, ob sie selbst, die Lehrkräfte, die Fachbereichsleiter, ein ernanntes oder gewähltes Gremium oder die Erziehungsbehörden die Hauptverantwortung für folgende Aufgaben tragen: Einstellung von Lehrkräften, Entlassung von Lehrkräften, Festlegung der Anfangsgehälter der Lehrkräfte, Entscheidung über die Beförderung bzw. Höhergruppierung von Lehrkräften, Festlegung des Schulbudgets, Entscheidung über die Verwendung des Budgets innerhalb der Schulen, Festlegung von disziplinären Regeln für die Schülerinnen und Schüler, Festlegung von Kriterien für die Schülerbeurteilung, Aufnahme von Schülerinnen und Schülern in die Schulen, Wahl der verwendeten Lehrbücher, Bestimmung des Lehrstoffs und Entscheidung über das Fächer- und Kursangebot.

Aus Tabelle 7.11 ist der Prozentsatz der Schülerinnen und Schüler ersichtlich, die sich in Schulen befinden, in denen die Schulleitung zumindest eine gewisse Verantwortung für verschiedene Aspekte der Schulverwaltung trägt (Daten abrufbar unter www.pisa.oecd.org $)^{14}$.

Anders als Privatunternehmen haben die Schulen in den meisten Ländern kaum Einfluss auf die Höhe der Anfangsgehälter ihrer Lehrkräfte. In allen Ländern außer der Tschechischen Republik, Griechenland, den Niederlanden, dem Vereinigten Königreich und den Vereinigten Staaten besuchen mindestens zwei Drittel der 15-Jährigen Schulen, die laut Aussage der Schulleitungen kein Mitspracherecht bei der Festsetzung der Anfangsgehälter ihrer Lehrkräfte haben. Auch der Spielraum der Schulen für eine leistungsorientierte Anpassung der Gehälter der bereits bei ihnen beschäftigten Lehrkräfte ist begrenzt. Nur in der Tschechischen Republik, Griechenland, Schweden, dem Vereinigten Königreich und den Vereinigten Staaten besuchen mehr als zwei Drittel der Schülerinnen und Schüler Schulen, in denen die Schulleitungen zumindest gewisse Befugnisse bei Entscheidungen über die Beförderung bzw. Höhergruppierung ihrer Lehrkräfte haben.

Was die Einstellung oder Entlassung von Lehrkräften anbelangt, scheinen die Schulen über mehr Flexibilität zu verfügen. Nur in Deutschland und Italien werden mindestens 90\% der 15-Jährigen in Schulen unterrichtet, die laut Aussage ihrer Leitungen keinerlei Befugnisse in diesem Bereich besitzen. In Belgien, der Tschechischen Republik, Dänemark, Island, Schweden, der Schweiz, dem Vereinigten Königreich und den Vereinigten Staaten besuchen hingegen zwischen 93\% und 99\% der Schülerinnen und Schüler Schulen, die einen gewissen Einfluss auf die Einstellung von Lehrkräften ausüben können (OECD-Durchschnitt: 61\%). In der Mehrzahl der Länder geben die Schulleitungen an, dass ihre Schule ein größeres Mitspracherecht bei 


\section{Abbildung 7.8}

Schulautonomie und Schülerleistungen

Verteilung der mittleren Prozentsätze der Schülerinnen und Schüler in Schulen, die nach Angaben der Schulleitungen eine gewisse Verantwortung für folgende Aspekte der Schulpolitik und -verwaltung tragen

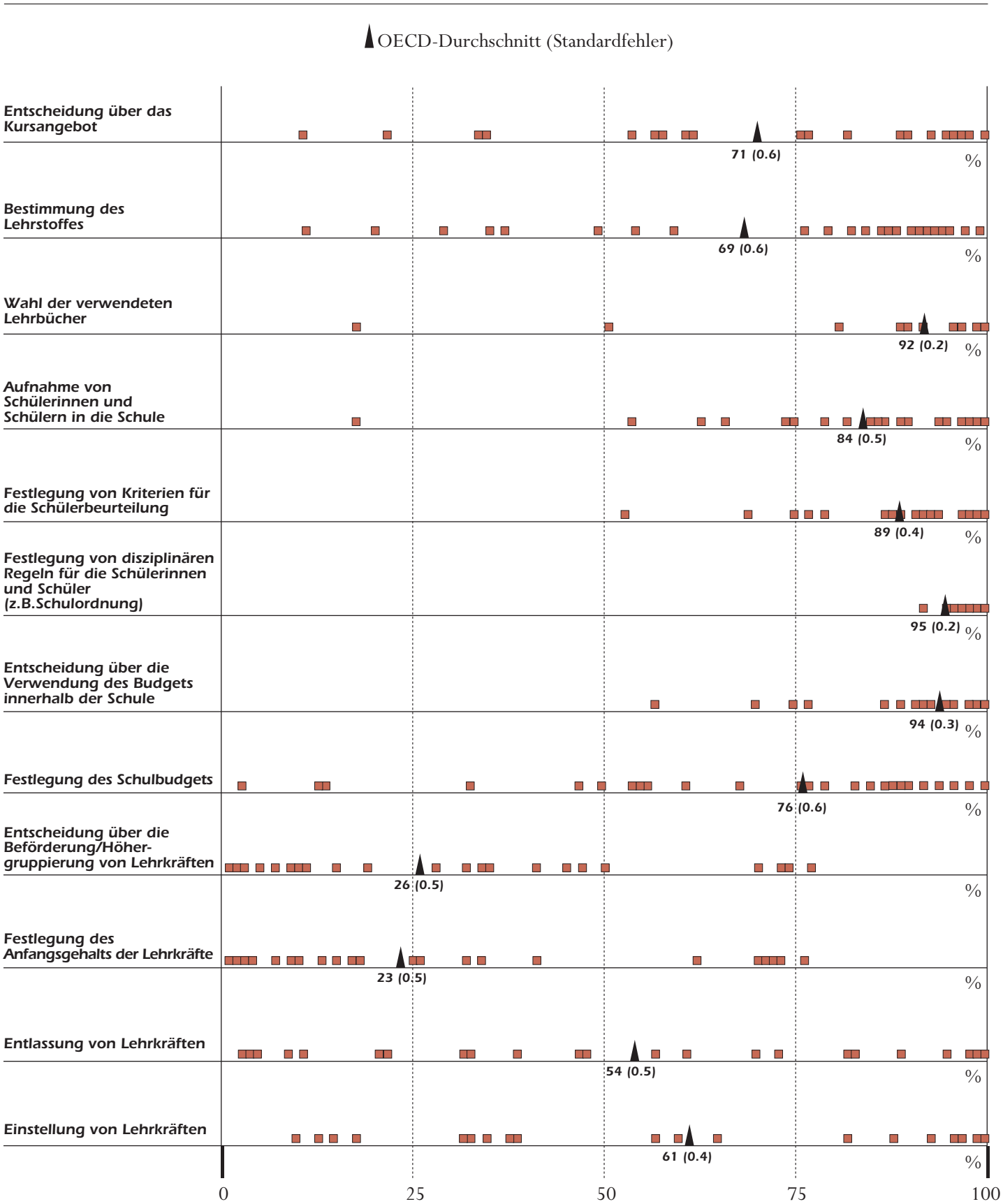

Anmerkung: Die Länder sind dargestellt durch die Quadrate.

Quelle: OECD PISA Datenbank, 2001. Daten abrufbar unter www.pisa.oecd.org. 
KAPITEL 7 Das Lernumfeld und die Schulorganisation

Größsere Unterschiede bestehen hinsichtlich der Befugnisse der Schulen bei der Festlegung der Budgets und der Entscheidung über deren Verwendung.

Die meisten Schulen haben einen gewissen Einfluss darauf, welche Schüler bei ihnen aufgenommen werden und

können auch über das

Fächerangebot, die disziplinären Regeln, die Beurteilungsverfahren und die verwendeten Lehrbücher entscheiden.

Es lassen sich nur schwer Zusammenhänge zwischen dem Grad der schulischen

Autonomie und den Schülerleistungen herstellen,...

... im Durchschnitt erzielen die Schülerinnen und Schüler jedoch in solchen Ländern höhere Ergebnisse, in denen die Schulen über mehr Autonomie verfügen, vor allem in Bezug auf das Fächerangebot und die Verwendung der zugebilligten Mittel. der Einstellung als bei der Entlassung von Lehrkräften hat, wobei die diesbezüglichen Unterschiede in Kanada und Dänemark am größten sind (21 bzw. 40 Prozentpunkte). In Belgien, der Tschechischen Republik, Ungarn, Island, Lettland, den Niederlanden, Neuseeland, der Russischen Föderation und den Vereinigten Staaten befinden sich über 95\% der Schülerinnen und Schüler in Schulen, deren Schulleitungen ein gewisses Mitspracherecht bei der Entlassung von Lehrkräften angeben (OECD-Durchschnitt: 54\%).

Auch hinsichtlich der Befugnisse der Schulen zur Festlegung der Schulbudgets bestehen Unterschiede, wobei der Einfluss der österreichischen und deutschen Schulen in diesem Bereich am geringsten ist. In Australien, Belgien, Italien, Luxemburg, den Niederlanden, Neuseeland, dem Vereinigten Königreich und den Vereinigten Staaten genießen die Schulen bei der Budgetfestlegung eine vergleichsweise große Autonomie. In den meisten Ländern haben die Schulen laut Aussage der Schulleitungen hingegen großen Einfluss auf die Mittelverwendung innerhalb ihrer Schule (OECD-Durchschnitt: 94\%).

In allen OECD-Ländern besucht die Mehrzahl der 15-Jährigen Schulen, die bis zu einem gewissen Grad selbst über die Aufnahme ihrer Schülerinnen und Schüler entscheiden können (OECD-Durchschnitt: 84\%).

Außer in Deutschland, Italien und der Schweiz wird die Mehrzahl der 15-Jährigen in Schulen unterrichtet, die Einfluss auf Entscheidungen bezüglich der von ihnen angebotenen Fächer haben (OECD-Durchschnitt: 71\%). Die meisten Schulleitungen (rund 90\% im OECD-Durchschnitt) geben schließlich an, dass die Festlegung der disziplinären Regeln für die Schülerinnen und Schüler, die Kriterien für die Schülerbeurteilung und die Auswahl des Lehrmaterials in den Zuständigkeitsbereich der Schulen fallen.

Inwieweit wirkt sich die Aufteilung der Entscheidungsbefugnisse nun auf die Schülerleistungen aus? In diesem Bereich bestehen innerhalb der einzelnen Länder oft nur schwache Zusammenhänge zwischen den verschiedenen Aspekten schulischer Autonomie und den Schülerleistungen. Dies ist einleuchtend, da die Verteilung der Entscheidungskompetenzen häufig durch die nationale Gesetzgebung geregelt wird. Folglich bestehen kaum Unterschiede innerhalb der einzelnen Länder.

Gleichwohl lässt sich aus den vorliegenden Daten schließen, dass in jenen Ländern, in denen die Schulen laut Aussage der Schulleitungen im Durchschnitt über ein höheres Maß an Autonomie in Bezug auf das Fächerangebot verfügen, die durchschnittlichen Leistungen auf der Gesamtskala Lesekompetenz im Allgemeinen höher sind (die Korrelation zwischen den Ländermitteln der Schülerleistungen und dem jeweiligen Prozentsatz der Schulen, die an Entscheidungen bezüglich des Fächerangebots beteiligt sind, beträgt 0,51). Ein ähnliches, wenngleich weniger deutliches Bild zeichnet sich für andere Aspekte schulischer Autonomie ab, z.B. für den Zusammenhang zwischen den 
durchschnittlichen Leistungen und dem Grad der Autonomie der Schulen im Hinblick auf die Verwendung der ihnen zugebilligten Mittel (Korrelation auf Länderebene 0,37) (Tabelle 7.11).

Aus Tabelle 7.12 ist der Prozentsatz der Schülerinnen und Schüler ersichtlich, die Schulen besuchen, in denen den Lehrkräften die Hauptverantwortung in den verschiedenen Entscheidungsbereichen zukommt. In den meisten Ländern konzentriert sich die Zuständigkeit der Lehrkräfte bei den erfragten Aspekten auf die Auswahl der Lehrbücher (OECD-Durchschnitt: 70\%), des Lehrstoffs (OECD-Durchschnitt: 55\%), der Beurteilungsverfahren (OECD-Durchschnitt: $57 \%$ ) und der disziplinären Regeln für die Schülerinnen und Schüler (OECD-Durchschnitt: 49\%). In wenigen Schulen kommt den Lehrkräften die Hauptverantwortung bei Entscheidungen in Bezug auf die Gehaltspolitik, die Einstellung und Entlassung von Lehrkräften und die Festlegung der Schulbudgets zu.

Auch hier zeigt sich wieder, dass die durchschnittlichen Leistungen auf der Gesamtskala Lesekompetenz in solchen Ländern offenbar höher sind, in denen die Lehrkräfte stark in die Verwaltung der Schulen einbezogen werden. Die Korrelationen zwischen dem Prozentsatz der Schulen, in denen die Lehrkräfte die Hauptverantwortung für Entscheidungen bezüglich des Lehrstoffs und der Fächerwahl haben, und den Mittelwerten auf der Gesamtskala Lesekompetenz betragen auf Länderebene 0,46 bzw. 0,55 (Tabelle 7.12).

Wie in anderen Analysen dieser Art können solche Korrelationen nicht als Kausalzusammenhänge interpretiert werden, da zahlreiche andere Faktoren mitspielen können. Dennoch legen die Ergebnisse den Schluss nahe, dass eine größere Autonomie der Schulen und stärkere Einbeziehung der Lehrkräfte in die Entscheidungsprozesse in der Regel, zumindest im Ländervergleich, in einem positiven Zusammenhang mit den durchschnittlichen Ergebnissen im Bereich der Lesekompetenz stehen.

\section{Öffentliche oder private Trägerschaft}

Das Schulwesen ist großenteils eine staatliche Unternehmung. In zwei Dritteln der OECD-Länder liegt der Anteil der in die Primar- und Sekundarschulbildung investierten privaten Mittel unter 10\%; in Italien, Norwegen, Portugal und Schweden stammen sogar weniger als 2\% der schulischen Mittel aus privaten Quellen (vgl. OECD, 2001). Die private Finanzierung gewinnt jedoch mehr und mehr an Bedeutung, und im Zuge einer wachsenden Vielfalt an Bildungsmöglichkeiten, -programmen und -anbietern gehen die staatlichen Stellen neue Partnerschaften ein, um finanzielle Mittel für das Bildungswesen zu mobilisieren und innovative Politiken für eine stärkere Einbindung der verschiedenen Akteure und eine gerechtere Verteilung von Kosten und Nutzen auszuarbeiten.
In den meisten Ländern tragen die Lehrkräfte die Hauptverantwortung für die Wahl der Lehrbücher, den Lehrstoff, die Beurteilungskriterien und die disziplinären Regeln.

In Ländern, in denen die Lehrkräfte mehr Einfluss auf die Wahl der Lehrinhalte haben, schneiden die Schülerinnen und Schüler im Allgemeinen besser ab, ...

... wenngleich daraus nicht geschlossen werden darf, dass eine größsere Autonomie der Schulen die Schülerleistungen steigen lässt.

Dem privaten Sektor kommt eine kleine, doch wachsende Rolle im Schulwesen zu,... 
KAPITEL 7 Das Lernumfeld und die Schulorganisation

... wobei im Durchschnitt

$6 \%$ der Schülerinnen und schüler überwiegend privat

finanzierte und verwaltete

Schulen besuchen, ...

... was in zwei Ländern

für rund ein Drittel der Schülerschaft der Fall ist.

Andere Formen privater Schulbildung sind in den $O E C D$-Ländern jedoch stärker verbreitet, ..

besonders vom Stad abhängige Privatschulen.
Im Durchschnitt der 24 OECD-Länder, für die diesbezügliche Daten zur Verfügung stehen, besuchen 6\% der 15-jährigen Schülerinnen und Schüler privat verwaltete und überwiegend privat finanzierte Schulen (im Folgenden unabhängige Privatschulen genannt) (Tabelle 7.13). Die Leiterinnen und Leiter dieser Schulen geben an, dass sie von nicht-staatlichen Stellen, wie Kirchen, Gewerkschaften oder Unternehmen, verwaltet werden und/oder Aufsichtsgremien unterstehen, deren Mitglieder in der Mehrzahl von nichtöffentlichen Stellen ernannt wurden. Mindestens 50\% ihrer Mittel stammen aus privaten Quellen, z.B. aus von den Eltern bezahlten Schulgebühren, aus Spenden von Stiftungen oder Sponsoren, aus von den Eltern gesammelten Geldern oder aus sonstigen nicht öffentlichen Quellen.

Ein solches Modell der privaten Trägerschaft ist jedoch nur in wenigen Ländern üblich. Der Prozentsatz der Schülerinnen und Schüler, die an unabhängigen Privatschulen unterrichtet werden, übersteigt nur in Korea (34\%), Japan (30\%) und Mexiko (15\%) die 10\%-Grenze (Tabelle 7.13). In vielen Ländern hingegen wird die Tatsache, dass solche Schulen von den Schülerinnen und Schülern bzw. ihren Eltern finanziert werden müssen, als potentielle Zugangsbarriere angesehen. In 9 der 24 Länder, für die Daten verfügbar sind, sind beispielsweise weniger als $1 \%$ der 15 -Jährigen an unabhängigen Privatschulen eingeschrieben.

Das private Schulwesen stellt nicht nur ein Mittel dar, um ein breiteres Spektrum von Finanzierungsquellen zu erschließen, sondern wird gelegentlich auch als eine Möglichkeit für eine kosteneffizientere Erfüllung der Bildungsaufgaben angesehen. Öffentlich finanzierte Schulen müssen nicht zwangsläufig von öffentlichen Stellen verwaltet werden. Der Staat kann öffentlichen und privaten Bildungseinrichtungen auch über verschiedene Allokationsmechanismen Mittel zukommen lassen (vgl. OECD, 2001). Indem er die Finanzierung von Bildungseinrichtungen von der Entscheidung der Eltern, ihre Kinder dort anzumelden, abhängig macht, kann der Staat Anreize schaffen, die die Bildungseinrichtungen dazu bewegen sollen, ihr Lehrprogramm und ihr Unterrichtsangebot besser auf die Bedürfnisse und Interessen der Schülerinnen und Schüler abzustimmen und so die Kosten von schulischem Versagen und bedarfsfremden Lehrangeboten zu reduzieren. Ein mögliches Modell hierfür ist die Finanzierung schulischer Einrichtungen durch den Staat auf Grundlage der Zahl der angemeldeten Schülerinnen und Schüler oder der von diesen besuchten Unterrichtseinheiten. Eine andere Methode besteht darin, den Schülern bzw. ihren Familien Geld zukommen zu lassen (z.B. in Form von Stipendien oder Bildungsgutscheinen), mit denen sie die Ausbildung an öffentlichen oder privaten Bildungseinrichtungen ihrer Wahl finanzieren können.

Schulen, die zwar privat verwaltet sind, aber überwiegend aus öffentlichen Mitteln finanziert werden - im Folgenden vom Staat abhängige Privatschulen genannt - sind in den OECD-Ländern wesentlich stärker verbreitet als die unabhängigen Privatschulen. Im Durchschnitt der 24 OECD-Länder, für die 
Vergleichsdaten zur Verfügung stehen, besuchen 10\% der 15-Jährigen vom Staat abhängige Privatschulen; in Irland und den Niederlanden sind es zwischen 58\% und $75 \%$.

In welchem Zusammenhang stehen diese institutionellen Regelungen nun mit den Schülerleistungen? Im Durchschnitt der 17 in diesen Vergleich einbezogenen Länder liegen die Ergebnisse der Schülerinnen und Schüler von unabhängigen Privatschulen im Bereich Lesekompetenz in 10 Ländern statistisch signifikant über denen der Schülerinnen und Schüler öffentlicher Schulen. Der Leistungsunterschied zwischen vom Staat abhängigen Privatschulen und öffentlichen Schulen ist nur rund halb so groß, wobei die privaten Schulen wiederum besser abschneiden (Abbildung 7.9).

Bei der Interpretation dieser Zahlen muss berücksichtigt werden, dass die Verteilung der Schülerinnen und Schüler auf private und öffentliche Schulen im Allgemeinen nicht nach dem Zufallsprinzip erfolgt. Unzureichende Finanzmittel ihrer Familien können z.B. für Schülerinnen und Schüler ein großes Hindernis darstellen, wenn sie unabhängige Privatschulen besuchen wollen, die hohe Schulgebühren verlangen. Zudem können auch vom Staat abhängige Privatschulen, die kein Schulgeld erheben, auf eine besondere Schülerschaft ausgerichtet sein oder strengere Kriterien für den Schulwechsel bzw. die Aufnahme anlegen.

Um dies zu untersuchen, empfiehlt es sich, einen Blick auf den sozioökonomischen Hintergrund der an den verschiedenen Schultypen eingeschriebenen Schülerinnen und Schüler zu werfen. In Abbildung 7.9 sind die Prozentsätze der Schülerinnen und Schüler dargestellt, die öffentliche und private Schulen besuchen, wobei jeweils die mittleren Punktzahlen und die durchschnittlichen Einstufungen auf dem sozioökonomischen Index der beruflichen Stellung angegeben sind.

Wie sich zeigt, bestehen hinsichtlich des mittleren internationalen sozioökonomischen Index der beruflichen Stellung zwischen vom Staat abhängigen Privatschulen und öffentlichen Schulen nur geringe Unterschiede, während die sozioökonomischen Unterschiede zwischen öffentlichen Schulen und unabhängigen Privatschulen zugunsten der Privatschulen grösser sind.
Die Schülerinnen und

Schüler beider

Privatschultypen erzielen

hohe Leistungen, ...

... doch in einigen Ländern sind sie nicht repräsentativ für die gesamte Schülerschaft,...

... denn bei der Untersuchung ihres sozialen Hintergrunds zeigt sich, ...

... dass Privatschulen stärker von sozial besser gestellten Schülerinnen und schülern besucht werden, was jedoch auf die vom Staat abhängigen

Privatschulen etwas weniger stark zutrifft. 
KAPITEL 7 Das Lernumfeld und die Schulorganisation

\section{Abbildung 7.9}

Schülerleistungen und staatliche oder private Trägerschaft der Schulen

Unterschiede zwischen öffentlichen Schulen und von staatlichen Mitteln abhängigen Privatschulen bei Schülerleistungen auf der Gesamtskala Lesekompetenz und dem internationalen soziö̈konomischen Index der beruflichen Stellung
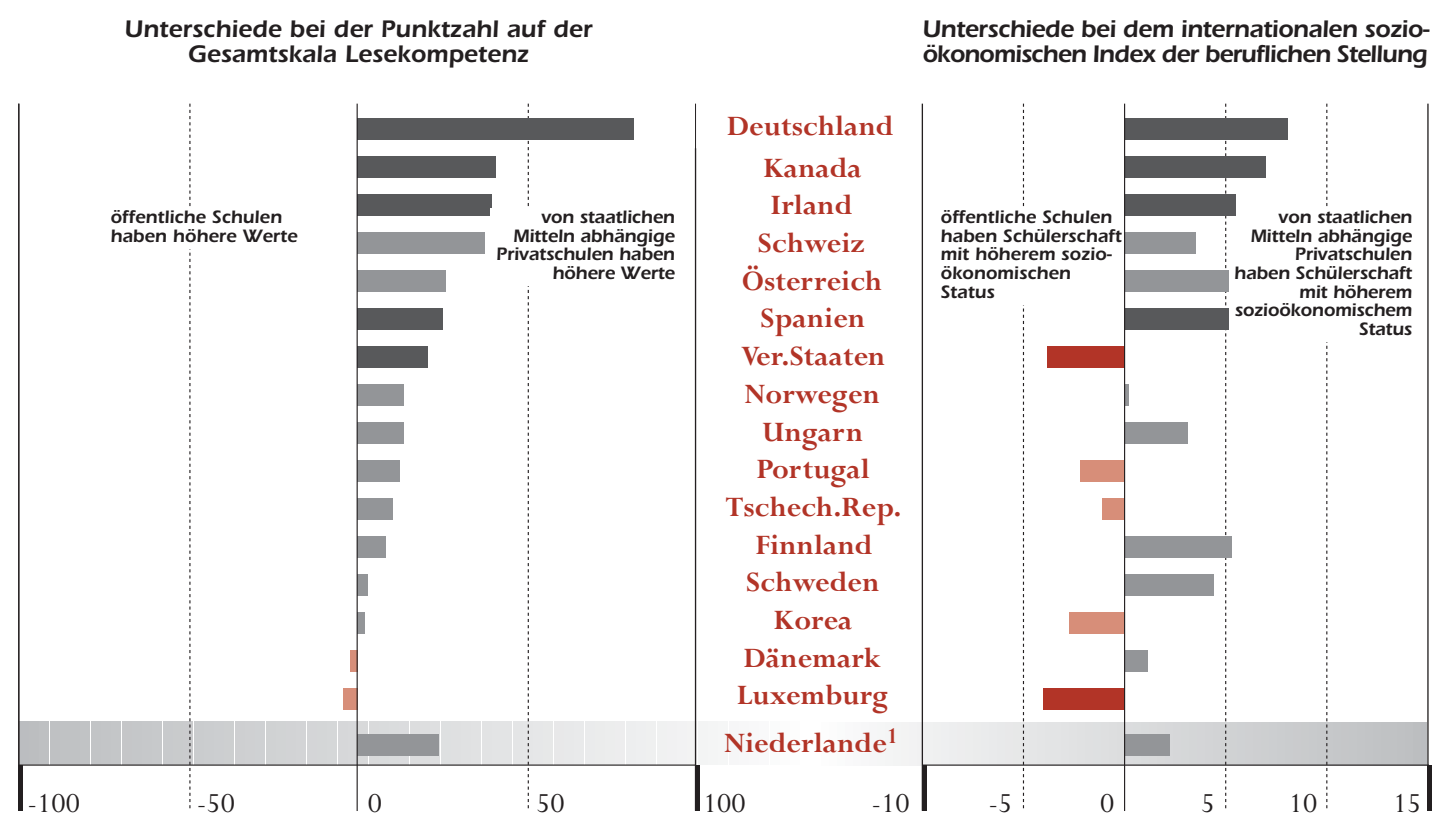

1. Die Beteiligungsquote ist zu niedrig, um die Vergleichbarkeit zu gewährleisten (vgl. Anhang A3). Anmerkung: Statistisch signifikante Unterschiede sind in schwarz und rot gekennzeichnet.

Quelle: OECD PISA Datenbank, 2001. Tabelle 7.13.

\section{Schlussbetrachtungen}

Die Schülerleistungen

werden ebenso von

Schulcharakteristika wie vom

familiären Hintergrund

beeinflusst.

Einige Schulleiterinnen und schulleiter beklagen einen

Mangel an schulischen

Ressourcen, ...
So wichtig sozioökonomische Faktoren im Hinblick auf die Schülerleistungen auch sein mögen, können Schulpolitik und -praxis doch einen entscheidenden Einfluss ausüben, denn schließlich hängt die Effizienz des Lernprozesses wesentlich vom Zugang zu hochwertigen Lernmöglichkeiten ab. Schulpolitik und -praxis können sowohl direkte Auswirkungen auf die Bildungsergebnisse als auch indirekte Effekte haben, indem sie den Einfluss der sozioökonomischen Faktoren mindern (vgl. Kapitel 6 und 8).

Obwohl alle Länder erhebliche Summen in ihr Bildungswesen investieren, beklagen die Schulleiterinnen und Schulleiter in einigen Ländern Mängel, was die Qualität der ihnen zurVerfügung stehenden Bildungs- und Humanressourcen anbelangt. In vielen Ländern scheint zudem ein Zusammenhang zwischen diesen Defiziten und geringeren Schülerleistungen zu bestehen. 
Allerdings sind nicht alle Hindernisse, die sich nach Ansicht der Schulleitungen negativ auf den Lernprozess auswirken, materieller Art. Ein weiterer Faktor, der laut PISA in engem Zusammenhang mit den Schülerleistungen steht, ist die Schuldisziplin. Zu den von den Schulleitungen am häufigsten genannten Hindernissen für den Lernprozess gehören häufiges Schuleschwänzen, Stören im Unterricht, mangelnder Respekt für die Lehrkräfte sowie das Schikanieren von Mitschülern. Aus Schülersicht schaden der Disziplin vor allem die vergeudete Zeit zu Beginn der Stunde, Lärm und Durcheinander während des Unterrichts sowie die Unaufmerksamkeit von Schülerinnen und Schülern, die ihren Lehrkräften nicht zuhören. Diese Probleme zu lösen, ist nicht allein eine Frage des Geldes.

Der Lernprozess fängt nicht mit Schulbeginn an und endet auch nicht mit Schulschluss. Ein großer Teil der von den Schülerinnen und Schülern auf das Lernen verwendeten Zeit entfällt oft auf Hausaufgaben sowie den in einigen Ländern üblichen Ergänzungs- bzw. Nachhilfeunterricht; gewöhnlich sind diese Aktivitäten mit einer Verlängerung der regulären Unterrichtsdauer in den drei PISA-Bereichen um bis zu einem Drittel verbunden. Angesichts des entsprechend großen Zeitaufwands ist es wichtig, dass diese Zeit effizient genutzt wird und die entsprechenden Lernmöglichkeiten in geeigneter Weise organisiert sind. Hierbei stellt sich u.a. das Problem, dass Hausaufgaben und außerschulischer Unterricht bereits bestehende Leistungsunterschiede verstärken können, die auf sozioökonomische Faktoren oder die ungleiche Ausstattung mit Bildungsressourcen bzw. die unterschiedliche Unterstützung, die die Schülerinnen und Schüler im Elternhaus finden, zurückzuführen sind. Allerdings legen die Ergebnisse aus einigen Ländern, in denen ein positiver Zusammenhang zwischen Hausaufgaben und Schülerleistungen nachgewiesen wurde und in denen offenbar zwischen dem familiären Hintergrund und den Leistungen keine Beziehung besteht, den Schluss nahe, dass der Lernprozess außerhalb der Schule fortgesetzt werden kann, ohne dass dadurch zwangsläufig ein zusätzliches Risiko für benachteiligte Schüler entstünde.

An letzter Stelle ist noch festzustellen, dass u.U. mehr Freiraum für Innovationen bei der Verwaltung der Schulen bestehen könnte. In den meisten Ländern haben anscheinend nur wenige Schulen ein Mitspracherecht bei der Festlegung der Gehälter der Lehrkräfte oder deren Beförderung. Etwas größer ist die Flexibilität der Schulen offenbar bei der Einstellung und Entlassung von Lehrkräften sowie der Festlegung und Verwendung des Schulbudgets. Allerdings ist der den Schulen und Lehrkräften jeweils zugebilligte Freiraum auch hier wieder in vielen Ländern begrenzt. Da Länder mit mehr Schulautonomie in der Tendenz bessere durchschnittliche Schülerleistungen vorweisen können, könnte die Förderung der Autonomie der Schulen u.U. eine Möglichkeit zur Verbesserung ihrer Leistungen darstellen.

\author{
... ein wesentlich stärkerer \\ Zusammenhang besteht \\ jedoch zwischen den \\ Schülerleistungen und dem \\ Schul-und \\ Unterrichtsklima...
}

... bzw. dem
außerschulischen Lernen.

Ein weiterer wichtiger

Faktor könnte in diesem

Zusammenhang die

Autonomie der Schulen

sein. 
KAPITEL 7 Das Lernumfeld und die Schulorganisation

Kapitel 8 erläutert ausführlicher, wie diese Faktoren miteinander in Zusammenhang stehen.
Insofern die in diesem Kapitel untersuchten Faktoren in einerWechselbeziehung miteinander stehen, kann ihre relative Bedeutung nur schwer abgeschätzt werden. Im nächsten - abschließenden - Kapitel soll die relative Bedeutung der schulischen Ressourcen sowie der schulischen Politik und Praxis in verschiedenen Schulsystemtypen beurteilt werden. Dies könnte Hinweise dafür liefern, wie die Bildungspolitik zugleich die Durchschnittsleistungen der Schülerinnen und Schüler anheben und die Auswirkungen des familiären Hintergrunds auf die Schülerleistungen mindern könnte. 


\section{Anmerkungen}

1. Zur Bestätigung der theoretisch erwarteten Dimensionen der Indizes und Überprüfung ihrer Eignung für den Ländervergleich wurden Strukturgleichungsmodelle verwendet. Zu diesem Zweck wurde jeweils eine Modellrechnung separat für jedes Land sowie für alle OECD-Länder zusammen geschätzt.

2. Es ist zu beachten, dass die Schülerinnen und Schüler gebeten wurden, eine bestimmte Gruppe von Lehrkräften in einem einzigen Schuljahr zu beurteilen. Die Ergebnisse sollten daher nicht als eine Charakterisierung der Gesamtheit der Lehrkräfte angesehen werden, mit denen die 15-Jährigen während ihrer gesamten Schulzeit konfrontiert waren.

3. Im Durchschnitt der OECD-Länder erklärt der Index 0,5\% der Varianz der Schülerleistungen auf der Gesamtskala Lesekompetenz, wobei der entsprechende Anteil nur in 7 Ländern 1\% übersteigt (vgl. Anhang A2).

4. Im Durchschnitt der OECD-Länder erklärt der Index der schülerbezogenen Faktoren für das Schulklima aus Sicht der Schulleitungen 5,8\% der Varianz der Schülerleistungen auf der Gesamtskala Lesekompetenz (vgl. Anhang A2).

5. Im Durchschnitt der OECD-Länder erklärt der Index der Schuldisziplin aus Schülersicht 1,6\% der Varianz der Schülerleistungen auf der Gesamtskala Lesekompetenz (vgl. Anhang A2).

6. Im Durchschnitt der OECD-Länder erklärt der Index 1,2\% der Varianz der Schülerleistungen auf der Gesamtskala Lesekompetenz (vgl. Anhang A2).

7. Im Durchschnitt der OECD-Länder erklärt der Index 1,9\% der Varianz der Schülerleistungen auf der Gesamtskala Lesekompetenz (vgl. Anhang A2).

8. Für jedes der drei Fächer wurden die Schüler gefragt, ob sie „keine Zeit“, „weniger als 1 Stunde“, „zwischen 1 und 3 Stunden“ oder „mehr als 3 Stunden pro Woche“ auf die Hausaufgaben verwenden. Die insgesamt auf die Hausaufgaben verwendete wöchentliche Zeit wurde geschätzt, indem die Antworten addiert wurden, wobei „keine Zeit“ der Wert 0 , „weniger als 1 Stunde“ der Wert 0,5, „zwischen 1 und 3 Stunden“ der Wert 2 und „3 Stunden und mehr in der Woche“ der Wert 4 zugeordnet wurde. Schülerinnen und Schüler, die für mindestens eines der drei Fächer keine Antwort gaben, wurden aus dem Vergleich herausgenommen.

9. Im Durchschnitt der OECD-Länder erklärt der Index 4,5\% der Varianz der Schülerleistungen auf der Gesamtskala Lesekompetenz (vgl. Anhang A2).

10. Im Durchschnitt der OECD-Länder erklärt der Index 1,7\% der Varianz der Schülerleistungen auf der Gesamtskala Lesekompetenz (vgl. Anhang A2).

11. Im Durchschnitt der OECD-Länder erklärt der Index 1,0\% der Varianz der Schülerleistungen auf der Gesamtskala Lesekompetenz (vgl. Anhang A2).

12. Im Durchschnitt der OECD-Länder erklärt der Index 1,3\% der Varianz der Schülerleistungen auf der Gesamtskala Lesekompetenz (vgl. Anhang A2).

13. In 14 Ländern sind diese Unterschiede statistisch signifikant.

14. Konkret wurde dieser Prozentsatz abgeleitet, indem der gewichtete Prozentsatz der Schulleitungen, die bei der entsprechenden Frage die Antwort „nicht im Verantwortungsbereich der Schule“ angekreuzt hatten, von 100 abgezogen wurde. 



\section{Kapitel}

8

ENTSCHEIDENDE ERKENNTNISSE AUS

DEN PISA-RESULTATEN -

HINWEISE FÜR

DIE POLITIKGESTALTUNG

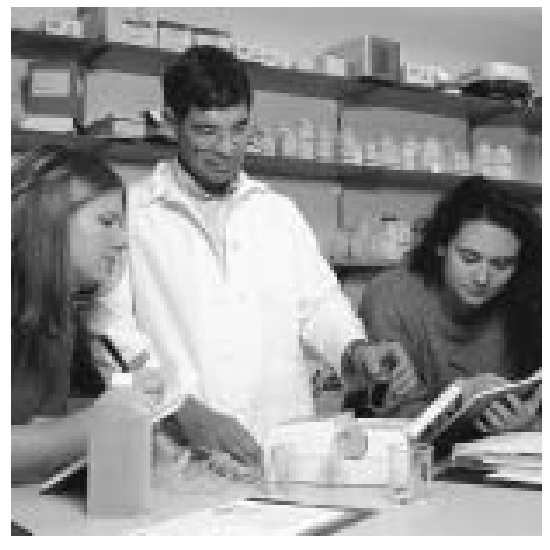


KAPITEL 8 Entscheidende Erkenntnisse aus den PISA-Resultaten - Hinweise für die Politikgestaltung

\section{Einführung: Einflüsse innerhalb und außerhalb der Schule}

Inner-undauferschulische Ausschlaggebend für die schulische Leistung eines Schülers ist nicht nur das Einflüsse aufdie Geschehen in der Schule selbst. Das familiäre Umfeld, die Nachbarschaft und

Leistungen müssen die gesamte Gemeinde, in der der Schüler lebt, spielen ebenfalls eine wichtige zusammen untersucht Rolle, wenngleich der Einfluss dieser Faktoren nicht vollkommen von dem werden, ... der Schule zu isolieren ist. Die inner- und außerschulischen Einflüsse müssen zusammen untersucht werden, um die möglichen Ursachen und Folgen hoher oder niedriger schulischer Leistungen identifizieren zu können.

... was in diesem Kapitel getan wird, ...

In den Kapiteln 2 bis 5 dieses Berichts wurde darauf eingegangen, wie erfolgreich die verschiedenen Länder im Hinblick auf die Ausbildung ihrer Schülerinnen und Schüler sind. In Kapitel 6 und 7 standen die Zusammenhänge zwischen den Hintergrundmerkmalen der Schülerinnen und Schüler mit den schulischen Leistungen im Vordergrund. Das vorliegende Kapitel führt diese Themen weiter aus und geht dabei der Frage nach, welcher Art die zwischen der schulischen Leistung, dem familiären Hintergrund der Schülerinnen und Schüler und den Schulen bestehenden Zusammenhänge sind. Durch eine Analyse der Zusammenhänge zwischen verschiedenen Variablen sowohl familiärer als auch schulischer Faktoren auf dem Hintergrund eines breiten Spektrums unterschiedlicher schulischer Rahmenbedingungen in verschiedenen Ländern versucht dieses Kapitel, die relative Bedeutung der den Schulen zur Verfügung stehenden Mittel sowie der Schulpolitik und -praxis in verschiedenen Bildungssystemen zu bewerten. Das Ziel dieser Analyse ist es, Anhaltspunkte für mögliche Maßnahmen der Bildungspolitik sowohl im Hinblick auf eine Verbesserung der durchschnittlichen Leistungen als auch auf die Gewährleistung einer ausgewogeneren Verteilung der Bildungschancen und -erträge zu liefern.

... indem zuerst der einzelne Schüler...

... danach die Effekte des Hintergrunds der gesamten Schülerschaft einer Schule...

... und zuletzt die Einflussmöglichkeiten der Schulen untersucht werden.
- Das Kapitel beginnt mit einer Analyse der Zusammenhänge zwischen den Leistungen des einzelnen Schülers und dessen familiärem Hintergrund. Das Verständnis dieser Zusammenhänge ist von grundlegender Bedeutung für die Bildungspolitik, da sich daraus ableiten lässt, wie gut Schülerinnen und Schüler mit unterschiedlichem familiären Hintergrund in der Schule zurecht kommen.

- Anschließend wird untersucht, inwieweit die Leistungsunterschiede zwischen den Schulen mit sozioökonomischen Faktoren in Zusammenhang stehen. Diese Information ist wichtig, um die Auswirkungen der institutionellen Rahmenbedingungen auf die Verteilung der Bildungserträge und für die Ausarbeitung entsprechender Bildungsreformen abzuschätzen.

- Schließlich versucht das Kapitel politische Instrumente und Merkmale auf Schulebene zu identifizieren, dank denen es u.U. möglich ist, das Leistungsniveau der Schülerinnen und Schüler insgesamt anzuheben und eine ausgewogenere Verteilung der Bildungschancen und -erträge zu erzielen. 
Bei den in diesem Kapitel durchgeführten Analysen stehen die Schülerleistungen auf der Gesamtskala Lesekompetenz im Mittelpunkt. Auf ihre Leistungen im Bereich der mathematischen und naturwissenschaftlichen Grundbildung wird nur dann Bezug genommen, wenn sich die Analysen und die Schlussfolgerungen zwischen den verschiedenen Grundbildungsbereichen deutlich unterscheiden.

\section{Zusammenhänge zwischen dem sozioökonomischen Hintergrund der Schülerinnen und Schüler und ihren schulischen Leistungen}

\section{Gesamtüberblick}

Wie in Kapitel 6 dargelegt, stehen die Leistungen der Schülerinnen und Schüler bei den PISA-Tests zur Beurteilung der Lesekompetenz sowie der mathematischen und naturwissenschaftlichen Grundbildung in engem Zusammenhang mit dem sozioökonomischen Hintergrund ihrer Familien. Schülerinnen und Schüler, deren Eltern Tätigkeiten nachgehen, die wenig Ansehen genießen, und ein vergleichsweise geringes Bildungsniveau besitzen, erreichen in der Regel niedrigere schulische Leistungen als Schülerinnen und Schüler, deren Eltern einen hohen Bildungsstand haben und hoch angesehene Berufe ausüben. Zur Vereinfachung der Analyse wurden die verschiedenen wirtschaftlichen, sozialen und kulturellen Aspekte des familiären Hintergrunds, die in Kapitel 6 jeweils gesondert untersucht wurden, in diesem Kapitel in einem einzigen Index zusammengefasst, dem PISA-Index des wirtschaftlichen, sozialen und kulturellen Status, manchmal auch kurz sozioökonomischer Status der Schüler ${ }^{1}$ genannt.

In Abbildung 8.1 ist der Zusammenhang zwischen den Schülerleistungen und dem Index des wirtschaftlichen, sozialen und kulturellen Status der Schülerinnen und Schüler für den gesamten OECD-Raum dargestellt ${ }^{2}$. Diese Beziehung wird als sozioökonomische Gradiente bezeichnet. Die Abbildung beschreibt, wie Schülerinnen und Schüler mit unterschiedlichem sozioökonomischem Hintergrund auf der Gesamtskala Lesekompetenz abschneiden. Der Zusammenhang wird sowohl von der Leistungsfähigkeit des jeweiligen Bildungssystems als auch von allgemeineren wirtschaftlichen, sozialen und kulturellen Faktoren beeinflusst (vgl. Kasten 8.1).

Die Analyse dieser Zusammenhänge liefert einen nützlichen Ausgangspunkt für die Untersuchung der Verteilung der Bildungschancen. Die Anhebung des Leistungsniveaus der Schülerinnen und Schüler insgesamt und die Abmilderung der Auswirkungen des sozioökonomischen Hintergrunds auf ihren Bildungserfolg sind entscheidende Ziele für die Bildungssysteme in allen OECD-Ländern. Aus schulpolitischer Sicht ist das Verständnis dieser Zusammenhänge auch deshalb wichtig, weil sie Aufschluss darüber geben, wie gerecht die Erträge aus der Schulbildung zwischen Schülern mit unterschiedlichem sozioökonomischen Hintergrund verteilt sind.
In diesem Kapitel wird ein zusammengesetzter Index für den wirtschaftlichen, kulturellen und sozialen Hintergrund der Schülerinnen und Schüler verwendet... ... und zu den
Schülerleistungen in
Beziehung gesetzt.

Die Kenntnis dieses Zusammenhangs ist ein Ausgangspunkt für die Untersuchung der Verteilung der Bildungschancen. 
KAPITEL 8 Entscheidende Erkenntnisse aus den PISA-Resultaten - Hinweise für die Politikgestaltung

\section{Abbildung 8.1}

Zusammenhang zwischen Schülerleistungen und sozioökonomischem Hintergrund, OECD-Raum insgesamt

Schülerleistungen auf der Gesamtskala Lesekompetenz, Index des wirtschaftlichen, sozialen und kulturellen Status* und sozioökonomische Gradiente für den gesamten OECD-Raum.

Leistungen auf der Gesamtskala Lesekompetenz

800 (PISA-Skalenpunkte)

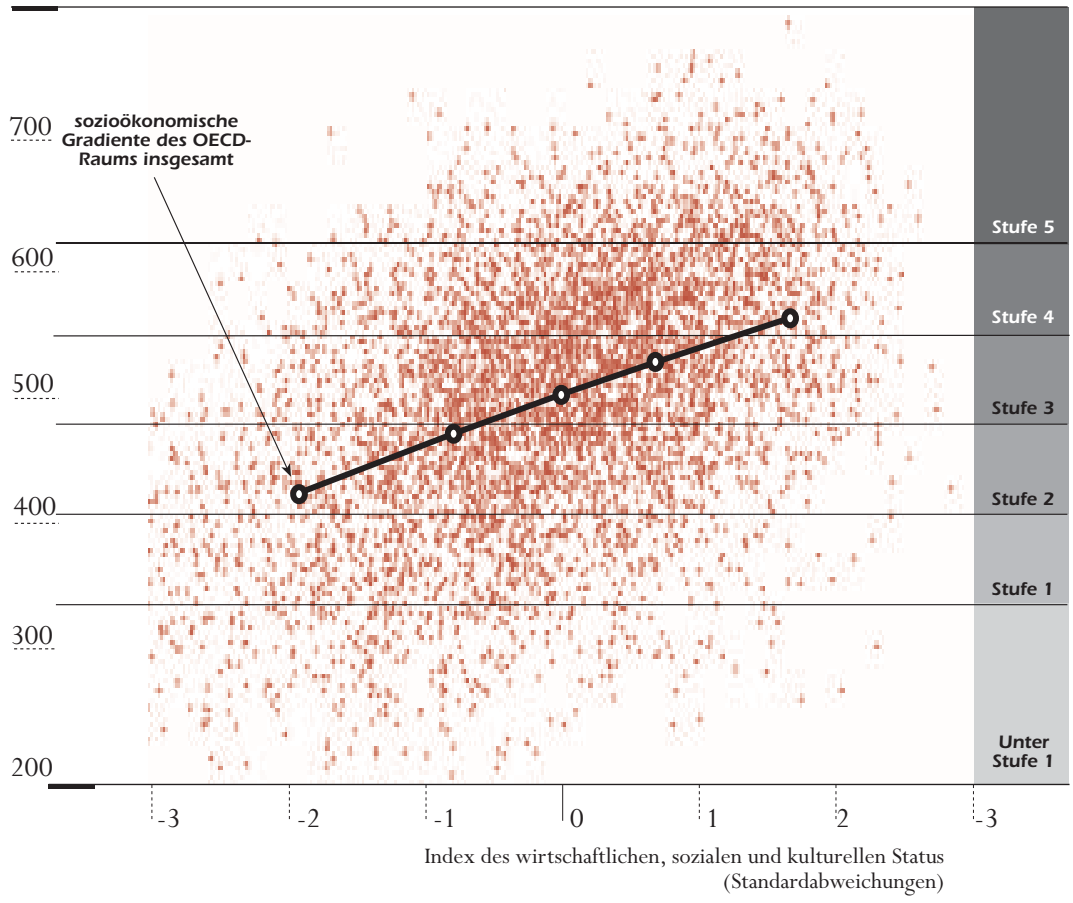

* Jeder Punkt steht für 2000 Schülerinnen und Schüler aus dem OECD-Raum.

Quelle: OECD PISA Datenbank, 2001. Tabelle 8.1.

Aus Abbildung 8.1 lassen sich mehrere Folgerungen ableiten:

Privilegierte Schülerinnen und Schüler erzielen

höhere Leistungen ...

... in jeweils ähnlichem

umfang auf

verschiedenen Stufen des

vorteils...
- Schülerinnen und Schüler, die einem in sozioökonomischer Hinsicht privilegierteren Milieu entstammen, erzielen in der Regel bessere Ergebnisse. Dies zeigt sich an der Steigung der Gradienten.

- Ein gegebener Unterschied im sozioökonomischen Status entspricht einer bestimmten Differenz in der Schülerleistung auf der Skala für Lesekompetenz, die für das gesamte Verteilungsspektrum in etwa identisch ist. Das bedeutet, dass der Grenznutzen eines zusätzlichen sozioökonomischen Vorteils bei steigendem sozialem Niveau weder zu- noch abnimmt, was sich daran zeigt, dass die sozioökonomische Gradiente eine nahezu gerade Linie ist ${ }^{3}$.

- Die Leistungsunterschiede bei Schülerinnen und Schülern mit geringem sozioökonomischem Status sind ein wenig stärker ausgeprägt als die zwischen 
Kasten 8.1 Interpretation von Abbildung 8.1

Jeder Punkt in dieser Graphik entspricht 2000 15-jährigen Schülerinnen und Schülern aus dem gesamten OECD-Raum. Die Graphik stellt deren Leistung im Bereich der Lesekompetenz im Verhältnis zu ihrem wirtschaftlichen, sozialen und kulturellen Status dar.

Die vertikale Achse entspricht den Ergebnissen der Schülerinnen und Schüler auf der Gesamtskala Lesekompetenz, deren Mittelwert 500 Punkte beträgt. Da die Standardabweichung bei der Konstruktion der PISA-Skala auf 100 gesetzt wurde, liegen zwei Drittel der Punkte zwischen 400 und 600. Die Bereiche mit unterschiedlichem Schattierungsgrad entsprechen den fünf Lesekompetenzstufen.

Auf der horizontalen Achse sind die Werte des PISA-Index des wirtschaftlichen, sozialen und kulturellen Status eingetragen. Bei der Konstruktion dieses Index wurde der Mittelwert auf 0 und die Standardabweichung auf 1 gesetzt, so dass zwei Drittel der Schülerinnen und Schüler zwischen +1 und -1 liegen.

Die dunkle Linie entspricht der internationalen sozioökonomischen Gradienten, die die Verbindung zwischen Leseleistung und sozioökonomischem Status in den OECD-Ländern am besten darstellt. Diese Linie reicht von dem Punkt, unterhalb dessen die fünf am stärksten benachteiligten Prozent der Schülerinnen und Schüler liegen, bis zu dem Punkt, oberhalb dessen die 5\% der Schülerinnen und Schüler liegen, die nach dem Index des wirtschaftlichen, sozialen und kulturellen Status am höchsten eingestuft sind. Die drei Markierungspunkte auf der Linie entsprechen jeweils dem 25., dem 50. und dem 75. Perzentil, d.h. den Punkten, unter denen 25\%, 50\% bzw. $75 \%$ der Schülerinnen und Schüler nach ihrem sozioökonomischen Status liegen.

Da Abbildung 8.1 nicht auf einen Vergleich der Bildungssysteme abzielt, sondern auf die Darstellung eines im gesamten OECD-Raum zu beobachtenden Zusammenhangs, wurde jeder Schüler gleich stark gewichtet, was bedeutet, dass größere Länder, in denen mehr Schüler an PISA teilnahmen z.B. Japan, Mexiko und die Vereinigten Staaten - den Verlauf der internationalen Gradienten stärker beeinflussen als kleinere Länder wie Island oder Luxemburg.

den Schülerinnen und Schülern mit höherem sozioökonomischen Status. Dies zeigt sich daran, dass die vertikale Streuung der Punkte im rechten Bereich der Graphik (die der Bandbreite der Lesekompetenzen der Schülerinnen und Schüler aus sozioökonomisch privilegierten Milieus entspricht) geringer ist als die im linken Bereich der Graphik (Bandbreite der Lesekompetenzen der sozioökonomisch benachteiligten Schülerinnen und Schüler).

- Zwischen den Schülerleistungen und dem Index des wirtschaftlichen, sozialen und kulturellen Status besteht kein Verhältnis von eins zu eins. Viele Schüler aus in sozialer Hinsicht benachteiligten Milieus (die auf der linken Seite der Graphik dargestellt sind) erzielen wesentlich bessere Ergebnisse, als die internationale Gradiente vermuten ließe .

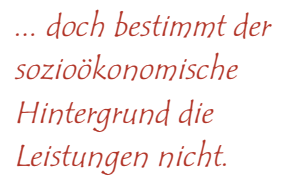

... doch bestimmt der sozioökonomische Hintergrund die Leistungen nicht. 
KAPITEL 8 Entscheidende Erkenntnisse aus den PISA-Resultaten - Hinweise für die Politikgestaltung

Die Kernfrage lautet, ob hohe Gesamtleistungen und geringe

Leistungsdifferenzen gleichzeitig erzielt werden können.

In jedem Land kann der Einfluss des sozioökonomischen Hintergrunds auf die Leistungen bestimmt werden ...

\section{Die Stärke des Einflusses sozioökonomischer Faktoren in den verschiedenen Ländern}

Inwieweit unterscheiden sich die Zusammenhänge zwischen den Leistungen der Schülerinnen und Schüler und deren sozioökonomischem Hintergrund zwischen den verschiedenen Ländern? Ein Weg zur Beantwortung dieser Frage stellen sozioökonomische Gradienten auf Länderebene dar. Sie sind ebenfalls ein nützlicher Ausgangspunkt für ein Verständnis der Faktoren, die zum Erfolg eines Bildungssystems im Hinblick auf die Gewährleistung von Chancengleichheit für alle Schülerinnen und Schüler beitragen. Ziel der Bildungspolitik ist gewöhnlich die Anhebung des Gesamtleistungsniveaus, wobei zugleich die Leistungsdifferenzen zwischen verschiedenen Verwaltungseinheiten, zwischen sozioökonomischen Gruppen und zwischen den Geschlechtern minimiert werden sollen. Im Mittelpunkt dieser Untersuchung steht die Frage, ob es möglich ist, zugleich eine hohe Durchschnittsleistung und geringe Leistungsdisparitäten zwischen den Schülerinnen und Schülern mit unterschiedlichem sozioökonomischem Hintergrund zu erzielen.

In Abbildung 8.2 ist für jedes Land das Verhältnis zwischen den Schülerleistungen auf der Gesamtskala Lesekompetenz und dem Index des wirtschaftlichen, sozialen und kulturellen Status wiedergegeben. Die Gradienten der verschiedenen Länder sind durch hellgraue Linien dargestellt. Die dunkle Linie entspricht der Gradienten des gesamten OECD-Raums ${ }^{5}$, die aus Abbildung 8.1 übernommen wurde.

\section{Abbildung 8.2}

Zusammenhang zwischen Schülerleistungen und sozioökonomischem Hintergrund in den einzelnen Ländern

Sozioökonomische Gradienten für jedes Land

Leistungen auf der Gesamtskala Lesekompetenz (PISA-Skalenpunkte)

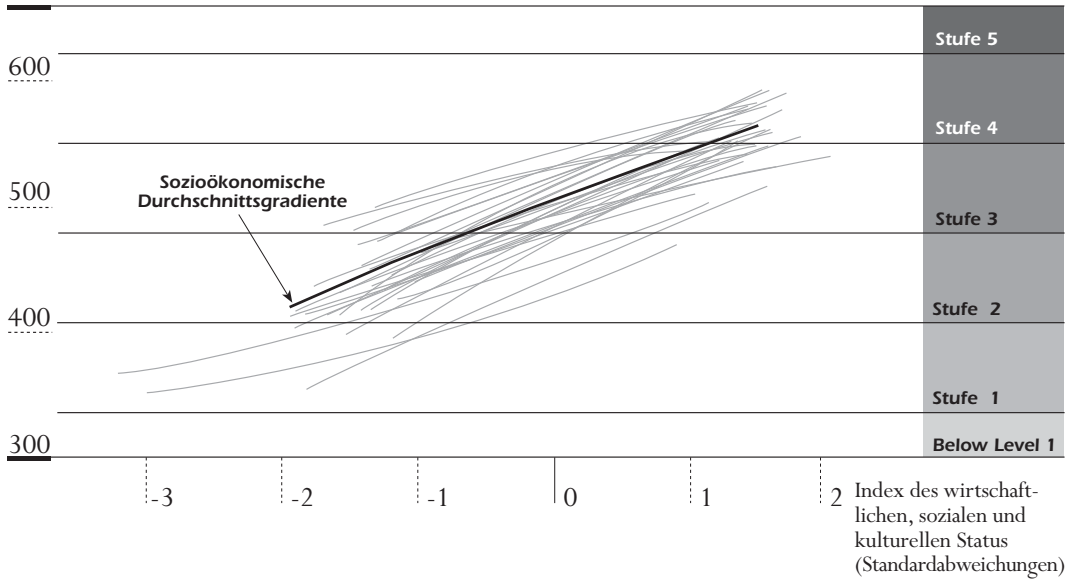

Anmerkung: Zur Identifizierung der einzelnen Länder siehe Tabelle 8.1 und Abb. 8.3.a, b und c. Quelle: OECD PISA Datenbank, 2001. Tabelle 8.1. 
Die Gradienten, mit denen das Verhältnis zwischen den Schülerleistungen und dem Index des wirtschaftlichen, sozialen und kulturellen Status in den einzelnen Ländern zusammenfassend dargestellt werden, sind durch ihre Höhe, ihre Steigung, ihre Länge und die Stärke des durch sie beschriebenen Zusammenhangs gekennzeichnet. Diese verschiedenen Faktoren sind wie folgt in Abbildung 8.2 und der dazugehörigen Tabelle 8.1 dargestellt $^{6}$.

- Die Höhe der Gradienten aus Abbildung 8.2, und zwar ihre durchschnittliche Höhe, ist Spalte 2 von Tabelle $8.1 \mathrm{zu}$ entnehmen. An ihr lässt sich das durchschnittliche Lesekompetenzergebnis der Schülerinnen und Schüler der einzelnen Länder ablesen, deren wirtschaftlicher, sozialer und kultureller Hintergrund dem durchschnittlichen sozioökonomischen Hintergrund sämtlicher OECD-Länder entspricht. Die Höhe der Gradienten eines Landes kann als Anhaltspunkt dafür dienen, wie hoch das Gesamtleistungsniveau des Bildungssystems eines Landes wäre, wenn der wirtschaftliche, soziale und kulturelle Hintergrund seiner Schülerschaft dem OECD-Durchschnitt entspräche. Im Durchschnitt liegt die Gradientenhöhe der OECD-Länder bei 505 Punkten ${ }^{7}$.

- Die Steigung der Gradienten liefert einen Anhaltspunkt für das Ausmaß der Ungleichheit im Bereich der Lesekompetenz, das auf sozioökonomische Faktoren zurückzuführen ist (Spalte 3, Tabelle 8.1). Steilere Gradienten deuten auf einen stärkeren Einfluss des wirtschaftlichen, sozialen und kulturellen Status auf die Schülerleistungen hin, d.h. auf größere Ungleichheiten. Eine schwächere Steigung der Gradienten bedeutet hingegen, dass der sozioökonomische Hintergrund weniger Einfluss auf die Schülerleistungen ausübt, die Ungleichheiten also weniger ausgeprägt sind. Im Durchschnitt der OECD-Länder beträgt die Steigung der Gradienten 41, was bedeutet, dass die Schülerpunktzahlen auf der Gesamtskala Lesekompetenz für jede zusätzliche Einheit auf dem PISA-Index des wirtschaftlichen, sozialen und kulturellen Status um 41 Punkte ansteigt ${ }^{8}$. Eine Einheit des Index des wirtschaftlichen, sozialen und kulturellen Status beträgt eine Standardabweichung, so dass bei einer Normalverteilung zwei Drittel der Population innerhalb des Bereichs von plus/minus einer Standardabweichung vom Mittelwert liegen. Im Fall Norwegens beispielsweise, dessen Gradiente dieselbe Steigung wie die Gradiente des OECD-Durchschnitts (41 Punkte) aufweist, beträgt die durchschnittliche Lesekompetenzpunktzahl der Schülerinnen und Schüler, die eine Einheit unter dem sozioökonomischen Mittelwert liegen, 464 Punkte, d.h. 41 Punkte weniger als das norwegische Durchschnittsergebnis von 505 Punkten; die durchschnittliche Lesekompetenzpunktzahl der Schülerinnne und Schüler, die eine Einheit über dem sozioökonomischen Mittelwert liegen, beläuft sich auf 546 Punkte, d.h. 41 Punkte mehr als die norwegische Durchschnittspunktzahl. Anders lässt sich dies mit der Feststellung ausdrücken, dass zwischen der durchschnittlichen Lesekompetenzpunktzahl der Schülerinnen und Schüler des am stärksten benachteiligten Populationssechstels und der des privilegiertesten OECD-Populationssechstels
... und zwar anhand von vier Eigenschaften der "Gradienten": ...

... anhand der Gesamtleistung: die Durchschnittspunktzahl, korrigiert um die sozioökonomische Zusammensetzung des Landes, ...

... anhand der Differenz zwischen den von den mehr und den weniger privilegierten schülergruppen erreichten Punktzahlen,... 


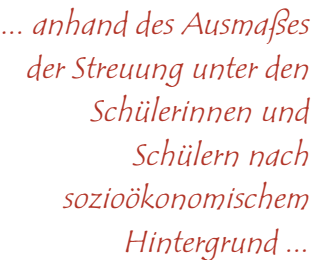

... und anhand der Stärke des Zusammenhangs zwischen Hintergrund und Leistungen. (die jeweils unter- bzw. oberhalb der mittleren zwei Drittel liegen) in Norwegen ein Abstand von über 82 Punkten besteht, d.h. von mehr als einer Kompetenzstufe. In Deutschland, wo die Gradiente am steilsten ist (60 Punkte), liegen über 120 Punkte zwischen dem Lesekompetenzergebnis des obersten und der des untersten Populationssechstels (international) nach dem sozioökonomischen Status, was nahezu zwei Kompetenzstufen entspricht.

- Die Länge der Gradienten hängt von der Bandbreite der sozioökonomischen Werte der mittleren 90\% der Schülerpopulation (vom 5. bis zum 95. Perzentil) in den einzelnen Ländern sowie von ihrer Steigung ab. In Spalte 5 von Tabelle 8.1 ist die Streuungsbreite der Werte nach dem Index des wirtschaftlichen, sozialen und kulturellen Status angegeben, die durch die Gradiente erfasst wird. Diese Information gibt Aufschluss darüber, wie breit die Streuung der Schülerpopulation hinsichtlich ihres sozioökonomischen Hintergrunds ist. Längere Gradienten stehen für größere Diskrepanzen innerhalb der Schülerpopulation des betreffenden Landes, was deren sozioökonomischen Hintergrund anbelangt.

- Die Stärke des Zusammenhangs zwischen den Ergebnissen im Bereich Lesekompetenz und dem sozioökonomischen Hintergrund bezieht sich darauf, wie stark die Leistung einzelner Schüler nach unten oder oben von der Gradiente abweicht. Dies ist für alle Länder insgesamt aus der Streuung der Punkte unter- und oberhalb der Linie in Abbildung 8.1 ersichtlich, in Abbildung 8.2 jedoch nicht dargestellt. Stattdessen gibt Tabelle 8.1 in Spalte 4 die erklärte Varianz wieder, d.h. einen statistischen Indikator, der die Stärke des Zusammenhangs ausdrückt, indem er angibt, welcher Prozentsatz der beobachteten Differenz zwischen den Ergebnissen der Schülerinnen und Schüler auf den durch die Gradiente dargestellten Zusammenhang zurückzuführen ist. Ist diese Zahl niedrig, geht nur ein vergleichsweise geringer Teil der Leistungsunterschiede zwischen Schülern auf deren sozioökonomischen Hintergrund zurück; ist sie hoch, ist es umgekehrt. Im OECD-Raum insgesamt erklären die Werte auf dem Index des wirtschaftlichen, sozialen und kulturellen Status 20\% der Unterschiede zwischen den Schülerleistungen, doch gibt es zwischen den Werten für die einzelnen Länder starke Schwankungen, die von 5\% in Island als tiefstem bis zu 26\% in Ungarn als höchstem Wert reichen.

Aus Abbildung 8.2 und Tabelle 8.1 lassen sich folgende Schlüsse ziehen:

Der Leistungsabstand zwischen mehr oder weniger begünstigten

Schülern variiert zwischen den Ländern um den Faktor drei, ...
- Erstens unterscheiden sich die sozioökonomischen Gradienten der einzelnen Länder. Aus der Abbildung gehen nicht nur die Länder hervor, die auf der Gesamtskala Lesekompetenz vergleichsweise hohe oder niedrige Ergebnisse erzielen, sondern auch jene, in denen die Leistungsdifferenzen zwischen Schülern mit unterschiedlichem sozioökonomischem Hintergrund mehr oder weniger stark ausgeprägt sind. Hierbei muss die Aufmerksamkeit insbesondere auf das erhebliche Ausmaß dieser Unterschiede gelenkt werden. Nehmen wir 
das Beispiel zweier Schüler. Der eine stammt aus einem relativ benachteiligten Milieu, so dass fünf von sechs Schülern im internationalen Vergleich nach dem PISA-Index des wirtschaftlichen, sozialen und kulturellen Status höher platziert sind. Der andere stammt aus einem relativ privilegierten Milieu, insofern nur einer von sechs Schülern eine höhere Wertung auf dem sozioökonomischen Index hat. Der Leistungsabstand zwischen diesen beiden Schülern variiert zwischen den verschiedenen Ländern um einen Faktor von nahezu drei. In Japan und Korea beträgt dieser Abstand 42 Punkte bzw. 0,6 Kompetenzstufen, in Deutschland aber 120 Punkte bzw. 1,7 Kompetenzstufen (in jedem Fall das Doppelte des Gradientenwerts, der einer Standardabweichung entspricht). Die Abbildung zeigt auch deutlich, dass ein hohes Leistungsniveau nicht zwangsläufig mit großen Ungleichheiten einhergehen muss, da die Gradienten einiger der leistungsstärksten Länder nur relativ schwache Steigungen aufweisen.

- Zweitens bestehen auch hinsichtlich der Streuung der von den Gradienten erfassten Werte auf dem Index des wirtschaftlichen, sozialen und kulturellen Status große Unterschiede zwischen den verschiedenen Ländern: Sie reicht von einem Abstand von weniger als 2,7 Indexpunkten zwischen den Werten der fünf am stärksten benachteiligten und der fünf am meisten privilegierten Prozent der Schülerinnen und Schüler in Österreich, der Tschechischen Republik, Japan und Schweden bis zu einem Abstand von 4 Punkten oder mehr in Brasilien und Mexiko. Die Probleme, mit denen sich die Bildungssysteme auf Grund der sozialen und wirtschaftlichen Disparitäten innerhalb ihrer Schülerpopulation konfrontiert sehen, sind daher von Land zu Land sehr unterschiedlich. Die Bildungssysteme Brasiliens und Mexikos sind wesentlich stärker gefordert, die Chancenungleichheit im Bildungswesen anzugehen, als die Bildungssysteme Japans oder Schwedens.

- Drittens handelt es sich bei den Gradienten der meisten Länder um nahezu gerade Linien. In den meisten Ländern entspricht daher jede Erhöhung auf dem Index des wirtschaftlichen, sozialen und kulturellen Status um eine Stufe einer konstanten Zunahme der Punktwerte auf der Gesamtskala Lesekompetenz. Man hätte erwarten können, dass die Gradienten bei niedrigerem wirtschaftlichen, sozialen und kulturellen Status steil verlaufen und sich mit steigendem sozioökonomischem Status abflachen ${ }^{9}$. In einigen Ländern folgen die Gradienten der Ergebnisse auf der Gesamtskala Lesekompetenz zwar diesem Muster, gleichwohl ist die Steigungsänderung bei wachsendem wirtschaftlichem, sozialem und kulturellem Status gering und in Abbildung 8.2 sogar kaum zu erkennen. Darüber hinaus folgen die Gradienten in den beiden Ländern, deren wirtschaftlicher, sozialer und kultureller Status besonders gering ist -Brasilien und Mexiko -, dem genau umgekehrten Muster: Ihre Steigung ist relativ gering bei sehr niedrigem Niveau des wirtschaftlichen, sozialen und kulturellen Status, nimmt aber mit steigendem sozioökonomischen Status einen steileren Verlauf. Da in diesen beiden Ländern ein vergleichsweise hoher Prozentsatz der Schülerinnen und
... doch weisen die einzelnen Länder verschiedene Sozialprofile auf.
Ein zusätzlicher sozioökonomischer Vorteil bringt über die ganze Skala bis ganz nach oben ähnliche Zuwächse... 
... so dass es in Bezug auf soziale Benachteiligung keinen bestimmten Punkt gibt, an dem Interventionen am wirksamsten wären.

Die Unterschiede zwischen den Ländern sind unter den benachteiligten schülerinnen und Schülern stärker ausgeprägt als unter den besser gestellten.
Schüler weit unter den unteren 5\% der Schülerinnen und Schüler der meisten OECD-Länder liegt, und zwar sowohl in Bezug auf die Leseleistung als auch auf den sozioökonomischen Hintergrund, könnte dies darauf hindeuten, dass es eine Art „Sockel“ gibt, der einem wirtschaftlichen, sozialen und kulturellen Mindeststandard entspricht, unterhalb dessen sozioökonomische Unterschiede kaum noch Einfluss auf die Fähigkeit der Schülerinnen und Schüler haben, die Art der in PISA gestellten Fragen zu beantworten.

Die Feststellung, dass die Gradienten über die gesamte Breite der Werte des wirtschaftlichen, sozialen und kulturellen Status in allen Ländern linear verlaufen, ist für die staatliche Politik von großer Bedeutung. Viele sozialpolitische Maßnahmen zielen auf eine Erhöhung der Ressourcen der sozial Schwächsten ab, entweder durch Steuervorteile oder durch die Ausrichtung von Sozialleistungen oder -programmen auf bestimmte Bevölkerungsgruppen. Die PISA-Resultate legen den Schluss nahe, dass sich nicht einfach ein „unteres Basisniveau des wirtschaftlichen, sozialen und kulturellen Status“ festlegen lässt, unterhalb dessen die Ergebnisse deutlich abfallen. Wird der wirtschaftliche, soziale und kulturelle Status außerdem als Ersatz für Entscheidungen und Aktionen seitens der Eltern genommen, die ihren Kindern ein kulturell reicheres Umfeld bieten wollen - z.B. indem sie ihnen aus Büchern vorlesen oder sich für ihre schulische Arbeit interessieren -, lässt sich aus diesen Ergebnissen schließen, dass auf der gesamten Breite des sozioökonomischen Spektrums Raum für Verbesserungen besteht. Aus der Tatsache, dass sich nur schwer ein unteres Basisniveau definieren lässt, darf jedoch nicht gefolgert werden, dass differenzierte Unterstützungsmaßnahmen für bestimmte Schülergruppen fehl am Platze wären. Die Erfolge, die viele Länder beim Abbau der Leistungsunterschiede zwischen den Geschlechtern erzielt haben, sind ein gutes Beispiel dafür, dass auf bestimmte Gruppen abzielende Anstrengungen einen sehr wirkungsvollen Beitrag zur Verringerung der Leistungsunterschiede leisten können.

- Viertens nähern sich die Gradienten in den oberen Bereichen des wirtschaftlichen, sozialen und kulturellen Index tendenziell an: Die Linien in Abbildung 8.2 liegen auf der rechten Seite näher beieinander als auf der linken. Diese, wenn auch nur schwach ausgeprägte ${ }^{10}$ Tendenz bedeutet, dass die Gradienten der Länder, die auf der Gesamtskala Lesekompetenz hohe Ergebnisse erzielen, in der Regel eine schwächere Steigung aufweisen. Sie bedeutet auch, dass zwischen der Lesekompetenz der Schülerinnen und Schüler mit einem hohen wirtschaftlichen, sozialen und kulturellen Status im Allgemeinen etwas weniger starke Unterschiede bestehen als zwischen der Lesekompetenz der Schülerinnen und Schüler mit einem vergleichsweise niedrigen wirtschaftlichen, sozialen und kulturellen Status. Die Auswirkungen verschiedener Bildungserfahrungen auf die Schülerleistung könnten daher bei Schülerinnen und Schülern, die in sozioökonomischer Hinsicht benachteiligt sind, am größten sein. 
Abbildung 8.2 zeigt sehr umfassend, dass zwischen den Ländern sowohl hinsichtlich der Art als auch der Stärke der Zusammenhänge zwischen dem sozioökonomischen Hintergrund und den schulischen Leistungen große Unterschiede bestehen. Bei einer genaueren Untersuchung kristallisieren sich verschiedene Muster heraus. In Abbildung 8.3a bis 8.3c sind die Länder in Gruppen eingeteilt, und zwar entsprechend der Kombination der Faktoren „durchschnittliche Bildungsqualität“ (in Bezug auf die durchschnittliche Gesamtleistung der Schülerinnen und Schüler des jeweiligen Landes) und „Bildungsgleichheit“ (gemessen am Einfluss des wirtschaftlichen, sozialen und kulturellen Status der Schülerinnen und Schüler auf deren Leistung, d.h. der Steigung der Gradienten).

In Abbildung 8.3a sind die zwölf Länder dargestellt, die auf der Gesamtskala Lesekompetenz überdurchschnittliche Ergebnisse erzielen. In sechs dieser Länder - Kanada, Finnland, Island, Japan ${ }^{11}$, Korea und Schweden - geht eine relativ hohe Qualität mit einer relativ hohen Gleichheit der Leistungen unterschiedlicher sozioökonomischer Gruppen einher (Tabelle 8.1) ${ }^{12}$. Das Durchschnittsergebnis der Ländergruppe dieser Kategorie auf der Gesamtskala Lesekompetenz liegt bei rund 525 Punkten, also weit über dem OECDDurchschnitt von 500, während die durchschnittliche Steigung ihrer Gradienten 30 beträgt, wesentlich weniger also als der OECD-Durchschnitt von 41. Daran zeigt sich, dass es durchaus möglich ist, ein vergleichsweise hohes Leistungsniveau und zugleich ein relativ geringes $\mathrm{Ma}$ an Bildungsungleichheit zwischen in sozioökonomischer Hinsicht privilegierten und benachteiligten Gruppen zu erzielen. In drei anderen Ländern - Australien, Belgien und dem Vereinigten Königreich - geht ein hohes Leistungsniveau mit überdurchschnittlich stark ausgeprägten Ungleichheiten zwischen den Leistungen der Schülerinnen und Schüler aus verschiedenen sozioökonomischen Gruppen einher ${ }^{13}$. In den übrigen in dieser Abbildung dargestellten Ländern - Österreich, Irland und Neuseeland - liegt das Leistungsniveau ebenfalls über dem OECDDurchschnitt, doch weichen die Gradienten statistisch nicht signifikant von der Durchschnittsgradienten der OECD-Länder ab.

In Abbildung 8.3b sind die fünf Länder dargestellt, deren Punktzahlen nicht wesentlich vom OECD-Durchschnitt abweichen. Unter diesen Ländern weisen Frankreich, die Schweiz und die Vereinigten Staaten überdurchschnittliche Ungleichheiten zwischen den verschiedenen sozioökonomischen Gruppen auf, während die sozioökonomischen Gradienten in Dänemark und Norwegen nicht signifikant von der OECD-Durchschnittsgradienten abweichen.

In Abbildung 8.3c sind die 13 Länder dargestellt, die auf der Gesamtskala Lesekompetenz unterdurchschnittliche Ergebnisse erreichten. In vier dieser Länder - der Tschechischen Republik, Deutschland, Ungarn und Luxemburg - geht eine überdurchschnittliche Bildungsungleichheit mit unterdurchschnittlichen Leistungen einher. Daraus lässt sich schließen, dass diese Länder ihre Durchschnittsleistungen anheben könnten, wenn es ihnen gelänge, die
Länder können

entsprechend der

Kombinationen von

Gesamtleistung und

Gleichheit in Gruppen

eingeteilt werden:

Länder mit hohem

Leistungsnivedu weisen jeweils ein unterschiedliches Majs an Gleichheit zwischen den Schülern auf,...

... in Ländern mit durchschnittlichem Leistungsniveau sind möglicherweise großse Unterschiede zwischen sozialen Gruppen vorhanden ...

... und einige Länder mit unterdurchschnittlichem Leistungsniveau weisen ebenfalls ein hohes Maßs an Ungleichheit auf. 
KAPITEL 8 Entscheidende Erkenntnisse aus den PISA-Resultaten - Hinweise für die Politikgestaltung

\section{Abbildung 8.3}

Zusammenhang zwischen Schülerleistungen und sozioökonomischem Hintergrund in den einzelnen Ländern

Sozioökonomische Gradiente für jedes Land

Unterdurchschnittlich starke Effekte des sozioökonomischen Hintergrunds auf die Schülerleistungen

Keine statistisch signifikanten Effekte des sozioökonomischen Hintergrunds auf die Schülerleistungen im

Vergleich zum OECD-

Durchschnitt

Überdurchschnittlich starke Effekte des sozio-

ökonomischen Hintergrunds auf die Schülerleistungen

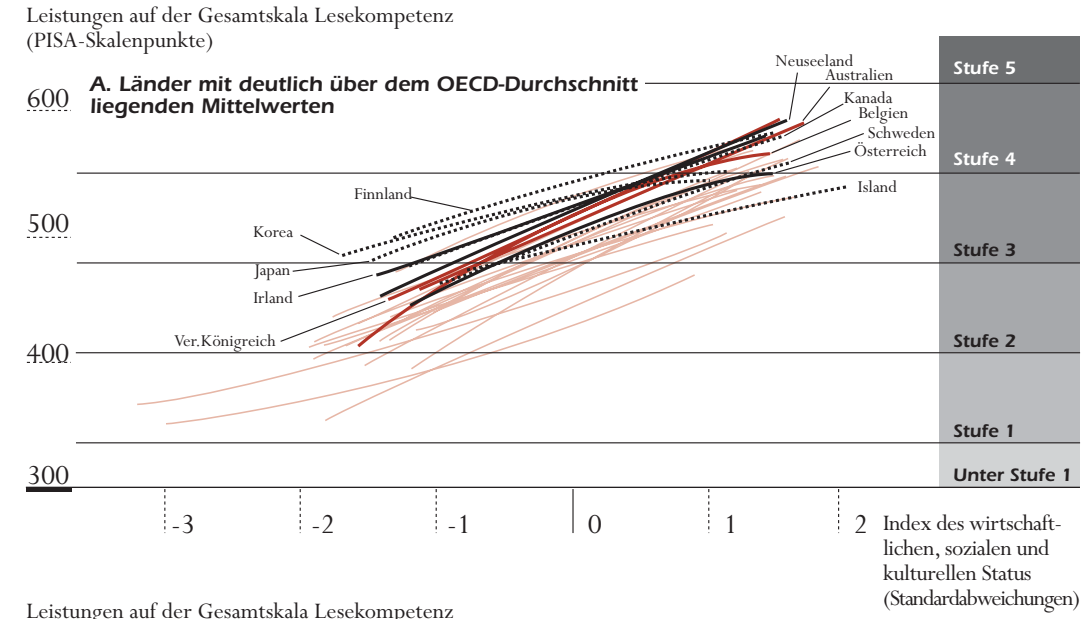

Leistungen auf der Gesamtskala Lesekompeten (PISA-Skalenpunkte)

Stufe 5

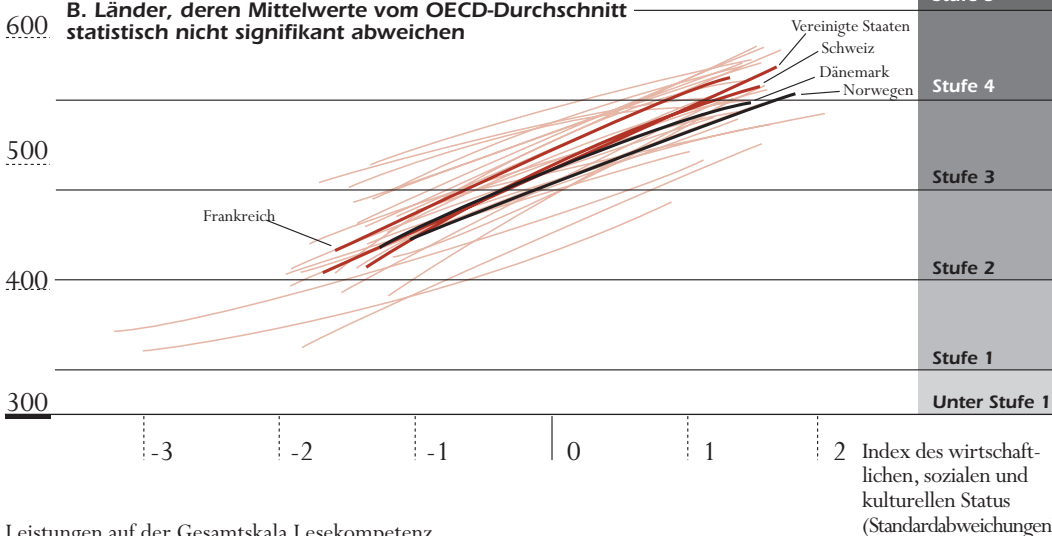

Leistungen auf der Gesamtskala Lesekompetenz

(Standardabweichungen)

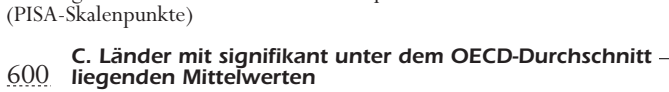

600 liegenden Mittelwerten

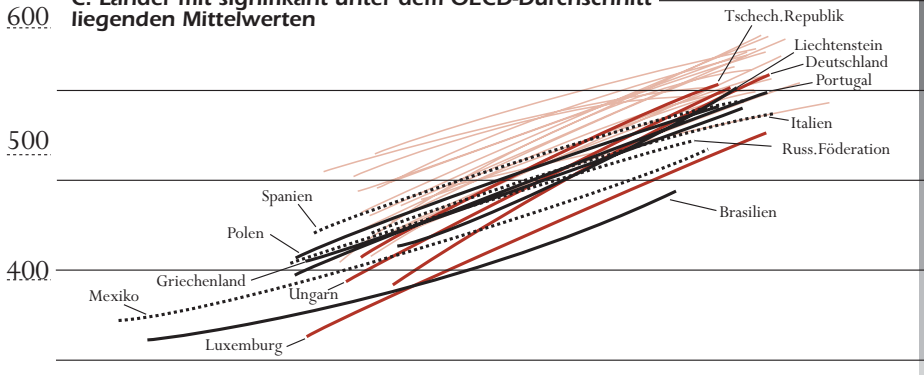

Stufe 5

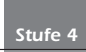

Stufe 3

Stufe 2

tufe 1

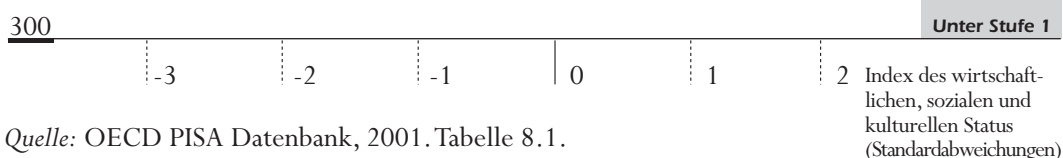

Quelle: OECD PISA Datenbank, 2001. Tabelle 8.1.

(Standardabweichungen) 
Auswirkungen des soziö̈konomischen Hintergrunds auf die Schülerleistungen zu mildern. In Italien, Mexiko, der Russischen Föderation und Spanien hingegen gehen unterdurchschnittliche Ergebnisse mit einer überdurchschnittlichen Ausgewogenheit der Leistungen der verschiedenen sozioökonomischen Gruppen einher. Die Ergebnisse der übrigen in dieser Abbildung wiedergegebenen Länder - Brasilien, Griechenland, Liechtenstein, Polen und Portugal - liegen unter dem OECD-Durchschnitt, doch unterscheiden sich ihre sozioökonomischen Gradienten nicht signifikant von der OECD-Durchschnittsgradienten.

Offensichtlich müssen der Gesamtkontext, in dem die Bildungssysteme tätig sind, und insbesondere die Verteilung der wirtschaftlichen und sozialen Variablen innerhalb eines Landes, bei der Interpretation dieser Zusammenhänge berücksichtigt werden.

\section{Komponenten des sozioökonomischen Hintergrunds}

Bislang wurde für die in diesem Kapitel durchgeführten Analysen ein zusammengesetzter Index des wirtschaftlichen, sozialen und kulturellen Status verwendet. Es ist jedoch auch sinnvoll, die unterschiedlichen Auswirkungen der Hintergrundmerkmale der einzelnen Schülerinnen und Schüler im Verhältnis zu ihren PISA-Leistungen getrennt zu prüfen, um zu einem präziseren Urteil darüber zu gelangen, wie und warum die zwischen dem sozioökonomischen Hintergrund und der Leistung der Schülerinnen und Schüler bestehenden Zusammenhänge von Land zu Land abweichen. In den folgenden Abschnitten wird dies untersucht, indem sowohl die einzelnen Bestandteile des Index des wirtschaftlichen, sozialen und kulturellen Status als auch die mit ihnen verknüpften Variablen, wie die Familienstruktur und die Frage, ob der jeweilige Schüler aus dem Ausland stammt, unter die Lupe genommen werden. ${ }^{14}$

Der einfachste Weg, die unterschiedlichen Einflüsse der einzelnen Aspekte auf einer einheitlichen Basis für alle Länder zu betrachten, besteht darin, von einer fiktiven Gruppe von 1000 Schülerinnen und Schülern in allen Ländern auszugehen, die das gleiche Profil aufweist wie eine Zufallsstichprobe im gesamten OECD-Raum ${ }^{15}$ (wobei aus jedem Land die gleiche Zahl an Schülern gezogen wird).

Diese Gruppe von 1000 Schülerinnen und Schülern müsste im Durchschnitt folgendermaßen zusammengesetzt sein:

- 501 Schüler männlichen und 499 Schüler weiblichen Geschlechts (Daten abrufbar unter www.pisa.oecd.org);

- 162 Schülerinnen und Schüler aus Ein-Eltern-Familien (Tabelle 6.9);

- 41 Schülerinnen und Schüler, die nicht im Erhebungsland geboren sind (Tabelle 6.10);

\author{
Es ist auch möglich, \\ die von den einzelnen \\ Aspekten des \\ Hintergrunds der \\ Schülerinnen und \\ Schüler ausgehenden \\ Effekte zu \\ unterscheiden ...
}

\author{
... indem zunächst von \\ einer fiktiven \\ schülergruppe in allen \\ Ländern mit einem \\ "typischen" \\ sozioökonomischen \\ Profil ausgegangen wird.
}


KAPITEL 8 Entscheidende Erkenntnisse aus den PISA-Resultaten - Hinweise für die Politikgestaltung

In der zweiten Spalte von Tabelle 8.2 sind die mittleren Punktzahlen um sozioökonomische Merkmale berichtigt ...

... und die übrigen

Spalten weisen die unabhängigen Effekte der einzelnen Merkmale aus.
- 55 Schülerinnen und Schüler, bei denen sich die zu Hause normalerweise gesprochene Sprache von der Testsprache, anderen offiziellen Landessprachen oder nationalen Dialekten unterscheidet (Tabelle 6.11);

- jeweils ein bis zwei Geschwister pro Schüler (Daten abrufbar unter www.pisa.oecd.org);

- Schülerinnen und Schüler, deren Eltern im Durchschnitt 12,3 Bildungsjahre absolviert haben (vgl. Anhang A1);

- Schülerinnen und Schüler, bei denen der bestplatzierte Elternteil nach dem sozioökonomischen PISA-Index der beruflichen Stellung einen Durchschnittswert von 49 Punkten aufweist (Tabelle 6.1a), sowie Schülerinnen und Schüler mit einer Durchschnittswertung von null auf dem PISA-Index des Besitzes von klassischen Kulturgütern im Elternhaus(vgl. Tabelle 6.3), was durch die Konstruktionsmethode dieser Indizes bedingt ist (vgl. Anhang A1).

Bei der Untersuchung der zu erwartenden Leistung einer solchen Schülerstichprobe eines Landes besteht der erste Analyseschritt in der Anpassung der PISA-Ergebnisse an die Zusammensetzung der Schülerpopulation als einem Erklärungsfaktor der Leistungsunterschiede. Anschließend werden die Auswirkungen bestimmter Merkmale auf die zu erwartenden Ergebnisse der Schülerinnen und Schüler analysiert. So kann besser unterschieden werden, wo höhere oder niedrigere Ergebnisse eher auf Unterschiede in der relativen Leistung bestimmter Gruppen zurückzuführen sind oder auf die unterschiedliche Zahl der Schülerinnen und Schüler, die diesen Gruppen in den jeweiligen Ländern angehören.

In Tabelle 8.2 sind die Ergebnisse zusammengefasst. Die erste Spalte von Tabelle 8.2 zeigt die tatsächlichen Ergebnisse der Schülerinnen und Schüler auf der Gesamtskala Lesekompetenz, wie in Kapitel 2 beschrieben. Die zweite Spalte von Tabelle 8.2 liefert einen Schätzwert dafür, welche Leistung eine repräsentative Gruppe gemäß der obigen Beschreibung in den verschiedenen Ländern erzielen würde. Im Durchschnitt der OECD-Länder beträgt das berichtigte Ergebnis auf der Gesamtskala Lesekompetenz 505 Punkte. Die berichtigten Ergebnisse weichen zwischen den OECD-Ländern jedoch erheblich ab: Sie reichen von 450 Punkten in Mexiko bis zu 543 Punkten in Finnland. Dies bedeutet, dass hinsichtlich der Durchschnittsleistung selbst nach Berücksichtigung einer Reihe von Hintergrundvariablen wie Besitztümer im Elternhaus, Zuwanderung usw. zwischen den Ländern noch immer erhebliche Unterschiede bestehen.

Des Weiteren ist in Tabelle 8.2 der Unterschied angegeben, der sich in jedem Land auf Grund der individuellen Merkmale des sozioökonomischen Hintergrunds für die Punktzahl der Lesekompetenz ergibt. 
In Spalte 3 von Tabelle 8.2 ist der Leistungsunterschied zwischen Schülern aus Ein-Eltern-Familien und solchen aus anderen Familientypen angegeben (vgl. auch Tabelle 6.9). Die Werte in Fettdruck stehen für statistisch signifikante Zusammenhänge. Im Durchschnitt der OECD-Länder beträgt der Leistungsunterschied auf der Gesamtskala Lesekompetenz nahezu 11 Punkte, wobei Schülerinnen und Schüler aus Zweielternhaushalten (bei sonst gleichen Bedingungen) besser abschneiden. In Finnland, dem Vereinigten Königreich und den Vereinigten Staaten beträgt dieser Abstand zwischen 14 und 17 Punkten, in Frankreich, Irland, Korea und Schweden zwischen 11 und 12 Punkten. Außer in Italien(8 Punkte) sind die Unterschiede in allen übrigen Ländern statistisch nicht signifikant.

Im Ausland geborene Schülerinnen und Schüler (vgl. auch Tabelle 6.10) erzielen in der Regel in den meisten Ländern niedrigere Ergebnisse als ihre im Inland geborenen Mitschüler, und zwar auch nach Berücksichtigung sämtlicher anderen Einflussfaktoren. Dies geht aus Spalte 5 von Tabelle 8.2 hervor. Im OECDDurchschnitt beträgt der Leistungsunterschied rund 26 Punkte. Das Ausmaß der Differenz variiert jedoch stark. In Österreich, Liechtenstein, Mexiko und der Schweiz beläuft sich der Abstand auf über 50 Punkte, d.h. mehr als die Hälfte der Standardabweichung auf der Gesamtskala Lesekompetenz. In der Tschechischen Republik, Portugal, der Russischen Föderation und den Vereinigten Staaten hingegen ist der Unterschied kleiner als 10 Punkte und folglich statistisch nicht signifikant ${ }^{16}$. Wie gut ausländische Schüler und Schülerinnen abschneiden, hängt neben den in PISA gemessenen und in dieser Analyse untersuchten Hintergrundmerkmalen natürlich von zahlreichen anderen Faktoren ab. Ihre Leistungen werden zusätzlich zu der Qualität des Bildungssystems ihres Gastlandes auch durch die Umstände ihrer Umsiedlung und ihre schulische Vorbildung beeinflusst.

Spalte 6 von Tabelle 8.2 ist zu entnehmen, dass im Durchschnitt der OECD-Länder ein Aufstieg auf dem internationalen sozioökonomischen Index der beruflichen Stellung um eine Standardabweichung ${ }^{17}$ mit einer Leistungssteigerung von rund 28 Punkten $^{18}$ auf der Gesamtskala Lesekompetenz einhergeht, allerdings sind die Einflüsse auch hier wieder sehr unterschiedlich. Die Steigerung reicht von 67 Punkten in Deutschland bis zu kleinen und statistisch nicht signifikanten Unterschieden in Brasilien, Dänemark, Finnland, Griechenland, Island, Irland, Korea, Norwegen und Schweden ${ }^{19}$.

Jedes zusätzliche Bildungsjahr der Eltern (vgl. Anhang A1) kann im OECDDurchschnitt mit einem Zuwachs um 5 Punkte auf der Gesamtskala Lesekompetenz in Zusammenhang gebracht werden. Dieser Durchschnittswert ist statistisch signifikant, was auch für die entsprechenden Werte fast aller Länder der Fall ist (Spalte 7, Tabelle 8.2). Ein Zuwachs um einen Punkt (d.h. eine Standardabweichung) bei den PISA-Indizes der Bildungsressourcen und des Besitzes von Kulturgütern im Elternhaus (vgl. Tabelle 6.3) ist im Durchschnitt mit einem Anstieg der Lesekompetenz um 12 bzw. 13 Punkte verbunden
Nur einen Elternteil

zu haben, hat lediglich

in einer Minderheit der

Länder einen

unabhängigen Effekt...

... im Ausland geboren

zu sein, macht einen

größseren Unterschied,

aber nicht in allen

Ländern ...

... ein Elternteil mit einer hoch angesehenen beruflichen Stellung ist in einigen Ländern von enormer Bedeutung, in anderen aber wesentlich weniger...

... gebildetere Eltern zu haben, ist nahezu überall vorteilhaft. 
Der sozioökonomische Hintergrund ist wichtig, aber in unterschiedlichem Ausmaß...

... und PISA stellt den Ländern ein

Instrumentarium zur Verfügung, um dieses Phänomen weiter zu untersuchen.

Benachteiligungen sind nicht leicht abzubauen.

Aber können die

schulen ihre Auswirkungen mildern?

Es ist notwendig herauszufinden, ob die Unterschiede innerhalb der Schulen oder zwischen ihnen bestehen.
(Spalte 8 und 9, Tabelle 8.2). Wie bei den anderen Variablen bestehen hinsichtlich der Auswirkungen dieser Variablen jeweils große Differenzen zwischen den verschiedenen Teilnehmerländern.

Alles in allem haben viele der sozioökonomischen Hintergrundfaktoren, die in PISA gemessen wurden, erheblichen Einfluss auf die Leistungen der Schülerinnen und Schüler in den OECD-Ländern, doch sind Art und die Stärke dieses Einflusses von Land zu Land sehr unterschiedlich. Die Analyse zeigt, dass die in den verschiedenen PISA-Ländern gemessenen Leistungsdifferenzen zwischen den Schülern auch dann nicht verschwinden, wenn die Unterschiede in Bezug auf den familiären Hintergrund der Schülerinnen und Schüler berücksichtigt werden.

Zwar hängen die geeigneten politischen Antworten auf derartige Unterschiede in den Schülerleistungen natürlich auch von wirtschaftlichen und sozialen Faktoren ab, auf die Pädagogen keinen Einfluss nehmen können, die Analyse der Gradienten bietet jedoch Möglichkeiten, um die Leistungen der Schulen zu beschreiben und so nützliche Anhaltspunkte für die Gestaltung der Bildungspolitik zu liefern. Ein zentrales Ziel von PISA ist es, den Ländern Instrumente zur Überprüfung ihrer Leistungen im Bildungssektor an die Hand zu geben. Ebenso wie Vergleiche zwischen Mittelwerten, Verteilungen und sozioökonomischen Gradienten der verschiedenen Länder angestellt werden können, ist es auch möglich, entsprechende Vergleiche innerhalb der einzelnen Länder zwischen verschiedenen Bundesstaaten oder Provinzen oder anderen Schülergruppen durchzuführen. Den Ländern wird zudem die Möglichkeit gegeben, die Entwicklung der Indexwerte zwischen PISA 2000 und künftigen PISA-Erhebungszyklen zu vergleichen.

\section{Möglichkeiten der Schulen zur Minderung der Effekte sozioökonomischer Benachteiligung}

Viele Faktoren einer sozioökonomischen Benachteiligung lassen sich nicht direkt durch die Bildungspolitik beeinflussen, zumindest nicht auf kurze Sicht. Beispielsweise kann das Bildungsniveau der Eltern nur ganz allmählich angehoben werden, und der Wohlstand der Familien hängt von den langfristigen wirtschaftlichen und sozialen Entwicklungen im jeweiligen Land sowie den jeweils gebildeten Ersparnissen ab. So erhebt sich für die politischen Entscheidungsträger eine entscheidende Frage: Bis zu welchem Grad ist es den Schulen und der Bildungspolitik möglich, die Auswirkungen einer sozioökonomischen Benachteiligung auf die Schülerleistungen zu mildern?

Zur Bestimmung der politischen Maßnahmen, mit denen die Gesamtleistung der Schülerinnen und Schüler angehoben und die Auswirkungen des sozioökonomischen Hintergrunds gemindert - d.h. die sozioökonomische Gradienten der jeweiligen Länder erhöht und abgeflacht - werden können, muss die Verteilung der Ergebnisse innerhalb und zwischen den Schulen 
untersucht werden. Zu diesem Zweck kann die Gradiente eines Landes zerlegt werden in eine Gradiente der Leistungsunterschiede innerhalb der Schulen, die den Zusammenhang zwischen der Leistung und dem familiären Hintergrund verschiedener Schülerinnen und Schüler einer selben Schule beschreibt, und eine Gradiente der Leistungsunterschiede zwischen den Schulen, die den Zusammenhang zwischen den durchschnittlichen Leistungsniveaus der Schulen und der durchschnittlichen wirtschaftlichen, sozialen und kulturellen Stellung ihrer Schülerinnen und Schüler beschreibt.

In einigen Ländern ist eine starke Segregation der Schülerinnen und Schüler nach sozioökonomischen Merkmalen zu beobachten, was z.T. auf eine entsprechende Trennung bei den Wohngebieten sowie auf wirtschaftliche Faktoren zurückzuführen ist, aber auch durch die besonderen Merkmale der jeweiligen Bildungssysteme bedingt sein kann. Diese Länder weisen in der Regel steile Gesamtgradienten auf. In diesen Ländern tragen Privatschulen oder besonders anspruchsvolle Schultypen oder Bildungszweige innerhalb des öffentlichen Bildungssystems u.U. zur sozioökonomischen Segregation bei. Um die Qualität und Gleichheit im Bildungswesen in solchen Ländern zu steigern (d.h. die Gradiente zu erhöhen und abzuflachen), müsste den Unterschieden zwischen den Schulen besondere Aufmerksamkeit gewidmet werden. Der Abbau der sozioökonomischen Segregation zwischen den Schulen stellt eine mögliche Strategie dar, um diesem Problem beizukommen; unabhängig von der Anwendung dieser Strategie bestünden weitere mögliche Ansatzpunkte in einer differenzierten Ressourcenzuweisung für verschiedene Schultypen und Lehrprogramme sowie in dem Versuch, den Schülerinnen und Schülern differenzierte, angemessene Bildungsmöglichkeiten $\mathrm{zu}$ bieten. In den betreffenden Ländern stellt sich die Frage, inwieweit die schulische Ressourcenzuweisung innerhalb eines Landes mit dem sozioökonomischen Hintergrund der aufgenommenen Schülerinnen und Schüler der einzelnen Schulen in Zusammenhang steht.

In anderen Ländern ist die sozioökonomische Segregation zwischen den verschiedenen Schulen relativ schwach ausgeprägt, d.h. der durchschnittliche sozioökonomische Hintergrund der Schülerinnen und Schüler der einzelnen Schulen ist in der Regel ähnlich. In diesen Ländern hängen Qualität (die Höhe) und Gleichheit (die Steigung der Gradienten) hauptsächlich vom Zusammenhang zwischen den Schülerleistungen und dem sozioökonomischen Hintergrund der einzelnen Schülerinnen und Schüler der jeweiligen Schulen ab. Um die Qualität und die Gleichheit in diesen Ländern zu erhöhen, muss vor allem innerhalb der Schulen gehandelt werden. Eine mögliche Strategie wäre hier die Reduzierung der schulinternen Segregation der Schülerinnen und Schüler entsprechend ihres unterschiedlichen wirtschaftlichen, sozialen und kulturellen Status, wobei wahrscheinlich die Einteilung in Klassen mit unterschiedlichem Leistungsniveau überdacht werden müsste. Eine direktere Unterstützung für leistungsschwache Schülerinnen und Schüler könnte ebenfalls erforderlich sein. In den betroffenen Ländern ist es wichtig, festzustellen,
Länder mit einem hohen Grad an sozialer

Segregation zwischen

den Schulen sollten die

Mittelzuweisung und die

Selektionsmechanismen

genauer unter die Lupe

nehmen, um die sozialen

Gradienten zu

verbessern ...

\author{
... während weniger \\ gegliederte Schulsysteme \\ schulinterne \\ Verbesserungen erwägen \\ könnten.
}


KAPITEL 8 Entscheidende Erkenntnisse aus den PISA-Resultaten - Hinweise für die Politikgestaltung

in welchem Zusammenhang die schulinterne Ressourcenzuweisung mit dem sozioökonomischen Hintergrund der Schüler steht.

Im folgenden Abschnitt soll genauer untersucht werden, welche Rolle die Bildungspolitik bei der Lösung dieser Probleme spielen kann. Die Analyse stützt sich hier auf die in Kapitel 2 durchgeführte Untersuchung des Ausmaßes, wie stark sich die Leistungen auf der Gesamtskala Lesekompetenz zwischen den Schülern und innerhalb der Schulen und zwischen den Schulen voneinander unterscheiden (vgl. Tabelle 2.4). Zunächst wird das Ausmaß untersucht, in dem diese beiden Formen der Varianz - zwischen den Schülern und zwischen den Schulen - auf den Einfluss geschlechtsspezifischer Unterschiede oder wirtschaftlicher, sozialer und kultureller Faktoren zurückzuführen sind. Abschließend wird analysiert, ob die zwischen den Schulen bestehenden Leistungsunterschiede, die nicht auf die Merkmale oder den familiären Hintergrund der Schülerinnen und Schüler zurückzuführen sind, mit dem sozioökonomischen Hintergrund der Schule, mit charakteristischen Merkmalsfaktoren des Bildungssystems oder mit der Schulpolitik und -praxis in Zusammenhang stehen.

\section{Sozioökonomischer Hintergrund und Varianz der Leistungen zwischen den Schulen}

Gut ein Drittel der

Unterschiede bei den

Schülerleistungen ist auf

Differenzen zwischen

den Schulen

zurückzuführen.
In Kapitel 2 wurde aufgezeigt, dass in den meisten Ländern bei den Leistungen der 15-jährigen Schülerinnen und Schüler zwischen den Schulen große Unterschiede bestehen. Im OECD-Durchschnitt entfallen 36\% der Gesamtvarianz der Schülerleistungen auf der Gesamtskala Lesekompetenz auf Unterschiede zwischen den Schulen (Tabelle 2.4). Um die Leistung der Schulen verbessern zu können, ist es besonders wichtig zu wissen, warum manche Schulen oder einzelne Teile des Bildungssystems eines Landes besser abschneiden als andere. Dazu bedarf es einer Analyse, bei der die Auswirkungen schülerwie auch schulspezifischer Faktoren auf die Leistungen der Schülerinnen und Schüler einzelner Schulen sowie verschiedener Schulen desselben Landes untersucht werden.

Es ist möglich, den Anteil der Varianz der Leistungen von Schülerinnen und Schülern innerhalb von einzelnen Schulen sowie zwischen verschiedenen Schulen zu schätzen, der auf den familiären Hintergrund der Schüler zurückgeht, wie er anhand der in der Analyse von Tabelle 8.2 verwendeten Hintergrundfaktoren gemessen wird. Die entsprechenden Ergebnisse sind in Tabelle 8.3 dargestellt. In Australien entfallen beispielsweise 16\% der Leistungsunterschiede innerhalb der Schulen und 64\% der Leistungsunterschiede zwischen den Schulen auf die in den Spalten 3 bis 9 von Tabelle 8.2 aufgeführten familiären Hintergrundfaktoren. Diese Prozentsätze unterscheiden sich z.B. deutlich von denen Polens, wo der familiäre Hintergrund der Schüler nur für 2\% der schulinternen Leistungsdifferenzen und für 10\% der Leistungsdifferenzen zwischen den Schulen verantwortlich ist. 
Beim Vergleich des Ausmaßes, in dem Leistungsunterschiede zwischen den Schulen in den verschiedenen Ländern auf familiäre Hintergrundfaktoren der Schülerinnen und Schüler zurückzuführen sind, muss unbedingt auch die Höhe der Unterschiede zwischen den Schulen berücksichtigt werden (vgl. Tabelle 2.4). In Schweden beispielsweise erklären die familiären Hintergrundfaktoren der Schülerinnen und Schüler die Leistungsunterschiede zwischen den Schulen in einem Ausmaß wie sonst in keinem anderen Land (73\%, vgl. Tabelle 8.3), doch sind in Schweden die Leistungsdifferenzen zwischen den Schulen geringer als in jedem anderen Land mit Ausnahme Islands (9\%, vgl. Tabelle 2.4). Familiäre Hintergrundfaktoren wirken sich in Polen weniger stark auf die Leistungsdifferenzen zwischen den Schulen aus als in jedem anderen Land (10\%, vgl. Tabelle 8.3), doch zugleich sind die Leistungsunterschiede zwischen den Schulen in Polen größer als in allen außer vier Ländern (Österreich, Deutschland, Ungarn und die Schweiz, vgl. Tabelle 2.4). Generell lässt sich feststellen, dass der Anteil der auf den familiären Hintergrund der Schüler zurückzuführenden Leistungsunterschiede zwischen den Schulen umso geringer ist, je größer die Leistungsunterschiede zwischen den Schulen sind.

Es bestehen auch deutliche Unterschiede zwischen den Ländern im Hinblick auf den Anteil derVarianz innerhalb der Schulen, der auf Unterschiede im familiären Hintergrund der Schülerinnen und Schüler zurückzuführen ist, wenngleich diese Anteile wesentlich kleiner sind als die bei den Leistungsunterschieden zwischen den Schulen. Im Allgemeinen gilt jedoch, dass der Anteil der durch den familiären Hintergrund der Schülerinnen und Schüler erklärten Leistungsunterschiede umso höher ist (Tabelle 8.3), je größer die Leistungsunterschiede innerhalb der Schulen sind (Tabelle 2.4). In Norwegen und Neuseeland, den beiden Ländern mit den größten Leistungsunterschieden innerhalb der Schulen, sind beispielsweise 20\% dieser Unterschiede auf familiäre Hintergrundfaktoren zurückzuführen. Nur in Finnland und Luxemburg spielt der familiäre Hintergrund im Hinblick auf die schulinternen Leistungsunterschiede (rund 20\%) eine noch größere Rolle. Familiäre Hintergrundfaktoren wirken sich in Polen weniger stark auf die schulinternen Leistungsdifferenzen aus als in jedem anderen Land (2\%), doch in Polen sind diese Leistungsunterschiede auch geringer als in allen außer drei Ländern (Ungarn, Korea und Mexiko).

\section{Die Verstärkung wie der vom familiären Hintergrund der einzelnen Schülerinnen und Schüler ausgehenden Effekte durch die soziale Struktur der Schulen}

Im vorigen Abschnitt wurde aufgezeigt, dass ein erheblicherTeil der Unterschiede in den Leistungen auf der Gesamtskala Lesekompetenz zwischen den Schulen mit Unterschieden in Bezug auf den sozioökonomischen Hintergrund der Schülerinnen und Schüler in Zusammenhang steht. Dieser Effekt kann sich auf zweierlei Weise auswirken: Erstens kann der familiäre Hintergrund des einzelnen Schülers dessen Leistung beeinflussen. Zweitens kann zusätzlich aber
Differenzen in den Hintergrundfaktoren der schülerinnen und schülern erklären unterschiedlich hohe Anteile der Varianz zwischen den Schulen bzw. innerhalb von schulen...

... dort aber, wo die Unterschiede innerhalb von Schulen grofs sind, könnte dies durch sozioökonomische Unterschiede bedingt sein.
Der Effekt sozialer Unterschiede zwischen den Schulen kann durch die Gesamtvorteile der besser gestellten Schulen noch verstärkt werden. 
In den meisten Ländern ist es eindeutig ein Vorteil, eine Schule zu besuchen, deren schülerschaft einen günstigeren familiären Hintergrund aufweist, ... auch der sozioökonomische Hintergrund der Gesamtheit aller Schülerinnen und Schüler einer Schule einen Einfluss auf die Schülerleistungen ausüben. Ein Verständnis dieser Zusammenhänge ist von entscheidender Bedeutung für die politischen Entscheidungsträger, wenn sie allen Schülern gleiche Bildungschancen bieten wollen.

Die Art und Weise, wie die Schülerinnen und Schüler innerhalb eines Verwaltungsbezirks oder einer Region auf verschiedene Schulen oder innerhalb der Schulen auf verschiedene Klassen und Programme verteilt sind, hat weitreichende Konsequenzen für die Lehr- und Lernbedingungen in den Schulen und somit auch für die Bildungserträge. Eine Reihe von Untersuchungen hat gezeigt, dass Schulen, die in sozioökonomischer Hinsicht im Durchschnitt besser gestellt sind, in der Regel mehrere Vorteile haben. Sie erhalten in der Regel mehr Unterstützung durch die Eltern, haben weniger Disziplinprobleme, bessere Beziehungen zwischen Lehrkräften und Schülern, Stimmung und Arbeitshaltung der Lehrkräfte sind besser und das gesamte Klima an der Schule ist im Allgemeinen auf die Erzielung höherer Leistungen ausgerichtet ${ }^{20}$. Häufig sieht auch der Lehrplan eine raschere Vorgehensweise vor. Begabte und motivierte Lehrkräfte geben in der Regel Schulen den Vorzug, die in sozioökonomischer Hinsicht besser gestellt sind, und tendieren dann auch seltener dazu, die Schule oder gar den Beruf zu wechseln. Ein Teil der „Umfeldeffekte”, die sich aus einem hohen sozioökonomischen Status der Schulen ergeben, können auch auf Peer-Interaktionen zurückzuführen sein, indem begabte Schülerinnen und Schüler zusammenarbeiten. Der von den Mitschülern ausgehende Leistungsdruck, der Wettbewerb zwischen den Mitschülern und die Konzentration einiger Schulen oder Bildungszweige auf ein späteres Hochschulstudium können ebenfalls eine Rolle spielen.

Abbildung 8.4 enthält Schätzwerte für die Effekte des wirtschaftlichen, sozialen und kulturellen Status sowie anderer in Tabelle 8.2 angegebener Hintergrundvariablen auf die Lesekompetenz der Schülerinnen und Schüler, wobei zwischen den vom Hintergrund der einzelnen Schüler ausgehenden Effekte zum einen und denen des Hintergrunds der gesamten Schülerschaft einer Schule zum anderen unterschieden wird. Diese Schätzwerte wurden anhand eines mehrschichtigen Modells ermittelt, bei dem der wirtschaftliche, soziale und kulturelle Status, das Geschlecht, die ethnische Zugehörigkeit und die Familienstruktur auf Ebene der einzelnen Schülerinnen und Schüler sowie der durchschnittliche wirtschaftliche, soziale und kulturelle Status auf Schulebene berücksichtigt wurden. Aus der Höhe der Balken in Abbildung 8.4 sind die Leistungsunterschiede auf der Gesamtskala Lesekompetenz ersichtlich, die einem Unterschied im Umfang von einer halben Standardabweichung auf dem sozioökonomischen Index für den einzelnen Schüler sowie für den Durchschnitt der Schülerinnen und Schüler einer Schule entsprechen (vgl. Angaben aus Tabelle 8.4). 


\section{Abbildung 8.4}

Effekte des sozioökonomischen Hintergrundes der Schüler und der Schulen auf die Schülerleistungen auf der Gesamtskala Lesekompetenz

Leistungsveränderung auf der Gesamtskala Lesekompetenz, die einer Veränderung um eine halbe Standardabweichung auf Schülerebene des wirtschaftichen, sozialen und kulturellen Status entspricht

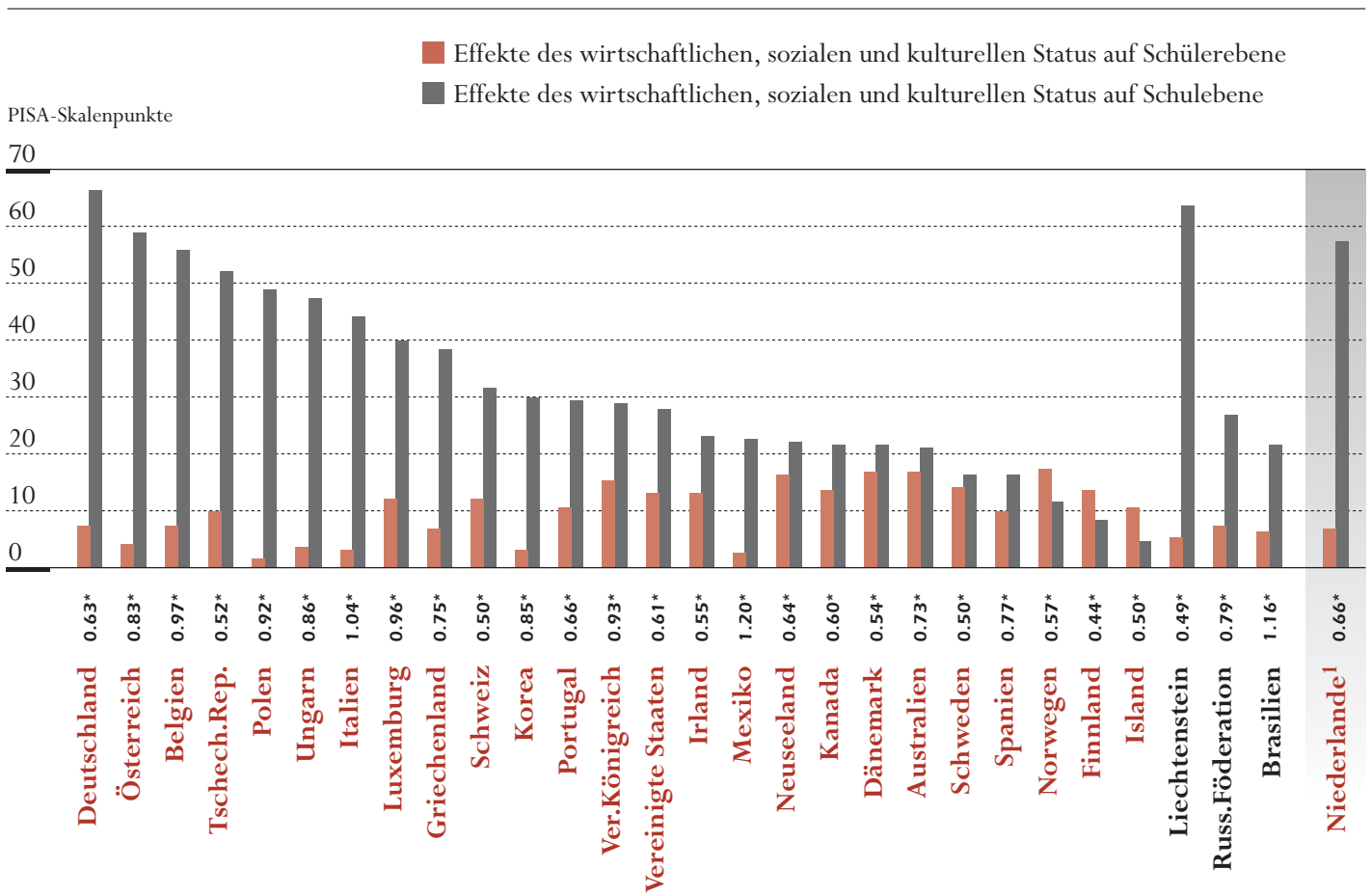

* Abstand zwischen den Quartilen nach dem durchschnittlichen Index des wirtschaftlichen, sozialen und kulturellen Status der Schulen.

1. Die Beteiligungsquote ist zu niedrig, um die Vergleichbarkeit zu gewährleisten (vgl. Anhang A3).

Quelle: OECD PISA Datenbank, 2001. Tabelle 8.4.

In fast allen Ländern scheinen die Schülerinnen und Schüler durchweg klar im Vorteil zu sein, wenn sie Schulen besuchen, deren Schülerschaft im Durchschnitt aus eher privilegierten Milieus stammt. Im Durchschnitt der OECD-Länder entspricht der Effekt einerVerbesserung der sozioökonomischen Zusammensetzung der Schülerschaft einer Schule um eine halbe Standardabweichung auf Schülerebene einem Leistungsanstieg um rund 32 Punkte, d.h. nahezu einer halben Kompetenzstufe auf der Gesamtskala Lesekompetenz $^{21}$. Der sozioökonomische Hintergrund der Gesamtheit aller Schülerinnen und Schüler einer Schule hat somit erhebliche Auswirkungen auf die Schülerleistungen auf der Gesamtskala Lesekompetenz, die wesentlich stärker sind als die vom familiären Hintergrund der einzelnen Schülerinnen und Schüler ausgehenden Effekte. In der Mehrzahl der OECD-Länder übersteigt der vom durchschnittlichen wirtschaftlichen, sozialen und kulturellen Status der Schülerinnen und Schüler einer Schule ausgehende Effekt bei weitem den 
... und in vielen Fällen ist der vom sozioökonomischen Hintergrund der gesamten Schule ausgehende Effekt auf die Schülerleistungen wesentlich stärker als der

auf Einzelpersonen zurückzuführende... auf den familiären Hintergrund des einzelnen Schülers zurückzuführenden Effekt. Da PISA keine Angaben über die früheren Leistungen der Schüler liefert, kann nicht festgestellt werden, ob und inwieweit der sozioökonomische Hintergrund der Schulen sich direkt oder indirekt - z.B. durch Selektionsund Autoselektionsphänomene - in den Schülerleistungen niederschlägt. Bei der Interpretation dieser Ergebnisse muss ferner bedacht werden, dass die Differenzen hinsichtlich des durchschnittlichen sozioökonomischen Hintergrunds der Schulen natürlich wesentlich geringer sind als die entsprechenden Unterschiede zwischen den einzelnen Schülern, da die Zusammensetzung der Schülerschaft jeder Schule bezüglich der sozioökonomischen Variablen gemischt ist. Um die Interpretation der Daten zu erleichtern, wurde in Abbildung 8.4 auch die typische Bandbreite des durchschnittlichen sozioökonomischen Status der verschiedenen Schulen dargestellt ${ }^{22}$.

Österreich und Deutschland sind Länder, in denen vom durchschnittlichen wirtschaftlichen, sozialen und kulturellen Status der Schulen ein erheblicher Einfluss auf die Schülerleistungen ausgeht. Nehmen wir das Beispiel zweier fiktiver Schüler aus Österreich oder Deutschland, die über ähnliche Fähigkeiten verfügen und in Familien aufwachsen, deren sozioökonomischer Hintergrund gemessen am Index des wirtschaftlichen, sozialen und kulturellen Status dem Durchschnitt entspricht. Einer dieser beiden Schüler besucht eine Schule in einer relativ gut situierten Wohngegend, in der das Indexmittel für den wirtschaftlichen, sozialen und kulturellen Status der Schülerschaft insgesamt (auf Schülerebene) eine viertel Standardabweichung über dem OECD-Durchschnitt liegt. Die meisten Mitschülerinnen und Mitschüler dieses Schülers stammen somit aus wirtschaftlich besser gestellten Familien als er selbst. Der andere Schüler besucht eine Schule in einer weniger gut situierten Wohngegend, in der das Indexmittel für den wirtschaftlichen, sozialen und kulturellen Status eine viertel Standardabweichung unter dem OECD-Durchschnitt liegt, so dass dieser Schüler folglich aus einer wohlhabenderen Familie kommt als die Mehrzahl seiner Mitschülerinnen und Mitschüler. Die Angaben in Abbildung 8.4 zeigen, dass der erste Schüler in Bezug auf seine Lesekompetenz wahrscheinlich wesentlich besser abschneiden würde als der zweite (und zwar um 66 Punkte auf der Gesamtskala Lesekompetenz in Deutschland und um 59 Punkte in Österreich). Für zwei Schüler hingegen, deren wirtschaftlicher, sozialer und kultureller Status nach dem Index jeweils eine viertel Standardabweichung unter bzw. über dem Durchschnitt liegt und die dieselbe Schule besuchen, deren sozialer Hintergrund dem Durchschnitt entspricht, würde der zu erwartende Leistungsunterschied wesentlich geringer ausfallen (8 Punkte in Deutschland und 4 Punkte in Österreich).

Im Bereich Lesekompetenz schlägt sich eineVerbesserung des sozioökonomischen Status einer Schule um eine halbe Standardabweichung auf Schülerebene in einem Leistungsanstieg nieder, der im Durchschnitt für Jungen um rund 2 Punkte höher ist als für Mädchen ${ }^{23}$. Auf der Skala für mathematische 
Grundbildung beläuft sich dieser Unterschied auf 4 Punkte und auf der Skala für naturwissenschaftliche Grundbildung auf 3 Punkte. Daraus lässt sich schließen, dass Jungen durch die sozioökonomischen Segregation zwischen den Schulen stärker im Vorteil bzw. im Nachteil sind als die Mädchen, deren Leistungen etwas weniger deutlich durch den wirtschaftlichen, sozialen und kulturellen Status ihrer Mitschülerinnen und Mitschüler beeinflusst werden.

Ein Teil des beobachteten „Umfeldeffekts” könnte auf Qualitätsaspekte der Schulen zurückzuführen sein, die mit den oben erwähnten Faktoren in Verbindung stehen. Beispielsweise könnte der „Umfeldeffekt” des sozioökonomischen Status in dem Maße durch systematische Lehrplanunterschiede verstärkt werden, wie manche Schulen auf ein späteres Hochschulstudium vorbereiten und andere nicht. Bei einem Teil des „Umfeldeffekts” könnten zudem Peer-Effekte eine Rolle spielen. Ein anderer Teil des Umfeldeffekts könnte aber auch auf hier nicht erfasste Faktoren zurückzuführen sein. Beispielsweise besteht die Möglichkeit, dass der Schüler aus dem obigen Beispiel, der die in sozioökonomischer Hinsicht besser gestellte Schule besucht, im Durchschnitt von seinen Eltern zu Hause stärker bei seiner Arbeit unterstützt wird, obwohl deren sozioökonomischer Hintergrund mit dem des Schülers vergleichbar ist, der die weniger gut gestellte Schule besucht. Hinzu kommt auch - und dies ist vielleicht noch wichtiger -, dass in diesem Beispiel in etwa gleiche Fähigkeiten und Motivation der beiden Schüler unterstellt wurde. In vielen Bildungssystemen erfolgt die Verteilung der Schülerinnen und Schüler auf verschiedene Schulen oder Programme jedoch auf der Grundlage von mehreren Kriterien, zu denen u.a. auch ihre Leistungen gehören. In den beiden Ländern des obigen Beispiels, Österreich und Deutschland, sind z.B. die Leistungsunterschiede zwischen den Schulen hauptsächlich auf die Verteilung der Schüler auf allgemeinbildende oder berufsbildende Schulen zurückzuführen. Die Verteilung der Schüler auf verschiedene Schultypen bzw. -zweige hängt von deren Leistungen ab, die wiederum in einem engen Zusammenhang mit ihrem sozioökonomischen Hintergrund stehen. So wichtig diese Ergebnisse für die Politikgestaltung auch sein mögen, es darf nicht voreilig darauf geschlossen werden, dass es ausreichen würde, eine Schülergruppe aus einer Schule mit ungünstigem sozioökonomischem Hintergrund in eine Schule mit einer sozial besser gestellten Schülerschaft umzusiedeln, um bei dieser Schülergruppe automatisch den in Abbildung 8.4 dargestellten Leistungsanstieg zu erzielen. Anders ausgedrückt: Die geschätzten Umfeldeffekte aus Abbildung 8.4 sind beschreibende Faktoren der Verteilung der Schulleistungen und sollten nicht als Kausalfaktoren verstanden werden.

Bei der Konzipierung bildungspolitischer Maßnahmen unter Berücksichtigung der vorgenannten Ergebnisse ist es in jedem Fall wichtig, sich eine Vorstellung von der Art der formellen und informellen Selektionsmechanismen zu machen, die zu der sozioökonomischen Segregation zwischen den Schulen beitragen,

\author{
... ein Phänomen, das \\ komplexe Ursachen hat \\ und z. T. mit der \\ Konzentration von \\ schülern nach ihren \\ Fähigkeiten \\ zusammenhängen \\ könnte...
}

. und im Kontext damit gesehen werden sollte, welche Mechanismen zu einer sozioökonomischen Segregation führen. 
KAPITEL 8 Entscheidende Erkenntnisse aus den PISA-Resultaten - Hinweise für die Politikgestaltung

Die wichtigste Aufgabe besteht im Abbau von segregationsbedingten Ungleichheiten, aber ohne Nivellierung nach unten.

PISA liefert Anhaltspunkte für die Einschätzung der Effekte einiger Schulmerkmale auf die Leistungen. sowie von deren Auswirkungen auf die Schülerleistungen. In einigen Ländern kann die sozioökonomische Segregation auf Grund der Unterteilung der großen Städte in besser und schlechter gestellte Viertel oder einer starken Kluft zwischen den ländlichen und städtischen Gebieten tief verwurzelt sein. In anderen Ländern sorgen die strukturellen Merkmale der Bildungssysteme dafür, dass bestimmte Schüler in bestimmte Schultypen gelenkt werden, deren Lehrinhalte und Unterrichtsmethoden u.U. abweichen. In dem Maße, wie die Verteilung der Schüler auf verschiedene Schultypen oder-zweige mit dem sozioökonomischen Hintergrund der Schüler verknüpft ist, besteht in solchen Systemen die Möglichkeit, dass Schüler aus sozial benachteiligten Milieus ihr Potential nicht voll ausschöpfen können.

Aus PISA ergeben sich zwei unterschiedliche Schlussfolgerungen, wenn es darum geht, sowohl die Bildungsqualität als auch die Bildungsgleichheit zu erhöhen. Einerseits hat sich gezeigt, dass soziale Segregation für die Privilegierteren Vorteile bringt, dank denen die Leistung einer Elite und damit u.U. auch die durchschnittliche Leistung angehoben werden kann. Andererseits geht aus diesem Bericht hervor, dass die Bildungsungleichheit durch die schulische Segregation wahrscheinlich noch zunimmt. Es gibt allerdings auch stichhaltige Belege dafür, dass dieses Dilemma gelöst werden kann. Dies zeigt sich an jenen Ländern, denen es gelungen ist, sowohl ein hohes Maß an Bildungsqualität als auch an Bildungsgleichheit zu erzielen. Die Frage ist nur, wie dieses Problem in anderen Ländern gelöst werden kann. Die Umsiedlung sämtlicher Schüler in Schulen mit gehobenem sozioökonomischem Status ist logischerweise unmöglich. Der Politik bieten sich somit nur zwei Optionen: entweder zu versuchen, die sozioökonomische Segregation zu beseitigen, oder deren Effekte abzumildern. Im Mittelpunkt muss dabei in jedem Fall die Bemühung stehen, die mit der sozialen Segregation verbundenen Qualitätsgewinne zu reproduzieren und zugleich die Vorteile einer stärkeren Bildungsgleichheit zu realisieren, wie sie sich aus einer größeren sozialen Heterogenität ergeben.

\section{Schulische Faktoren, mit denen die Leistungsniveaus angehoben und die Auswirkungen des sozioökonomischen Hintergrunds gemildert werden können}

Was können Schulen tun, um die erwünschten Effekte zu erzielen? Untersuchungen wie PISA können diese Frage nur bis zu einem gewissen Grad beantworten, da viele wichtige Umfeldfaktoren in internationalen Vergleichsstudien von Schülerleistungen nicht erfasst und Entwicklungen im zeitlichen Verlauf in solchen Studien auch nicht mit hinreichender Genauigkeit analysiert werden können, um Ursache und Wirkung jeweils eindeutig zu bestimmen. Gleichwohl ermöglicht es PISA, die Effekte einiger wichtiger Schulmerkmale auf die Schülerleistungen einzuschätzen.

Die unten stehende Analyse liefert Schätzwerte des jeweils gesonderten Einflusses von schulischen Faktoren und familiären Hintergrundfaktoren sowie 
deren kombinierter Wirkung. In jedem der drei getesteten Kompetenzbereiche werden die Auswirkungen dreier Gruppen von schulspezifischen Faktoren auf die Schülerleistungen untersucht: die den Schulen zur Verfügung stehenden Mittel, die Schulpolitik und -praxis sowie die Unterrichtspraktiken. Die Schätzwerte basieren auf dem kombinierten Effekt der Variablen auf Schüler-, Schul- und Landesebene. Die Ergebnisse dieser Analyse, die für die Gesamtheit der Schülerpopulation des OECD-Raums durchgeführt wurde (wobei alle Länder in etwa gleich stark gewichtet wurden), sind Tabelle 8.5 zu entnehmen.

Der Vergleich zeigt, dass einige der Faktoren bezüglich der den Schulen zur Verfügung stehenden Mittel bei einer Gesamtbetrachtung aller OECDLänder statistisch signifikant mit den Schülerleistungen zusammenhängen: Der Umfang, in dem die Schüler die Ressourcen ihrer Schule nutzen, die Schüler/ Lehrer-Quote, die Größe der Schule sowie der Anteil der Lehrkräfte mit Hochschulabschluss ${ }^{24}$ und Studienschwerpunkt im jeweiligen Unterrichtsfach.

Obwohl aus den PISA-Ergebnissen nicht automatisch auf Kausalbeziehungen geschlossen werden kann, sind die Schülerleistungen im Bereich Lesekompetenz im OECD-Durchschnitt in solchen Schulen höher, in denen die Schülerinnen und Schüler häufiger von den schulischen Ressourcen Gebrauch machen (was in der Häufigkeit zum Ausdruck kommt, mit der die Schüler laut eigener Angabe die Bibliothek, die Computer, die Taschenrechner, die Labors und den Internetanschluss der Schule benutzen). Ein Anstieg um eine Einheit (die hier einer internationalen Standardabweichung entspricht) auf dem PISA-Index der Schulressourcennutzung durch die Schüler (wegen der Definition des Index vgl. Anhang A1) entspricht einem durchschnittlichen Leistungsunterschied auf der Gesamtskala Lesekompetenz von 18 Punkten. Die Qualität der räumlichen Bedingungen der Schulen (vgl. Tabelle 7.9), gemessen an den Schulleiterangaben darüber, inwieweit das Lernen der 15-Jährigen durch verschiedene ressourcenbezogene Faktoren behindert wird, hat offenbar keine statistisch signifikanten Effekte auf die Lesekompetenz, und dasselbe gilt auch für den Prozentsatz der pro Schüler verfügbaren Computer (nach Berücksichtigung anderer Variablen, wie z.B. der Benutzung der Computer durch die Schüler laut deren eigenen Angaben).

In Abbildung 8.5 ist der durchschnittliche Effekt der Schüler/Lehrer-Quote auf Schülerleistungen auf der Gesamtskala Lesekompetenz dargestellt ${ }^{25}$. Die Schüler/Lehrer-Quote wurde auf der Grundlage der Schulleiterangaben über die Zahl der in ihrer Schule angemeldeten Schüler und die Zahl der dort beschäftigten Lehrkräfte in Vollzeitäquivalenten berechnet (Daten abrufbar unter www.pisa.oecd.org). Die Abbildung zeigt, dass der Effekt der Schüler/ Lehrer-Quote nicht linear ist, d.h. dass sich deren Auswirkungen auf die Schülerleistungen mit zunehmender Höhe der Quote verändern.
Mehrere schulbezogene Faktoren stehen mit den Leistungen in Zusammenhang, ...

.. so z. B. die Tatsache, wie häufig Schülerinnen und Schüler Bibliotheken, Computer, Taschenrechner und Labors der Schule benutzen.

Die Schüler/LehrerQuote ist ebenfalls von Bedeutung, ... 
KAPITEL 8 Entscheidende Erkenntnisse aus den PISA-Resultaten - Hinweise für die Politikgestaltung

Abbildung 8.5

Durchschnittliche Auswirkung der Schüler/Lehrer-Quote auf die Schülerleistungen auf der Gesamtskala Lesekompetenz im gesamten OECD-Raum

Leistung auf der Gesamtskala Lesekompetenz

(PISA-Skalenpunkte)

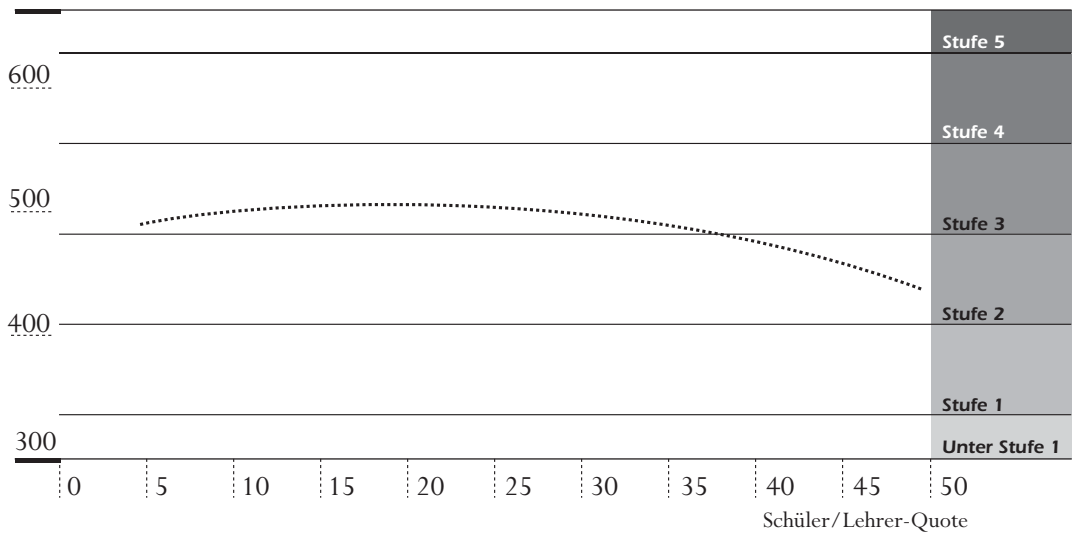

Quelle: OECD PISA Datenbank, 2001. Daten abrufbar unter www.pisa.oecd.org.

Die Ergebnisse der Schulen, in denen die Schüler/Lehrer-Quote weniger als 10 beträgt, liegen rund 5 bis 10 Punkte unter dem OECD-Durchschnitt. Dies könnte darauf zurückzuführen sein, dass es sich hier häufig um Sonderschulen oder vergleichsweise kleine Schulen auf dem Land handelt.

... doch nehmen die Leistungen erst bei Quoten über 25 mit jedem zusätzlichen Schüler je Lehrkraft kontinuierlich ab.
Unterschiede in der Schüler/Lehrer-Quote innerhalb einer Bandbreite von 10 bis 25 haben nur vergleichsweise geringe Auswirkungen. Erhöht sich die Schüler/Lehrer-Quote jedoch über 25, ist bei einer Gesamtuntersuchung aller OECD-Länder ein kontinuierlicher Rückgang der Leistungen in allen PISATestbereichen festzustellen. Dabei ist zu beachten, dass sich diese Ergebnisse (ebenso wie die anderen Ergebnisse aus Tabelle 8.5) auf den durchschnittlichen Effekt innerhalb der einzelnen Länder beziehen. Obwohl der Zusammenhang zwischen Schülerleistungen und Schüler/Lehrer-Quote von Land zu Land verschieden stark ausgeprägt ist, sind die Ergebnisse aus Abbildung 8.5 somit kein Artefakt einiger besonders schlecht abschneidender Schulen in einigen wenigen Ländern ${ }^{26}$.

Ebenfalls zu erwähnen ist, dass es sich bei der Schüler/Lehrer-Quote um ein anderes Konzept handelt als bei der Klassengröße. Wie oben erwähnt, errechnet sich die Schüler/Lehrer-Quote aus der Schülerzahl einer Schule und der Zahl ihrer Lehrkräfte in Vollzeitäquivalenten. In vielen Ländern werden zum Lehrkörper auch die Schulleiter sowie sonstige Kräfte wie Bibliothekare oder Sonderpädagogen hinzugerechnet. Die Schüler/Lehrer-Quote ist insofern wichtig, als sie Aufschluss darüber gibt, in welchem Umfang personelle 
Ressourcen direkt oder indirekt auf die Ausbildung jedes einzelnen Schülers verwendet werden. Sie steht zudem in engem Zusammenhang mit den Gesamtausbildungskosten für einen Schüler innerhalb des Bildungssystems. Die Klassengröße bezieht sich auf die tatsächliche Zahl der Schülerinnen und Schüler, die in einer Klasse unterrichtet werden. Sie lässt sich auf Sekundarstufenebene in nationalen und internationalen Studien nur schwer messen, da die Schüler für verschiedene Fächer unterschiedliche Klassen besuchen und die Klassengröße auch davon abhängig ist, wie viele Schüler an einem Tag jeweils anwesend sind.

Die Beziehung zwischen der Lesekompetenz und der Schulgröße verläuft in den OECD-Ländern ebenfalls nicht linear. Eine Zunahme um 100 Schüler entspricht in Schulen mit bis zu 1000 Schülern einem Leistungsanstieg um ca. drei bis vier Punkte. Bei Schulen mit über 1000 Schülern lässt sich jedoch kaum noch ein Zusammenhang nachweisen.

In allen PISA-Testbereichen sind die Schülerleistungen in solchen Schulen höher, in denen ein größerer Prozentsatz der Lehrkräfte eine besondere Ausbildung für die von ihnen unterrichteten Fächer erhalten haben (dieses Ergebnis stützt sich auf die Schulleiterangaben über die Zahl der Lehrkräfte mit Hochschulabschluss in ihrem jeweiligen Unterrichtsfach). Im Bereich der Lesekompetenz z.B. entspricht ein Anstieg um 10 Prozentpunkte des Anteils der Lehrkräfte mit Hochschulqualifikation im entsprechenden Fach einem Leistungsanstieg um ca. 3,7 Punkte auf der Gesamtskala Lesekompetenz ${ }^{27}$. Aus den Angaben der Schulleiter darüber, ob Lehrkräfte während der drei vorangegangenen Monate an offiziellen Programmen teilgenommen haben, die auf eine Verbesserung ihrer pädagogischen Qualifikationen oder Unterrichtspraktiken abzielten, ließen sich hingegen keine nennenswerten Auswirkungen auf die Schülerleistungen ablesen. Dabei ist allerdings zu vermerken, dass die Teilnahme an Fortbildungsprogrammen in einem Drei-Monats-Zeitraum ein wesentlich schwächerer Indikator für berufsbegleitende Fortbildungeist als es der Hochschulabschluss für die Berufsausbildung ist.

Drei Aspekte der Schulpolitik und -praxis wirken sich im OECD-Durchschnitt in statistisch signifikanter Weise auf die Schülerleistungen in den drei Testbereichen aus: Die Einschätzungen der Schulleitungen in Bezug auf a) die lehrkräftebezogenen Faktoren für das Schulklima (vgl. Tabelle 7.4), b) die Stimmung und Arbeitshaltung der Lehrkräfte (vgl. Tabelle 7.5) sowie c) die Autonomie der Schule (vgl. Tabelle 7.11). Eine Erhöhung auf diesen Indizes um eine Einheit, d.h. um eine internationale Standardabweichung, entspricht einem Leistungsanstieg auf der Geamtskala Lesekompetenz um rund 6, 2 bzw. 5 Punkte. Hierbei ist anzumerken, dass diese Effekte relativ gering sind im Vergleich zu dem Leistungsanstieg um 18 Punkte, der sich aus einer Erhöhung um eine Einheit auf dem Index der Nutzung der Schulressourcen ergibt. Die Schulleiterangaben über die Lehrerautonomie sowie über die Nutzung formeller Schülerbeurteilungen (vgl. Anhang A1) haben keine statistisch signifikanten Auswirkungen, wenn alle OECD-Länder insgesamt betrachtet werden.
Schülerinnen und schüler erreichen bessere Leistungen in größseren Schulen mit bis zu 1000 Schülern, ...

... an denen auch mehr Lehrkräfte eine bessere und spezialisierte Erstausbildung absolviert haben. 
KAPITEL 8 Entscheidende Erkenntnisse aus den PISA-Resultaten - Hinweise für die Politikgestaltung

Gute Desgleichen weisen drei Aspekte der Unterrichtspraktiken statistisch signifikante

Unterrichtspraktiken,

darunter positive

Schüler-Lehrer-

Verhältnisse, und gute

Disziplin machen einen

deutlichen Unterschied.

Die obigen Faktoren

können zwar die

Unterschiede zwischen

den Schulen teilweise

erklären, ... erklärt sich jedoch durch den sozioökonomischen Hintergrund. positive Zusammenhänge mit den Schülerleistungen in den drei bei PISA getesteten Bereichen auf: die Einschätzungen der Schülerinnen und Schüler bezüglich a) des Schüler-Lehrer-Verhältnisses, b) der Schuldisziplin (vgl. Tabelle 7.3) und c) des Leistungsdrucks ${ }^{28}$. Das Schüler-Lehrer-Verhältnis und die Schuldisziplinweisen die höchsten Zusammenhänge auf: Einer Differenz im Umfang von einem Punkt auf den entsprechenden Indizes, die jeweils einer internationalen Standardabweichung entspricht, entspricht ein Leistungsunterschied auf der Gesamtskala Lesekompetenz in Höhe von rund 18 bzw. 10 Punkten. Der Leistungsdruck, der daran gemessen wurde, wie stark die Lehrkräfte nach Schülereinschätzung akademische Leistungen betonen und hohe Anforderungen an die Schüler stellen, wirkt sich nur schwach auf die Leistungen aus und die Zusammenhänge zwischen Leistungsdruck und Leistungen auf den Skalen für mathematische Grundbildung und Lesekompetenz sind im Durchschnitt aller OECD-Länder statistisch nicht signifikant.

Insgesamt erklären diese schulbezogenen Variablen zusammengenommen für alle drei Testbereiche 31\% der Leistungsunterschiede zwischen den Schulen innerhalb der jeweiligen Länder sowie 21\% der Leistungsunterschiede zwischen den verschiedenen Ländern (Tabelle 8.5). Für eine solch groß angelegte internationale Studie ist dies zwar ein respektables Ergebnis, doch bedeutet dies andererseits auch, dass ein Großteil der Varianz der Schülerleistungen durch die in PISA untersuchten schulischen Faktoren nicht erklärt wird.

Die vorangegangene Analyse legte den Schluss nahe, dass der familiäre Hintergrund und der durchschnittliche sozioökonomische Status einer Schule zu den Erklärungsfaktoren für die Leistungsunterschiede zwischen den Schülern gehören. Im Länderdurchschnitt erklärt der familiäre Hintergrund der einzelnen Schülerinnen und Schüler sowie der durchschnittliche sozioökonomischen Status der Schule rund 11\% bis 12\% der Leistungsunterschiede zwischen den Schülern derselben Schule in den drei Testbereichen. Auf der anderen Seite erklären sie 66\% der Leistungsdifferenzen zwischen den verschiedenen Schulen im Bereich Lesekompetenz und 62\% bzw. 63\% der Leistungsunterschiede zwischen den Schulen in den Bereichen mathematische und naturwissenschaftliche Grundbildung (vgl. zweites Spaltenset in Tabelle 8.5). Sie erklären auch ein Drittel der Leistungsdifferenzen zwischen den Ländern in den Bereichen Lesekompetenz und mathematische Grundbildung.

Kasten 8.2 liefert einen Anhaltspunkt für die relative Größenordnung der Effekte einiger der oben beschriebenen Hauptfaktoren, die sich auf die Schülerleistungen in PISA auswirken.

Es sind vor allem familiäre Hintergrund- und schulische Faktoren, die den Großteil der Leistungsunterschiede zwischen den Schulen erklären. Hinzu kommt, dass sich schulische Faktoren und sozioökonomische Hintergrundfaktoren natürlich gegenseitig beeinflussen. Leistungsunterschiede 
Kasten 8.2 Vergleich der Bedeutung verschiedener Faktoren

Der Effekt der verschiedenen Faktoren, die zu einer Verbesserung der Schülerleistungen beitragen können, lässt sich nur schwer vergleichen, da diese Faktoren jeweils unterschiedlich gemessen werden. Gleichwohl werden in dem hier verwendeten Modell einige dieser Faktoren in einem weitgehend dem Gleichwertigkeitsprinzip folgenden Ansatz miteinander verglichen. In diesem Kasten wird auf die Gesamtskala Lesekompetenz Bezug genommen, die Ergebnisse auf den Skalen für mathematische und naturwissenschaftliche Grundbildung sind allerdings sehr ähnlich.

Es ist möglich, die Effekte dreier wichtiger Variablen zu vergleichen, die in allen Ländern mit gleichen Methoden gemessen werden können: die Zahl der Schülerinnen und Schüler pro Mitglied des Lehrkörpers, die Zahl der Schülerinnen und Schüler pro Schule und der Prozentsatz der Lehrkräfte einer Schule mit Hochschulabschluss und Studienschwerpunkt im jeweiligen Unterrichtsfach. Mit Hilfe von Berechnungen kann vorhergesagt werden, dass ein um zehn Punkte höheres Ergebnis der Schülerinnen und Schüler einer Schule im Vergleich zu denen einer anderen in Zusammenhang steht mit durchschnittlich:

- 3,3 weniger Schülern pro Lehrer

- 207 mehr Schülern in der Schule

- 27\% mehr Lehrkräften mit Hochschulabschluss und Studienschwerpunkt in ihrem Unterrichtsfach.

Anhand dieser Ergebnisse lässt sich vergleichen, wie stark eine Veränderung jedes dieser Faktoren mit einem gegebenen Leistungsunterschied in Zusammenhang steht.

Im Rahmen von PISA wurde zudem eine Reihe von Faktoren untersucht, die sich weniger leicht messen lassen, insbesondere auf internationaler Ebene. Der hier verwendete Ansatz besteht darin, Indizes zu konstruieren, die sich nicht auf die inhärenten Merkmale des Gemessenen beziehen, sondern auf die Verteilung der einzelnen Merkmale in den Schulen der verschiedenen OECDLänder. Im Einzelnen wurde ein Mittelwert von 0 mit einer Standardabweichung von 1 festgelegt. Auf dieser Grundlage entspricht in den OECD-Ländern insgesamt eine Veränderung um eine Einheit (eine Standardabweichung) einem Anstieg der Schülerleistungen um:

- 18 Punkte bei einem häufigeren Gebrauch der Schulressourcen;

- 6 Punkte bei einem laut Schulleiterangaben besseren schulischen Klima bezüglich der lehrkräftebezogenen Faktoren;

- 5 Punkte bei einer laut Schulleiterangaben größeren Autonomie der Schule;

- 18 Punkte bei laut Schülerangaben besserem Schüler-Lehrer-Verhältnis;

- 10 Punkte bei laut Schülerangaben mehr Disziplin;

- 67 Punkte bei einem höheren durchschnittlichen wirtschaftlichen, sozialen und kulturellen Index der Schüler.

Die Tabellen 8.5 und 8.5a enthalten die entsprechenden Daten. 
KAPITEL 8 Entscheidende Erkenntnisse aus den PISA-Resultaten - Hinweise für die Politikgestaltung

Ein Teil des durch den sozioökonomischen Hintergrund bedingten Vorteils ist darauf zurückzuführen, dass privilegiertere Schülerinnen und schüler Schulen mit erfolgsversprechenden Charakteristiken besuchen, ...

... während umgekehrt Schulen mit diesen Merkmalen z.T. wegen ihrer besser gestellten Schülerschaft höhere Ergebnisse erzielen. zwischen den Schulen sind das Gesamtresultat der Differenzen zwischen den Schulen und den dort jeweils eingesetzten pädagogischen Prozessen und zwischen dem unterschiedlichen sozialen Hintergrund ihrer Schülerschaft. Schätzungsweise rund 70\% der beobachteten Leistungsunterschiede können durch die Kombination der beschriebenen schulischen Faktoren und familiären Hintergrundfaktoren erklärt werden (vgl. drittes Spaltenset in Tabelle 8.5), was für alle drei in PISA getesteten Bereiche zutrifft. Mit diesen Faktoren lässt sich auch ein Teil der zwischen den Ländern beobachteten Leistungsunterschiede erklären. Auf Länderebene beläuft sich der Anteil der Leistungsunterschiede in den Bereichen Lesekompetenz, mathematische und naturwissenschaftliche Grundbildung, der durch schulischen Faktoren und soziale Hintergrundfaktoren zusammengenommen erklärt werden kann, auf 43\%, 32\% bzw. 16\% $\%^{29}$.

Wie sich zeigt, handelt es sich beim kombinierten Effekt von schulspezifischen und Hintergrundfaktoren auf die Leistungsunterschiede zwischen den Schulen nicht einfach um die Summe des Einflusses der schulischen Faktoren und des Einflusses der familiären Hintergrundfaktoren. Zurückzuführen ist dies darauf, dass viele der Merkmale der Schulen eng mit den Merkmalen der Familien ihrer Schüler verknüpft sind. Das bedeutet, dass ein Teil der Effekte des familiären Hintergrunds auf die Schulleistungen durch die Merkmale der Schule vermittelt wird. Nehmen wir das Beispiel des im Bereich Lesekompetenz in PISA zu erwartenden Unterschieds zwischen den Ergebnissen zweier Schulen, deren Schülerinnen und Schüler einem unterschiedlichen Milieu entstammen, wobei sich die Differenz ihrer durchschnittlichen Bewertung nach dem Index des wirtschaftlichen, sozialen und kulturellen Status eine Einheit beträgt. Insgesamt ist zu erwarten, dass die Schülerinnen und Schüler der besser gestellten Schule im OECD-Durchschnitt 67 Punkte mehr erzielen. Ein Teil dieses Leistungsunterschieds ist darauf zurückzuführen, dass besser gestellte Schülerinnen und Schüler im Durchschnitt Schulen besuchen, deren Merkmale als leistungsfördernd angesehen werden können: Hierbei handelt es sich um den vermittelten Teil des Leistungsunterschieds, auf den ca. 10 Punkte der Ergebnisdifferenz von insgesamt 67 Punkten entfallen. Die übrigen Effekte des sozialen Hintergrunds der Schülerinnen und Schüler, die nicht mit den Merkmalen der Schule verknüpft sind, machen 57 Punkte aus. Die Differenz von 10 Punkten kann als ein $\mathrm{Maß}$ dafür angesehen werden, inwieweit die Schulsysteme im Durchschnitt dazu tendieren, den Vorteil der sozial bereits besser gestellten Schüler weiter zu verstärken.

Umgekehrt kann auch untersucht werden, inwieweit das Zusammenspiel von schulspezifischen Faktoren (z.B. eine bessere Mittelausstattung) und höherer Leistung auf den günstigeren Hintergrund der Schüler zurückzuführen sein kann, die Schulen mit vorteilhafteren Merkmalen besuchen. In den meisten Fällen schwächen sich die gesonderten Auswirkungen der schulbezogenen Faktoren nach Berücksichtigung des familiären Hintergrunds der Schülerinnen und Schüler $a b$, weil viele der Faktoren, die für die Qualität der Schule ausschlaggebend sind, mit deren wirtschaftlichem, sozialem und kulturellem 
Status korrelieren. Im OECD-Durchschnitt stehen beispielsweise die Hälfte der erfassten Unterschiede hinsichtlich der Ressourcenausstattung der Schulen sowie zwei Drittel der aus der Schulgröße und der Schüler/Lehrer-Quote resultierenden Effekte mit dem familiären Hintergrund in Zusammenhang. Bei den Variablen für Schulpolitik und -praxis ist dieser Zusammenhang sogar noch stärker. Der Großteil der Effekte des Schüler-Lehrer-Verhältnisses sowie der Schuldisziplin ist hingegen unabhängig vom familiären Hintergrund.

Hierbei muss betont werden, dass diese Ergebnisse nicht den Schluss nahe legen, schulspezifische Faktoren seien weniger wichtig, sondern nur, dass es nicht möglich ist, sie ganz von den Hintergrundfaktoren abzukoppeln. Dies kann an einem hypothetischen Extremfall verdeutlicht werden, in dem nur Schüler aus besser gestellten Familien Schulen mit besonders günstigen Merkmalen besuchen und diese Schulen besser abschneiden als andere. Ein Teil der besseren Leistungen könnte auch unabhängig von der Qualität der Schule erzielt werden als unmittelbares Ergebnis des familiären Hintergrunds der Schüler. Ein anderer Teil könnte jedoch durch die bessere Qualität der Schule bedingt sein. Je stärker sich diese zwei Arten von Vorteilen überschneiden, umso schwieriger ist es festzustellen, in welchem Ausmaß jeder von ihnen, für sich genommen, zur Leistungssteigerung beiträgt.

Aus der obigen Analyse ergeben sich zwei Folgerungen:

- Erstens stehen wichtige schulspezifische Faktoren, die die Leistungen der Schulen und der Länder insgesamt beeinflussen, in einer Wechselbeziehung mit dem sozioökonomischen Status der Schulen, wie er anhand des durchschnittlichen Index des wirtschaftlichen, sozialen und kulturellen Status der PISA-Populationen der jeweiligen Schulen geschätzt wird. Das bedeutet, dass ein Teil der bei der Analyse der sozioökonomischen Gradienten beobachteten Ungleichheit der Bildungserträge mit Chancenungleichheit in Zusammenhang steht.

- Zweitens befindet sich unter den in PISA untersuchten Faktoren keiner, mit dem allein erklärt werden könnte, warum manche Schulen oder manche Länder besser abschneiden als andere. Gute Leistungen scheinen vielmehr das Resultat des Zusammenspiels einer Reihe von Faktoren zu sein, zu denen die den Schulen zur Verfügung stehenden Mittel, die Schulpolitik und -praxis sowie die Unterrichtspraktiken gehören.

In der obigen Analyse standen die Einflüsse bestimmter Faktoren auf die Schülerleistungen insgesamt, d.h. in allen OECD-Ländern, im Mittelpunkt. Im folgenden Abschnitt soll nun untersucht werden, wie diese Faktoren auf die verschiedenen Länder verteilt sind. Steht einmal fest, welche Faktoren in allen Ländern von Bedeutung sind, ist es sinnvoll zu untersuchen, welche dieser Faktoren in den Bildungssystemen der einzelnen Länder eine hervorragende Rolle spielen. In diesem Zusammenhang ist es nicht nur notwendig zu untersuchen, ob und inwieweit die Schulen eines gegebenen Landes bestimmte
Soziale Nachteile werden durch ungleiche Bildungschancen verstärkt, ...

... jedoch in unterschiedlicher Weise

In den einzelnen

Ländern lässt sich das

vorhandensein der

beschriebenen

Erfolgsfaktoren aufeiner

Zehnpunkteskala

kategorisieren, ... 
KAPITEL 8 Entscheidende Erkenntnisse aus den PISA-Resultaten - Hinweise für die Politikgestaltung

\section{Kasten 8.3 Interpretation von Tabelle 8.6}

Für jede Schulvariable ist in der Tabelle ein Indexwert auf einer Skala von 1 bis 10 angegeben. Höhere Werte sind positiv, d.h. auf internationaler Ebene mit einer höheren Schülerleistung in den PISA-Tests verknüpft. Folgende Werte wurden zugeteilt:

\begin{tabular}{|c|c|c|}
\hline Variable & Wert & Indexwert \\
\hline Schüler/Lehrer-Quote & $\begin{array}{l}\text { unter } 25 \\
25 \text { bis } 27.5 \\
27.5 \text { bis } 30 \\
\ldots \\
\text { größer als } 47.5\end{array}$ & $\begin{array}{l}10^{30} \\
9 \\
8 \\
\cdots \\
0\end{array}$ \\
\hline Schulgröße & $\begin{array}{l}1000 \text { bis } 2500 \\
900 \text { bis } 999 \\
800 \text { bis } 899 \\
\ldots \\
\text { unter } 100\end{array}$ & $\begin{array}{l}10 \\
9 \\
8 \\
\cdots \\
10\end{array}$ \\
\hline $\begin{array}{l}\text { Prozentsatz der Lehrkräfte } \\
\text { mit Hochschulabschluss } \\
\text { und Studienschwerpunkt im } \\
\text { jeweiligen Unterrichtsfach }\end{array}$ & $\begin{array}{l}90 \text { bis } 100 \\
80 \text { bis } 90 \\
\ldots \\
0 \text { bis } 10\end{array}$ & $\begin{array}{l}10 \\
9 \\
\cdots \\
0\end{array}$ \\
\hline $\begin{array}{l}\text { Sonstige Variablen ( mit Skalen auf } \\
\text { Basis internationaler Standard- } \\
\text { abweichungen) }\end{array}$ & $\begin{array}{l}\text { Neu skaliert, so } \\
\text { Standardabweicl } \\
\text { davon mit null u }\end{array}$ & $\begin{array}{l}\text { ittelwert bei } 5 \text { und die } \\
\text { iegt und die Werte jenseits } \\
\text { setzt werden. }\end{array}$ \\
\hline
\end{tabular}

Der Indexwert der einzelnen Länder entspricht dem Durchschnittswert ihrer Schulen. Aus den Korrelationswerten für die einzelnen Länder in Tabelle 8.6 ist der Grad ersichtlich, in dem Unterschiede im jeweiligen Faktor innerhalb des Landes positiv mit Unterschieden hinsichtlich der Schülerschaft der Schulen verknüpft sind, z.B. wie häufig eine günstigere Schüler/Lehrer-Quote in Schulen anzutreffen ist, deren Schüler aus sozial besser gestellten Milieus stammen. Eine negative Korrelation bedeutet einen umgekehrten Zusammenhang. Ein Korrelationswert 0 würde bedeuten, dass Schüler mit unterschiedlichem Hintergrund alle die gleichen Chancen haben, Schulen mit günstigeren Rahmenbedingungen zu besuchen. Das Ausmaß dieses Effekts kann zur Beurteilung seiner relativen Bedeutung mit dem OECD-Durchschnitt verglichen werden.

positive Merkmale aufweisen, sondern auch, in welchem Umfang sich diese Merkmale auf Schulen konzentrieren, deren Schülerschaft einem privilegierteren Milieu entstammen. Tabelle 8.6 sind diesbezügliche Schätzwerte zu entnehmen. Für jede von zehn verschiedenen schulspezifischen Variablen sind darin die Werte der einzelnen Länder (auf einer Skala von 1 bis 10) sowie der Grad des Zusammenhangs der jeweiligen Variablen mit dem sozioökonomischen Profil der Schulen angegeben (vgl. Kasten 8.3). 
Nehmen wir das Beispiel der Vereinigten Staaten, die einen Indexwert von 10 haben, was die Auswirkung der Schüler/Lehrer-Quote auf die Leistungen im Bereich Lesekompetenz anbelangt. Dies deutet darauf hin, dass die durchschnittliche Schüler/Lehrer-Quote in den dortigen Schulen unter 25 liegt. Der Wert für die Schulgröße beträgt hier 3,6 woraus sich schließen lässt, dass es in den USA z.T. sehr kleine Schulen gibt. In Anbetracht der Ergebnisse für den gesamten OECD-Raum könnten diese Schulen weniger erfolgreich sein als andere Schulen mittlerer Größe. Auf dem Index der Lehrkräftequalifikationen erreichen die Vereinigten Staaten einen Wert von 7,5. Im Durchschnitt ist der Prozentsatz der Lehrkräfte mit einer einschlägigen Hochschulausbildung hier höher als im OECD-Durchschnitt. Bei den übrigen sieben Indikatoren (mit Ausnahme der Stimmung und Arbeitshaltung der Lehrkräfte) liegen die Vereinigten Staaten über dem OECD-Durchschnittswert von 5,0 .

$\mathrm{Zu}$ beachten ist, dass die vorstehende Analyse nicht die Effekte dieser Variablen auf die Leistungsunterschiede innerhalb der einzelnen Länder untersucht. Gleichwohl liefert Tabelle 8.5a die Ergebnisse für ein ähnliches Modell, das jeweils separat an die Daten jedes einzelnen Landes angepasst wurde, sowie eine statistische Zusammenfassung (Meta-Effekte) der Ergebnisse für den gesamten OECD-Raum. Die Zusammenfassung dieser länderspezifischen Analysen erbringt ähnliche Resultate wie die in Tabelle 8.5 dargestellten $^{31}$; sie zeigt allerdings auch, dass die Effekte zahlreicher Variablen zwischen den einzelnen Ländern abweichen und in vielen Fällen von den globalen Zusammenhängen differieren ${ }^{32}$. Griechenland, Italien und die Niederlande beispielsweise haben alle relativ niedrige Werte auf dem 10-Punkte-Index der Schuldisziplin, jedoch wirkt sich die Schuldisziplin in Italien offenbar erheblich auf die Schülerleistungen aus, während die Effekte in Griechenland und den Niederlanden eher unerheblich sind. Es ist jedoch möglich, dass in diesen Ländern noch andere Faktoren ins Spiel kommen, die dafür sorgen, dass Schulen mit relativ wenig Klassendisziplin dort relativ gut abschneiden und somit nicht dem allgemeinen Schema entsprechen. Diese Erkenntnisse sind ein deutlicher Hinweis darauf, dass es weiterer Analysen innerhalb jedes einzelnen Landes bedarf.

Neben den Indexwerten sind in Tabelle 8.6 die Korrelationen der verschiedenen Variablen auf Schulebene mit dem durchschnittlichen Index des wirtschaftlichen, sozialen und kulturellen Status angegeben. Eine positive Korrelation bedeutet, dass die Bedingungen in Bezug auf die hier untersuchten Politikvariablen in Schulen, deren Schülerinnen und Schüler einem privilegierteren Milieu entstammen, in der Regel günstiger sind als in solchen, die von Schülerinnen und Schülern aus weniger gut gestellten Milieus besucht werden (vgl. Kasten 8.3).

\author{
... wobei ein Wert von 10 \\ für günstige \\ Bedingungen und ein \\ Wert von O für \\ ungünstige \\ Bedingungen steht...
} ... jedoch ist zu
beachten, dass sich
hinter den
Durchschnittswerten
erhebliche Unterschiede
hinsichtlich der
Bedeutung der
verschiedenen Faktoren
verbergen. 
KAPITEL 8 Entscheidende Erkenntnisse aus den PISA-Resultaten - Hinweise für die Politikgestaltung

In Tabelle 8.6 sind folgende Ergebnisse auffällig:

Die Schüler/ - Für die Gesamtheit aller OECD-Länder zeigen die Ergebnisse, dass die Lehrer-Quoten liegen zumeist unterhalb der schwelle, ab der sie einen Unterschied machen.

In einigen Ländern ist eine Konzentration der höher qualifizierten Lehrkräfte in Schulen mit einer Schülerschaft aus privilegierten Milieus zu beobachten.

Sozial begünstigte schüler erhalten nicht

mehr Ressourcen, nutzen diese aber besser.

In einigen Fällen sind

Stimmung und Arbeitshaltung der Lehrkräfte an Schulen mit weniger privilegierten

schülerinnen und schülern weniger gut. Mehrzahl der Schulen Schüler/Lehrer-Quoten von unter 25 aufweist. Ausgehend von den PISA-Ergebnissen ist nicht zu erwarten, dass die Schülerleistungen bei einer weiteren Reduzierung dieser Quote nennenswert steigen würden. Andererseits liegt der Durchschnittswert auf der Zehnpunkteskala der Schulgröße bei 4,3 und damit weit unter dem Niveau, ab dem eine maximale Schülerleistung zu erwarten wäre. Die Ergebnisse deuten auch darauf hin, dass hinsichtlich der durchschnittlichen Schulgröße in allen Teilnehmerländern erhebliche Unterschiede bestehen. Differenzen in Bezug auf die Schüler/Lehrer-Quote stehen innerhalb der einzelnen Länder im Allgemeinen nicht in einem engen Zusammenhang mit dem sozioökonomischen Hintergrund der Schülerinnen und Schüler.

- Der Durchschnittswert auf der Zehnpunkteskala der Lehrkräftequalifikationen beträgt 7,1. Das bedeutet, dass rund zwei Drittel der Lehrkräfte einen Hochschulabschluss in ihrem Unterrichtsfach besitzen. In Belgien, Deutschland und Luxemburg ist die Korrelation zwischen dem Prozentsatz der Lehrkräfte mit Hochschulabschluss im jeweiligen Unterrichtsfach und dem durchschnittlichen sozioökonomischen Status der Schulen am größten (zwischen 0,47 und 0,58), woraus sich schließen lässt, dass auf ihr Fach spezialisierte Lehrkräfte in der Regel eher in Schulen oder Schultypen mit günstigem sozioökonomischen Hintergrund tätig sind.

- Der Umfang der den Schulen zur Verfügung gestellten Mittel steht in den meisten Fällen in keinem engen Zusammenhang mit dem Hintergrund ihrer Schülerschaft. Umgekehrt ist das Ergebnis jedoch, wenn der Umfang der Nutzung dieser Ressourcen durch die Schüler untersucht wird. Zwischen dem PISA-Index der Nutzung der Schulressourcen durch die Schülerinnen und Schüler und dem wirtschaftlichen, sozialen und kulturellen Status der Schülerinnen und Schüler besteht eine engere Verbindung als mit jeder anderen schulspezifischen Variablen. Daraus lässt sich schließen, dass Schülerinnen und Schüler aus weniger privilegierten Milieus gewöhnlich weniger regelmäßig von den Ressourcen ihrer Schule Gebrauch machen als Schülerinnen und Schüler mit einem höheren wirtschaftlichen, sozialen und kulturellen Status.

- In einigen Ländern besteht ein positiver Zusammenhang zwischen den lehrkräftebezogenen Faktoren für das Schulklima nach Angaben der Schulleitungen und dem sozioökonomischen Status der Schulen, vor allem in Korea, Spanien und dem Vereinigten Königreich (Korrelationswerte zwischen 0,41 und 0,58). In einigen wenigen Ländern besteht ein positiver Zusammenhang zwischen der Stimmung und Arbeitshaltung der Lehrkräfte und dem sozioökonomischen Status der Schulen. Hier sticht insbesondere Polen hervor, wo Stimmung und Arbeitshaltung der Lehrkräfte von den 
Schulleitungen generell als niedrig eingestuft werden $(3,8)$, dies aber ganz besonders in Schulen mit niedrigem sozioökonomischem Status der Fall ist (Korrelationswert 0,51).

- In Schulen mit in soziö̈konomischer Hinsicht besser gestellter Schülerschaft, ist die Schuldisziplin aus Schülersicht in der Regel besser. Dies gilt insbesondere für Italien, Japan, Spanien, das Vereinigte Königreich und die Vereinigten Staaten. Unter diesen Ländern sticht Italien deutlich als Land hervor, in dem die Schülerinnen und Schüler die Schuldisziplin generell als gering einstufen, ganz besonders aber in Schulen mit ungünstigem sozioökonomischem Hintergrund. Es gibt aber auch einige Gegenbeispiele in Form von Ländern, in denen die Schuldisziplin von den Schülerinnen und Schülern an weniger gut gestellten Schulen als besser angesehen wird: Dies ist der Fall für Mexiko und Neuseeland, wo die Korrelationswerte bei - 0,33 und $-0,36$ liegen.

\section{Schlussbetrachtungen}

Obwohl PISA zeigt, dass schlechte schulische Leistungen keine automatische Folge eines ungünstigen sozioökonomischen Hintergrunds sind, scheinen die sozialen Hintergrundfaktoren doch zu denjenigen zu gehören, die sich am stärksten auf die Leistungen der Schülerinnen und Schüler auf den PISA-Skalen Lesekompetenz sowie mathematische und naturwissenschaftliche Grundbildung auswirken.

Dies bedeutet eine große Herausforderung für die staatliche Politik, die allen Schülerinnen und Schülern unabhängig von ihrem familiären Hintergrund Lernmöglichkeiten bieten will. Die Ergebnisse von Untersuchungen in einzelnen Ländern waren diesbezüglich im Allgemeinen entmutigend. Der Einfluss der Schulen galt oft als kaum nennenswert. Sei es, weil Familien aus privilegierten Milieus eher in der Lage sind, den Effekt der Schulen zu verstärken und zu verbessern, oder weil es den Schulen besser gelingt, junge Menschen zu erziehen und zu fördern, die einem privilegierten Milieu entstammen. Häufig hat sich gezeigt, dass die Schulen die bereits existierenden sozialen Muster reproduzieren, anstatt allen gleiche Möglichkeiten zu bieten und auf diese Weise eine ausgewogenere Verteilung der Bildungserträge zu gewährleisten.

Die internationalen Ergebnisse von PISA sind ermutigender. Zwar besteht in allen Ländern ein klarer Zusammenhang zwischen dem familiären Hintergrund der Schülerinnen und Schüler und den Bildungserträgen, doch zeigen einige Länder, dass eine hohe durchschnittliche Bildungsqualität mit einer ausgewogenen Verteilung der Bildungserträge einhergehen kann: In Kanada, Finnland, Island, Japan, Korea und Schweden liegt das Leistungsniveau der Schülerinnen und Schüler auf der Gesamtskala Lesekompetenz über dem Durchschnitt, während die Effekte des wirtschaftlichen, sozialen und kulturellen Status auf die Schülerleistungen zugleich unterdurchschnittlich stark ausgeprägt
Zwischen Schuldisziplin und sozioökonomischem Hintergrund besteht ein enger Zusammenhang.

PISA zeigt den großen Einfluss des familiären Hintergrunds, ...

... doch während die Möglichkeiten der schulen, für einen Ausgleich zu sorgen, begrenzt sind, ...

... gelingt es einigen

Ländern, ausgewogenere Bildungserträge mit hoher Qualität zu kombinieren. 
Die Herausforderung wird größer angesichts der kombinierten

Wirkung des sozioökonomischen

Hintergrunds der Schülerschaft einer schule...

... und angesichts der

Tatsache, dass

privilegiertere

schülerinnen und schüler eher Schulen mit weiteren Vorteilen

besuchen,...

... was weitere Chancenungleichheit nach sich zieht. sind. Umgekehrt liegen die durchschnittlichen Ergebnisse der Schüler auf der Gesamtskala Lesekompetenz in der Tschechischen Republik, Deutschland, Ungarn und Luxemburg deutlich unter dem OECD-Durchschnitt, während die Leistungsunterschiede zwischen in sozioökonomischer Hinsicht privilegierten und benachteiligten Schülern dort zugleich überdurchschnittlich groß sind.

Eines der wichtigsten Ergebnisse von PISA ist, dass der familiäre Hintergrund des einzelnen Schülers nur ein Teil des Gesamtkomplexes der sozioökonomisch bedingten Ungleichheiten im Bildungsbereich ist - in den meisten Ländern sogar nur der kleinere Teil. Die kombinierte Wirkung des sozioökonomischen Hintergrunds der Gesamtheit der Schülerschaft einer Schule kann sich merklich in der Leistung des einzelnen Schülers niederschlagen und hat generell größeren Einfluss auf die zu erwartenden Ergebnisse des Schülers als dessen eigener familiärer Hintergrund.

Ein zweites besonders wichtiges Ergebnis der Analyse der PISA-Resultate lautet, dass leistungsfördernde schulische Effekte durch den soziö̈konomischen Hintergrund verstärkt werden. In Schulen, die über mehr Mittel verfügen und deren pädagogische Maßnahmen und Praxis zu besseren Leistungen führen, scheinen die Schüler im Allgemeinen - wenn auch nicht immer in gleichem Umfang - einem besser gestellten Milieu zu entstammen. In Belgien, Deutschland und Luxemburg sind auf ihre Fächer spezialisierte Lehrkräfte häufiger an Schulen tätig, deren sozioökonomischer Hintergrund eher günstig ist. In Schulen, in denen die Schülerinnen und Schüler einem in sozioökonomischer Hinsicht besser gestellten Milieu entstammen, ist die Schuldisziplin aus Schülersicht offenbar ebenfalls besser, insbesondere in Italien, Japan, Spanien, dem Vereinigten Königreich und den Vereinigten Staaten. Schließlich ist noch festzustellen, dass die Schülerinnen und Schüler in Schulen mit niedrigem sozioökonomischen Status in der Regel weniger regelmäßig von den Ressourcen ihrer Schulen Gebrauch machen als Schülerinnen und Schüler besser gestellter Schulen.

Im Endergebnis führen diese Effekte dazu, dass in Ländern, in denen ein hoher Grad an schulischer Segregation nach sozioökonomischen Merkmalen besteht, Schülerinnen und Schüler aus sozial benachteiligten Milieus schlechtere Leistungen erzielen. Dies bedeutet wiederum, dass ein Teil der Ungleichheit in Bezug auf die Bildungserträge, die bei der Analyse der sozioökonomischen Gradienten zu beobachten war, auf Chancenungleichheit zurückzuführen ist. Unter solchen Umständen bleiben Begabungen ungenutzt und werden menschliche Ressourcen vergeudet.

In einigen Ländern besteht eine starke Segregation der Schülerinnen und Schüler nach sozioökonomischen Merkmalen, die z.T. auf Unterschiede in den Wohngebieten und auf wirtschaftliche Faktoren zurückzuführen ist, aber auch auf Charakteristiken des Schulsystems. In solchen Ländern könnte die Bildungspolitik versuchen, die Auswirkungen des sozioökonomischen 
Hintergrunds auf die Schülerleistungen abzuschwächen, und zwar entweder durch eine Verringerung der sozioökonomischen Segregation oder durch eine differenzierte Mittelallokation für die verschiedenen Schulen. In diesen Ländern ist es u.U. erforderlich, die Zusammenhänge zwischen der Mittelverteilung auf die verschiedenen Schulen des Landes und dem sozioökonomischen Hintergrund der Schülerinnen und Schüler dieser Schulen zu untersuchen. In anderen Ländern gibt es relativ wenig sozioökonomische Segregation, d.h. dass der durchschnittliche Hintergrund der Schülerinnen und Schüler der verschiedenen Schulen in etwa ähnlich ist. In solchen Ländern könnte die Bildungspolitik danach streben, die Auswirkungen des sozioökonomischen Hintergrunds durch Maßnahmen zu mindern, die auf eine Verbesserung der schulischen Ressourcen und eineVerringerung der Segregation der Schülerinnen und Schüler innerhalb der Schulen entsprechend ihrem wirtschaftlichen, sozialen und kulturellen Status abzielen. Am wichtigsten dabei ist natürlich letzten Endes, dass die Ressourcen wirkungsvoll eingesetzt werden. Dazu kann es u.U. erforderlich sein, die Einteilung in Klassen mit unterschiedlichen Leistungsniveaus aufzuheben oder leistungsschwachen Schülern mehr Unterstützung zukommen zu lassen.

In Ländern, in denen sich der sozioökonomische Hintergrund nur in bescheidenem Umfang auf die Schülerleistungen auswirkt, können nicht alle Erfolge allein dem Bildungssystem zugeschrieben werden, ebenso wie in Ländern mit steil verlaufenden Gradienten nicht alle Probleme den Schulen angekreidet werden dürfen. Die Analyse hat gezeigt, dass die Herausforderungen, vor denen die Bildungssysteme auf Grund der unterschiedlichen Verteilung der familiären Hintergrundfaktoren innerhalb ihrer Schülerpopulation stehen, ganz unterschiedlich sind. In Brasilien, Mexiko, Polen, Portugal und den Vereinigten Staaten ist es z.B. wegen der ungleichen Vermögenssituation der Familien, wie sie in PISA gemessen wurde (vgl. Kapitel 6), wesentlich schwieriger, allen Schülern gleiche Bildungschancen zu bieten, als in Finnland, Japan oder Korea, wo der Wohlstand gleichmäßiger verteilt ist. Viele Faktoren einer sozioökonomischen Benachteiligung lassen sich auch nicht unmittelbar durch die Bildungspolitik beeinflussen, zumindest nicht auf kurze Sicht. Das Bildungsniveau der Eltern kann z.B. nur ganz allmählich angehoben werden. Desgleichen ist die familiäre Vermögenssituation von der langfristigen wirtschaftlichen Entwicklung eines Landes abhängig.

Die PISA-Ergebnisse lassen jedoch vermuten, dass die Schulpolitik und die Schulen selbst eine entscheidende Rolle bei der Minderung der Effekte sozioökonomischer Benachteiligung auf die Schülerleistungen spielen können. Die Ergebnisse lassen eine Reihe von an die schulischen Ressourcen geknüpften Faktoren, von schulpolitischen Maßnahmen und Unterrichtspraktiken erkennen, mit denen die Schülerleistungen offenbar beeinflusst werden können. Wie intensiv die Schülerinnen und Schüler von den schulischen Ressourcen Gebrauch machen und wie viele auf ihr Fach spezialisierte Lehrkräfte zurVerfügung stehen, sind beides Faktoren, die sich auf die Schülerleistungen auswirken können. Nach
Bildungspolitische Masnahmen hängen z. T. davon ab, ob und wie Schulen nach sozioökonomischem Hintergrund segregiert sind ...

... sowie von den sozioökonomischen Gesamtprofilen der einzelnen Länder.

PISA identifiziert einige schulinterne Faktoren, die einen Unterschied machen können. 
KAPITEL 8 Entscheidende Erkenntnisse aus den PISA-Resultaten - Hinweise für die Politikgestaltung

Kein einzelner Faktor ist allein entscheidend. Analysen werden darauf ausgerichtet sein, die

Ursachen besser zu verstehen. den Einschätzungen der Schulleiter scheinen auch lehrkräftebezogene Faktoren für das Schulklima, die Stimmung und die Arbeitshaltung der Lehrkräfte sowie die Autonomie der Schule Einfluss zu haben. Schließlich gibt es auch einige Aspekte der Unterrichtspraktiken, z.B. das Schüler-Lehrer-Verhältnis und die Schuldisziplin, die sich positiv auf die Schülerleistungen auswirken.

Aus den PISA-Resultaten geht hervor, dass kein einziger Faktor allein erklären kann, warum manche Schulen oder Länder besser abschneiden als andere. Hohe Leistungen sind vielmehr dem Zusammenspiel einer Reihe von Faktoren zu verdanken, darunter die Ressourcenausstattung der Schulen, die Schulpolitik und -praxis und die Unterrichtspraktiken. Weitere umfangreiche Forschungsarbeiten und Analysen sind erforderlich, um zu verdeutlichen, wie sich diese Faktoren auswirken, in welcher Wechselbeziehung sie mit dem familiären Hintergrund der Schülerinnen und Schüler stehen und wie sie deren schulische Leistungen beeinflussen.

Im Interesse eines solchen besseren Verständnisses ist im Rahmen von PISA für 2002 und 2003 die Veröffentlichung einer Reihe thematischer Berichte geplant, in denen die Auswirkungen schul- und bildungssystembezogener Faktoren auf die Schülerleistungen ausführlicher untersucht werden. Dabei soll auch genauer herausgefunden werden, warum einige Länder bessere und zugleich ausgewogener verteilte Bildungserträge erzielen als andere. Nach dem derzeitigen Stand ist schon allein die Tatsache ermutigend, dass in einigen Ländern gute Bildungsergebnisse für die meisten Schülerinnen und Schüler bereits eine Realität sind; denn sie zeigt, dass die bevorstehenden Herausforderungen durchaus bewältigt werden können. 


\section{Anmerkungen}

1. Zur Definition dieses Index vgl. Anhang A1.

2. Gemeint sind sämtliche an PISA teilnehmenden OECD-Länder mit Ausnahme der Niederlande (vgl. Anhang A3).

3. Es besteht ein statistisch signifikanter kurvilinearer Zusammenhang, der jedoch vergleichsweise gering und in Abbildung 8.1 kaum erkennbar ist.

4. Statistisch gesehen ist der Anteil der Varianz, der sich anhand des PISA-Index des wirtschaftlichen, sozialen und kulturellen Status für den OECD-Raum insgesamt erklären lässt (20\%), wesentlich geringer als bei einem vollkommenen Zusammenhang (100\%).

5. Die Gradiente des gesamten OECD-Raums aus Abbildung 8.1 deckt sich weitgehend mit der Durchschnittsgradienten der einzelnen Länder.

6. Zur Behandlung der fehlenden Daten vgl. Anhang A1. Der Prozentsatz der Fälle, in denen Daten für den Index des wirtschaftlichen, sozialen und kulturellen Status fehlen, ist Tabelle 8.1 zu entnehmen.

7. Der Hauptgrund dafür, dass dieser Wert über 500 Punkten liegt - d.h. dem OECD-Durchschnittsergebnis auf der Gesamtskala Lesekompetenz -, besteht darin, dass die Ergebnisse der größeren Länder, die sich stärker im gewichteten Mittel niederschlagen, über dem OECD-Durchschnitt liegen. Ein weiterer Grund für die Abweichung ist im Fehlen sozioökonomischer Daten zu sehen.

8. In diese Analyse wurde auch das Quadrat des Index des wirtschaftlichen, sozialen und kulturellen Status (X) einbezogen, um auch nichtlineare Effekte in diesem Zusammenhang zu berücksichtigen. Der Koeffizient ist allerdings relativ gering: -1,21. Die sozioökonomischen Gradienten für die mathematische und naturwissenschaftliche Grundbildung weichen geringfügig von der Gradienten der Lesekompetenz ab:Y $=506,42+40,58 \mathrm{X}-4,14$ X2 für die mathematische Grundbildung und Y $=507,66+38,50 \mathrm{X}-1,37 \mathrm{X} 2$ für die naturwissenschaftliche Grundbildung.

9. Dies ist der Fall für Gradienten, die den Zusammenhang zwischen dem Einkommen und zahlreichen Variablen des Gesundheitszustands der Bevölkerung darstellen, z.B. der Lebenserwartung (House et al., 1990; Mirowsky und Hu, 1996; Wolfson, et al., 1993): Wenn die Grundbedürfnisse gestillt sind, wirken sich weitere Einkommenszuwächse nur noch geringfügig auf den Gesundheitszustand der Bevölkerung aus.

10. Statistisch ausgedrückt beträgt die Korrelation zwischen der Höhe und der Steigung bei einem durchschnittlichen wirtschaftlichen, sozialen und kulturellen Status - 0,38.

11. Für Japan fehlten über 50\% der Angaben zum beruflichen Status und Bildungsstand der Eltern. Wenn für Schülerinnen und Schüler keine diesbezüglichen Angaben verfügbar waren, wurde der Index des wirtschaftlichen, sozialen und kulturellen Status stattdessen aus den Daten des Index des relativen Wohlstands der Familien, des Index des Besitzes klassischer Kulturgüter im Elternhaus und des Index der Bildungsressourcen im Elternhaus abgeleitet. Die Steigung der Gradienten ist für Schülerinnen und Schüler, die nach diesen Ersatzindizes eingestuft wurden, nahezu identisch mit der Steigung der Gradienten der Schülerinnen und Schüler, bei denen alle fünf Variablen berücksichtigt werden konnten, was darauf hindeutet, dass die Schätzungen durch diese Berechnungsmethode nicht zusätzlich verzerrt wurden.

12. In diesen Ländern liegen die Schülerleistungen statistisch signifikant über dem OECD-Durchschnitt, und die sozioökonomische Gradiente verläuft statistisch signifikant flacher als die OECD-Durchschnittsgradiente. 
KAPITEL 8 Entscheidende Erkenntnisse aus den PISA-Resultaten - Hinweise für die Politikgestaltung

13. In diesen Ländern liegen die Schülerleistungen statistisch signifikant über dem OECD-Durchschnitt, während die sozioökonomische Gradiente statistisch signifikant steiler verläuft als die OECD-Durchschnittsgradiente.

14. Diese Analysen gründen sich auf eine gewöhnliche Regression nach der Methode der kleinsten Quadrate der Ergebnisse auf der Gesamtskala Lesekompetenz nach a) dem internationalen sozioökonomischen Index der beruflichen Stellung (Tabelle 6.1) sowie dessen Quadrat, b) dem Bildungstand der Eltern gemessen in Jahren (vgl. Anhang A1), c) dem Index der Bildungsressourcen im Elternhaus, d) dem Index des Besitzes klassischer Kulturgüter im Elternhaus (Tabelle 6.3), e) einer Dummy-Variablen für das Geschlecht des Schülers, f) zwei Dummy-Variablen dafür, ob der Schüler in einem Ein-Eltern- oder einem Zwei-Eltern-Haushalt oder in anderen Familientypen aufwächst (vgl. Tabelle 6.9), g) der Zahl der Geschwister (Daten abrufbar unter www.pisa.oecd.org), h) einer Dummy-Variablen dafür, ob der Schüler bzw. die Schülerin im In- oder im Ausland geboren ist (Tabelle 6.10) sowie i) drei Dummy-Variablen für fehlende Daten für den Internationalen sozioökonomischen Index der beruflichen Stellung, die Bildungsjahre der Eltern und die Zahl der Geschwister. In vorläufigen Analysen wurde auch der Index des relativen Wohlstandes der Familien berücksichtigt (Tabelle 6.2), doch in den meisten Ländern erwies sich diese Variable als kolinear mit den anderen im Index des wirtschaftlichen, sozialen und kulturellen Status inbegriffenen Faktoren, weshalb auf sie in der Folge verzichtet wurde. Die Resultate, die sich auf Unterschiede zwischen männlichen und weiblichen Schülern beziehen, entsprachen jenen aus Kapitel 6 und wurden daher in Tabelle 8.2 nicht nochmals aufgeführt. Die Koeffizienten für die anderen Familientypen waren in den meisten Fällen statistisch signifikant, da sie jedoch nur eine vergleichsweise kleine Gruppe betreffen, wurden sie ebenfalls nicht in Tabelle 8.2 aufgenommen.

15. Alle OECD-Länder, die an PISA teilgenommen haben, mit Ausnahme der Niederlande.

16. In einigen Ländern ist es auf Grund des geringen Anteils der ausländischen Schülerinnen und Schüler in Anbetracht der Größe der PISA-Stichproben sehr schwierig, statistisch signifikante Unterschiede festzustellen.

17. Eine Standardabweichung auf dem sozioökonomischen PISA-Index der beruflichen Stellung entspricht im OECDDurchschnitt 16,3 Punkten.

18. Hier ist darauf hinzuweisen, dass dieser Wert von den Angaben in Tabelle 6.1a abweicht. Dies ist dadurch bedingt, dass in Tabelle 8.2 nur die Effekte des Index des wirtschaftlichen, sozialen und kulturellen Status dargestellt sind, wobei für die anderen Faktoren von einer identischen Verteilung in allen OECD-Ländern ausgegangen wurde. Tabelle 6.1a verdeutlicht hingegen die Auswirkungen des Index des wirtschaftlichen, sozialen und kulturellen Status ohne Berücksichtigung der Unterschiede in Bezug auf andere Faktoren.

19. Weitere Länder, in denen diese Auswirkungen nicht als statistisch signifikant angesehen werden können, sind Ungarn, Polen, das Vereinigte Königreich und die Vereinigten Staaten.

20. Vgl. Brookover et al., 1978; Henderson et al., 1978; Rumberger et al., 1992; Shavit et al., 1985; Summers et al., 1977; Willms, 1986.

21. Das Maß 0,5 einer Standabweichung auf Schülerebene wurde für diesen Vergleich gewählt, weil dieser Wert einen realistischen Unterschied zwischen Schulen im Hinblick auf ihre sozioökonomische Zusammensetzung beschreibt. Im OECD-Durchschnitt beträgt der Unterschied zwischen dem 75. und dem 25. Perzentil des durchschnittlichen Indexwerts des wirtschaftlichen, sozialen und kulturellen Status der Schulen 0,72 einer Standardabweichung auf Schülerebene; in allen außer einem OECD-Land ist dieser Unterschied auf dem sozioökonomischen Index größer als eine halbe Standardabweichung auf Schülerebene. 
22. Die typische Bandbreite ist durch den Unterschied zwischen dem 75. und dem 25. Perzentil der Mittelwerte der Schulen nach dem PISA-Index des wirtschaftlichen, sozialen und kulturellen Status dargestellt.

23. Folgende Effekte können mit einem Anstieg des Mittelwerts der Schulen auf dem Index des wirtschaftlichen, sozialen und kulturellen Status um eine halbe Standardabweichung auf Schülerebene in Zusammenhang gebracht werden: Im Bereich der Lesekompetenz 34,1 für Jungen und 31,8 für Mädchen, im Bereich der mathematischen Grundbildung 34,8 für Jungen und 30,5 für Mädchen, im Bereich der naturwissenschaftlichen Grundbildung 33,7 für Jungen und 30,7 für Mädchen.

24. Gemeint sind hier alle Qualifikationen der Stufe 5A nach der Internationalen Standardklassifizierung des Bildungswesens (ISCED).

25. Um der Möglichkeit eines nicht linearen Zusammenhangs zwischen der Schüler/Lehrer-Quote und den Leistungen Rechnung zu tragen, wurden in diesem Modell lineare und quadratische Glieder verwendet. Zudem waren die Schüler/ Lehrer-Quoten in einigen Schulen sehr hoch, was sich unverhältnismäßig stark auf die Zusammenhänge auswirkt. Um den Effekt der Schüler/Lehrer-Quoten in diesen Schulen zu erfassen, wurde in das Modell ein gesondertes Glied aufgenommen.

26. In einigen Ländern gibt es Schulen, in denen die Schüler/Lehrer-Quote bei über 50 liegt. Diese Schulen wurden in der Analyse gesondert behandelt, indem sie durch eine eigene Dummy-Variable modelliert wurden. Die Resultate zeigen, dass die Durchschnittsergebnisse auf der Gesamtskala Lesekompetenz in diesen Schulen ebenfalls deutlich unter dem OECDDurchschnitt lagen.

27. Es muss berücksichtigt werden, dass es in vielen Ländern nur wenig Unterschiede im Hinblick auf die Qualifikation der Lehrkräfte gibt, weshalb dort nur mit einem sehr schwachen Zusammenhang zu rechnen ist.

28. Die Variablen für Leistungsdruck und die Schüler-Lehrer-Verhältnisse stehen mit den Schülerleistungen in einem nicht linearen Zusammenhang. Um dieser Situation Rechnung zu tragen, beziehen sich die in dieser Analyse verwendeten Messgrößen für Leistungsdruck auf Extremfälle, in denen die Schülerinnen und Schüler Fragen wie „unser Lehrer/unsere Lehrerin sagt, dass wir eigentlich besser sein könnten“ mit „nie“ beantworten. Desgleichen bezieht sich die in diesem Kapitel verwendete Messgröße für die Schüler-Lehrer-Verhältnisse auf den Anteil der Antworten, bei dem die Schülerinnen und Schüler bei Aussagen, die auf ein positives Schüler-Lehrer-Verhältnis abzielten, „stimme überhaupt nicht zu“ angaben.

29. Die zwischen den Ländern auf der Skala für naturwissenschaftliche Grundbildung bestehenden Leistungsunterschiede, die sich aus der kombinierten Wirkung schulspezifischer und sozioökonomischer Faktoren erklären, sind geringer als diejenigen, die allein schulspezifischen Faktoren zuzuschreiben sind. Bei den hier verwendeten Modellen wurden zwischen den Ländern bestehende Unterschiede hinsichtlich der Effekte des durchschnittlichen Indexwerts des wirtschaftlichen, sozialen und kulturellen Status der Schulen berücksichtigt. Die Komponenten der Variationsbreite wurden errechnet für einen Schüler mit durchschnittlichen Werten auf dem Index des wirtschaftlichen, sozialen und kulturellen Status, der eine Schule besucht, deren mittlerer Index des wirtschaftlichen, sozialen und kulturellen Status bei 0 liegt (was dem Durchschnitt aller Schüler des OECD-Raums entspricht). Die Abnahme der Variationsbreite nach Einrechnung des mittleren Indexwerts des wirtschaftlichen, sozialen und kulturellen Status legt den Schluss nahe, dass zwischen den schulischen Ressourcen und dem durchschnittlichen wirtschaftlichen, sozialen und kulturellen Status der Schulen signifikante Wechselbeziehungen bestehen.

30. Auf internationaler Ebene werden die Effekte der Schüler/Lehrer-Quote ab Werten über 25 bedeutungslos, weshalb diese Quote als oberste Kategorie des Index gewählt wurde. 
KAPITEL 8 Entscheidende Erkenntnisse aus den PISA-Resultaten - Hinweise für die Politikgestaltung

31. Die Analyse ergab ein zweistufiges hierarchisches Modell für jedes Land. Das Modell stimmt mit dem für Tabelle 8.5 verwendeten dreistufigen Modell überein, mit Ausnahme der Tatsache, dass die zwei Messgrößen für die Schüler/LehrerQuote durch separate Dummy-Variablen ersetzt wurden, die für die Effekte von Schüler/Lehrer-Quoten von 20-25, 25-30 und über 30 stehen, verglichen mit Schüler/Lehrer-Quoten unter 20. Dies ermöglichte einen direkteren Vergleich des nicht linearen Effekts innerhalb der einzelnen Länder. Nicht in dem Modell enthalten war zudem die Schulgröße, da die Effekte zwischen den Ländern erheblich voneinander abwichen und in den meisten Fällen statistisch nicht signifikant waren. Die Ergebnisse der Meta-Analyse ergaben Erkenntnisse, die mit den aus dem Dreistufenmodell abgeleiteten Resultaten vergleichbar waren, mit Ausnahme der Tatsache, dass die Effekte des Leistungsdrucks geringer und statistisch nicht signifikant waren.

32. Zwischen den Koeffizienten für die landesspezifischen Analysen bestehen erhebliche Unterschiede, wie in der Größenordnung ihrer Standardfehler zum Ausdruck kommt. Die Koeffizienten können von Land zu Land variieren, was zum einen auf die „echte“ Varianz ihrer Effekte in jedem Land, zum anderen aber auch auf Mess- und Stichprobenfehler zurückzuführen ist. In diesen Analysen spielen Stichprobenfehler eine signifikante Rolle, da die Genauigkeit der Koeffizienten für Variablen auf Schulebene vorwiegend von der Zahl der in jedem Land beteiligten Schulen abhängt und weniger von der Schülerzahl. Die Zahl der in dieser Analyse berücksichtigten Schulen reichte von 24 Schulen in Luxemburg bis zu 1111 Schulen in Kanada, mit einem Durchschnitt von rd. 214 Schulen in den OECD-Ländern. Dieses Problem wirkt sich insbesondere auf die Koeffizienten für die Schüler/Lehrer-Quoten aus, da diese in einigen Fällen auf den Ergebnissen einer sehr kleinen Anzahl von Schulen basieren. 
Audas, R. und J.D. Willms (2000), Engagement and dropping out of school: A life-course perspective. Für Human Resources Development, Kanada, erstellter Bericht.

Baumert, J., S. Heyn und O. Köller (1994), Das Kieler Lernstrategien-Inventar (KSI). Kiel: Institut für die Pädagogik der Naturwissenschaften an der Universität Kiel.

Baumert, J., S. Gruehn, S. Heyn, O.Köller und K.U. Schnabel (1997), Bildungsverläufe und Psychosoziale Entwicklung im Jugendalter (BIJU): Dokumentation - Band 1. Berlin: Max-Planck-Institut für Bildungsforschung.

Beaton, A.E., I.V. Mullis, M.O. Martin, E.J. Gonzalez, D.L. Kelly und T.A. Smith (1996), Mathematics Achievement in the Middle School Years. Center for the Study of Testing, Evaluation and Educational Policy, Boston College.

Brookover,W.B., J.H. Schweitzer, J.M. Schneider, C.H. Beady, P.K. Flood und J.M.Wisenbaker (1978), Elementary school social climate and school achievement. American Educational Research Journal, 15, 301-318.

Deutsches PISA-Konsortium (Hrsg.) (2000), Schülerleistungen im internationalen Vergleich - Eine neue Rahmenkonzeption für die Erfassung vonWissen und Fähigkeiten. Berlin: Max-Planck-Institut für Bildungsforschung.

Eignor, D., C. Taylor, I. Kirsch und J. Jamieson (1998), Development of a scale for assessing the level of computer familiarity of TOEFL students. (TOEFL Research Report No. 60). Princeton, NJ: Educational Testing Service.

Ganzeboom, H.B.G., P. De Graaf und D.J.Treiman (mit J. De Leeuw) (1992), A Standard International Socio-Economic Index of Occupational Status. Social Science Research, 21(1), 1-56.

Ganzeboom, H.B.G., D.J. Treiman und J. Donald (1996), Internationally Comparable Measures of Occupational Status for the 1988 International Standard Classification of Occupations. Social Science Research 25, 201-239.

Henderson, V., P. Mieszkowski und Y. Sauvageau (1978), Peer group effects and educational production functions. Journal of Public Economics, 10, 97-106.

House, J., R. Kessler, R. Herzog, R.P. Mero, A.M. Kinney und M.J. Breslow (1990), Age, socioeconomic status, and health. The Millbank Quarterly, 68, 383-411.

INSEE-DPD. (1999), Etude de la fiabilité des déclarations des élèves de 15 ans dans le cadre de l'opération PISA 2000, internes Dokument, DPD-INSEE.

Marsh, H.W., R.J. Shavelson und B.M. Byrne (1992), A multidimensional, hierarchical self-concept. In R.P. Lipka und T.M. Brinthaupt (Hrsg.), Studying the self: Self-perspectives across the life-span. Albany: State University of New York Press.

Mirowsky, J. und P. Hu (1996), Physical impairment and the diminishing effects of income. Social Forces, 74(3), 1073-1096.

Mullis, I.V., M.O. Martin, A.E. Beaton, E.J. Gonzales, D.L. Kelly und T.A. Smith (1998), Mathematics and Science Achievement in the Final Year of Secondary School. Center for the Study of Testing, Evaluation and Educational Policy, Boston College.

OECD (1996), Bildung auf einen Blick - OECD Indikatoren. Paris.

OECD (1997), Bildung auf einen Blick - OECD Indikatoren. Paris.

OECD (1999a), Measuring Student Knowledge and Skills - A New Framework for Assesement. Paris.

OECD (1999b), Classifying Educational Programmes. Manual for ISCED-97 Implementation in OECD Countries. Paris.

OECD (2001), Bildung auf einen Blick - OECD Indikatoren. Paris.

OECD und Statistics Canada (1995), Literacy, Economy and Society: Results of the First International Adult Literacy Survey. Paris und Ottawa.

OECD und Statistics Canada (2000), Literacy in the Information Age. Paris und Ottawa.

Owens, L. und J. Barnes (1992), Learning preferences scales. Victoria, Australien: ACER. 
Pelgrum,W.J. und Anderson, R.E. (1999), ICT and the Emerging Paradigm for Lifelong Learning: a worldwide educational assessment of infrastrucure, goals, and practices. Amsterdam: International Association for the Evaluation of Educational Achievment (IEA).

Pintrich, P.R., D.A.F. Smith, T. Garcia und W.J. McKeachie (1993), Reliability and predictive validity of the motivated strategies for learning questionnaire (MLSQ). Educational and Psychological Measurement, 53, 801-813.

Rumberger, R. und J.D. Willms (1992), The impact of racial and ethnic segregation on the achievement gap in California high schools. Educational Evaluation and Policy Analysis 14(4), 377-396.

Shavit, Y. und R.A. Williams (1985), Ability grouping and contextual determinants of educational expectations in Israel. American Sociological Review, 50, 62-73.

Summers, A.A. und B.L.Wolfe (1977), Do schools make a difference? American Economic Review, 67, 639-652.

Warm, T.A. (1985), Weighted maximum likelihood estimation of ability in Item Response Theory with tests of finite length. Technical Report CGI-TR-85-08. Oklahoma City: U.S. Coast Guard Institute.

Willms, J.D. (1986), Social class segregation and its relationship to pupils' examination results in Scotland. American Sociological Review, 51, 224-241.

Willms, J.D. und M. Chen (1989), The effects of ability grouping on the ethnic achievement gap in Israeli elementary schools. American Journal of Education, 97(3), 237-257.

Wolfson, M., G. Rowe, J.F. Gentleman und M.Tomiak (1993), Career earnings and death: A longitudinal analysis of older Canadian men. Journal of Gerontology, 48(4), S167-S179. 


\section{$A N H A N G$}

A

\section{TECHNISCHE HINWEISE}

Anhang A1: Konstruktion der Indizes und anderer von den Kontextfragebogen für Schülerinnen und Schüler und für Schulen abgeleiteten Messgrößen

Anhang A2: Erklärte Varianz der Schülerleistungen

Anhang A3: PISA-Zielpopulation und PISA-Stichproben

Anhang A4: Standardfehler, Signifikanztests und Ländervergleich

Anhang A5: Qualitätssicherung

Anhang A6: Entwicklung der PISA-Erhebungsinstrumente

Anhang A7: Reliabilität der Kodierung offener Items 


\section{Anhang A1: Konstruktion der Indizes und anderer von den Kontextfragebogen für Schülerinnen und Schüler und für Schulen abgeleiteten Messgrößen}

Bei mehreren PISA-Messgrößen handelt es sich um Indizes, die Antworten von Schülern bzw. Schulvertretern (in der Regel Schulleitungen) auf eine Reihe miteinander verknüpfter Fragen zusammenfassen. Die Fragen wurden auf der Basis theoretischer Überlegungen und vorangegangener Forschungen aus einem umfassenderen Konstrukt ausgewählt. Zur Bestätigung des theoretisch erwarteten Verhaltens der Indizes und zur Validierung ihrer Vergleichbarkeit zwischen den Ländern wurden Strukturgleichungsmodelle verwendet. Zu diesem Zweck wurde für jedes Land separat und für alle OECD-Länder insgesamt ein Modell geschätzt.

Dieser Abschnitt erklärt die von den Kontextfragebogen für Schüler und Schulen abgeleiteten Indizes, die in diesem Bericht verwendet werden. Eine Beschreibung anderer PISA-Indizes und Einzelheiten über die gewählten Methoden enthält der PISA 2000 Technical Report, der Anfang 2002 im Internet verfügbar sein wird.

Sofern nicht anders vermerkt, wurde ein Index, der mehrere Fragen und Schülerantworten umfasst, mit Hilfe einer gewichteten Maximum-Likelihood-Schätzung auf der Basis eines dreistufigen einparametrigen Item-Response-Modells (die so genannte WARM-Schätzfunktion; vgl. Warm, 1985) skaliert:

- Die Fragenparameter wurden auf der Basis gleich grosser Teilstichproben von Schülerinnen und Schülern aus jedem OECD-Land geschätzt.

- Die Schätzungen wurden für alle Schüler und alle Schulen durch die im vorangegangenen Schritt ermittelten Fragenparameter geankert.

- Die Indizes wurden dann standardisiert, so dass der mittlere Indexwert für die OECD-Schülerpopulation gleich 0 und die Standardabweichung gleich 1 gesetzt wurde (wobei den Ländern bei dem Standardisierungsprozess dieselbe Gewichtung gegeben wurde).

Es ist zu beachten, dass negative Werte bei einem Index nicht zwangsläufig auf negative Antworten der Schülerinnen und Schüler auf die gestellten Fragen schließen lassen. Ein negativer Wert weist lediglich darauf hin, dass eine Gruppe von Schülern (oder alle Schüler in einem Land zusammengenommen) oder eine Gruppe von Schulleitern weniger positiv antworteten, als das beim Durchschnitt aller Schüler oder Schulleiter in den OECD-Ländern der Fall war. Dementsprechend bedeutet ein positiver Wert bei einem Index, dass eine Gruppe von Schülern oder Schulleitern positivere Antworten gab, als dies beim Durchschnitt der Schüler oder Schulleiter in den OECD-Ländern der Fall war.

Die bei den folgenden Beschreibungen in Klammern $<>$ gesetzten Begriffe wurden in den nationalen Fassungen der Schülerund Schulleiterfragebogen durch den entsprechenden nationalen Ausdruck ersetzt. So wurde z.B. der Begriff <Abschluss entsprechend ISCED-Niveau 5A> in den Vereinigten Staaten übersetzt in „Bachelor's Degree, post-graduate certificate program, Master's degree program or first professional degree program“. Desgleichen wurde der Ausdruck <Testsprachenunterricht> in Luxemburg übersetzt in „Deutschunterricht“ oder „Französischunterricht“, je nachdem ob die Schülerinnen und Schüler die deutsche oder die französische Fassung der Erhebungsinstrumente erhielten.

Nähere Einzelheiten zur Reliabilität dieser Indizes enthält der PISA 2000 Technical Report.

\section{Schülermerkmale und familiärer Hintergrund}

Familienstruktur

Die Schülerinnen und Schüler wurden gebeten anzugeben, wer normalerweise mit ihnen zusammenwohnt. Die Antworten wurden in vier Kategorien unterteilt: a) Familien mit einem Elternteil (Schülerinnen und Schüler, die angaben, mit einer der folgenden Personen zusammenzuleben: Mutter, Vater, weiblichem oder männlichem Erziehungsberechtigten); $\boldsymbol{b}$ ) Kernfamilien (Schülerinnen und Schüler, die angaben, mit Mutter und Vater zusammenzuleben); c) zusammengesetzte Familien (Schülerinnen und Schüler, die angaben, mit der Mutter und einem männlichen Erziehungsberechtigten, dem Vater und einem weiblichen Erziehungsberechtigten oder mit zwei Erziehungsberechtigten zusammenzuleben) und $d$ ) andere Antwortkombinationen. 


\section{Zahl der Geschwister}

Die Schülerinnen und Schüler wurden gebeten, die Zahl der älteren, jüngeren oder gleichaltrigen Geschwister anzugeben. Für die Analysen in Kapitel 8 wurden die Zahlen aus jeder Kategorie addiert.

\section{Geburtsland}

Die Schülerinnen und Schüler wurden gefragt, ob sie selber, ihre Mutter und ihr Vater im Inland oder in einem anderen Land geboren sind. Die Antworten wurden in drei Kategorien unterteilt: a) Gruppe 1 (die im Inland geborenen Schüler mit mindestens einem im Inland geborenen Elternteil), b) Gruppe 2 (im Inland geborene Schüler mit im Ausland geborenen Eltern) und c) Gruppe 3 (im Ausland geborene Schüler mit im Ausland geborenen Eltern).

\section{Zu Hause gesprochene Sprache}

Die Schülerinnen und Schüler wurden gefragt, ob es sich bei der normalerweise zu Hause gesprochenen Sprache um die Testsprache, eine andere offizielle Landessprache, einen landesüblichen Dialekt oder um andere Sprachen handelt. Die Antworten wurden in zwei Kategorien unterteilt: a) die normalerweise zu Hause gesprochene Sprache unterscheidet sich von der Testsprache, von anderen offiziellen Landessprachen und anderen nationalen Dialekten und b) die normalerweise zu Hause gesprochene Sprache ist identisch mit der Testsprache, anderen offiziellen Landessprachen oder nationalen Dialekten.

\section{Wirtschaftlicher, sozialer und kultureller Status}

Die Schülerinnen und Schüler wurden gebeten, den Beruf ihrer Mutter und ihres Vaters zu nennen und anzugeben, ob er/sie voll- oder teilzeitbeschäftigt ist, ob er/sie nicht berufstätig, aber auf Arbeitssuche ist oder etwas anderes macht. Die offenen Antworten wurden dann entsprechend der Internationalen Standardklassifikation der Berufe (ISCO 1988) kodiert.

Der in der PISA-Studie verwendete Internationale sozioökonomische Index der beruflichen Stellung (ISEI) wurde von den Schülerantworten auf die Frage nach dem Beruf der Eltern abgeleitet. Der Index erfasst die Attribute von Berufen, die die Bildungsabschlüsse der Eltern in Einkommen umsetzen. Zur Konstruktion dieses Index wurden die Berufsgruppen auf einer linearen Skala so angeordnet, dass der indirekte Einfluss der Bildung auf das Einkommen mittels der Bildungsabschlüsse maximiert und der direkte Einfluss der Bildung auf das Einkommen, unabhängig vom Beruf, minimiert wurde (bei beiden Effekten wurde dem Alter nicht Rechnung getragen). Weitere Informationen über die Methodik finden sich in Ganzeboom et al. (1992). Der in PISA verwendete Internationale sozioökonomische Index der beruflichen Stellung basiert auf dem Beruf des Vaters oder der Mutter, je nachdem wer die höhere Stellung hat.

Die Werte auf dem Index reichen von 0 bis 90, niedrige Werte repräsentieren eine niedrige und hohe Werte eine hohe sozioökonomische Stellung.

Um neben der beruflichen Stellung auch allgemeinere Aspekte des familiären und häuslichen Umfelds der Schülerinnen und Schüler zu erfassen, wurde der PISA-Index des wirtschaftlichen, sozialen und kulturellen Status auf der Basis folgender Variablen konstruiert: dem Internationalen sozioökonomischen Index der beruflichen Stellung (ISEI) (vgl. Tabelle 6.1); dem höchsten Bildungsabschluss der Eltern der Schülerin oder des Schülers, umgerechnet in Schuljahre (Angaben zum elterlichen Bildungsstand enthält Tabelle 6.7, wegen der Umrechnung der Koeffizienten vgl. Tabelle A1.1); dem PISA-Index des relativen Wohlstands der Familie (vgl. Tabelle 6.2); dem PISA-Index der Bildungsressourcen im Elternhaus und dem PISA-Index des Besitzes von „klassischen“ Kulturgütern im Elternhaus (vgl. Tabelle 6.3). Der ISEI stellt die erste Hauptkomponente der oben beschriebenen Faktoren dar. Der Index wurde so konstruiert, dass der Mittelwert gleich 0 und die Standardabweichung gleich 1 ist.

Soweit bei den verschiedenen Komponenten Daten fehlen, handelt es sich gewöhnlich um solche, die den Internationalen sozioökonomischen Index der beruflichen Stellung (ISEI), den Bildungsstand der Eltern oder beides betreffen. Deshalb wurden getrennte Faktoranalysen für alle Schülerinnen und Schüler durchgeführt, für die gültige Daten für jeweils die folgenden Komponenten vorlagen: a) sozioökonomischer Index der beruflichen Stellung, Index des relativen Wohlstands der Familie, Index der Bildungsressourcen im Elternhaus, Index des Besitzes von „klassischen“ Kulturgütern im Elternhaus; b) Bildungsjahre der Eltern, Index des relativen Wohlstands der Familie, Index der Bildungsressourcen im Elternhaus und Index des Besitzes von „klassischen“ Kulturgütern im Elternhaus und c) Index des relativen Wohlstands der Familie, Index der Bildungsressourcen im Elternhaus und Index des Besitzes von „klassischen“ Kulturgütern im Elternhaus. Den Schülerinnen und Schülern wurde dann 
je nach dem Umfang der verfügbaren Daten eine Faktorpunktzahl gegeben. Es mussten Angaben zu mindestens drei Variablen vorliegen. Im Fall Frankreichs bestehen nach wie vor Unsicherheiten hinsichtlich der Reliabilität der Schülerantworten auf die Fragen nach dem Beruf und dem Bildungsabschluss der Eltern (vgl. INSEE, 1999).

\section{Bildungsabschluss der Eltern}

Die Schülerinnen und Schüler wurden gebeten, den höchsten Bildungsabschluss ihrer Mutter und ihres Vaters (entsprechend dem jeweiligen nationalen System) anzugeben, der dann gemäß der Internationalen Standardklassifikation des Bildungswesens (ISCED 1997) kodiert wurde, um international vergleichbare Kategorien der erreichten Bildungsabschlüsse zu erhalten. Daraus ergaben sich folgende Kategorien: hat keine Schule besucht, hat <ISCED Niveau 1 (Grundschule) $>$, hat <ISCED Niveau 2 (Abschluss der Sekundarstufe 1) $>$, hat <ISCED Niveau 3B oder 3C (Abschluss der Sekundarstufe 2, der in den meisten Ländern zum Erwerb der Berufsreife führt) $>$, hat $<$ ISCED Niveau 3A (Abschluss der Sekundarstufe 2, der in den meisten Ländern zum Erwerb der Hochschulreife führt) $>$ und hat <ISCED Niveau 5A, 5B oder 6 (Hochschulabschluss) $>$.

Eine Liste der nationalen institutionellen Kategorien, die in den oben angeführten Klammern $<>$ verwendet wurden, enthält der PISA 2000 Technical Report.

Für die Analyse in Kapitel 8 wurde eine Umwandlung des höchsten Bildungsabschlusses der Eltern in eine Schätzung der entsprechenden Schuljahre unter Zugrundelegung der in der folgenden Tabelle angegebenen Koeffizienten vorgenommen.

Tabelle A.1.1

Bildungsabschluss der Eltern, umgerechnet in Schuljahre

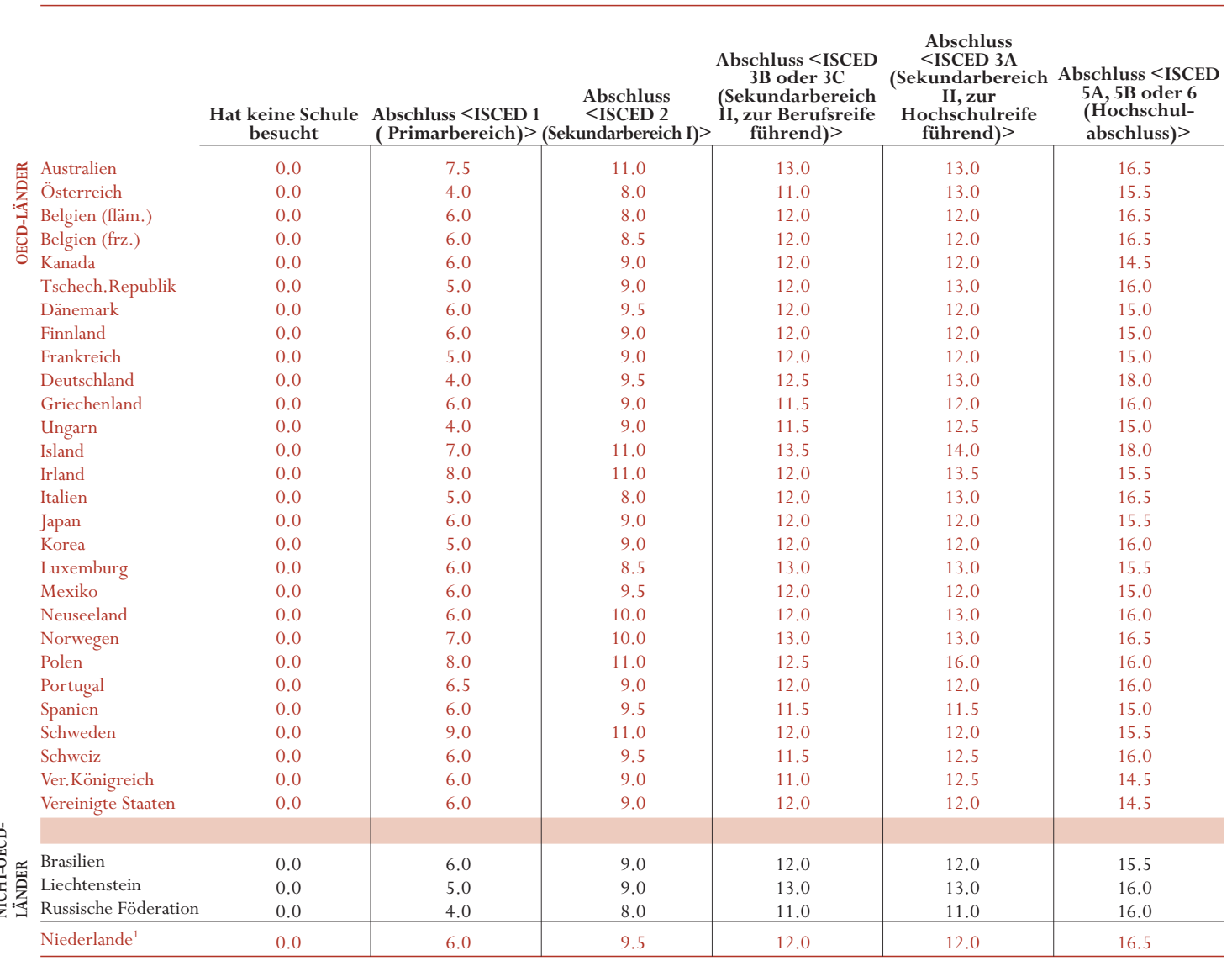

1. Die Beteiligungsquote ist zu niedrig, um die Vergleichbarkeit zu gewährleisten (vgl. Anhang A3). 
Das elterliche Interesse

Der PISA-Index der kulturellen Kommunikation wurde abgeleitet von den Angaben der Schülerinnen und Schüler, wie häufig sie mit ihren Eltern (oder Erziehungsberechtigten) gemeinsam politische oder soziale Fragen erörtern, über Bücher, Filme oder Fernsehsendungen diskutieren oder klassische Musik hören.

Der PISA-Index der sozialen Kommunikation wurde abgeleitet von den Angaben der Schülerinnen und Schüler, wie häufig ihre Eltern (oder Erziehungsberechtigten) mit ihnen über ihre Schulleistungen sprechen, mit ihnen < die Hauptmahlzeit> gemeinsam am Tisch einnehmen, sich Zeit nehmen, um einfach nur mit ihnen zu reden.

Die Schülerinnen und Schüler antworteten auf einer Fünfpunkteskala mit folgenden Antwortkategorien: ,nie oder fast nie ', ,ein paar Mal im Jahr', ,etwa einmal im Monat', ,mehrmals im Monat' und ,mehrmals in der Woche‘. Beide Indizes wurden mit Hilfe der weiter oben beschriebenen WARM-Schätzfunktion konstruiert.

\section{Teilnahme an zusätzlichem Unterricht}

Die Schülerinnen und Schüler wurden gefragt, ob sie in den vergangenen drei Jahren gelegentlich oder regelmäßig an irgendwelchen Sonderkursen außerhalb der Schule teilgenommen haben, um ihre Leistungen zu verbessern. Die Antworten wurden in zwei Kategorien unterteilt: a) Schülerinnen und Schüler, die außerhalb der Schule zusätzlichen Unterricht in der $<$ Testsprache $>$, Kurse in anderen Fächern oder sonstigen Ergänzungsunterricht hatten und b) Schülerinnen und Schüler, die außerhalb der Schule an Förder- bzw. Stützkursen in der $<$ Testsprache $>$, bzw. in anderen Fächern, oder an Kursen zur Verbesserung ihrer Lern- oder Arbeitstechniken teilgenommen oder private Nachhilfestunden gehabt hatten.

\section{Kulturelle Aktivitäten}

Der PISA-Index der „klassischen “ kulturellen Aktivitäten wurde abgeleitet von den Angaben der Schülerinnen und Schüler, wie oft sie im vergangenen Jahr ein Museum oder eine Kunstausstellung besucht haben, in die Oper, ins Ballet oder in ein Symphoniekonzert gegangen sind oder einer Theateraufführung beigewohnt haben. Die Schülerinnen und Schüler trugen ihre Angaben auf einer Vierpunkteskala mit folgenden Antwortkategorien ein: ,nie oder fast nie', ,ein- bis zweimal im Jahr', ,drei- bis viermal im Jahr', , mehr als viermal im Jahr'. Der Index wurde mit Hilfe der weiter oben beschriebenen WARMSchätzfunktion konstruiert.

\section{Familiärer Besitzstand}

Der PISA-Index des relativen Wohlstands der Familie wurde abgeleitet von den Angaben der Schülerinnen und Schüler, ob sie bei sich zu Hause a) eine Geschirrspülmaschine, ein eigenes Zimmer, Lern-Software und einen Internetanschluss haben und b) wie viele Handys, Fernseher, Computer, Autos und Badezimmer sie zu Hause haben.

Der PISA-Index der Bildungsressourcen im Elternhaus wurde abgeleitet von den Angaben der Schülerinnen und Schüler über das Vorhandensein bzw. die Anzahl folgender Dinge im Elternhaus: ein Wörterbuch, ein ruhiger Platz zum Lernen, ein Schreibtisch zum Lernen, Schulbücher und Taschenrechner.

Der PISA-Index des Besitzes von „klassischen“ Kulturgütern im Elternhaus wurde abgeleitet von den Angaben der Schülerinnen und Schüler über das Vorhandensein folgender Güter im Elternhaus: klassische Literatur (es wurden Beispiele gegeben), Gedichtbände und Kunstwerke (es wurden Beispiele gegeben).

Diese Indizes wurden mit Hilfe der weiter oben beschriebenen WARM-Schätzfunktion (Warm, 1985) konstruiert.

\section{Lernstrategien und Einstellung zum Lernen}

Freude am Lesen

Der PISA-Index Freude am Lesen wurde davon abgeleitet, inwieweit die Schülerinnen und Schüler folgenden Aussagen zustimmten: Ich lese nur, wenn ich muss; Lesen ist eines meiner liebsten Hobbys; ich spreche gern mit anderen Leuten über Bücher; es fällt mir schwer, Bücher zu Ende zu lesen; ich freue mich, wenn ich ein Buch geschenkt bekomme; für mich ist Lesen Zeitverschwendung; ich gehe gern in Buchhandlungen oder Büchereien; ich lese nur, um Informationen zu bekommen, die ich brauche; ich kann nicht länger als ein paar Minuten stillsitzen und lesen. Es wurde eine Vierpunkteskala mit folgenden 
Antwortkategorien verwendet: ,stimme überhaupt nicht zu', ,stimme eher nicht zu', ,stimme eher zu', ,stimme ganz zu'. Die Indizes wurden mit Hilfe der weiter oben beschriebenen WARM-Schätzfunktion konstruiert.

Interesse der Schüler am Lesen

Der PISA-Index des Leseinteresses wurde davon abgeleitet, inwieweit die Schülerinnen und Schüler folgenden Aussagen zustimmten: Weil mir das Lesen Spaß macht, würde ich es nicht gerne aufgeben, ich lese in meiner Freizeit, und wenn ich lese, vergesse ich manchmal alles um mich herum. Es wurde eine Vierpunkteskala mit folgenden Antwortkategorien verwendet: ,stimme überhaupt nicht zu', ,stimme eher nicht zu', ,stimme eher zu', ,stimme ganz zu'. Die Indizes wurden mit Hilfe der weiter oben beschriebenen WARM-Schätzfunktion konstruiert. Wegen Informationen über die konzeptuellen Grundlagen des Index siehe Baumert et al. (1997).

\section{Interesse der Schüler an Mathematik}

Der PISA-Index des Mathematikinteresses wurde davon abgeleitet, inwieweit die Schülerinnen und Schüler folgenden Aussagen zustimmten: Wenn ich mich mit Mathematik beschäftige, vergesse ich manchmal alles um mich herum, Mathematik ist mir persönlich wichtig, und weil mir die Beschäftigung mit Mathematik Spaß macht, würde ich das nicht gerne aufgeben. Es wurde eine Vierpunkteskala mit folgenden Antwortkategorien verwendet: ,stimme überhaupt nicht zu', ,stimme eher nicht zu', ,stimme eher zu', ,stimme ganz zu'. Die Indizes wurden mit Hilfe der weiter oben beschriebenen WARM-Schätzfunktion konstruiert. Wegen Informationen über die konzeptuellen Grundlagen des Index siehe Baumert et al. (1997).

\section{Kontrollstrategien}

Der PISA-Index der Kontrollstrategien wurde davon abgeleitet, wie häufig die Schülerinnen und Schüler folgenden Strategien beim Lernen nutzen: Ich überlege mir zuerst, was genau ich lernen muss; ich zwinge mich zu prüfen, ob ich das Gelernte auch behalten habe; ich versuche herauszufinden, was ich noch nicht richtig verstanden habe; ich passe genau auf, dass ich das Wichtigste behalte; und wenn ich lerne und etwas nicht verstehe, suche ich nach zusätzlichen Informationen, um das Problem zu klären. Es wurde eine Vierpunkteskala mit folgenden Antwortkategorien verwendet: ,fast nie', ,manchmal', ,oft', ,fast immer'. Die Indizes wurden mit Hilfe der weiter oben beschriebenen WARM-Schätzfunktion konstruiert. Wegen Informationen über die konzeptuellen Grundlagen des Index siehe Baumert et al. (1994).

\section{Memorieren}

Der PISA-Index der Memorierstrategien wurde davon abgeleitet, wie häufig die Schülerinnen und Schüler folgende Strategien beim Lernen nutzen: Ich versuche, alles auswendig zu lernen, was drankommen könnte; ich lerne so viel wie möglich auswendig; ich präge mir alles Neue so ein, dass ich es aufsagen kann; und ich lerne, indem ich den Stoff immer wieder aufsage. Es wurde eine Vierpunkteskala mit folgenden Antwortkategorien verwendet: ,fast nie', ,manchmal', ,oft', ,fast immer'. Die Indizes wurden mit Hilfe der weiter oben beschriebenen WARM-Schätzfunktion konstruiert. Wegen Informationen über die konzeptuellen Grundlagen des Index siehe Baumert et al. (1994) und Pintrich et al. (1993).

\section{Elaborieren}

Der PISA-Index der Elaborationsstrategien wurde davon abgeleitet, wie häufig die Schülerinnen und Schüler folgende Strategien beim Lernen nutzen: Ich versuche, den neuen Stoff mit Dingen zu verbinden, die ich in anderen Fächern gelernt habe; ich überlege, inwiefern die Information im wirklichen Leben nützlich sein könnte; ich versuche, den Stoff besser zu verstehen, indem ich Verbindungen zu Dingen herstelle, die ich schon kenne; und ich überlege, wie der Stoff mit dem zusammenhängt, was ich schon gelernt habe. Es wurde eine Vierpunkteskala mit folgenden Antwortkategorien verwendet: ,fast nie', ,manchmal', , oft', ,fast immer'. Die Indizes wurden mit Hilfe der weiter oben beschriebenen WARM-Schätzfunktion konstruiert. Wegen Informationen über die konzeptuellen Grundlagen des Index siehe Baumert et al. (1994).

\section{Kooperatives und wettbewerbsorientiertes Lernen}

Der PISA-Index des kooperativen Lernens wurde davon abgeleitet, inwieweit die Schülerinnen und Schüler folgende Aussagen für zutreffend hielten: Ich arbeite gern mit anderen Schülerinnen und Schülern zusammen; ich lerne am meisten, wenn ich mit anderen Schülerinnen und Schülern zusammenarbeite; ich leiste die beste Arbeit, wenn ich mit anderen Schülerinnen und Schülern zusammenarbeite; ich helfe anderen gern dabei, in einer Gruppe gute Arbeit zu leisten; und wenn man an einem Projekt arbeitet, ist es nützlich, die Ideen von allen zusammen zu bringen. Es wurde eine Vierpunkteskala mit folgenden 
Antwortkategorien verwendet: ,trifft nicht zu', ,trifft eher nicht zu', ,trifft eher zu', ,trifft zu'. Die Indizes wurden mit Hilfe der weiter oben beschriebenen WARM-Schätzfunktion konstruiert. Wegen Informationen über die konzeptuellen Grundlagen des Index siehe Owens und Barnes (1992).

Der PISA-Index des wettbewerbsorientierten Lernens wurde davon abgeleitet, inwieweit die Schülerinnen und Schüler folgende Aussagen für zutreffend hielten: Ich versuche gerne, besser zu sein als andere Schülerinnen und Schüler; wenn ich versuche, besser als andere zu sein, leiste ich gute Arbeit; ich wäre gerne in irgendeinem Bereich die/der Beste; und ich lerne schneller, wenn ich versuche, besser zu sein als die anderen. Es wurde eine Vierpunkteskala mit folgenden Antwortkategorien verwendet: ,trifft nicht zu', ,trifft eher nicht zu', ,trifft eher zu', ,trifft zu'. Die Indizes wurden mit Hilfe der weiter oben beschriebenen WARM-Schätzfunktion konstruiert. Wegen Informationen über die konzeptuellen Grundlagen des Index siehe Owens und Barnes (1992).

\section{Selbstkonzept der Schülerinnen und Schüler in Bezug auf Lesen}

Der PISA-Index des Selbstkonzepts Lesen wurde davon abgeleitet, inwieweit die Schülerinnen und Schüler folgende Aussagen für zutreffend hielten: Im < Testsprachenfach $>$ bin ich ein hoffnungsloser Fall, im $<$ Testsprachenfach $>$ lerne ich schnell, im $<$ Testsprachenfach $>$ bekomme ich gute Noten. Es wurde eine Vierpunkteskala mit folgenden Antwortkategorien verwendet: ,trifft nicht zu', ,trifft eher nicht zu', ,trifft eher zu', ,trifft zu'. Die Indizes wurden mit Hilfe der weiter oben beschriebenen WARM-Schätzfunktion konstruiert. Wegen Informationen über die konzeptuellen Grundlagen des Index siehe Marsh et al. (1992).

\section{Selbstkonzept der Schülerinnen und Schüler in Bezug auf Mathematik}

Der PISA-Index des Selbstkonzepts Mathematik wurde davon abgeleitet, inwieweit die Schülerinnen und Schüler folgende Aussagen als zutreffend bezeichneten: Im Fach Mathematik bekomme ich gute Noten, Mathematik ist eins meiner besten Fächer, und ich war schon immer gut in Mathematik. Es wurde eine Vierpunkteskala mit folgenden Antwortkategorien verwendet: ,trifft nicht zu', ,trifft eher nicht zu', ,trifft eher zu', ,trifft zu'. Die Indizes wurden mit Hilfe der weiter oben beschriebenen WARM-Schätzfunktion konstruiert. Wegen Informationen über die konzeptuellen Grundlagen des Index siehe Marsh et al. (1992).

\section{Interesse an Computern}

Der PISA-Index des Interesses an Computern wurde davon abgeleitet, inwieweit die Schülerinnen und Schüler folgenden Aussagen zustimmten: Es ist mir sehr wichtig, mit dem Computer zu arbeiten; am Computer zu spielen oder zu arbeiten, macht richtig Spaß; ich benutze den Computer, weil mich das sehr interessiert; wenn ich am Computer arbeite, merke ich nicht, wie die Zeit vergeht. Es wurde eine Zweipunkteskala mit den Antwortkategorien ,ja' und ,nein 'verwendet. Die Indizes wurden mit Hilfe der weiter oben beschriebenen WARM-Schätzfunktion konstruiert. Wegen Informationen über die konzeptuellen Grundlagen des Index siehe Eignor et al. (1998).

\section{Vertrautheit und Selbsteinschätzung der Fähigkeit im Umgang mit Computern}

Der PISA-Index der Vertrautheit und Selbsteinschätzung der Fähigkeit im Umgang mit Computern wurde davon abgeleitet, wie die Schülerinnen und Schüler folgende Fragen beantworten:Wie gut bist Du im Umgang mit dem Computer; wie gut bist Du beim Schreiben eines Aufsatzes mit dem Computer; wie gut wärst Du, wenn Du eine Klassenarbeit am Computer schreiben müsstest, und wenn Du dich mit anderen 15-Jährigen vergleichst, wie würdest Du Deine Fähigkeit im Umgang mit dem Computer beurteilen. Für die ersten drei Fragen wurde eine Vierpunkteskala mit folgenden Antwortkategorien verwendet: ,sehr gut', ,gut', ,einigermaßen gut' und ,überhaupt nicht gut'. Für die letzte Frage wurde eine Vierpunkteskala mit folgenden Antwortkategorien eingesetzt: ,sehr gut', ,gut', ,durchschnittlich' und ,schlecht‘. Die Indizes wurden mit Hilfe der weiter oben beschriebenen WARM-Schätzfunktion konstruiert. Wegen Informationen über die konzeptuellen Grundlagen des Index siehe Eignor et al. (1998).

\section{Zeitaufwand für Hausaufgaben}

Der PISA-Index des Zeitaufwands für Hausaufgaben wurde abgeleitet von den Angaben der Schülerinnen und Schüler, wie viel Zeit sie für die Hausaufgaben in den Fächern <Testsprache>, Mathematik und Naturwissenschaften brauchen. Die Schülerinnen und Schüler konnten den Zeitaufwand auf einer Vierpunkteskala mit folgenden Antwortkategorien bewerten: 
,keine Zeit', ,weniger als 1 Stunde in derWoche', ,1-3 Stunden in derWoche', , 3 und mehr Stunden in der Woche'. Die Indizes wurde mit Hilfe der weiter oben beschriebenen WARM-Schätzfunktion konstruiert.

\section{Schulpolitik und -praktiken}

\section{Durchführung von Schülerbeurteilungen}

Die Schulleitungen gaben an, wie häufig in ihren Schulen die 15-Jährigen durch Folgendes beurteilt werden: standardisierte Tests, von Lehrkräften entwickelte Tests, Beurteilung durch die Lehrkraft, von den Schülern zusammengestellte $<$ Portfolios $>$, Schülerarbeiten/Projektarbeiten/Hausaufgaben. Die Schulleiterinnen und Schulleiter kreuzten für jede Form der Beurteilung Antworten auf einer Fünfpunkteskala mit folgenden Kategorien an: ,nie', ,einmal im Jahr', ,zweimal im Jahr', ,dreimal im Jahr', ,viermal im Jahr oder häufiger'. Die Schulleitungen machten ebenfalls Angaben darüber, ob die Beurteilung der 15-jährigen Schülerinnen und Schüler für folgende Zwecke genutzt werden: Um die Schule mit < regionalen oder nationalen> Leistungsstandards zu vergleichen, um die Entwicklung des Leistungsniveaus der Schule von Jahr zu Jahr zu beobachten und um die Effektivität der Lehrkräfte zu beurteilen.

Der PISA-Index der Nutzung formeller Beurteilungen wurde abgeleitet von den Angaben der Schulleitungen, wie häufig standardisierte Tests durchgeführt werden und welchem Zweck diese Leistungsbeurteilungen dienen. Die Indizes wurden mit Hilfe der weiter oben beschriebenen WARM-Schätzfunktion konstruiert. Hohe Werte bei diesem Index bezeichnen Schulen, bei denen standardisierte Beurteilungen eine wichtige Rolle als Kontrollinstrument spielen.

Der PISA-Index der Nutzung informeller Beurteilungen wurde abgeleitet von den Antworten der Schulleitungen, wie häufig die Schüler durch Folgendes beurteilt wurden: von Lehrkräften entwickelte Tests, Beurteilungen durch die Lehrkraft, von den Schülern zusammengestellte <Portfolios>, Schülerarbeiten/Projektarbeiten/Hausaufgaben, sowie den Angaben der Schulleitungen bezüglich der Nutzung dieser Leistungsbeurteilungen. Die Indizes wurden mit Hilfe der weiter oben beschriebenen WARM-Schätzfunktion konstruiert. Hohe positive Werte bei diesem Index bezeichnen Schulen, bei denen informelle Leistungsbeurteilungen eine wichtige Rolle als Kontrollinstrument spielen.

\section{Autonomie der Schulen und der Lehrkräfte}

Die Schulleitungen wurden gebeten anzugeben, ob die Lehrkräfte, Fachbereichsleiter, Schulleiterinnen und Schulleiter, ein ernanntes oder ein gewähltes Gremium oder Bildungsbehörden auf einer höheren Ebene die Hauptverantwortung für die folgenden Bereiche tragen: Einstellung von Lehrkräften, Entlassung von Lehrkräften, Festlegung des Anfangsgehalts der Lehrkräfte, Entscheidung über die Beförderung/Höhergruppierung von Lehrkräften, Festlegung des Schulbudgets, Entscheidung über die Verwendung des Budgets innerhalb der Schule, Festlegung von disziplinären Regeln für Schülerinnen und Schüler, Festlegung von Kriterien für die Schülerbeurteilung, Aufnahme von Schülerinnen und Schülern in die Schule, Wahl der verwendeten Lehrbücher, Bestimmung des Lehrstoffes und Entscheidung über das Fächer- und Kursangebot.

Der in Kapitel 8 verwendete PISA-Index der Schulautonomie wurde abgeleitet von den Angaben der Schulleitungen über die Zahl der Bereiche, für die die Schulen keine Verantwortung tragen. Die Skala wurde dann so gepolt, dass hohe Werte auf einen hohen Autonomiegrad hinweisen.

Der in Kapitel 8 verwendete PISA-Index der Lehrerautonomie wurde abgeleitet von den Angaben der Schulleitungen über die Zahl der Hauptverantwortungsbereiche der Lehrkräfte.

Die Indizes wurden mit Hilfe der weiter oben beschriebenen WARM-Schätzfunktion konstruiert.

\section{Berufliche Fortbildung der Lehrkräfte}

Die Schulleitungen gaben an, wie viel Prozent der Lehrkräfte an ihrer Schule an einer Fortbildungsveranstaltung teilgenommen hatten. Zu Fortbildungsveranstaltungen zählen offizielle Angebote, die dazu dienen, didaktische oder pädagogische Kompetenzen zu verbessern. Solche Programme müssen nicht unbedingt zu einer anerkannten Qualifikation führen. Bei dieser Frage sollten nur Veranstaltungen berücksichtigt werden, die zumindest einen Tag dauerten und sich mit pädagogischen und Bildungsfragen befassten. 
Lehrkräftebezogene Faktoren für das Schulklima aus der Sicht der Schulleitungen

Der PISA-Index lehrkräftebezogener Faktoren für das Schulklima aus der Sicht der Schulleitungen wurde abgeleitet von den Angaben der Schulleiterinnen und Schulleiter, inwieweit das Lernen von 15-Jährigen durch Folgendes beeinträchtigt wird: niedrige Erwartungen der Lehrkräfte, schlechtes Schüler-Lehrer-Verhältnis, die Lehrkräfte gehen nicht auf die individuellen Schülerbedürfnisse ein, häufige Abwesenheit von Lehrkräften, Widerstand des Kollegiums gegen Veränderungen, die Lehrkräfte sind mit den Schülern zu streng, die Schülerinnen und Schüler werden nicht ermutigt, ihr Potential voll auszuschöpfen. Es wurde eine Vierpunkteskala mit folgenden Antwortkategorien verwendet: ,gar nicht', ,kaum', ,etwas', ,sehr'. Die Indizes wurden mit Hilfe der weiter oben beschriebenen WARM-Schätzfunktion konstruiert. Dieser Index wurde so gepolt, dass niedrigere Werte auf ein schlechteres Schulklima hinweisen.

\section{Stimmung und Arbeitshaltung der Lehrkräfte aus der Sicht der Schulleitungen}

Der PISA-Index der Stimmung und Arbeitshaltung der Lehrkräfte aus der Sicht der Schulleitungen wurde davon abgeleitet, inwieweit die Schulleiterinnen und Schulleiter den folgenden Aussagen zustimmten: Stimmung und Arbeitshaltung der Lehrkräfte sind in dieser Schule gut, die Lehrkräfte arbeiten mit großem Engagement, die Lehrkräfte sind stolz auf diese Schule und die Lehrkräfte legen Wert auf schulische Leistung. Es wurde eine Vierpunkteskala mit folgenden Antwortkategorien verwendet: ,stimme überhaupt nicht zu', stimme eher nicht zu', ,stimme eher zu', stimme ganz zu'. Die Indizes wurden mit Hilfe der weiter oben beschriebenen WARM-Schätzfunktion konstruiert.

\section{Lehrermangel}

Der PISA-Index des Lehrermangels wurde davon abgeleitet, inwieweit nach Ansicht der Schulleitungen das Lernen der 15-Jährigen in ihrer Schule durch den Mangel bzw. die unzureichende Lehrbefähigung von Lehrkräften generell sowie für die Fächer $<$ Testsprache $>$ Mathematik oder Naturwissenschaften beeinträchtigt wird. Der Index wurde mit Hilfe der weiter oben beschriebenen WARM-Schätzfunktion konstruiert. Dieser Index wurde so gepolt, dass niedrige Werte auf Probleme im Zusammenhang mit Lehrermangel hinweisen.

\section{Unterrichtspraktiken}

\section{Unterstützung durch die Lehrkräfte}

Der PISA-Index der Unterstützung durch die Lehrkräfte wurde abgeleitet von den Angaben der Schülerinnen und Schüler, wie oft es vorkommt, dass der Lehrer/die Lehrerin sich für den Lernfortschritt jedes einzelnen Schülers interessiert; den Schülerinnen und Schülern Gelegenheit gibt, ihre Meinung zu sagen; den Schülerinnen und Schülern bei der Arbeit hilft; etwas so lange erklärt, bis es die Schülerinnen und Schüler verstanden haben; viel tut, um den Schülerinnen und Schülern zu helfen und den Schülerinnen und Schülern beim Lernen hilft. Es wurde eine Vierpunkteskala mit folgenden Antwortkategorien verwendet: ,nie', ,in einigen Stunden', ,in den meisten Stunden', ,in jeder Stunde'. Der Index wurde mit Hilfe der weiter oben beschriebenen WARM-Schätzfunktion konstruiert.

\section{Schuldisziplin}

Der PISA-Index der Schuldisziplin fasst die Angaben der Schülerinnen und Schüler zusammen, wie häufig in ihrem $<$ Testsprachenunterricht $>$ Folgendes vorkommt: Der Lehrer/die Lehrerin muss lange warten, bis < Ruhe eintritt>; die Schülerinnen und Schüler können nicht ungestört arbeiten; die Schülerinnen und Schüler hören nicht auf das, was der Lehrer/ die Lehrerin sagt; die Schülerinnen und Schüler fangen erst lange nach Beginn der Stunde an zu arbeiten; es ist laut und alles geht durcheinander; und es vergehen zu Beginn der Stunde mehr als fünf Minuten, in denen gar nichts passiert. Es wurde eine Vierpunkteskala mit folgenden Antwortkategorien verwendet: ,nie', ,in einigen Stunden', ,in den meisten Stunden', ,in jeder Stunde‘. Dieser Index wurde so gepolt, dass niedrige Werte auf eine schlechte Schuldisziplin hinweisen.

Der PISA-Index schülerbezogener Faktoren für das Schulklima aus der Sicht der Schulleitungen wurde abgeleitet von den Angaben der Schulleitungen, wie sehr das Lernen der 15-Jährigen in ihren Schulen durch Folgendes beeinträchtigt wird: häufige Abwesenheit von Schülerinnen und Schülern, Störung des Unterrichts durch Schülerinnen und Schüler, Schwänzen des Unterrichts, fehlender Respekt der Schülerinnen und Schüler vor den Lehrkräften, Konsum von Alkohol oder illegalen Drogen und Einschüchtern oder Schikanieren von Schülerinnen und Schüler durch Mitschüler. Es wurde eine Vierpunkteskala mit folgenden Antwortkategorien verwendet: ,gar nicht', ,kaum', ,etwas', ,sehr'. Dieser Index wurde so gepolt, dass niedrige 
Werte auf eine schlechte Schuldisziplin hinweisen. Die Indizes wurden mit Hilfe der weiter oben beschriebenen WARMSchätzfunktion konstruiert.

Leistungsdruck

Der PISA-Index des Leistungsdrucks wurde abgeleitet von den Angaben der Schülerinnen und Schüler, wie häufig in ihrem $<$ Testsprachenunterricht> Folgendes vorkommt: der Lehrer/die Lehrerin will, dass sie sich richtig anstrengen; ihnen sagt, dass sie eigentlich besser sein könnten; unzufrieden ist, wenn sie < nachlässig $>$ arbeiten; von ihnen verlangt, dass sie viel lernen. Es wurde eine Vierpunkteskala mit folgenden Antwortkategorien verwendet: ,nie', ,in einigen Stunden', ,in den meisten Stunden', ,in jeder Stunde'. Der Index wurde mit Hilfe der weiter oben beschriebenen WARM-Schätzfunktion konstruiert, wobei die Antwortkategorie ,nie' als 1 und alle anderen als 0 kodiert wurden.

\section{Schüler-Lehrer-Verhältnis}

Der PISA-Index des Schüler-Lehrer-Verhältnisses wurde abgeleitet von den Angaben der Schülerinnen und Schüler, inwieweit sie den folgenden Aussagen zustimmten: Die Schülerinnen und Schüler kommen mit den meisten Lehrern/Lehrerinnen gut aus; den meisten Lehrern/Lehrerinnen ist es wichtig, dass die Schülerinnen und Schüler sich wohlfühlen; die meisten meiner Lehrer/Lehrerinnen interessieren sich für das, was ich zu sagen habe; wenn ich zusätzliche Hilfe brauche, bekomme ich sie von meinen Lehrern/Lehrerinnen; und die meisten meiner Lehrer/Lehrerinnen behandeln mich fair. Es wurde eine Vierpunkteskala mit folgenden Antwortkategorien verwendet: ,stimme überhaupt nicht zu', ,stimme eher nicht zu', ,stimme eher zu', ,stimme ganz zu'. Die Indizes wurden mit Hilfe der weiter oben beschriebenen WARM-Schätzfunktion konstruiert, wobei die Antwortkategorie ,stimme ganz zu' als 1 und alle anderen als 0 kodiert wurden.

\section{Schulressourcen und Schultypen}

\section{Qualität der räumlichen Bedingungen der Schulen}

Der PISA-Index der Qualität der räumlichen Bedingungen der Schulen wurde abgeleitet von den Angaben der Schulleitungen, inwieweit das Lernen der 15-Jährigen in ihren Schulen durch folgende Probleme beeinträchtigt wird: schlechter Zustand der Gebäude, schlechte Heiz-, Klima- oder Beleuchtungsanlagen und Mangel an Unterrichtsräumen (z.B. Klassenzimmer).

Es wurde eine Vierpunkteskala mit folgenden Antwortkategorien verwendet: ,gar nicht', ,kaum', ,etwas', ,sehr'. Der Index wurde mit Hilfe der weiter oben beschriebenen WARM-Schätzfunktion konstruiert. Dieser Index wurde so gepolt, dass niedrige Werte auf eine geringe Qualität der räumlichen Bedingungen hinweisen.

\section{Qualität der Ausstattung mit Lehr- und Sachmitteln}

Der PISA-Index der Qualität der Ausstattung mit Lehr- und Sachmitteln wurde abgeleitet von den Angaben der Schulleitungen, inwieweit das Lernen der 15-Jährigen durch folgende Probleme beeinträchtigt wird: nicht genügend Computer für den Unterricht, Mangel an Unterrichtsmaterialien in der Bibliothek, Mangel an Multimedia-Ausstattung für den Unterricht, unzureichende Laborausstattung und unzureichende Ausstattung für den Kunstunterricht.

Es wurde eine Vierpunkteskala mit folgenden Antwortkategorien verwendet: , gar nicht', ,kaum`, ,etwas', ,sehr‘. Der Index wurde mit Hilfe der weiter oben beschriebenen WARM-Schätzfunktion konstruiert. Dieser Index wurde so gepolt, dass niedrige Werte auf eine geringe Qualität der Austattung mit Lehr- und Sachmitteln hinweisen.

\section{Verfügbarkeit von Computern}

Die Schulleiterinnen und Schulleiter machten Angaben über die Gesamtzahl der in ihren Schulen vorhandenen Computer und im einzelnen auch darüber, wie viele Computer: für 15-jährige Schülerinnen und Schüler verfügbar sind; nur für Lehrerinnen und Lehrer verfügbar sind; nur für die Verwaltung/Schulleitung verfügbar sind; einen Internetanschluss besitzen und an das lokale Netzwerk angeschlossen sind. Der PISA-Index der Verfügbarkeit von Computern wurde anhand einer Division der Gesamtzahl der für die 15-jährigen Schülerinnen und Schüler verfügbaren Computer durch die Gesamtzahl der in der Schule vorhandenen Computer konstruiert. 


\section{Schüler/Lehrer-Quote und Klassengröße}

Die Schulleitungen nannten die Zahl der in ihrer Schule beschäftigten Vollzeit- und Teilzeitlehrkräfte. Sie machten auch Angaben darüber, wie viele Lehrkräfte die $<$ Testsprache $>$ und wie viele Mathematik und Naturwissenschaften unterrichten, wie viele Lehrkräfte über eine ordentliche Lehrbefähigung (ausgestellt von der < zuständigen nationalen Behörde $>$ ) verfügen und wie viele Lehrkräfte den erforderlichen Abschluss $<$ ISCED Niveau 5A $>$ in $<$ Pädagogik $>,<$ ISCED Niveau $5 \mathrm{~A}>$ in der $<$ Testsprache $>$, $<$ ISCED Niveau 5A $>$ in $<$ Mathematik $>$ und $<$ ISCED Niveau $5 \mathrm{~A}>$ in $<$ Naturwissenschaften $>$ besitzen. Der Anteil der Lehrkräfte in den jeweiligen Kategorien wurde in Kapitel 8 verwendet.

Die Schüler/Lehrer-Quote wurde definiert als das Verhältnis der Zahl der Lehrkräfte (ausgedrückt in Vollzeitäquivalenten) zur Zahl der Schülerinnen und Schüler. Bei Umrechnung der absoluten Zahlen in Vollzeitäquivalente erhielten Vollzeitlehrkräfte, die definitionsgemäß mit mindestens 90\% der Lehrverpflichtung im Unterricht eingesetzt sind, eine Gewichtung von 1, und Teilzeitlehrkräfte, die definitionsgemäß mit weniger als $90 \%$ im Unterricht eingesetzt sind, eine Gewichtung von 0.5.

Eine Schätzung der Klassengröße wurde auf der Basis der Schülerangaben über die Zahl der Schülerinnen und Schüler in den Fächern $<$ Testsprache $>$, Mathematik und Naturwissenschaften vorgenommen.

\section{Nutzung der Schulressourcen}

Der PISA-Index der Schulressourcennutzung wurde abgeleitet von den Angaben der Schülerinnen und Schüler, wie häufig sie die folgenden Ressourcen in ihren Schulen benutzen: die Schulbücherei, einen Taschenrechner, das Internet und $<$ naturwissenschaftliche> Labors. Die Schülerinnen und Schüler verwendeten hierfür eine Fünfpunkteskala mit folgenden Antwortkategorien: ,nie oder fast nie', , ein paar Mal im Jahr', ,etwa einmal im Monat', ,mehrmals im Monat', ,mehrmals in der Woche‘. Der Index wurde mit Hilfe der weiter oben beschriebenen WARM-Schätzfunktion konstruiert.

\section{Unterrichtszeit}

Der PISA-Index der Unterrichtszeit pro Jahr wurde abgeleitet von den Angaben der Schulleitungen über die Zahl der Unterrichtswochen in einem normalen Schuljahr, die Zahl der < Unterrichtsstunden $>$ in einer Schulwoche und die Zahl von Minuten einer einzelnen < Unterrichtsstunde> . Der Index wurde berechnet als das Produkt dieser drei Faktoren, geteilt durch 60.

\section{Schultyp}

Eine Schule wurde entweder als öffentliche oder als private Einrichtung eingestuft, je nachdem, ob die letzte Entscheidungsgewalt über ihre Angelegenheiten einer staatlichen Behörde oder einem privaten Träger obliegt. Eine Schule wurde als öffentliche Einrichtung eingestuft, wenn sie nach Angaben der Schulleitung der direkten Kontrolle und Verwaltung folgender Stellen unterstand: einer Behörde oder einem Amt für das Bildungswesen, einer Regierungsbehörde selbst oder einem Direktorium (Rat, Ausschuss usw.), dessen Mitglieder zumeist von einer öffentlichen Stellen ernannt oder öffentlich gewählt wurden. Eine Schule wurde als private Einrichtung eingestuft, wenn sie nach Angaben der Schulleitung der Kontrolle und Verwaltung einer nicht staatlichen Organisation (z.B. einer Kirche, einer Gewerkschaft oder einem Unternehmen) unterstand oder wenn sich ihr Direktorium hauptsächlich aus Mitgliedern zusammensetzte, die nicht von einer öffentlichen Stelle ausgewählt worden waren.

Entsprechend dem Grad der Abhängigkeit einer privaten Bildungseinrichtung von öffentlichen Finanzmitteln wurde zwischen „vom Staat abhängigen“ und „vom Staat unabhängigen“ Privatschulen unterschieden. Die Schulleitungen wurden gebeten anzugeben, wie sich die von ihrer Schule in einem normalen Schuljahr insgesamt empfangenen Finanzmittel prozentual auf die folgenden Quellen verteilen: öffentliche Haushalte, Schulgeld, das von den Eltern entrichtet wird, Spenden von Förderern, Geld aus Stiftungen, Sponsoring, Elternspenden sowie andere Quellen. Bildungseinrichtungen wurden als vom Staat abhängige private Schulen eingestuft, wenn mindestens 50\% ihrer Kernfinanzierung aus staatlichen Quellen stammten. Bildungseinrichtungen wurden als unabhängige private Schulen eingestuft, wenn sie weniger als $50 \%$ ihrer Kernfinanzierung von staatlichen Stellen bezogen. 


\section{Anhang A2: Erklärte Varianz der Schülerleistungen}

In mehreren Tabellen der Kapitel 4, 6 und 7 wurde die Veränderung der Schülerleistungen im Zusammenhang mit der Veränderung einer Einheit einer gegebenen Messgröße anhand einer Regressionsanalyse geschätzt. Die Varianz der Schülerleistungen, die mittels dieser Regression erklärt wird, ist in der nachstehendenTabelle veranschaulicht und üblicherweise mit $\mathrm{R}^{2}$ bezeichnet. Zur Definition der Indizes siehe Anhang A1.

Tabelle A2.1

Erklärte Varianz der Schülerleistungen $\left(\mathbf{R}^{2}\right)$ DieWerte sind in Prozent ausgedrückt

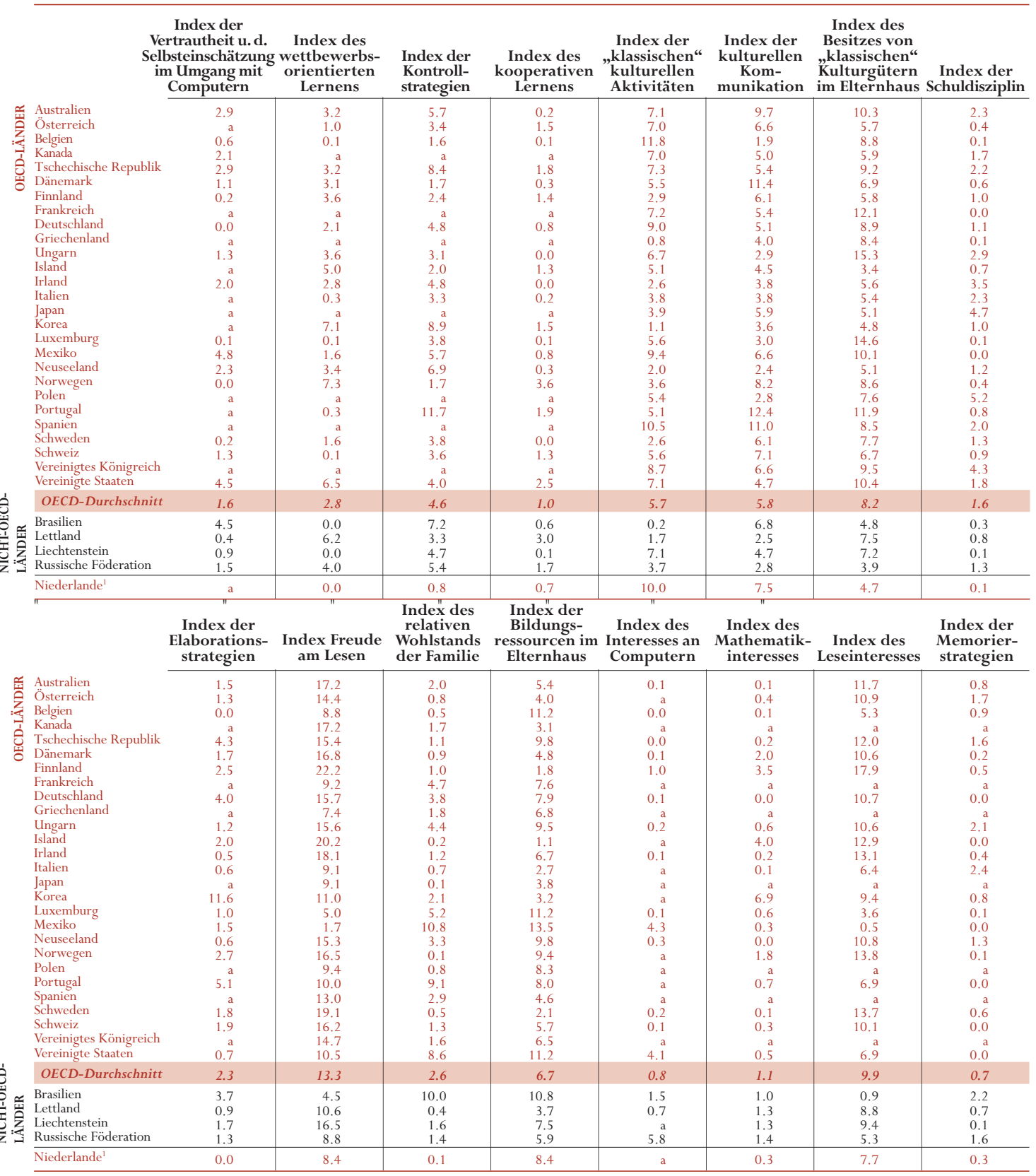

1. Die Beteiligungsquote ist zu niedrig, um die Vergleichbarkeit zu gewährleisten (vgl. Anhang A3). 
Tabelle A2. 1 (Fortsetzung)

Erklärte Varianz der Schülerleistungen $\left(\mathbf{R}^{2}\right)$ DieWerte sind in Prozent ausgedrückt

\begin{tabular}{|c|c|c|c|c|c|c|c|c|c|}
\hline \multirow{28}{*}{ : } & $\begin{array}{r}\text { Ind } \\
\text { mun } \\
\text { haltt } \\
\text { krä } \\
\text { der S }\end{array}$ & $\begin{array}{l}\text { ex der Stim- } \\
\text { g u. Arbeits- } \\
\text { ung der Lehr- } \\
\text { fte aus Sicht } \\
\text { chulleitungen }\end{array}$ & $\begin{array}{c}\text { Index der } \\
\text { Schul- } \\
\text { autonomie }\end{array}$ & $\begin{array}{c}\text { Index der } \\
\text { Schul- } \\
\text { selektivität }\end{array}$ & $\begin{array}{c}\text { Index des } \\
\text { Selbst- } \\
\text { konzepts } \\
\text { Mathematik }\end{array}$ & $\begin{array}{c}\text { Index des } \\
\text { Selbst- } \\
\text { konzepts } \\
\text { Lesen } \\
\end{array}$ & $\begin{array}{c}\text { Index der } \\
\text { sozialen } \\
\text { Kom- } \\
\text { munikation } \\
\end{array}$ & $\begin{array}{c}\text { Index schüler- } \\
\text { bezogener } \\
\text { Faktoren für } \\
\text { das Schul- } \\
\text { klima }\end{array}$ & $\begin{array}{c}\text { Index der } \\
\text { Lehrer- } \\
\text { autonomie }\end{array}$ \\
\hline & Australien & 2.7 & 2.5 & 0.0 & 2.5 & 1.1 & 2.9 & 4.9 & 0.7 \\
\hline & Österreich & 0.5 & 0.2 & 13.4 & 0.9 & 2.3 & 1.3 & 1.8 & 3.6 \\
\hline & Belgien & 8.0 & 0.7 & 0.5 & 0.0 & 0.0 & 0.9 & 18.4 & 0.0 \\
\hline & Kanada & 0.3 & 0.2 & 0.1 & a & $\mathrm{a}$ & 2.0 & 1.1 & 0.1 \\
\hline & Tschechische Republik & 0.2 & 0.0 & 1.3 & 2.3 & 1.9 & 1.2 & 8.5 & 0.6 \\
\hline & Dänemark & 0.6 & 0.0 & 0.0 & 7.1 & 1.8 & 4.0 & 1.7 & 0.5 \\
\hline & Finnland & 0.3 & 0.0 & 0.1 & 8.9 & 5.5 & 0.4 & 0.1 & 0.0 \\
\hline & Frankreich & $\mathrm{m}$ & $\mathrm{m}$ & $\mathrm{m}$ & $\mathrm{a}$ & $\mathrm{a}$ & 1.6 & $\mathrm{~m}$ & $\mathrm{~m}$ \\
\hline & Deutschland & 1.1 & 0.0 & 4.6 & 0.6 & 1.7 & 0.5 & 12.3 & 2.9 \\
\hline & Griechenland & 0.9 & 0.1 & 3.9 & $\mathrm{a}$ & $\mathrm{a}$ & 0.9 & 0.0 & 0.1 \\
\hline & Ungarn & 2.7 & 0.4 & 3.6 & 2.7 & 2.3 & 1.7 & 13.5 & 0.1 \\
\hline & Island & 0.1 & 0.0 & 0.0 & 11.1 & 4.4 & 1.5 & 0.4 & 0.0 \\
\hline & Irland & 0.6 & 1.9 & 0.1 & 2.7 & 0.2 & 1.1 & 3.3 & 0.0 \\
\hline & Italien & 0.1 & 0.1 & a & 2.2 & 1.5 & 0.5 & 13.3 & 0.2 \\
\hline & Japan & 7.5 & 0.0 & 4.9 & $\mathrm{a}$ & $\mathrm{a}$ & 6.3 & 16.0 & 1.3 \\
\hline & Korea & 2.4 & 0.0 & 1.0 & 4.2 & 4.5 & 9.0 & 10.2 & 0.1 \\
\hline & Luxemburg & 4.0 & a & 6.9 & 0.0 & 1.3 & 1.3 & 0.6 & a \\
\hline & Mexiko & 0.5 & 8.2 & 6.8 & 0.3 & 0.1 & 3.4 & 0.3 & 0.1 \\
\hline & Neuseeland & 1.1 & 0.3 & 0.3 & 4.3 & 0.2 & 1.7 & 3.5 & 0.3 \\
\hline & Norwegen & 0.0 & $\mathrm{a}$ & 0.0 & 9.1 & 5.1 & 2.4 & 0.1 & a \\
\hline & Polen & 6.5 & $\mathrm{a}$ & 0.1 & $\mathrm{a}$ & $\mathrm{a}$ & 2.4 & 16.7 & $\mathrm{a}$ \\
\hline & Portugal & 0.8 & 1.0 & 2.0 & 1.7 & 1.0 & 4.6 & 1.3 & 0.1 \\
\hline & Spanien & 2.9 & 4.1 & 0.2 & $\mathrm{a}$ & a & 1.7 & 4.8 & 1.8 \\
\hline & Schweden & 0.4 & 0.0 & 0.1 & 5.3 & 1.9 & 0.3 & 1.4 & 0.3 \\
\hline & Schweiz & 0.2 & 0.0 & 2.4 & 0.0 & 0.0 & 1.6 & 2.4 & 0.1 \\
\hline & Vereinigtes Königreich & 2.8 & 0.5 & 4.5 & $\mathrm{a}$ & a & 1.9 & 11.8 & 0.2 \\
\hline & Vereinigte Staaten & 1.4 & 0.4 & 0.1 & 3.6 & 2.0 & 1.8 & 1.3 & 0.5 \\
\hline \multirow{7}{*}{ 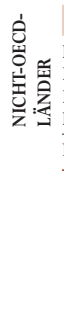 } & OECD-Durchschnitt & 1.9 & 0.9 & 2.3 & 3.5 & 1.9 & 2.2 & 5.8 & 0.6 \\
\hline & Brasilien & 0.7 & 10.4 & 4.3 & 1.0 & 0.3 & 2.9 & 2.9 & 0.1 \\
\hline & Lettland & 0.3 & 0.2 & 4.5 & 0.8 & 1.5 & 0.8 & 0.6 & 0.1 \\
\hline & Liechtenstein & 0.0 & 1.3 & 9.7 & 0.1 & 0.1 & 1.6 & 1.9 & 13.0 \\
\hline & Russische Föderation & 3.5 & 1.1 & 0.0 & 4.8 & 4.4 & 1.8 & 1.1 & 0.1 \\
\hline & Niederlande $^{1}$ & 0.8 & 0.1 & 0.0 & 0.2 & 0.0 & 7.1 & 21.3 & 1.6 \\
\hline & & $\begin{array}{l}\text { Index des } \\
\text { Mangels an } \\
\text { Lehrkräften }\end{array}$ & $\begin{array}{c}\text { Index der } \\
\text { Unterstützung } \\
\text { durch die } \\
\text { Lehrkräfte }\end{array}$ & $\begin{array}{c}\text { Index } \\
\text { lehrkräfte- } \\
\text { bezogener } \\
\text { Faktoren für } \\
\text { das Schulklima }\end{array}$ & $\begin{array}{c}\text { Index des } \\
\text { Schüller-Lehrer- } \\
\text { a Verhältnisses }\end{array}$ & $\begin{array}{l}\text { Index der } \\
\text { Qualität der } \\
\text { Ausstattung } \\
\text { mit Lehr- und } \\
\text { Sachmitteln }\end{array}$ & $\begin{array}{l}\text { Index der } \\
\text { Qualität der } \\
\text { räumlichen } \\
\text { Bedingungen }\end{array}$ & $\begin{array}{c}\text { Index des } \\
\text { Zeitaufwands } \\
\text { für } \\
\text { Hausaufgaben }\end{array}$ & $\begin{array}{l}\text { Internatio- } \\
\text { naler sozioöko- } \\
\text { nomischer } \\
\text { Index der } \\
\text { beruflichen } \\
\text { Stellung (ISEI) }\end{array}$ \\
\hline \multirow{27}{*}{ 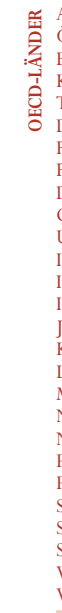 } & Australien & 1.8 & 0.5 & 2.1 & 2.6 & 0.9 & 0.0 & 7.0 & 10.2 \\
\hline & Österreich & 3.5 & 0.0 & 1.5 & 0.1 & 0.1 & 0.1 & 0.1 & 11.0 \\
\hline & Belgien & 3.8 & 0.2 & 5.1 & 0.0 & 0.7 & 1.3 & 8.6 & 14.0 \\
\hline & Kanada & 0.1 & 0.2 & 0.2 & 2.1 & 0.2 & 0.0 & 4.5 & 7.4 \\
\hline & Tschechische Republik & 7.2 & 0.0 & 0.0 & 0.3 & 0.0 & 0.2 & 2.0 & 15.0 \\
\hline & Dänemark & 0.4 & 1.1 & 0.3 & 3.0 & 0.3 & 0.1 & 0.4 & 9.3 \\
\hline & Finnland & 0.0 & 0.3 & 0.1 & 1.4 & 0.2 & 0.1 & 1.5 & 5.5 \\
\hline & Frankreich & $\mathrm{m}$ & $\mathrm{m}$ & $\mathrm{m}$ & 0.1 & $\mathrm{~m}$ & $\mathrm{~m}$ & 9.0 & 12.8 \\
\hline & Deutschland & 11.9 & 1.5 & 1.2 & 0.1 & 4.1 & 1.2 & 0.9 & 15.8 \\
\hline & Griechenland & 0.2 & 0.0 & 0.1 & 0.0 & 0.7 & 1.2 & 15.2 & 10.3 \\
\hline & Ungarn & 1.5 & 0.1 & 1.3 & 1.0 & 1.0 & 0.9 & 7.8 & 16.8 \\
\hline & Island & 0.3 & 0.8 & 0.4 & 3.4 & 0.3 & 0.0 & 0.2 & 4.7 \\
\hline & Irland & 0.1 & 0.0 & 0.0 & 1.4 & 0.0 & 0.2 & 2.2 & 9.9 \\
\hline & Italien & 0.1 & 1.2 & 0.8 & 0.2 & 1.6 & 0.5 & 7.0 & 8.1 \\
\hline & Japan & 2.1 & 0.6 & 4.0 & 3.8 & 1.9 & 0.2 & 6.5 & 0.7 \\
\hline & Korea & 0.9 & 0.5 & 1.3 & 0.9 & 0.0 & 0.2 & 6.2 & 3.5 \\
\hline & Luxemburg & 0.2 & 0.3 & 0.5 & 0.1 & 2.5 & 6.3 & 0.1 & 16.2 \\
\hline & Mexiko & 0.2 & 0.1 & 0.0 & 0.1 & 13.0 & 4.9 & 1.4 & 14.9 \\
\hline & Neuseeland & 1.3 & 0.2 & 1.8 & 1.5 & 1.1 & 0.1 & 3.3 & 9.7 \\
\hline & Norwegen & 0.2 & 1.8 & 0.2 & 3.1 & 0.4 & 0.0 & 1.9 & 7.6 \\
\hline & Polen & 0.6 & 0.7 & 1.3 & 0.2 & 0.6 & 3.0 & 8.8 & 12.4 \\
\hline & Portugal & 0.0 & 0.0 & 0.4 & 0.3 & 0.3 & 2.4 & 1.6 & 15.4 \\
\hline & Spanien & 0.1 & 0.1 & 1.3 & 0.6 & 1.6 & 1.3 & 8.8 & 10.2 \\
\hline & Schweden & 0.7 & 0.4 & 0.1 & 1.7 & 0.4 & 0.4 & 0.0 & 8.8 \\
\hline & Schweiz & 2.0 & 1.6 & 0.1 & 0.1 & 0.5 & 0.8 & 0.1 & 15.9 \\
\hline & Vereinigtes Königreich & 3.8 & 0.4 & 4.9 & 2.9 & 2.8 & 0.8 & 7.9 & 14.7 \\
\hline & Vereinigte Staaten & 1.5 & 0.5 & 1.1 & 3.5 & 0.0 & 0.0 & 7.6 & 11.3 \\
\hline \multirow{6}{*}{ 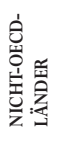 } & OECD-Durchschnitt & 1.7 & 0.5 & 1.2 & 1.3 & 1.3 & 1.0 & 4.5 & 10.8 \\
\hline & Brasilien & 0.5 & 0.3 & 0.8 & 0.1 & 3.1 & 1.2 & 2.6 & 10.4 \\
\hline & Lettland & 0.3 & 1.6 & 0.4 & 0.6 & 0.7 & 0.0 & 2.4 & 5.8 \\
\hline & Liechtenstein & 22.7 & 1.7 & 9.6 & 0.0 & 0.1 & 5.3 & 0.2 & 11.1 \\
\hline & Russische Föderation & 0.0 & 0.4 & 0.5 & 0.1 & 1.0 & 2.5 & 9.9 & 9.2 \\
\hline & Niederlande $^{1}$ & 2.8 & 0.3 & 6.8 & 0.5 & 1.8 & 0.1 & 1.0 & 11.6 \\
\hline
\end{tabular}

1. Die Beteiligungsquote ist zu niedrig, um die Vergleichbarkeit zu gewährleisten (vgl. Anhang A3). 


\section{Anhang A3: PISA-Zielpopulation und PISA-Stichproben}

\section{Das PISA-Konzept der "Erträge“ und die Definition der PISA-Zielpopulation}

PISA 2000 erfasst die kumulativen Bildungserträge und Lernergebnisse zu einem Zeitpunkt, in dem sich die meisten Jugendlichen noch in der Phase der Erstausbildung befinden.

Eine große Herausforderung bei einer internationalen Erhebung besteht darin, ein solches Konzept operationell so umzusetzen, dass die internationale Vergleichbarkeit der Zielpopulationen in den verschiedenen Ländern gewährleistet ist.

Auf Grund länderspezifischer Unterschiede bei Art und Verbreitung von Einrichtungen des Elementarbereichs, dem regulären Einschulungsalter und der institutionellen Struktur der Bildungssysteme ist die Definition über bestimmte Klassenstufen für internationale Vergleiche ungeeignet. Daher werden bei internationalen Vergleichen von Schulleistungen die Populationen in der Regel in Bezug auf ein Zielalter definiert. Einige frühere internationale Erhebungen definierten ihre Zielpopulationen auf der Basis der Klassenstufe, die eine maximale Erfassung einer bestimmten Alterskohorte gewährleistet. Dieser Ansatz weist den Nachteil auf, dass leichte Abweichungen bei der altersmäßigen Verteilung der Schülerinnen und Schüler auf die einzelnen Klassenstufen sowie auch Unterschiede zwischen den Bildungssystemen innerhalb der einzelnen Länder häufig dazu führen, dass in den verschiedenen Ländern unterschiedliche Zielklassen ausgewählt werden, was die Vergleichbarkeit der Ergebnisse zwischen den einzelnen Ländern und zuweilen auch innerhalb der Länder ernsthaft in Frage stellt. Darüber hinaus kann es, da gewöhnlich nicht alle Schülerinnen und Schüler einer gewünschten Altersgruppe bei klassenspezifischen Stichproben repräsentiert sind, zu potentiell gravierenderen Verzerrungen der Ergebnisse kommen, wenn die sich unterrepräsentierten Schülerinnen und Schüler in einigen Ländern typischerweise in der nächst höheren Klasse, in anderen Ländern wiederum in der gerade darunter liegenden Klasse befinden. Dadurch blieben Schülerinnen und Schüler mit einem potentiell höheren Leistungsniveau in den erstgenannten Ländern und Schülerinnen und Schüler mit einem potentiell niedrigeren Leistungsniveau in den an zweiter Stelle genannten Ländern unberücksichtigt.

Um dieses Problem zu vermeiden, geht PISA von einer altersspezifischen Definition ihrer Zielpopulation aus, d.h. einer Definition, die unabhängig ist von der institutionellen Struktur der Bildungssysteme der jeweiligen Länder: PISA erfasste Schülerinnen und Schüler, die zu Beginn der Testperiode im Alter von 15 Jahren und 3 (vollen) Monaten bis zu 16 Jahren und 2 (vollen) Monaten alt waren, gleichgültig welche Klasse oder Art von Bildungseinrichtung sie besuchten und unabhängig davon, ob es sich um eine Ganztags- oder Halbtagsschule handelte (15-Jährige, die sich in der Klassenstufe 6 oder darunter befanden, wurden in PISA nicht miteinbezogen, aber solche Schülerinnen und Schüler gibt es innerhalb der OECD-Länder in größerer Anzahl nur in Brasilien). Bildungseinrichtungen werden in dieser Veröffentlichung generell mit dem Begriff Schulen bezeichnet, obwohl einige davon (insbesondere manche Formen von berufsbildenden Einrichtungen) im landesüblichen Sprachgebrauch vielleicht nicht als Schulen bezeichnet werden. Wie aufgrund dieser Definition zu erwarten, beträgt das Durchschnittsalter der Schülerinnen und Schüler OECD-weit 15 Jahre und 8 Monate, und dieser Wert schwankte zwischen den einzelnen Teilnehmerländern um weniger als 0,2 Jahre.

Auf Grund dieser Populationsdefinition gelangt PISA 2000 zu Feststellungen über die Kenntnisse und Fähigkeiten einer Gruppe von Personen, die in einer vergleichbaren Referenzperiode geboren sind, die aber über unterschiedliche inner- wie außerschulische Bildungserfahrungen verfügen. Im Rahmen von PISA werden diese Kenntnisse und Fähigkeiten als Erträge der Bildung in einem für die verschiedenen Länder gleichen Alter bezeichnet. Je nach der von den einzelnen Ländern verfolgten Politik in Bezug auf Einschulung und Versetzung können sich diese Schülerinnen und Schüler auf ein engeres oder ein breiteres Spektrum von Klassenstufen verteilen. In einigen Ländern sind die Schülerinnen und Schüler der PISA-Zielpopulation zudem unterschiedlichen Bildungssystemen, Bildungsgängen oder -zweigen zuzuordnen.

Wenn ein Land auf den Skalen für Lesekompetenz, mathematische und naturwissenschaftliche Grundbildung eine wesentlich höhere Punktzahl erreicht als ein anderes, ist daraus nicht automatisch zu folgern, dass die Schulen oder bestimmte Teile des Bildungssystems in diesem Land effektiver sind als in dem anderen. Gleichwohl lässt sich aber durchaus die Schlussfolgerung ableiten, dass der kumulative Effekt der Lernerfahrungen in dem Land mit der höheren Punktzahl von der frühen Kindheit bis zum Alter von 15 Jahren, und zwar sowohl im schulischen als auch im außerschulischen Umfeld, zu positiveren Ergebnissen in den von PISA getesteten Grundbildungsbereichen geführt hat.

In der PISA-Zielpopulation nicht enthalten sind Schülerinnen und Schüler des jeweiligen Landes, die eine Schule im Ausland besuchen. 
Um den Ländern entgegenzukommen, die zum Zweck nationaler Analysen nach Klassenstufen aufgeschlüsselte Ergebnisse wünschten, stand bei PISA 2000 eine internationale Option zur Ergänzung der altersbezogenen Stichprobe durch eine klassenstufenbezogene Stichprobe offen.

\section{Erfassungsbereich der PISA-Population}

Alle Länder waren um eine möglichst breite Erfassung der 15-jährigen Schülerinnen und Schüler in ihren nationalen Stichproben bemüht, darunter auch solche in Sondereinrichtungen. Daher erreichte PISA 2000 einen für internationale Erhebungen dieser Art bisher beispiellosen Standard der Populationserfassung.

Die PISA-Stichprobenstandards erlaubten es den Ländern, bis zu insgesamt 5\% der relevanten Population auszuschließen, sei es durch Ausschluss von Schulen oder durch Ausschluss von Schülern innerhalb der Schulen. Mit Ausnahme von drei Ländern erreichten alle Länder mindestens den geforderten Erfassungsgrad von 95\% der national angestrebten Grundgesamtheit, und die Hälfte der Länder erreichte einen Grad von 98\% und darüber. Mit der Festsetzung eines Höchstwerts von 5\% für Populationsausklammerungen wird sichergestellt, dass potentielle Verzerrungen auf Grund von Ausschlüssen innerhalb der Grenzen eines Stichproben-Standardfehlers bleiben dürften.

Tabelle A3.1

PISA-Zielpopulationen und -Stichproben

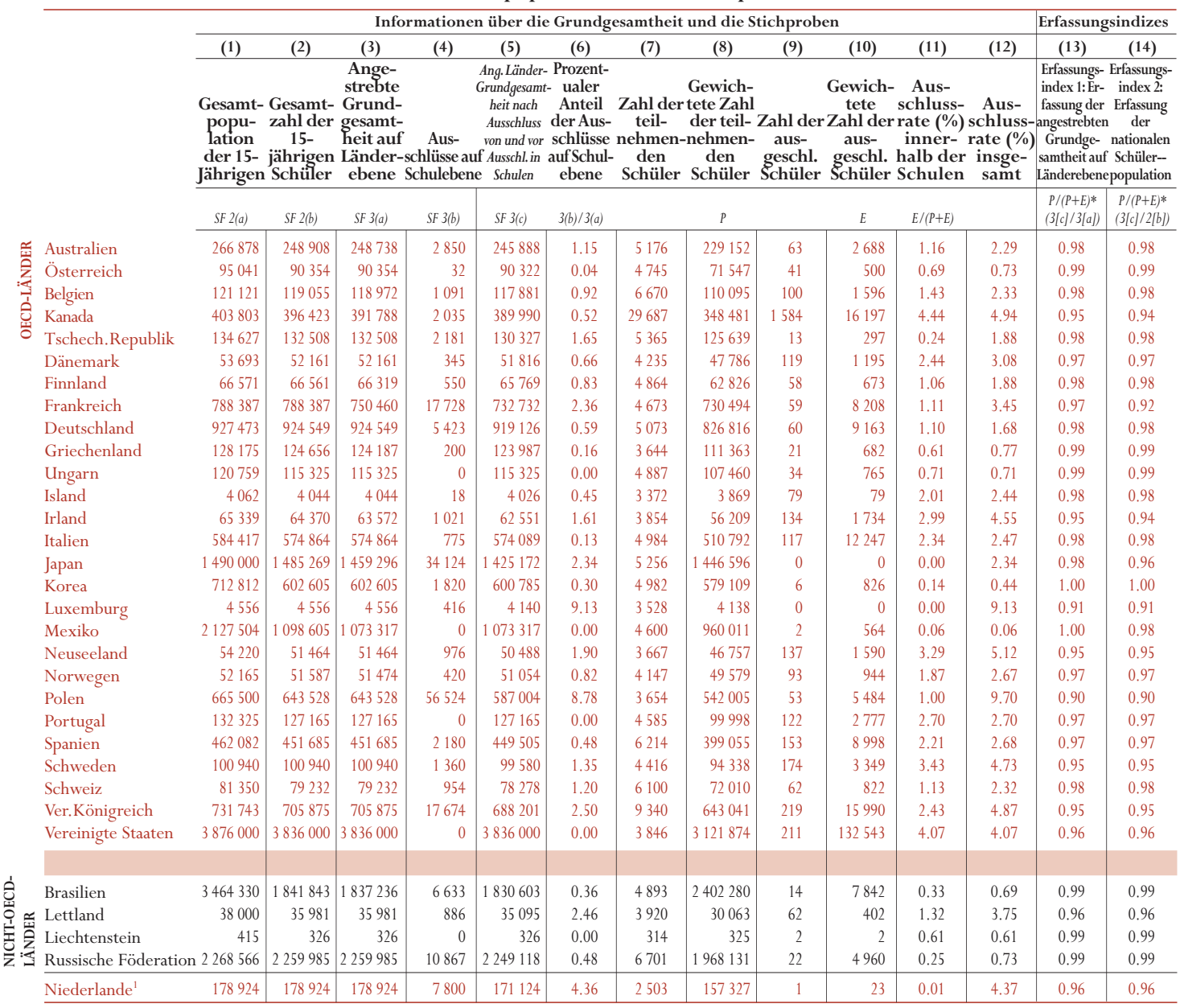

Nähere Einzelheiten hierzu enthält der PISA 2000 Technical Report.

1. Die Beteiligungsquote ist zu niedrig, um die Vergleichbarkeit zu gewährleisten (vgl. Anhang A3). 
Zu den Ausklammerungen innerhalb der oben genannten Grenzen zählen:

- Auf Schulebene: a) Schulen, die geographisch unzugänglich waren oder wo die Durchführung der PISA-Erhebung als nicht praktikabel angesehen wurde; sowie b) Schulen, deren Unterrichtsangebot sich auf die Kategorien beschränkt, die unter der Rubrik „Ausschlüsse innerhalb der Schulen“ definiert sind, wie z.B. Blindenschulen. Der Prozentsatz der 15-Jährigen, die solche Schulen besuchten, musste unter 2,5\% der angestrebten Grundgesamtheit auf Länderebene liegen. Der PISA 2000 Technical Report enthält Angaben über die Größenordnung, Art und Begründung von Ausschlüssen auf Schulebene.

- Auf Schülerebene: a) Schülerinnen und Schüler, die nach dem fachlichen Urteil der Schulleitung oder anderer qualifizierter Mitglieder des Lehrkörpers bzw. auf Grund psychologischer Tests trotz einer gewissen geistigen Behinderung am Unterricht teilnehmen können (darunter auch Schülerinnen und Schüler, die in emotionaler oder geistiger Hinsicht unfähig waren, den allgemeinen PISA-Anweisungen zu folgen); b) Schülerinnen und Schüler mit einer bleibenden körperlichen Behinderung dergestalt, dass sie nicht an der Erhebung unter den PISA-Testbedingungen teilnehmen konnten (funktionsgestörte Schülerinnen und Schüler, die zur Teilnahme in der Lage waren, wurden in die Erhebung einbezogen); und c) Schülerinnen und Schüler mit einer anderen Muttersprache, die weniger als ein Jahr Unterricht in der Testsprache hatten. Aufgrund normaler Disziplinprobleme konnten Schülerinnen und Schüler nicht ausgeschlossen werden. Der Prozentsatz der innerhalb der Schulen ausgeklammerten 15-Jährigen musste weniger als 2,5\% der auf Länderebene angestrebten Grundgesamtheit betragen.

Tabelle A3.1 beschreibt die Zielpopulation der an PISA 2000 teilnehmenden Länder. Nähere Einzelheiten über die Zielpopulation und die Anwendung der PISA-Stichprobenstandards enthält der PISA 2000 Technical Report.

- Spalte 1 zeigt die Gesamtzahl der 15-Jährigen gemäß den nationalen Bevölkerungsregistern für das Jahr 2000.

- Spalte 2 zeigt die Zahl der eine Schule (gemäß obiger Definition) besuchenden 15-Jährigen, also die in Betracht kommende Population.

- Spalte 3 zeigt die angestrebte Grundgesamtheit auf Länderebene. Im Rahmen der Ausschlüsse auf Schulebene konnten die Länder a priori bis zu 0,5\% der Schülerinnen und Schüler von der in Betracht kommenden Population ausschließen, im Wesentlichen aus praktischen Gründen. In folgenden Fällen wurde diese Höchstgrenze überschritten, was aber im Einvernehmen mit dem PISA-Konsortium geschah: In Kanada wurden 1,17\% der in Betracht kommenden Population ausgeschlossen, 0,73\% davon besuchten Schulen in den föderalen Indianerreservaten und 0,43\% Schulen in den Territorien Yukon, Northwest und Nunuvuk. Im Fall Frankreichs umfasste die in Betracht kommende Population auch Schülerinnen und Schüler in den Überseeterritorien (Territoires d'Outre-Mer). Da von den Ländern aber nicht verlangt wurde, die Erhebung auf Schüler in Überseegebieten auszudehnen, die nicht unter die nationalen Bildungssysteme fallen, war es zulässig, diese Schülerinnen und Schüler von der Erhebung auszuschließen. Französische Schülerinnen und Schüler in außerhalb liegenden Departements wurden in PISA 2000 - wie verlangt - einbezogen. In Irland wurden 1,61\% der in Betracht kommenden Population nicht berücksichtigt. Davon entfielen 1,15\% auf Schülerinnen und Schüler in Schulen, die nicht vom Ministerium für Bildung und Wissenschaften gefördert wurden, 0,36\% auf sehr kleine Schulen und 0,12\% auf „Schulen in sozialen Brennpunkten“. Im Fall Japans wurden 4,0\% der in Betracht kommenden Population ausgeschlossen, dabei handelte es sich bei 1,7\% um Schülerinnen und Schüler, die an Fernunterricht oder „anderen kleinen Bildungszweigen (Bekka, Koto-senmon-gakko)“ teilnahmen und bei 2,3\% um Schülerinnen und Schüler in Teilzeitbildung (Teiji-sei). Bei Mexiko wurden 2,3\% der Schülerinnen und Schüler auf Grund der geographischen Abgelegenheit der von ihnen besuchten Schulen nicht berücksichtigt. In Brasilien wurden 15-Jährige in den Klassen 1 bis 6 nicht berücksichtigt, die dort 16\% der 15-jährigen Schulbesucher ausmachen. Diese Ausklammerung ist insofern gerechtfertigt, als diese Schülerinnen und Schüler nicht Teil der PISA-Zielpopulation sind. Wird die Zahl der a priori ausgeschlossenen Schülerinnen und Schüler von der in Betracht kommenden Population subtrahiert, ergibt sich die angestrebte Grundgesamtheit auf Länderebene in Spalte 3.

- Spalte 4 zeigt die Zahl der Schülerinnen und Schüler in Schulen, die von der angestrebten Grundgesamtheit auf Länderebene ausgeschlossen wurde.

- Spalte 5 zeigt die Größe der angestrebten Grundgesamtheit auf Länderebene nach Abzug der Schülerinnen und Schüler in den ausgeschlossenen Schulen. Sie ergibt sich durch Subtraktion der Spalte 4 von Spalte 3. 
- Spalte 6 zeigt den prozentualen Anteil der Schülerinnen und Schüler in den ausgeschlossenen Schulen. Er wird durch Division der Spalte 4 durch Spalte 3 ermittelt.

- Spalte 7 zeigt die Zahl der an PISA 2000 teilnehmenden Schülerinnen und Schüler. Dabei ist zu beachten, dass dieser Wert die im Rahmen nationaler Optionen zusätzlich einbezogenen Schülerinnen und Schüler nicht berücksichtigt. In allen Ländern wurden insgesamt 82105 weitere Schülerinnen und Schüler im Rahmen der nationalen Option zusätzlich getestet.

- Spalte 8 zeigt die gewichtete Zahl der teilnehmenden Schülerinnen und Schüler, d.h. die Zahl der Schülerinnen und Schüler in der definierten Grundgesamtheit auf Länderebene, die die PISA-Stichprobe repräsentiert.

- Jedes Land war bestrebt, den Erfassungsbereich der PISA-Zielpopulation bei den in die Stichprobe einbezogenen Schulen zu maximieren. Für jede einbezogene Schule wurden alle in Betracht kommenden Schülerinnen und Schüler, d.h. die 15-Jährigen, unabhängig von ihrer Klasse, zuerst aufgelistet. Auch die von der Stichprobe auszuschließenden Schülerinnen und Schüler mussten in der Stichprobendokumentation aufgeführt sein, und auf einer Liste waren die Gründe für ihren Ausschluss darzulegen. Spalte 9 gibt die Zahl der ausgeschlossenen Schülerinnen und Schüler an, d.h. jener Schülerinnen und Schüler, die unter eine der weiter oben beschriebenen Kategorien fallen; Spalte 10 enthält die gewichtete Zahl der ausgeschlossenen Schülerinnen und Schüler, d.h. die Gesamtzahl der Schüler in der definierten Grundgesamtheit auf Länderebene, die die Zahl der von der Stichprobe ausgeschlossenen Schülerinnen und Schüler repräsentiert.

- Spalte 11 zeigt den prozentualen Anteil der Schülerinnen und Schüler, die innerhalb der Schulen ausgeschlossen wurden. Er ist berechnet als die gewichtete Zahl der ausgeschlossenen Schülerinnen und Schüler (Spalte 10), dividiert durch die gewichtete Zahl der ausgeschlossenen und der teilnehmenden Schülerinnen und Schüler (Spalte 8 plus Spalte 10).

- Spalte 12 zeigt die Ausschlussrate insgesamt, die dem gewichteten Prozentsatz der angestrebten Grundgesamtheit auf Länderebene entspricht, der von PISA entweder auf Schulebene oder auf innerschulischer Ebene von der Erhebung ausgeschlossen wurde. Sie ergibt sich durch Multiplikation des Prozentsatzes der Ausschlüsse auf Schulebene (Spalte 6) mit 100, abzüglich des Prozentsatzes der innerhalb der Schulen ausgeschlossenen Schülerinnen und Schüler (Spalte 11), und Addition des Prozentsatzes der innerhalb der Schulen ausgeschlossenen Schülerinnen und Schüler (Spalte 11).

- Spalte 13 zeigt einen Index für den Erfassungsgrad der angestrebten Grundgesamtheit auf Länderebene durch die PISA-Stichprobe. Der Index ist ausgedrückt in Prozent der erfassten angestrebten Grundgesamtheit auf Länderebene. Luxemburg, Polen und Brasilien sind die einzigen Länder, in denen weniger als 95\% der Population, die von der PISAErhebung erfasst werden sollte, durch die PISA-Stichproben repräsentiert wurden. Im Fall Polens beläuft sich die Ausschlussrate auf 10\%. Das schließt 6,7\% der 15-Jährigen ein, die Grundschulen besuchten. Da diese Schülerinnen und Schüler bei den PISA-Tests wahrscheinlich schlechter abgeschnitten hätten als die 15-Jährigen in Sekundarschulen, könnte dieser Ausschluss bedeuten, dass die Leistungen der polnischen Schülerinnen und Schüler auf der Gesamtskala Lesekompetenz um zwei Rangpositionen und auf der Skala für naturwissenschaftliche Grundbildung um etwa drei Rangpositionen zu hoch bewertet sind. Dagegen dürfte es bei der Skala für mathematische Grundbildung zu keinerlei Verschiebungen der Rangordnung kommen. Luxemburg hatte eine Ausschlussrate von 9,1\%, was weitgehend darauf zurückzuführen war, dass die Schülerinnen und Schüler in Sprachen unterrichtet wurden, die sich nicht mit den Testsprachen für Luxemburg deckten. Zulässige Ausschlüsse umfassten 28 Sonderschüler, 297 Schüler, die auf die Europäische Schule, und 32 Schüler, die auf die Amerikanische Internationale Schule gingen, 45 Schüler, die andere, nicht in den Zuständigkeitsbereich des Bildungsministeriums fallende Bildungseinrichtungen besuchten sowie 14 Schüler, die auf kleine Schulen gingen. Es steht nicht zu vermuten, dass die Rangposition Luxemburgs auf den PISA-Skalen auf Grund dieser Ausschlüsse zu hoch geschätzt ist. Von den Nicht-OECD-Ländern hatte Brasilien eine Ausschlussrate auf Schulebene von 18\%, was sich aber größtenteils aus dem Anteil der 15-Jährigen in der 5. und 6. Klasse erklärt, die nicht zur PISA-Zielpopulation gehören. Auf Grund der in Brasilien vorgenommenen Ausschlüsse dürften sich keine Verschiebungen in der Rangordnung ergeben. Nähere Einzelheiten hierzu enthält der PISA 2000 Technical Report.

- Spalte 14 zeigt einen Index für den Erfassungsgrad der 15-jährigen Schülerinnen und Schüler durch die PISAStichprobe. Der Index misst den Gesamtanteil der nationalen Schülerpopulation, der durch die Schülerstichprobe erfasst wird. Bei dem Index wurden Ausschlüsse sowohl auf Schulebene als auch auf Schülerebene berücksichtigt. Die nahe bei 100 liegenden Werte weisen darauf hin, dass die PISA-Stichprobe das gesamte Schulsystem nach Definition für PISA 2000 repräsentiert. Der Index ergibt sich aus der gewichteten Zahl der teilnehmenden Schülerinnen und Schüler 
(Spalte 9), dividiert durch die gewichtete Zahl der teilnehmenden und der ausgeschlossenen Schüler (Spalte 9 plus Spalte 11), multipliziert mit der definierten Grundgesamtheit auf Länderebene (Spalte 5), dividiert durch die angestrebte Grundgesamtheit auf Länderebene (mal 100).

\section{Stichprobenziehung und Beteiligungsquoten}

Die Genauigkeit jeglicher Untersuchungsergebnisse hängt von der Qualität der Informationen ab, auf denen die nationalen Stichproben basieren, sowie von der Stichprobenziehung. Für PISA wurden Qualitätsstandards, Verfahren, Instrumente und Verifikationsmechanismen entwickelt, die gewährleisteten, dass die nationalen Stichproben vergleichbare Daten ergaben und dass die Ergebnisse ohne Vorbehalte miteinander verglichen werden können.

Die meisten PISA-Stichproben wurden nach dem Konzept der zweistufigen geschichteten Stichproben konzipiert (soweit die Länder unterschiedliche Stichprobenkonzepte anwandten, sind diese im PISA 2000 Technical Report dokumentiert). Die erste Stufe bestand darin, eine Stichprobe einzelner Schulen zu ziehen, die von 15-jährigen Schülerinnen und Schülern besucht wurden. Die Schulstichprobe wurde systematisch gezogen, mit Wahrscheinlichkeiten proportional zur Größe, die wiederum in Abhängigkeit zur geschätzten Zahl der in Betracht kommenden (15-jährigen) Schülerinnen und Schüler stand. In jedem Land wurden mindestens 150 Schulen (sofern vorhanden) ausgewählt, wobei die Anforderungen für die nationalen Analysen häufig eine etwas größere Stichprobe bedingten. Gleichzeitig mit der Stichprobenziehung wurden Ersatzschulen ermittelt für den Fall, dass eine der ausgewählten Schulen nicht an der PISA-2000-Erhebung teilnehmen wollte.

In Island, Liechtenstein und Luxemburg wurden alle Schulen und alle innerhalb der Schulen in Betracht kommenden Schülerinnen und Schüler in die Stichprobe aufgenommen. Da jedoch nicht alle zu den PISA-Stichproben gehörenden Schülerinnen und Schüler in mathematischer und naturwissenschaftlicher Grundbildung getestet wurden, liegt mit diesen nationalen Stichproben für die Erfassung der Lesekompetenz eine Vollerhebung vor, nicht aber für mathematische und die naturwissenschaftliche Grundbildung.

Experten des PISA-Konsortiums überwachten den Prozess der Stichprobenziehung in allen Teilnehmerländern.

In der zweiten Phase des Auswahlprozesses wurden Schülerstichproben innerhalb der teilnehmenden Schulen gezogen. Nach der Auswahl der Schulen wurde eine Liste aller 15-jährigen Schülerinnen und Schüler an den für die Stichprobe gezogenen Schulen erstellt. Aus dieser Liste wurden 35 Schülerinnen und Schüler mit gleicher Wahrscheinlichkeit ausgewählt (wenn es weniger als 35 gab, wurden alle 15-jährigen Schülerinnen und Schüler ausgewählt).

Die Qualitätsstandards der PISA-Daten setzten eine Mindestteilnahmequote sowohl für die Schulen als auch für die Schülerinnen und Schüler voraus. Diese Standards wurden aufgestellt, um mögliche beteiligungsbedingte Verzerrungen zu minimieren. Bei den Ländern, die diese Standards erfüllten, dürften etwaige Verzerrungen infolge von Nichtbeteiligung unerheblich, d.h. in der Regel geringer als der Stichprobenfehler sein.

Für die ursprünglich ausgewählten Schulen galt eine Mindestbeteiligungsquote von $85 \%$ als erforderlich. Wenn die ursprüngliche Beteiligungsquote auf Schulebene zwischen 65\% und 85\% lag, konnte eine akzeptable Quote jedoch immer noch durch die Einbeziehung von Ersatzschulen erzielt werden. Dieses Verfahren brachte die Gefahr eines erhöhten Beteiligungsbias mit sich. Daher wurden die Teilnehmerländer ersucht, so viele der ursprünglich für die Stichprobe gezogenen Schulen wie möglich zu einer Teilnahme zu veranlassen. Wenn die Beteiligungsquote der Schülerinnen und Schüler zwischen 25\% und 50\% lag, galt die betreffende Schule nicht als teilnehmende Schule, aber die diesbezüglichen Daten wurden in die Datenbank aufgenommen und bei den verschiedenen Schätzungen berücksichtigt. Daten von Schulen mit einer Schülerbeteiligungsquote von weniger als 25\% wurden nicht in die Datenbank aufgenommen.

Für PISA 2000 wurde auch eine minimale Schülerbeteiligungsquote von 80\% innerhalb der teilnehmenden Schulen (ursprünglich ausgewählte und Ersatzschulen) festgesetzt. Diese Mindestquote musste auf Landesebene und nicht zwangsläufig von jeder teilnehmenden Schule erreicht werden. Folgesitzungen waren in Schulen erforderlich, in denen zu wenig Schülerinnen und Schüler an der ursprünglichen Testsitzung teilgenommen hatten. Die Schülerbeteiligungsquoten wurden unter Einbeziehung aller teilnehmenden Schulen - ursprünglich ausgewählte oder Ersatzschulen - und auf der Basis der Teilnahme der Schülerinnen und Schüler an der ursprünglichen Testsitzung wie auch an etwaigen Folgesitzungen errechnet. Schüler, die nicht an der ersten Testsitzung teilgenommen hatten, galten nicht als Teilnehmer, wurden aber in die internationale 
Tabelle A3.2

Beteiligungsquoten

\begin{tabular}{|c|c|c|c|c|c|c|c|c|c|c|c|}
\hline & \multicolumn{3}{|c|}{$\begin{array}{c}\text { Ursprüngl. Stichprobe- } \\
\text { vor Einbeziehung von Ersatzschulen }\end{array}$} & \multicolumn{3}{|c|}{$\begin{array}{c}\text { Endgültige Stichprobe - } \\
\text { nach Einbeziehung von Ersatzschulen }\end{array}$} & \multicolumn{5}{|c|}{$\begin{array}{c}\text { Endgültige Stichprobe -Schüler innerhalb } \\
\text { der Schulen nach Einbeziehung von Ersatzschulen }\end{array}$} \\
\hline & (1) & $(2)$ & (3) & (4) & (5) & (6) & (7) & $(8)$ & (9) & (10) & (11) \\
\hline & $\begin{array}{c}\text { Gewichtete } \\
\text { Schulbetei- } \\
\text { ligungs- } \\
\text { quote vor } \\
\text { Einbeziehung } \\
\text { von Ersatz- } \\
\text { schulen (\%) }\end{array}$ & $\begin{array}{c}\text { Zahl } \\
\text { der } \\
\text { teilnehmen- } \\
\text { den Schulen } \\
\text { g (gewichtet } \\
\text { nach Schüler- } \\
\text { zahlen) }\end{array}$ & $\begin{array}{l}\text { Zahl der Stich- } \\
\text { probenschulen } \\
\text { (effektiv teilnehm- } \\
\text { ende u. nicht } \\
\text { teilnehmende) } \\
\text { (gew. nach } \\
\text { Schülerzahlen) }\end{array}$ & \begin{tabular}{|c} 
Gewichtete \\
Schulbe- \\
teiligungs- \\
quote nach \\
Einbeziehung \\
von Ersatz- \\
schulen (\%)
\end{tabular} & $\begin{array}{c}\text { Zahl } \\
\text { der } \\
\text { teilnehmen-(e } \\
\text { den Schulen } \\
\text { (gewichtet } \\
\text { nach Schüler- } \\
\text { zahlen) }\end{array}$ & $\begin{array}{l}\text { Zahl der Stich- } \\
\text { probenschulen } \\
\text { effektiv teilnehm- } \\
\text { ende u. nicht } \\
\text { teilnehmende) } \\
\text { (gew. nach } \\
\text { Schülerzahlen) }\end{array}$ & \begin{tabular}{|c|} 
Gewichtete \\
Schülerbetei- \\
ligungs- \\
quote nach \\
Einbeziehung \\
von Ersatz- \\
schulen (\%)
\end{tabular} & $\begin{array}{c}\text { Zahl der } \\
\text { getesteten } \\
\text { Schüler } \\
\text { (gewichtet) }\end{array}$ & $\begin{array}{c}\text { Zahl der } \\
\text { Stichproben- } \\
\text { schüler } \\
\text { (teilneh- } \\
\text { mende u. } \\
\text { abwesende) } \\
\text { (gewichtet) }\end{array}$ & $\begin{array}{c}\text { Zahl der } \\
\text { getesteten } \\
\text { Schüler } \\
\text { (ungewichtet) }\end{array}$ & $\begin{array}{c}\text { Zahl der } \\
\text { Stichproben- } \\
\text { schüler } \\
\text { (teilneh- } \\
\text { mende u. } \\
\text { abwesende) } \\
\text { t)(ungewichtet) }\end{array}$ \\
\hline Australien & 80.95 & 197639 & 244157 & 93.65 & 228668 & 244175 & 84.24 & 161607 & 191850 & 5154 & 6173 \\
\hline Österreich & 99.38 & 86062 & 86601 & 100.00 & 86601 & 86601 & 91.64 & 65562 & 71547 & 4745 & 5164 \\
\hline Belgien & 69.12 & 81453 & 117836 & 85.52 & 100833 & 117911 & 93.30 & 88816 & 95189 & 6648 & 7103 \\
\hline Kanada & 87.91 & 335100 & 381165 & 93.31 & 355644 & 381161 & 84.89 & 276233 & 325386 & 29461 & 33736 \\
\hline Tschech.Republik & 95.30 & 123345 & 129422 & 99.01 & 128551 & 129841 & 92.76 & 115371 & 124372 & 5343 & 5769 \\
\hline Dänemark & 83.66 & 42027 & 50236 & 94.86 & 47689 & 50271 & 91.64 & 37171 & 40564 & 4212 & 4592 \\
\hline Finnland & 96.82 & 63783 & 65875 & 100.00 & 65875 & 65875 & 92.80 & 58303 & 62826 & 4864 & 5237 \\
\hline Frankreich & 94.66 & 704971 & 744754 & 95.23 & 709454 & 744982 & 91.19 & 634276 & 695523 & 4657 & 5115 \\
\hline Deutschland & 94.71 & 885792 & 935222 & 94.71 & 885792 & 935222 & 85.65 & 666794 & 778516 & 4983 & 5788 \\
\hline Griechenland & 83.91 & 92824 & 110622 & 99.77 & 130555 & 130851 & 96.83 & 136919 & 141404 & 4672 & 4819 \\
\hline Ungarn & 98.67 & 209153 & 211969 & 98.67 & 209153 & 211969 & 95.31 & 100807 & 105769 & 4883 & 5111 \\
\hline Island & 99.88 & 4015 & 4020 & 99.88 & 4015 & 4020 & 87.09 & 3372 & 3872 & 3372 & 3872 \\
\hline Irland & 85.56 & 53164 & 62138 & 87.53 & 54388 & 62138 & 85.59 & 42088 & 49172 & 3786 & 4424 \\
\hline Italien & 97.90 & 550932 & 562763 & 100.00 & 562755 & 562755 & 93.08 & 475446 & 510792 & 4984 & 5369 \\
\hline Japan & 82.05 & 1165576 & 1420533 & 90.05 & 1279121 & 1420533 & 96.34 & 1267367 & 1315462 & 5256 & 5450 \\
\hline Korea & 100.00 & 589018 & 589018 & 100.00 & 589018 & 589018 & 98.84 & 572767 & 579470 & 4982 & 5045 \\
\hline Luxemburg & 93.04 & 3852 & 4140 & 93.04 & 3852 & 4140 & 89.19 & 3434 & 3850 & 3434 & 3850 \\
\hline Mexiko & 92.69 & 985745 & 1063524 & 100.00 & 1063524 & 1063524 & 93.95 & 903100 & 961283 & 4600 & 4882 \\
\hline Neuseeland & 77.65 & 39328 & 50645 & 86.37 & 43744 & 50645 & 88.23 & 35616 & 40369 & 3667 & 4163 \\
\hline Norwegen & 85.95 & 43207 & 50271 & 92.25 & 46376 & 50271 & 89.28 & 40908 & 45821 & 4147 & 4665 \\
\hline Polen & 79.11 & 432603 & 546842 & 83.21 & 455870 & 547847 & 87.70 & 393675 & 448904 & 3639 & 4169 \\
\hline Portugal & 95.27 & 120521 & 126505 & 95.27 & 120521 & 126505 & 86.28 & 82395 & 95493 & 4517 & 5232 \\
\hline Spanien & 95.41 & 423900 & 444288 & 100.00 & 444288 & 444288 & 91.78 & 366301 & 399100 & 6214 & 6764 \\
\hline Schweden & 99.96 & 100534 & 100578 & 99.96 & 100534 & 100578 & 87.96 & 82956 & 94312 & 4416 & 5017 \\
\hline Schweiz & 91.81 & 89208 & 97162 & 95.84 & 92888 & 96924 & 95.13 & 65677 & 69037 & 6084 & 6389 \\
\hline Ver.Königreich & 61.27 & 400737 & 654095 & 82.14 & 537219 & 654022 & 80.97 & 419713 & 518358 & 9250 & 11300 \\
\hline Vereinigte Staaten & 56.42 & 2013101 & 3567961 & 70.33 & 2503666 & 3559661 & 84.99 & 1801229 & 2119392 & 3700 & 4320 \\
\hline Lettland & 82.39 & 29354 & 35628 & 88.51 & 31560 & 35656 & 90.73 & 24403 & 26895 & 3915 & 4305 \\
\hline Liechtenstein & 100.00 & 327 & 327 & 100.00 & 327 & 327 & 96.62 & 314 & 325 & 314 & 325 \\
\hline Russische Föderation & 98.84 & 4445841 & 4498235 & 99.29 & 4466335 & 4498235 & 96.21 & 1903348 & 1978266 & 6701 & 6981 \\
\hline Niederlande $^{1}$ & 27.13 & 49019 & 180697 & 55.50 & 100283 & 180697 & 84.03 & 72656 & 86462 & 2503 & 2958 \\
\hline
\end{tabular}

1. Die Beteiligungsquote ist zu niedrig, um die Vergleichbarkeit zu gewährleisten (s.o.).

Datenbank aufgenommen und bei den Statistiken in dieser Veröffentlichung berücksichtigt, wenn sie bei der zweiten Testsitzung anwesend waren und zumindest Angaben über die berufliche Tätigkeit des Vaters oder der Mutter gemacht hatten.

Tabelle A3.2 gibt Auskunft über die Beteiligungsquoten auf Schüler- und auf Schulebene, vor und nach Einbeziehung von Ersatzschulen.

- Spalte 1 zeigt die gewichtete Beteiligungsquote der Schulen vor Einbeziehung von Ersatzschulen. Sie wird durch Division von Spalte 2 durch Spalte 3 ermittelt. Die Niederlande, das Vereinigte Königreich und die Vereinigten Staaten wurden den PISA-Anforderungen im Hinblick auf die Beteiligungsquoten vor Einbeziehung von Ersatzschulen nicht gerecht. Im Vereinigten Königreich lag die ursprüngliche Beteiligungsquote um 3,7\% und in den Vereinigten Staaten um 8,6\% unter dem erforderlichen Schwellenwert. Beide Länder legten dem PISA-Konsortium umfangreiche Belege vor, die eine Evaluierung der erwarteten Leistungen der nicht teilnehmenden Schulen erlaubten. Auf der Basis dieser Daten entschied die Technische Beratungsgruppe von PISA, dass der Effekt dieser Abweichungen auf die Erhebungsergebnisse unerheblich sei. Die Ergebnisse für diese beiden Länder wurden in sämtlichen Analysen berücksichtigt. Für die Niederlande betrug die ursprüngliche Beteiligungsquote lediglich 27\%. Aus diesem Grund leitete das PISA-Konsortium ergänzende Analysen ein, die bestätigten, dass die niederländischen Daten hinreichend verlässlich sein und für gewisse relationale Analysen verwendet werden könnten. Trotz dieser Folgerung war die Beteiligungsquote zu niedrig, als dass darauf vertraut werden 
konnte, dass die Stichprobenergebnisse die Resultate für die gesamte niederländische Bevölkerung zu dem für PISA 2000 erforderlichen Grad an Genauigkeit und Präzision widerspiegelt. Wenn unterstellt wird, dass die durch Nichtbeteiligung bedingten Verzerrungen unerheblich bis moderat sind, könnten die Rangposition der Niederlande unter den Ländern mit einem Konfidenzgrad von 95\% auf der Gesamtskala Lesekompetenz zwischen der 2. und der 14. Stelle, auf der Skala für mathematische Grundbildung zwischen der 1. und der 4. Stelle und auf der Skala für naturwissenschaftliche Grundbildung zwischen der 3. Und der 14. Stelle angesiedelt werden (nähere Einzelheiten hierzu enthält der PISA 2000 Technical Report). Daher können die mittleren Punktzahlen für die Niederlande nicht mit jenen der anderen Länder verglichen werden. Somit wurden die Niederlande in den Tabellen, in denen das Schwergewicht auf einem Vergleich der Mittelwerte liegt, nicht aufgeführt. Wo die Leistungen von Untergruppen wiedergegeben sind, sollten lediglich die relativen Leistungsunterschiede zwischen den relevanten Untergruppen innerhalb der Niederlande berücksichtigt werden, und die Mittelwerte sollten nicht mit denen aus anderen Ländern verglichen werden.

- Spalte 2 zeigt die gewichtete Zahl der teilnehmenden Schulen vor Einbeziehung von Ersatzschulen (gewichtet nach der Schülerzahl).

- Spalte 3 zeigt die gewichtete Zahl der für die Stichprobe gezogenen Schulen vor Einbeziehung von Ersatzschulen (sowohl effektiv teilnehmende als auch nicht teilnehmende Schulen).

- Spalte 4 zeigt die gewichtete Schulbeteiligungsquote nach Einbeziehung von Ersatzschulen. Sie ergibt sich durch Division von Spalte 5 durch Spalte 6.

- Spalte 5 zeigt die gewichtete Zahl der teilnehmenden Schulen, nach Einbeziehung von Ersatzschulen (gewichtet nach der Schülerzahl).

- Spalte 6 zeigt die gewichtete Zahl der für die Stichprobe gezogenen Schulen nach Einbeziehung von Ersatzschulen (sowohl effektiv teilnehmende als auch nicht teilnehmende Schulen).

- Spalte 7 zeigt die gewichtete Schülerbeteiligungsquote nach Einbeziehung von Ersatzschulen. Sie ergibt sich durch Division von Spalte 8 durch Spalte 9.

- Spalte 8 zeigt die gewichtete Zahl der getesteten Schülerinnen und Schüler.

- Spalte 9 zeigt die gewichtete Zahl der Stichprobenschüler (sowohl an der Testsitzung teilnehmende als auch dabei abwesende Schülerinnen und Schüler).

- Spalte 10 zeigt die ungewichtete Zahl der getesteten Schülerinnen und Schüler.

- Spalte 11 zeigt die ungewichtete Zahl der Stichprobenschüler (sowohl an der Testsitzung teilnehmende als auch dabei abwesende Schülerinnen und Schüler). 


\section{Anhang A4: Standardfehler, Signifikanztests und Ländervergleich}

Die in diesem Bericht enthaltenen Statistiken stellen Schätzwerte der nationalen Leistung auf der Basis der Schülerstichproben dar, und nicht etwa aus den Antworten sämtlicher Schülerinnen und Schüler eines Landes auf sämtliche Fragen errechnete Werte. Daher ist es wichtig, die mögliche Höhe des Messfehlers dieser Schätzungen zu kennen. In PISA 2000 wird bei jeder Schätzung ein Messfehler angegeben, der durch den Standardfehler (S.E.) ausgedrückt ist. Die Verwendung von Konfidenzintervallen ermöglicht es, Schlüsse in Bezug auf die Populationsdurchschnittswerte und -prozentsätze zu ziehen und dabei den an die Stichprobenschätzungen geknüpften Messfehler zu berücksichtigen. Es kann davon ausgegangen werden, dass das tatsächlich beobachtete statistische Ergebnis einer gegebenen Population in 95 von 100 Wiederholungsmessungen mit unterschiedlichen Stichproben derselben Population innerhalb des Konfidenzintervalls liegen würde.

Die Leser sind häufig in erster Linie daran interessiert, ob sich ein bestimmter Wert für ein gegebenes Land von einem zweiten Wert für dasselbe Land oder für ein anderes Land unterscheidet, z.B. ob in einem bestimmten Land Mädchen bessere Leistungen als Jungen aufweisen. In den Tabellen und Abbildungen dieses Berichts werden Unterschiede als statistisch signifikant bezeichnet, wenn ein Unterschied dieser Größe oder darüber in weniger als 5\% der Fälle beobachtet würde, in denen bei den entsprechenden Populationswerten ein solcher Unterschied tatsächlich nicht vorhanden wäre. Entsprechend wird das Risiko, eine Korrelation als signifikant zu bezeichnen, wenn effektiv keine Korrelation zwischen zwei Messgrößen besteht, auf 5\% begrenzt.

Zwar ist die Wahrscheinlichkeit, bestimmte Differenzen fälschlicherweise für statistisch signifikant zu erklären, bei jedem einzelnen Vergleich gering (5\%), sie nimmt jedoch zu, wenn mehrere Vergleiche gleichzeitig gemacht werden.

Hier kann eine Anpassung vorgenommen werden, die die maximale Wahrscheinlichkeit, dass zumindest einmal bei allen vorgenommenen Vergleichen Unterschiede fälschlicherweise als statistisch signifikant bezeichnet werden, auf 5\% reduziert. Eine solche Anpassung wurde auf der Basis der Bonferroni-Methode bei den in den Kapiteln 2 und 3 enthaltenen Abbildungen mit Ländermittelwerten durchgeführt, da in einem solchen Kontext das Interesse der Leser wahrscheinlich darauf gerichtet ist, das Ergebnis eines Landes mit den Ergebnissen aller anderen Länder zu vergleichen.

Bei allen anderen Tabellen und Abbildungen sollte der Leser beachten, dass die Vergleiche der Länder in Verbindung mit einem Signifikanzniveau von 5\%, wenn bei einer gegebenen Messgröße keine wirklichen Differenzen bestünden, fälschlicherweise bei 0,05-mal der Zahl der vorgenommenen Vergleiche Differenzen ermitteln würden. Obwohl die in PISA angewandten Signifikanztests zur Ermittlung geschlechtsspezifischer Unterschiede gewährleisten, dass die Wahrscheinlichkeit einer fälschlichen Ermittlung von geschlechtsspezifischen Unterschieden für jedes Land weniger als $5 \%$ beträgt, würden z.B. bei einem Vergleich, der Unterschiede für 27 Länder zeigt, durchschnittlich 1,4 Fälle $(0,05$ x 27) mit signifikanten geschlechtsspezifischen Unterschieden ermittelt, selbst wenn in keinem der Länder wirklich geschlechtsspezifische Unterschiede vorlägen. Dasselbe gilt für andere Statistiken, für die im Rahmen dieser Veröffentlichung Signifikanztests durchgeführt wurden, wie z.B. Korrelation und Regressionskoeffizienten. 


\section{Anhang A5: Qualitätssicherung}

Qualitätssicherungsverfahren wurden in sämtlichen Teilen von PISA durchgeführt.

Die einheitliche Qualität und sprachliche Äquivalenz der PISA-Erhebungsinstrumente wurden dadurch erleichtert, dass den Ländern äquivalente Originalfassungen der Erhebungsinstrumente in Englisch und Französisch vorgelegt und die Länder (soweit es sich um solche handelte, in denen die Schülerleistungen nicht in diesen zwei Sprachen erhoben wurden) aufgefordert wurden, ausgehend von den beiden Originalversionen zwei unabhängige Übersetzungen anzufertigen und dann zusammenzufassen. Es wurden auch genaue Übersetzungsrichtlinien vorgegeben mit einer Beschreibung dessen, was mit jedem Item gemessen werden sollte, und Anwei-sungen hinsichtlich der Auswahl und Ausbildung der Übersetzer erteilt. Für jedes Land wurden die Übersetzung und das Format der Erhebungsinstrumente durch Experten des PISA-Konsortiums überprüft (deren Muttersprache die Unterrichtssprache in dem betreffenden Land war, und die ausreichende Kenntnisse bezüglich der Bildungssysteme hatten), bevor sie im PISA-Feldtest und in der PISA-Haupterhebung eingesetzt wurden. Experten aus den Teilnehmerländern wurden damit beauftragt, die Kodieranweisungen zu übersetzen und zur Prüfung vorzulegen. Nähere Informationen über die PISA-Übersetzungsverfahren enthält der PISA 2000 Technical Report.

Die Erhebung wurde mit Hilfe standardisierter Verfahren durchgeführt. Das PISA-Konsortium stellte umfassende Handbücher zur Verfügung, die den Ablauf der Erhebung erklärten und u.a. präzise Anweisungen für die Arbeit der Schulkoordinatoren sowie Vorlagen für die Testleiter zum Gebrauch bei den Testsitzungen enthielten. Das PISA-Konsortium überprüfte die nationalen Übersetzungen und die Anpassung dieser Handbücher.

Um die Glaubwürdigkeit von PISA im Hinblick auf Validität und Unvoreingenommenheit zu gewährleisten, und einen einheitlichen Ablauf der Testsitzungen zu fördern, wurden die Testleiterinnen und Testleiter in den Teilnehmerländern nach folgenden Kriterien ausgewählt:Vorgeschrieben wurde, dass die Leiterinnen und Leiter der vorgesehenen PISA-Testsitzungen nicht zugleich die Fachlehrer der teilnehmenden Schülerinnen und Schüler in den Bereichen Lesen, Mathematik und Naturwissenschaften sein durften; empfohlen wurde, dass die Testleiterinnen und Testleiter nicht Mitglied des Kollegiums einer Schule sein sollten, an der sie die Leitung der PISA-Tests übernahmen; als wünschenswert wurde erachtet, dass die Testleiterinnen und Testleiter nicht zum Kollegium einer für die PISA-Stichprobe gezogenen Schule gehören sollten. Die Teilnehmerländer organisierten für die Testleiterinnen und Testleiter einen individuellen Trainingskurs.

Die Teilnehmerländer waren nicht befugt, ohne vorherige Genehmigung des PISA-Konsortiums Änderungen an der Vorlage für die Testsitzung und an den im Testleitungshandbuch beschriebenen Anweisungen vorzunehmen. Es war Aufgabe der Teilnehmerländer sicherzustellen, dass: die Testleiterinnen und Testleiter mit den Schulkoordinatoren bei der Vorbereitung der Testsitzung zusammenarbeiteten, u.a. bei der Aktualisierung der Unterlagen über den bisherigen Bildungsweg der Schülerinnen und Schüler sowie der Identifizierung ausgeschlossener Schülerinnen und Schüler; für die kognitiven Items keine zusätzliche Zeit eingeräumt wurde (während es erlaubt war, für die Beantwortung des Schülerfragebogens mehr Zeit zuzugestehen); kein Instrument vor Beginn der zwei einstündigen Teile der kognitiven Sitzung bekannt gegeben wurde; die Testleiterinnen und Testleiter den Stand der Schülerbeteiligung auf den Unterlagen über die Bildungswege der Schülerinnen und Schüler festhielten und ein Formular für den Sitzungsbericht ausfüllten; kein kognitives Instrument von den Testleiterinnen oder Testleitern vor der Testsitzung fotokopiert oder an irgendjemand ausgeliehen wurde; und die Testleiterinnen und Testleiter das Material unmittelbar nach Abschluss der Testsitzungen an das nationale Zentrum zurückgaben.

Die nationalen Projektmanager wurden dazu angehalten, eine Folgesitzung zu organisieren, wenn mehr als 15\% der PISA-Stichprobe nicht an der ursprünglichen Testsitzung teilnehmen konnten.

Für das Qualitätsmonitoring zuständige nationaleVertreter des PISA-Konsortiums besuchten alle nationalen Zentren, um die Verfahren der Datenerhebung zu überprüfen. Schließlich besuchten „School Quality Monitors“ aus dem PISA-Konsortium eine Stichprobe von 25\% der Schulen während der Erhebung. Nähere Informationen über die Feldoperationen enthält der PISA 2000 Technical Report.

Eine speziell für PISA 2000 konzipierte Software erleichterte die Dateneingabe, deckte häufig gemachte Fehler während der Dateneingabe auf und machte den Prozess der Datenbereinigung einfacher. Durch Trainingskurse wurden die nationalen Projektleiter mit diesem Verfahren vertraut gemacht. Eine Beschreibung der für PISA angewandten Verfahren zur Qualitätssicherung und deren Ergebnisse enthält der PISA 2000 Technical Report. 


\section{Anhang A6: Entwicklung der PISA-Erhebungsinstrumente}

Die Erhebungsinstrumente für PISA 2000 wurden in einem interaktiven Prozess zwischen dem PISA-Konsortium, den verschiedenen Expertenausschüssen, den Regierungen der OECD-Länder sowie nationalen Sachverständigen entwickelt. Eine internationale Expertengruppe übernahm - in enger Konsultation mit den Teilnehmerländern - die Führungsrolle bei der Ermittlung von Kenntnissen und Kompetenzen, die in den jeweiligen Erhebungsbereichen als unabdingbar für die Fähigkeit des Einzelnen erachtet werden, in vollem Umfang an einer erfolgreichen modernen Gesellschaft teilzuhaben und einen Beitrag dazu zu leisten. Eine Beschreibung der Erhebungsbereiche - die Rahmenkonzeption - diente den Teilnehmerländern und anderen Fachleuten dann für die Entwicklung von Testaufgaben als Grundlage für Beiträge zum Aufgabenpool. Die Erarbeitung dieser Rahmenkonzeption umfasste folgende Schritte:

- Entwicklung einer Arbeitsdefinition für den jeweiligen Bereich und Beschreibung der Annahmen, die dieser Definition zu Grunde liegen;

- Evaluierung von Möglichkeiten der Organisation der Aufgabensets in einer Weise, die gewährleistet, dass die Ergebnisberichte Politikern und Forschern Aufschluss über die Leistungen von 15-jährigen Schülerinnen und Schülern der Teilnehmerländer in jedem Erhebungsbereich geben;

- Festlegung einer Reihe von Schlüsselmerkmalen, die bei der Entwicklung von international einsetzbaren Testaufgaben zu berücksichtigen sind;

- Operationalisierung der bei der Testkonstruktion zu berücksichtigenden Schlüsselmerkmale, wobei den Definitionen die vorhandene Literatur sowie die Erfahrungen aus anderen großen Leistungsstudien zu Grunde gelegt werden;

- Validierung der Variablen und Bestimmung ihres Beitrags zur Erklärung der Schwierigkeit der Aufgaben in den Teilnehmerländern; und

- Entwicklung eines Interpretationsschemas für die Ergebnisse.

Tabelle A6.1

Itemverteilung nach den Dimensionen der PISA-Rahmenkonzeption auf der Gesamtskala Lesekompetenz

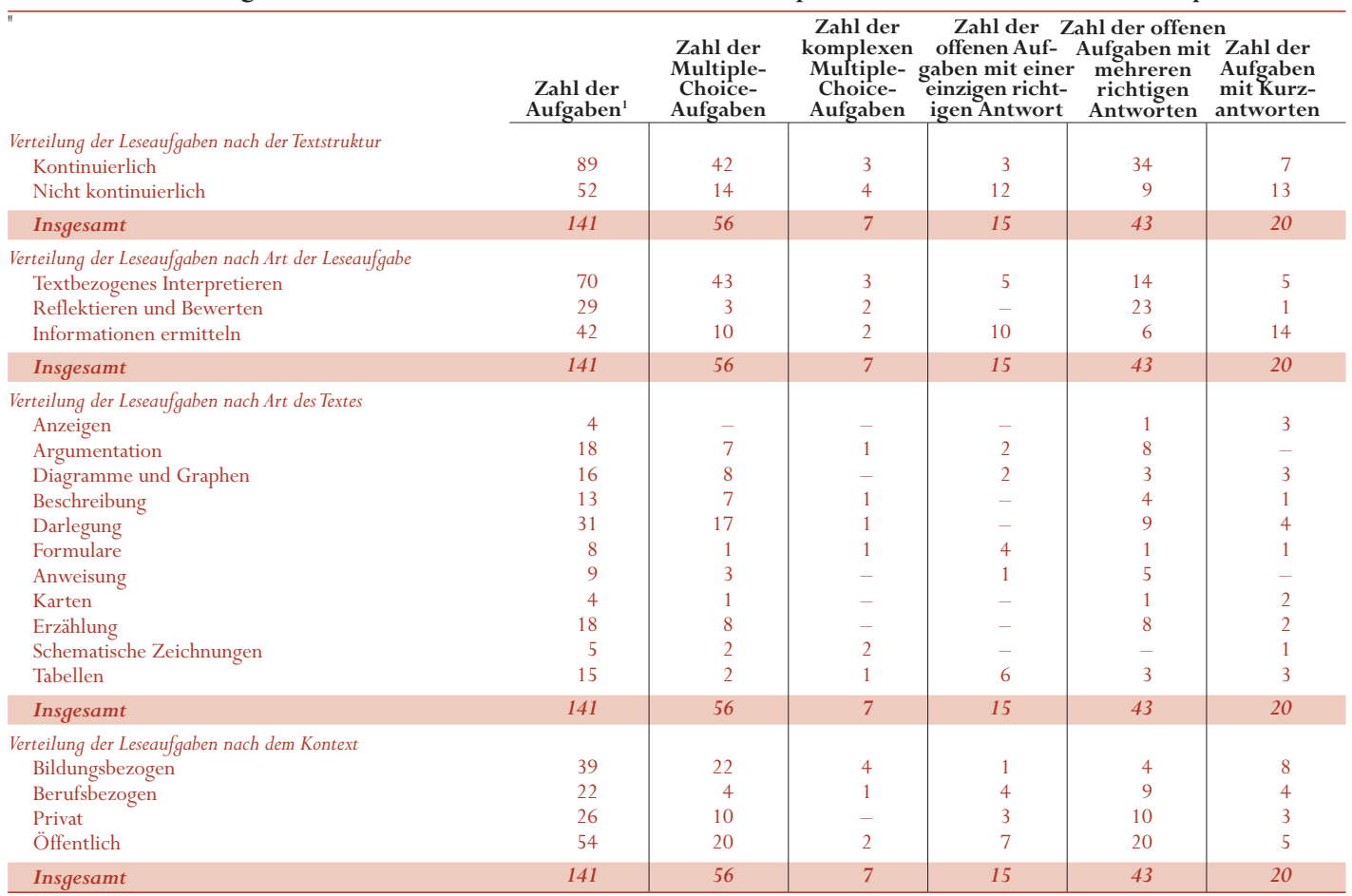

1. Neun Aufgaben wurden bei späteren Analysen eliminiert. 
Die Rahmenkonzeptionen wurden auf wissenschaftlicher wie auch politischer Ebene verabschiedet und bildeten dann die Grundlage für die Entwicklung der Erhebungsinstrumente (OECD, 2000). Sie lieferten eine gemeinsame Sprache und boten den Teilnehmerländern eine Grundlage zur Konsensbildung im Hinblick auf die Ziele der PISA-Messung.

Danach wurden Erhebungsitems entsprechend den Intentionen der Rahmenkonzeptionen entworfen und in einem Feldtest in allen Teilnehmerländern erprobt, bevor ein endgültiger Aufgabenset für die PISA-2000-Haupterhebung ausgewählt wurde. Die Tabellen A6.1 bis A6.3 zeigen die Verteilung der Erhebungsitems für PISA 2000 nach den verschiedenen Dimensionen der PISA-Rahmenkonzeptionen.

Es wurde darauf geachtet, der nationalen, kulturellen und sprachlichen Vielfalt unter den OECD-Ländern gebührend Rechnung zu tragen. Im Zuge dieser Bemühungen hat das PISA-Konsortium zusätzlich zu den von ihm entwickelten Items von den Teilnehmerländern übermitteltes Erhebungsmaterial aufgenommen, insofern das vom Konsortium eingesetzte multinationale Expertenteam dieses für die Aufgabenentwicklung von den Anforderungen der PISA-Rahmenkonzeptionen her für geeignet hielt. Infolgedessen wurden in den Aufgabenpool Items aus Australien, Österreich, Belgien, der Tschechischen Republik, Dänemark, Finnland, Frankreich, Deutschland, Griechenland, Irland, Italien, Japan, Korea, Neuseeland, Norwegen, der Russischen Föderation, Schweden, der Schweiz, dem Vereinigten Königreich und den Vereinigten Staaten aufgenommen. Der Anteil der von den Teilnehmerländern eingereichten Items betrug sowohl für den Feldtest als auch für die Haupterhebung etwas mehr als $50 \%$.

Für den Feldtest wurden ungefähr 290 Einheiten und 1169 Items beigesteuert bzw. entwickelt, darunter etwa 150 Leseeinheiten mit rund 781 Items für Lesekompetenz. Nach Abschluss des ersten Konsultationsprozesses mit den Ländern wurden im Feldtest 69 Leseeinheiten mit 342 Lese-Items eingesetzt. Bei diesen Leseeinheiten stammte das Stimulusmaterial in 24 Fällen aus nationalen Beiträgen, während 26 Einheiten auf das PISA-Konsortium und weitere 19 auf die Internationale Erhebung über Grad und Verteilung elementarer Grundqualifikationen Erwachsener (IALS) zurückgingen. Material wurde aus

Tabelle A6.2

Itemverteilung nach den Dimensionen der PISA-Rahmenkonzeption auf der Skala für mathematische Grundbildung

\begin{tabular}{|c|c|c|c|c|}
\hline & Zahl der Aufgaben $^{1}$ & $\begin{array}{l}\text { Zahl der Multiple- } \\
\text { Choice-Aufgaben }\end{array}$ & $\begin{array}{c}\text { Zahl der offenen } \\
\text { Aufgaben mit einer } \\
\text { einzigen richtigen } \\
\text { Antwort }\end{array}$ & $\begin{array}{c}\text { Zahl der offenen } \\
\text { Aufgaben mit } \\
\text { mehreren richtigen } \\
\text { Antworten }\end{array}$ \\
\hline \multicolumn{5}{|c|}{ Verteilung der Mathematikaufgaben nach "Leitideen" } \\
\hline Wachstum und Veränderung & 18 & 6 & 9 & 3 \\
\hline Raum und Form & 14 & 5 & 9 & - \\
\hline Insgesamt & 32 & 11 & 18 & 3 \\
\hline \multicolumn{5}{|c|}{ Verteilung der Mathematikaufgaben nach Stoffgebieten } \\
\hline Algebra & 5 & - & 4 & 1 \\
\hline Funktionen & 5 & 4 & - & 1 \\
\hline Geometrie & 8 & 3 & 5 & - \\
\hline Größen und Größenordnung & 7 & 3 & 4 & - \\
\hline Arithmetik & 1 & - & 1 & - \\
\hline Statistik & 6 & 1 & 4 & 1 \\
\hline Insgesamt & 32 & 11 & 18 & 3 \\
\hline \multicolumn{5}{|c|}{ Verteilung der Mathematikaufgaben nach Kompetenzklasse } \\
\hline Klasse 1 & 10 & 4 & 6 & - \\
\hline Klasse 2 & 20 & 7 & 11 & 2 \\
\hline Klasse 3 & 2 & - & 1 & 1 \\
\hline Insgesamt & 32 & 11 & 18 & 3 \\
\hline \multicolumn{5}{|c|}{ Verteilung der Mathematikaufgaben nach dem Kontext } \\
\hline Gemeinschaft & 4 & - & 2 & 2 \\
\hline Bildungsbezogen & 6 & 2 & 3 & 1 \\
\hline Berufsbezogen & 3 & 1 & 2 & - \\
\hline Privat & 12 & 6 & 6 & - \\
\hline Wissenschaftlich & 7 & 2 & 5 & - \\
\hline Insgesamt & 32 & 11 & 18 & 3 \\
\hline
\end{tabular}

1. Eine Aufgabe wurde bei späteren Analysen eliminiert. 
Tabelle A6.3

Itemverteilung nach den Dimensionen der PISA-Rahmenkonzeption auf der Skala für naturwissenschaftliche Grundbildung

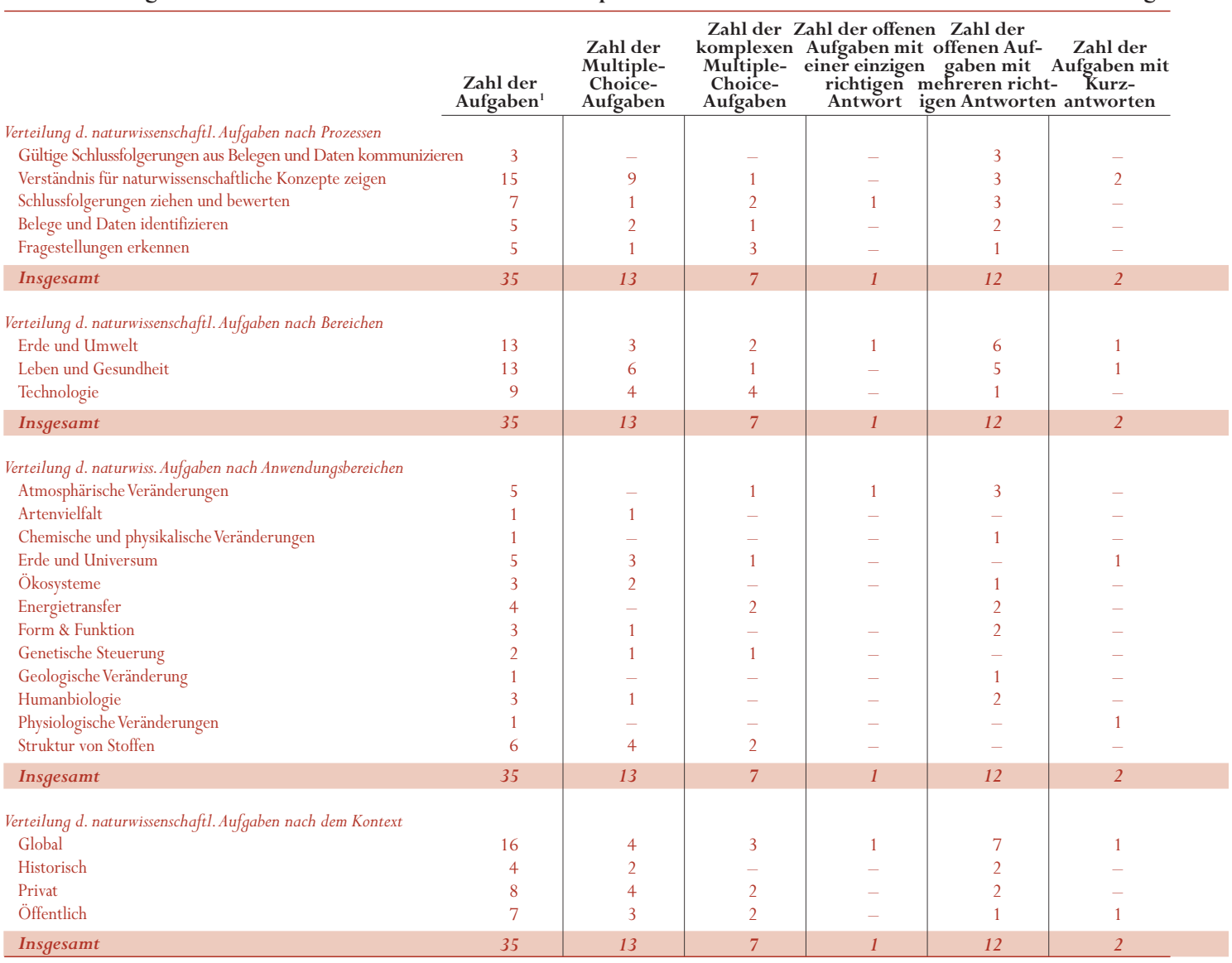

1. Eine Aufgabe wurde bei späteren Analysen eliminiert.

der IALS-Erhebung entnommen, weil die Länder die Möglichkeit haben wollten, deren Ergebnisse mit denen der PISA-Studie zu vergleichen.

Jedes in den Aufgabenpool einbezogene Item wurde dann von jedem Land unter folgenden Gesichtspunkten bewertet: der kulturellen, geschlechtsspezifischen oder sonstiger Akzeptanz; der Relevanz für 15-Jährige im schulischen und außerschulischen Kontext sowie der Vertrautheit und dem Grad des Interesses. Im Rahmen des Prozesses der Entwicklung der Erhebungsinstrumente für den Feldtest wurde eine erste Konsultation der Länder im Hinblick auf die Aufgabensammlung durchgeführt. Eine zweite Konsultation fand nach dem Feldtest als Hilfe für die endgültige Aufgabenauswahl für die Haupterhebung statt und wurde durch eine Überprüfung des Erhebungsmaterials durch eine internationale Gruppe für kulturelle Fairness ergänzt.

Im Anschluss an den Feldtest, bei dem sämtliche Items in allen Teilnehmerländern getestet wurden, betrachteten die Testentwickler und die Expertengruppen bei der Aufgabenauswahl für die Haupterhebung eine Reihe von Aspekten: a) die Ergebnisse des Feldtests, b) die Resultate der Itemüberprüfungen durch die Länder und c) die während der Bewertung des Feldtests eingegangenen Anfragen. Die Testentwickler und Expertengruppen trafen im Oktober 1999 eine endgültige Auswahl der Aufgabensets, die nach einer Verhandlungsrunde von den Teilnehmerländern auf wissenschaftlicher wie auch politischer Ebene aufgenommen wurde. 
Die Haupterhebung umfasste 37 Leseeinheiten mit 141 Items (wobei verschiedene Aufgabenteile als separate Items gezählt wurden), bei denen der Stimulus für 14 Einheiten von nationalen Beiträgen, bei 13 Einheiten vom PISA-Konsortium und bei 10 Einheiten von IALS herrührte. Die bei der Haupterhebung eingesetzten Instrumente enthielten auch 16 Mathematikeinheiten (32 Items) und 14 naturwissenschaftliche Einheiten (35 Items).

Für den PISA-Fragenkatalog wurden 5 unterschiedliche Arten von Items verwendet:

- Multiple-Choice-Aufgaben: Bei diesen Aufgaben mussten die Schülerinnen und Schüler einen Buchstaben umkreisen, um unter vier oder fünf Alternativen eine Antwort auszuwählen, wobei es sich jeweils um eine Zahl, ein Wort, einen Ausdruck oder einen Satz handeln konnte. Diese Items wurden dichotom kodiert.

- Komplexe Multiple-Choice-Aufgaben: Bei diesen Aufgaben hatten die Schülerinnen und Schüler mehrere - gewöhnlich binäre - Antwortmöglichkeiten. Sie beantworteten die jeweiligen Fragen, indem sie ein Wort oder einen kurzen Ausdruck (z.B. ja oder nein) für jeden Punkt umkreisten. Diese Items wurden für jede Wahl dichotom bewertet, wobei für die Antworten die volle oder eine Teilpunktzahl gegeben werden konnte, je nachdem, ob die Aufgabe vollständig oder teilweise gelöst worden war.

- Aufgaben mit einer richtigen Antwort: Bei diesen Aufgaben mussten die Schülerinnen und Schüler ihre eigene Antwort unter einer begrenzten Auswahl akzeptabler Antworten formulieren. Die meisten dieser Items wurden dichotom bewertet, wobei einige Items im Kodierungsprozess zu beurteilen waren.

- Aufgaben mit kurzen Antworten: Ähnlich wie bei den Items mit nur einer richtigen Antwort mussten die Schülerinnen und Schüler eine kurze Antwort geben, wobei ihnen eine umfassende Reihe möglicher Antworten offen stand. Diese Items wurden per Hand kodiert, sie erlaubten daher sowohl eine dichotome Bewertung als auch abgestufte Punktwerte.

- Aufgaben mit mehreren richtigen Antworten: Bei diesen Aufgaben formulierten die Schülerinnen und Schüler eine ausführlichere Antwort, womit der Möglichkeit einer Vielfalt divergierender individueller Antworten und gegensätzlicher Auffassungen Rechnung getragen wurde. Bei diesen Aufgaben wurde von den Schülerinnen und Schüler gewöhnlich verlangt, Informationen oder Ideen aus dem Stimulustext zu ihren eigenen Erfahrungen oder Auffassungen in Beziehung zu setzen, wobei die Bewertung ihrer Antwort weniger von dem jeweils vertretenen Standpunkt abhing als vielmehr von der Fähigkeit, diesen Standpunkt unter Hinweise auf das Gelesene zu begründen und zu erläutern. Bei diesen Items waren häufig für teilweise richtige oder weniger ausgefeilte Antworten abgestufte Punktwerte vorgesehen, und sie wurden durchweg per Hand kodiert.

PISA 2000 zielte darauf ab, gruppenspezifische Informationen über ein breites Spektrum von Inhalten zu erhalten. Im Bereich Lesekompetenz wurden Aufgaben mit einer Testdauer von insgesamt 270 Minuten eingesetzt, wovon 45\% auf Fragen entfielen, für die es mehrere richtige Antworten gab. Die mathematischen und naturwissenschaftlichen Aufgaben waren auf eine Testzeit von 60 Minuten ausgelegt, wobei es sich bei 35\% um offene Items handelte. Alle Schülerinnen und Schüler nahmen jedoch an Testsitzungen von insgesamt 120 Minuten teil.

Um das beabsichtigte breite Spektrum von Inhalten abzudecken und gleichzeitig die auf 120 Minuten beschränkte individuelle Testzeit einzuhalten, wurden die Aufgaben in jedem Bereich in Blöcke untergliedert und auf neun Testhefte verteilt. Es gab neun 30-minütige Leseeinheiten, vier 15-minütige Mathematikeinheiten und vier 15-minütige Naturwissenschaftseinheiten. Im Rahmen von PISA 2000 beantwortete jeder Schüler Lese-Items, und über die Hälfte der Schüler bearbeitete Items in Naturwissenschaften und Mathematik.

Diese Testkonzeption wies eine Reihe besonderer Merkmale auf. Erstens wurde der größte Teil des Lesematerials in einer ausgewogenen Weise präsentiert, um Positionseffekte zu vermeiden und sicherzustellen, dass jedes Item dieselbe Gewichtung in der Erhebung erhielt. Zweitens begannen sieben der neun Testhefte mit Leseaufgaben, und bei allen Testheften beanspruchten solche Aufgaben mindestens 60 Minuten der verfügbaren Zeit. FünfTesthefte enthielten zudem naturwissenschaftliche und fünf weitere mathematische Aufgaben. Drittens stellte Pisa 2000 insofern eine Verbindung zwischen den PISA- und IALS-Studien her, als zwei Leseeinheiten lediglich IALS-Items enthielten, die in sechs der neun Testhefte aufgenommen wurden. Schließlich wurde mit diesem Konzept gewährleistet, dass eine repräsentative Schülerstichprobe jede der Testeinheiten beantwortete.

Nähere Einzelheiten über die Entwicklung der PISA-Erhebungsinstrumente und das PISA-Erhebungskonzept enthält der PISA 2000 Technical Report. 


\section{Anhang A7: Reliabilität der Kodierung offener Items}

Der Prozess der Kodierung offener Items stellte einen wichtigen Schritt zur Gewährleistung der Qualität und Vergleichbarkeit der Ergebnisse der PISA-Erhebung dar.

Detaillierte Richtlinien trugen dazu bei, dass der Kodierungsprozess richtig und in den einzelnen Ländern in gleicher Weise durchgeführt wurde. Die Richtlinien für die Kodierung umfassten Kodieranweisungen, Ausbildungsmaterial für die Einstellung von Kodierern und Material aus Fachseminaren für die Ausbildung nationaler Kodierer. Vor nationalen Schulungen organisierte das PISA-Konsortium Ausbildungssitzungen zur Vorstellung des Materials und zur Ausbildung der in den Teilnehmerländern zuständigen Koordinatoren, die später die Ausbildung der Kodierer in ihren jeweiligen Ländern übernahmen.

Für jedes Item wurde in der relevanten Kodieranweisung der Zweck der Frage beschrieben und erklärt, wie die Antworten der Schülerinnen und Schüler auf jede Aufgabe zu kodieren sind. Hinsichtlich der verschiedenen möglichen Antwortkategorien wurde auch die Abstufung der Punktwerte beschrieben, je nachdem, ob es sich um vollständig bzw. teilweise richtige Antworten oder falsche Antworten handelte. PISA 2000 sah ferner ein zweistelliges Kodiersystem für die mathematischen und naturwissenschaftlichen Items vor, wobei die erste Zahl die erreichte Punktzahl wiedergibt und die zweite Zahl die unterschiedlichen Strategien oder Konzepte, die die Schülerinnen und Schüler zur Lösung des Problems anwendeten. Mit Hilfe der zweistelligen Kodierung konnten nationale Profile der Lösungsstrategien und der Fehlkonzeptionen von Schülerinnen und Schülern erstellt werden. Die Kodieranweisungen enthielten zur Veranschaulichung zudem echte Beispiele der Antworten von Schülerinnen und Schülern (aus dem Feldtest), mit denen eine Begründung ihrer Einstufung einherging.

In jedem Land wurde eine Teilstichprobe von Testheften unabhängig von vier Kodierern bewertet und vom PISA-Konsortium geprüft. Um die Konsistenz des Kodierungsprozesses in den einzelnen Ländern eingehender zu untersuchen und den Umfang der Varianzkomponenten im Zusammenhang mit dem Einsatz der Kodierer zu schätzen, führte das PISA-Konsortium bei einer Teilstichprobe von Testheften eine Reliabiltätsanalyse auf der Ebene der Kodierer durch. Hinsichtlich der nationalen Sets der multiplen Kodierung wurden Homogenitätsanalysen vorgenommen und die Ergebnisse mit denen des Feldtests verglichen. Nähere Einzelheiten hierzu enthält der PISA 2000 Technical Report.

Was die zwischenstaatliche Ebene betrifft, so wurde eine ländervergleichende Reliabilitätsanalyse bei einer Untergruppe von Items durchgeführt. Ihr Ziel bestand darin festzustellen, ob die von den nationalen Kodierern für die Aufgaben insgesamt sowie auch für bestimmte Items gegebenen Bewertungen in den einzelnen Ländern gleich streng waren. Bei diesem Prozess wurde von ausgebildetem mehrsprachigem Personal eine unabhängige Kodierung der Originaltesthefte vorgenommen und mit den Bewertungen der nationalen Kodierer in den verschiedenen Ländern verglichen. Die Ergebnisse ließen eine sehr große Konsistenz der in den einzelnen Ländern vorgenommenen Bewertungen erkennen. Der Durchschnittsindex der „Übereinstimmung“ betrug bei den Reliabilitätsanalysen in den Ländern 92\% (bei insgesamt 41796 Schülerantworten, die von internationalen Prüfern unabhängig bewertet wurden). Als ,übereinstimmend“ galten sowohl Fälle, in denen die internationalen Prüfer mit mindestens drei der nationalen Kodierer übereinstimmten, als auch Fälle, in denen der Prüfer nicht derselben Meinung war wie die nationalen Kodierer, die Testentwickler des PISA-Konsortiums aber zu dem Urteil kamen, dass die nationalen Kodierer die richtige Bewertung gegeben hatten. Lediglich acht Länder hatten Übereinstimmungsquoten von weniger als 90\%. Im Durchschnitt wurde die Kodierung in 1\% der Fälle als zu streng befunden (mit einem Maximum von 2,5\% in Lettland), in 2,5\% der Fälle als zu positiv (mit einem Maximum von 9,4\% für Lettland). Eine umfassende Beschreibung dieses Prozesses und der Ergebnisse enthält der PISA 2000 Technical Report. 

ANHANG

B

\section{Datentabellen}

Anhang B1: Datentabellen zu den Kapiteln

(Ländereinträge in der Reihenfolge

der englischen Länderbezeichnungen sortiert)

Anhang B2: Leistungsunterschiede zwischen den flämischen und französischen

Gemeinschaften in Belgien sowie den Sprachgemeinschaften in der Schweiz 
Anhang B1: Datentabellen zu den Kapiteln

Tabelle 2.1a Prozentsatz der Schülerinnen und Schüler auf den jeweiligen Kompetenzstufen der Gesamtskala Lesekompetenz

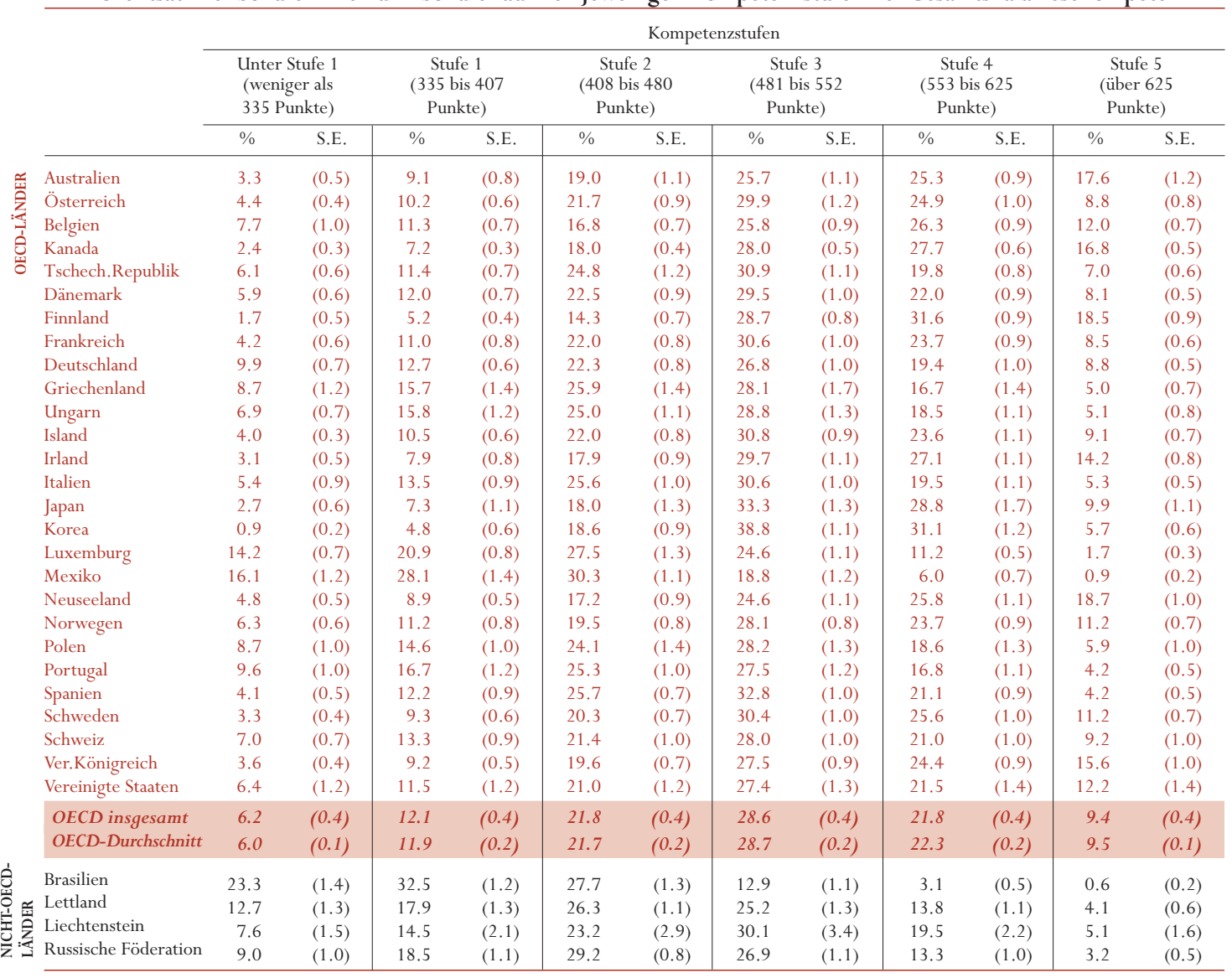


Tabelle $2.1 b$

Prozentsatz der Schülerinnen und Schüler auf den jeweiligen Kompetenzstufen der Lesekompetenz-Subskala Informationen Ermitteln

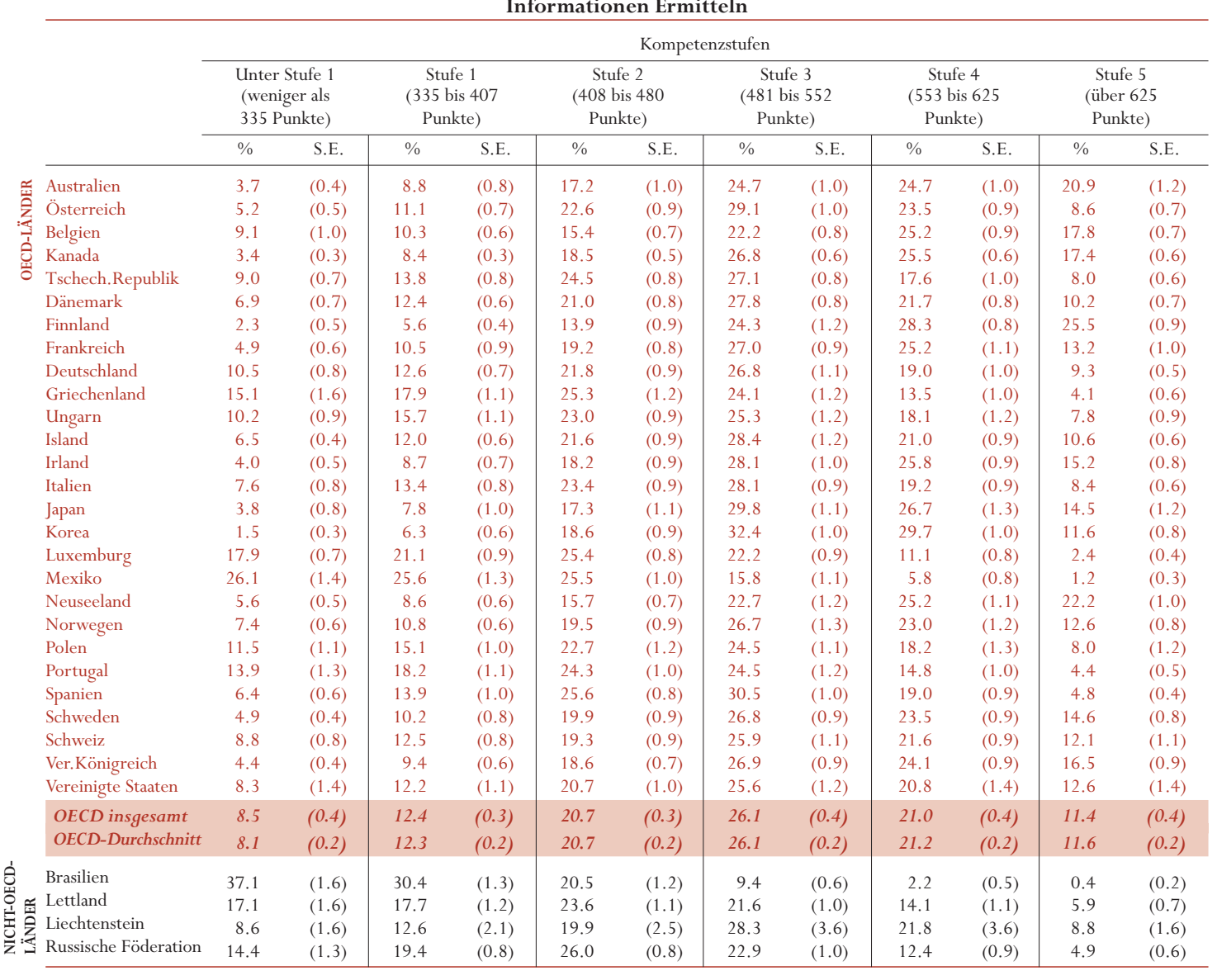


ANHANG B1

Tabelle 2.1c

Prozentsatz der Schülerinnen und Schüler auf den jeweiligen Kompetenzstufen der Lesekompetenz-Subskala Textbezogenes Interpretieren

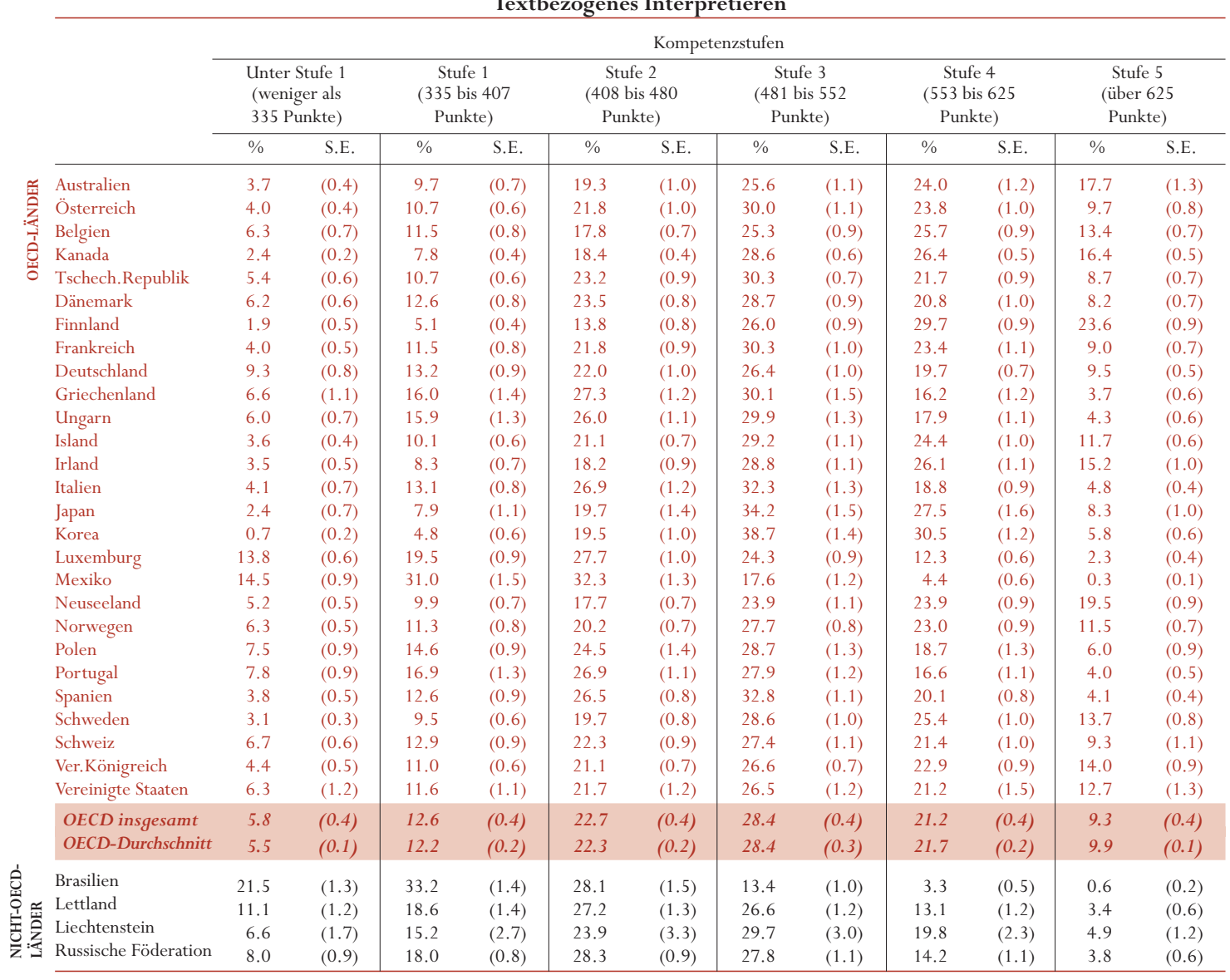


Tabelle 2.1d

Prozentsatz der Schülerinnen und Schüler auf den jeweiligen Kompetenzstufen der Lesekompetenz-Subskala

Reflektieren und Bewerten

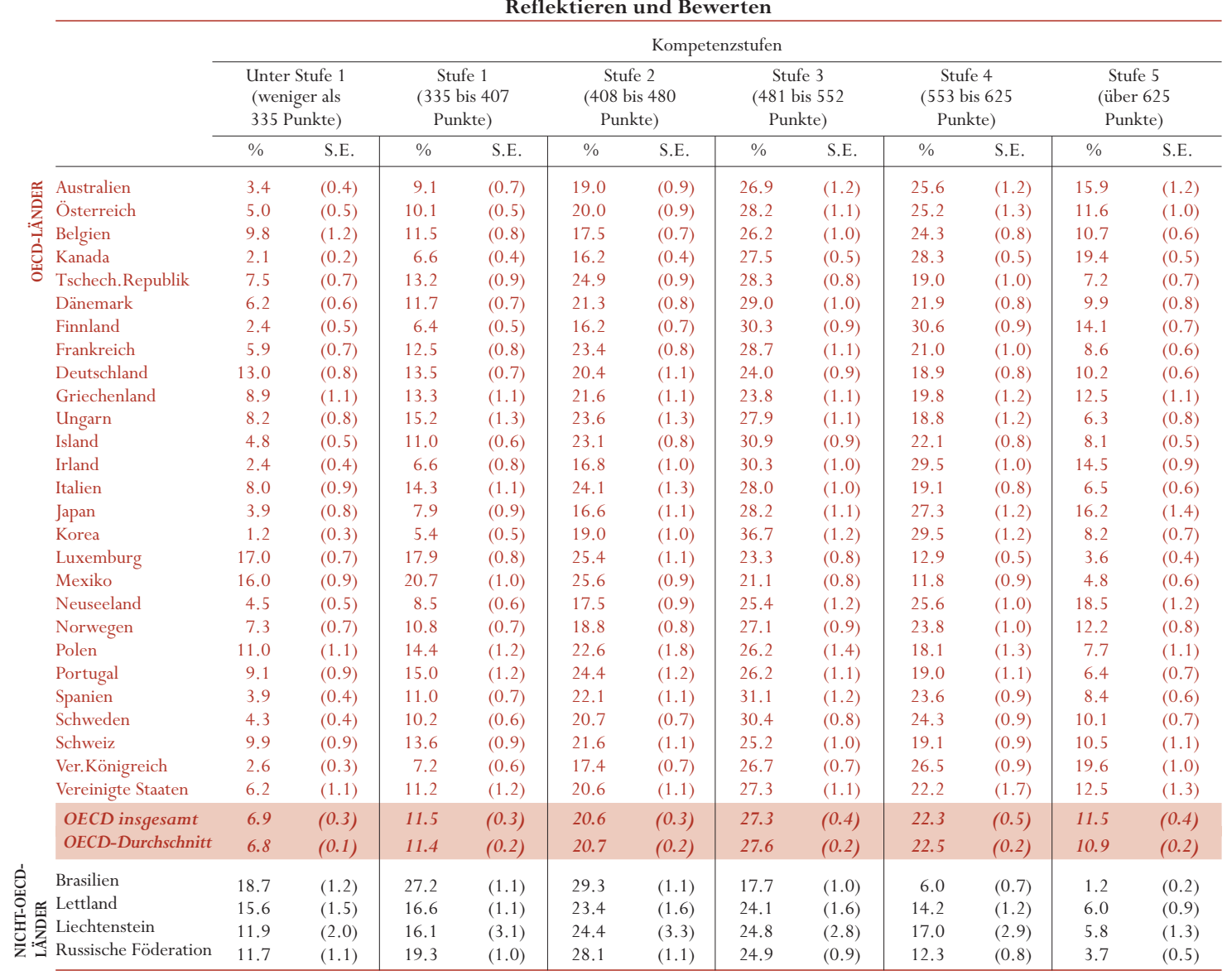


Tabelle $2.2 a$

Vergleich der Durchschnittsergebnisse der Länder auf der Subskala Informationen Ermitteln

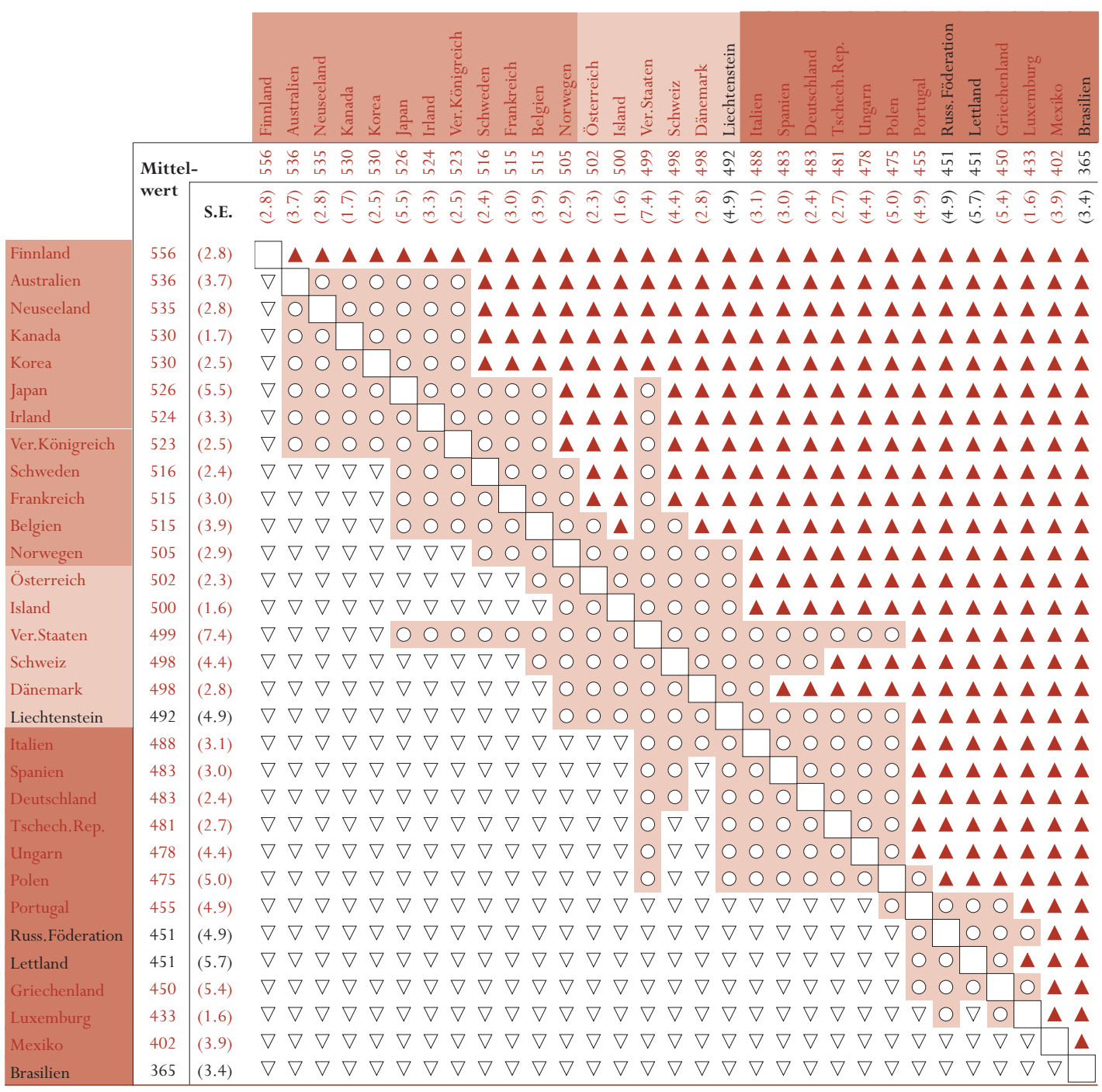

Erläuterungen

Zum Vergleich der Ergebnisse eines Landes mit denen der Länder im Tabellenkopf ist die Zeile des betreffenden Landes zu lesen. Die Symbole zeigen, ob die Durchschnittsergebnisse des Landes in der jeweiligen Zeile signifikant über denen des Vergleichslandes oder darunter liegen oder ob zwischen den Durchschnittsergebnissen beider Länder kein statistisch signifikanter Unterschied besteht.

D Durchschnittsergebnisse statistisch signifikant höher als im Vergleichsland

Kein statistisch signifikanter Unterschied gegenüber dem Vergleichsland

$\nabla$ Durchschnittsergebnisse statistisch signifikant niedriger als im Vergleichsland

Statistisch signifikant über dem OECD-Durchschnitt

Kein statistisch signifikanter Unterschied zum OECD-Durchschnitt

Statistisch signifikant unter dem OECD-Durchschnitt 


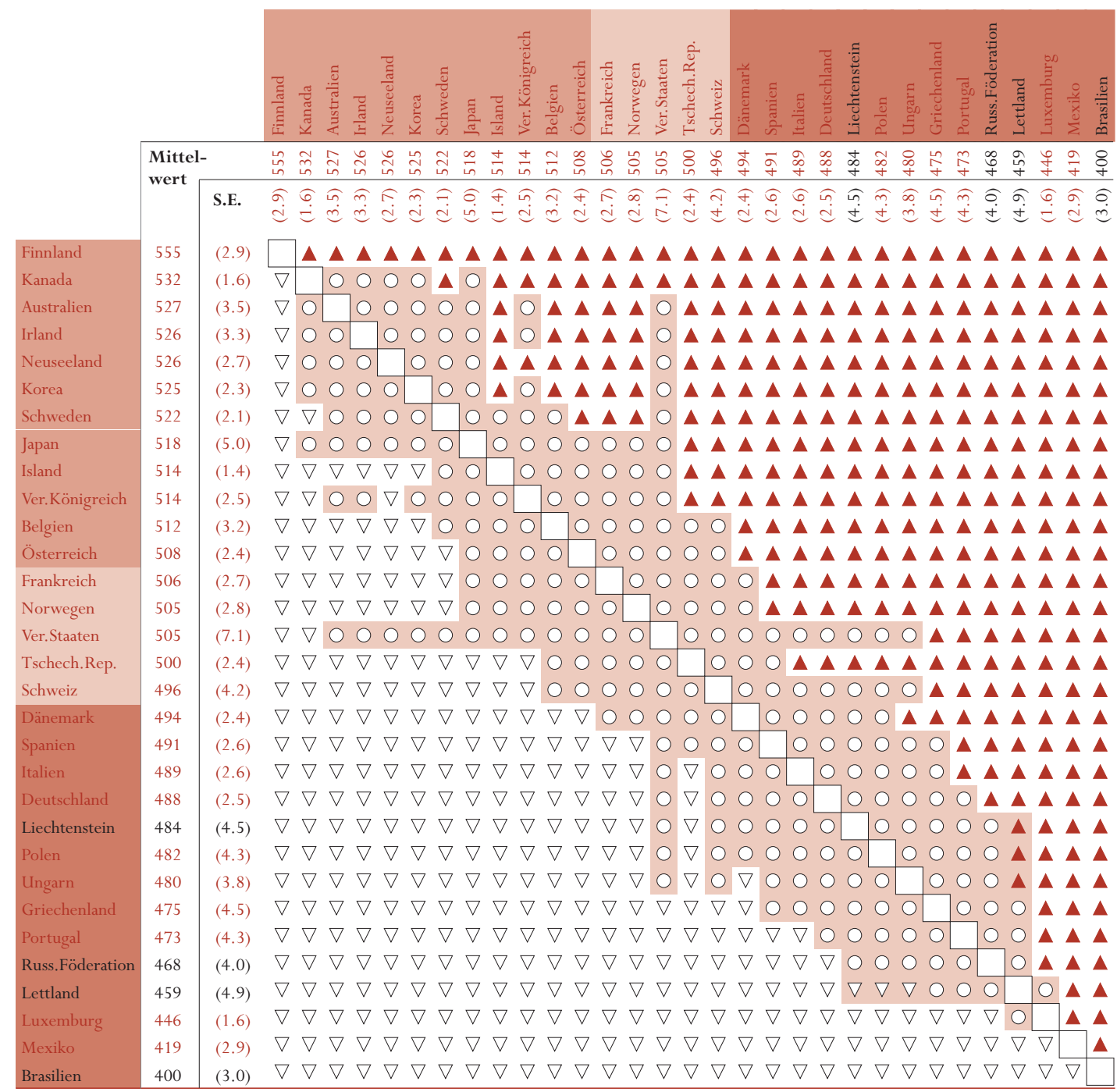

Erläuterungen

Zum Vergleich der Ergebnisse eines Landes mit denen der Länder im Tabellenkopf ist die Zeile des betreffenden Landes zu lesen. Die Symbole zeigen, ob die Durchschnittsergebnisse des Landes in der jeweiligen Zeile signifikant über denen des Vergleichslandes oder darunter liegen oder ob zwischen den Durchschnittsergebnissen beider Länder kein statistisch signifikanter Unterschied besteht.

Durchschnittsergebnisse statistisch signifikant höher als im Vergleichsland

$\bigcirc$ Kein statistisch signifikanter Unterschied gegenüber dem Vergleichsland

$\nabla$ Durchschnittsergebnisse statistisch signifikant niedriger als im Vergleichsland

Statistisch signifikant über dem OECD-Durchschnitt

Kein statistisch signifikanter Unterschied zum OECD-Durchschnitt

Statistisch signifikant unter dem OECD-Durchschnitt 
Tabelle $2.2 c$

Vergleich der Durchschnittsergebnisse der Länder auf der Subskala Reflektieren und Bewerten

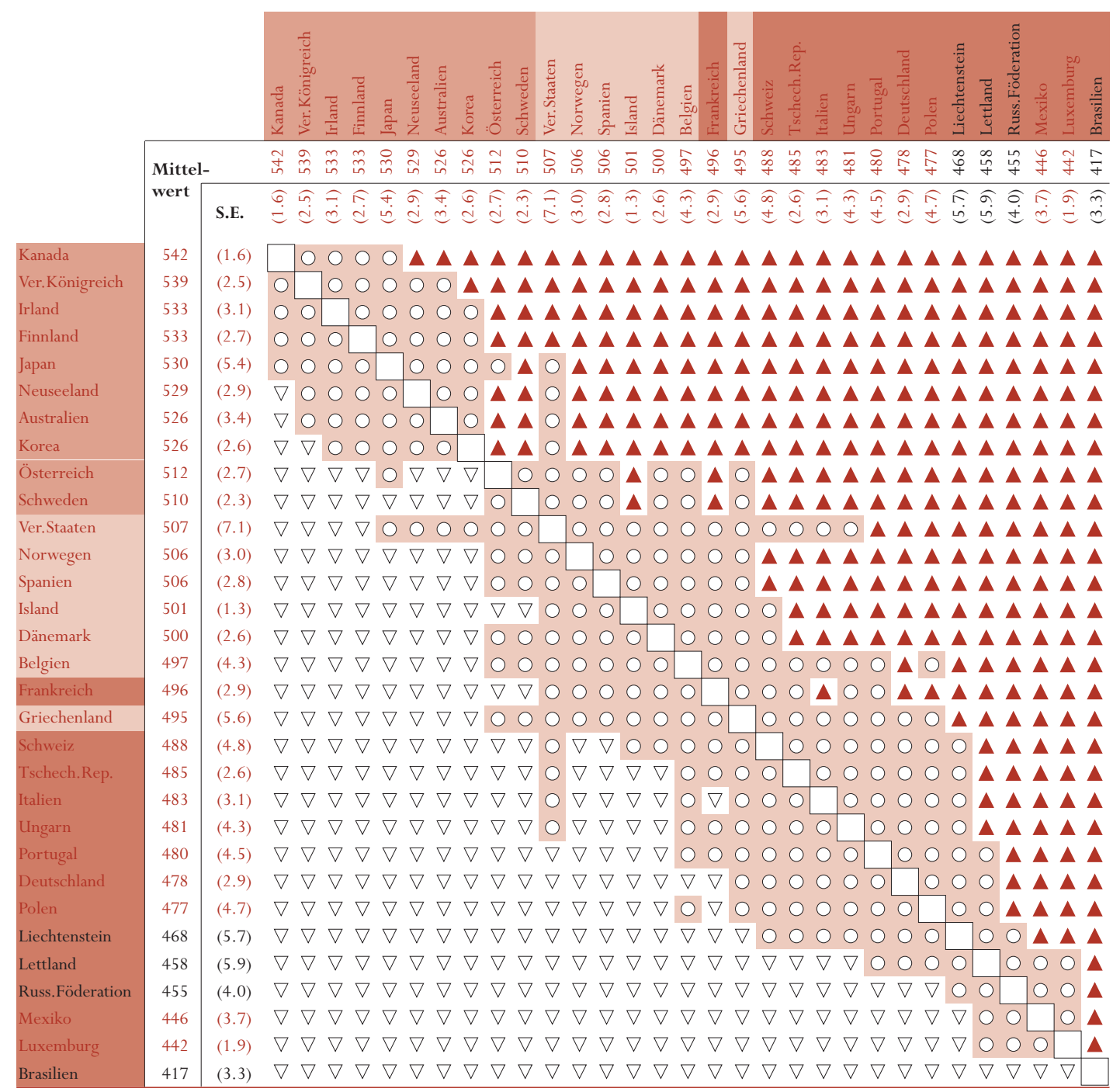

Erläuterungen

Zum Vergleich der Ergebnisse eines Landes mit denen der Länder im Tabellenkopf ist die Zeile des betreffenden Landes zu lesen. Die Symbole zeigen, ob die Durchschnittsergebnisse des Landes in der jeweiligen Zeile signifikant über denen des Vergleichslandes oder darunter liegen oder ob zwischen den Durchschnittsergebnissen beider Länder kein statistisch signifikanter Unterschied besteht.

A Durchschnittsergebnisse statistisch signifikant höher als im Vergleichsland

Kein statistisch signifikanter Unterschied gegenüber dem Vergleichsland

$\nabla$ Durchschnittsergebnisse statistisch signifikant niedriger als im Vergleichsland

Statistisch signifikant über dem OECD-Durchschnitt

Kein statistisch signifikanter Unterschied zum OECD-Durchschnitt Statistisch signifikant unter dem OECD-Durchschnitt 
ANHANG B1

Tabelle $2.3 a$

Verteilung der Schülerleistungen auf der Gesamtskala Lesekompetenz

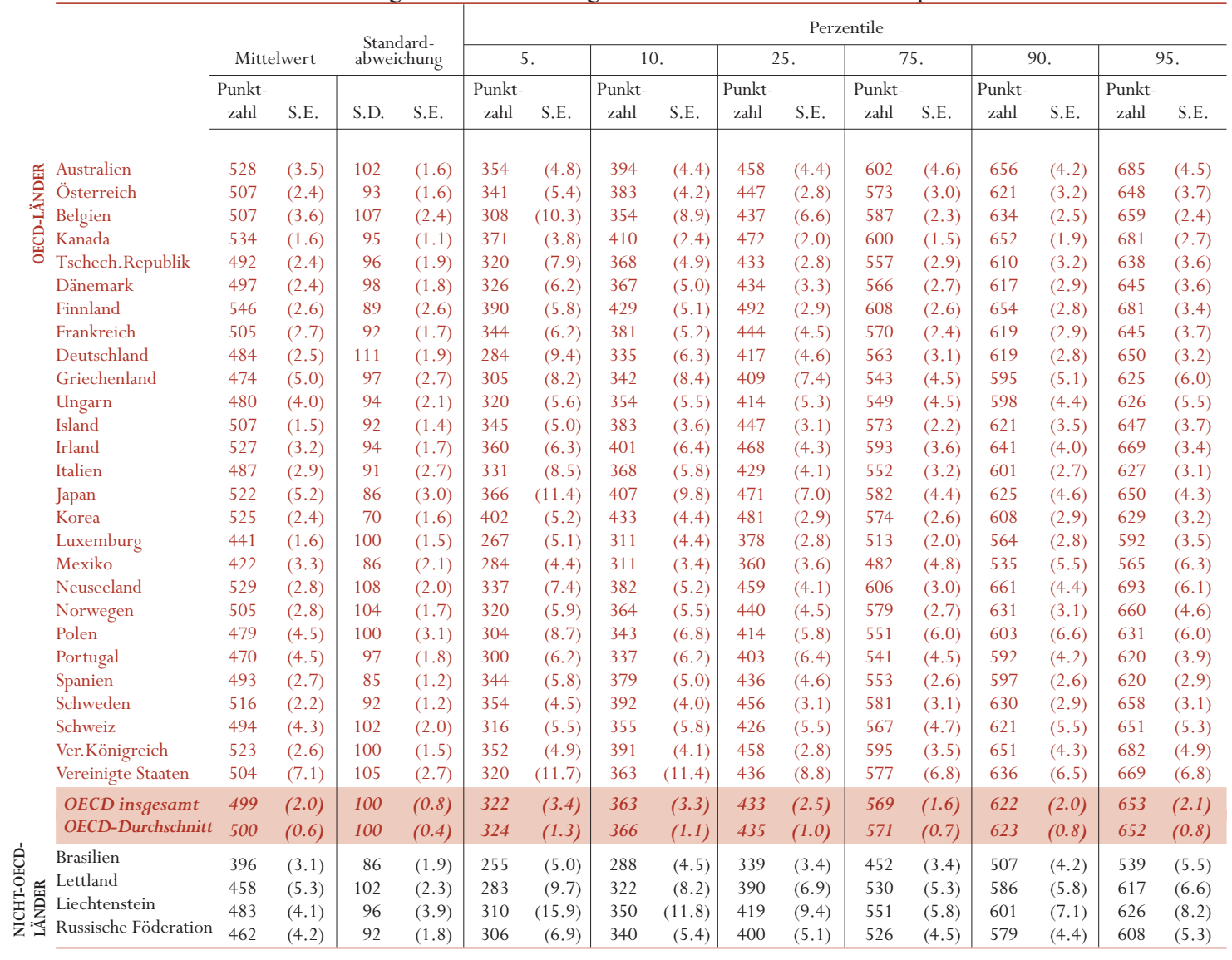


Tabelle 2.3b

Verteilung der Schülerleistungen auf der Lesekompetenz-Subskala Informationen Ermitteln

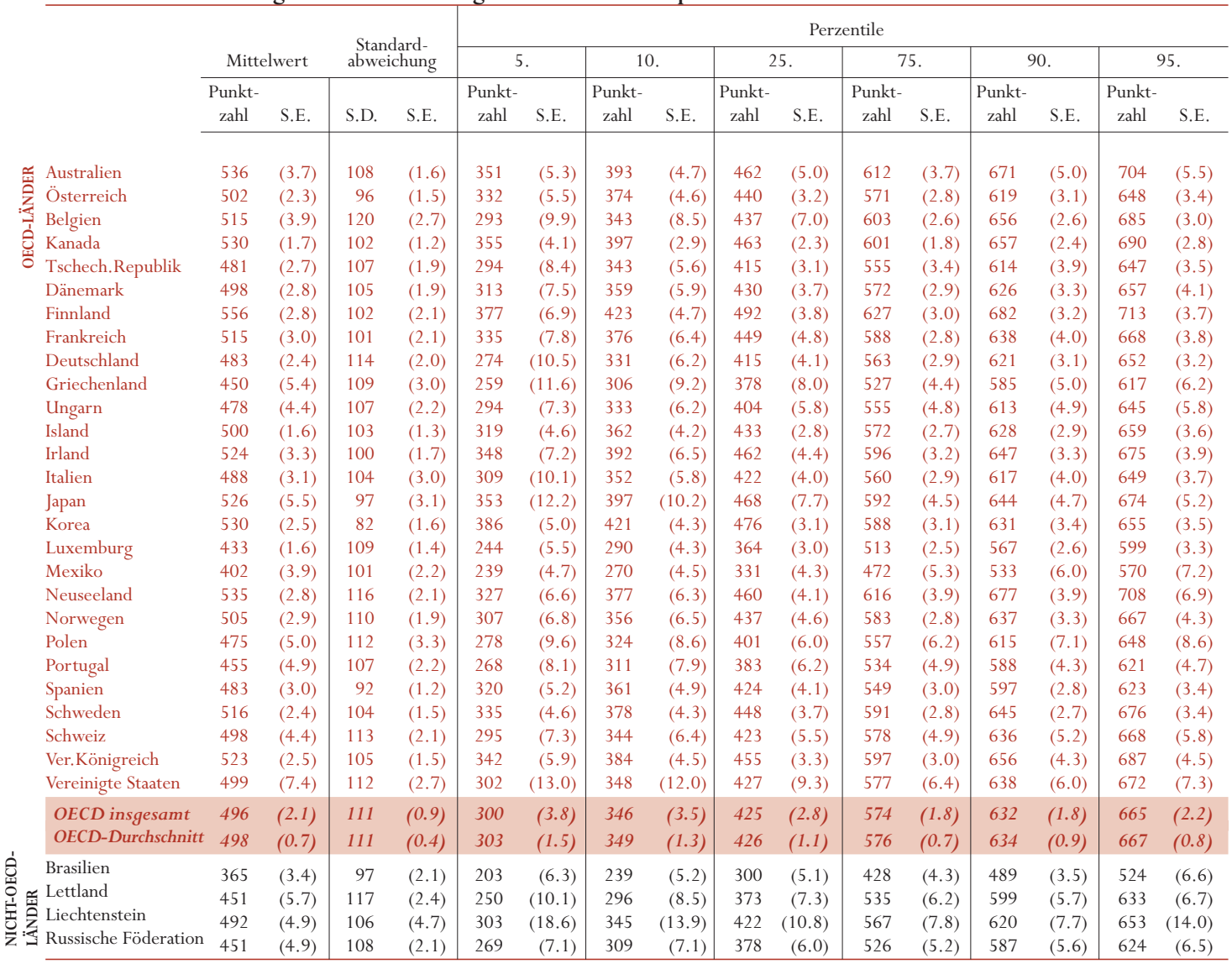


Tabelle 2.3c

Verteilung der Schülerleistungen auf der Lesekompetenz-Subskala Textbezogenes Interpretieren

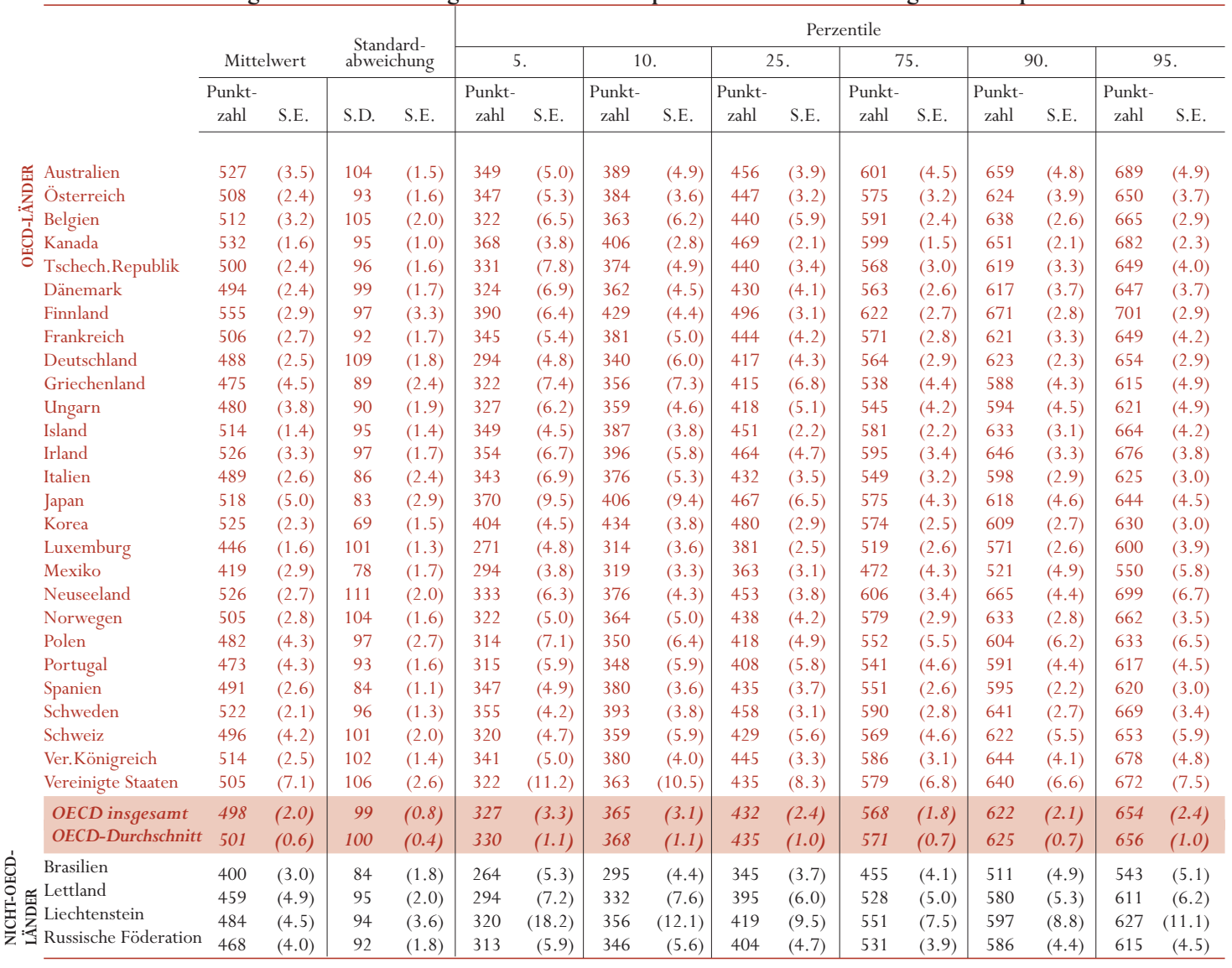


Tabelle $2.3 d$

Verteilung der Schülerleistungen auf der Lesekompetenz-Subskala Reflektieren und Bewerten

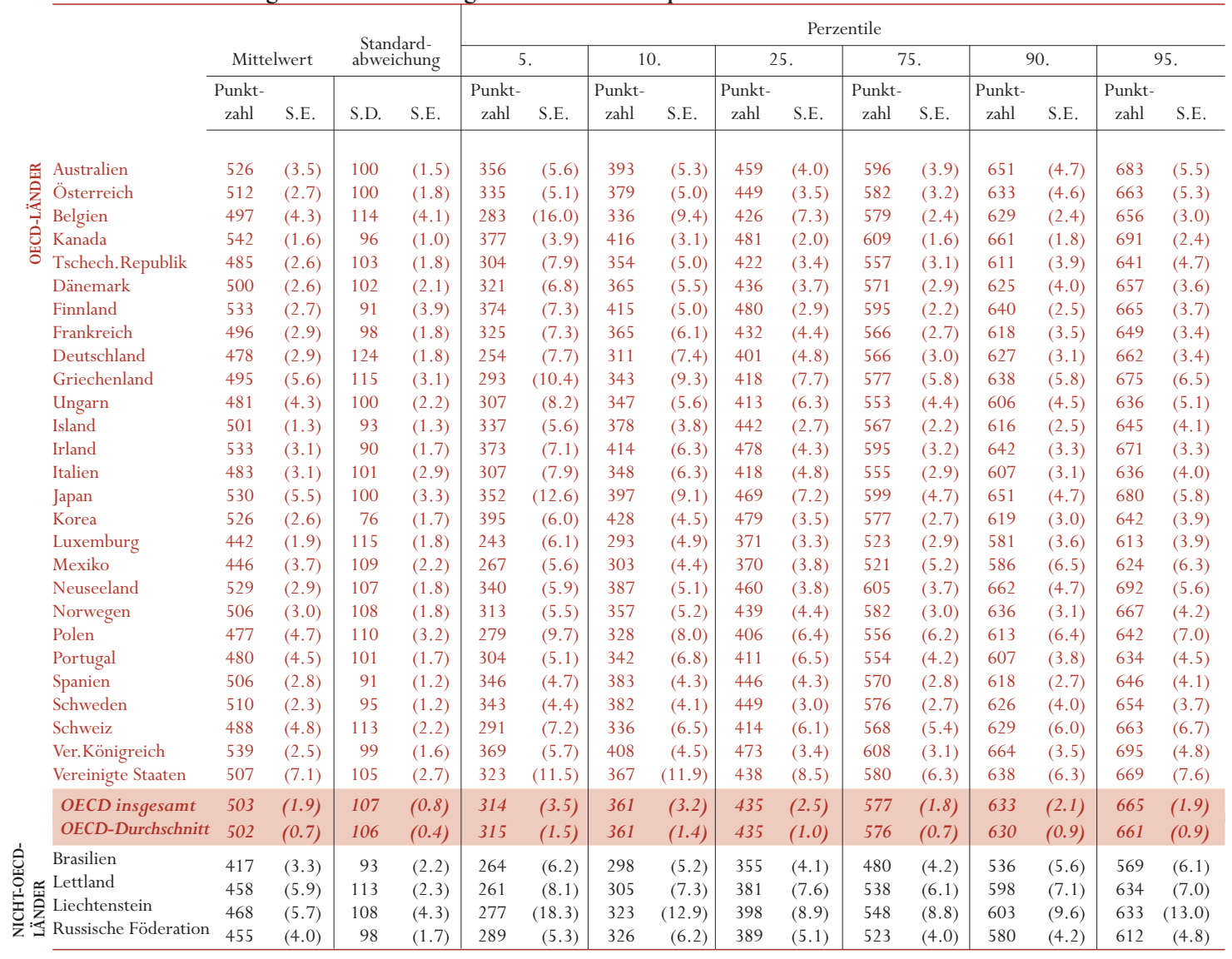


Tabelle 2.4

Varianz der Schülerleistungen zwischen Schulen und innerhalb von Schulen auf der Gesamtskala Lesekompetenz Varianz, ausgedrückt in Prozent

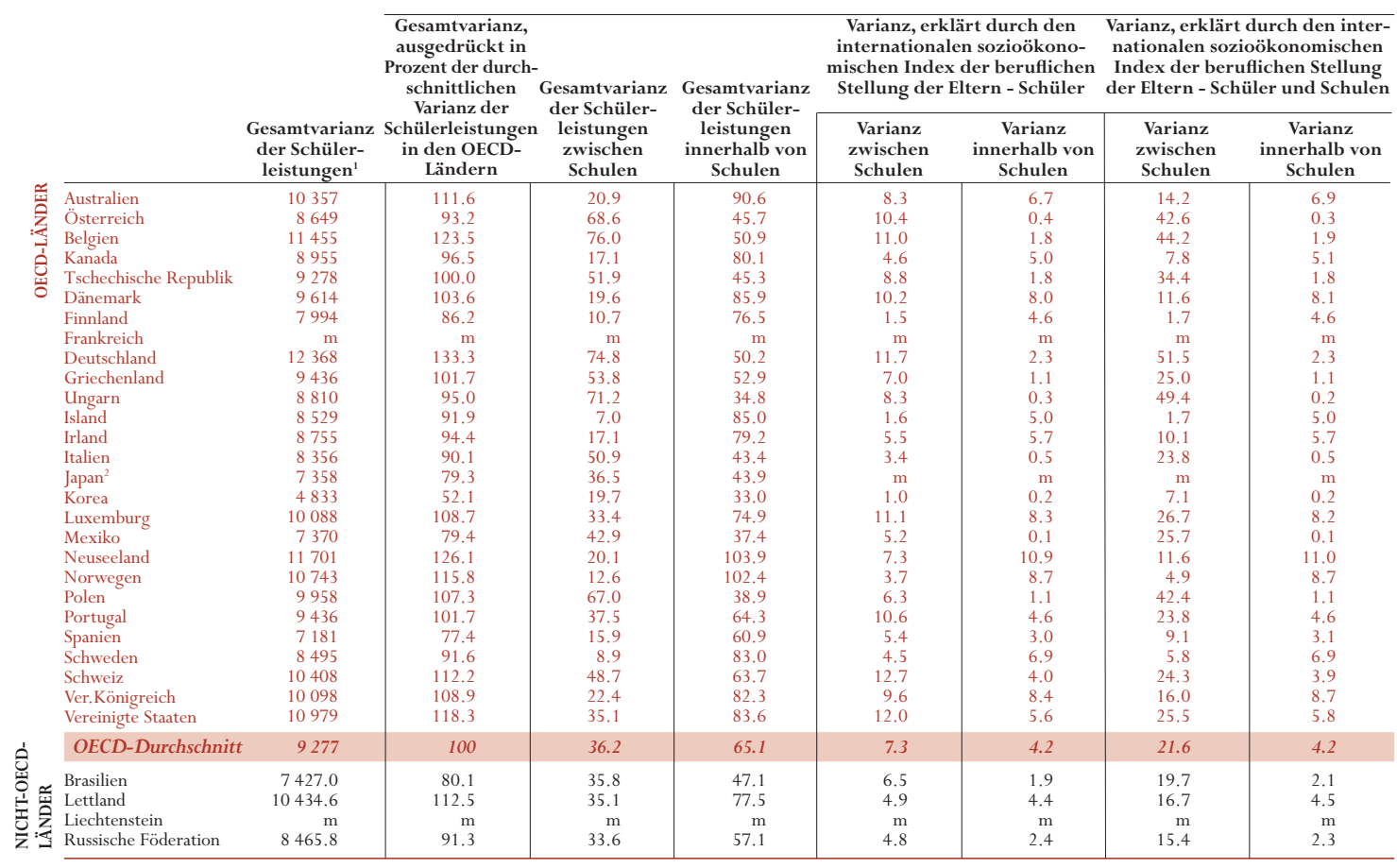

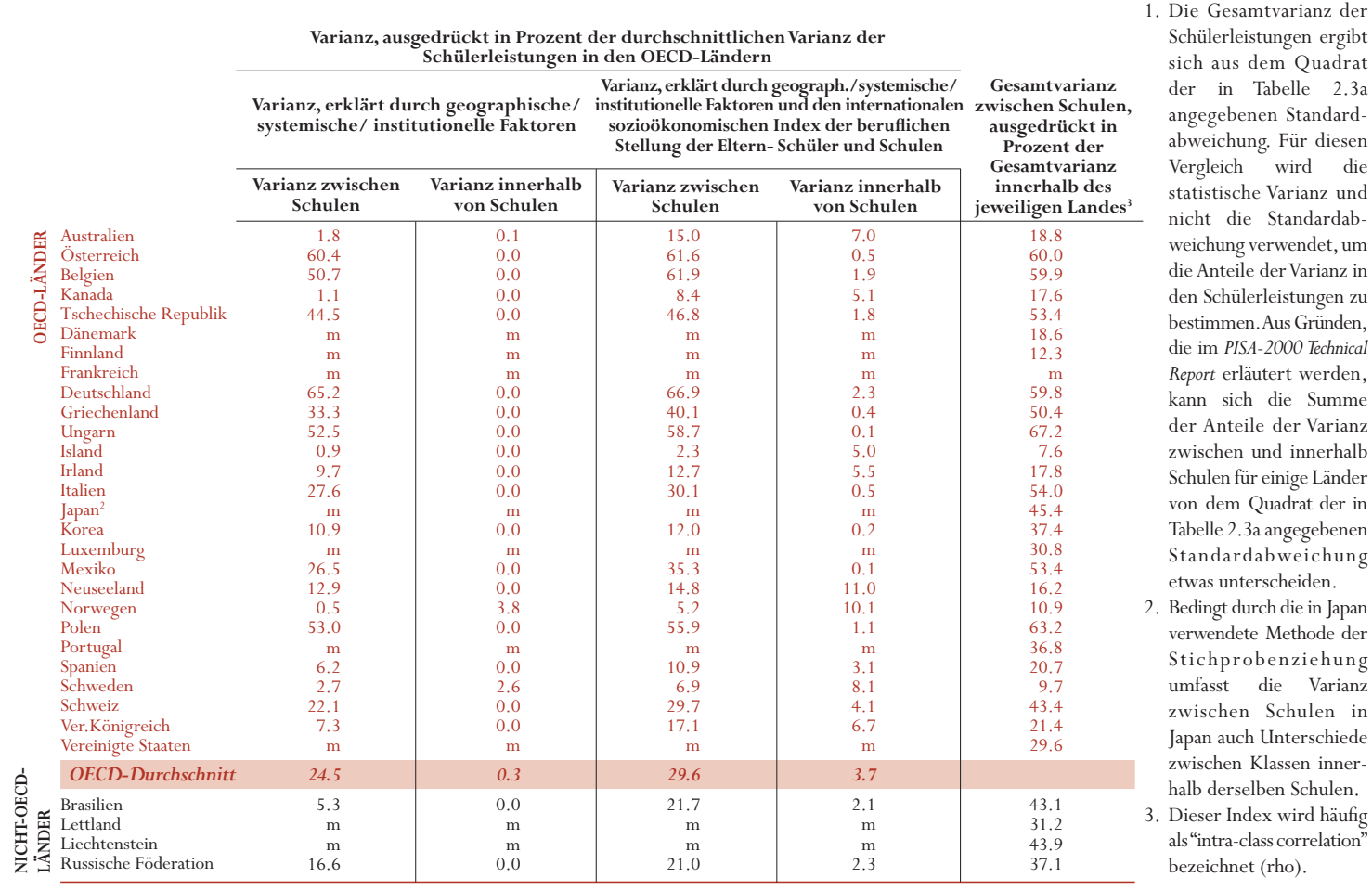


Tabelle 2.5

Festgelegte Mindestnote für die Versetzung und Leistungen auf der Gesamtskala Lesekompetenz

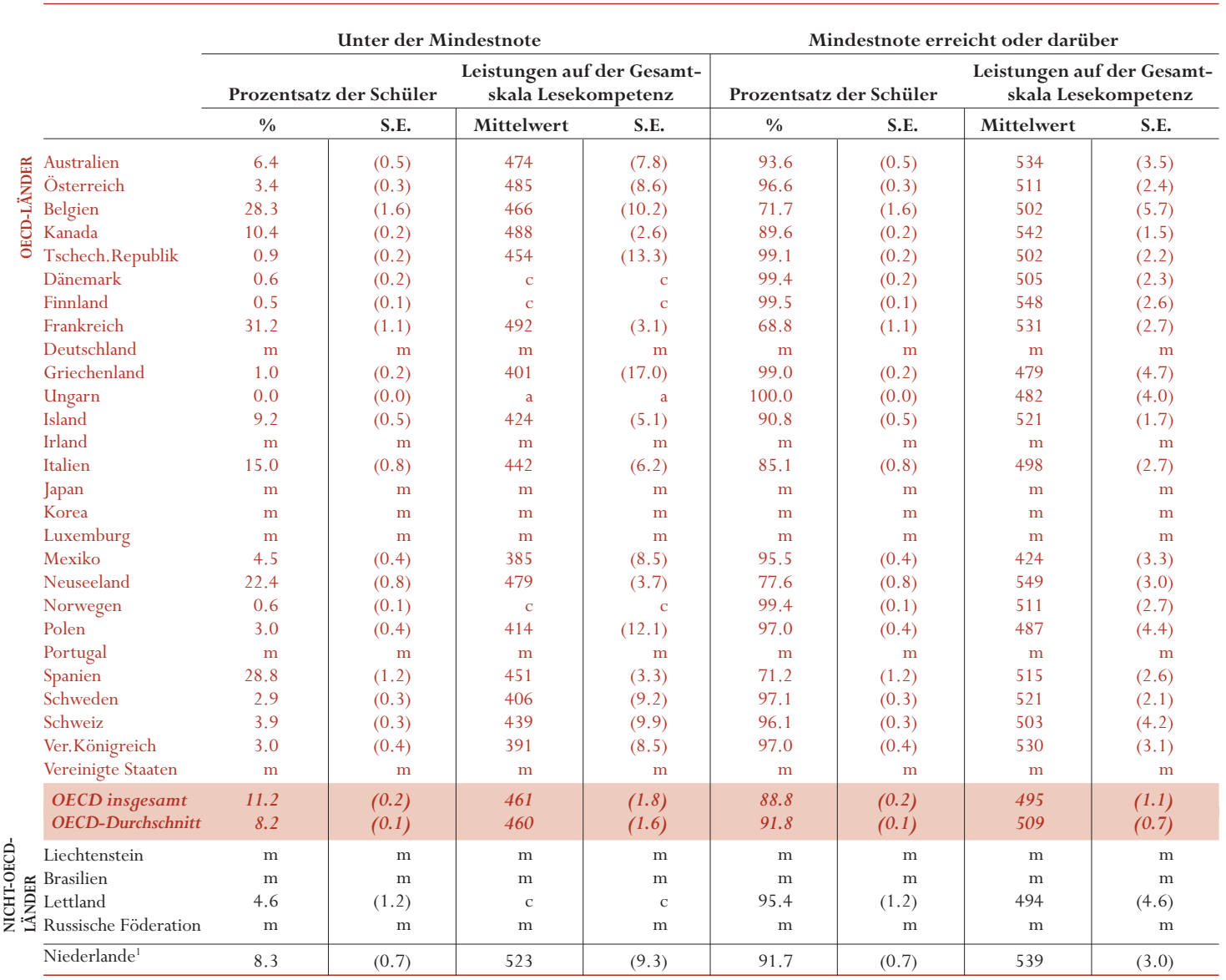

1. Die Beteiligungsquote ist zu niedrig, um die Vergleichbarkeit zu gewährleisten (vgl. Anhang A3). 
Tabelle 3.1

Verteilung der Schülerleistungen auf der Skala für mathematische Grundbildung

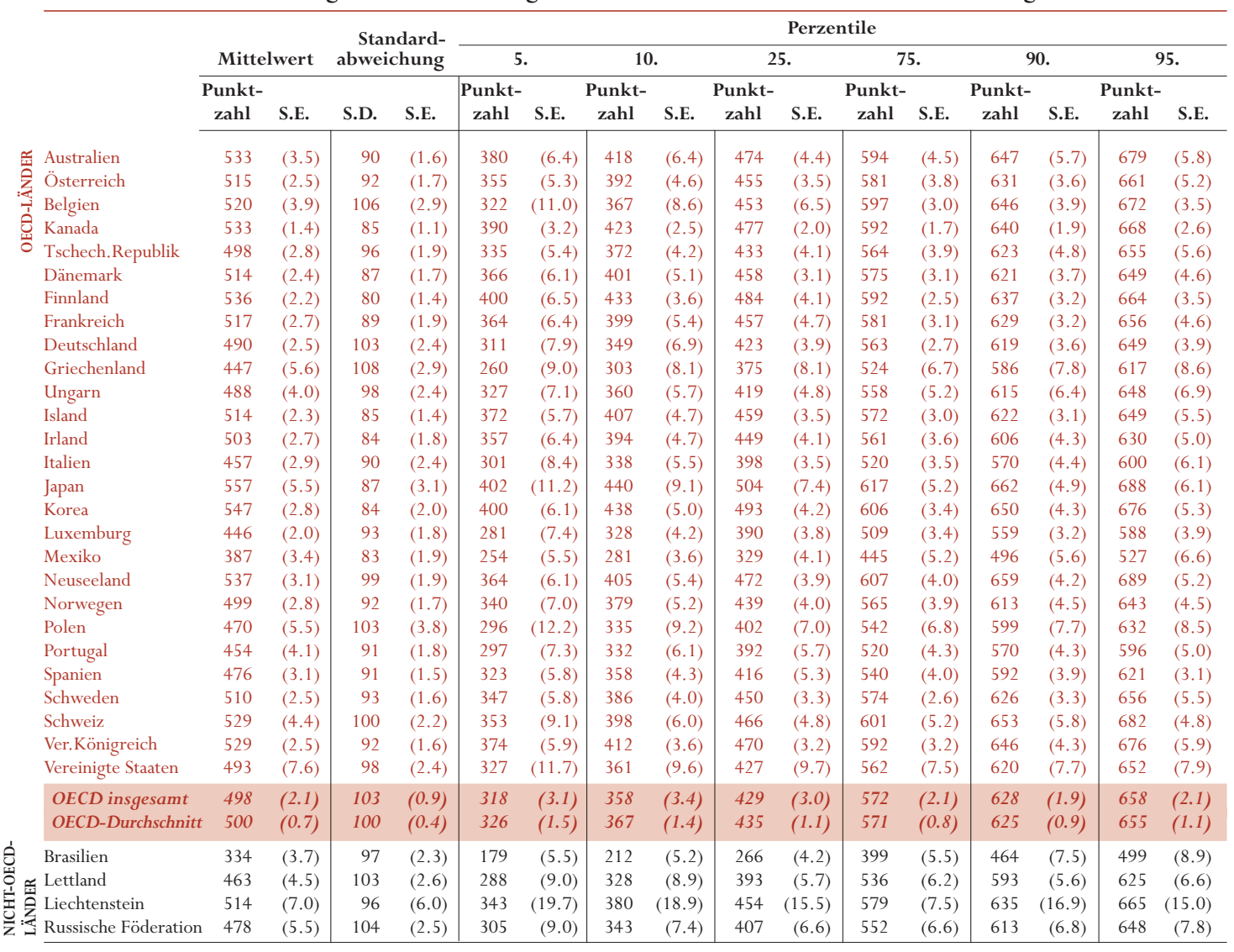


Tabelle 3.2

Festgelegte Mindestnote für die Versetzung und Leistungen auf der Skala für mathematische Grundbildung

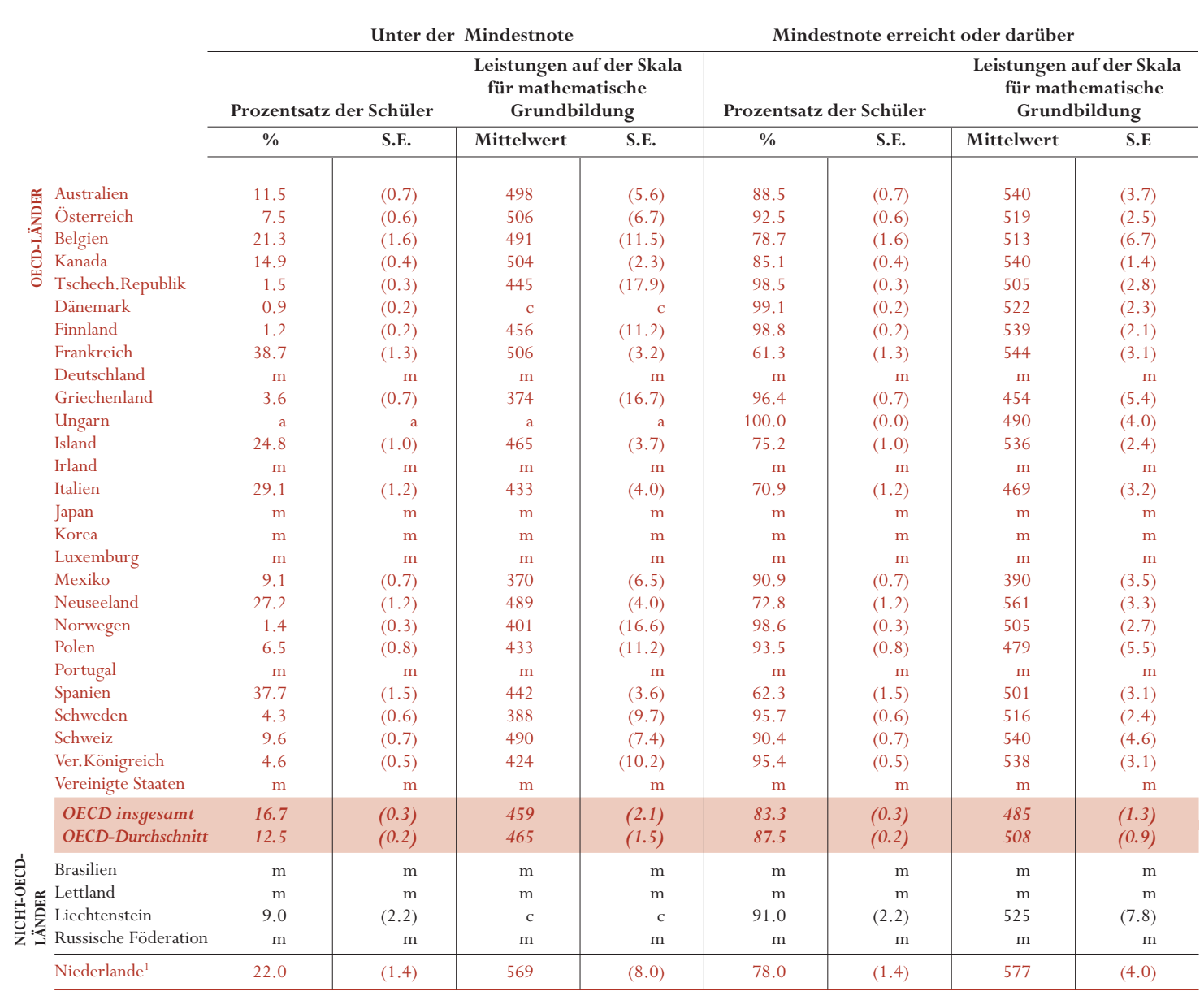

1. Die Beteiligungsquote ist zu niedrig, um die Vergleichbarkeit zu gewährleisten (vgl. Anhang A3). 
Tabelle 3.3

Verteilung der Schülerleistungen auf der Skala für naturwissenschaftliche Grundbildung

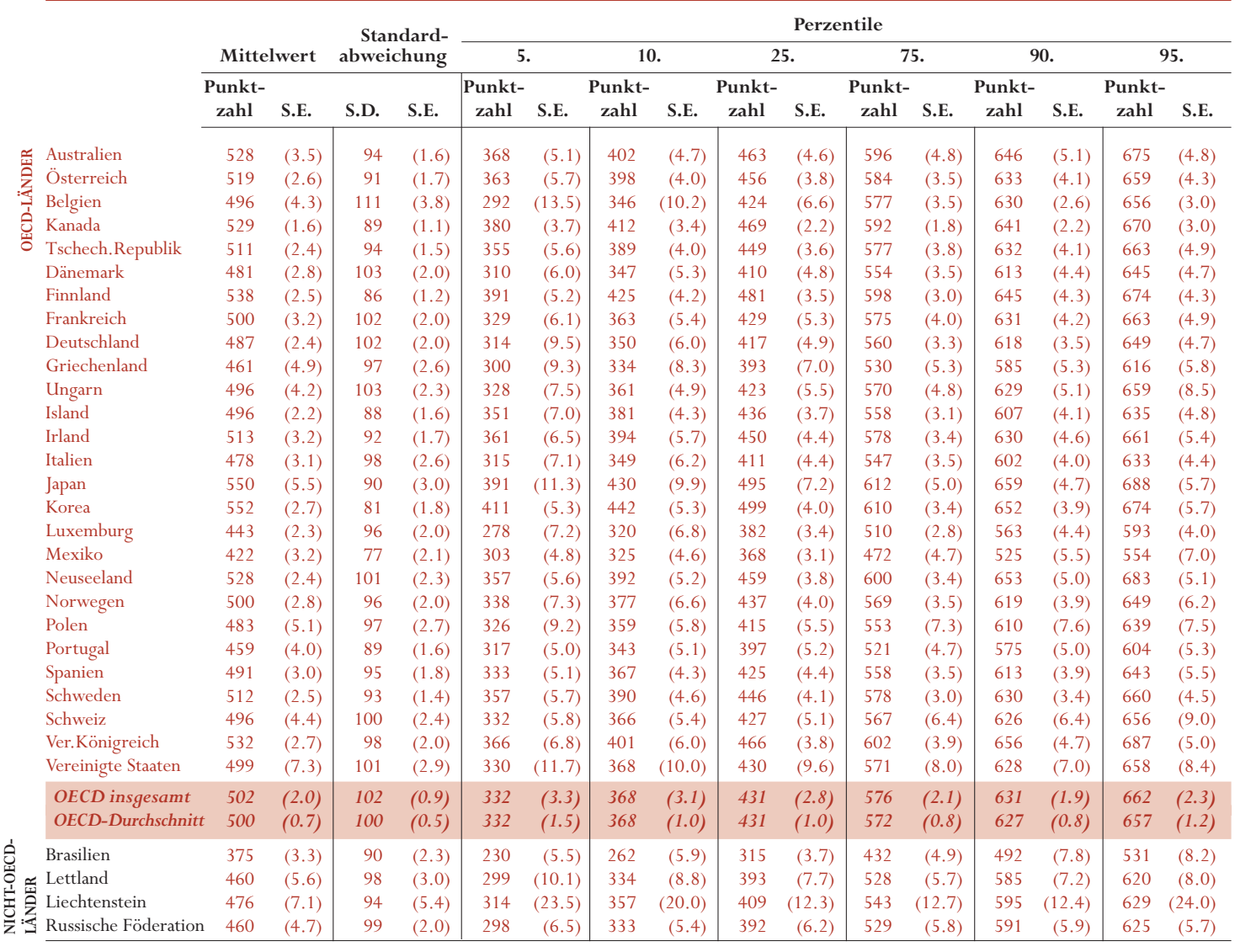


ANHANG B1

Tabelle 3.4

Festgelegte Mindestnote für die Versetzung und Leistungen auf der Skala für naturwissenschaftliche Grundbildung

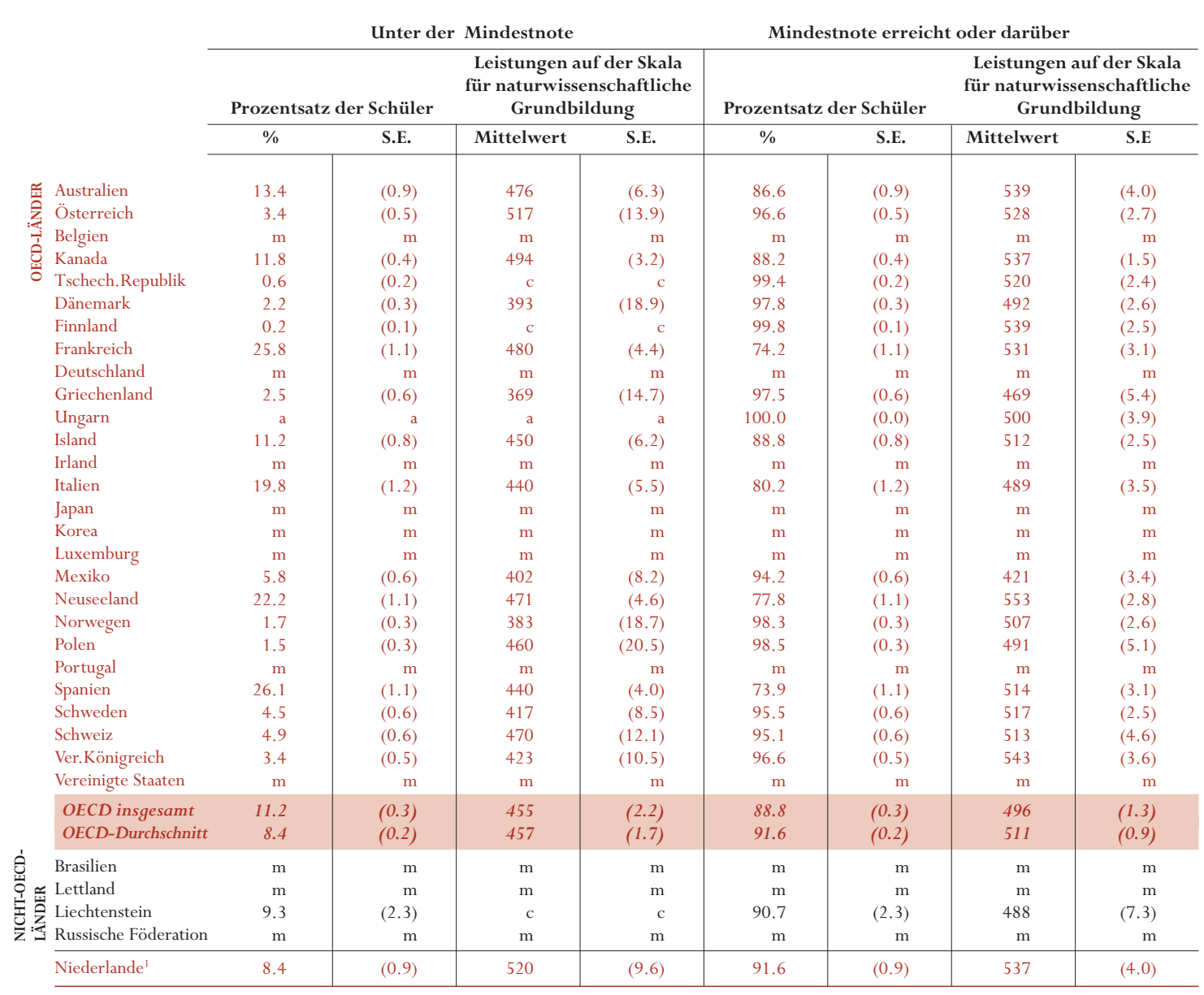

1. Die Beteiligungsquote ist zu niedrig, um die Vergleichbarkeit zu gewährleisten (vgl. Anhang A3). 
Tabelle 3.5

Varianz der Schülerleistungen zwischen Schulen und innerhalb von Schulen auf den Skalen für mathematische und für naturwissenschaftliche Grundbildung

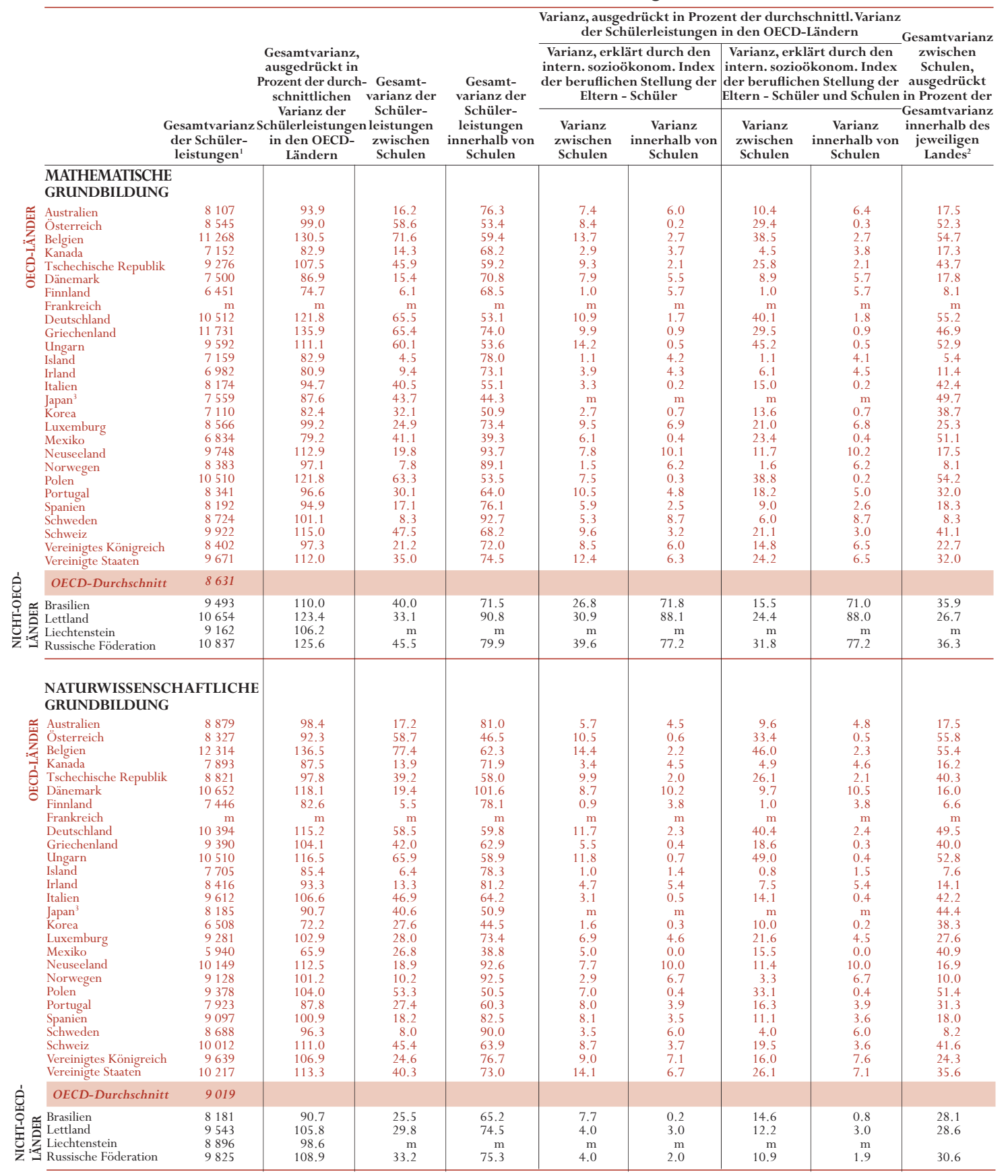

1. Die Gesamtvarianz der Schülerleistungen ergibt sich aus dem Quadrat der in Tabelle 3.1 für mathematische Grundbildung und in Tabelle 3.3 für naturwissenschaftliche Grundbildung angegebenen Standardabweichung. Für diesen Vergleich wird die statistische Varianz und nicht die Standardabweichung verwendet, um die Anteile der Varianz in den Schülerleistungen zu bestimmen. Aus Gründen, die im PISA-2000 Technical Report erläutert werden, kann sich die Summe der Anteile der Varianz zwischen und innerhalb Schulen für einige Länder von dem Quadrat der in Tabellen 3.1 und 3.3 angegebenen Standardabweichung etwas unterscheiden.

2. Dieser Index wird häufig als "intra-class correlation" bezeichnet (rho).

3. Bedingt durch die in Japan verwendete Methode der Stichprobenziehung umfasst die Varianz zwischen Schulen in Japan auch Unterschiede zwischen Klassen innerhalb derselben Schulen. 
ANHANG B1

Tabelle 3.6

Schülerleistungen auf der Gesamtskala Lesekompetenz, der Skala für naturwissenschaftliche Grundbildung und der Skala für mathematische Grundbildung und Volkseinkommen

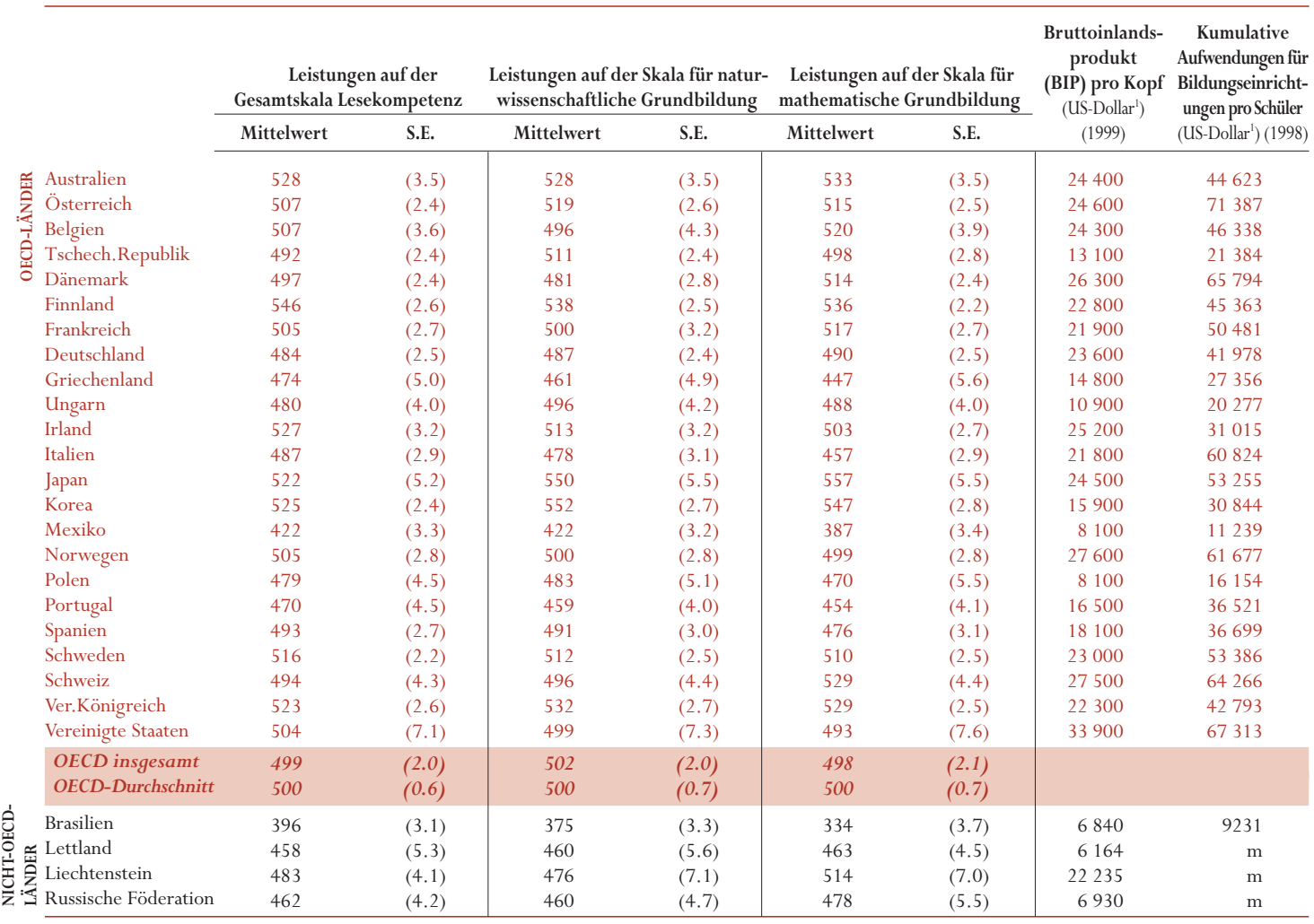

1. Umrechnung in US-\$ nach Kaufkraftparitäten. 
Tabelle 4.1

Index des Leseinteresses und Leistungen auf der Gesamtskala Lesekompetenz, nach Indexquartilen für das jeweilige Land Die Ergebnisse basieren auf Schülerangaben

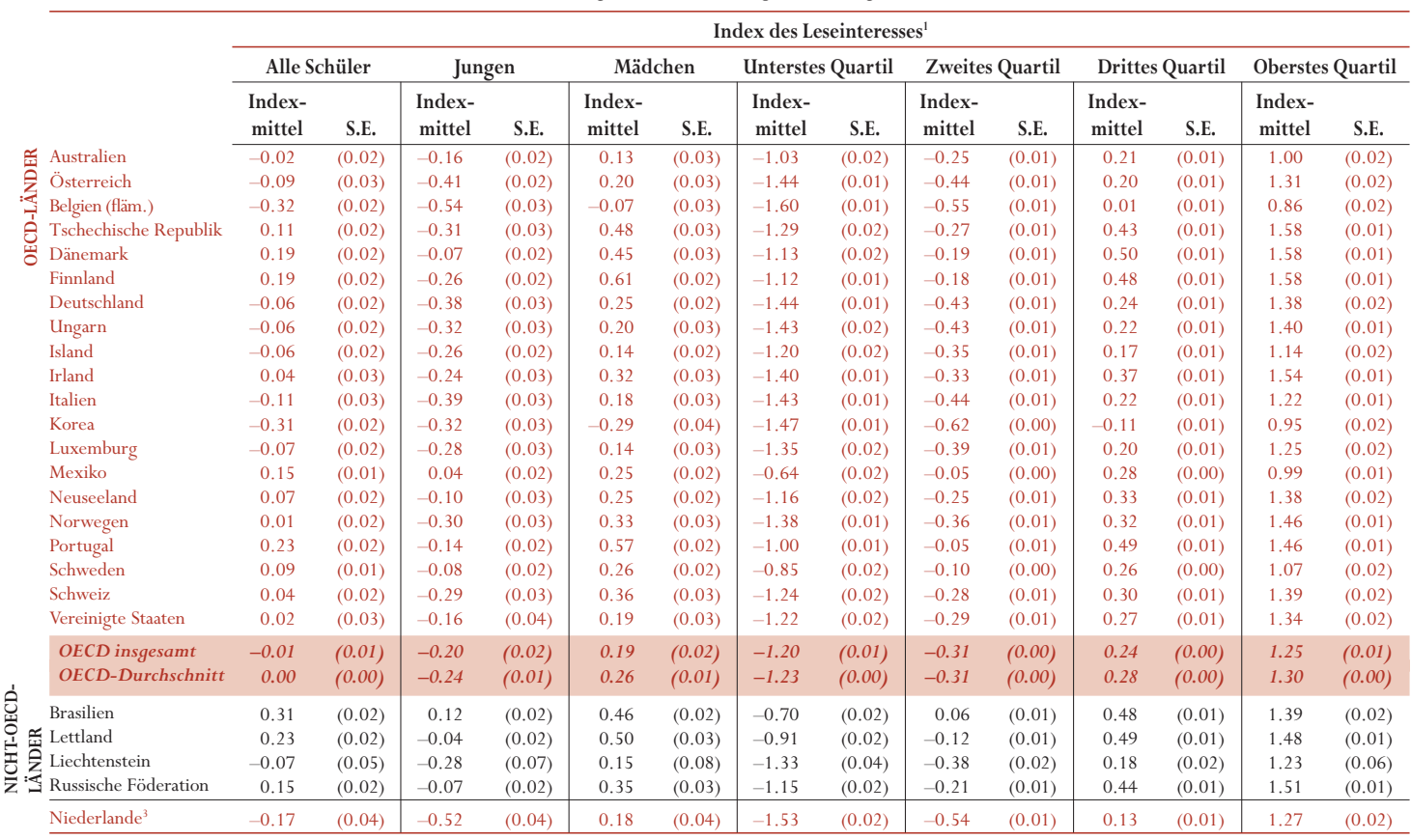

\begin{tabular}{|c|c|c|c|c|c|c|c|c|c|c|}
\hline & \multicolumn{8}{|c|}{$\begin{array}{l}\text { Leistungen auf der Gesamtskala Lesekompetenz, } \\
\text { nach Indexquartilen für das jeweilige Land }{ }^{2}\end{array}$} & \multirow{2}{*}{\multicolumn{2}{|c|}{$\begin{array}{c}\text { Punktzahlveränderung auf der Gesamtskala } \\
\text { Lesekompetenz, je Indexeinheit }{ }^{2}\end{array}$}} \\
\hline & \multicolumn{2}{|c|}{ Unterstes Quartil } & \multicolumn{2}{|c|}{ Zweites Quartil } & \multicolumn{2}{|c|}{ Drittes Quartil } & \multicolumn{2}{|c|}{ Oberstes Quartil } & & \\
\hline & $\begin{array}{c}\text { Mittel- } \\
\text { wert }\end{array}$ & S.E. & $\begin{array}{c}\text { Mittel- } \\
\text { wert }\end{array}$ & S.E. & $\begin{array}{c}\text { Mittel- } \\
\text { wert }\end{array}$ & S.E. & $\begin{array}{c}\text { Mittel- } \\
\text { wert }\end{array}$ & S.E. & Veränderung & S.E. \\
\hline Australien & 495 & $(4.0)$ & 505 & $(4.3)$ & 540 & (4.7) & 588 & (4.6) & 41.1 & $(2.37)$ \\
\hline Österreich & 481 & $(3.1)$ & 485 & (3.5) & 514 & (3.4) & 557 & (3.5) & 28.5 & $(1.48)$ \\
\hline Belgien (fläm.) & 515 & $(4.5)$ & 516 & $(6.1)$ & 544 & (5.3) & 570 & $(6.0)$ & 22.6 & $(2.05)$ \\
\hline Finnland & 502 & (2.7) & 527 & $(4.6)$ & 564 & $(2.8)$ & 599 & (3.2) & 36.1 & (1.18) \\
\hline Deutschland & 468 & (3.7) & 471 & (3.7) & 500 & $(4.2)$ & 552 & (3.8) & 30.0 & (1.65) \\
\hline Ungarn & 451 & (3.8) & 466 & (5.4) & 489 & $(5.2)$ & 529 & (4.4) & 27.8 & $(1.55)$ \\
\hline Island & 475 & $(3.2)$ & 493 & $(3.1)$ & 514 & (3.4) & 560 & (3.3) & 35.3 & $(1.85)$ \\
\hline Irland & 495 & (3.8) & 503 & $(4.0)$ & 536 & $(4.1)$ & 580 & (3.7) & 30.0 & $(1.46)$ \\
\hline Italien & 463 & $(4.2)$ & 474 & $(4,2)$ & 490 & (3.5) & 524 & (3.3) & 22.5 & $(1.51)$ \\
\hline Korea & 493 & $(2,8)$ & 519 & $(3.2)$ & 536 & (3.5) & 551 & (2.6) & 22.6 & $(1.27)$ \\
\hline Schweden & 479 & (3.1) & 501 & $(3.2)$ & 524 & $(3.3)$ & 568 & $(2.8)$ & 43.8 & $(2.01)$ \\
\hline Schweiz & 464 & $(4.2)$ & 479 & $(4,6)$ & 499 & $(5.7)$ & 548 & $(5.1)$ & 31.5 & (1.94) \\
\hline Vereinigte Staaten & 488 & $(8.1)$ & 495 & (6.5) & 507 & (7.9) & 558 & (6.9) & 27.0 & $(2.37)$ \\
\hline$O E C D$ insgesamt & 474 & $(3.2)$ & 482 & (3.0) & 497 & $(3.4)$ & 537 & (3.3) & 23.6 & $(1.16)$ \\
\hline OECD-Durchschnitt & 474 & $(0.8)$ & 485 & (1.1) & 508 & $(1.1)$ & 549 & $(0.9)$ & 27.9 & $(0.41)$ \\
\hline Brasilien & 397 & $(4.3)$ & 395 & $(3.5)$ & 399 & $(4.5)$ & 419 & $(4.3)$ & 10.0 & $(2.11)$ \\
\hline Lettland & 429 & $(6.6)$ & 440 & $(6.5)$ & 464 & (5.3) & 512 & (5.6) & 32.5 & $(2.21)$ \\
\hline Liechtenstein & 454 & $(10.0)$ & 463 & (9.6) & 494 & $(10.2)$ & 528 & $(10.8)$ & 29.5 & $(5.13)$ \\
\hline Russische Föderation & 440 & $(5.1)$ & 453 & (3.5) & 468 & $(4.8)$ & 498 & $(5.3)$ & 20.4 & $(1.16)$ \\
\hline Niederlande $^{3}$ & 510 & $(4.8)$ & 516 & (4.9) & 534 & $(4.7)$ & 572 & $(4.6)$ & 22.8 & $(1.93)$ \\
\hline
\end{tabular}

1. Zur Definition des Index siehe Anhang A1.

2. Zur erklärten Varianz siehe Anhang A2. In Indexeinheiten gemessene Veränderungen sind, soweit fettgedruckt, statistisch signifikant. Wenn die untersten und obersten Quartile durch Fettdruck gekennzeichnet sind, weist dies auf eine statistisch signifikante Differenz zwischen diesen Quartilen hin.

3. Die Beteiligungsquote ist zu niedrig, um die Vergleichbarkeit zu gewährleisten (vgl. Anhang A3). 
Tabelle 4.2

Index des Mathematikinteresses und Leistungen auf der Skala für mathematische Grundbildung, nach Indexquartilen für das jeweilige Land

Die Ergebnisse basieren auf Schülerangaben

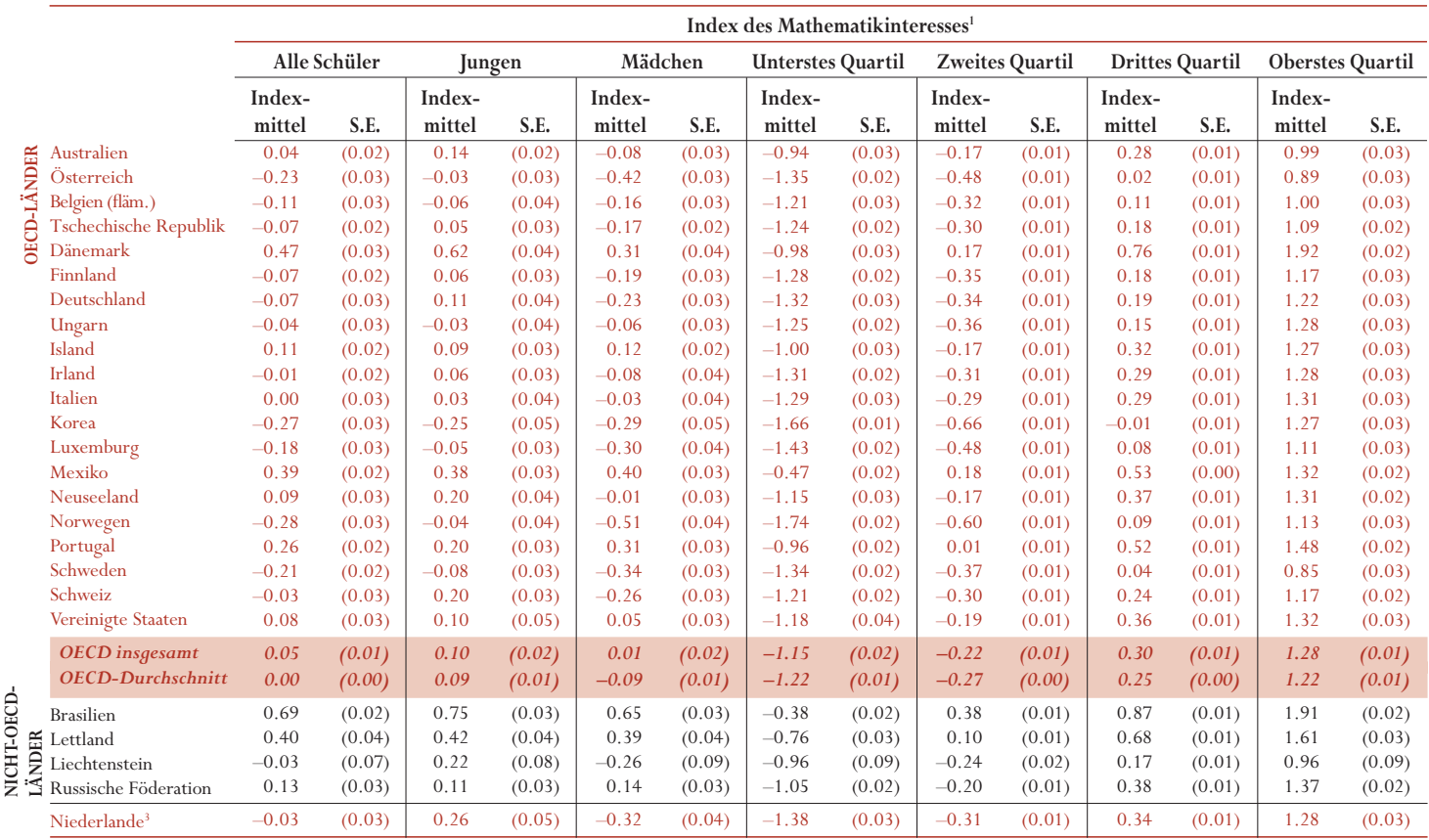

\begin{tabular}{|c|c|c|c|c|c|c|c|c|c|c|c|}
\hline & \multicolumn{8}{|c|}{$\begin{array}{l}\text { Leistungen auf der Skala für mathem. Grundbildung, } \\
\text { nach Indexquartilen für das jeweilige Land }{ }^{2}\end{array}$} & \multicolumn{2}{|c|}{$\begin{array}{l}\text { Punktzahlveränderung auf für mathem } \\
\text { Grundbildung, je Indexeinheit }{ }^{2}\end{array}$} \\
\hline & & $\begin{array}{c}\text { Mittel- } \\
\text { wert }\end{array}$ & S.E. & $\begin{array}{c}\text { Mittel- } \\
\text { wert }\end{array}$ & S.E. & $\begin{array}{c}\text { Mittel- } \\
\text { wert }\end{array}$ & S.E. & $\begin{array}{c}\text { Mittel- } \\
\text { wert }\end{array}$ & S.E. & Veränderung & S.E. \\
\hline \multirow{13}{*}{ 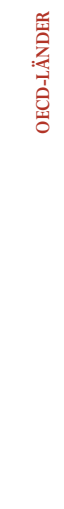 } & Australien & 529 & $(6.1)$ & 525 & $(4.8)$ & 530 & $(4.8)$ & 560 & $(5.3)$ & 15.0 & $(3.10)$ \\
\hline & Österreich & 510 & $(4.3)$ & 519 & (4.4) & 510 & $(5.0)$ & 526 & $(4.8)$ & 7.8 & $(2.37)$ \\
\hline & Belgien (fläm.) & 533 & $(5.1)$ & 546 & (5.9) & 545 & (6.6) & 564 & (7.5) & 12.5 & $(3.12)$ \\
\hline & Finnland & 508 & (3.5) & 527 & (3.8) & 541 & (3.7) & 575 & (3.4) & 25.0 & $(1.75)$ \\
\hline & Deutschland & 497 & $(4.4)$ & 487 & $(4.0)$ & 494 & $(5.0)$ & 514 & (4.7) & 8.9 & $(1.97)$ \\
\hline & Ungarn & 477 & $(5.6)$ & 483 & (4.7) & 492 & $(5.5)$ & 513 & (5.9) & 14.0 & $(2.33)$ \\
\hline & Island & 499 & (4.1) & 502 & $(4.1)$ & 520 & $(4.1)$ & 549 & $(4.3)$ & 22.9 & $(2.16)$ \\
\hline & Irland & 501 & (3.9) & 500 & $(4.4)$ & 499 & (5.1) & 519 & (4.6) & 7.8 & $(2.08)$ \\
\hline & Italien & 447 & $(4.1)$ & 455 & $(5,4)$ & 454 & $(4.5)$ & 475 & $(5.1)$ & 9.3 & $(2.29)$ \\
\hline & Korea & 503 & $(4,0)$ & 537 & (3.6) & 564 & $(4.6)$ & 584 & $(4.1)$ & 26.7 & $(1.74)$ \\
\hline & Schweden & 495 & $(3.3)$ & 509 & $(4,2)$ & 508 & $(4.4)$ & 534 & (4.6) & 16.0 & $(2.10)$ \\
\hline & Schweiz & 525 & (6.5) & 533 & $(6.0)$ & 531 & (5.5) & 541 & $(5.6)$ & 6.9 & $(2.54)$ \\
\hline & Vereinigte Staaten & 491 & $(9.5)$ & 493 & $(6.2)$ & 489 & $(10.8)$ & 525 & $(9.8)$ & 12.0 & $(3.09)$ \\
\hline \multirow{7}{*}{ 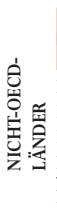 } & $O E C D$ insgesamt & 476 & (3.9) & 481 & $(3.0)$ & 484 & $(4.3)$ & 509 & $(4.5)$ & 6.1 & $(1.55)$ \\
\hline & OECD-Durchschnitt & 489 & $(1.2)$ & 496 & $(1.2)$ & 501 & $(1.1)$ & 524 & (1.3) & 10.7 & $(0.63)$ \\
\hline & Brasilien & 328 & $(4.9)$ & 334 & (5.4) & 340 & $(5.8)$ & 359 & (7.0) & 13.4 & $(2.95)$ \\
\hline & Lettland & 442 & $(6.0)$ & 463 & $(7.9)$ & 465 & $(6.4)$ & 492 & (6.1) & 18.3 & $(3.16)$ \\
\hline & Liechtenstein & 511 & $(13.9)$ & 511 & $(16.9)$ & 506 & $(15.0)$ & 532 & $(15.3)$ & 7.6 & $(10.26)$ \\
\hline & Russische Föderation & 460 & $(6.8)$ & 466 & $(5.8)$ & 482 & $(7.2)$ & 513 & $(5.3)$ & 20.3 & $(2.75)$ \\
\hline & Niederlande $^{3}$ & 555 & (6.1) & 555 & (6.5) & 569 & (6.4) & 580 & (5.8) & 9.6 & $(3.28)$ \\
\hline
\end{tabular}

1. Zur Definition des Index siehe Anhang A1.

2. Zur erklärten Varianz siehe Anhang A2. In Indexeinheiten gemessene Veränderungen sind, soweit fett gedruckt, statistisch signifikant. Wenn die untersten und obersten Quartile durch Fettdruck gekennzeichnet sind, weist dies auf eine statistisch signifikante Differenz zwischen diesen Quartilen hin.

3. Die Beteiligungsquote ist zu niedrig, um die Vergleichbarkeit zu gewährleisten (vgl. Anhang A3). 
Tabelle 4.3

Index Freude am Lesen und Leistungen auf der Gesamtskala Lesekompetenz, nach Indexquartilen für das jeweilige Land Die Ergebnisse basieren auf Schülerangaben

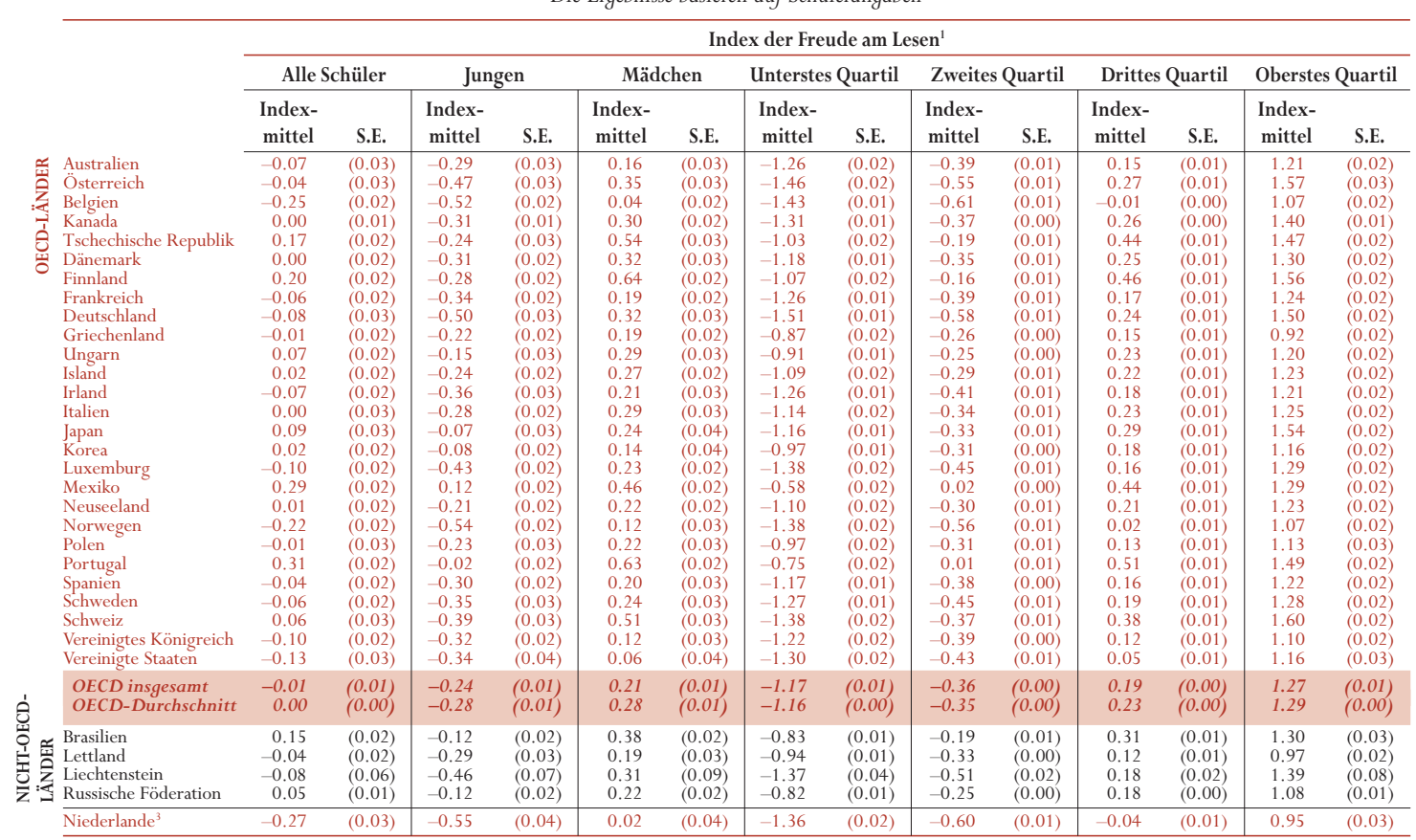

\begin{tabular}{|c|c|c|c|c|c|c|c|c|c|c|}
\hline & \multicolumn{8}{|c|}{$\begin{array}{l}\text { Leistungen auf der Gesamtskala Lesekompetenz, } \\
\text { nach Indexquartilen für das jeweilige Land }\end{array}$} & \multirow{2}{*}{\multicolumn{2}{|c|}{$\begin{array}{c}\text { Punktzahlveränderung auf der Gesamtskala } \\
\text { Lesekompetenz, je Indexeinheit }{ }^{2}\end{array}$}} \\
\hline & \multicolumn{2}{|c|}{ Unterstes Quartil } & \multicolumn{2}{|c|}{ Zweites Quartil } & \multicolumn{2}{|c|}{ Drittes Quartil } & \multicolumn{2}{|c|}{ Oberstes Quartil } & & \\
\hline & $\begin{array}{c}\text { Mittel- } \\
\text { wert }\end{array}$ & S.E. & $\begin{array}{c}\text { Mittel- } \\
\text { wert }\end{array}$ & S.E. & $\begin{array}{c}\text { Mittel- } \\
\text { wert }\end{array}$ & S.E. & $\begin{array}{c}\text { Mittel- } \\
\text { wert }\end{array}$ & S.E. & Veränderung & S.E. \\
\hline $\begin{array}{l}\text { Australien } \\
\text { Österreich } \\
\text { Belgien } \\
\text { Kanada } \\
\text { Tschechische Republik } \\
\text { Dänemark } \\
\text { Finnland } \\
\text { Frankreich } \\
\text { Deutschland } \\
\text { Griechenland } \\
\text { Ungarn } \\
\text { Island } \\
\text { Irland } \\
\text { Italien } \\
\text { Japan } \\
\text { Korea } \\
\text { Luxemburg } \\
\text { Mexiko } \\
\text { Neuseeland } \\
\text { Norwegen } \\
\text { Polen } \\
\text { Portugal } \\
\text { Spanien } \\
\text { Schweden } \\
\text { Schweiz } \\
\text { Vereinigtes Königreich } \\
\text { Vereinigte Staaten }\end{array}$ & $\begin{array}{l}479 \\
468 \\
483 \\
486 \\
459 \\
452 \\
493 \\
479 \\
453 \\
452 \\
440 \\
456 \\
482 \\
463 \\
499 \\
494 \\
436 \\
413 \\
487 \\
461 \\
460 \\
436 \\
460 \\
469 \\
447 \\
481 \\
474\end{array}$ & $\begin{array}{l}(3.8) \\
(3.3) \\
(3.8) \\
(1.9) \\
(3.0) \\
(3.7) \\
(3.2) \\
(3.2) \\
(4.1) \\
(6.3) \\
(4.0) \\
(2.9) \\
(3.9) \\
(4.0) \\
(5.2) \\
(2.9) \\
(2.8) \\
(5.3) \\
(3.1) \\
(5.1) \\
(5.7) \\
(4.6) \\
(3.5) \\
(2.8) \\
(4.2) \\
(2.7) \\
(6.2)\end{array}$ & $\begin{array}{l}496 \\
483 \\
489 \\
514 \\
476 \\
476 \\
526 \\
489 \\
466 \\
454 \\
453 \\
488 \\
505 \\
468 \\
509 \\
513 \\
434 \\
408 \\
501 \\
484 \\
454 \\
449 \\
476 \\
496 \\
470 \\
503 \\
481\end{array}$ & $\begin{array}{l}(4.4) \\
(3.3) \\
(4.2) \\
(2.1) \\
(2.8) \\
(3.4) \\
(4.5) \\
(3.9) \\
(3.2) \\
(5.7) \\
(5.3) \\
(2.5) \\
(4.0) \\
(3.3) \\
(5.9) \\
(3.1) \\
(3.0) \\
(3.8) \\
(4.5) \\
(3.9) \\
(5.2) \\
(5.9) \\
(3.4) \\
(3.3) \\
(4.7) \\
(3.3) \\
(9.8)\end{array}$ & $\begin{array}{l}551 \\
519 \\
513 \\
552 \\
518 \\
511 \\
566 \\
518 \\
505 \\
478 \\
493 \\
526 \\
536 \\
491 \\
526 \\
535 \\
436 \\
420 \\
548 \\
514 \\
483 \\
483 \\
501 \\
527 \\
509 \\
536 \\
514\end{array}$ & $\begin{array}{l}(3.8) \\
(3.2) \\
(4.7) \\
(2.0) \\
(3.0) \\
(3.4) \\
(3.6) \\
(4.1) \\
(4.7) \\
(5.4) \\
(5.1) \\
(2.6) \\
(4.1) \\
(4.2) \\
(5.9) \\
(2.7) \\
(3.7) \\
(4.1) \\
(4.4) \\
(3.9) \\
(4.9) \\
(4.7) \\
(3.4) \\
(3.5) \\
(4.9) \\
(3.3) \\
(8.6)\end{array}$ & $\begin{array}{l}591 \\
560 \\
562 \\
590 \\
550 \\
555 \\
604 \\
552 \\
555 \\
520 \\
539 \\
566 \\
588 \\
532 \\
562 \\
558 \\
494 \\
445 \\
591 \\
570 \\
537 \\
521 \\
539 \\
576 \\
556 \\
583 \\
566\end{array}$ & $\begin{array}{l}(4.2) \\
(3.1) \\
(5.3) \\
(1.6) \\
(3.0) \\
(3.5) \\
(2.5) \\
(2.9) \\
(3.5) \\
(5.2) \\
(4.1) \\
(2.9) \\
(3.2) \\
(3.0) \\
(4.4) \\
(2.6) \\
(3.4) \\
(4.5) \\
(4.2) \\
(3.1) \\
(5.9) \\
(4.5) \\
(2.9) \\
(3.2) \\
(5.1) \\
(3.8) \\
(6.2)\end{array}$ & $\begin{array}{l}41.8 \\
29.3 \\
30.8 \\
36.2 \\
33.8 \\
40.0 \\
40.0 \\
27.5 \\
33.3 \\
35.7 \\
43.0 \\
43.7 \\
40.1 \\
28.0 \\
23.8 \\
25.9 \\
19.4 \\
14.6 \\
43.7 \\
42.5 \\
34.1 \\
33.5 \\
31.3 \\
39.5 \\
34.8 \\
40.4 \\
33.0\end{array}$ & $\begin{array}{l}(2.06) \\
(1.35) \\
(1.71) \\
(0.70) \\
(1.48) \\
(1.69) \\
(1.13) \\
(1.29) \\
(1.59) \\
(3.51) \\
(2.10) \\
(1.59) \\
(1.63) \\
(1.75) \\
(1.55) \\
(1.26) \\
(1.66) \\
(2.59) \\
(1.95) \\
(2.12) \\
(3.09) \\
(1.72) \\
(1.42) \\
(1.55) \\
(1.74) \\
(1.55) \\
(2.22)\end{array}$ \\
\hline $\begin{array}{l}\text { OECD insgesamt } \\
\text { OECD-Durchschnitt }\end{array}$ & $\begin{array}{l}470 \\
465\end{array}$ & $\begin{array}{l}(1.7) \\
(0.8)\end{array}$ & $\begin{array}{l}480 \\
480\end{array}$ & $\begin{array}{l}(2.6) \\
(0.8)\end{array}$ & $\begin{array}{l}508 \\
512\end{array}$ & $\begin{array}{l}(2.4) \\
(0.9)\end{array}$ & $\begin{array}{l}550 \\
554\end{array}$ & $\begin{array}{l}(2.0) \\
(0.8)\end{array}$ & $\begin{array}{l}28.1 \\
32.4\end{array}$ & $\begin{array}{l}(0.84) \\
(0.39)\end{array}$ \\
\hline $\begin{array}{l}\text { Brasilien } \\
\text { Lettland } \\
\text { Liechtenstein } \\
\text { Russische Föderation }\end{array}$ & $\begin{array}{l}386 \\
422 \\
441 \\
431\end{array}$ & $\begin{array}{l}(4.3) \\
(6.8) \\
(8.7) \\
(4.9) \\
\end{array}$ & $\begin{array}{l}379 \\
439 \\
446 \\
446\end{array}$ & $\begin{array}{r}(4.1) \\
(5.5) \\
(10.5) \\
(4.0) \\
\end{array}$ & $\begin{array}{l}396 \\
467 \\
503 \\
470\end{array}$ & $\begin{array}{r}(3.9) \\
(5.8) \\
(10.4) \\
(4.2) \\
\end{array}$ & $\begin{array}{l}431 \\
511 \\
543 \\
504\end{array}$ & $\begin{array}{l}(4.5) \\
(6.4) \\
(7.4) \\
(5.8) \\
\end{array}$ & $\begin{array}{l}20.6 \\
42.3 \\
35.7 \\
34.9\end{array}$ & $\begin{array}{l}(2.24) \\
(3.33) \\
(4.01) \\
(1.71)\end{array}$ \\
\hline Niederlande $^{3}$ & 507 & $(3.7)$ & 512 & $(6.0)$ & 538 & $(5.2)$ & 572 & $(4.3)$ & 27.6 & $(2.13)$ \\
\hline
\end{tabular}

1. Zur Definition des Index siehe Anhang A1.

2. Zur erklärten Varianz siehe Anhang A2. In Indexeinheiten gemessene Veränderungen sind, soweit fett gedruckt, statistisch signifikant. Wenn die untersten und obersten Quartile durch Fettdruck gekennzeichnet sind, weist dies auf eine statistisch signifikante Differenz zwischen diesen Quartilen hin.

3. Die Beteiligungsquote ist zu niedrig, um die Vergleichbarkeit zu gewährleisten (vgl. Anhang A3). 
ANHANG B1

Tabelle 4.4

Täglicher Zeitaufwand für Lesen zum Vergnügen und Leistungen auf der Gesamtskala Lesekompetenz Die Ergebnisse basieren auf Schülerangaben

\begin{tabular}{|c|c|c|c|c|c|c|c|c|c|c|c|c|c|c|c|c|c|c|c|c|}
\hline & \multicolumn{4}{|c|}{$\begin{array}{l}\text { Schüler gibt an, } \\
\text { nicht zum Vergnügen } \\
\text { zu lesen }\end{array}$} & \multicolumn{4}{|c|}{$\begin{array}{l}\text { Schüler gibt an, } \\
\text { täglich bis zu } \\
30 \text { Min. zu lesen }\end{array}$} & \multicolumn{4}{|c|}{$\begin{array}{l}\text { Schüler gibt an, } \\
\text { täglich zwischen } 30 \\
\text { und } 60 \mathrm{Min} . \text { zu lesen }\end{array}$} & \multicolumn{4}{|c|}{$\begin{array}{c}\text { Schüler gibt an, } \\
\text { täglich 1-2 Std. } \\
\text { zu lesen }\end{array}$} & \multicolumn{4}{|c|}{$\begin{array}{l}\text { Schüler gibt an, } \\
\text { täglich mehr als } \\
\text { 2Std. zu lesen }\end{array}$} \\
\hline & \multicolumn{4}{|c|}{ Mittel- } & $\%$ & \multicolumn{3}{|c|}{ Mittel- } & $\%$ & \multicolumn{3}{|c|}{ Mittel- } & $\%$ & \multicolumn{3}{|c|}{ Mittel- } & $\%$ & S.E. & Mittel- & I- \\
\hline Australien & 33.1 & $(1.2)$ & 484 & 3.9) & 30.5 & .9) & 537 & (3.9) & 20.5 & 9) & 564 & $(4.7)$ & 1.8 & $(0.5)$ & 575 & (5.5) & 4.1 & $(0.3)$ & 558 & (9. \\
\hline Öster & 41.1 & $(1.1)$ & 477 & $(2.5)$ & 8.7 & .8) & & $(3.0)$ & 8.1 & $(0.7)$ & 539 & $(4.2)$ & 9.0 & & 540 & (5.6) & 3.1 & $(0.4)$ & 32 & (7.9) \\
\hline Tschech. & 26.2 & $(0.8)$ & 458 & $30)$ & 29.7 & & & & 5.7 & & 524 & (2.8) & 12.9 & & & (4.3) & 5.5 & & 8 & \\
\hline & & & 44 & & & & & & & & & & 94 & & & & 4.4 & & & \\
\hline & & & & & & & & & 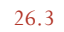 & & & & & & & & .1 & & 34 & \\
\hline & & & & & & & & & 6 & & & (3.1) & 6 & & & & .4 & & 14 & \\
\hline & & & & & & & & & 0 & & & & & & & & 6 & & 1 & \\
\hline & & & & & & & & & 7 & & & & 20.0 & & & (4 & 8.7 & & & \\
\hline & & & & & & & & & 4.2 & & & & 13.4 & & & (6 & 8.1 & & 68 & \\
\hline Kor & & & 2 & 7 & & & & & 9 & & & (3.2) & & & & (3. & & & 9 & \\
\hline & & & & & & & & & & & & & & & & (6 & & & & \\
\hline & & & & & & & & & & & & & & & & & & & & \\
\hline & & & & & & & & & & & & & & & & & & & 3 & \\
\hline & & & & & & & & & 20. & & & & & & & & 2 & & 6 & \\
\hline & & & & & , & & & & 28 & (0 & 2 & $(5$ & 16.5 & & 498 & (6 & .0 & & 497 & \\
\hline & & 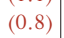 & - & ( & 1 & & 474 & (4 & 26.5 & $(0.9)$ & & (5.3) & 12.4 & & 494 & (6.0) & 3.7 & & 468 & $(10.7)$ \\
\hline & & (0. & 0 & 100 & 32.9 & & & & 24.2 & & 19 & $(3.0)$ & 8 & & 514 & (5.1) & 2.4 & & 499 & (10.1) \\
\hline & 36.0 & & 83 & $(2.8)$ & 30.8 & & & (3.6) & 21.0 & $(0.6)$ & 547 & (3.1) & 8 & $(0.5)$ & 556 & (4.9) & 3.4 & $(0.3)$ & 529 & $(8.8)$ \\
\hline & & & & $(4.1)$ & 33.0 & & & & 20.5 & & & $(4.7)$ & & & 533 & $(7.8)$ & .0 & & 499 & (12.8) \\
\hline & & & & $(3.0)$ & 35.7 & & & & & & & & & & 556 & & 2.9 & & 528 & $(9.8)$ \\
\hline Vereinigte Staaten & 40.7 & $(1$. & 479 & (7.0 & 31.2 & $(1.1)$ & 53 & $(7.3)$ & 16.2 & $(0.8)$ & 531 & $(8.4)$ & 8.1 & $(0.6)$ & 539 & $(12.2)$ & 3.9 & $(0.5)$ & 511 & (10. \\
\hline
\end{tabular}

1. Die Beteiligungsquote ist zu niedrig, um die Vergleichbarkeit zu gewährleisten (vgl. Anhang A3). 
Tabelle 4.5

Index der Kontrollstrategien und Leistungen auf der Gesamtskala Lesekompetenz, nach Indexquartilen für das jeweilige Land Die Ergebnisse basieren auf Schülerangaben

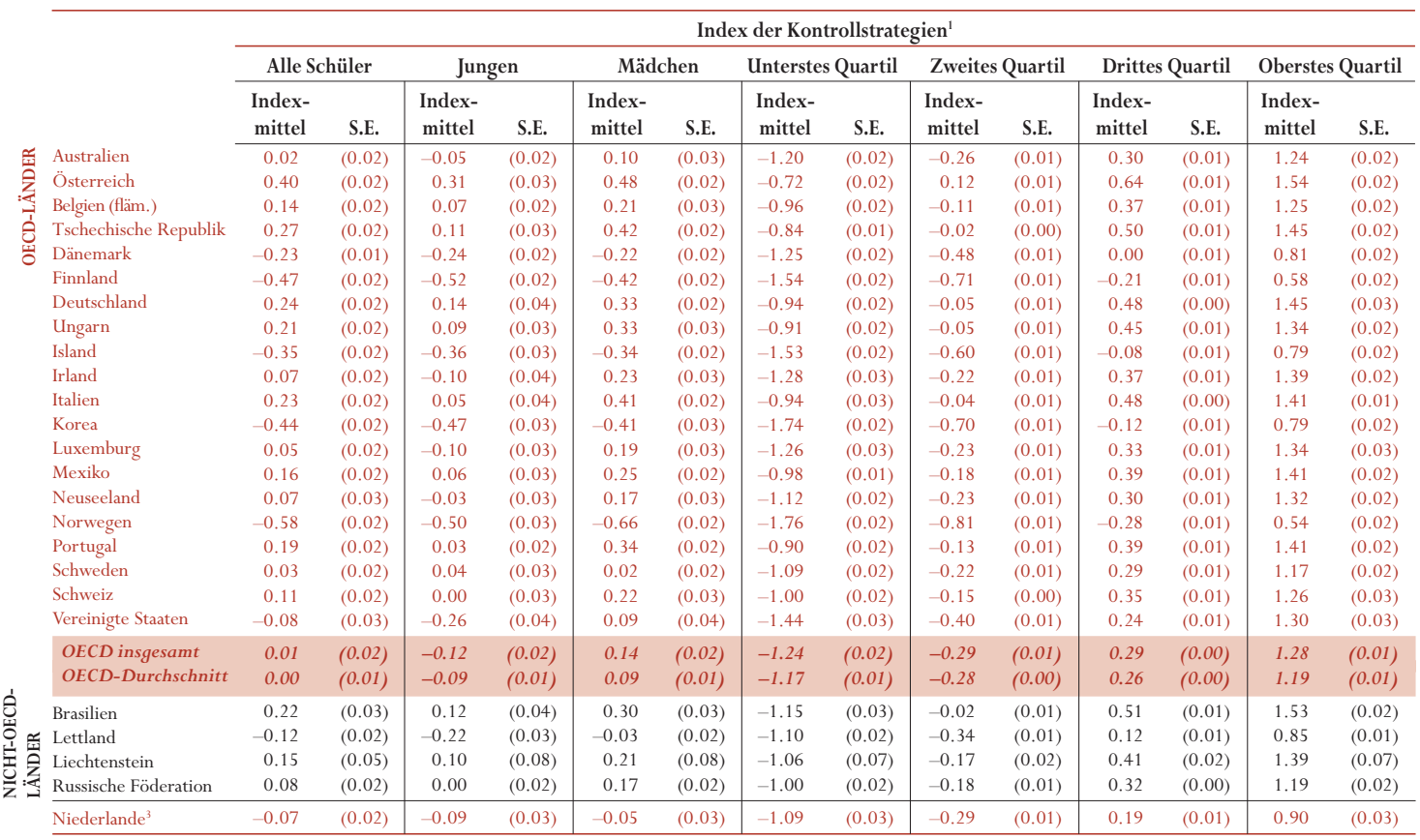

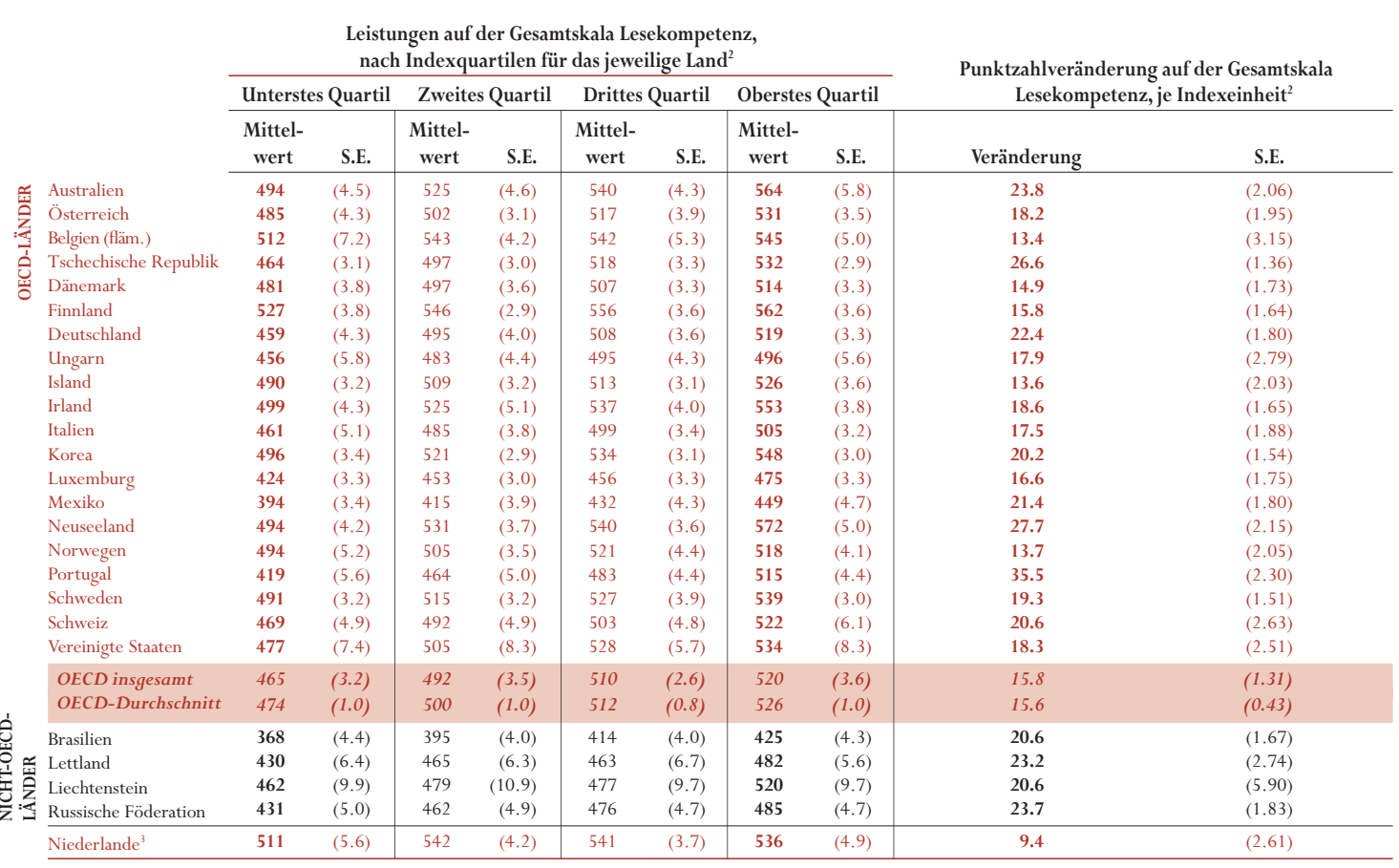

1. Zur Definition des Index siehe Anhang A1.

2. Zur erklärten Varianz siehe Anhang A2. In Indexeinheiten gemesseneVeränderungen sind, soweit fett gedruckt, statistisch signifikant. Wenn die untersten und obersten Quartile durch Fettdruck gekennzeichnet sind, weist dies auf eine statistisch signifikante Differenz zwischen diesen Quartilen hin.

3. Die Beteiligungsquote ist zu niedrig, um die Vergleichbarkeit zu gewährleisten (vgl. Anhang A3). 
Tabelle 4.6

Index der Memorierstrategien und Leistungen auf der Gesamtskala Lesekompetenz, nach Indexquartilen für das jeweilige Land Die Ergebnisse basieren auf Schülerangaben

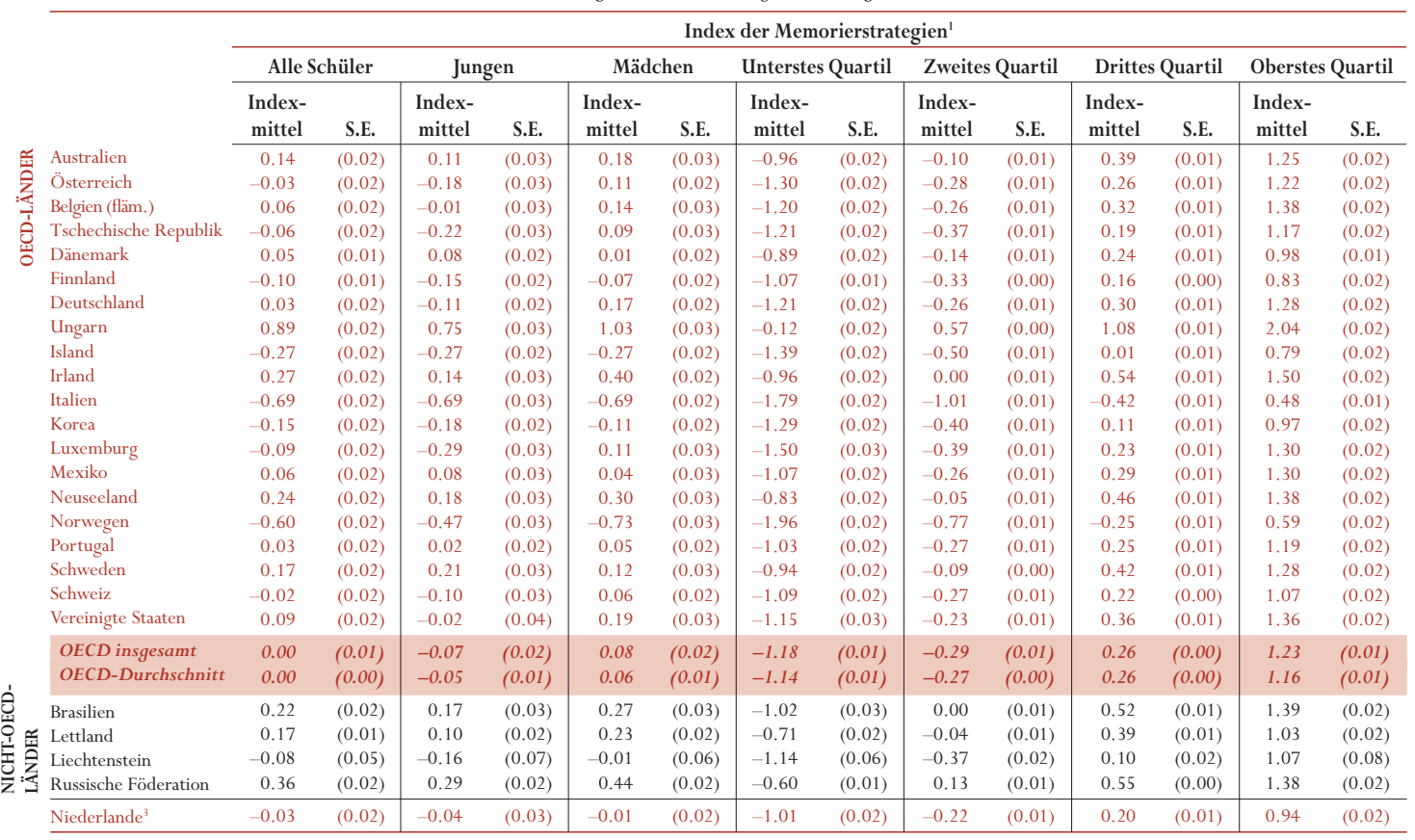

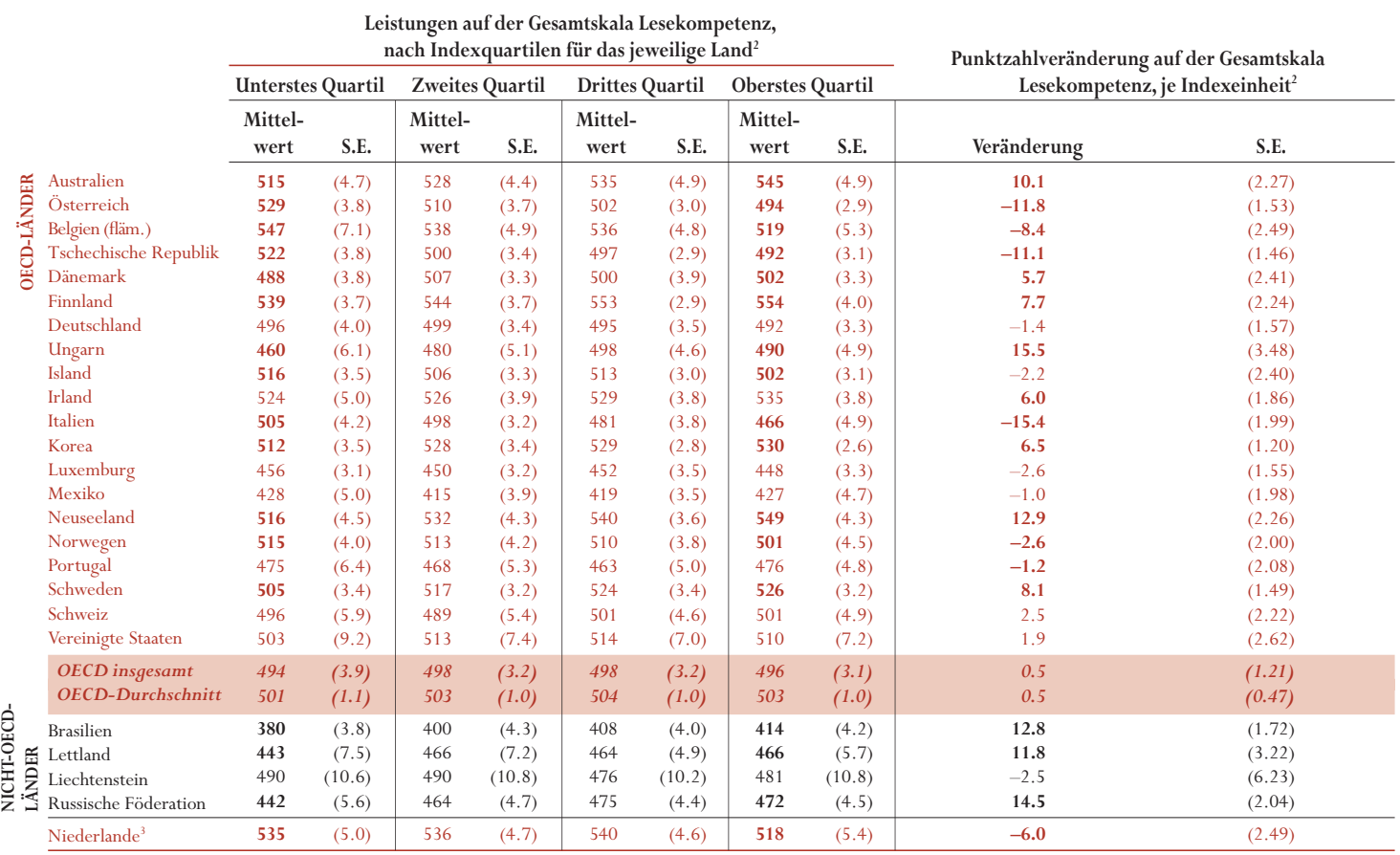

1. Zur Definition des Index siehe Anhang A1.

2. Zur erklärten Varianz siehe Anhang A2. In Indexeinheiten gemessene Veränderungen sind, soweit fett gedruckt, statistisch signifikant. Wenn die untersten und obersten Quartile durch Fettdruck gekennzeichnet sind, weist dies auf eine statistisch signifikante Differenz zwischen diesen Quartilen hin.

3. Die Beteiligungsquote ist zu niedrig, um die Vergleichbarkeit zu gewährleisten (vgl. Anhang A3). 
Tabelle 4.7

Index der Elaborationsstrategien und Leistungen auf der Gesamtskala Lesekompetenz, nach Indexquartilen für das jeweilige Land Die Ergebnisse basieren auf Schülerangaben

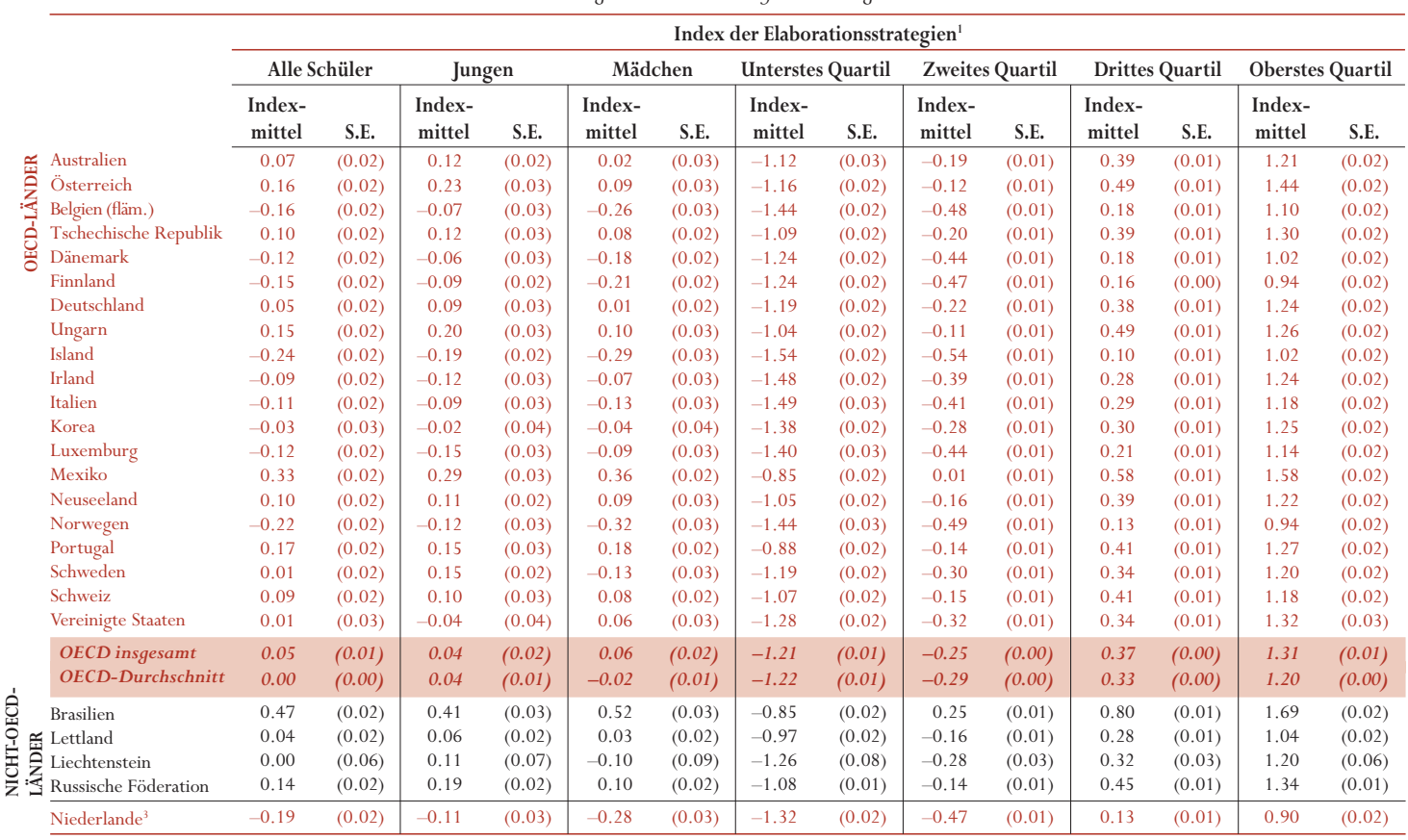

\begin{tabular}{|c|c|c|c|c|c|c|c|c|c|c|}
\hline & \multicolumn{8}{|c|}{$\begin{array}{l}\text { Leistungen auf der Gesamtskala Lesekompetenz, } \\
\text { nach Indexquartilen für das jeweilige Land }{ }^{2}\end{array}$} & \multirow{2}{*}{\multicolumn{2}{|c|}{$\begin{array}{c}\text { Punktzahlveränderung auf der Gesamtskala } \\
\text { Lesekompetenz, je Indexeinheit }^{2}\end{array}$}} \\
\hline & \multicolumn{2}{|c|}{ Unterstes Quartil } & \multicolumn{2}{|c|}{ Zweites Quartil } & \multicolumn{2}{|c|}{ Drittes Quartil } & \multicolumn{2}{|c|}{ Oberstes Quartil } & & \\
\hline Australien & 517 & $(4.4)$ & 523 & $(4.2)$ & 533 & $(4.6)$ & 551 & $(5.3)$ & 13.0 & $(1.96)$ \\
\hline Österreich & 501 & $(3.6)$ & 500 & (3.3) & 509 & $(3.2)$ & 526 & $(2.7)$ & 9.8 & $(1.44)$ \\
\hline Belgien (fläm.) & 532 & $(6.6)$ & 536 & $(4.7)$ & 540 & $(4.5)$ & 534 & $(6.1)$ & 0.4 & $(2.23)$ \\
\hline Tschechische Republik & 485 & (3.4) & 491 & $(3.0)$ & 506 & $(3.0)$ & 529 & $(3.2)$ & 18.2 & $(1.42)$ \\
\hline Dänemark & 482 & $(3.9)$ & 492 & $(4.1)$ & 514 & (3.1) & 514 & (3.3) & 13.6 & $(1.66)$ \\
\hline Finnland & 535 & (3.9) & 537 & $(3.2)$ & 553 & $(3.0)$ & 566 & $(4.6)$ & 15.4 & $(1.81)$ \\
\hline Deutschland & 474 & $(4.4)$ & 486 & $(3.4)$ & 499 & $(4.4)$ & 525 & (3.1) & 20.1 & $(1.77)$ \\
\hline Ungarn & 466 & $(5.7)$ & 484 & $(5.8)$ & 490 & (5.4) & 490 & $(5.0)$ & 10.7 & $(2.96)$ \\
\hline Island & 498 & $(3.5)$ & 501 & $(2.7)$ & 507 & (3.4) & 533 & (3.6) & 12.4 & $(1.95)$ \\
\hline Irland & 521 & $(4.5)$ & 527 & $(4.4)$ & 528 & $(3.8)$ & 539 & $(4.1)$ & 6.0 & $(1.62)$ \\
\hline Italien & 483 & $(4.1)$ & 480 & $(4.2)$ & 487 & (3.9) & 501 & (3.6) & 6.4 & $(1.71)$ \\
\hline Korea & 492 & $(3.1)$ & 518 & $(3.1)$ & 537 & $(2.5)$ & 552 & (3.1) & 22.4 & $(1.29)$ \\
\hline Luxemburg & 441 & $(3.2)$ & 449 & $(3.3)$ & 456 & $(3.2)$ & 467 & $(3.2)$ & 8.8 & $(1.69)$ \\
\hline Mexiko & 414 & $(3.8)$ & 413 & $(3.9)$ & 425 & $(4.4)$ & 439 & $(4.8)$ & 10.9 & $(1.76)$ \\
\hline Neuseeland & 525 & $(4.7)$ & 533 & $(4.4)$ & 538 & $(3.8)$ & 544 & $(5.3)$ & 8.7 & $(2.48)$ \\
\hline Norwegen & 490 & $(4.6)$ & 504 & (3.5) & 517 & $(4.2)$ & 529 & $(4.3)$ & 16.9 & $(1.77)$ \\
\hline Portugal & 441 & $(5.5)$ & 463 & $(4.8)$ & 476 & $(5.0)$ & 502 & (5.1) & 25.2 & $(2.37)$ \\
\hline Schweden & 504 & $(3.0)$ & 513 & $(3.3)$ & 518 & $(2.9)$ & 536 & (3.1) & 12.4 & $(1.39)$ \\
\hline Schweiz & 477 & $(4.7)$ & 493 & $(4.4)$ & 506 & $(5.4)$ & 513 & $(6,1)$ & 15.2 & $(2.19)$ \\
\hline Vereinigte Staaten & 500 & $(7.6)$ & 505 & $(8.5)$ & 520 & $(6.8)$ & 521 & $(8.1)$ & 7.7 & $(2.59)$ \\
\hline$O E C D$ insgesamt & 482 & $(3.2)$ & 490 & (3.7) & 503 & $(3.1)$ & 513 & (3.4) & 8.1 & $(1.24)$ \\
\hline OECD-Durchschnitt & 488 & $(1.1)$ & 497 & $(0.9)$ & 508 & $(0.9)$ & 521 & $(1.1)$ & 10.5 & $(0.46)$ \\
\hline Brasilien & 382 & $(4.3)$ & 394 & (3.4) & 410 & $(4.1)$ & 418 & (3.9) & 15.9 & $(1.70)$ \\
\hline Lettland & 447 & $(6.3)$ & 459 & $(6.2)$ & 463 & $(6.8)$ & 473 & $(6.0)$ & 11.8 & $(2.81)$ \\
\hline Liechtenstein & 473 & (9.6) & 485 & $(11.0)$ & 476 & $(11.0)$ & 505 & (10.3) & 12.3 & $(4.84)$ \\
\hline Russische Föderation & 450 & $(4.7)$ & 459 & $(5.1)$ & 468 & $(5.2)$ & 478 & $(4.3)$ & 10.6 & $(1.49)$ \\
\hline Niederlande $^{3}$ & 531 & $(4.3)$ & 528 & $(4.9)$ & 539 & $(4.2)$ & 533 & $(5.3)$ & 1.6 & $(2.00)$ \\
\hline
\end{tabular}

1. Zur Definition des Index siehe Anhang A1.

2. Zur erklärten Varianz siehe Anhang A2. In Indexeinheiten gemessene Veränderungen sind, soweit fett gedruckt, statistisch signifikant. Wenn die untersten und obersten Quartile durch Fettdruck gekennzeichnet sind, weist dies auf eine statistisch signifikante Differenz zwischen diesen Quartilen hin.

3. Die Beteiligungsquote ist zu niedrig, um die Vergleichbarkeit zu gewährleisten (vgl. Anhang A3). 
Tabelle 4.8

Index des kooperativen Lernens und Leistungen auf der Gesamtskala Lesekompetenz, nach Indexquartilen für das jeweilige Land Die Ergebnisse basieren auf Schülerangaben

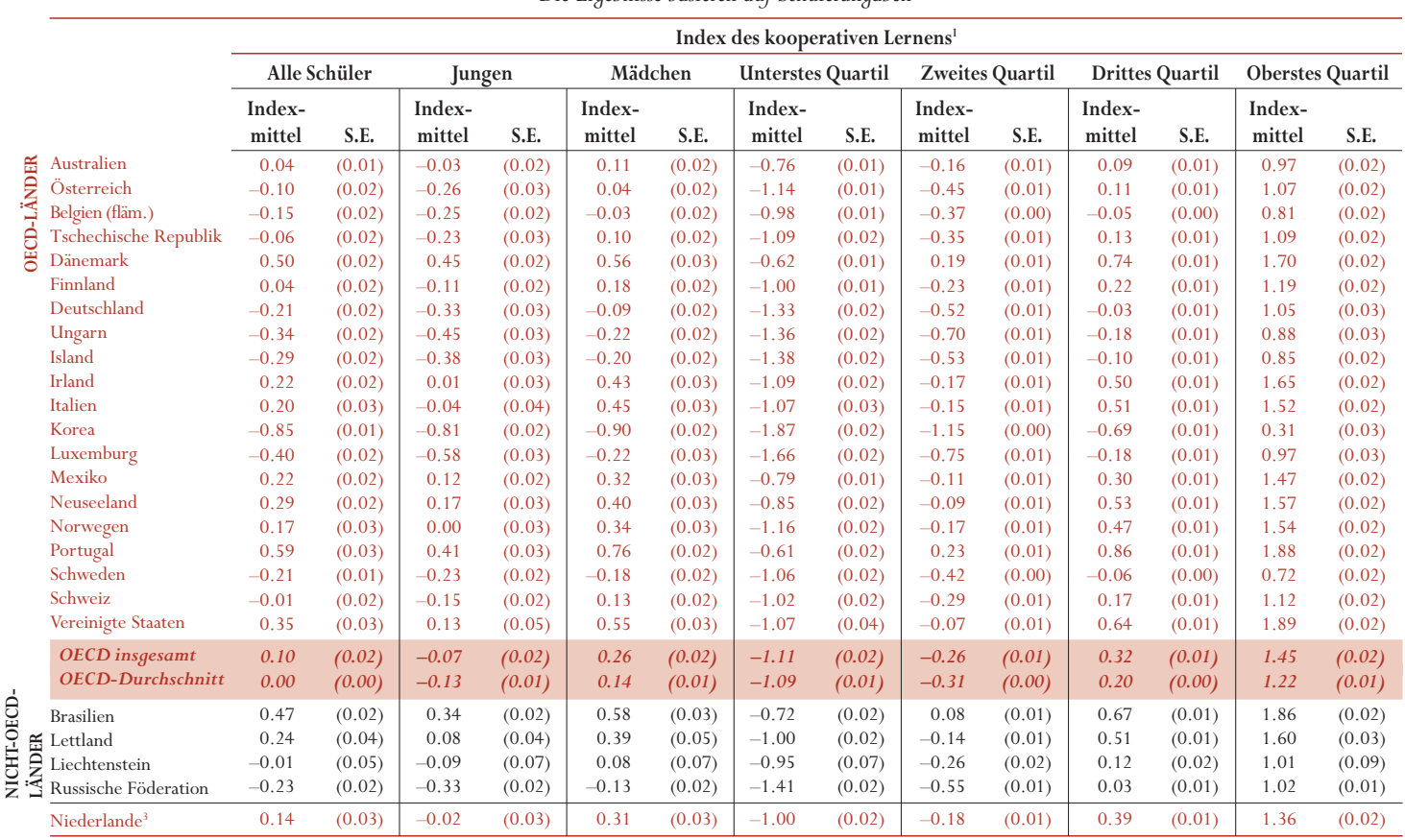

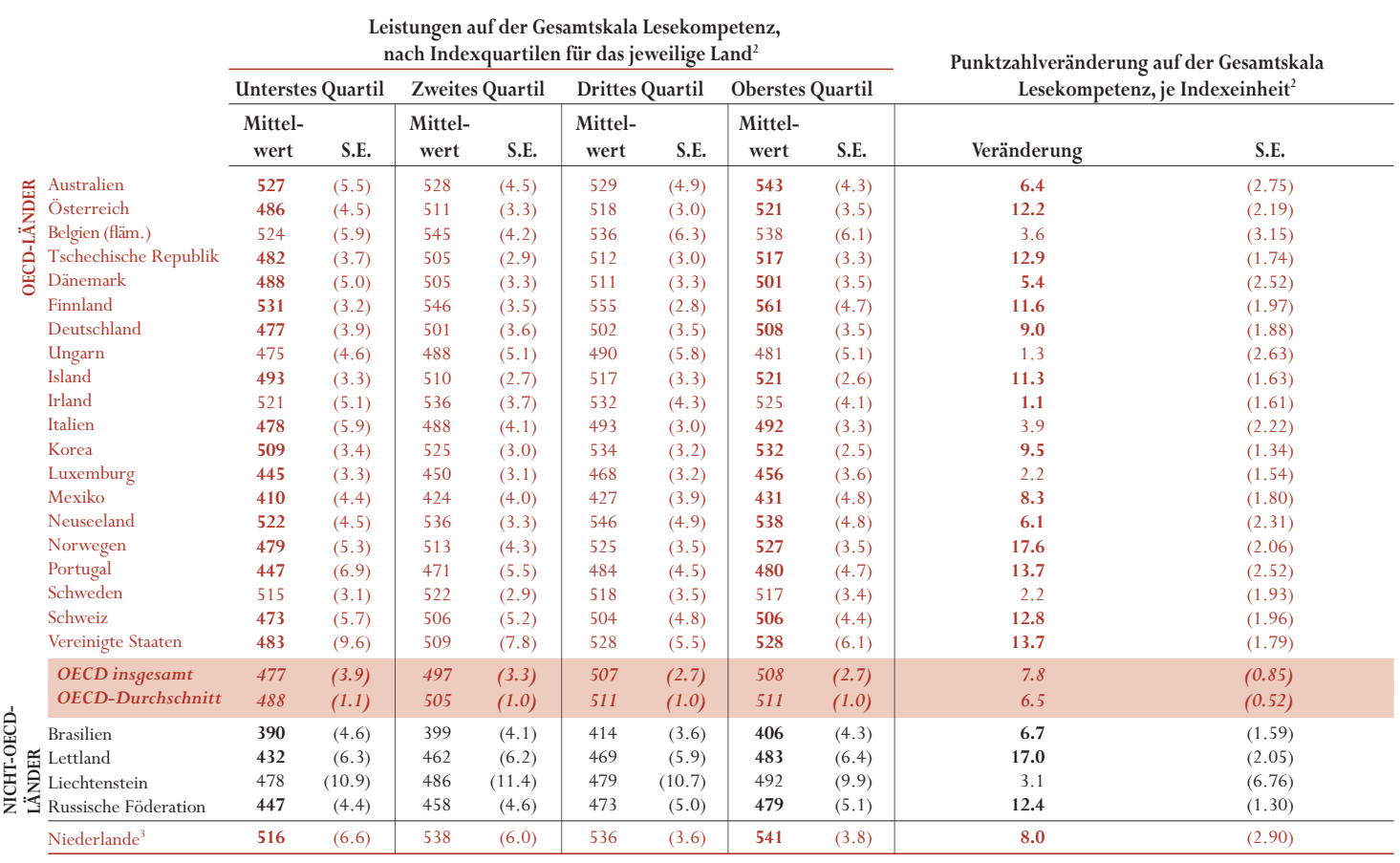

1. Zur Definition des Index siehe Anhang A1.

2. Zur erklärten Varianz siehe Anhang A2. In Indexeinheiten gemessene Veränderungen sind, soweit fett gedruckt, statistisch signifikant. Wenn die untersten und obersten Quartile durch Fettdruck gekennzeichnet sind, weist dies auf eine statistisch signifikante Differenz zwischen diesen Quartilen hin.

3. Die Beteiligungsquote ist zu niedrig, um die Vergleichbarkeit zu gewährleisten (vgl. Anhang A3). 
Tabelle 4.9

Index des wettbewerbsorientierten Lernens und Leistungen auf der Gesamtskala Lesekompetenz, nach Indexquartilen für das jeweilige Land

Die Ergebnisse basieren auf Schülerangaben

\begin{tabular}{|c|c|c|c|c|c|c|c|c|c|c|c|c|c|c|}
\hline & \multicolumn{14}{|c|}{ Index des wettbewerbsorientierten Lernens ${ }^{1}$} \\
\hline & \multicolumn{2}{|c|}{ Alle Schüler } & \multicolumn{2}{|c|}{ Jungen } & \multicolumn{2}{|c|}{ Mädchen } & \multicolumn{2}{|c|}{ Unterstes Quartil } & \multicolumn{2}{|c|}{ Zweites Quartil } & \multicolumn{2}{|c|}{ Drittes Quartil } & \multicolumn{2}{|c|}{ Oberstes Quartil } \\
\hline & $\begin{array}{l}\text { Index- } \\
\text { mittel }\end{array}$ & S.E. & $\begin{array}{l}\text { Index- } \\
\text { mittel }\end{array}$ & S.E. & $\begin{array}{l}\text { Index- } \\
\text { mittel }\end{array}$ & S.E. & $\begin{array}{l}\text { Index- } \\
\text { mittel }\end{array}$ & S.E. & $\begin{array}{l}\text { Index- } \\
\text { mittel }\end{array}$ & S.E. & $\begin{array}{l}\text { Index- } \\
\text { mittel }\end{array}$ & S.E. & $\begin{array}{l}\text { Index- } \\
\text { mittel }\end{array}$ & S.E. \\
\hline Australien & 0.10 & $(0.02)$ & 0.20 & $(0.02)$ & 0.00 & $(0.03)$ & -0.83 & $(0.01)$ & -0.20 & $(0.01)$ & 0.27 & $(0.01)$ & 1.17 & $(0.03)$ \\
\hline Österreich & -0.19 & $(0.02)$ & -0.13 & $(0.02)$ & -0.25 & $(0.03)$ & -1.32 & $(0.02)$ & -0.51 & $(0.01)$ & 0.04 & $(0.01)$ & 1.02 & $(0.02)$ \\
\hline Belgien (fläm.) & -0.38 & $(0.02)$ & -0.29 & $(0.02)$ & -0.48 & $(0.02)$ & -1.34 & $(0.02)$ & -0.65 & $(0.01)$ & -0.18 & $(0.01)$ & 0.66 & $(0.02)$ \\
\hline Tschechische Republik & 0.14 & $(0.02)$ & 0.14 & $(0.02)$ & 0.14 & $(0.02)$ & -0.94 & $(0.01)$ & -0.17 & $(0.01)$ & 0.35 & $(0.01)$ & 1.33 & $(0.02)$ \\
\hline Finnland & -0.25 & $(0.02)$ & -0.13 & $(0.02)$ & -0.35 & $(0.02)$ & -1.35 & $(0.02)$ & -0.55 & $(0.00)$ & -0.01 & $(0.01)$ & 0.92 & $(0.02)$ \\
\hline Deutschland & -0.07 & $(0.02)$ & -0.01 & $(0.03)$ & -0.14 & $(0.02)$ & -1.14 & $(0.02)$ & -0.38 & $(0.01)$ & 0.13 & $(0.01)$ & 1.11 & $(0.02)$ \\
\hline Ungarn & 0.10 & $(0.02)$ & 0.07 & $(0.03)$ & 0.13 & $(0.02)$ & -1.05 & $(0.02)$ & -0.23 & $(0.00)$ & 0.34 & $(0.01)$ & 1.32 & $(0.02)$ \\
\hline Island & 0.01 & $(0.02)$ & 0.13 & $(0.03)$ & -0.09 & $(0.03)$ & -1.20 & $(0.02)$ & -0.34 & $(0.01)$ & 0.26 & $(0.01)$ & 1.34 & $(0.02)$ \\
\hline Irland & 0.15 & $(0.02)$ & 0.35 & $(0.03)$ & -0.06 & $(0.03)$ & -1.25 & $(0.02)$ & -0.28 & $(0.01)$ & 0.47 & $(0.01)$ & 1.66 & $(0.02)$ \\
\hline Italien & -0.01 & $(0.02)$ & 0.06 & $(0.03)$ & -0.07 & $(0.03)$ & -1.33 & $(0.02)$ & -0.36 & $(0.01)$ & 0.31 & $(0.01)$ & 1.37 & $(0.02)$ \\
\hline Korea & -0.14 & $(0.02)$ & -0.10 & $(0.03)$ & -0.19 & $(0.03)$ & -1.31 & $(0.02)$ & -0.51 & $(0.01)$ & 0.07 & $(0.01)$ & 1.19 & $(0.02)$ \\
\hline Luxemburg & -0.18 & $(0.02)$ & -0.16 & $(0.03)$ & -0.20 & $(0.03)$ & -1.38 & $(0.02)$ & -0.49 & $(0.01)$ & 0.07 & $(0.01)$ & 1.07 & $(0.02)$ \\
\hline Mexiko & 0.54 & $(0.02)$ & 0.59 & $(0.02)$ & 0.49 & $(0.02)$ & -0.46 & $(0.01)$ & 0.23 & $(0.00)$ & 0.70 & $(0.01)$ & 1.70 & $(0.02)$ \\
\hline Neuseeland & 0.29 & $(0.02)$ & 0.40 & $(0.03)$ & 0.17 & $(0.03)$ & -0.94 & $(0.02)$ & -0.07 & $(0.01)$ & 0.53 & $(0.01)$ & 1.63 & $(0.02)$ \\
\hline Norwegen & -0.03 & $(0.02)$ & 0.12 & $(0.03)$ & -0.19 & $(0.03)$ & -1.38 & $(0.02)$ & -0.40 & $(0.01)$ & 0.24 & $(0.01)$ & 1.41 & $(0.02)$ \\
\hline Portugal & -0.22 & $(0.02)$ & -0.04 & $(0.03)$ & -0.39 & $(0.03)$ & -1.48 & $(0.02)$ & -0.58 & $(0.01)$ & 0.07 & $(0.01)$ & 1.10 & $(0.02)$ \\
\hline Schweden & -0.01 & $(0.02)$ & 0.09 & $(0.02)$ & -0.12 & $(0.02)$ & -1.02 & $(0.02)$ & -0.34 & $(0.01)$ & 0.20 & $(0.01)$ & 1.10 & $(0.02)$ \\
\hline Schweiz & -0.26 & $(0.02)$ & -0.14 & $(0.02)$ & -0.38 & $(0.02)$ & -1.34 & $(0.02)$ & -0.53 & $(0.01)$ & -0.02 & $(0.00)$ & 0.86 & $(0.02)$ \\
\hline Vereinigte Staaten & 0.27 & $(0.03)$ & 0.30 & $(0.04)$ & 0.25 & $(0.03)$ & -1.02 & $(0.02)$ & -0.08 & $(0.01)$ & 0.52 & $(0.01)$ & 1.68 & $(0.02)$ \\
\hline$O E C D$ insgesamt & 0.16 & $(0.01)$ & 0.21 & $(0.02)$ & 0.12 & $(0.01)$ & -1.02 & $(0.01)$ & -0.18 & $(0.01)$ & 0.39 & $(0.00)$ & 1.47 & $(0.01)$ \\
\hline OECD-Durchschnitt & 0.00 & $(0.00)$ & 0.10 & $(0.01)$ & -0.08 & $(0.01)$ & -1.16 & $(0.00)$ & -0.32 & $(0.00)$ & 0.25 & $(0.00)$ & 1.27 & $(0.01)$ \\
\hline Brasilien & -0.03 & $(0.02)$ & 0.09 & $(0.03)$ & -0.12 & $(0.03)$ & -1.23 & $(0.02)$ & -0.37 & $(0.01)$ & 0.22 & $(0.01)$ & 1.29 & $(0.03)$ \\
\hline Lettland & 0.22 & $(0.02)$ & 0.16 & $(0.03)$ & 0.27 & $(0.03)$ & -0.89 & $(0.02)$ & -0.07 & $(0.01)$ & 0.44 & $(0.01)$ & 1.38 & $(0.02)$ \\
\hline Liechtenstein & -0.20 & $(0.05)$ & -0.07 & $(0.08)$ & -0.34 & $(0.06)$ & -1.18 & $(0.06)$ & -0.48 & $(0.02)$ & 0.02 & $(0.02)$ & 0.81 & $(0.07)$ \\
\hline Russische Föderation & 0.13 & $(0.02)$ & 0.06 & $(0.02)$ & 0.21 & $(0.02)$ & -1.05 & $(0.02)$ & -0.20 & $(0.01)$ & 0.40 & $(0.01)$ & 1.38 & $(0.02)$ \\
\hline Niederlande $^{3}$ & -0.25 & $(0.03)$ & -0.07 & $(0.04)$ & -0.43 & $(0.04)$ & -1.55 & $(0.03)$ & -0.60 & $(0.01)$ & 0.05 & $(0.01)$ & 1.10 & $(0.03)$ \\
\hline
\end{tabular}

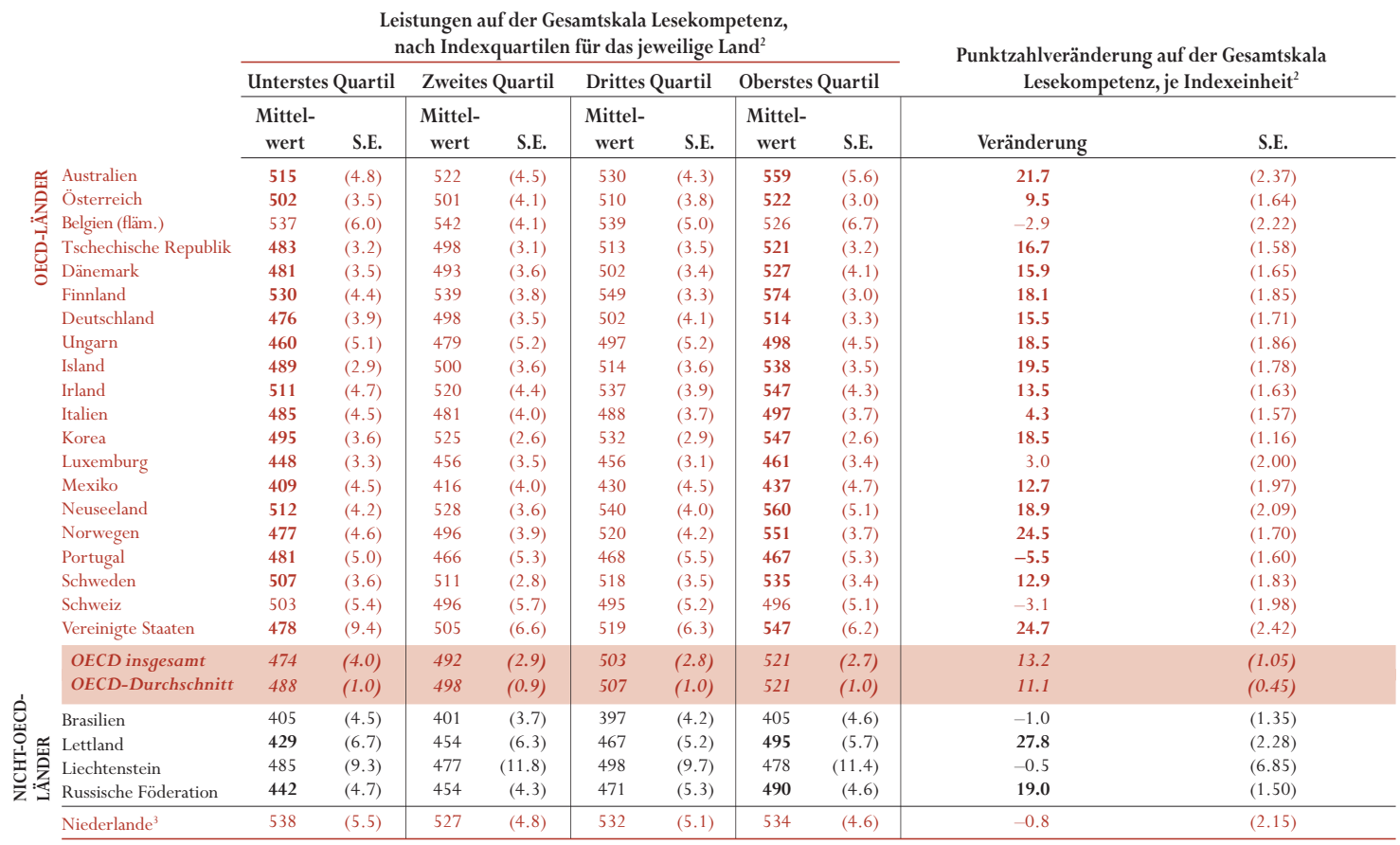

1. Zur Definition des Index siehe Anhang A1.

2. Zur erklärten Varianz siehe Anhang A2. In Indexeinheiten gemessene Veränderungen sind, soweit fett gedruckt, statistisch signifikant. Wenn die untersten und obersten Quartile durch Fettdruck gekennzeichnet sind, weist dies auf eine statistisch signifikante Differenz zwischen diesen Quartilen hin.

3. Die Beteiligungsquote ist zu niedrig, um die Vergleichbarkeit zu gewährleisten (vgl. Anhang A3). 
Tabelle 4.10

Index des Interesses an Computern und Leistungen auf der Gesamtskala Lesekompetenz, nach Indexquartilen für das jeweilige Land Die Ergebnisse basieren auf Schülerangaben

\begin{tabular}{|c|c|c|c|c|c|c|c|c|c|c|c|c|c|c|}
\hline & \multicolumn{14}{|c|}{ Index des Interesses an Computern $^{1}$} \\
\hline & \multicolumn{2}{|c|}{ Alle Schüler } & \multicolumn{2}{|c|}{ Jungen } & \multicolumn{2}{|c|}{ Mädchen } & \multicolumn{2}{|c|}{ Unterstes Quartil } & \multicolumn{2}{|c|}{ Zweites Quartil } & \multicolumn{2}{|c|}{ Drittes Quartil } & \multicolumn{2}{|c|}{ Oberstes Quartil } \\
\hline & $\begin{array}{c}\text { Index- } \\
\text { mittel }\end{array}$ & S.E. & $\begin{array}{c}\text { Index- } \\
\text { mittel }\end{array}$ & S.E. & $\begin{array}{c}\text { Index- } \\
\text { mittel }\end{array}$ & S.E. & $\begin{array}{c}\text { Index- } \\
\text { mittel }\end{array}$ & S.E. & $\begin{array}{c}\text { Index- } \\
\text { mittel }\end{array}$ & S.E. & $\begin{array}{c}\text { Index- } \\
\text { mittel }\end{array}$ & S.E. & $\begin{array}{c}\text { Index- } \\
\text { mittel }\end{array}$ & S.E. \\
\hline Australien & -0.21 & $(0.02)$ & -0.04 & $(0.03)$ & -0.41 & $(0.03)$ & -1.70 & $(0.02)$ & -0.40 & $(0.01)$ & 0.35 & $(0.02)$ & Max & \\
\hline Belgien & 0.00 & $(0.02)$ & 0.18 & $(0.02)$ & -0.22 & $(0.02)$ & -1.34 & $(0.02)$ & -0.21 & $(0.01)$ & 0.64 & $(0.01)$ & Max & \\
\hline Kanada & -0.09 & $(0.01)$ & 0.07 & $(0.01)$ & -0.24 & $(0.01)$ & -1.54 & $(0.01)$ & -0.28 & $(0.01)$ & 0.57 & $(0.01)$ & Max & \\
\hline Finnland & -0.15 & $(0.02)$ & 0.10 & $(0.02)$ & -0.39 & $(0.03)$ & -1.58 & $(0.02)$ & -0.33 & $(0.01)$ & 0.41 & $(0.01)$ & Max & \\
\hline Deutschland & 0.24 & $(0.02)$ & 0.43 & $(0.02)$ & 0.04 & $(0.03)$ & -1.09 & $(0.03)$ & 0.27 & $(0.02)$ & 0.90 & $(0.00)$ & Max & \\
\hline Ungarn & -0.02 & $(0.02)$ & 0.14 & $(0.03)$ & -0.21 & $(0.04)$ & -1.46 & $(0.02)$ & -0.19 & $(0.01)$ & 0.66 & $(0.02)$ & Max & \\
\hline Irland & 0.00 & $(0.02)$ & 0.02 & $(0.03)$ & -0.02 & $(0.02)$ & -1.17 & $(0.02)$ & -0.22 & $(0.01)$ & 0.51 & $(0.02)$ & Max & \\
\hline Luxemburg & 0.29 & $(0.02)$ & 0.45 & $(0.02)$ & 0.12 & $(0.03)$ & -1.04 & $(0.03)$ & 0.39 & $(0.02)$ & 0.90 & $(0.00)$ & Max & \\
\hline Mexiko & 0.28 & $(0.02)$ & 0.27 & $(0.03)$ & 0.29 & $(0.03)$ & -0.99 & $(0.04)$ & 0.30 & $(0.02)$ & 0.90 & $(0.00)$ & $\operatorname{Max}$ & \\
\hline Schweiz & 0.06 & $(0.02)$ & 0.24 & $(0.02)$ & -0.12 & $(0.03)$ & -1.43 & $(0.02)$ & -0.10 & $(0.01)$ & 0.89 & $(0.00)$ & Max & \\
\hline Vereinigte Staaten & 0.31 & $(0.02)$ & 0.29 & $(0.04)$ & 0.33 & $(0.02)$ & -0.92 & $(0.04)$ & 0.37 & $(0.02)$ & 0.90 & $(0.00)$ & Max & \\
\hline$O E C D$ insgesamt & 0.19 & $(0.01)$ & 0.26 & $(0.02)$ & 0.13 & $(0.01)$ & -1.11 & $(0.02)$ & 0.18 & $(0.01)$ & 0.81 & $(0.00)$ & 0.90 & $(0.00)$ \\
\hline OECD-Durchschnitt & 0.00 & $(0.00)$ & 0.17 & $(0.01)$ & -0.17 & $(0.01)$ & -1.39 & $(0.01)$ & -0.13 & $(0.00)$ & 0.62 & $(0.00)$ & 0.90 & $(0.00)$ \\
\hline Brasilien & 0.36 & $(0.02)$ & 0.39 & $(0.03)$ & 0.34 & $(0.03)$ & -0.66 & $(0.04)$ & 0.32 & $(0.03)$ & 0.90 & $(0.00)$ & Max & \\
\hline Lettland & 0.31 & $(0.02)$ & 0.34 & $(0.03)$ & 0.28 & $(0.03)$ & -0.92 & $(0.04)$ & 0.37 & $(0.02)$ & 0.90 & $(0.00)$ & Max & \\
\hline Liechtenstein & 0.17 & $(0.05)$ & 0.35 & $(0.06)$ & -0.02 & $(0.08)$ & -1.28 & $(0.08)$ & 0.20 & $(0.05)$ & 0.90 & $(0.00)$ & Max & \\
\hline Russische Föderation & 0.18 & $(0.03)$ & 0.18 & $(0.04)$ & 0.18 & $(0.04)$ & -1.44 & $(0.04)$ & 0.36 & $(0.02)$ & 0.90 & $(0.00)$ & Max & \\
\hline
\end{tabular}

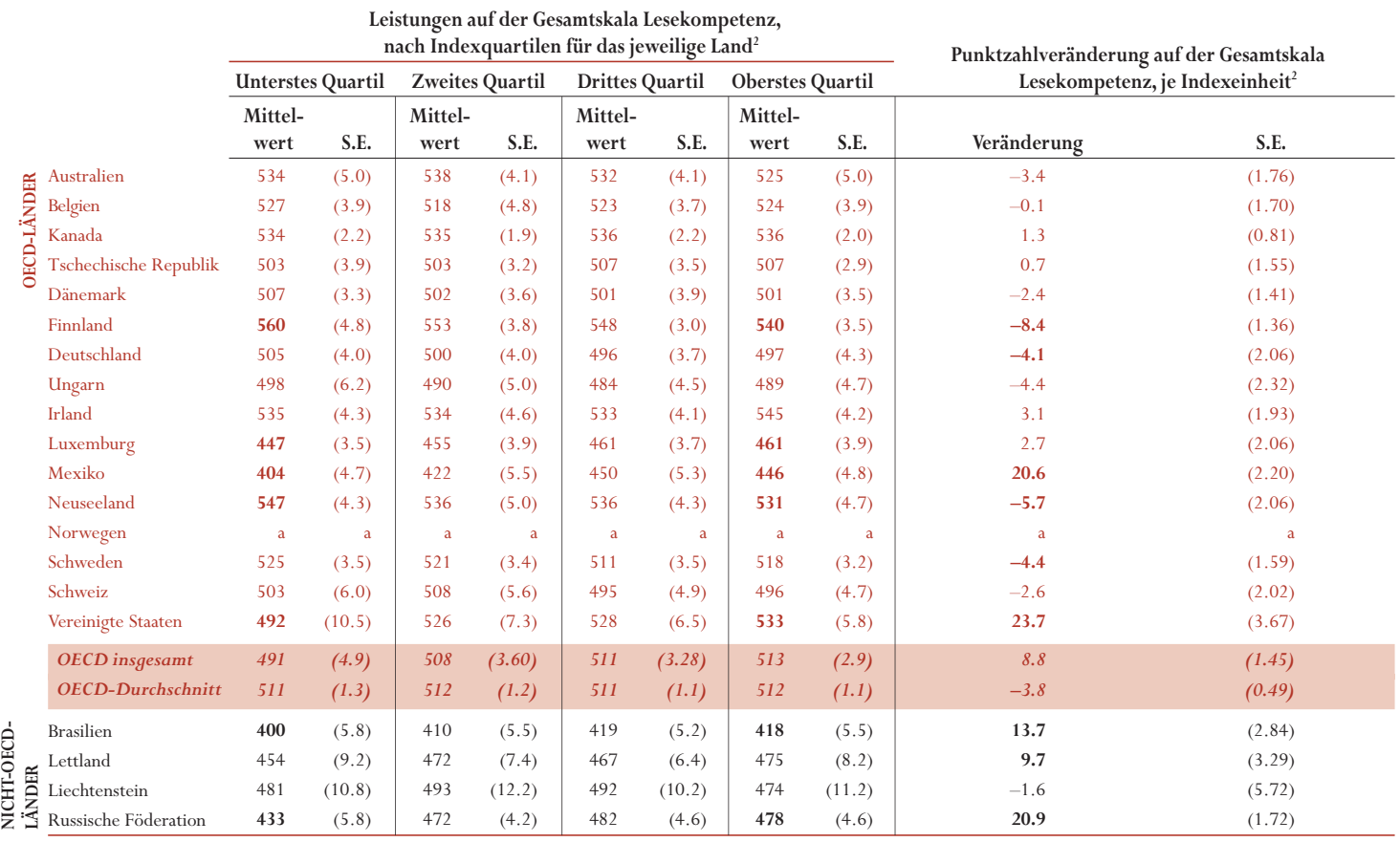

1. Zur Definition des Index siehe Anhang A1. "Max" wird verwendet, um Länder abzubilden, in denen mehr als $25 \%$ der Schülerinnen und Schüler den höchsten Wert dieses Index erreichen, der 0.90 beträgt.

2. Zur erklärten Varianz siehe Anhang A2. In Indexeinheiten gemessene Veränderungen sind, soweit fett gedruckt, statistisch signifikant. Wenn die untersten und obersten Quartile durch Fettdruck gekennzeichnet sind, weist dies auf eine statistisch signifikante Differenz zwischen diesen Quartilen hin. 
Tabelle 4.11

Index derVertrautheit und Selbsteinschätzung der Fähigkeit im Umgang mit Computern und Leistungen auf der Gesamtskala Lesekompetenz, nach Indexquartilen für das jeweilige Land

Die Ergebnisse basieren auf Schülerangaben

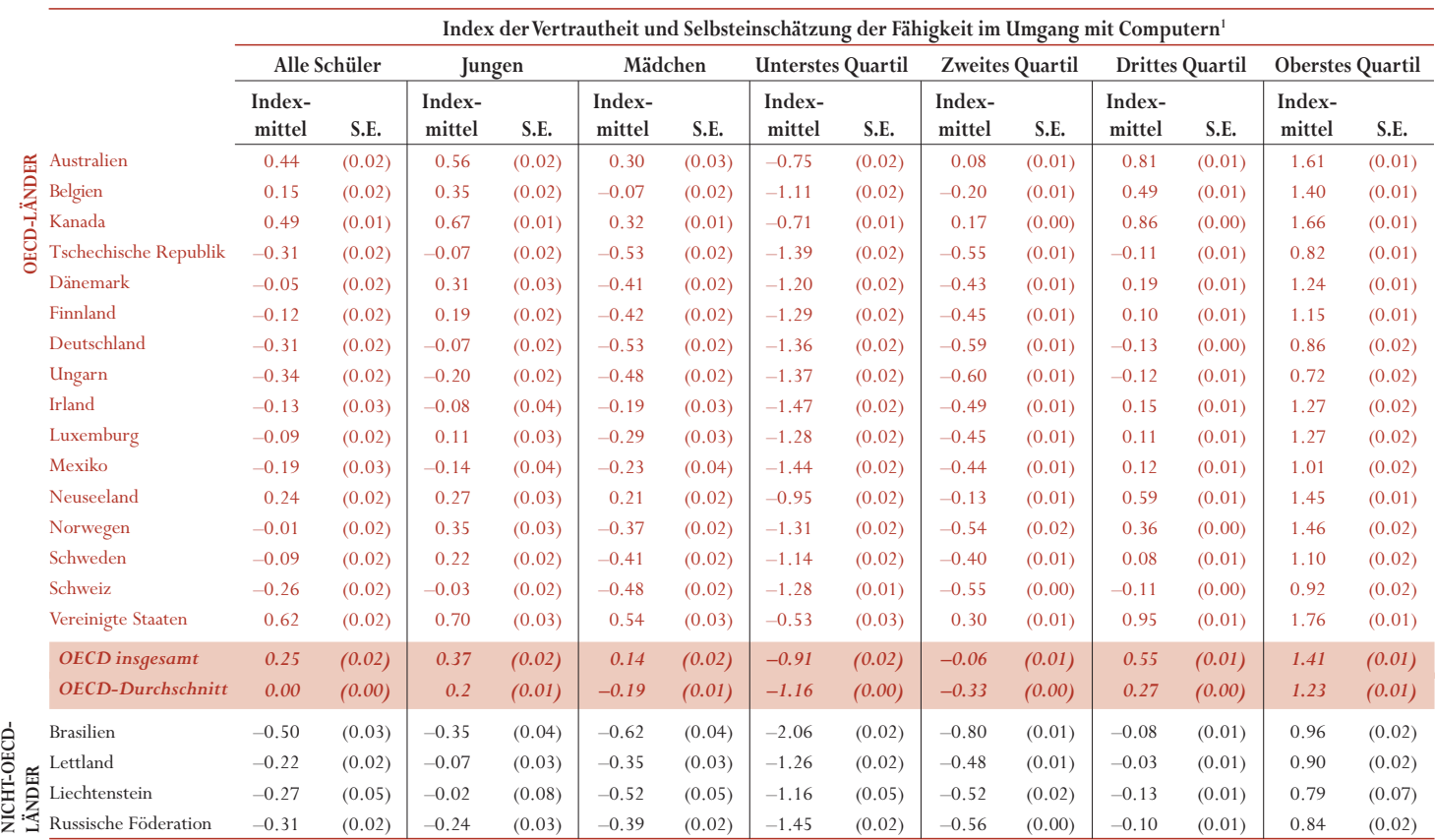

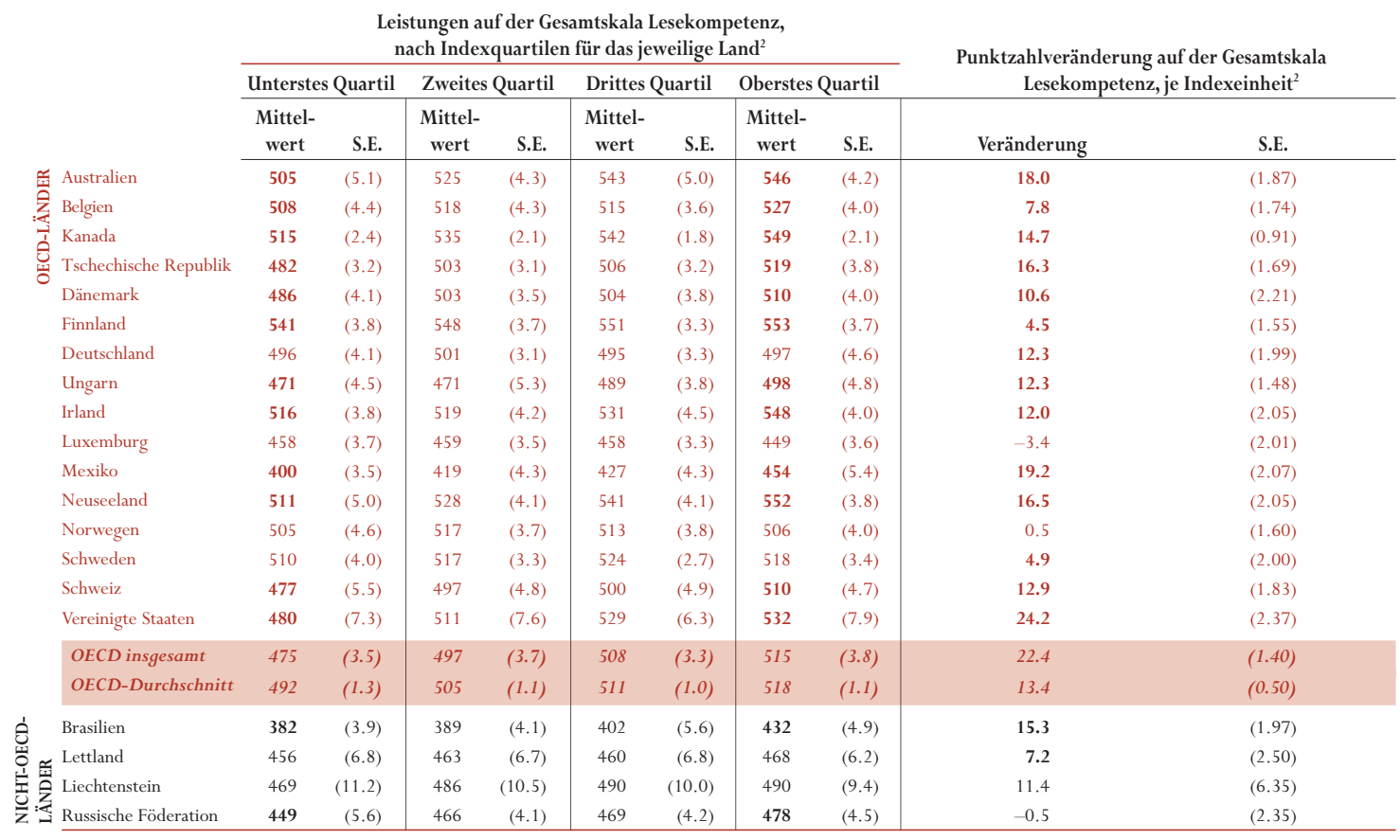

1. Zur Definition des Index siehe Anhang A1.

2. Zur erklärten Varianz siehe Anhang A2. In Indexeinheiten gemessene Veränderungen sind, soweit fett gedruckt, statistisch signifikant. Wenn die untersten und obersten Quartile durch Fettdruck gekennzeichnet sind, weist dies auf eine statistisch signifikante Differenz zwischen diesen Quartilen hin. 
ANHANG B1

Tabelle $5.1 a$

Schülerleistungen auf der Gesamtskala Lesekompetenz sowie den Skalen für mathematische und für naturwissenschaftliche Grundbildung, nach Geschlecht

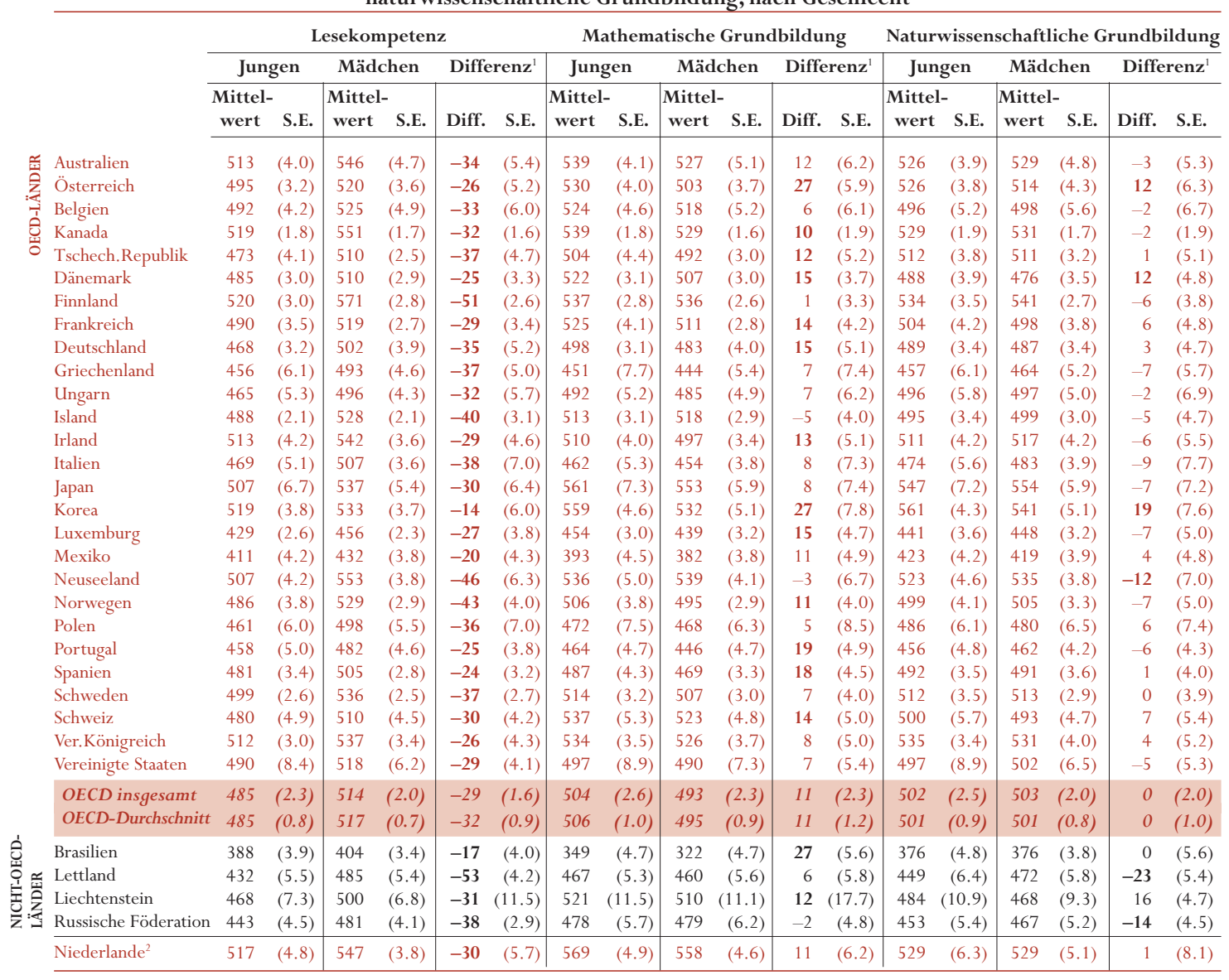

1. Positive Differenzen bedeuten, dass Jungen besser abschneiden als Mädchen, negative Differenzen bedeuten, dass Mädchen besser abschneiden als Jungen Statistisch signifikante Unterschiede sind durch Fettdruck gekennzeichnet.

2. Die Beteiligungsquote ist zu niedrig, um die Vergleichbarkeit zu gewährleisten (vgl. Anhang A3). 
Tabelle $5.1 b$

Schülerleistungen auf den Subskalen Informationen Ermitteln, Textbezogenes Interpretieren, Reflektieren und Bewerten, nach Geschlecht

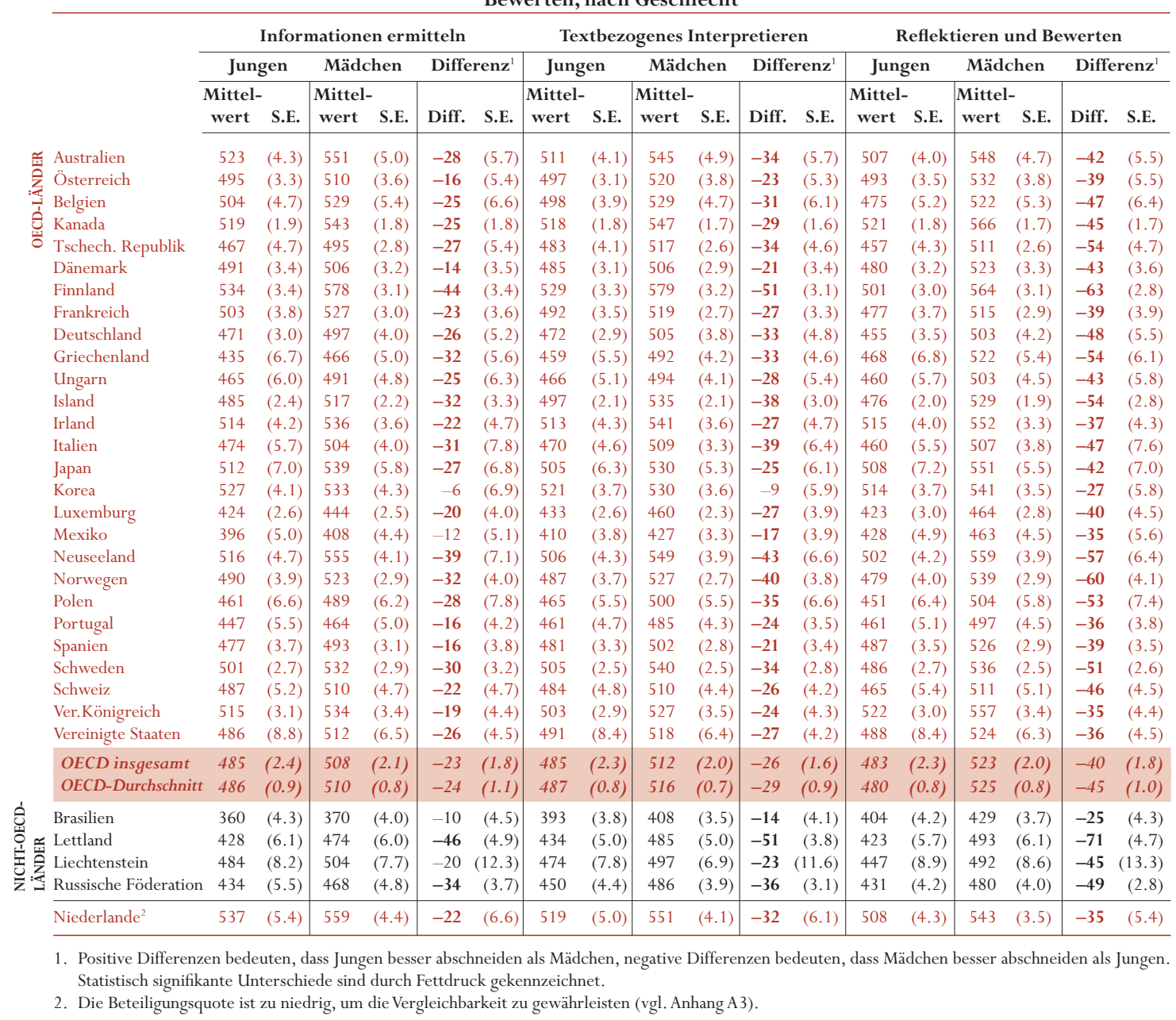


ANHANG B1

Tabelle $5.2 a$

Prozentsatz der Schülerinnen und Schüler auf den jeweiligen Kompetenzstufen der Gesamtskala Lesekompetenz, nach Geschlecht

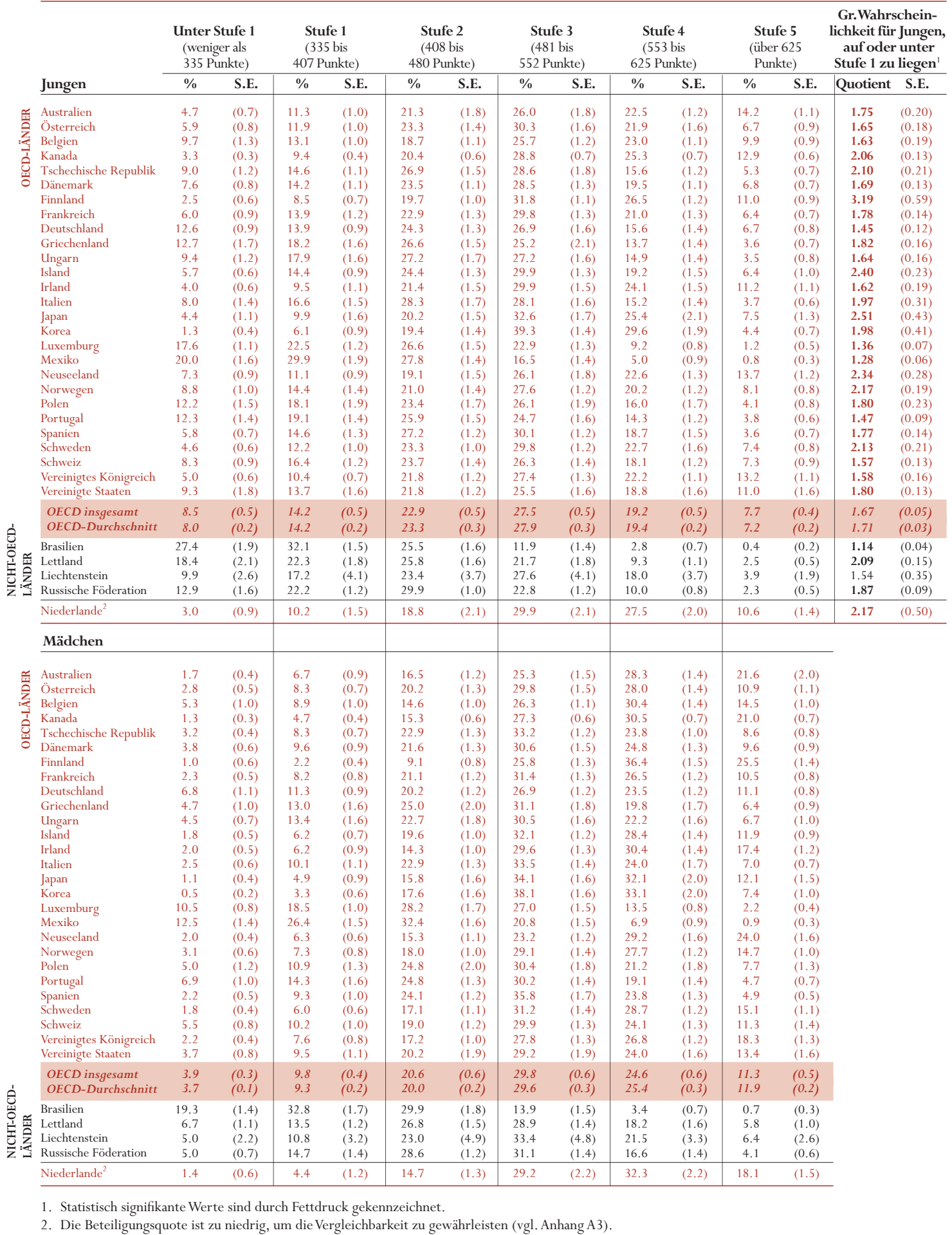


Tabelle $5.2 b$

Prozentsatz der Schülerinnen und Schüler mit weniger als 400 und mehr als 600 Punkten auf der Skala für mathematische Grundbildung

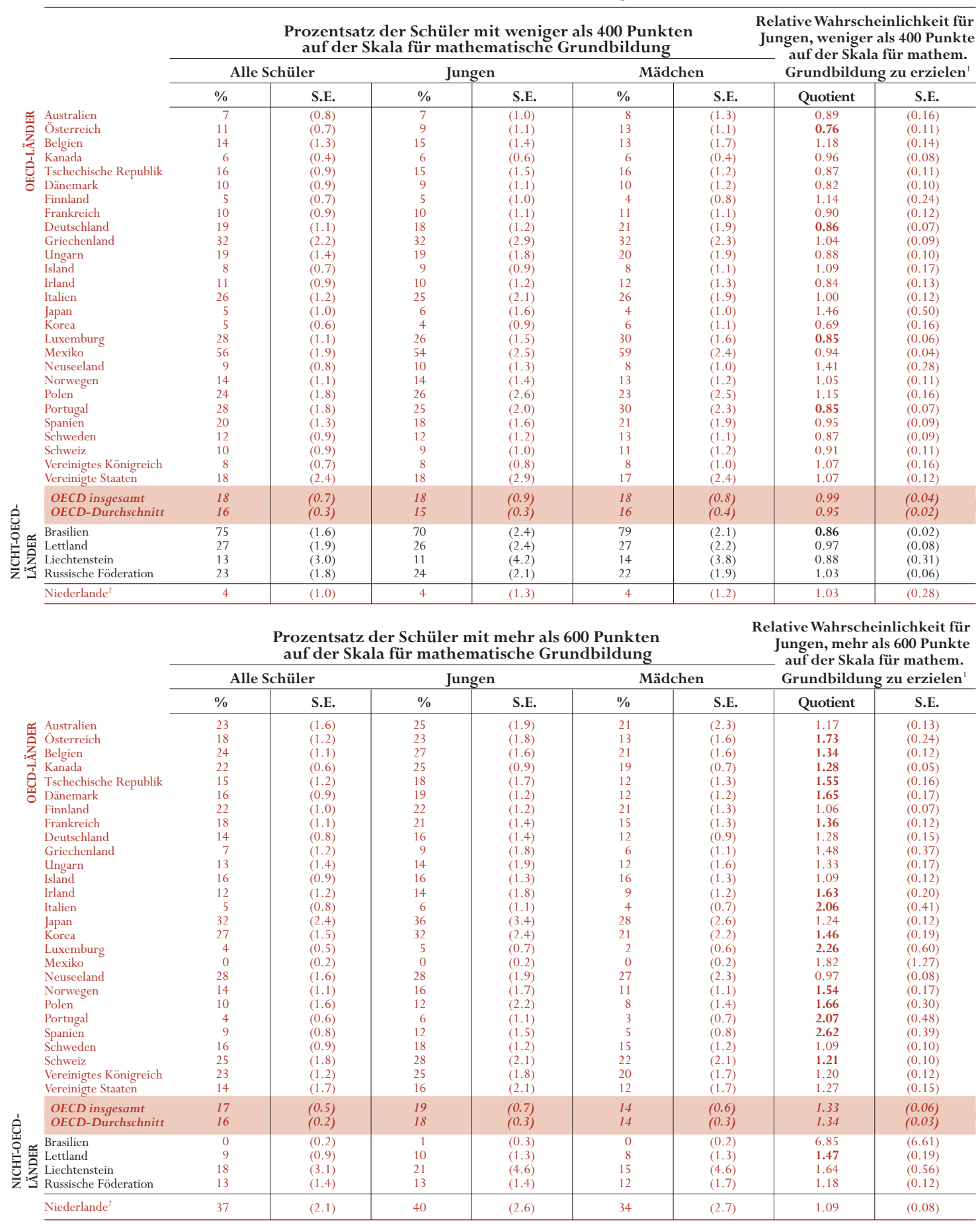

1. Statistisch signifikante Werte sind durch Fettdruck gekennzeichnet.

2. Die Beteiligungsquote ist zu niedrig, um die Vergleichbarkeit zu gewährleisten (vgl. Anhang A3). 
Tabelle 5.3

Täglicher Zeitaufwand für Lesen zum Vergnügen

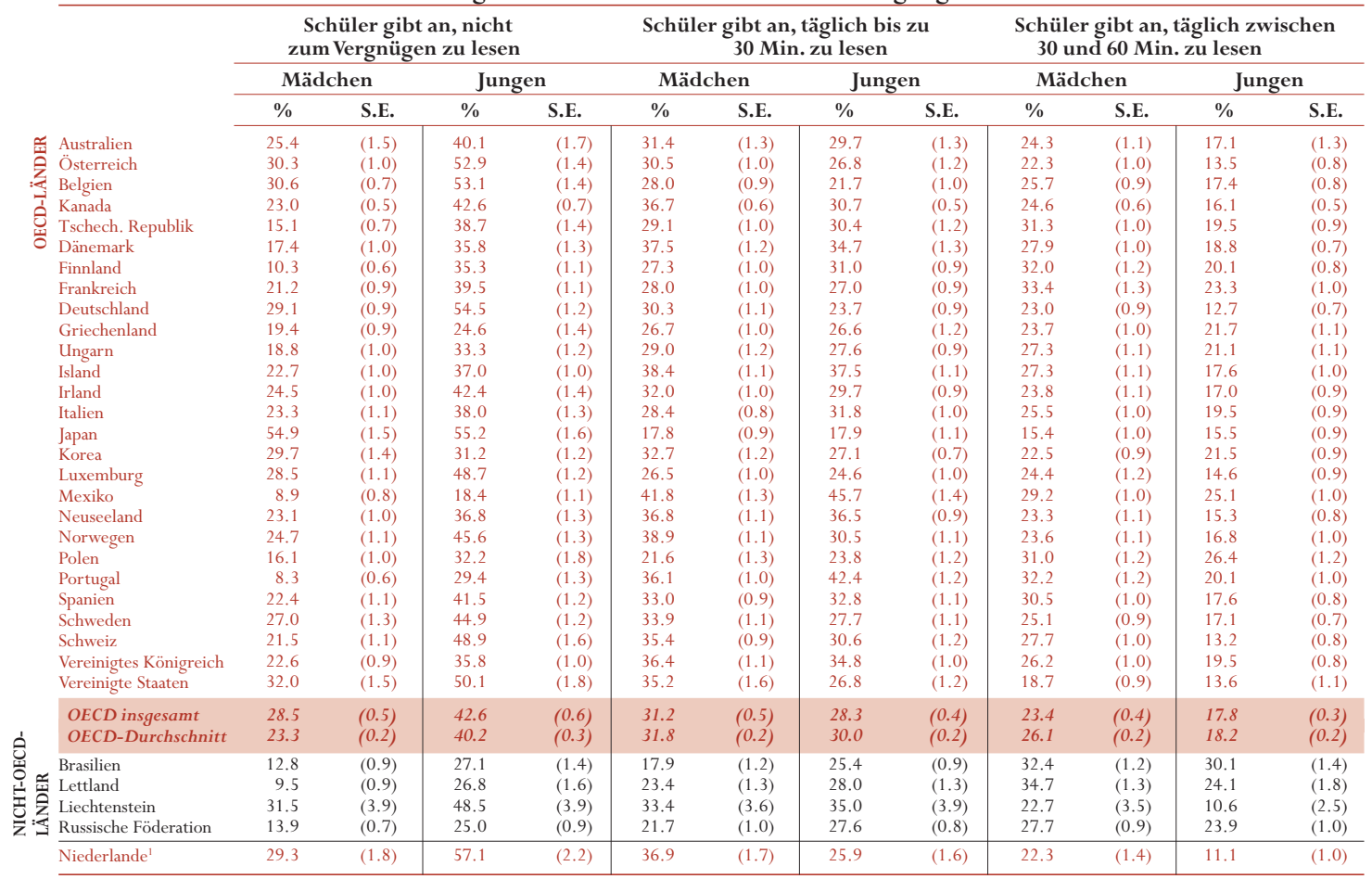

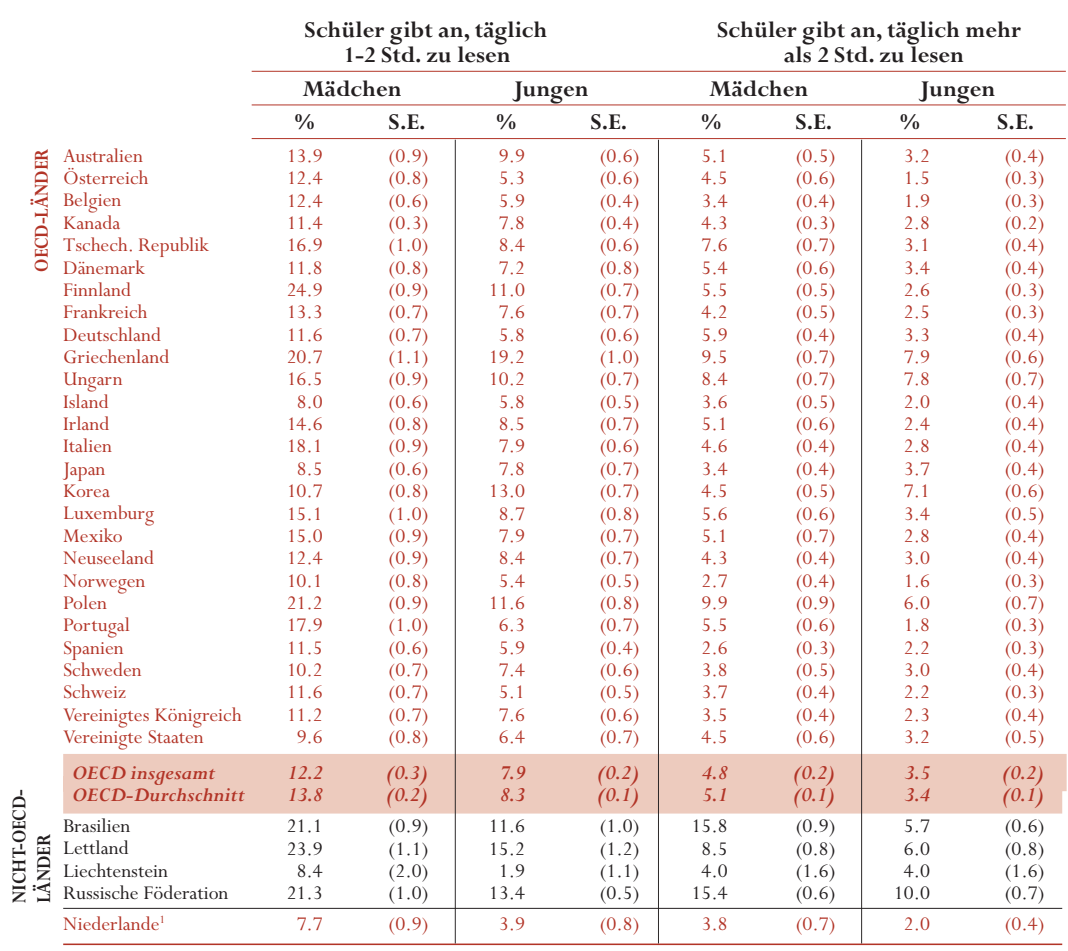

1. Die Beteiligungsquote ist zu niedrig, um die Vergleichbarkeit zu gewährleisten (vgl. Anhang A3). 
Tabelle $5.4 a$

Index des Selbstkonzepts Lesen, nach Geschlecht und Leistungen auf der Gesamtskala Lesekompetenz, nach Indexquartilen für das jeweilige Land

Die Ergebnisse basieren auf Schülerangaben

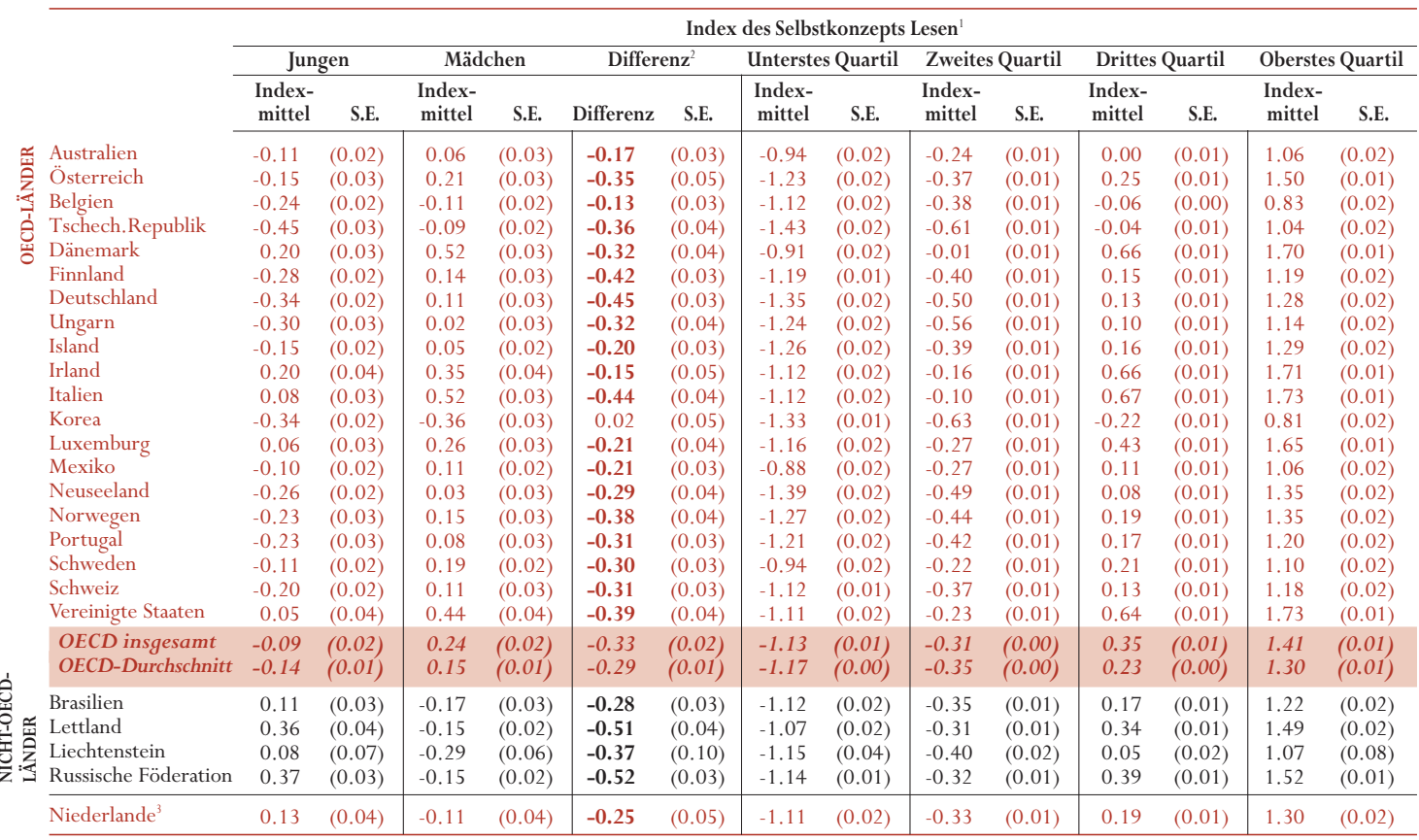

Leistungen auf der Gesamtskala Lesekompetenz,

nach Indexquartilen für das jeweilige Land

\begin{tabular}{|c|c|c|c|c|c|c|c|c|}
\hline & \multirow{2}{*}{\multicolumn{2}{|c|}{ Unterstes Quartil }} & \multirow{2}{*}{\multicolumn{2}{|c|}{ Zweites Quartil }} & \multirow{2}{*}{\multicolumn{2}{|c|}{ Drittes Quartil }} & \multirow{2}{*}{\multicolumn{2}{|c|}{ Oberstes Quartil }} \\
\hline & & & & & & & & \\
\hline & $\begin{array}{c}\text { Mittel- } \\
\text { wert }\end{array}$ & S.E. & $\begin{array}{c}\text { Mittel- } \\
\text { wert }\end{array}$ & S.E. & $\begin{array}{c}\text { Mittel- } \\
\text { wert }\end{array}$ & S.E. & $\begin{array}{c}\text { Mittel- } \\
\text { wert }\end{array}$ & S.E. \\
\hline Australien & 499 & $(4.6)$ & 519 & $(4.9)$ & 538 & $(4.4)$ & 572 & $(4.5)$ \\
\hline Österreich & 484 & $(3.8)$ & 492 & (4.1) & 513 & $(3.6)$ & 547 & (3.3) \\
\hline Belgien & 514 & (6.6) & 529 & $(5.2)$ & 553 & $(4.4)$ & 548 & (5.1) \\
\hline Tschech.Republik & 478 & $(3.8)$ & 490 & $(3.2)$ & 511 & (3.1) & 536 & (3.1) \\
\hline Dänemark & 456 & $(3.9)$ & 491 & $(3.5)$ & 510 & $(3.2)$ & 548 & (3.4) \\
\hline Finnland & 509 & (3.1) & 531 & (3.9) & 560 & $(3.2)$ & 593 & (3.6) \\
\hline Deutschland & 477 & $(3.8)$ & 476 & $(4.0)$ & 503 & $(3.6)$ & 534 & (3.8) \\
\hline Ungarn & 458 & $(4.1)$ & 464 & (5.4) & 491 & $(4.8)$ & 521 & $(5.0)$ \\
\hline Island & 474 & $(3.2)$ & 495 & $(3.4)$ & 522 & $(3.2)$ & 551 & (3.3) \\
\hline Irland & 513 & $(4.4)$ & 527 & $(3.8)$ & 533 & (4.3) & 542 & $(4.8)$ \\
\hline Italien & 452 & $(5.4)$ & 484 & $(3.6)$ & 501 & $(3.1)$ & 514 & (3.7) \\
\hline Korea & 498 & (3.1) & 518 & $(2.9)$ & 531 & (3.3) & 552 & (2.4) \\
\hline Luxemburg & 417 & $(3.3)$ & 442 & $(3.6)$ & 471 & $(2.9)$ & 492 & (3.2) \\
\hline Mexiko & 410 & $(4.9)$ & 415 & $(4.7)$ & 425 & $(4.0)$ & 441 & (4.2) \\
\hline Neuseeland & 514 & (3.9) & 510 & $(4.1)$ & 544 & $(4.8)$ & 573 & $(4.4)$ \\
\hline Norwegen & 470 & $(4.8)$ & 490 & $(4.5)$ & 523 & $(3.4)$ & 561 & (3.9) \\
\hline Portugal & 433 & $(5.9)$ & 454 & $(5.2)$ & 483 & $(4.5)$ & 512 & $(5.0)$ \\
\hline Schweden & 481 & $(3.0)$ & 503 & $(2.8)$ & 528 & (3.2) & 559 & (3.5) \\
\hline Schweiz & 473 & $(4.9)$ & 482 & $(5.6)$ & 511 & $(4.6)$ & 524 & (5.2) \\
\hline Vereinigte Staaten & 469 & $(9.0)$ & 496 & $(6.3)$ & 526 & (7.0) & 558 & (6.2) \\
\hline OECD insgesamt & 465 & (3.6) & 484 & $(2.9)$ & 507 & (3.1) & 533 & $(3.0)$ \\
\hline OECD-Durchschnitt & 473 & (1.0) & 490 & $(1.1)$ & 513 & (1.0) & 539 & (1.0) \\
\hline Brasilien & 391 & $(4.3)$ & 392 & $(3.8)$ & 401 & (3.4) & 424 & $(5.1)$ \\
\hline Lettland & 425 & $(6.8)$ & 441 & $(6.8)$ & 467 & $(5.6)$ & 513 & (5.4) \\
\hline Liechtenstein & 458 & $(10.6)$ & 472 & $(9.8)$ & 494 & $(11.1)$ & 515 & $(8.6)$ \\
\hline Russische Föderation & 435 & $(5.3)$ & 447 & $(4.0)$ & 471 & $(4.3)$ & 505 & (5.1) \\
\hline Niederlande $^{3}$ & 515 & $(6.8)$ & 529 & $(5.0)$ & 538 & $(4.6)$ & 549 & $(4.2)$ \\
\hline
\end{tabular}

1. Zur Definition des Index siehe Anhang A1.

2. Positive Differenzen bedeuten, dass Jungen höhereWerte beim Selbstkonzept Lesen haben als Mädchen, negative Differenzen bedeuten, dass Mädchen höhereWerte beim Selbstkonzept Lesen haben als Jungen. Statistisch signifikante Unterschiede sind durch Fettdruck gekennzeichnet

3. Die Beteiligungsquote ist zu niedrig, um die Vergleichbarkeit zu gewährleisten (vgl. Anhang A3). 
Tabelle $5.4 b$

Index des Selbstkonzepts Mathematik, nach Geschlecht und Leistungen auf der Skala für mathematische Grundbildung, nach Indexquartilen für das jeweilige Land

Die Ergebnisse basieren auf Schülerangabens

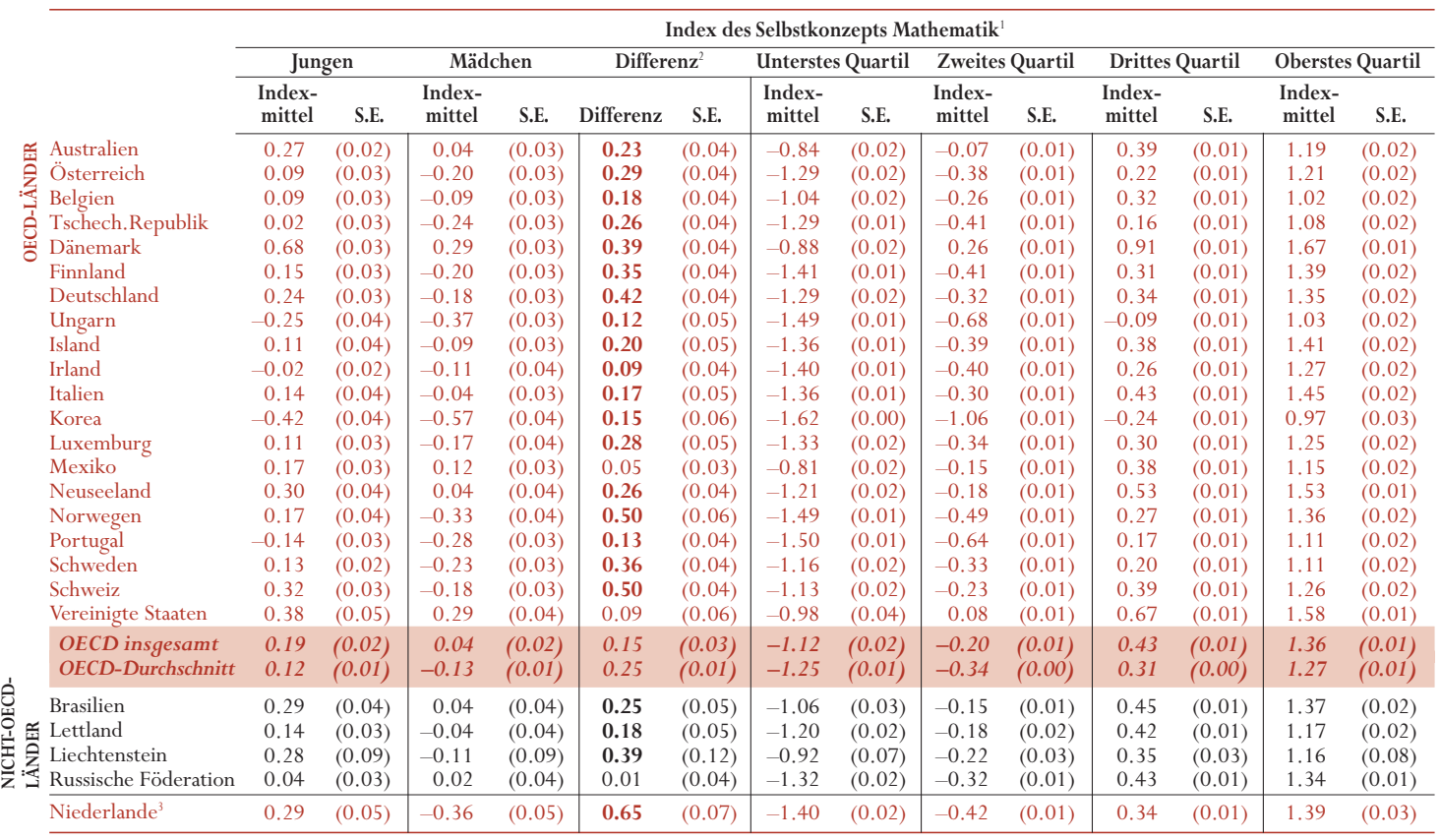

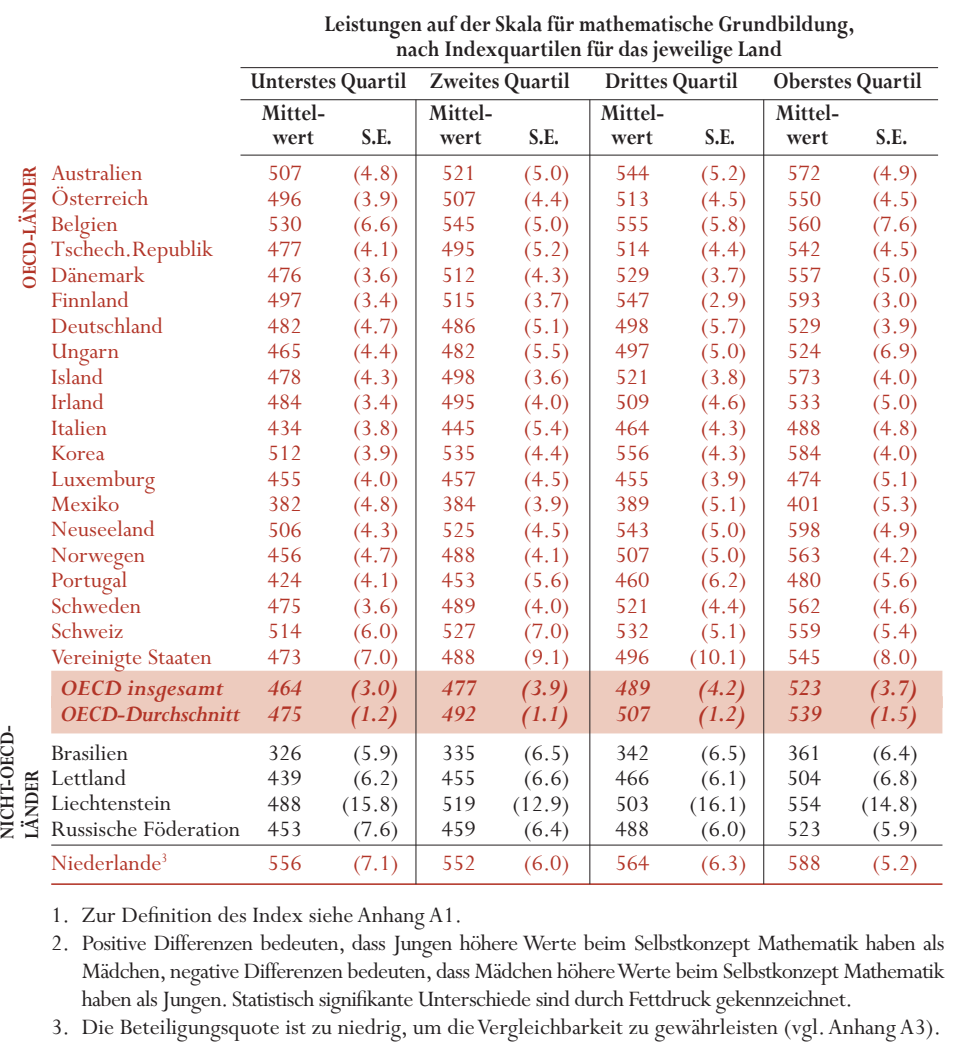

326 
Tabelle $6.1 a$

Internationaler sozioökonomischer Index der beruflichen Stellung der Eltern (ISEI) und Leistungen auf der Gesamtskala Lesekompetenz, nach Indexquartilen für das jeweilige Land

Die Ergebnisse basieren auf Schülerangaben

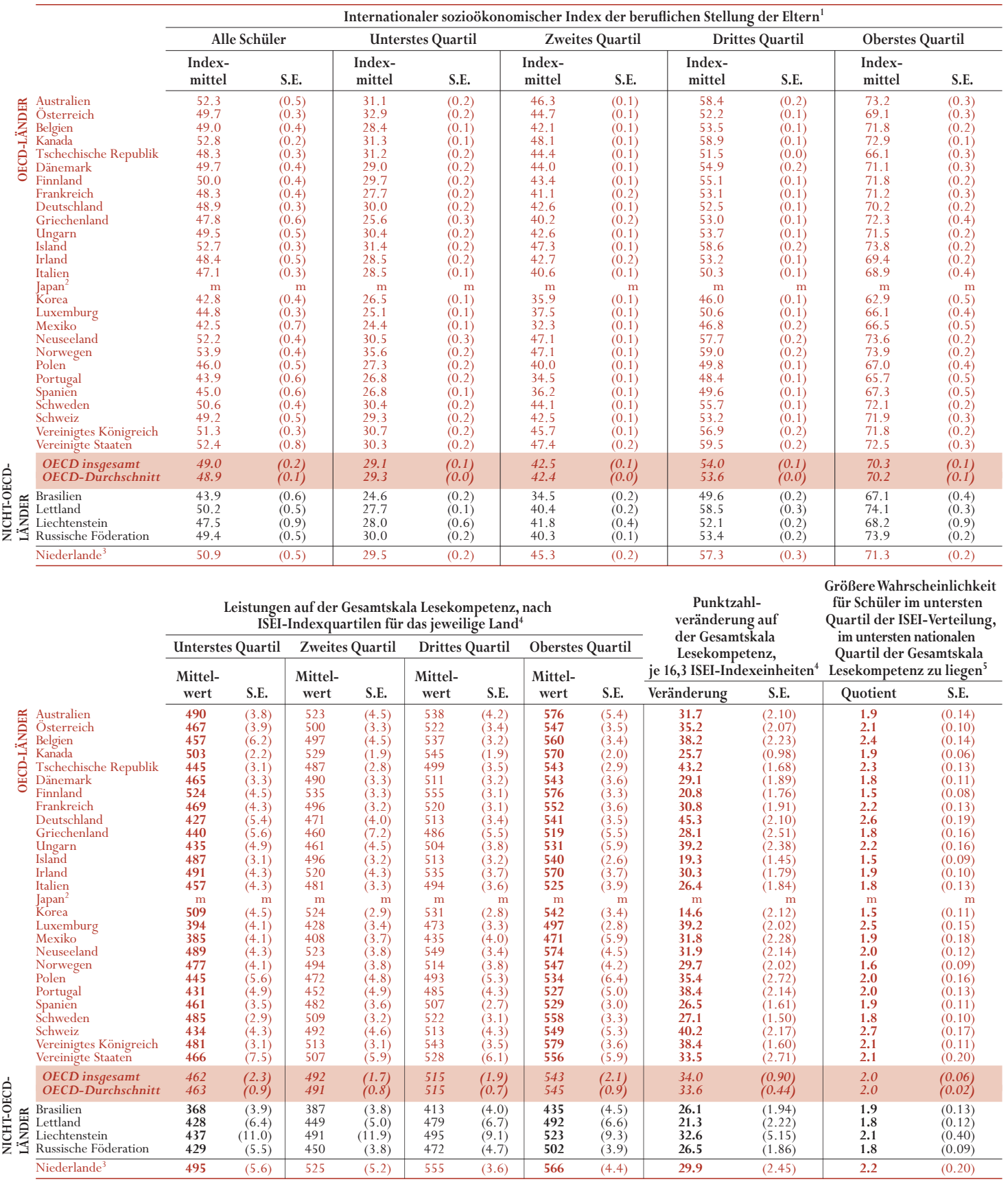

1. Zur Definition des Index siehe Anhang A1.

2. Japan wurde bei diesem Vergleich wegen großenteils fehlender Daten nicht berücksichtigt.

3. Die Beteiligungsquote ist zu niedrig, um die Vergleichbarkeit zu gewährleisten (vgl. Anhang A3).

4. Zur erklärten Varianz siehe Anhang A2. In Indexeinheiten gemessene Veränderungen sind, soweit fettgedruckt, statistisch signifikant. Wenn die untersten und obersten Quartile durch Fettdruck gekennzeichnet sind, weist dies auf eine statistisch signifikante Differenz zwischen diesen Quartilen hin. 16,3

5. Statistisch signifikante Quotienten von mehr als 1 sind durch Fettdruck gekennzeichnet. 
Tabelle $6.1 \mathrm{~b}$

Internationaler sozioökonomischer Index der beruflichen Stellung der Eltern (ISEI) und Leistungen auf der Skala für mathematische Grundbildung, nach Indexquartilen für das jeweilige Land

Die Ergebnisse basieren auf Schülerangaben

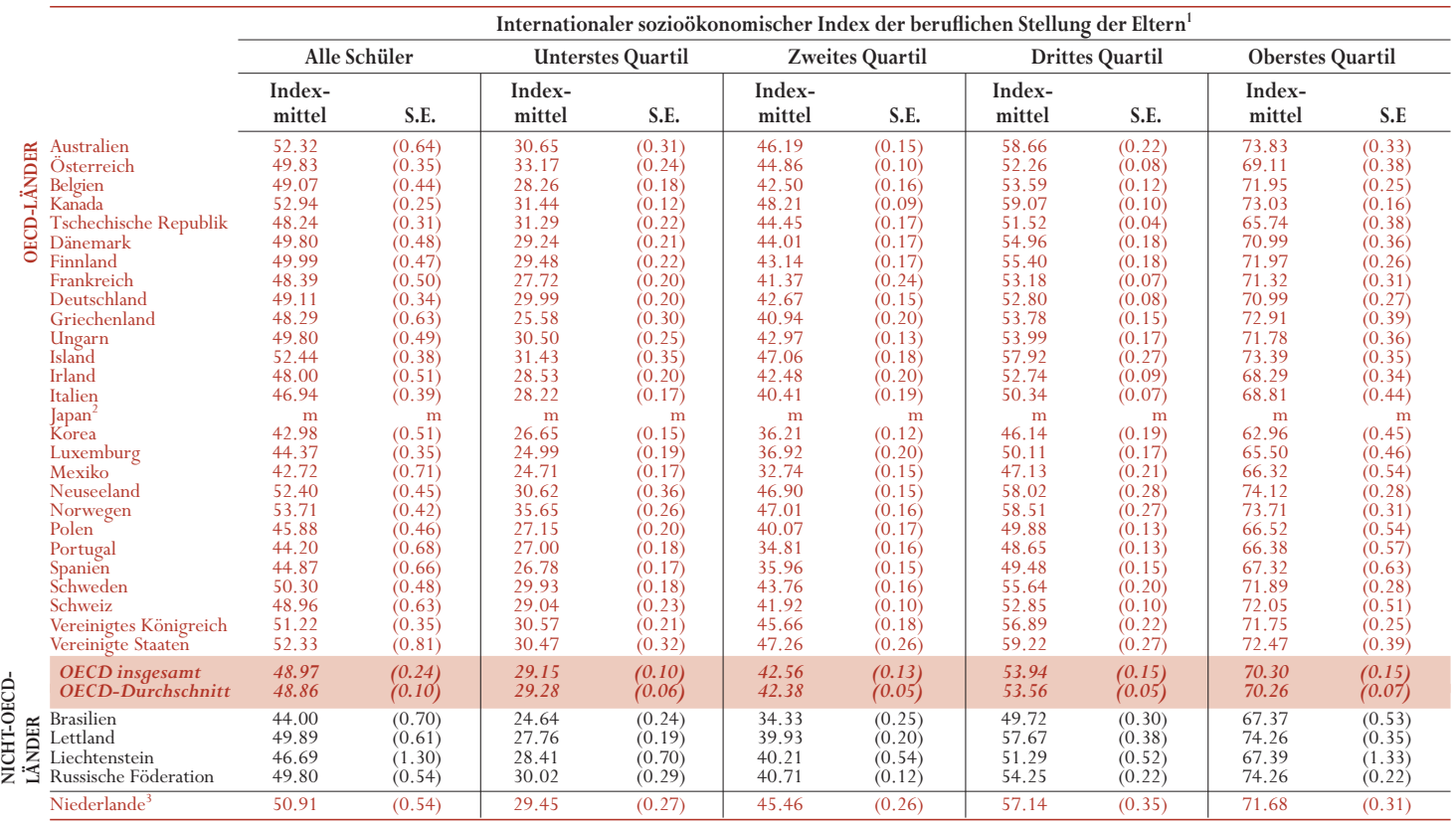

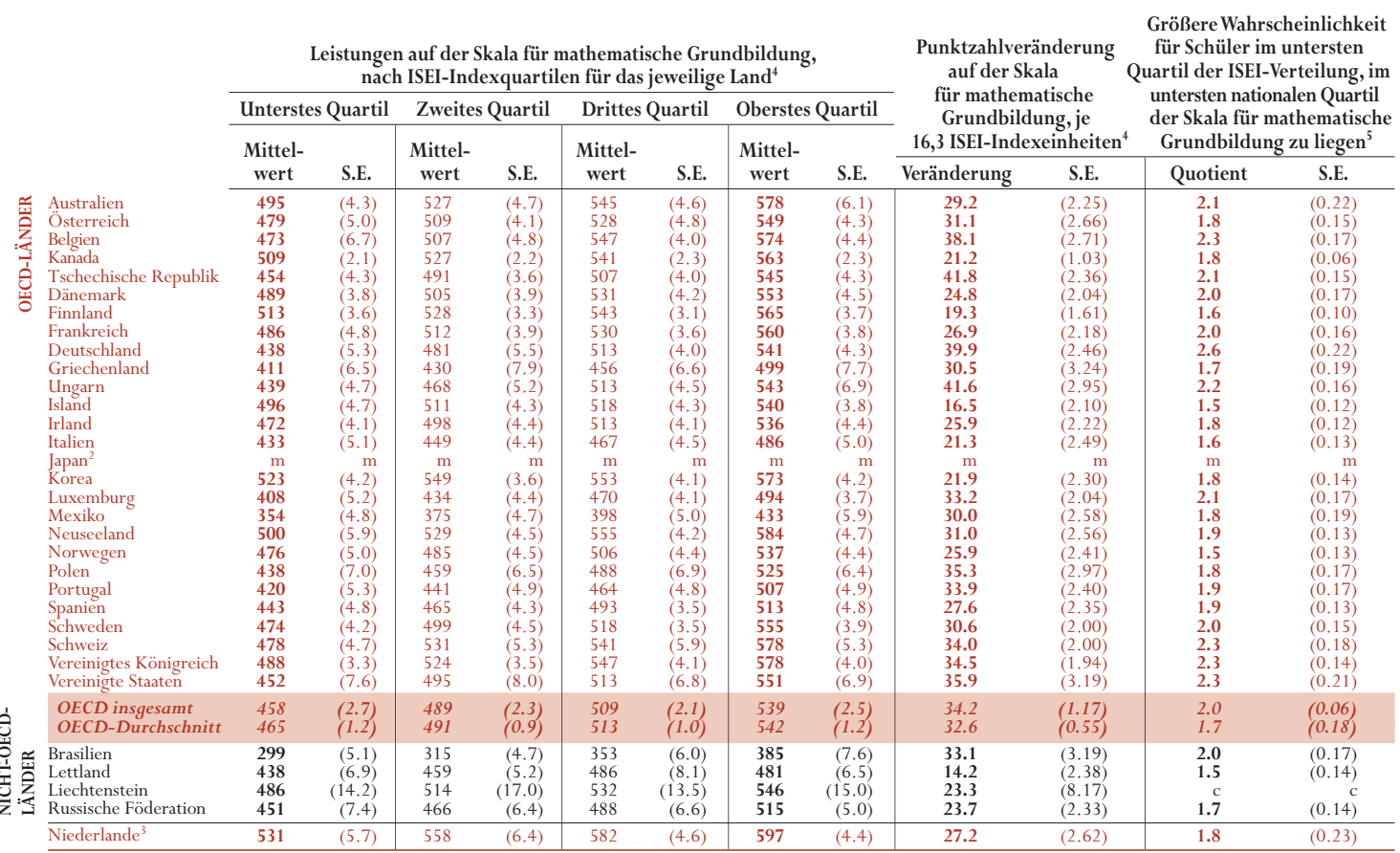

1. Zur Definition des Index siehe Anhang A1.

2. Japan wurde bei diesem Vergleich wegen großenteils fehlender Daten nicht berücksichtigt.

3. Die Beteiligungsquote ist zu niedrig, um die Vergleichbarkeit zu gewährleisten (vgl. Anhang A3).

4. Zur erklärten Varianz siehe Anhang A2. In Indexeinheiten gemessene Veränderungen sind, soweit fettgedruckt, statistisch signifikant. Wenn die untersten und obersten Quartile durch Fettdruck gekennzeichnet sind, weist dies auf eine statistisch signifikante Differenz zwischen diesen Quartilen hin. 16,3

5. Statistisch signifikante Quotienten von mehr als 1 sind durch Fettdruck gekennzeichnet.. 
Tabelle $6.1 c$

Internationaler sozioökonomischer Index der beruflichen Stellung der Eltern (ISEI) und Leistungen auf der Skala für naturwissenschaftliche Grundbildung, nach Indexquartilen für das jeweilige Land

Die Ergebnisse basieren auf Schülerangaben

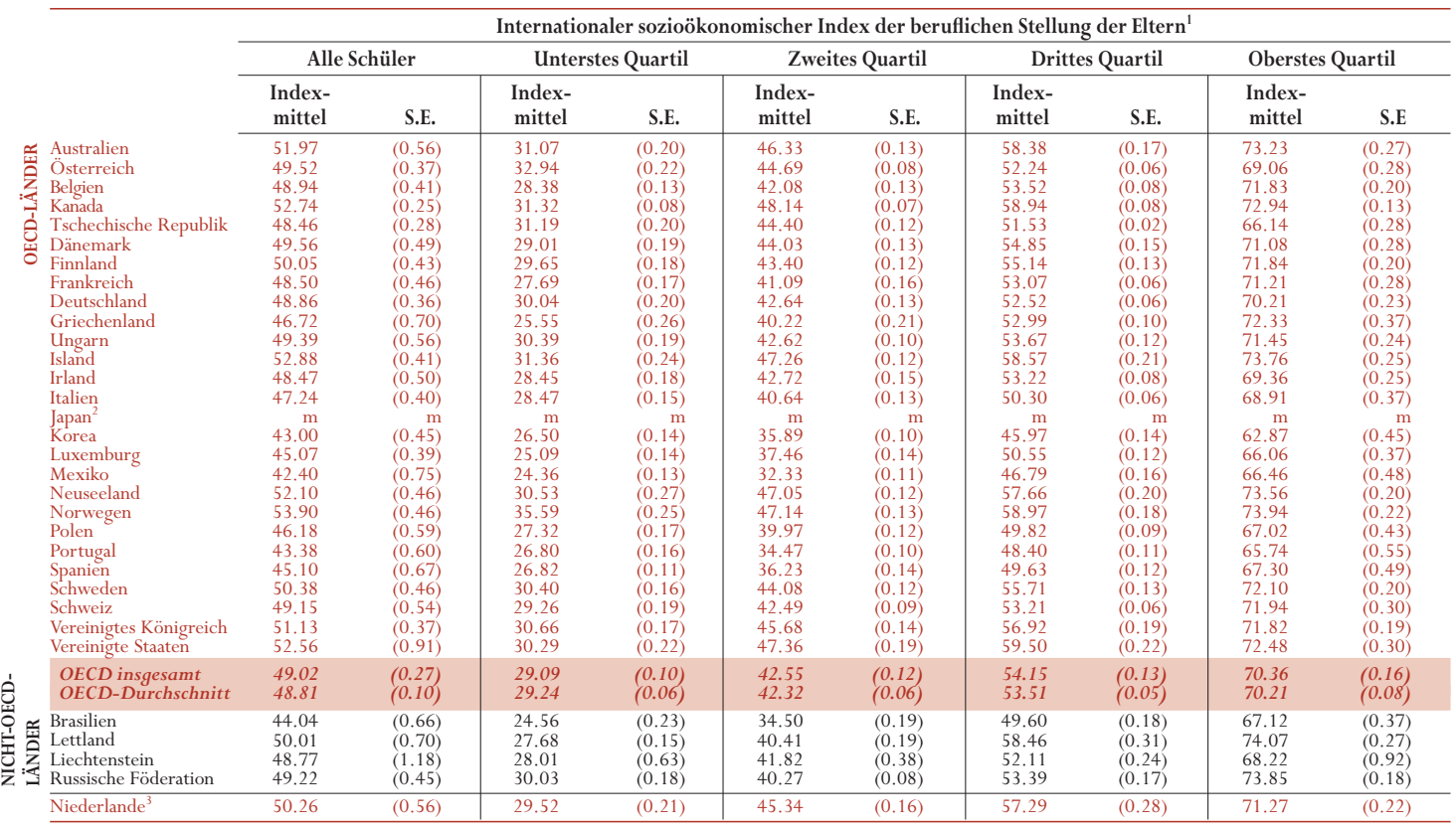

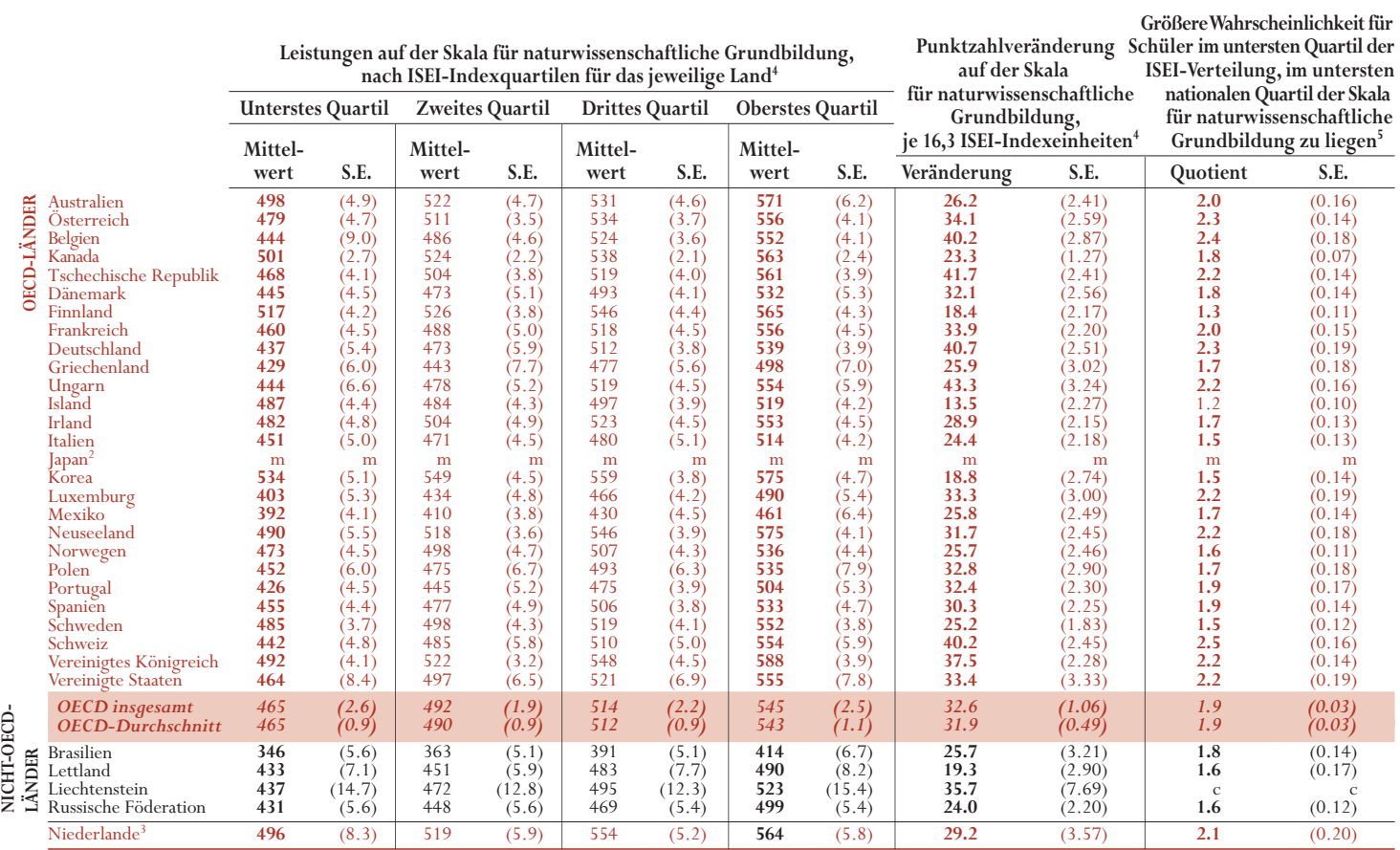

1. Zur Definition des Index siehe Anhang A1.

2. Japan wurde bei diesem Vergleich wegen großenteils fehlender Daten nicht berücksichtigt.

3. Die Beteiligungsquote ist zu niedrig, um die Vergleichbarkeit zu gewährleisten (vgl. Anhang A3).

4. Zur erklärten Varianz siehe Anhang A2. In Indexeinheiten gemessene Veränderungen sind, soweit fettgedruckt, statistisch signifikant. Wenn die untersten und obersten Quartile durch Fettdruck gekennzeichnet sind, weist dies auf eine statistisch signifikante Differenz zwischen diesen Quartilen hin. 16,3

5. Statistisch signifikante Quotienten von mehr als 1 sind durch Fettdruck gekennzeichnet. 
Tabelle 6.2

Index des relativen Wohlstands der Familie und Leistungen auf der Gesamtskala Lesekompetenz, nach Indexquartilen für das jeweilige Land

Die Ergebnisse basieren auf Schülerangaben

\begin{tabular}{|c|c|c|c|c|c|c|c|c|c|c|}
\hline & \multicolumn{10}{|c|}{ Index des relativen Wohlstands der Familie $^{1}$} \\
\hline & \multicolumn{2}{|c|}{ Alle Schüler } & \multicolumn{2}{|c|}{ Unterstes Quartil } & \multicolumn{2}{|c|}{ Zweites Quartil } & \multicolumn{2}{|c|}{ Drittes Quartil } & \multicolumn{2}{|c|}{ Oberstes Quartil } \\
\hline & $\begin{array}{l}\text { Index- } \\
\text { mittel }\end{array}$ & S.E. & $\begin{array}{l}\text { Index- } \\
\text { mittel }\end{array}$ & S.E. & $\begin{array}{l}\text { Index- } \\
\text { mittel }\end{array}$ & S.E. & $\begin{array}{l}\text { Index- } \\
\text { mittel }\end{array}$ & S.E. & $\begin{array}{l}\text { Index- } \\
\text { mittel }\end{array}$ & S.E \\
\hline $\begin{array}{l}\text { OECD insgesamt } \\
\text { OECD-Durchschnitt }\end{array}$ & $\begin{array}{l}0.00 \\
0.00\end{array}$ & $\begin{array}{l}(0.02) \\
(0.00)\end{array}$ & $\begin{array}{l}-1.08 \\
-1.04\end{array}$ & $\begin{array}{l}(0.02) \\
(0.01)\end{array}$ & $\begin{array}{l}-0.26 \\
-0.26\end{array}$ & $\begin{array}{l}(0.01) \\
(0.01)\end{array}$ & $\begin{array}{l}0.26 \\
0.25\end{array}$ & $\begin{array}{l}(0.02) \\
(0.01)\end{array}$ & $\begin{array}{l}1.09 \\
1.06\end{array}$ & $\begin{array}{l}(0.02) \\
(0.01)\end{array}$ \\
\hline $\begin{array}{l}\text { Brasilien } \\
\text { Lettland } \\
\text { Liechtenstein } \\
\text { Russische Föderation }\end{array}$ & $\begin{array}{r}-1.39 \\
-1.46 \\
0.26 \\
-1.79\end{array}$ & $\begin{array}{l}(0.04) \\
(0.03) \\
(0.05) \\
(0.03)\end{array}$ & $\begin{array}{l}-2.69 \\
-2.63 \\
-0.73 \\
-2.87\end{array}$ & $\begin{array}{l}(0.01) \\
(0.03) \\
(0.05) \\
(0.02)\end{array}$ & $\begin{array}{r}-1.79 \\
-1.71 \\
0.02 \\
-2.04\end{array}$ & $\begin{array}{l}(0.01) \\
(0.01) \\
(0.01) \\
(0.01)\end{array}$ & $\begin{array}{r}-1.13 \\
-1.18 \\
0.52 \\
-1.54\end{array}$ & $\begin{array}{l}(0.01) \\
(0.01) \\
(0.02) \\
(0.01)\end{array}$ & $\begin{array}{r}0.05 \\
-0.31 \\
1.27 \\
-0.70\end{array}$ & $\begin{array}{l}(0.03) \\
(0.02) \\
(0.05) \\
(0.02)\end{array}$ \\
\hline Niederlande $^{2}$ & 0.18 & $(0.03)$ & -0.69 & $(0.02)$ & -0.01 & $(0.01)$ & 0.40 & $(0.01)$ & 1.03 & $(0.02)$ \\
\hline
\end{tabular}

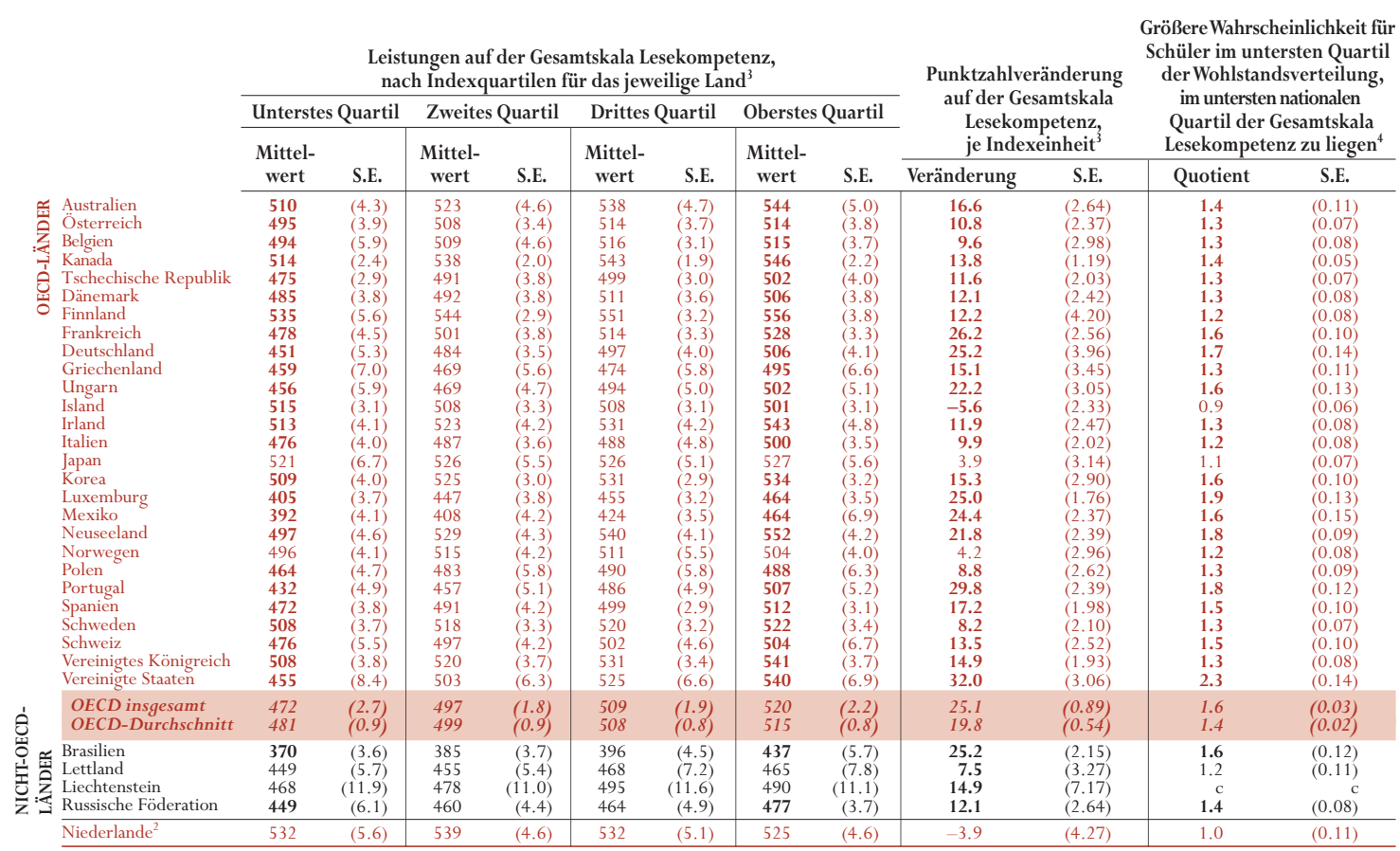

1. Zur Definition des Index siehe Anhang A1.

2. Die Beteiligungsquote ist zu niedrig, um die Vergleichbarkeit zu gewährleisten (vgl. Anhang A3).

3. Zur erklärten Varianz siehe Anhang A2. In Indexeinheiten gemessene Veränderungen sind, soweit fettgedruckt, statistisch signifikant. Wenn die untersten und obersten Quartile durch Fettdruck gekennzeichnet sind, weist dies auf eine statistisch signifikante Differenz zwischen diesen Quartilen hin.

4. Statistisch signifikante Quotienten von mehr als 1 sind durch Fettdruck gekennzeichnet. 
Tabelle 6.3

Index des Besitzes von „klassischen“ Kulturgütern im Elternhaus und Leistungen auf der Gesamtskala Lesekompetenz, nach Indexquartilen für das jeweilige Land

Die Ergebnisse basieren auf Schülerangaben

\begin{tabular}{|c|c|c|c|c|c|c|c|c|c|c|}
\hline & \multicolumn{10}{|c|}{ Index des Besitzes von Kulturgütern im Elternhaus ${ }^{1}$} \\
\hline & \multicolumn{2}{|c|}{ Alle Schüler } & \multicolumn{2}{|c|}{ Unterstes Quartil } & \multicolumn{2}{|c|}{ Zweites Quartil } & \multicolumn{2}{|c|}{ Drittes Quartil } & \multicolumn{2}{|c|}{ Oberstes Quartil } \\
\hline & $\begin{array}{l}\text { Index- } \\
\text { mittel }\end{array}$ & S.E. & $\begin{array}{l}\text { Index- } \\
\text { mittel }\end{array}$ & S.E. & $\begin{array}{l}\text { Index- } \\
\text { mittel }\end{array}$ & S.E. & $\begin{array}{l}\text { Index- } \\
\text { mittel }\end{array}$ & S.E. & $\begin{array}{l}\text { Index- } \\
\text { mittel }\end{array}$ & S.E \\
\hline $\begin{array}{l}\text { Australien } \\
\text { Osterreich } \\
\text { Belgien } \\
\text { Kanada } \\
\text { Tschechische Republik } \\
\text { Dänemark } \\
\text { Finnland } \\
\text { Frankreich } \\
\text { Deutschland } \\
\text { Griechenland } \\
\text { Ungarn } \\
\text { Island } \\
\text { Irland } \\
\text { Italien } \\
\text { Japan } \\
\text { Korea } \\
\text { Luxemburg } \\
\text { Mexiko } \\
\text { Neuseeland } \\
\text { Norwegen } \\
\text { Polen } \\
\text { Portugal } \\
\text { Spanien } \\
\text { Schweden } \\
\text { Schweiz } \\
\text { Vereinigtes Königreich } \\
\text { Vereinigte Staaten }\end{array}$ & $\begin{array}{r}-0.09 \\
0.01 \\
-0.41 \\
-0.12 \\
0.18 \\
-0.11 \\
0.12 \\
-0.30 \\
-0.02 \\
0.20 \\
0.33 \\
0.67 \\
-0.08 \\
0.34 \\
-0.27 \\
0.24 \\
-0.11 \\
-0.58 \\
-0.22 \\
0.14 \\
0.18 \\
-0.10 \\
0.17 \\
0.05 \\
-0.08 \\
-0.07 \\
-0.12\end{array}$ & $\begin{array}{l}(0.03) \\
(0.02) \\
(0.02) \\
(0.01) \\
(0.02) \\
(0.02) \\
(0.02) \\
(0.02) \\
(0.02) \\
(0.03) \\
(0.02) \\
(0.01) \\
(0.03) \\
(0.02) \\
(0.03) \\
(0.02) \\
(0.02) \\
(0.03) \\
(0.02) \\
(0.02) \\
(0.02) \\
(0.03) \\
(0.03) \\
(0.02) \\
(0.03) \\
(0.02) \\
(0.04)\end{array}$ & $\begin{array}{l}-1.38 \\
-1.24 \\
-1.65 \\
-1.39 \\
-1.06 \\
-1.31 \\
-1.18 \\
-1.65 \\
-1.30 \\
-0.96 \\
-0.84 \\
-0.44 \\
-1.39 \\
-0.98 \\
-1.63 \\
-1.02 \\
-1.50 \\
-1.65 \\
-1.51 \\
-1.21 \\
-1.03 \\
-1.44 \\
-1.16 \\
-1.18 \\
-1.37 \\
-1.50 \\
-1.49\end{array}$ & $\begin{array}{l}(0.02) \\
(0.02) \\
(0.00) \\
(0.01) \\
(0.02) \\
(0.02) \\
(0.02) \\
(0.00) \\
(0.02) \\
(0.02) \\
(0.03) \\
(0.02) \\
(0.02) \\
(0.02) \\
(0.00) \\
(0.02) \\
(0.01) \\
(0.00) \\
(0.01) \\
(0.02) \\
(0.02) \\
(0.01) \\
(0.02) \\
(0.02) \\
(0.01) \\
(0.01) \\
(0.02)\end{array}$ & $\begin{array}{r}-0.50 \\
-0.30 \\
-0.76 \\
-0.56 \\
-0.08 \\
-0.55 \\
-0.25 \\
-0.65 \\
-0.33 \\
-0.02 \\
0.12 \\
0.83 \\
-0.43 \\
0.12 \\
-0.62 \\
0.06 \\
-0.48 \\
-1.15 \\
-0.62 \\
-0.25 \\
0.00 \\
-0.38 \\
-0.04 \\
-0.34 \\
-0.42 \\
-0.43 \\
-0.51\end{array}$ & $\begin{array}{l}(0.01) \\
(0.01) \\
(0.01) \\
(0.00) \\
(0.01) \\
(0.01) \\
(0.01) \\
(0.01) \\
(0.01) \\
(0.01) \\
(0.00) \\
(0.02) \\
(0.01) \\
(0.00) \\
(0.00) \\
(0.01) \\
(0.01) \\
(0.01) \\
(0.00) \\
(0.01) \\
(0.01) \\
(0.01) \\
(0.01) \\
(0.01) \\
(0.01) \\
(0.01) \\
(0.01)\end{array}$ & $\begin{array}{r}0.38 \\
0.41 \\
-0.15 \\
0.33 \\
0.72 \\
0.28 \\
0.75 \\
0.12 \\
0.39 \\
0.63 \\
0.90 \\
1.15 \\
0.36 \\
1.07 \\
0.09 \\
0.75 \\
0.38 \\
-0.29 \\
0.10 \\
0.86 \\
0.60 \\
0.25 \\
0.73 \\
0.57 \\
0.31 \\
0.48 \\
0.35\end{array}$ & $\begin{array}{l}(0.02) \\
(0.02) \\
(0.01) \\
(0.01) \\
(0.02) \\
(0.01) \\
(0.02) \\
(0.00) \\
(0.01) \\
(0.02) \\
(0.01) \\
(0.00) \\
(0.01) \\
(0.01) \\
(0.00) \\
(0.02) \\
(0.01) \\
(0.01) \\
(0.01) \\
(0.02) \\
(0.02) \\
(0.01) \\
(0.02) \\
(0.02) \\
(0.01) \\
(0.02) \\
(0.02)\end{array}$ & $\begin{array}{l}\text { Max } \\
\text { Max } \\
0.93 \\
\text { Max } \\
\text { Max } \\
\text { Max } \\
\text { Max } \\
1.00 \\
\text { Max } \\
\text { Max } \\
\text { Max } \\
\text { Max } \\
\text { Max } \\
\text { Max } \\
1.09 \\
\text { Max } \\
\text { Max } \\
0.77 \\
\text { Max } \\
\text { Max } \\
\text { Max } \\
\text { Max } \\
\text { Max } \\
\text { Max } \\
\text { Max } \\
\text { Max } \\
\text { Max }\end{array}$ & $\begin{array}{l}(0.01) \\
(0.02)\end{array}$ \\
\hline $\begin{array}{l}\text { OECD insgesamt } \\
\text { OECD-Durchschnitt }\end{array}$ & $\begin{array}{r}-0.10 \\
0.00\end{array}$ & $\begin{array}{l}(0.01) \\
(0.00)\end{array}$ & $\begin{array}{l}-1.40 \\
-1.27\end{array}$ & $\begin{array}{l}(0.01) \\
(0.00)\end{array}$ & $\begin{array}{l}-0.45 \\
-0.31\end{array}$ & $\begin{array}{l}(0.01) \\
(0.00)\end{array}$ & $\begin{array}{l}0.35 \\
0.47\end{array}$ & $\begin{array}{l}(0.01) \\
(0.00)\end{array}$ & $\begin{array}{l}1.10 \\
1.12\end{array}$ & $\begin{array}{l}(0.00) \\
(0.00)\end{array}$ \\
\hline $\begin{array}{l}\text { Brasilien } \\
\text { Lettland } \\
\text { Liechtenstein } \\
\text { Russische Föderation }\end{array}$ & $\begin{array}{r}-0.41 \\
0.55 \\
-0.03 \\
0.44\end{array}$ & $\begin{array}{l}(0.02) \\
(0.02) \\
(0.05) \\
(0.03)\end{array}$ & $\begin{array}{l}-1.65 \\
-0.53 \\
-1.24 \\
-0.44\end{array}$ & $\begin{array}{l}(0.00) \\
(0.03) \\
(0.05) \\
(0.03)\end{array}$ & $\begin{array}{r}-0.68 \\
0.44 \\
-0.28 \\
0.12\end{array}$ & $\begin{array}{l}(0.01) \\
(0.02) \\
(0.04) \\
(0.00)\end{array}$ & $\begin{array}{r}-0.06 \\
1.15 \\
0.27 \\
0.95\end{array}$ & $\begin{array}{l}(0.01) \\
(0.00) \\
(0.04) \\
(0.01)\end{array}$ & $\begin{array}{l}0.77 \\
\text { Max } \\
\text { Max } \\
\text { Max }\end{array}$ & $(0.02)$ \\
\hline Niederlande $^{2}$ & -0.45 & $(0.02)$ & -1.65 & $(0.00)$ & -0.68 & $(0.01)$ & -0.21 & $(0.02)$ & 0.77 & $(0.03)$ \\
\hline
\end{tabular}

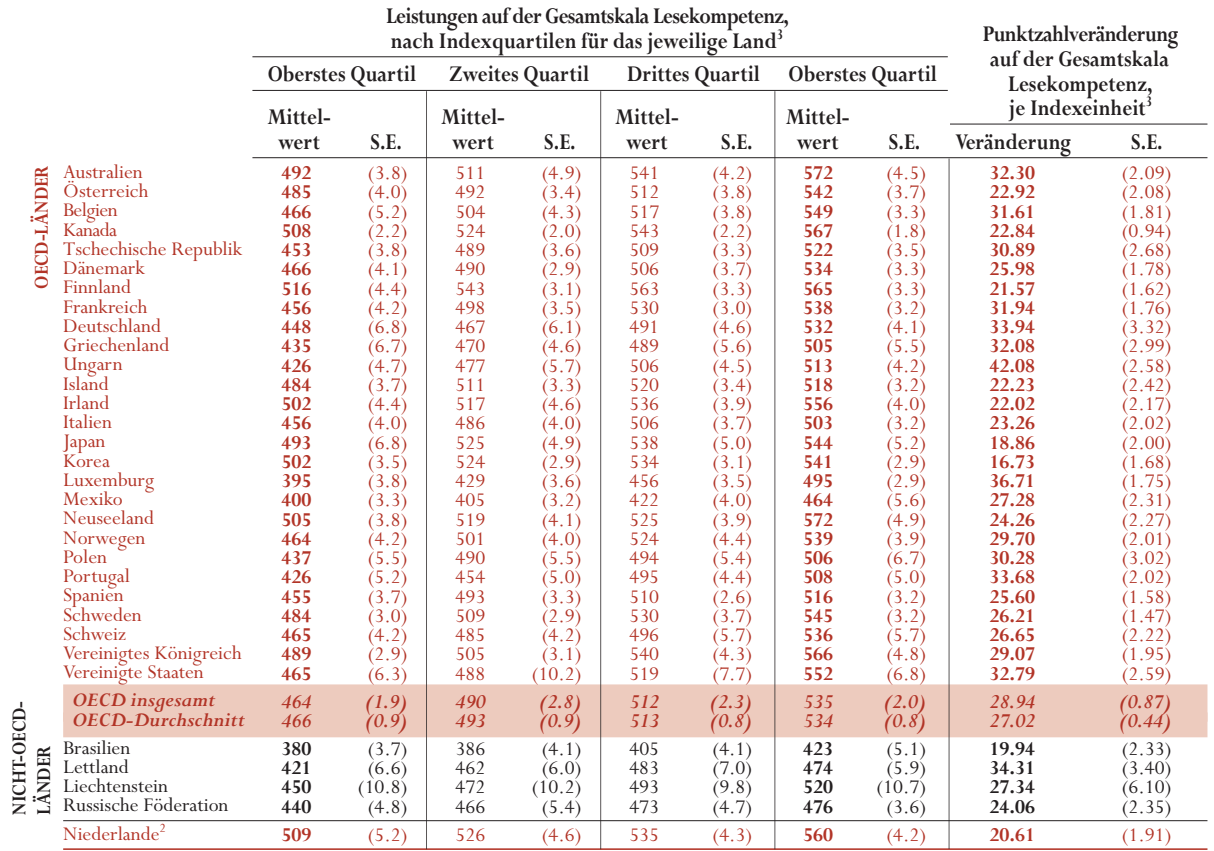

1. Zur Definition des Index siehe Anhang A1. "Max" wird für Länder verwendet, in denen mehr als $25 \%$ der Schülerinnen und Schüler den höchsten Wert dieses Index erreichen, der 1.15 beträgt.

2. Die Beteiligungsquote ist zu niedrig, um die Vergleichbarkeit zu gewährleisten (vgl. Anhang A3).

3. Zur erklärten Varianz siehe Anhang A2. In Indexeinheiten gemessene Veränderungen sind, soweit fettgedruckt, statistisch signifikant. Wenn die untersten und obersten Quartile durch Fettdruck gekennzeichnet sind, weist dies auf eine statistisch signifikante Differenz zwischen diesen Quartilen hin. 
Tabelle 6.4

Index der „klassischen“ kulturellen Aktivitäten und Leistungen auf der Gesamtskala Lesekompetenz, nach Indexquartilen für das jeweilige Land

Die Ergebnisse basieren auf Schülerangaben

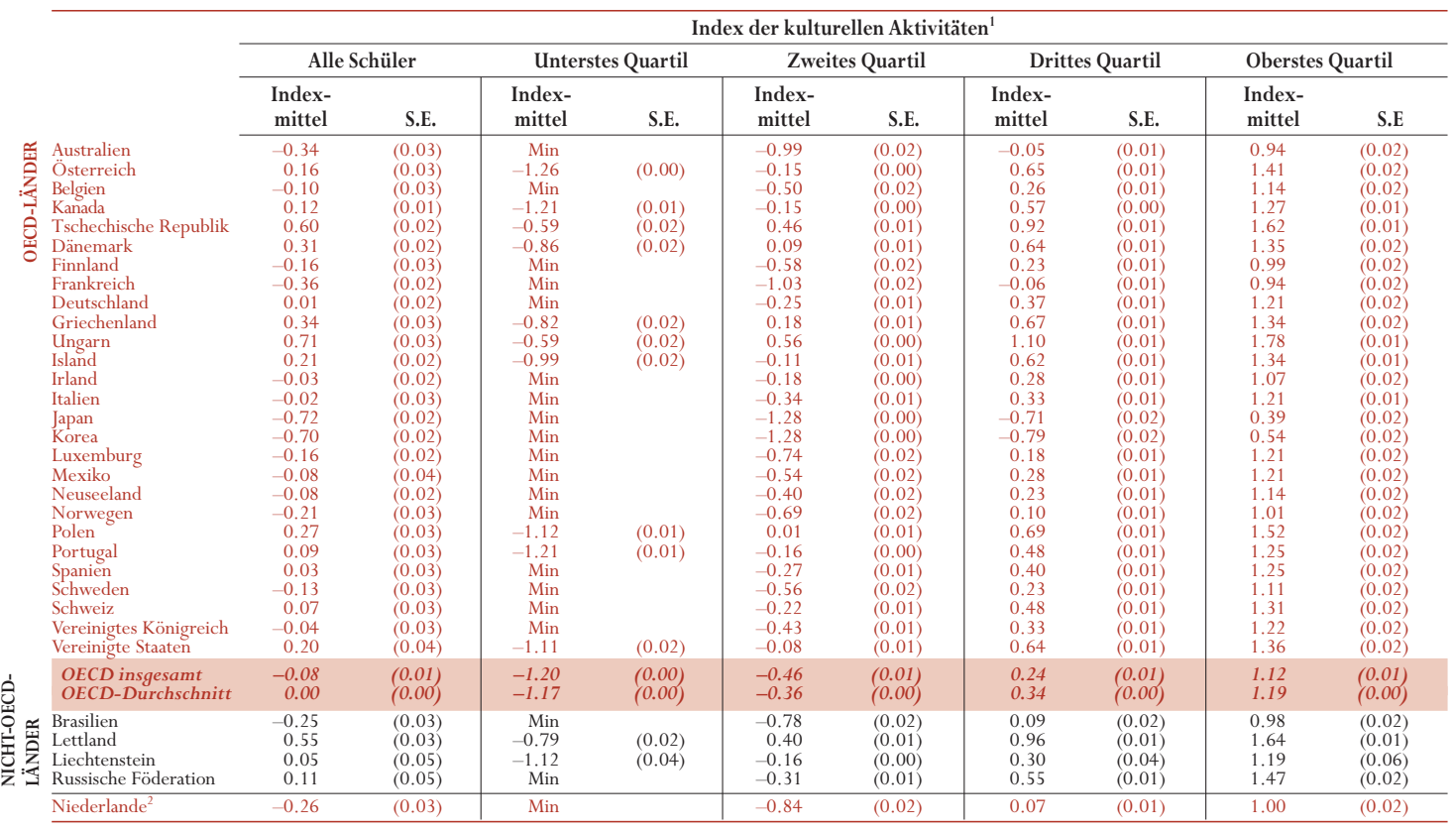

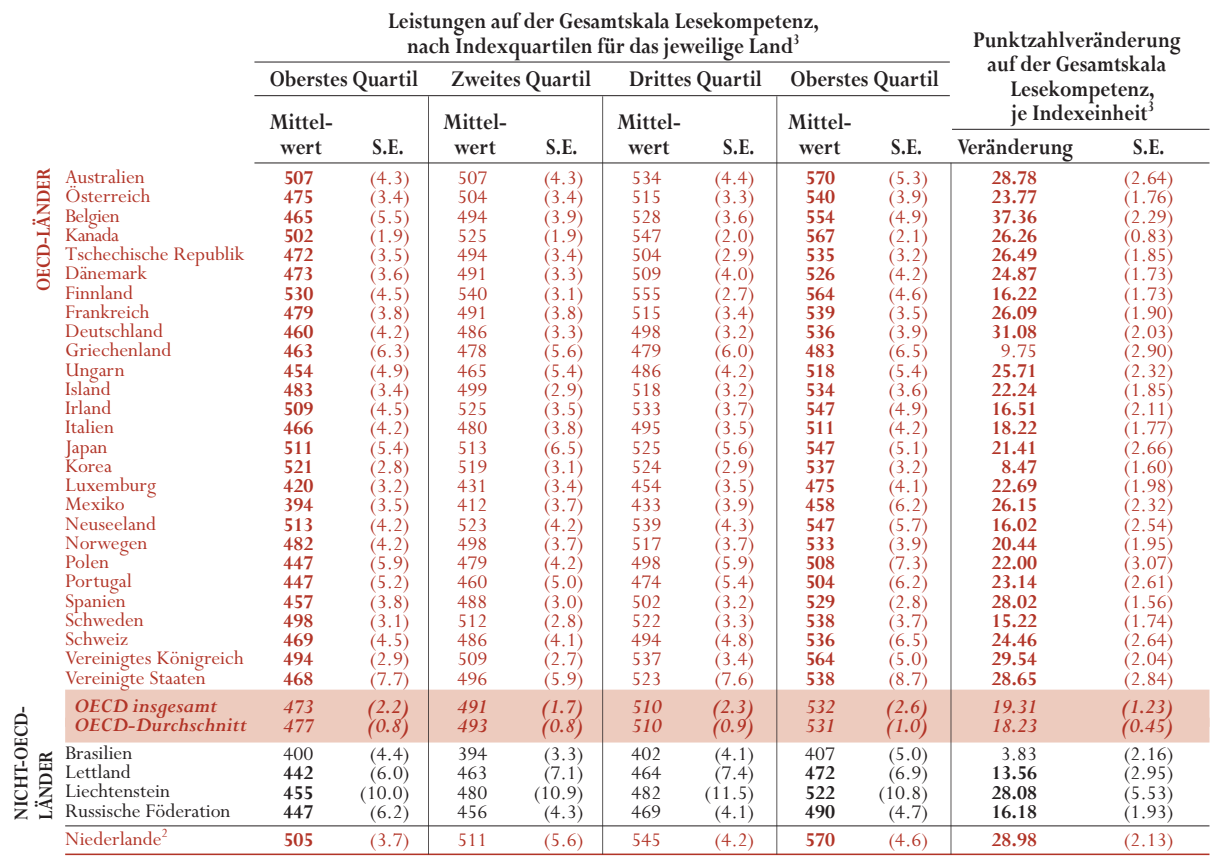

1. Zur Definition des Index siehe Anhang A1. "Min” wird für Länder verwendet, in denen mehr als $25 \%$ der Schülerinnen und Schüler den niedrigsten Wert dieses Index erreichen, der -1.28 beträgt.

2. Die Beteiligungsquote ist zu niedrig, um die Vergleichbarkeit zu gewährleisten (vgl. Anhang A3).

. Zur erklärten Varianz siehe Anhang A2. In Indexeinheiten gemessene Veränderungen sind, soweit fettgedruckt, statistisch signifikant. Wenn die untersten und obersten Quartile durch Fettdruck gekennzeichnet sind, weist dies auf eine statistisch
signifikante Differenz zwischen diesen Quartilen hin. 
Tabelle 6.5

Index der sozialen Kommunikation mit den Eltern und Leistungen auf der Gesamtskala Lesekompetenz, nach Indexquartilen für das jeweilige Land

Die Ergebnisse basieren auf Schülerangaben

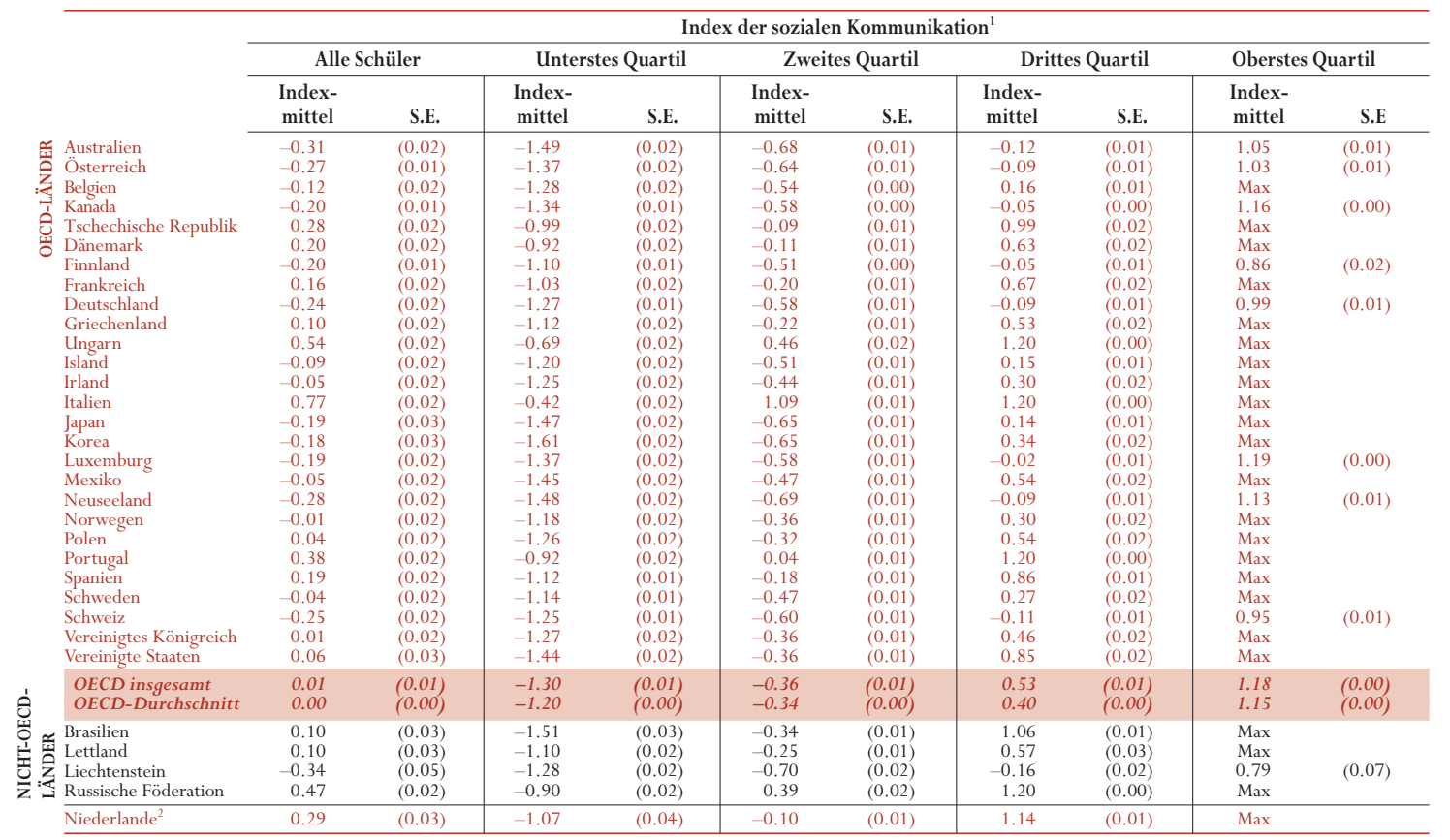

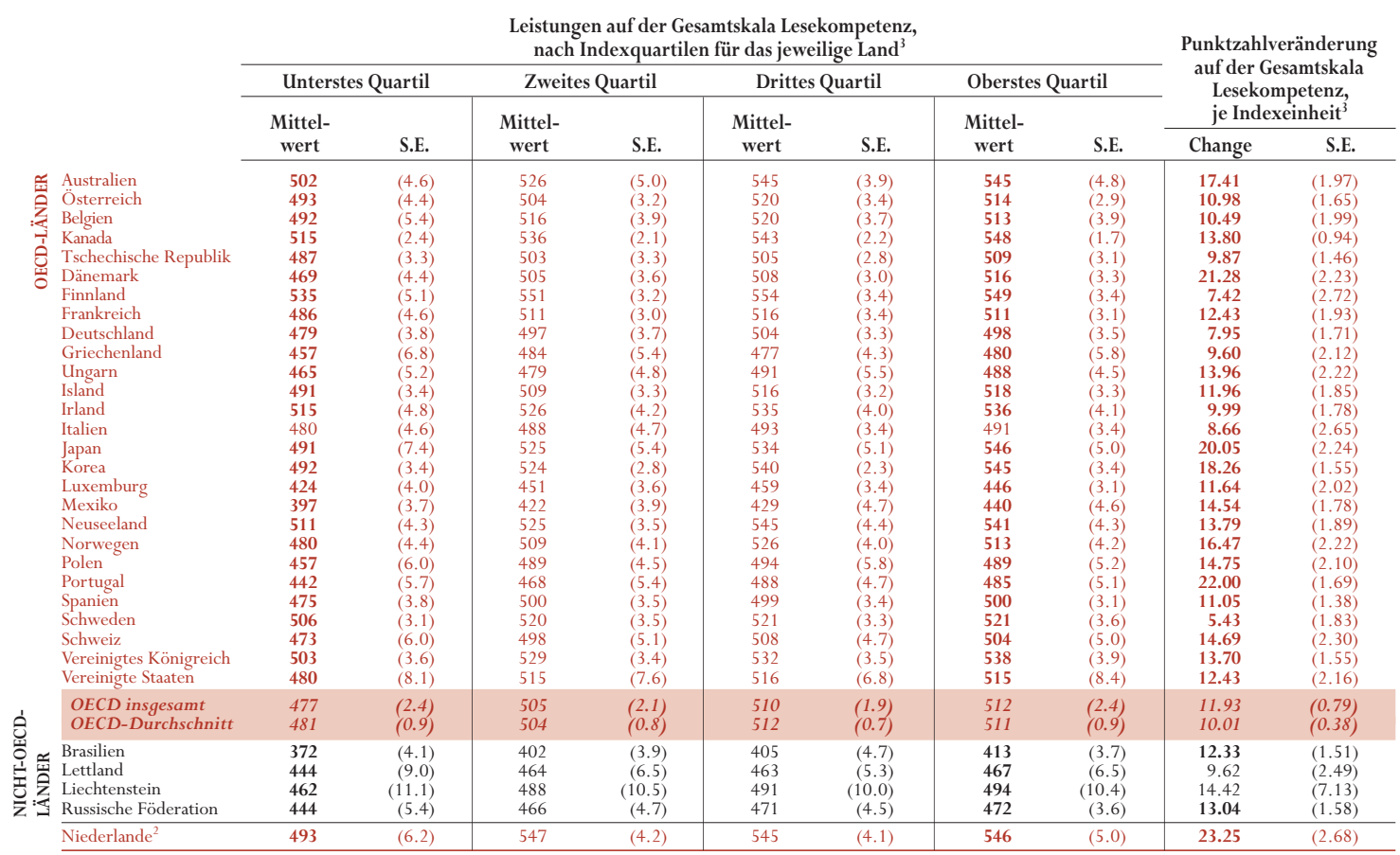

1. Zur Definition des Index siehe Anhang A1. "Max" wird für die Länder verwendet, in denen mehr als $25 \%$ der Schülerinnen und Schüler den höchsten Wert dieses Index erreichen, der 1.20 beträgt.

2. Die Beteiligungsquote ist zu niedrig, um die Vergleichbarkeit zu gewährleisten (vgl. Anhang A3).

3. Zur erklärten Varianz siehe Anhang A2. In Indexeinheiten gemessene Veränderungen sind, soweit fettgedruckt, statistisch signifikant. Wenn die untersten und obersten Quartile durch Fettdruck gekennzeichnet sind, weist dies auf eine statistisch signifikante Differenz zwischen diesen Quartilen hin. 
Tabelle 6.6

Index der kulturellen Kommunikation mit den Eltern und Leistungen auf der Gesamtskala Lesekompetenz, nach Indexquartilen für das jeweilige Land

Die Ergebnisse basieren auf Schülerangaben

\begin{tabular}{|c|c|c|c|c|c|c|c|c|c|c|}
\hline & \multicolumn{10}{|c|}{ Index der kulturellen Kommunikation $^{1}$} \\
\hline & \multicolumn{2}{|c|}{ Alle Schüler } & \multicolumn{2}{|c|}{ Unterstes Quartil } & \multicolumn{2}{|c|}{ Zweites Quartil } & \multicolumn{2}{|c|}{ Drittes Quartil } & \multicolumn{2}{|c|}{ Oberstes Quartil } \\
\hline & $\begin{array}{l}\text { Index- } \\
\text { mittel }\end{array}$ & S.E. & $\begin{array}{l}\text { Index- } \\
\text { mittel }\end{array}$ & S.E. & $\begin{array}{l}\text { Index- } \\
\text { mittel }\end{array}$ & S.E. & $\begin{array}{l}\text { Index- } \\
\text { mittel }\end{array}$ & S.E. & $\begin{array}{l}\text { Index- } \\
\text { mittel }\end{array}$ & S.E \\
\hline $\begin{array}{l}\text { OECD insgesamt } \\
\text { OECD-Durchschnitt }\end{array}$ & $\begin{array}{l}0.07 \\
0.00\end{array}$ & $\begin{array}{l}(0.01) \\
(0.00)\end{array}$ & $\begin{array}{l}-1.29 \\
-1.29\end{array}$ & $\begin{array}{l}(0.01) \\
(0.01)\end{array}$ & $\begin{array}{l}-0.15 \\
-0.23\end{array}$ & $\begin{array}{l}(0.00) \\
(0.00)\end{array}$ & $\begin{array}{l}0.47 \\
0.37\end{array}$ & $\begin{array}{l}(0.00) \\
(0.00)\end{array}$ & $\begin{array}{l}1.26 \\
1.15\end{array}$ & $\begin{array}{l}(0.01) \\
(0.00)\end{array}$ \\
\hline $\begin{array}{l}\text { Brasilien } \\
\text { Lettland } \\
\text { Liechtenstein } \\
\text { Russische Föderation }\end{array}$ & $\begin{array}{r}0.17 \\
0.25 \\
-0.20 \\
0.19\end{array}$ & $\begin{array}{l}(0.03) \\
(0.02) \\
(0.05) \\
(0.02)\end{array}$ & $\begin{array}{l}-1.31 \\
-1.01 \\
-1.43 \\
-1.22\end{array}$ & $\begin{array}{l}(0.02) \\
(0.02) \\
(0.07) \\
(0.02)\end{array}$ & $\begin{array}{r}-0.09 \\
0.02 \\
-0.46 \\
-0.06\end{array}$ & $\begin{array}{l}(0.01) \\
(0.01) \\
(0.02) \\
(0.01)\end{array}$ & $\begin{array}{l}0.63 \\
0.64 \\
0.14 \\
0.62\end{array}$ & $\begin{array}{l}(0.01) \\
(0.01) \\
(0.02) \\
(0.01)\end{array}$ & $\begin{array}{l}1.48 \\
1.36 \\
0.96 \\
1.43\end{array}$ & $\begin{array}{l}(0.02) \\
(0.02) \\
(0.05) \\
(0.01)\end{array}$ \\
\hline Niederlande $^{2}$ & -0.35 & $(0.03)$ & -1.85 & $(0.02)$ & -0.61 & $(0.01)$ & 0.11 & $(0.01)$ & 0.95 & $(0.02)$ \\
\hline
\end{tabular}

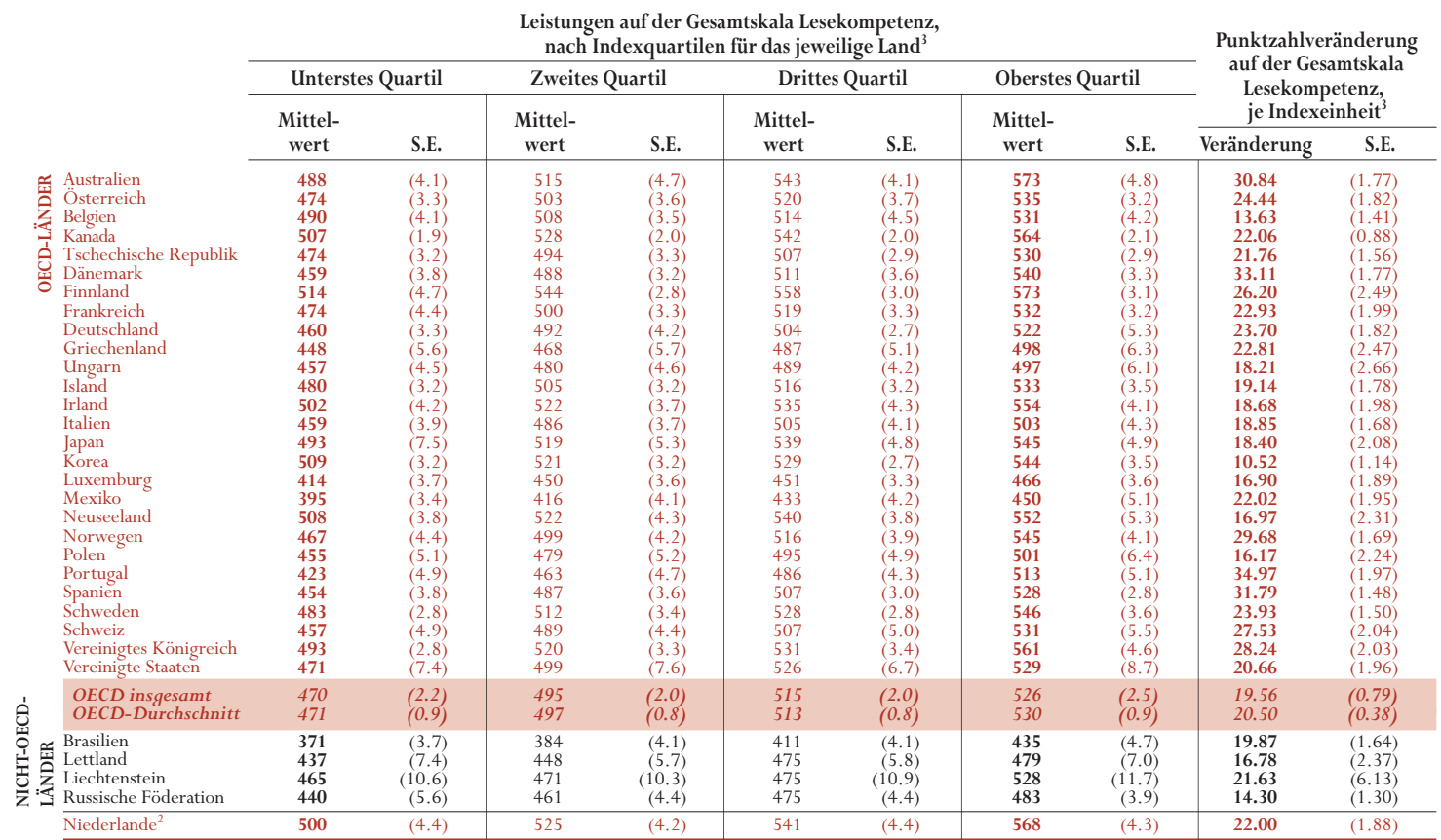

1. Zur Definition des Index siehe Anhang A1.

2. Die Beteiligungsquote ist zu niedrig, um die Vergleichbarkeit zu gewährleisten (vgl. Anhang A3).

3. Zur erklärten Varianz siehe Anhang A2. In Indexeinheiten gemessene Veränderungen sind, soweit fettgedruckt, statistisch signifikant. Wenn die untersten und obersten Quartile durch Fettdruck gekennzeichnet sind, weist dies auf eine statistisch signifikante Differenz zwischen diesen Quartilen hin. 
Tabelle 6.7

Prozentsatz der Schüler und Leistungen auf der Gesamtskala Lesekompetenz sowie den Skalen für mathematische und für naturwissenschaftliche Grundbildung, nach dem Bildungsabschluss der Mutter

Die Ergebnisse basieren auf Schülerangaben

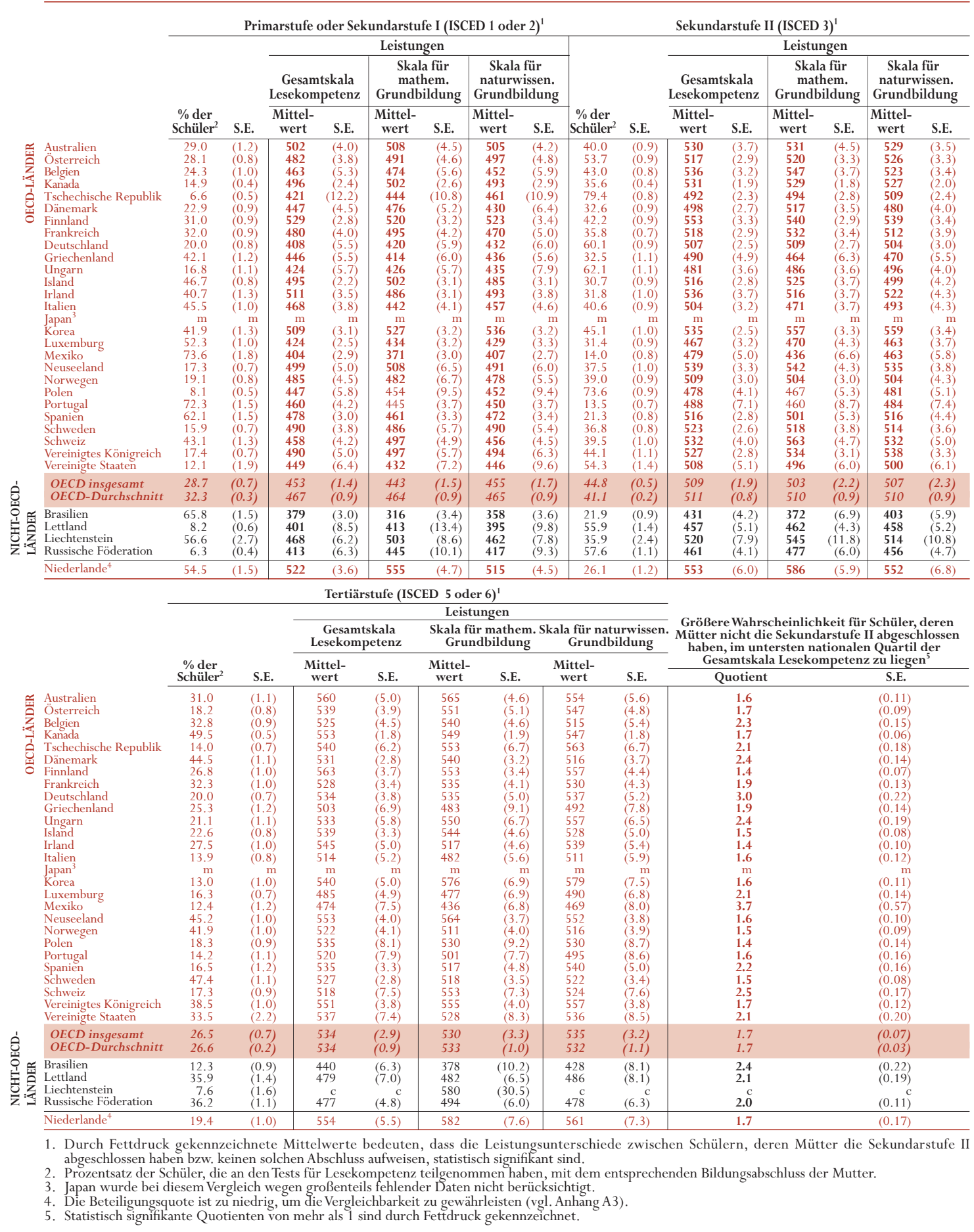


Tabelle 6.8

Prozentualer Anteil der Hochschulabsolventen an der erwachsenen Bevölkerung und Wahrscheinlichkeit, einen Hochschulabschluss zu erhalten, nach Bildungsstand der Eltern

\begin{tabular}{|c|c|c|c|c|c|c|}
\hline & \multicolumn{3}{|c|}{$\begin{array}{l}\text { Prozentualer Anteil der Bevölkerung im } \\
\text { Alter 16-65 Jahre mit abgeschlossener } \\
\text { Hochschulausbildung, nach Bildungsstand der Eltern }\end{array}$} & \multicolumn{3}{|c|}{$\begin{array}{c}\text { Größere Wahrscheinlichkeit von Personen, deren Eltern } \\
\text { Hochschulabsolventen sind, ebenfalls einen Hochschulabschluss } \\
\text { zu erwerben, im Vergleich zu Personen, deren } \\
\text { Eltern keinen Sekundarschulabschluss aufweisen }\end{array}$} \\
\hline & $\begin{array}{l}\text { Schulabschluss unter } \\
\text { Sekundarstufe II }\end{array}$ & $\begin{array}{l}\text { Schulabschluss } \\
\text { Sekundarstufe II }\end{array}$ & $\begin{array}{l}\text { Hochschul- } \\
\text { abschluss }\end{array}$ & $\begin{array}{c}\text { Insgesamt } \\
(16-65 \text { Jahre })\end{array}$ & 26- bis 35-Jährige & 46- bis 55-Jährige \\
\hline Australien & 20.0 & 25.7 & 39.2 & 2.0 & 2.4 & 1.9 \\
\hline Belgien (fläm.) & 15.3 & 32.8 & 49.7 & 3.3 & 2.6 & 4.4 \\
\hline Kanada & 23.7 & 41.5 & 57.2 & 2.4 & 2.9 & 2.2 \\
\hline Deutschland & 16.0 & 23.3 & 38.4 & 2.3 & 2.9 & 2.4 \\
\hline Irland & 12.0 & 36.1 & 57.4 & 4.8 & $\mathrm{~m}$ & $\mathrm{~m}$ \\
\hline Niederlande & 12.8 & 22.5 & 42.6 & 3.3 & 3.3 & 4.3 \\
\hline Neuseeland & 21.4 & 28.8 & 45.3 & 2.1 & 2.8 & 2.0 \\
\hline Polen & 9.2 & 26.0 & 53.8 & 5.8 & 6.6 & $\mathrm{~m}$ \\
\hline Schweden & 18.7 & 29.5 & 40.2 & 2.2 & 2.8 & 2.6 \\
\hline Schweiz & 8.8 & 19.6 & 37.8 & 4.3 & 4.3 & 5.6 \\
\hline Ver.Königreich & 16.5 & 38.2 & 47.0 & 2.9 & 3.3 & 3.1 \\
\hline Vereinigte Staaten & 19.7 & 35.7 & 64.2 & 3.3 & 3.6 & 4.6 \\
\hline
\end{tabular}

Quelle: OECD und Statistics Canada (1995).

Tabelle 6.9

Prozent der Schüler und Leistungen auf der Gesamtskala Lesekompetenz, nach Familientyp Die Ergebnisse basieren auf Schülerangaben

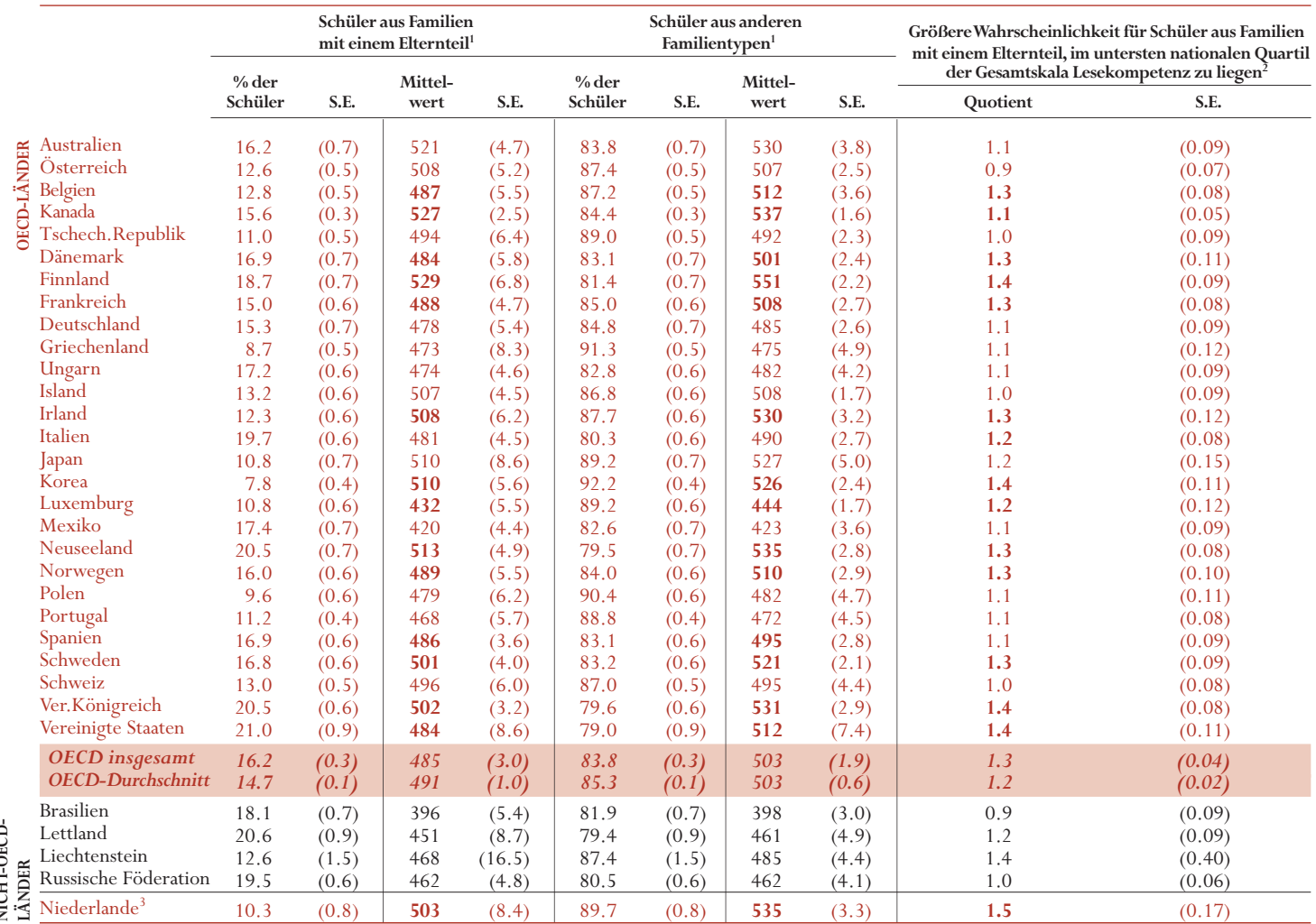

1. Zur Definition der Familientypen siehe Anhang A1. Durch Fettdruck gekennzeichnete Mittelwerte bedeuten, dass die Leistungsunterschiede auf der Gesamtskala für Lesekompetenz zwischen Schülern aus Familien mit einem Elternteil und Schülern aus anderen Familientypen statistisch signifikant sind.

2. Statistisch signifikante Quotienten von mehr als 1 sind durch Fettdruck gekennzeichnet.

3. Die Beteiligungsquote ist zu niedrig, um die Vergleichbarkeit zu gewährleisten (vgl. Anhang A3). 
Tabelle 6.10

Prozentsatz der Schüler und Leistungen auf der Gesamtskala Lesekompetenz sowie den Skalen für mathematische und für naturwissenschaftliche Grundbildung, nach Staatsangehörigkeit der Schüler und deren Eltern

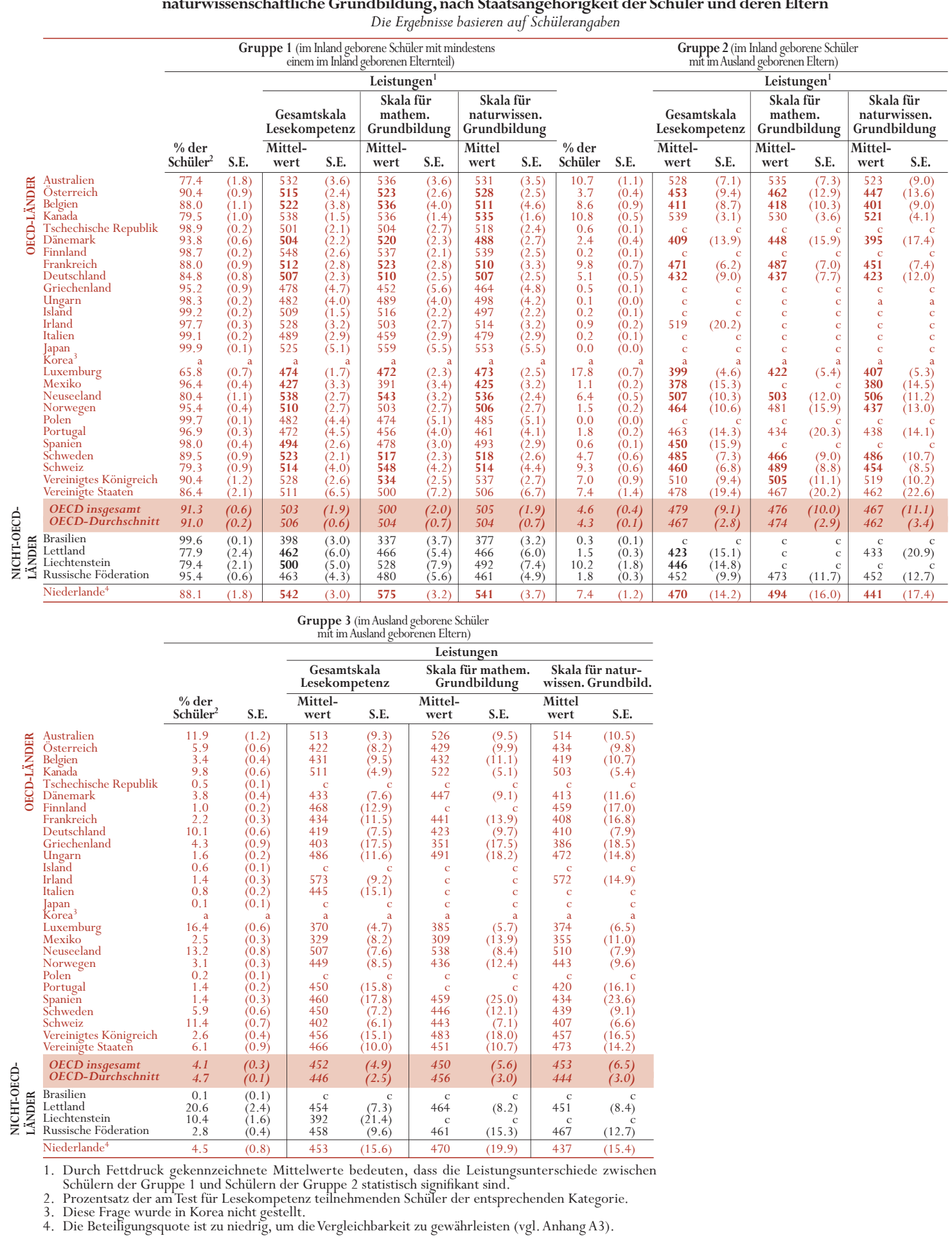


Tabelle 6.11

Schülerleistungen auf der Gesamtskala Lesekompetenz sowie den Skalen für mathematische und für naturwissenschaftliche Grundbildung, nach der zu Hause gesprochenen Sprache Die Ergebnisse basieren auf Schülerangaben

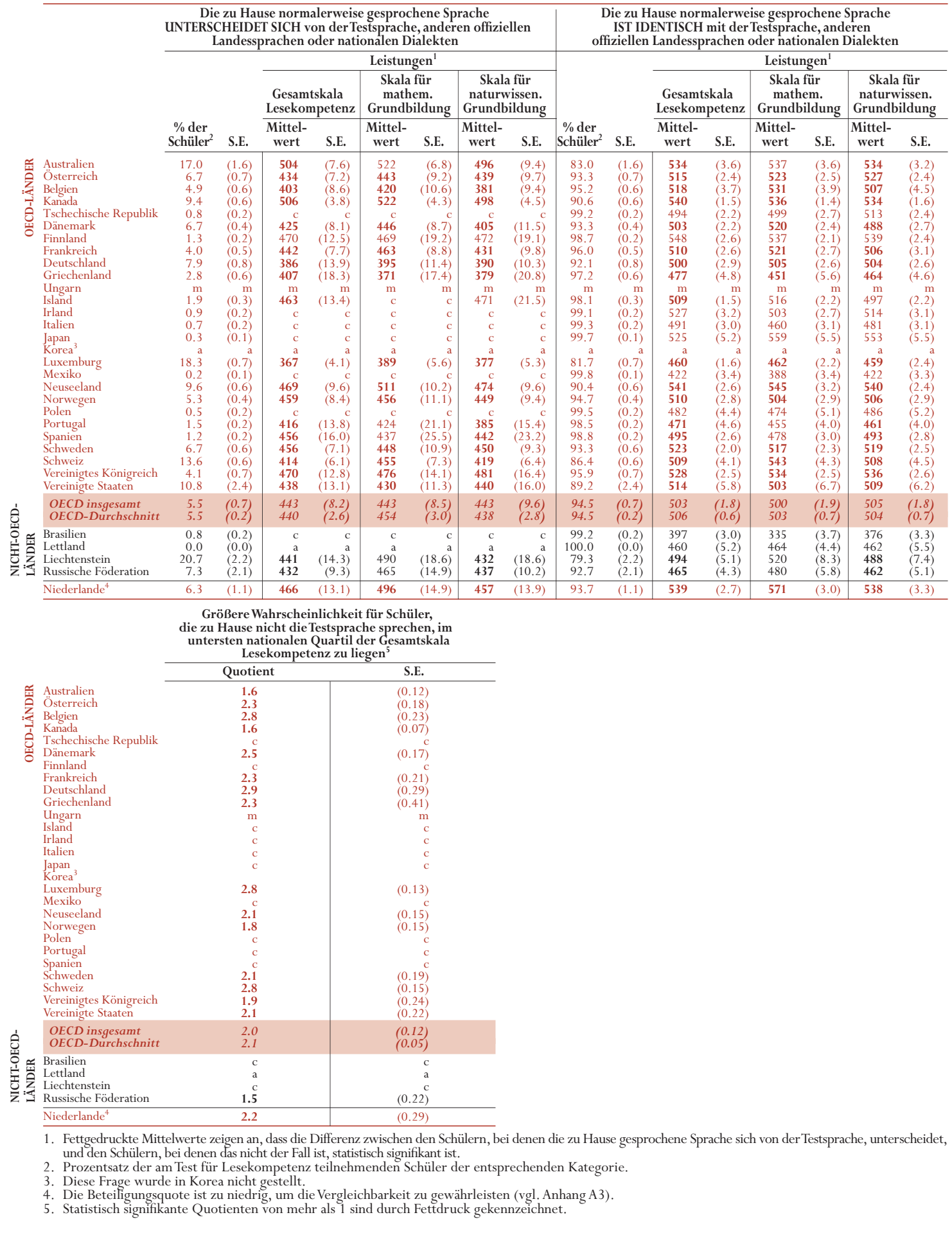

338 
Tabelle 7.1

Index der Unterstützung der Schüler durch die Lehrkräfte und Leistungen auf der Gesamtskala Lesekompetenz, nach Indexquartilen für das jeweilige Land

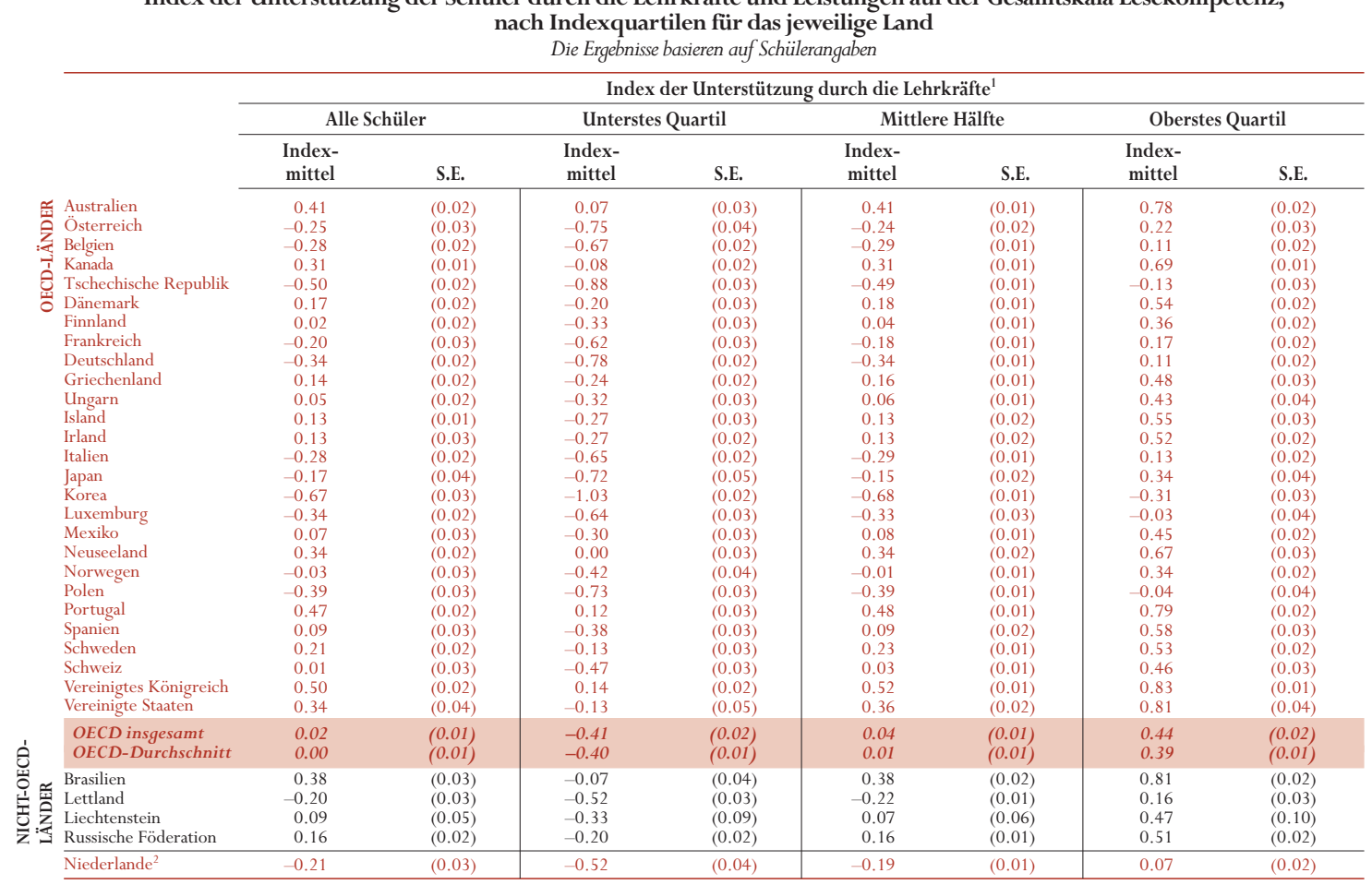

Leistungen auf der Gesamtskala Lesekompetenz,

\begin{tabular}{|c|c|c|c|c|c|c|c|}
\hline \multicolumn{2}{|c|}{ Unterstes Quartil } & \multicolumn{2}{|c|}{ Mittlere Hälfte } & \multicolumn{2}{|c|}{ Oberstes Quartil } & \multirow{2}{*}{\multicolumn{2}{|c|}{$\begin{array}{c}\text { Punktzahlveränderung } \\
\text { auf der Gesamtskala Lesekompetenz, } \\
\text { je Indexeinheit }{ }^{3}\end{array}$}} \\
\hline \multirow{2}{*}{$\begin{array}{c}\text { Mittel- } \\
\text { wert }\end{array}$} & \multirow[b]{2}{*}{ S.E. } & \multirow{2}{*}{$\begin{array}{c}\text { Mittel- } \\
\text { wert }\end{array}$} & \multirow[b]{2}{*}{ S.E. } & \multirow{2}{*}{$\begin{array}{c}\text { Mittel- } \\
\text { wert }\end{array}$} & \multirow[b]{2}{*}{ S.E. } & & \\
\hline & & & & & & Veränderung & S.E. \\
\hline 524 & $(8.1)$ & 533 & $(5.0)$ & 529 & $(7.9)$ & 7.37 & $(2.24)$ \\
\hline 528 & $(9.1)$ & 498 & (5.3) & 510 & $(9.1)$ & -0.62 & $(2.34)$ \\
\hline 526 & (8.8) & 514 & (5.9) & 485 & (14.0) & -4.83 & $(2.38)$ \\
\hline 536 & (2.3) & 536 & $(2.5)$ & 534 & $(3.4)$ & 4.42 & $(0.97)$ \\
\hline 514 & (8.1) & 498 & (4.9) & 494 & (6.5) & 0.77 & $(2.32)$ \\
\hline 490 & (6.4) & 500 & (3.0) & 507 & $(4,1)$ & 11.65 & $(2.34)$ \\
\hline 543 & (5.0) & 551 & $(2.7)$ & 543 & (7.0) & 5.48 & $(2.03)$ \\
\hline 524 & (8.2) & 501 & (5.7) & 499 & (9.4) & -2.53 & $(1.77)$ \\
\hline 532 & (7.2) & 500 & (5.1) & 443 & (9.6) & -12.55 & $(2.12)$ \\
\hline 466 & (12.5) & 489 & $(9.2)$ & 459 & $(9.0)$ & 2.20 & $(2.42)$ \\
\hline 490 & (11.3) & 491 & (7.6) & 453 & (12.9) & -2.43 & $(2.76)$ \\
\hline 507 & (3.0) & 506 & $(2.0)$ & 514 & $(2.8)$ & 8.87 & $(1.92)$ \\
\hline 530 & (6.1) & 530 & (5.1) & 521 & (6.9) & -0.13 & $(1.90)$ \\
\hline 523 & (7.1) & 492 & $(6.0)$ & 444 & (8.6) & -11.46 & $(2.21)$ \\
\hline 517 & $(12.2)$ & 522 & (8.3) & 537 & (7.5) & 6.23 & $(2.28)$ \\
\hline 516 & (6.3) & 526 & $(4.9)$ & 532 & (7.6) & 5.56 & $(1.61)$ \\
\hline 493 & $(2.4)$ & 426 & (2.3) & 430 & (2.9) & -5.13 & $(1.48)$ \\
\hline 435 & (10.1) & 422 & (6.3) & 410 & (8.5) & -2.60 & $(2.29)$ \\
\hline 524 & (6.5) & 534 & (4.1) & 530 & (8.3) & 5.26 & $(2.45)$ \\
\hline 502 & (5.1) & 511 & $(4.3)$ & 504 & (6.0) & 14.95 & $(2.26)$ \\
\hline 444 & $(12.5)$ & 493 & (8.7) & 499 & $(12.1)$ & 9.20 & $(2.96)$ \\
\hline 483 & $(12.5)$ & 469 & (6.3) & 462 & $(9.9)$ & -1.33 & $(2.78)$ \\
\hline 487 & (6.0) & 497 & $(4.0)$ & 494 & (6.4) & 2.53 & $(1.89)$ \\
\hline 516 & (5.0) & 514 & (3.5) & 524 & (3.9) & 6.20 & $(1.82)$ \\
\hline 546 & $(9.2)$ & 489 & (5.7) & 458 & (7.6) & -13.40 & $(2.24)$ \\
\hline 522 & (6.6) & 525 & (6.4) & 529 & (7.6) & 6.66 & $(1.45)$ \\
\hline 502 & (12.8) & 508 & $(8.3)$ & 507 & (12.3) & 6.87 & $(2.61)$ \\
\hline $\begin{array}{l}503 \\
508\end{array}$ & $\begin{array}{l}(3.7) \\
(1.8)\end{array}$ & $\begin{array}{l}502 \\
503\end{array}$ & $\begin{array}{l}(2.8) \\
(1.3)\end{array}$ & $\begin{array}{l}496 \\
495\end{array}$ & $\begin{array}{l}(4.4) \\
(2.0)\end{array}$ & $\begin{array}{l}2.96 \\
2.82\end{array}$ & $\begin{array}{l}(0.90) \\
(0.45)\end{array}$ \\
\hline 390 & (8.1) & 394 & $(4.7)$ & 410 & $(6.6)$ & 4.60 & $(1.79)$ \\
\hline 456 & (11.9) & 451 & (6.0) & 481 & (9.7) & 15.56 & $(2.42)$ \\
\hline 584 & (7.3) & 480 & (4.8) & 408 & $(10.3)$ & -14.19 & $(5.19)$ \\
\hline 465 & (5.8) & 462 & (7.3) & 463 & (8.0) & 6.40 & $(1.41)$ \\
\hline 563 & $(11.5)$ & 528 & (7.3) & 510 & $(13.3)$ & -5.54 & $(3.55)$ \\
\hline
\end{tabular}

Östrerreic

: Belgien

Tschechische Republik

Dänemark

Finnland

Frankreich

Deutschland
Griechenland

Ungarn

Island

Irland

Japan

Korea

Luxemburg

Mexiko

Neuseeland

Norwegen

Polen

Portugal

Spanien

Schweden

Vereinigtes Königreich

Vereinigte Staaten

OECD insgesamt

息藏

Liechtenstein

1. Zur Definition des Index siehe Anhang A1.

2. Die Beteiligungsquote ist zu niedrig, um die Vergleichbarkeit zu gewährleisten (vgl. Anhang A3).
3. Zur erklärten Varianz siehe Anhang A2. In Indexeinheiten gemessene Veränderungen sind, soweit fett gedruckt, statistisch signifikant. Wenn die untersten und obersten Quartile durch Fettdruck gekennzeichnet sind, weist dies auf eine statistisch signifikante Differenz zwischen diesen Quartilen hin. 
Tabelle 7.2

Index schülerbezogener Faktoren für das Schulklima und Leistungen auf der Gesamtskala Lesekompetenz, nach Indexquartilen für das jeweilige Land

Die Ergebnisse basieren auf Angaben der Schulleitungen und beziehen sich auf den jeweiligen zahlenmäßigen Anteil der 15-Jährigen in den Schulen

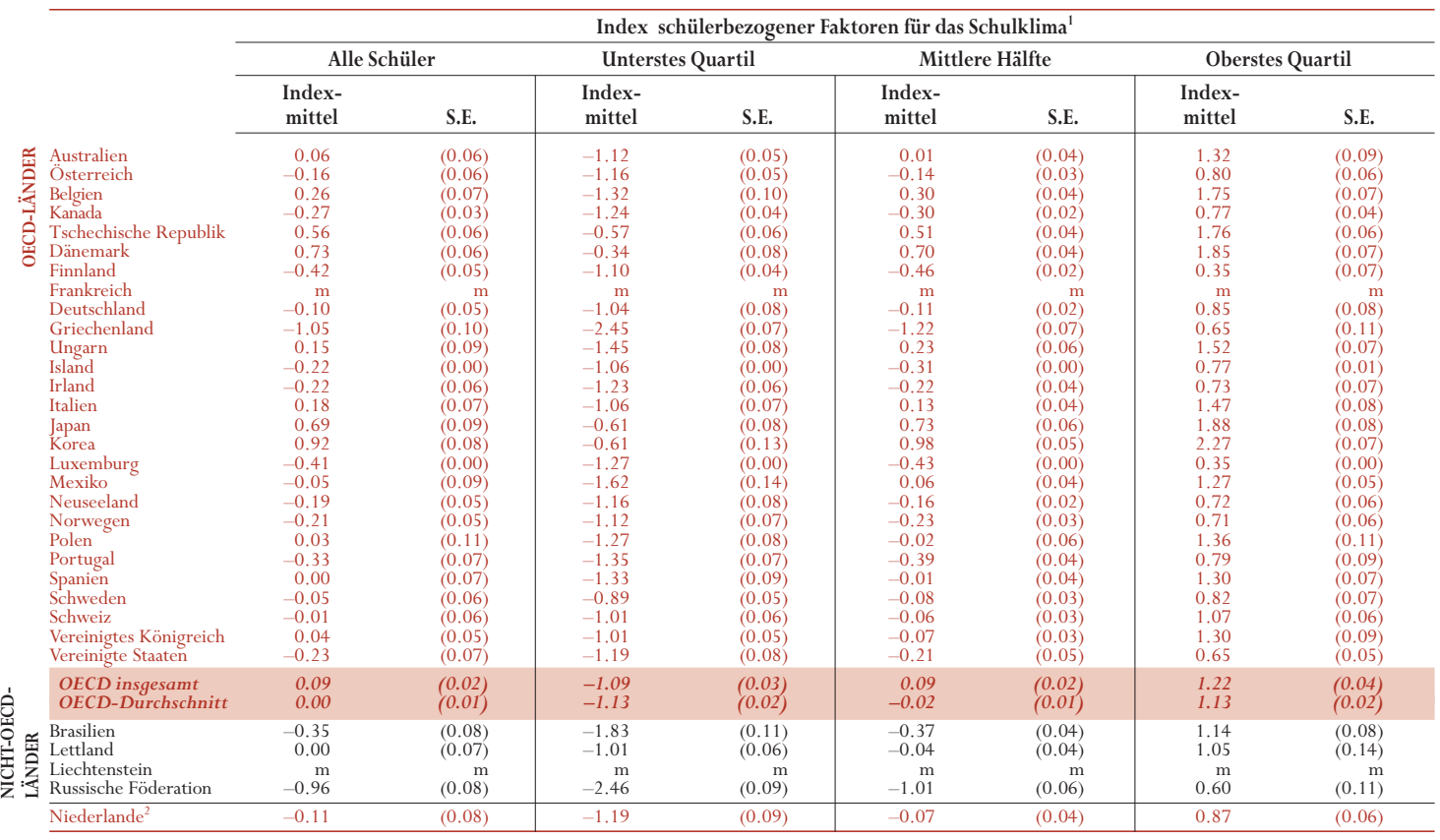

\begin{tabular}{|c|c|c|c|c|c|c|c|c|}
\hline & \multicolumn{6}{|c|}{$\begin{array}{l}\text { Leistungen auf der Gesamtskala Lesekompetenz, } \\
\text { nach Indexquartilen für das jeweilige Land }\end{array}$} & \multirow{3}{*}{\multicolumn{2}{|c|}{$\begin{array}{c}\text { Punktzahlveränderung } \\
\text { auf der Gesamtskala Lesekompetenz, } \\
\text { je Indexeinheit }{ }^{3}\end{array}$}} \\
\hline & \multicolumn{2}{|c|}{ Unterstes Quartil } & \multicolumn{2}{|c|}{ Mittlere Hälfte } & \multicolumn{2}{|c|}{ Oberstes Quartil } & & \\
\hline & \multirow{2}{*}{$\begin{array}{c}\text { Mittel- } \\
\text { wert }\end{array}$} & \multirow[b]{2}{*}{ S.E. } & \multirow{2}{*}{$\begin{array}{c}\text { Mittel- } \\
\text { wert }\end{array}$} & \multirow[b]{2}{*}{ S.E. } & \multirow{2}{*}{$\begin{array}{c}\text { Mittel- } \\
\text { wert }\end{array}$} & \multirow[b]{2}{*}{ S.E. } & & \\
\hline & & & & & & & Veränderung & S.E. \\
\hline & 503 & $(7.1)$ & 524 & (3.7) & 562 & $(8.4)$ & 23.35 & $(3.08)$ \\
\hline $\begin{array}{l}\text { Stserreich } \\
\text { Belgien }\end{array}$ & $\begin{array}{l}488 \\
443\end{array}$ & $(9.1)$ & $\begin{array}{l}513 \\
517\end{array}$ & $\begin{array}{l}(5.9) \\
5.6\end{array}$ & $\begin{array}{l}517 \\
554\end{array}$ & $\begin{array}{l}(7.5) \\
(95)\end{array}$ & $\begin{array}{l}16.37 \\
37.91\end{array}$ & $\begin{array}{l}(5.67) \\
(4.02)\end{array}$ \\
\hline Kanada & 519 & (3.7) & 539 & $(2.0)$ & 543 & $\begin{array}{l}(9.5) \\
(2.3)\end{array}$ & 12.41 & $\left.\begin{array}{l}(4.02) \\
(1.97)\end{array}\right)$ \\
\hline Tschechische Republik & 458 & (10.4) & 495 & $(4.9)$ & 519 & (7.7) & $\begin{array}{l}12.01 \\
31.03\end{array}$ & $(4.84)$ \\
\hline Dänemark & 480 & $(6.9)$ & 499 & $(4.0)$ & 505 & $(5.3)$ & 14.48 & $(4.70)$ \\
\hline $\begin{array}{l}\text { Finnland } \\
\text { Frankreich }\end{array}$ & 545 & $(4.1)$ & 544 & (4.3) & 554 & (5.1) & 4.30 & $(3.67)$ \\
\hline Deutschland & 412 & $\begin{aligned} \mathrm{m} \\
(11.1)\end{aligned}$ & $\begin{aligned} \mathrm{m} \\
506\end{aligned}$ & $\begin{array}{l}\mathrm{m} \\
(8.0)\end{array}$ & $\begin{aligned} \mathrm{m} \\
519\end{aligned}$ & $\begin{array}{r}\mathrm{m} \\
(8.5)\end{array}$ & $\begin{aligned} \mathrm{m} \\
50.74\end{aligned}$ & $\begin{aligned} \mathrm{m} \\
(709)\end{aligned}$ \\
\hline Griechenland & 474 & (14.1) & 472 & (8.1) & 478 & $(12.2)$ & $\begin{array}{r}0.74 \\
1.49\end{array}$ & (4.98) \\
\hline $\begin{array}{l}\text { Ungarn } \\
\text { Island }\end{array}$ & 429 & $(8.7)$ & $\begin{array}{l}480 \\
505\end{array}$ & $(7.2)$ & 527 & $(8.6)$ & $\begin{array}{l}29.41 \\
8>2\end{array}$ & $(3.80)$ \\
\hline $\begin{array}{l}\text { Island } \\
\text { Irland }\end{array}$ & 502 & $(3.1)$ & $\begin{array}{c}505 \\
528\end{array}$ & $(2.1)$ & $\begin{array}{l}517 \\
548\end{array}$ & $\begin{array}{l}(3.2) \\
(6.3)\end{array}$ & $\begin{array}{r}8.29 \\
21.87\end{array}$ & $(1.93)$ \\
\hline $\begin{array}{l}\text { Itanien } \\
\text { Itali }\end{array}$ & $\begin{array}{l}502 \\
445\end{array}$ & $\begin{array}{l}(8.2) \\
(12.0)\end{array}$ & $\begin{array}{l}528 \\
489\end{array}$ & $\begin{array}{r}(3.8) \\
(5.5)\end{array}$ & $\begin{array}{l}548 \\
526\end{array}$ & $\begin{array}{l}(6.3) \\
(7.3)\end{array}$ & $\begin{array}{l}21.87 \\
33.98\end{array}$ & $\begin{array}{l}(3.92) \\
(4.93)\end{array}$ \\
\hline Japan & 471 & $(11.1)$ & 532 & (6.5) & 553 & $(7.9)$ & 34.74 & $\begin{array}{l}(4.93) \\
(4.85)\end{array}$ \\
\hline & 489 & (7.1) & 531 & $(3.9)$ & 547 & $(3.7)$ & 19.55 & $(2.59)$ \\
\hline Luxemburg & 453 & $(2,5)$ & 419 & (2.6) & 481 & (2.9) & 11.44 & $(2.09)$ \\
\hline Mexiko & 407 & & 430 & (6.7) & 423 & $(9.5)$ & 4.31 & $(4.00)$ \\
\hline $\begin{array}{l}\text { Neuseeland } \\
\text { Norwegen }\end{array}$ & 504 & $\begin{array}{l}(6.5) \\
(5.6)\end{array}$ & 536 & $(4.6)$ & 546 & $(5.9)$ & $\begin{array}{l}26.17 \\
525\end{array}$ & $(4.03)$ \\
\hline $\begin{array}{l}\text { Norwegen } \\
\text { Polen }\end{array}$ & $\begin{array}{l}503 \\
421\end{array}$ & $\begin{array}{l}(5.6) \\
(12.2)\end{array}$ & 481 & $\begin{array}{l}(4.4) \\
(9.4)\end{array}$ & $\begin{array}{l}509 \\
530\end{array}$ & $\begin{array}{l}(3.3) \\
(8.5)\end{array}$ & $\begin{array}{r}5.25 \\
38.90\end{array}$ & $\begin{array}{l}3.45) \\
(6.53)\end{array}$ \\
\hline Portugal & 451 & $(9.5)$ & 473 & (6.2) & 484 & $(11.0)$ & 13.28 & $(5.50)$ \\
\hline $\begin{array}{l}\text { Spanien } \\
\text { SShweden }\end{array}$ & 471 & $(5.8)$ & 490 & $(4.1)$ & 519 & $(5.9)$ & 18.15 & $(2.53)$ \\
\hline $\begin{array}{l}\text { Schweden } \\
\text { Schwez }\end{array}$ & 498 & $(5.7)$ & 520 & $(3.2)$ & 526 & $(4.9)$ & $\begin{array}{l}15.70 \\
19.05\end{array}$ & $(3.74)$ \\
\hline $\begin{array}{l}\text { Schweiz } \\
\text { Vereinigtes Kö }\end{array}$ & $\begin{array}{l}463 \\
487\end{array}$ & $\begin{array}{l}(8.3) \\
(59)\end{array}$ & 521 & $(7.0)$ & 508 & $(8.1)$ & 19.05 & $\begin{array}{l}(5.15) \\
(3.12)\end{array}$ \\
\hline $\begin{array}{l}\text { Vereinigte Staaten } \\
\text {. }\end{array}$ & 489 & (13.1) & 505 & (7.7) & 520 & $(10.0)$ & $\begin{array}{l}36.92 \\
15.92\end{array}$ & $(9.23)$ \\
\hline $\begin{array}{l}\text { OECD insgesamt } \\
\text { OECD-Durchschnitt }\end{array}$ & 466 & $(3.9)$ & 503 & $(2.2)$ & 522 & (3.2) & 23.59 & $(1.78)$ \\
\hline & & & & & & & & \\
\hline $\begin{array}{l}\text { Lettland } \\
\text { Lets }\end{array}$ & 452 & (10.5) & 450 & $\begin{array}{l}(3.4) \\
(6.3)\end{array}$ & 484 & $(14.6)$ & 9.52 & (7.99) \\
\hline $\begin{array}{l}\text { Liechtenstein } \\
\text { Russische Föderation }\end{array}$ & $\underset{450}{m}$ & $\mathrm{~m}$ & $\underset{463}{m}$ & $\frac{\mathrm{m}}{\mathrm{m}}$ & $\mathrm{m}_{471}$ & $\frac{m}{m}$ & $\begin{aligned} m \\
7.83\end{aligned}$ & $\frac{m}{m}$ \\
\hline Niederlande $^{2}$ & 458 & $(13.2)$ & 545 & $(8.1)$ & 572 & $(7.6)$ & 52.09 & $(6.24)$ \\
\hline
\end{tabular}

1. Zur Definition des Index siehe Anhang A1. Die Skala wurde so gepolt, dass positive und hohe Werte auf ein positives Schulklima in Bezug

2. Die Beteiligungsquote ist zu niedrig, um die Vergleichbarkeit zu gewährleisten (vgl. Anhang A3).

3. Zur erklärten Varianz siehe Anhang A2. In Indexeinheiten gemessene Veränderungen sind, soweit fett gedruckt, statistisch signifikant. Wenn die untersten und obersten Quartile durch Fettdruck gekennzeichnet sind, weist dies auf eine statistisch signifikante Differenz zwischen diesen Quartilen hin. 
Tabelle 7.3

Index der Schuldisziplin und Leistungen auf der Gesamtskala Lesekompetenz, nach Indexquartilen für das jeweilige Land Die Ergebnisse basieren auf Schülerangaben

\begin{tabular}{|c|c|c|c|c|c|c|c|c|}
\hline & \multicolumn{8}{|c|}{ Index der Schuldisziplin $^{1}$} \\
\hline & \multicolumn{2}{|c|}{ Alle Schüler } & \multicolumn{2}{|c|}{ Unterstes Quartil } & \multicolumn{2}{|c|}{ Mittlere Hälfte } & \multicolumn{2}{|c|}{ Oberstes Quartil } \\
\hline & $\begin{array}{l}\text { Index- } \\
\text { mittel }\end{array}$ & S.E. & $\begin{array}{l}\text { Index- } \\
\text { mittel }\end{array}$ & S.E. & $\begin{array}{l}\text { Index- } \\
\text { mittel }\end{array}$ & S.E. & $\begin{array}{l}\text { Index- } \\
\text { mittel }\end{array}$ & S.E. \\
\hline $\begin{array}{l}\text { Australien } \\
\text { Osterreich } \\
\text { Belgien } \\
\text { Kanada } \\
\text { Tschechische Republik } \\
\text { Dänemark } \\
\text { Finnland } \\
\text { Frankreich } \\
\text { Deutschland } \\
\text { Griechenland } \\
\text { Ungarn } \\
\text { Island } \\
\text { Irland } \\
\text { Italien } \\
\text { Japan } \\
\text { Korea } \\
\text { Luxemburg } \\
\text { Mexiko } \\
\text { Neuseeland } \\
\text { Norwegen } \\
\text { Polen } \\
\text { Portugal } \\
\text { Spanien } \\
\text { Schweden } \\
\text { Schweiz } \\
\text { Vereinigtes Königreich } \\
\text { Vereinigte Staaten }\end{array}$ & $\begin{array}{r}-0.09 \\
0.19 \\
-0.12 \\
-0.14 \\
0.14 \\
-0.20 \\
-0.16 \\
-0.05 \\
0.10 \\
-0.42 \\
0.23 \\
-0.08 \\
0.09 \\
-0.24 \\
0.49 \\
0.20 \\
0.12 \\
0.17 \\
-0.15 \\
-0.36 \\
0.37 \\
-0.05 \\
-0.17 \\
-0.19 \\
0.30 \\
0.02 \\
0.03\end{array}$ & $\begin{array}{l}(0.03) \\
(0.04) \\
(0.03) \\
(0.01) \\
(0.03) \\
(0.02) \\
(0.03) \\
(0.03) \\
(0.02) \\
(0.02) \\
(0.04) \\
(0.01) \\
(0.03) \\
(0.03) \\
(0.05) \\
(0.03) \\
(0.02) \\
(0.03) \\
(0.02) \\
(0.03) \\
(0.04) \\
(0.02) \\
(0.03) \\
(0.02) \\
(0.03) \\
(0.03) \\
(0.03)\end{array}$ & $\begin{array}{l}-0.51 \\
-0.37 \\
-0.57 \\
-0.52 \\
-0.43 \\
-0.57 \\
-0.55 \\
-0.49 \\
-0.39 \\
-0.78 \\
-0.38 \\
-0.62 \\
-0.74 \\
-0.22 \\
-0.26 \\
-0.10 \\
-0.24 \\
-0.48 \\
-0.72 \\
-0.24 \\
-0.65 \\
-0.57 \\
-0.23 \\
-0.44 \\
-0.40\end{array}$ & $\begin{array}{l}(0.03) \\
(0.03) \\
(0.02) \\
(0.01) \\
(0.04) \\
(0.02) \\
(0.03) \\
(0.02) \\
(0.04) \\
(0.02) \\
(0.05) \\
(0.03) \\
(0.03) \\
(0.03) \\
(0.05) \\
(0.03) \\
(0.04) \\
(0.03) \\
(0.03) \\
(0.03) \\
(0.04) \\
(0.02) \\
(0.04) \\
(0.03) \\
(0.03) \\
(0.02) \\
(0.02)\end{array}$ & $\begin{array}{r}-0.10 \\
0.18 \\
-0.12 \\
-0.15 \\
0.11 \\
-0.20 \\
-0.18 \\
-0.07 \\
0.10 \\
-0.42 \\
0.23 \\
-0.07 \\
0.08 \\
-0.25 \\
0.51 \\
0.20 \\
0.09 \\
0.15 \\
-0.16 \\
-0.38 \\
0.39 \\
-0.05 \\
-0.16 \\
-0.19 \\
0.30 \\
0.01 \\
0.01\end{array}$ & $\begin{array}{l}(0.02) \\
(0.02) \\
(0.01) \\
(0.01) \\
(0.02) \\
(0.01) \\
(0.01) \\
(0.01) \\
(0.02) \\
(0.02) \\
(0.02) \\
(0.02) \\
(0.02) \\
(0.02) \\
(0.03) \\
(0.02) \\
(0.02) \\
(0.01) \\
(0.02) \\
(0.01) \\
(0.02) \\
(0.01) \\
(0.01) \\
(0.01) \\
(0.02) \\
(0.02) \\
(0.01)\end{array}$ & $\begin{array}{r}0.33 \\
0.76 \\
0.33 \\
0.27 \\
0.77 \\
0.18 \\
0.27 \\
0.42 \\
0.55 \\
-0.06 \\
0.80 \\
0.44 \\
0.54 \\
0.28 \\
1.14 \\
0.63 \\
0.37 \\
0.61 \\
0.21 \\
0.02 \\
0.91 \\
0.20 \\
0.27 \\
0.17 \\
0.83 \\
0.49 \\
0.47\end{array}$ & $\begin{array}{l}(0.02) \\
(0.04) \\
(0.02) \\
(0.01) \\
(0.03) \\
(0.02) \\
(0.04) \\
(0.03) \\
(0.02) \\
(0.03) \\
(0.03) \\
(0.03) \\
(0.04) \\
(0.03) \\
(0.05) \\
(0.04) \\
(0.04) \\
(0.03) \\
(0.03) \\
(0.03) \\
(0.04) \\
(0.02) \\
(0.03) \\
(0.02) \\
(0.03) \\
(0.04) \\
(0.04)\end{array}$ \\
\hline $\begin{array}{l}\text { OECD insgesamt } \\
\text { OECD-Durchschnitt }\end{array}$ & $\begin{array}{l}0.09 \\
0.00\end{array}$ & $\begin{array}{l}(0.01) \\
(0.01)\end{array}$ & $\begin{array}{l}-0.40 \\
-0.45\end{array}$ & $\begin{array}{l}(0.01) \\
(0.01)\end{array}$ & $\begin{array}{r}0.08 \\
-0.01\end{array}$ & $\begin{array}{l}(0.01) \\
(0.01)\end{array}$ & $\begin{array}{l}0.57 \\
0.45\end{array}$ & $\begin{array}{l}(0.02) \\
(0.01)\end{array}$ \\
\hline $\begin{array}{l}\text { Brasilien } \\
\text { Lettland } \\
\text { Liechtenstein } \\
\text { Russische Föderation }\end{array}$ & $\begin{array}{r}-0.34 \\
0.38 \\
0.35 \\
0.45\end{array}$ & $\begin{array}{l}(0.02) \\
(0.03) \\
(0.05) \\
(0.03)\end{array}$ & $\begin{array}{r}-0.72 \\
-0.07 \\
0.05 \\
0.01\end{array}$ & $\begin{array}{l}(0.02) \\
(0.04) \\
(0.10) \\
(0.02)\end{array}$ & $\begin{array}{r}-0.35 \\
0.40 \\
0.36 \\
0.42\end{array}$ & $\begin{array}{l}(0.01) \\
(0.01) \\
(0.08) \\
(0.02)\end{array}$ & $\begin{array}{l}0.06 \\
0.79 \\
0.52 \\
0.92\end{array}$ & $\begin{array}{l}(0.04) \\
(0.02) \\
(0.06) \\
(0.03)\end{array}$ \\
\hline Niederlande $^{2}$ & -0.33 & $(0.03)$ & -0.73 & $(0.05)$ & -0.31 & $(0.02)$ & 0.02 & $(0.03)$ \\
\hline
\end{tabular}

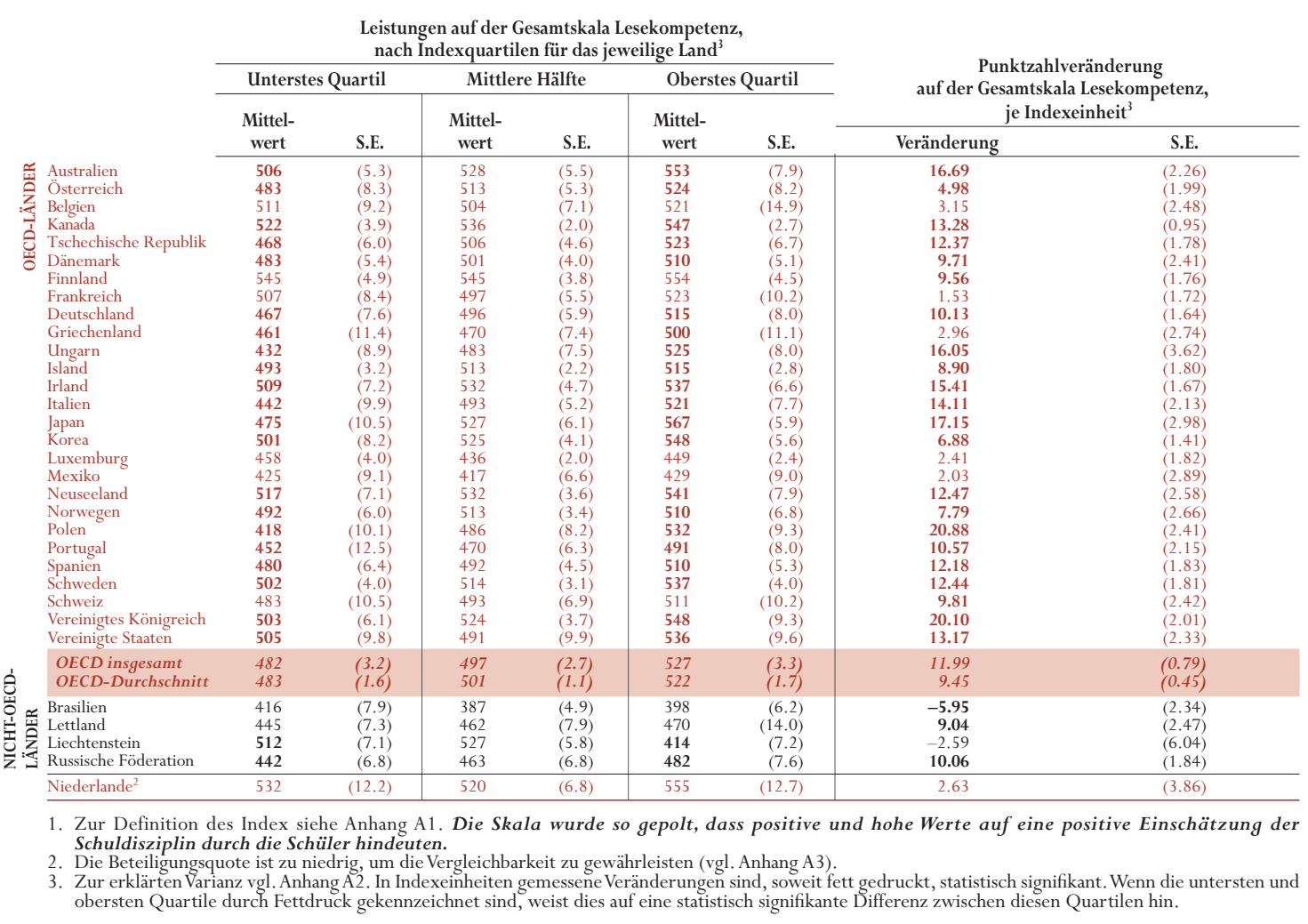


Tabelle 7.4

Index lehrkräftebezogener Faktoren für das Schulklima und Leistungen auf der Gesamtskala Lesekompetenz, nach Indexquartilen für das jeweilige Land

Die Ergebnisse basieren auf Angaben der Schulleitungen und beziehen sich auf den jeweiligen zahlenmäßigen Anteil der 15-Jährigen in den Schulen

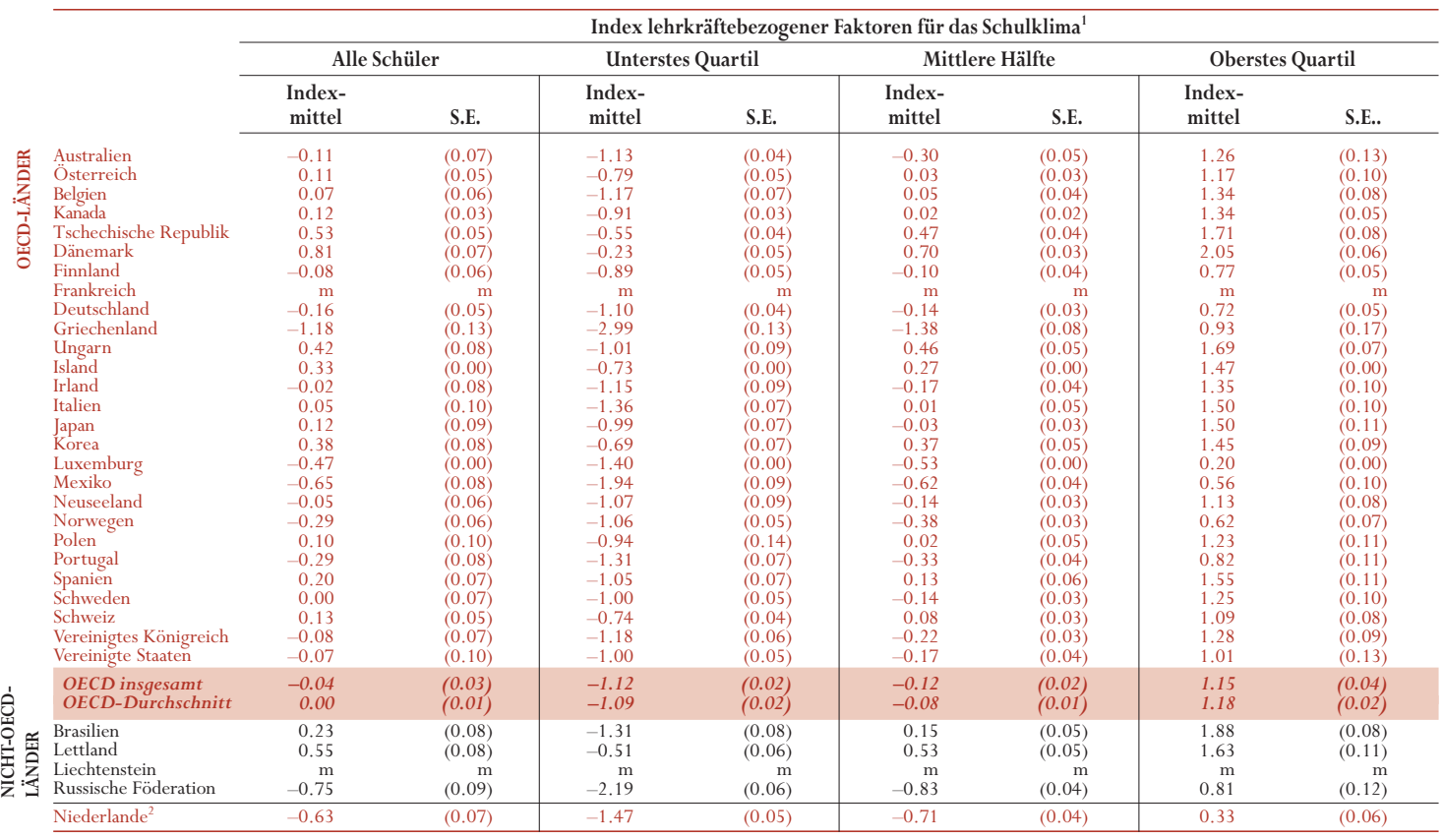

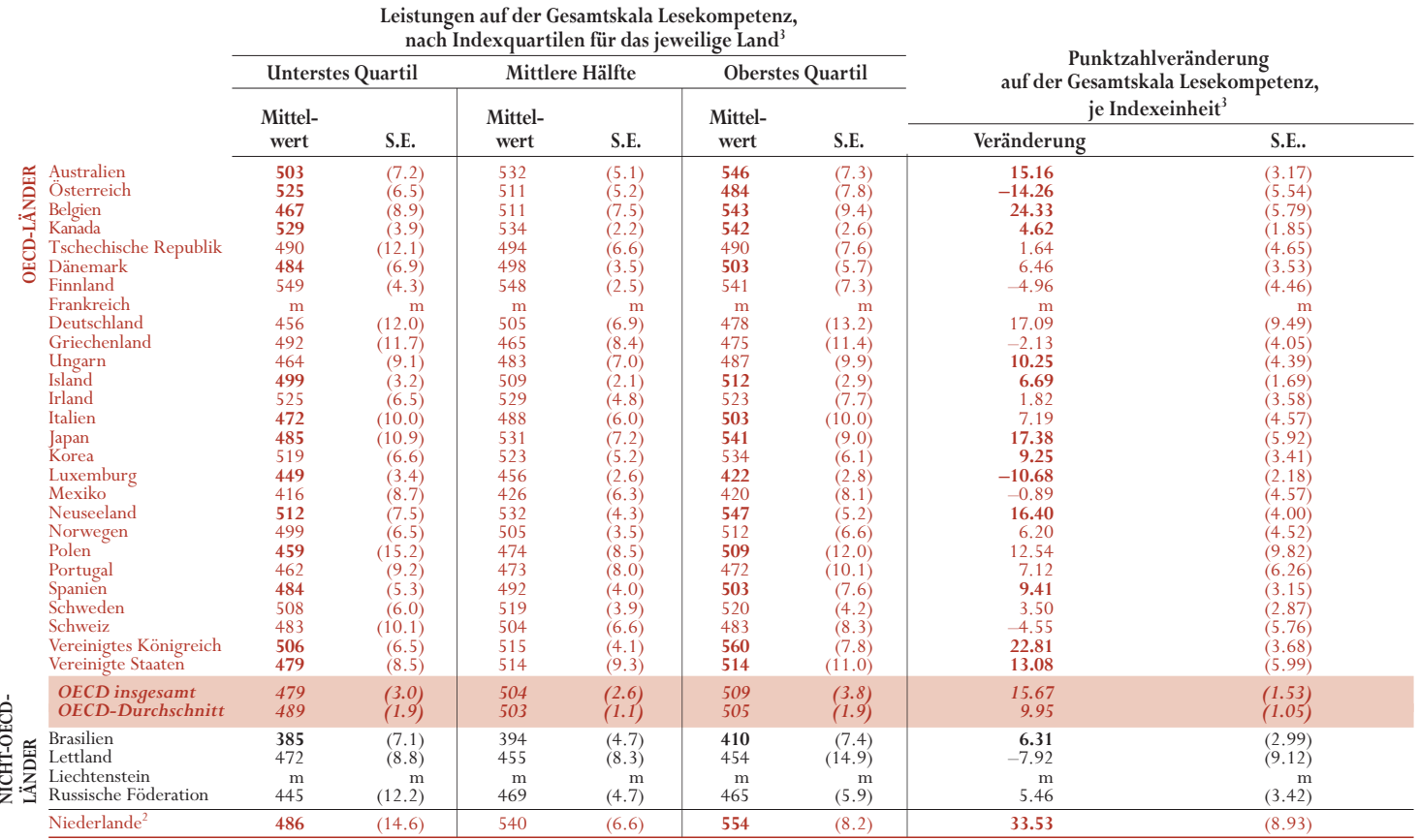

1. Zur Definition des Index siehe Anhang A1. Die Skala wurde so gepolt, dass positive und hohe Werte auf ein positives Schulklima in Bezug auf lehrkräftebezogene Faktoren hindeuten.

2. Die Beteiligungsquote ist zu niedrig, um die Vergleichbarkeit zu gewährleisten (vgl. Anhang A3).

3. Zur erklärten Varianz siehe Anhang A2. In Indexeinheiten gemessene Veränderungen sind, soweit fett gedruckt, statistisch signifikant. Wenn die untersten und obersten Quartile durch Fettdruck gekennzeichnet sind, weist dies auf eine statistisch signifikante Differenz zwischen diesen Quartilen hin. 
Tabelle 7.5

Index der Stimmung und Arbeitshaltung der Lehrkräfte aus der Sicht der Schulleitung und Leistungen auf der Gesamtskala Lesekompetenz, nach Indexquartilen für das jeweilige Land

Die Ergebnisse basieren auf Angaben der Schulleitungen und beziehen sich auf den jeweiligen zahlenmäßigen Anteil der 15-Jährigen in den Schulen

\begin{tabular}{|c|c|c|c|c|c|c|c|c|}
\hline & \multicolumn{8}{|c|}{ Index der Stimmung und Arbeitshaltung der Lehrkräfte $^{1}$} \\
\hline & \multicolumn{2}{|c|}{ Alle Schüler } & \multicolumn{2}{|c|}{ Unterstes Quartil } & \multicolumn{2}{|c|}{ Mittlere Hälfte } & \multicolumn{2}{|c|}{ Oberstes Quartil } \\
\hline & $\begin{array}{l}\text { Index- } \\
\text { mittel }\end{array}$ & S.E. & $\begin{array}{l}\text { Index- } \\
\text { mittel }\end{array}$ & S.E. & $\begin{array}{l}\text { Index- } \\
\text { mittel }\end{array}$ & S.E. & $\begin{array}{l}\text { Index- } \\
\text { mittel }\end{array}$ & S.E. \\
\hline $\begin{array}{l}\text { OECD insgesamt } \\
\text { OECD-Durchschnitt }\end{array}$ & $\begin{array}{r}-0.05 \\
0.00\end{array}$ & $\begin{array}{l}(0.03) \\
(0.01)\end{array}$ & $\begin{array}{l}-1.20 \\
-1.10\end{array}$ & $\begin{array}{l}(0.06) \\
(0.02)\end{array}$ & $\begin{array}{l}-0.09 \\
-0.04\end{array}$ & $\begin{array}{l}(0.02) \\
(0.01)\end{array}$ & $\begin{array}{l}1.22 \\
1.21\end{array}$ & $\begin{array}{l}(0.04) \\
(0.02)\end{array}$ \\
\hline $\begin{array}{l}\text { Brasilien } \\
\text { Lettland } \\
\text { Liechtenstein } \\
\text { Russische Föderation }\end{array}$ & $\begin{array}{r}-0.42 \\
-0.47 \\
\mathrm{~m} \\
-0.15\end{array}$ & $\begin{array}{r}(0.07) \\
(0.08) \\
\mathrm{m} \\
(0.07)\end{array}$ & $\begin{array}{r}-1.58 \\
-1.49 \\
\mathrm{~m} \\
-1.21\end{array}$ & $\begin{array}{r}(0.04) \\
(0.07) \\
\mathrm{m} \\
(0.06)\end{array}$ & $\begin{array}{r}-0.50 \\
-0.50 \\
\mathrm{~m} \\
-0.22 \\
\end{array}$ & $\begin{array}{r}(0.04) \\
(0.04) \\
\mathrm{m} \\
(0.04)\end{array}$ & $\begin{array}{r}0.95 \\
0.69 \\
\mathrm{~m} \\
1.08 \\
\end{array}$ & $\begin{array}{r}(0.09) \\
(0.12) \\
\mathrm{m} \\
(0.07) \\
\end{array}$ \\
\hline Niederlande $^{2}$ & -0.19 & $(0.09)$ & -0.93 & $(0.10)$ & -0.28 & $(0.06)$ & 0.75 & $(0.11)$ \\
\hline
\end{tabular}

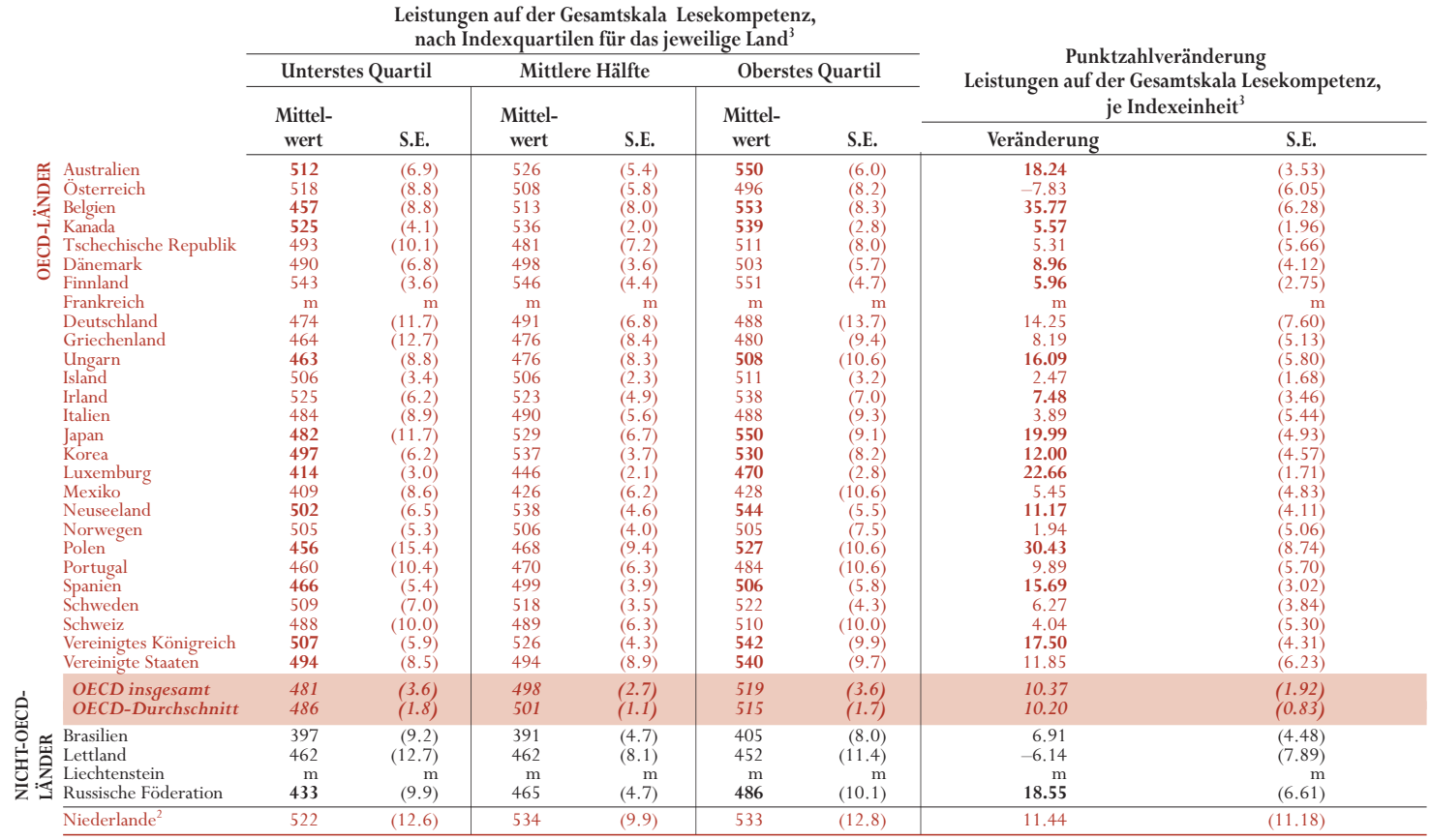

1. Zur Definition des Index siehe Anhang A1.

2. Die Beteiligungsquote ist zu niedrig, um die Vergleichbarkeit zu gewährleisten (vgl. Anhang A3).

3. Zur erklärten Varianz siehe Anhang A2. In Indexeinheiten gemessene Veränderungen sind, soweit fett gedruckt, statistisch signifikant. Wenn die untersten und obersten Quartile durch Fettdruck gekennzeichnet sind, weist dies auf eine statistisch signifikante Differenz zwischen diesen Quartilen hin. 
Tabelle 7.6

Index des wöchentlichen Zeitaufwands für Hausaufgaben in derTestsprache, in Mathematik und den Naturwissenschaften und Leistungen auf der Gesamtskala Lesekompetenz, nach Indexquartilen für das jeweilige Land

Die Ergebnisse basieren auf Schülerangaben

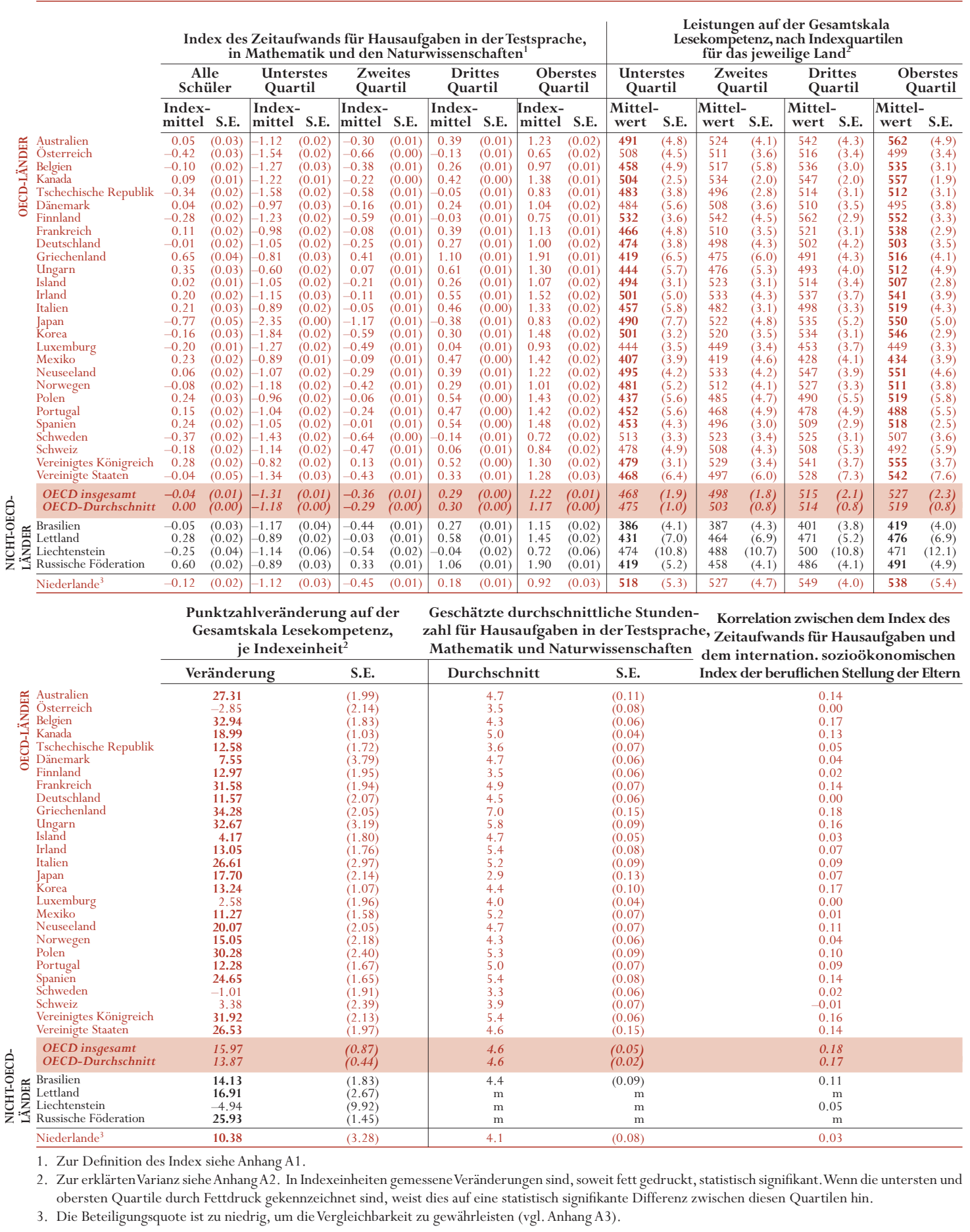

344 
Tabelle 7.7

Teilnahme der Schülerinnen und Schüler an Kursen ausserhalb des Unterrichts Die Ergebnisse basieren auf Schülerangaben

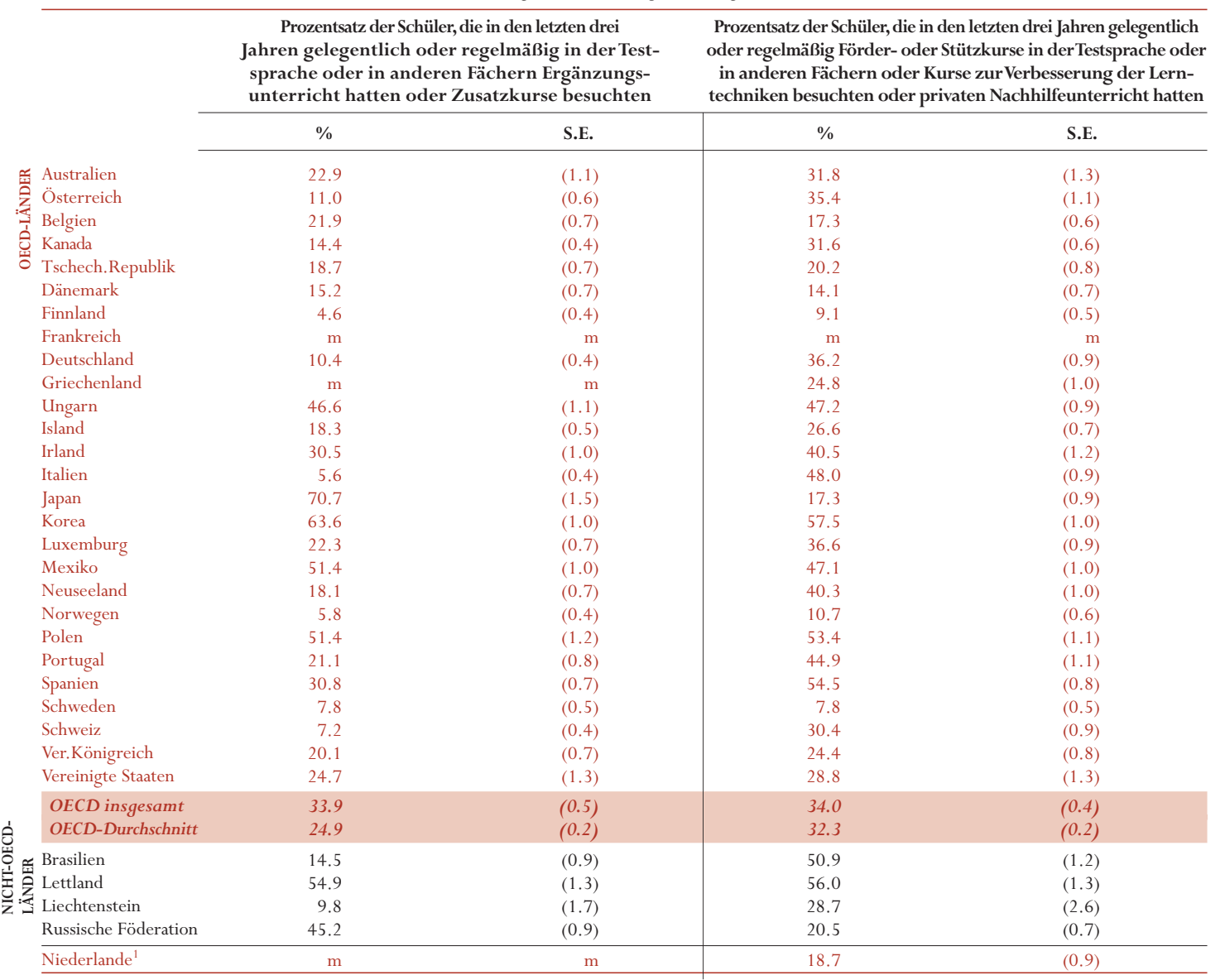

1. Die Beteiligungsquote ist zu niedrig, um die Vergleichbarkeit zu gewährleisten (vgl. Anhang A3). 
Tabelle 7.8

Index des Mangels an Lehrkräften und Leistungen auf der Gesamtskala Lesekompetenz, nach Indexquartilen für das jeweilige Land Die Ergebnisse basieren auf Angaben der Schulleitungen und beziehen sich auf den jeweiligen zahlenmäßigen Anteil der 15-Jährigen in den Schulen

\begin{tabular}{|c|c|c|c|c|c|c|c|c|}
\hline & \multicolumn{8}{|c|}{ Index des Mangels an Lehrkräften $^{1}$} \\
\hline & \multicolumn{2}{|c|}{ Alle Schüler } & \multicolumn{2}{|c|}{ Unterstes Quartil } & \multicolumn{2}{|c|}{ Mittlere Hälfte } & \multicolumn{2}{|c|}{ Oberstes Quartil } \\
\hline & $\begin{array}{c}\text { Index- } \\
\text { mittel }\end{array}$ & S.E. & $\begin{array}{c}\text { Index- } \\
\text { mittel }\end{array}$ & S.E. & $\begin{array}{c}\text { Index- } \\
\text { mittel }\end{array}$ & S.E. & $\begin{array}{c}\text { Index- } \\
\text { mittel }\end{array}$ & S.E. \\
\hline Australien & -0.18 & $(0.08)$ & -1.39 & $(0.04)$ & -0.16 & $(0.08)$ & $\operatorname{Max}$ & \\
\hline Österreich & 0.53 & $(0.05)$ & -0.43 & $(0.08)$ & 0.79 & $(0.04)$ & $\operatorname{Max}$ & \\
\hline Belgien & 0.25 & $(0.07)$ & -0.89 & $(0.08)$ & 0.47 & $(0.07)$ & $\operatorname{Max}$ & \\
\hline Kanada & -0.01 & $(0.04)$ & -1.41 & $(0.03)$ & 0.20 & $(0.04)$ & Max & \\
\hline Tschechische Republik & 0.51 & $(0.04)$ & -0.36 & $(0.06)$ & 0.71 & $(0.04)$ & $\operatorname{Max}$ & \\
\hline Dänemark & 0.31 & $(0.05)$ & -0.71 & $(0.04)$ & 0.50 & $(0.05)$ & $\operatorname{Max}$ & \\
\hline Finnland & 0.09 & $(0.06)$ & -0.88 & $(0.03)$ & 0.13 & $(0.04)$ & Max & \\
\hline Frankreich & $\mathrm{m}$ & $\mathrm{m}$ & $\mathrm{m}$ & $\mathrm{m}$ & $\mathrm{m}$ & $\mathrm{m}$ & $\mathrm{m}$ & $\mathrm{m}$ \\
\hline Deutschland & -0.23 & $(0.06)$ & -1.47 & $(0.04)$ & -0.23 & $(0.04)$ & $\operatorname{Max}$ & \\
\hline Griechenland & -0.73 & $(0.14)$ & -2.97 & $(0.15)$ & -0.52 & $(0.10)$ & $\operatorname{Max}$ & \\
\hline Ungarn & 0.29 & $(0.08)$ & -1.04 & $(0.12)$ & 0.61 & $(0.05)$ & $\operatorname{Max}$ & \\
\hline Island & -0.39 & $(0.00)$ & -1.59 & $(0.00)$ & -0.47 & $(0.00)$ & $\operatorname{Max}$ & \\
\hline Irland & -0.06 & $(0.08)$ & -1.35 & $(0.11)$ & 0.07 & $(0.07)$ & $\operatorname{Max}$ & \\
\hline Italien & -0.28 & $(0.09)$ & -1.53 & $(0.07)$ & -0.30 & $(0.08)$ & $\operatorname{Max}$ & \\
\hline Japan & -0.23 & $(0.07)$ & -1.51 & $(0.07)$ & -0.19 & $(0.09)$ & $\operatorname{Max}$ & \\
\hline Korea & 0.32 & $(0.06)$ & -0.90 & $(0.09)$ & 0.62 & $(0.06)$ & $\operatorname{Max}$ & \\
\hline Luxemburg & -0.10 & $(0.01)$ & -1.66 & $(0.00)$ & -0.14 & $(0.01)$ & c & \\
\hline Mexiko & -0.53 & $(0.09)$ & -1.88 & $(0.08)$ & -0.60 & $(0.05)$ & 0.94 & $(0.01)$ \\
\hline Neuseeland & -0.18 & $(0.07)$ & -1.42 & $(0.06)$ & -0.15 & $(0.07)$ & $\operatorname{Max}$ & \\
\hline Norwegen & -0.32 & $(0.07)$ & -1.42 & $(0.07)$ & -0.41 & $(0.05)$ & 0.92 & $(0.03)$ \\
\hline Polen & 0.30 & $(0.10)$ & -1.05 & $(0.20)$ & 0.64 & $(0.05)$ & $\operatorname{Max}$ & \\
\hline Portugal & 0.03 & $(0.08)$ & -0.97 & $(0.03)$ & 0.05 & $(0.10)$ & $\operatorname{Max}$ & \\
\hline Spanien & 0.52 & $(0.06)$ & -0.59 & $(0.11)$ & 0.85 & $(0.03)$ & $\operatorname{Max}$ & \\
\hline Schweden & -0.25 & $(0.07)$ & -1.54 & $(0.06)$ & -0.21 & $(0.06)$ & $\operatorname{Max}$ & \\
\hline Schweiz & 0.35 & $(0.06)$ & -0.78 & $(0.07)$ & 0.61 & $(0.05)$ & $\operatorname{Max}$ & \\
\hline Vereinigtes Königreich & -0.40 & $(0.07)$ & -1.71 & $(0.06)$ & -0.42 & $(0.06)$ & Max & \\
\hline Vereinigte Staaten & 0.20 & $(0.08)$ & -1.18 & $(0.09)$ & 0.48 & $(0.07)$ & $\operatorname{Max}$ & \\
\hline $\begin{array}{l}\text { OECD insgesamt } \\
\text { OECD-Durchschnitt }\end{array}$ & $\begin{array}{r}-0.01 \\
0.00\end{array}$ & $\begin{array}{l}(0.03) \\
(0.01)\end{array}$ & $\begin{array}{l}-1.32 \\
-1.24\end{array}$ & $\begin{array}{l}(0.03) \\
(0.03)\end{array}$ & $\begin{array}{l}0.14 \\
0.12\end{array}$ & $\begin{array}{l}(0.02) \\
(0.01)\end{array}$ & $\begin{array}{l}\text { Max } \\
\text { Max }\end{array}$ & \\
\hline \multirow{5}{*}{ Niederlande $^{2}$} & -0.07 & $(0.07)$ & -1.32 & $(0.06)$ & 0.03 & $(0.07)$ & Max & \\
\hline & -0.05 & $(0.10)$ & -1.18 & $(0.12)$ & 0.00 & $(0.04)$ & $\operatorname{Max}$ & \\
\hline & $\mathrm{m}$ & & $\mathrm{m}$ & $\mathrm{m}$ & $\mathrm{m}$ & $\mathrm{m}$ & $\mathrm{m}$ & \\
\hline & -0.75 & $(0.10)$ & -2.52 & $(0.10)$ & -0.70 & $(0.06)$ & 0.90 & $(0.03)$ \\
\hline & -0.37 & $(0.09)$ & -1.37 & $(0.06)$ & -0.41 & $(0.06)$ & 0.67 & $(0.08)$ \\
\hline
\end{tabular}

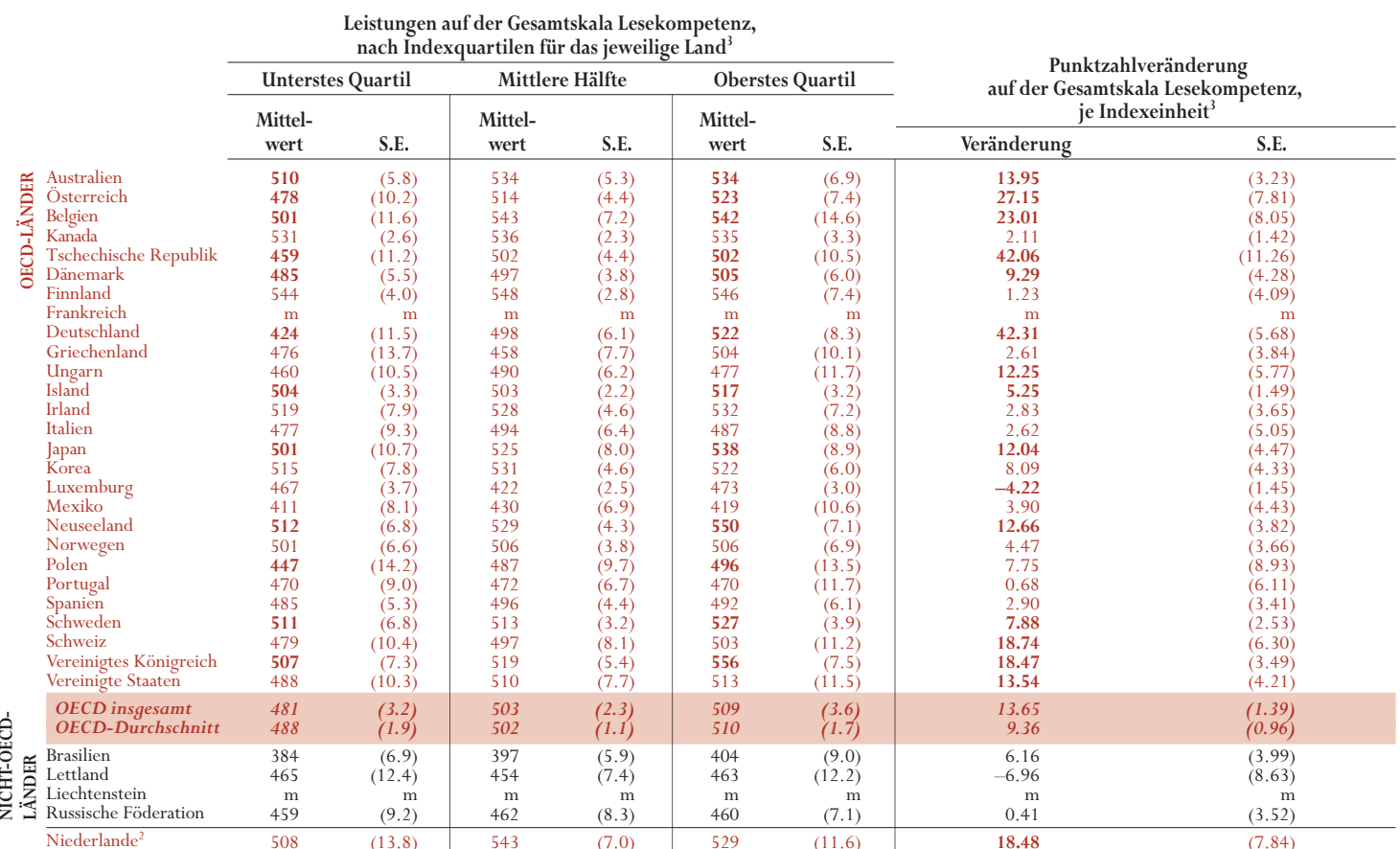

1. Zur Definition des Index siehe Anhang A1. Die Skala wurde umgepolt, so dass positive und hohe Werte bedeuten, dass Lehrermangel weniger als Problem eingeschätzt wird als im OECD-Durchschnitt. "Max" wird in Fällen verwendet, in denen sich mehr als $25 \%$ der Schülerinnen und Schüler in Schulen befinden, wo die Angaben der Schulleitungen dem höchsten Indexwert entsprechen, der 0.95 beträgt.

2. Die Beteiligungsquote ist zu niedrig, um die Vergleichbarkeit zu gewährleisten (vgl. Anhang A3)

3. Zur erklärten Varianz siehe Anhang A2. In Indexeinheiten gemessene Veränderungen sind, soweit fett gedruckt, statistisch signifikant. Wenn die untersten und obersten Quartile durch Fettdruck gekennzeichnet sind, weist dies auf eine statistisch signifikante Differenz zwischen diesen Quartilen hin. 
Tabelle 7.9

Index der Qualität der räumlichen Bedingungen der Schulen und Leistungen auf der Gesamtskala Lesekompetenz, nach Indexquartilen für das jeweilige Land

Die Ergebnisse basieren auf Angaben der Schulleitungen und beziehen sich auf den jeweiligen zahlenmäßigen Anteil der 15-Jährigen in den Schulen

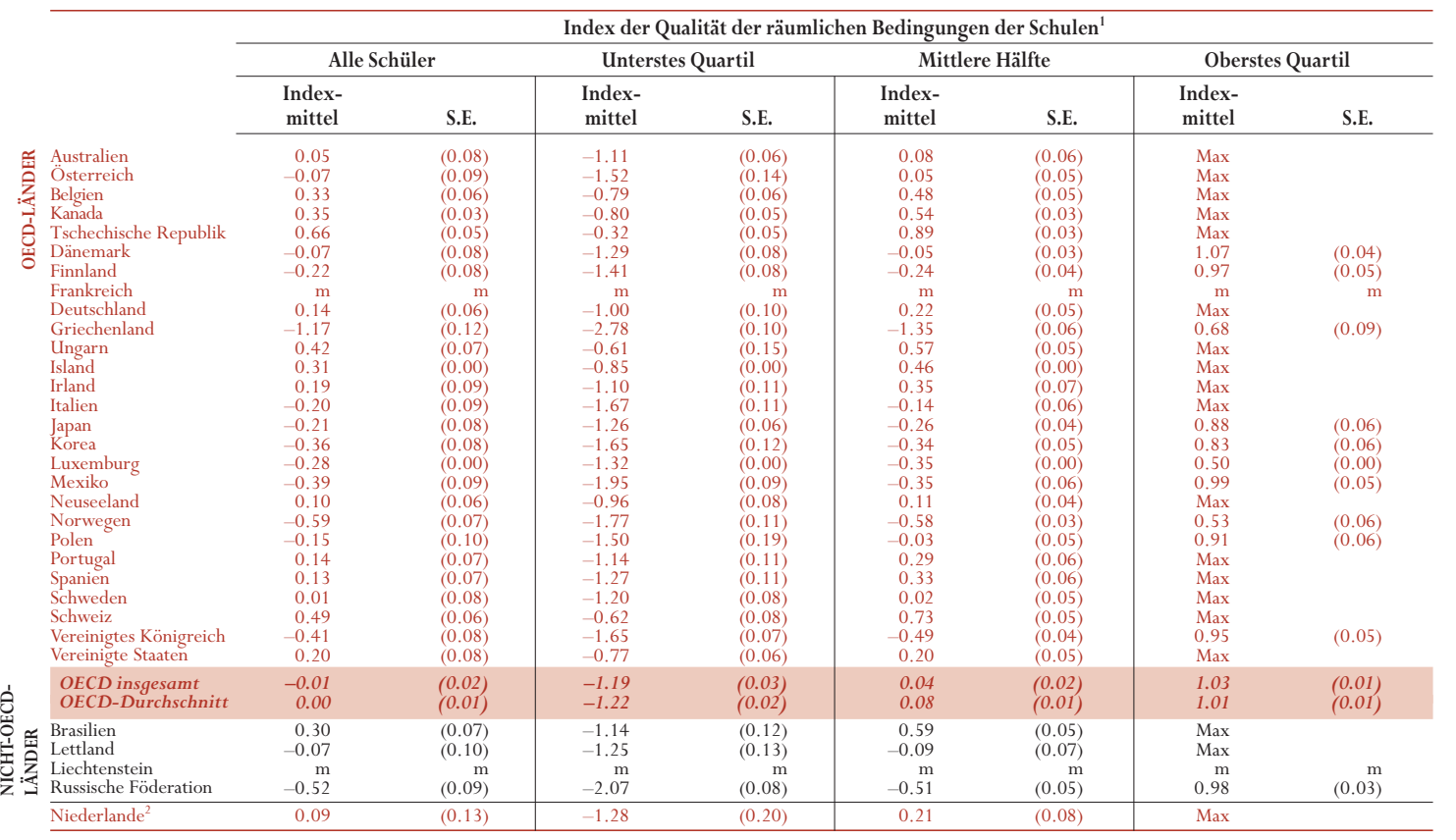

Leistungen auf der Gesamtskala Lesekompetenz,

nach Indexquartilen für das jeweilige Land ${ }^{3}$

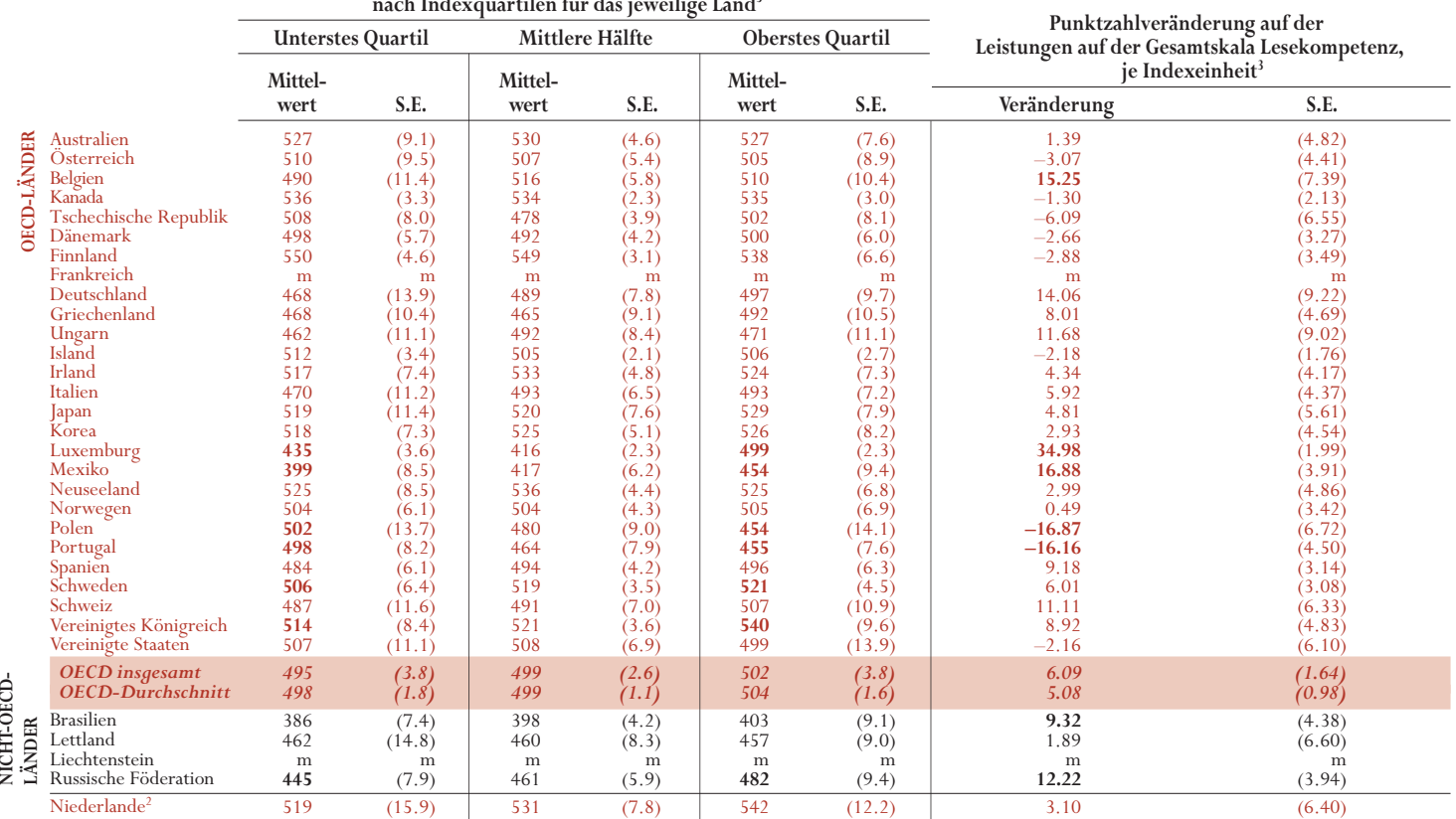

1. Zur Definition des Index siehe Anhang A1. Die Skala wurde umgepolt, so dass positive und hohe Werte bedeuten, dass die räumlichen Bedingungen der Schule weniger als ein Problem eingeschätzt werden als im OECD-Durchschnitt." Max" wird in Fällen verwendet, in denen sich mehr als $25 \%$ der Schülerinnen und Schüler in Schulen befinden, wo die Angaben der Schulleitungen dem höchsten Indexwert entsprechen, der 1.12 beträgt.

2. Die Beteiligungsquote ist zu niedrig, um die Vergleichbarkeit zu gewährleisten (vgl. Anhang A3)

3. Zur erklärten Varianz siehe Anhang A2. In Indexeinheiten gemessene Veränderungen sind, soweit fett gedruckt, statistisch signifikant. Wenn die untersten und obersten Quartile durch Fettdruck gekennzeichnet sind, weist dies auf eine statistisch signifikante Differenz zwischen diesen Quartilen hin. 
Tabelle 7.10

Index der Qualität der Ausstattung mit Lehr- und Sachmitteln und Leistungen auf der Gesamtskala Lesekompetenz, nach Indexquartilen für das jeweilige Land

Die Ergebnisse basieren auf Angaben der Schulleitungen und beziehen sich auf den jeweiligen zahlenmäßigen Anteil der 15-Jährigen in den Schulen

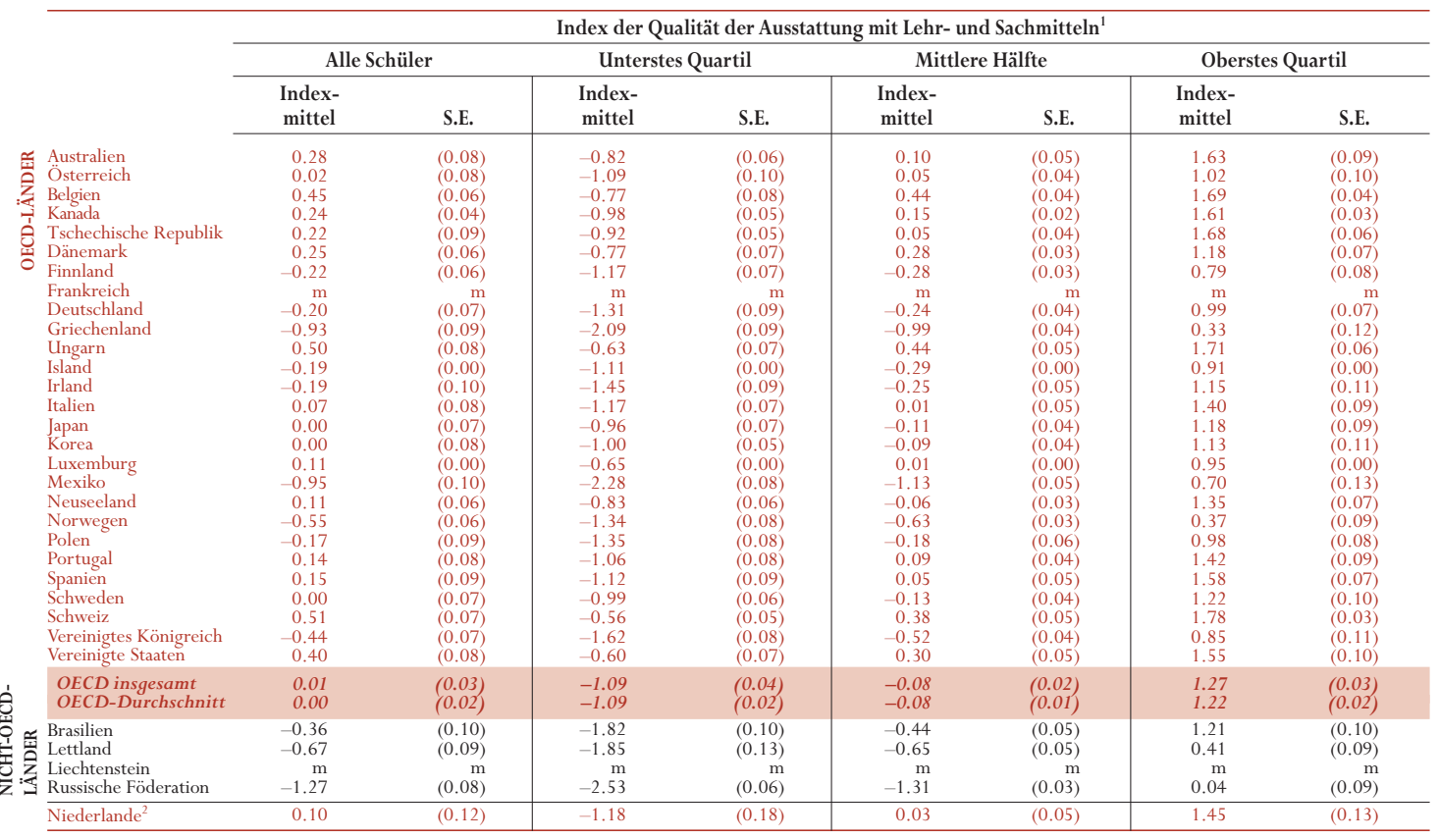

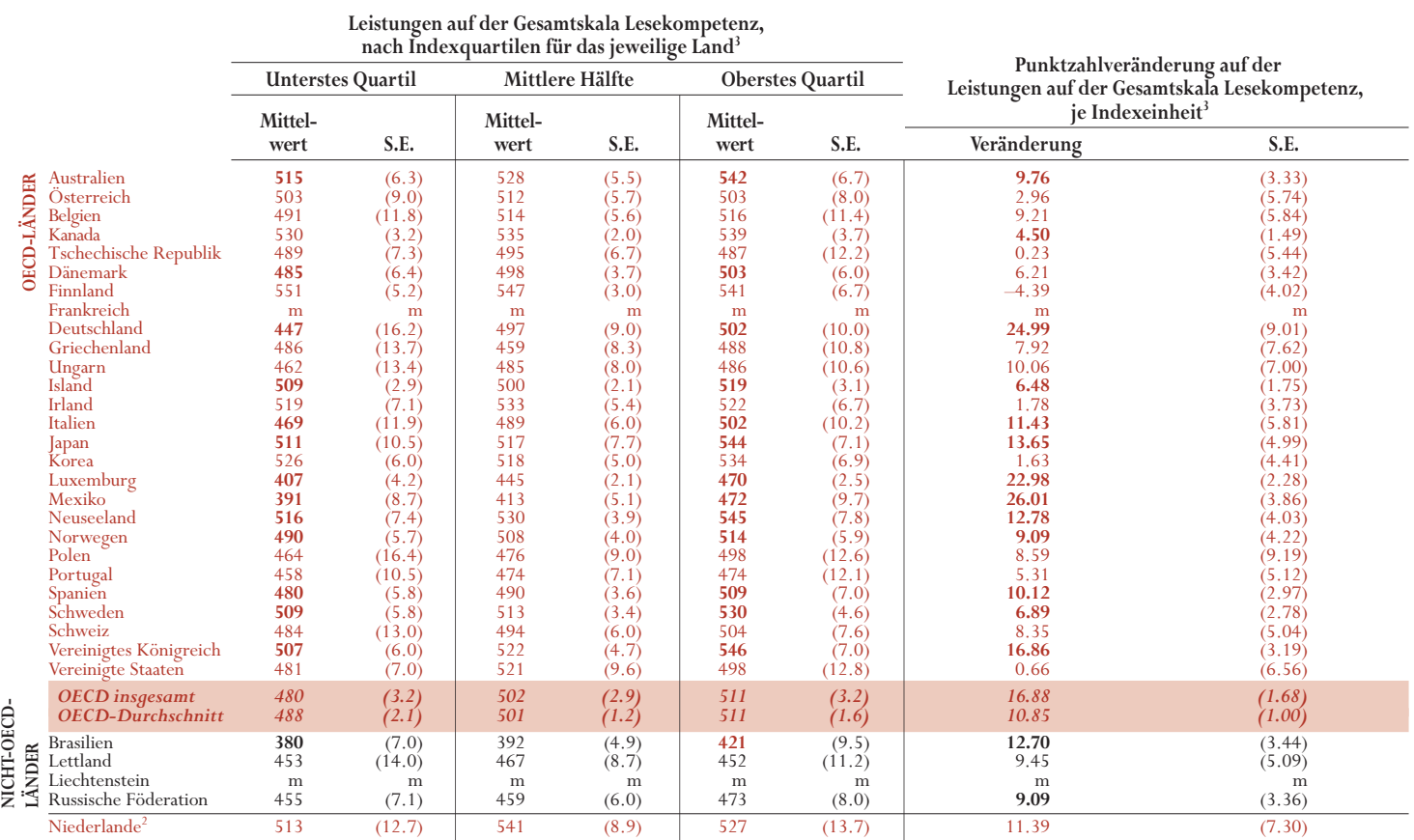

1. Zur Definition des Index siehe Anhang A1. Die Skala wurde umgepolt, so dass positive und hohe Werte bedeuten, dass die Ausstattung mit Lehrund Sachmitteln nicht als wichtiges Problem eingeschätzt wird.

. Die Beteiligungsquote ist zu niedrig, um die Vergleichbarkeit zu gewährleisten (vgl. Anhang A3).

3. Zur erklärten Varianz siehe Anhang A2. In Indexeinheiten gemessene Veränderungen sind, soweit fett gedruckt, statistisch signifikant. Wenn die untersten und obersten Quartile durch Fettdruck gekennzeichnet sind, weist dies auf eine statistisch signifikante Differenz zwischen diesen Quartilen hin. 
Tabelle 7.11

Prozentsatz der Schülerinnen und Schüler in Schulen, in denen die Schulen bei folgenden Aspekten der Schulpolitik und -verwaltung eine gewisse Verantwortung tragen

Die Ergebnisse basieren auf Angaben der Schulleitungen und beziehen sich auf den jeweiligen zahlenmäßigen Anteil der 15-Jährigen in den Schulen

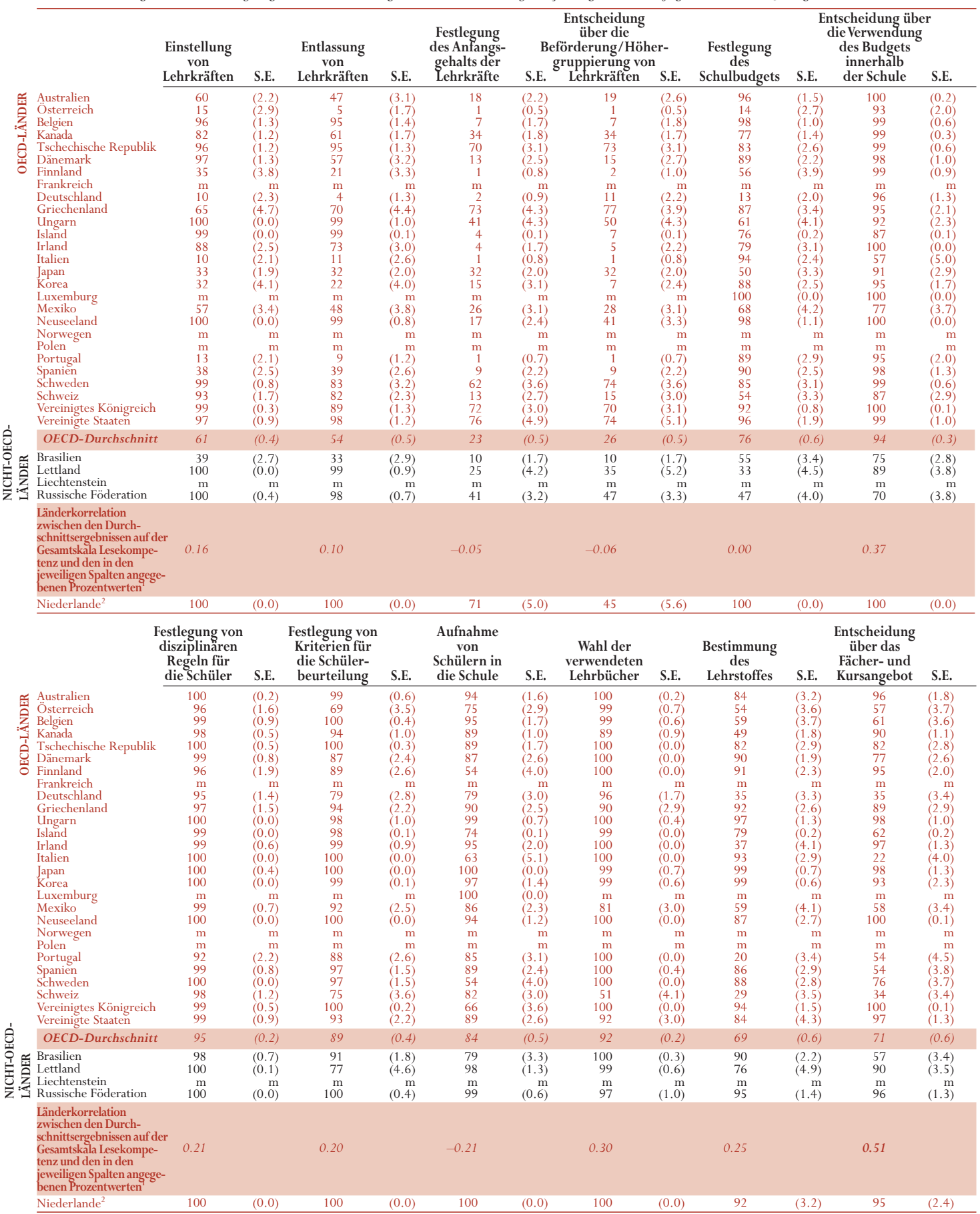

1. Durch Fettdruck gekennzeichnete Korrelationswerte sind statistisch signifikant.

2. Die Beteiligungsquote ist zu niedrig, um die Vergleichbarkeit zu gewährleisten (vgl. Anhang A3). 
Tabelle 7.12

Prozentsatz der Schülerinnen und Schüler in Schulen, in denen die Lehrkräfte bei folgenden Aspekten der Schulpolitik und -verwaltung die Hauptverantwortung tragen

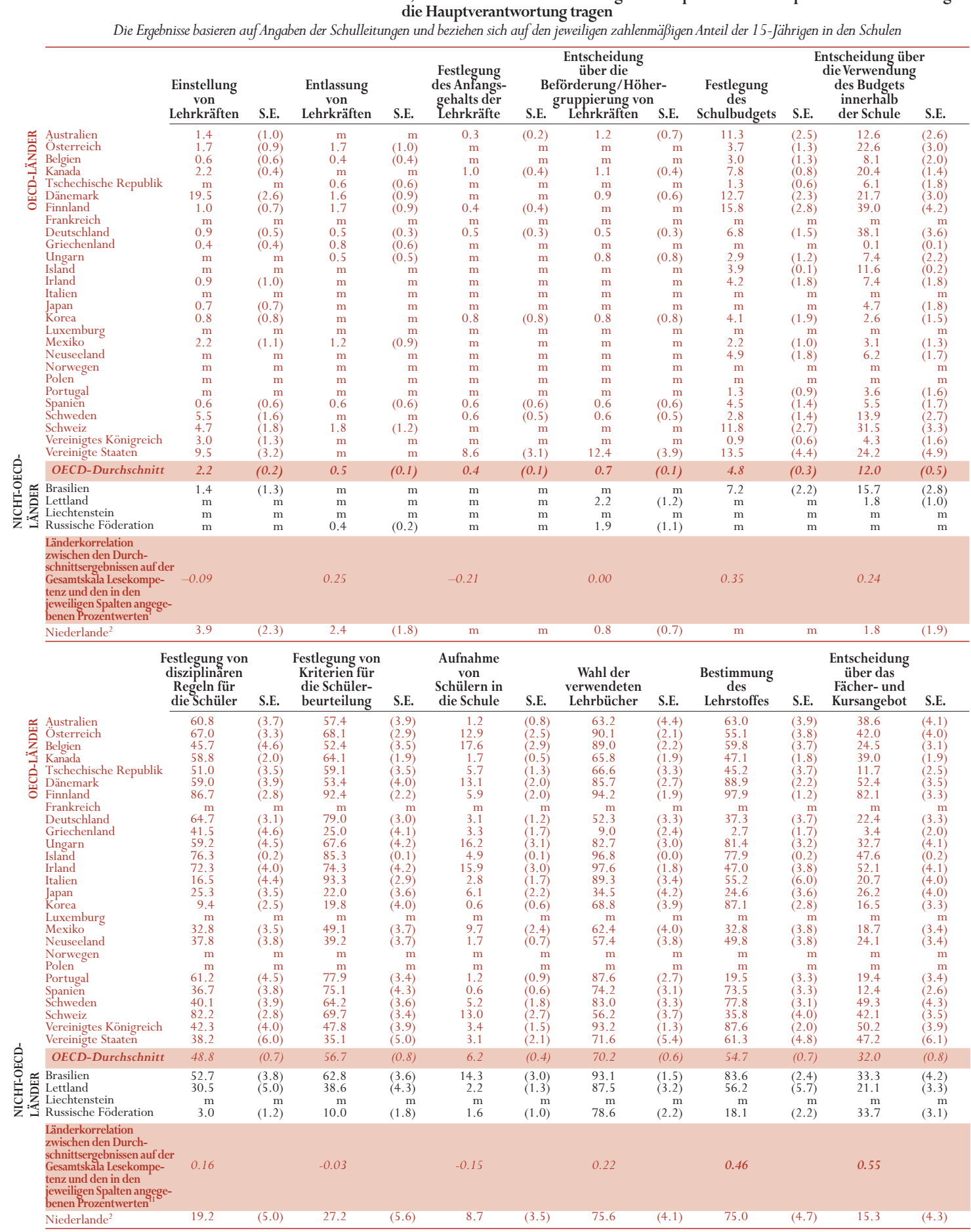

1. Durch Fettdruck gekennzeichnete Korrelationswerte sind statistisch signifikant.

2. Die Beteiligungsquote ist zu niedrig, um die Vergleichbarkeit zu gewährleisten (vgl. Anhang A3). 
Tabelle 7.13

Prozent der Schüler und Leistungen auf der Gesamtskala Lesekompetenz, nach Schultyp Die Ergebnisse basieren auf Angaben der Schulleitungen und beziehen sich auf den jeweiligen zahlenmäßigen Anteil der 15-jährigen in den Schulen

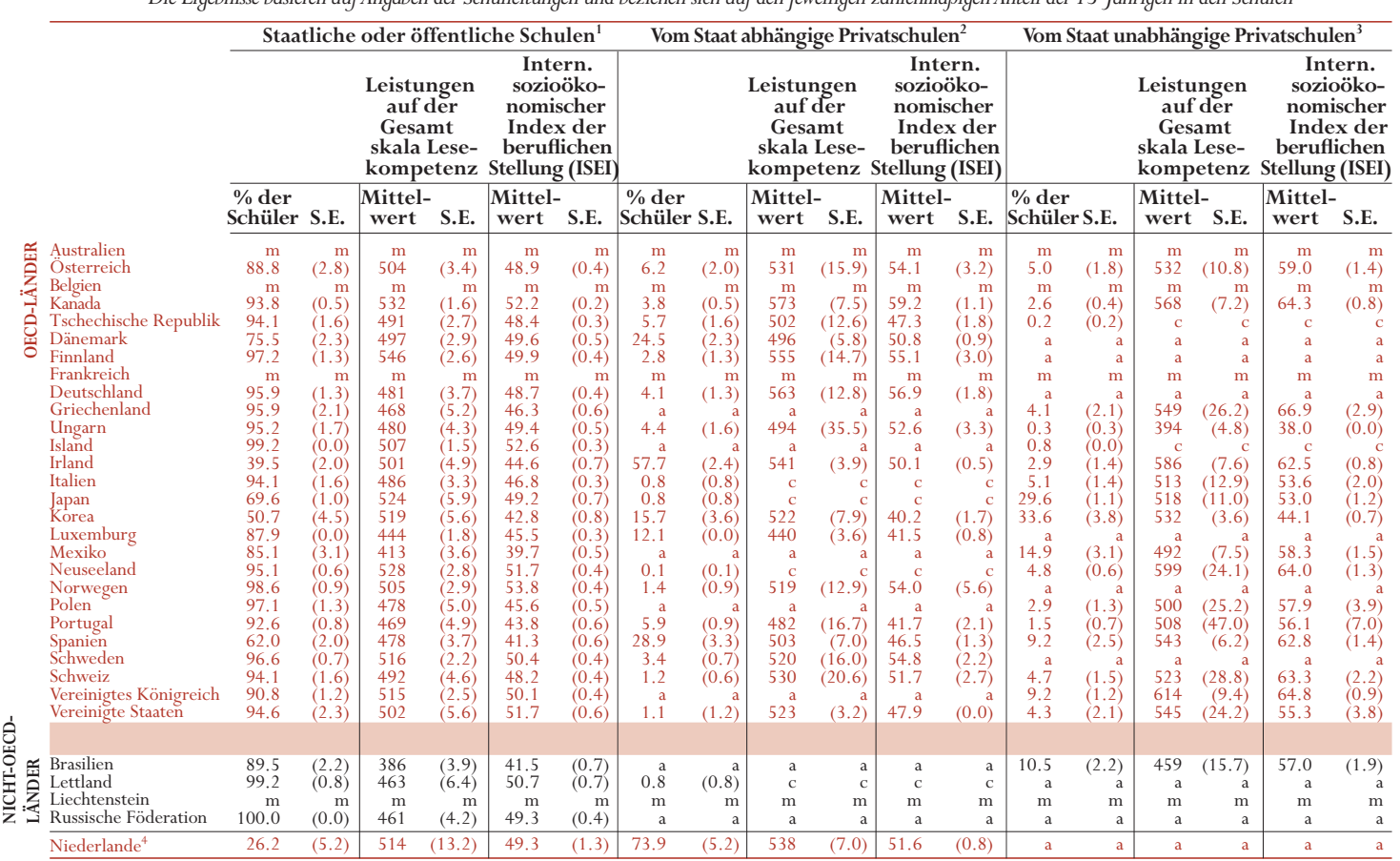

\begin{tabular}{|c|c|c|c|c|c|c|c|c|}
\hline & \multicolumn{4}{|c|}{$\begin{array}{l}\text { Leistungsunterschiede auf der } \\
\text { Gesamtskala Lesekompetenz }\end{array}$} & \multicolumn{4}{|c|}{$\begin{array}{c}\text { Unterschiede bei dem } \\
\text { sozioökonomischen Index der } \\
\text { beruflichen Stellung der Eltern (ISEI) }\end{array}$} \\
\hline & \multicolumn{2}{|c|}{$\begin{array}{l}\text { Vom Staat abhängige } \\
\text { Privatschulen und } \\
\text { offfentliche Schulen } \\
\end{array}$} & \multicolumn{2}{|c|}{$\begin{array}{l}\text { Vom Staat unabhängige } \\
\text { Privatschulen und } \\
\text { offentliche Schulen }\end{array}$} & \multicolumn{2}{|c|}{$\begin{array}{l}\text { Vom Staat abhängige } \\
\text { Privatschulen und } \\
\text { offentliche Schulen }\end{array}$} & \multicolumn{2}{|c|}{$\begin{array}{l}\text { Vom Staat unabhängige } \\
\text { Privatschulen und } \\
\text { offentliche Schulen }\end{array}$} \\
\hline & Differenz & S.E. & Differenz & S.E. & Differenz & S.E. & Differenz & S.E. \\
\hline $\begin{array}{l}\text { Australien } \\
\text { Osterreich }\end{array}$ & $\begin{array}{l}\mathrm{m} \\
26\end{array}$ & $(16.5)$ & $\underset{28}{m}$ & $\underset{(11.8)}{m}$ & $\begin{array}{r}\mathrm{m} \\
5.2\end{array}$ & $\underset{(3.3)}{\mathrm{m}}$ & 10.1 & $(1.5)$ \\
\hline $\begin{array}{l}\text { Belgien } \\
\text { Kanada }\end{array}$ & $\mathrm{m}$ & $\mathrm{m}$ & $\mathrm{m}$ & $\mathrm{m}$ & $\mathrm{m}$ & $\mathrm{m}$ & $\mathrm{m}$ & $\mathrm{m}$ \\
\hline $\begin{array}{l}\text { Ranada } \\
\text { Tschechische Republik }\end{array}$ & $\begin{array}{l}41 \\
11 \\
11\end{array}$ & (13.5) & $\begin{array}{r}36 \\
\mathrm{c}\end{array}$ & $(7.3)$ & $\begin{array}{c}7.0 \\
-1.1\end{array}$ & $\begin{array}{l}(1.1) \\
(1.9)\end{array}$ & $\begin{array}{l}12.1 \\
\mathrm{c}\end{array}$ & $\underset{\mathrm{C}}{(0.8)}$ \\
\hline $\begin{array}{l}\text { Dänemark } \\
\text { Finnland }\end{array}$ & $\begin{array}{r}-2 \\
9\end{array}$ & $\begin{array}{l}(6.7) \\
(15.0)\end{array}$ & a & a & $\begin{array}{l}1.2 \\
5.3\end{array}$ & $\begin{array}{l}(1.1) \\
(3.1)\end{array}$ & $\mathrm{a}$ & $\begin{array}{l}\mathrm{a} \\
\mathrm{a}\end{array}$ \\
\hline $\begin{array}{l}\text { Frankreich } \\
\text { Doutschland }\end{array}$ & $\mathrm{m}$ & $\mathrm{m}$ & $\mathrm{m}$ & $\frac{\mathrm{a}}{\mathrm{m}}$ & m & $\mathrm{m}$ & $\begin{array}{c}\mathrm{a} \\
\mathrm{m}\end{array}$ & $\begin{array}{c}\mathrm{a} \\
\mathrm{m}\end{array}$ \\
\hline $\begin{array}{l}\text { Deutschhland } \\
\text { Griechenland }\end{array}$ & $\begin{array}{r}83 \\
\mathrm{a}\end{array}$ & $(13.8)$ & 81 & $(26.7)^{a}$ & $\begin{array}{r}8.2 \\
\mathrm{a}\end{array}$ & $\begin{array}{r}(1.9) \\
\mathrm{a}\end{array}$ & $\begin{array}{r}a \mathrm{a} \\
20.6\end{array}$ & $(3.0)^{\mathrm{a}}$ \\
\hline $\begin{array}{l}\text { Ungarn } \\
\text { Island }\end{array}$ & 14 & $(36,2)$ & -85 & $(6.3)$ & 3.2 & (3.4) & -11.4 & $(0.5)$ \\
\hline $\begin{array}{l}\text { Irland } \\
\text { Irland }\end{array}$ & 41 & $(6 .)^{\mathrm{a}}$ & 86 & $(9.0)^{c}$ & 5.5 & $(0.9)^{\mathrm{a}}$ & 18.0 .0 & $(1.1)^{\mathrm{c}}$ \\
\hline $\begin{array}{l}\text { Ittalien } \\
\text { Japan }\end{array}$ & $\begin{array}{l}\mathrm{c} \\
\mathrm{c}\end{array}$ & & $\begin{array}{l}27 \\
-6\end{array}$ & $\begin{array}{l}(13.3) \\
(12.5)\end{array}$ & ${ }_{\mathrm{c}}^{\mathrm{c}}$ & $\begin{array}{l}\mathrm{c} \\
\mathrm{c}\end{array}$ & $\begin{array}{l}6.8 \\
3.8\end{array}$ & $\begin{array}{l}(2.1) \\
(1.4)\end{array}$ \\
\hline Korea & 3 & $(11.4)$ & 13 & (7.1) & -2.6 & $(2.1)^{\mathrm{C}}$ & $\begin{array}{l}.0 \\
1.3\end{array}$ & (1.1) \\
\hline $\begin{array}{l}\text { Luxemburg } \\
\text { Mexiko }\end{array}$ & -4 & $(3.8)$ & 79 & $(8.9)^{\mathrm{a}}$ & -4.0 & $(0.9)$ & 18.5 & $(1.7)^{\mathrm{a}}$ \\
\hline $\begin{array}{l}\text { Neuseeland } \\
\text { Norwegen }\end{array}$ & ${ }_{14}^{c}$ & (13, c & 71 & $(24.4)$ & c & & 12.3 & (1.4) \\
\hline $\begin{array}{l}\text { Norwegen } \\
\text { Polen }\end{array}$ & $\begin{array}{c}14 \\
\mathrm{a}\end{array}$ & $(13.3)$ & 22 & $(26.2)^{a}$ & $\begin{array}{l}0.2 \\
a\end{array}$ & $(5.7)$ & $12 .{ }^{\mathrm{a}}$ & $(4.0)^{\mathrm{a}}$ \\
\hline $\begin{array}{l}\text { Portugal } \\
\text { Sanien }\end{array}$ & $\begin{array}{l}13 \\
25\end{array}$ & $\begin{array}{c}(17.5) \\
(79)\end{array}$ & $\begin{array}{l}39 \\
65\end{array}$ & $\left(\begin{array}{l}47.3) \\
(71)\end{array}\right.$ & -2.1 & $\begin{array}{l}(2.2) \\
(14)\end{array}$ & $\begin{array}{l}12.3 \\
21.5\end{array}$ & $(7.1)$ \\
\hline Schweden & 3 & (16.3) & a & a & 4.3 & $(2.3)$ & a & a \\
\hline $\begin{array}{l}\text { Schweiz } \\
\text { Vereinigtes Königreich }\end{array}$ & $\begin{array}{c}38 \\
\mathrm{a}\end{array}$ & (22.6) & $\begin{array}{l}31 \\
98\end{array}$ & $\begin{array}{l}(29.4) \\
(98)\end{array}$ & 3.5 & $(2.8)$ & 15.1 & (2.3) \\
\hline $\begin{array}{l}\text { Vereinigte Staaten } \\
\text { Stite }\end{array}$ & 22 & $(6.1)^{a}$ & 43 & $(26.2)$ & -3.9 & $(0.6)^{a}$ & $\begin{array}{l}14.5 \\
3.5\end{array}$ & $(4.0)$ \\
\hline \multicolumn{9}{|l|}{ : } \\
\hline $\begin{array}{l}\text { 울 魚 Brasilien } \\
\text { Lettland }\end{array}$ & a & ${ }_{c}^{a}$ & $\begin{array}{l}74 \\
\mathrm{a}\end{array}$ & $(16.4)$ & $\mathrm{a}$ & $\mathrm{a}$ & 15.4 & $(2.1)$ \\
\hline $\begin{array}{l}\text { Liechtenstein } \\
\text { Russische Föderation }\end{array}$ & $\mathrm{m}$ & $\mathrm{m}$ & $\mathrm{m}$ & $\mathrm{m}$ & $\mathrm{m}$ & $\mathrm{m}$ & $\mathrm{m}$ & $\mathrm{m}$ \\
\hline Russische Föderation & $\mathrm{a}$ & $\mathrm{a}$ & $\mathrm{a}$ & a & $\mathrm{a}$ & $\mathrm{a}$ & a & a \\
\hline Niederlande $^{4}$ & 24 & $\begin{array}{l}(18.5) \\
\end{array}$ & $\mathrm{a}$ & $a$ & 2.3 & (1.7) & $a$ & a \\
\hline $\begin{array}{l}\text { 1. Staatlich oder öffentlich } \\
\text { oder inem Direktorium } \\
\text { 2. Privat, vom Statat abhära } \\
\text { 3. Privat, vom Staat unabh } \\
\text { 4. Die Beteiligungsquote } \\
\text { 5. Positive Differenzen bed } \\
\text { 6. Positive Differenzen be } \\
\text { signifikant. }\end{array}$ & 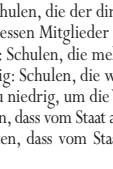 & $\begin{array}{l}\text { trolle oder } \\
\text { n einer off } \\
\text { ihrer Kert } \\
\text { 50\% ihrer } \\
\text { arkit zu g } \\
\text { rivatschule } \\
\text { gige Privat }\end{array}$ & $\begin{array}{l}\text { ung folgender Eir } \\
\text { n Stelle errannt } \\
\text { rung (Mitel zur } \\
\text { antierung (Mittel } \\
\text { sten (vgl. Anhang } \\
\text { abschneiden, nega } \\
\text { abeser abschneid }\end{array}$ & $\begin{array}{l}\text { en unterste } \\
\text { tlich gewäl } \\
\text { ung der gru } \\
\text { zierung de }\end{array}$ & $\begin{array}{l}\text { einer Behörde o } \\
\text { len. } \\
\text { nden Bildungslei } \\
\text { legenden schulis } \\
\text { söffentliche Sch } \\
\text { seuten, dass offer }\end{array}$ & $\begin{array}{l}\text { Amt fuir das } \\
\text { r Institution } \\
\text { ungen) von } \\
\text { abschneiden } \\
\text { ulcen besser }\end{array}$ & $\begin{array}{l}\text { gswesen, b) einer } \\
\text { taatlichen Stellen } \\
\text { hen Stellen erhalt } \\
\text { edruckte Werte sin } \\
\text { eiden. Fett gedru }\end{array}$ & $\begin{array}{l}\text { signifikant. } \\
\text { sind statistisch }\end{array}$ \\
\hline
\end{tabular}


Tabelle 8.1

Zusammenhang zwischen Schülerleistungen und sozioökonomischem Hintergrund Geschätzte Höhe, Steigung und Stärke der Gradienten zwischen Schülerleistungen auf der Gesamtskala Lesekompetenz und dem PISA-Index des wirtschaftlichen, sozialen und kulturellen Status (ESCS)

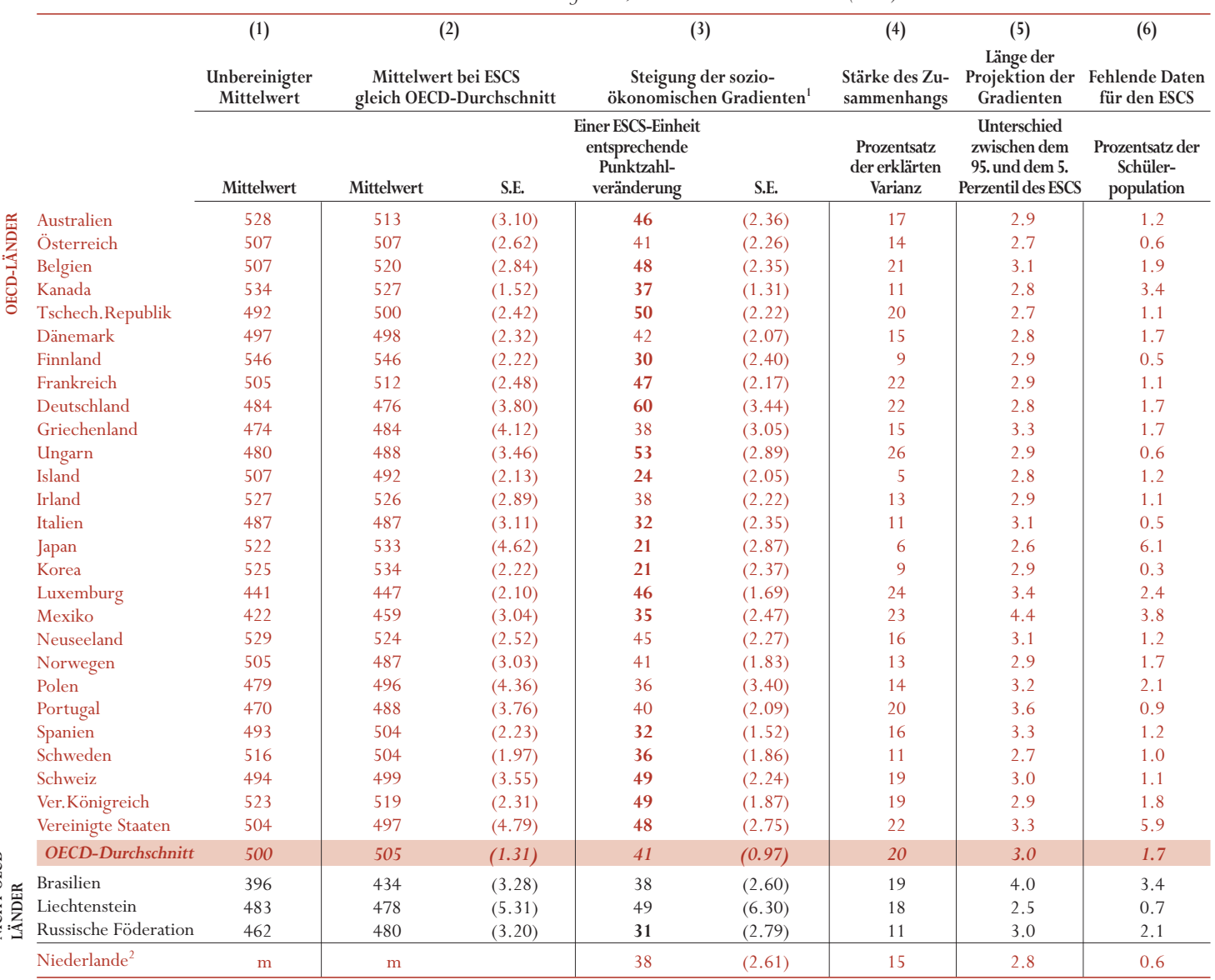

1. Fett gedruckte Steigungsgrade weisen einen statistisch signifikanten Unterschied zum OECD-Durchschnitt aus.

2. Die Beteiligungsquote ist zu niedrig, um die Vergleichbarkeit zu gewährleisten (vgl. Anhang A3). 
Tabelle 8.2

Effekte ausgewählter familiärer und demographischer Faktoren auf die Schülerleistungen auf der Gesamtskala Lesekompetenz

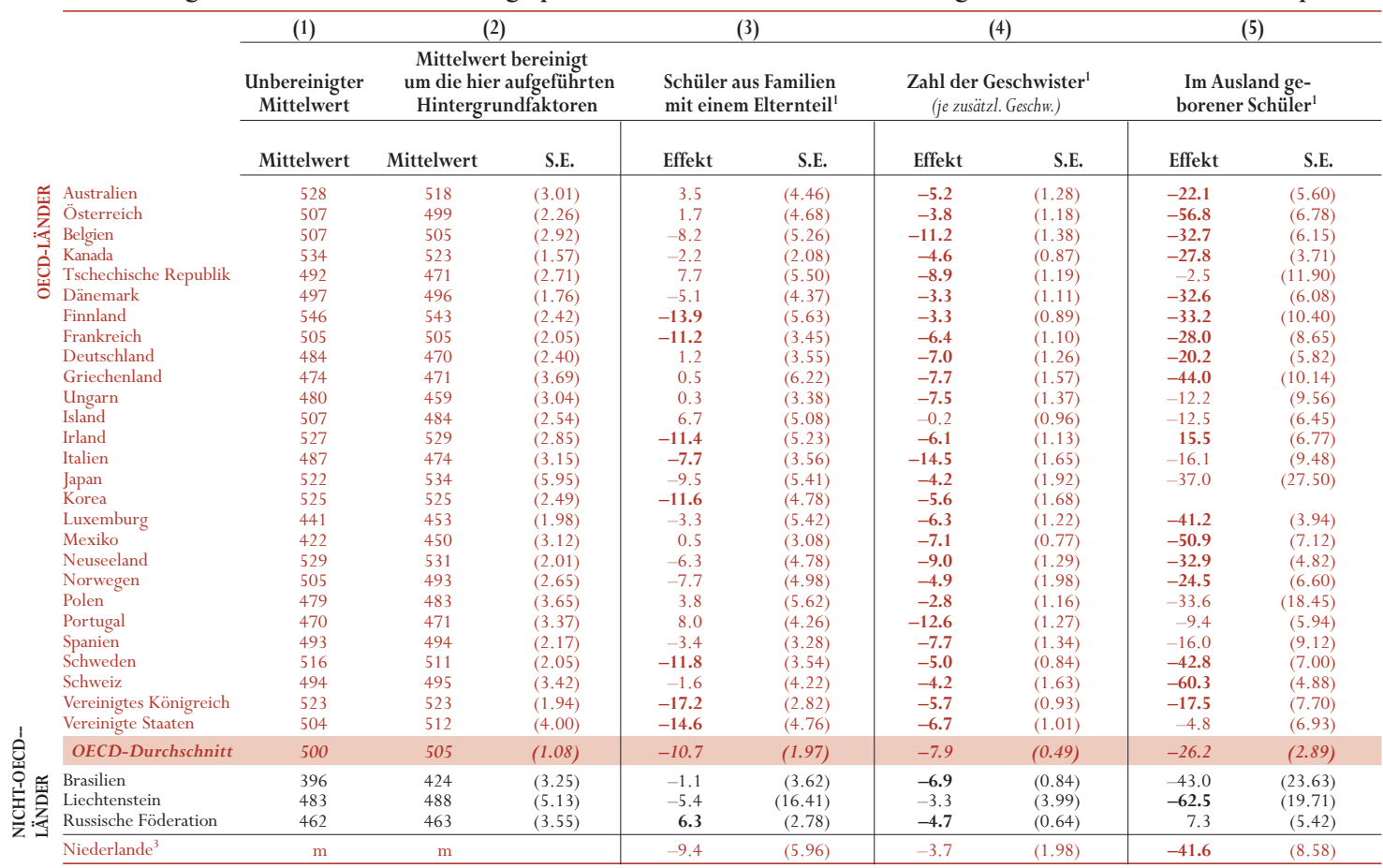

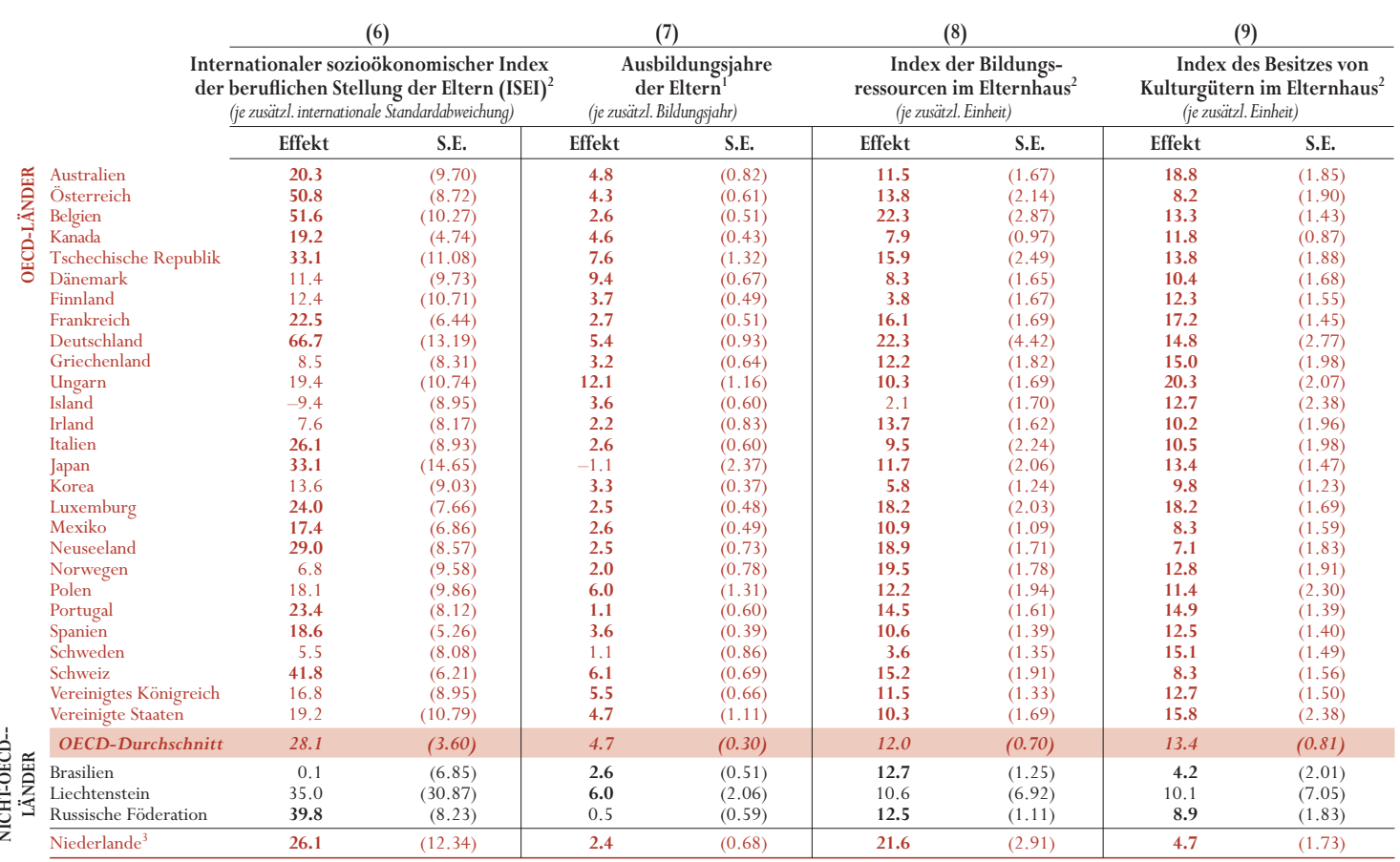

Fett gedruckte Effekte sind statistisch signifikant

1. Zur Definition der Maße siehe Anhang A1.

3. Die Beteiligungsquote ist zu niedrig, um die Vergleichbarkeit zu gewährleisten (vgl. Anhang A3). 
ANHANG B1

Tabelle 8.3

Durch den soziö̈konomischen Hintergrund erklärte Varianz der Leistungen auf der Gesamtskala Lesekompetenz

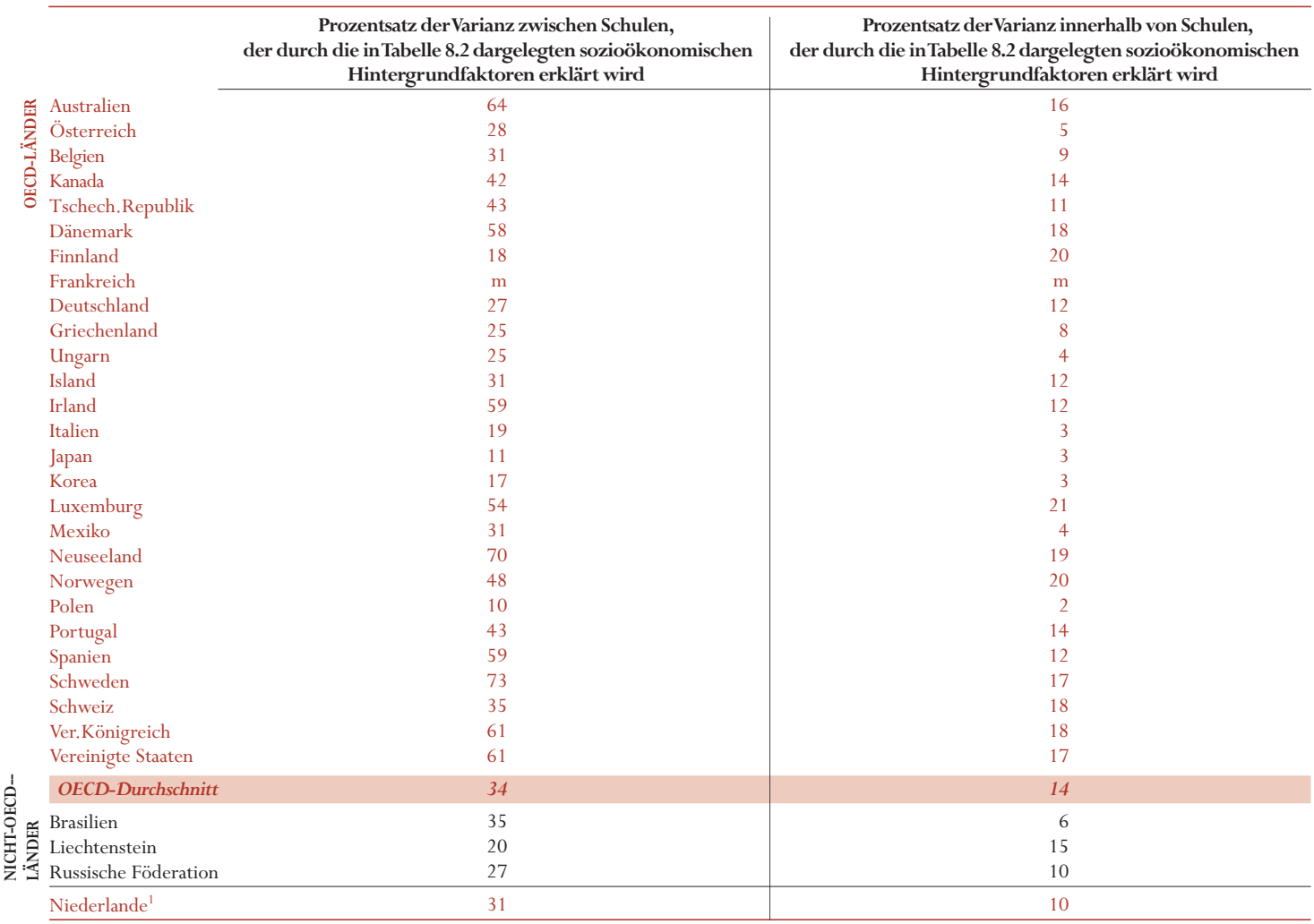

1. Die Beteiligungsquote ist zu niedrig, um die Vergleichbarkeit zu gewährleisten (vgl. Anhang A3). 
Tabelle 8.4

Effekte des soziö̈konomischen Hintergrunds von Schülern und von Schulen auf die Schülerleistungen auf der Gesamtskala Lesekompetenz

Effekt einer Erhöhung um eine halbe Standardabweichung auf Schülerebene des Index des wirtschaftichen, sozialen und kulturellen Status

\begin{tabular}{|c|c|c|c|}
\hline \multirow[b]{2}{*}{ Australien } & $\begin{array}{c}\text { Abstand zwischen den Quartilen } \\
\text { des Indexmittels des } \\
\text { wirtschaftlichen, sozialen und } \\
\text { kulturellen Status der Schulen }{ }^{1}\end{array}$ & $\begin{array}{l}\text { Effekt des wirtschaftlichen, } \\
\text { sozialen und kulturellen } \\
\text { Status der Schüler } \\
\text { auf deren Leistung } \\
\end{array}$ & $\begin{array}{l}\text { Effekt des wirtschaftlichen, } \\
\text { sozialen und kulturellen } \\
\text { Status der Schulen } \\
\text { auf die Schülerleistungen }{ }^{1}\end{array}$ \\
\hline & 0.73 & 17 & 21 \\
\hline Österreich & 0.83 & 4 & 59 \\
\hline Belgien & 0.97 & 7 & 56 \\
\hline Kanada & 0.60 & 14 & 22 \\
\hline Finnland & 0.44 & 13 & 8 \\
\hline Frankreich & $\mathrm{m}$ & $\mathrm{m}$ & $\mathrm{m}$ \\
\hline Deutschland & 0.63 & 8 & 66 \\
\hline Griechenland & 0.75 & 7 & 39 \\
\hline Ungarn & 0.86 & 4 & 47 \\
\hline Island & 0.50 & 11 & 5 \\
\hline Luxemburg & 0.96 & 12 & 40 \\
\hline Mexiko & 1.20 & 3 & 22 \\
\hline Neuseeland & 0.64 & 16 & 22 \\
\hline Norwegen & 0.57 & 17 & 12 \\
\hline Polen & 0.92 & 2 & 49 \\
\hline Portugal & 0.66 & 11 & 29 \\
\hline Spanien & 0.77 & 10 & 16 \\
\hline Schweden & 0.50 & 14 & 16 \\
\hline Schweiz & 0.50 & 12 & 32 \\
\hline Ver.Königreich & 0.93 & 15 & 29 \\
\hline Vereinigte Staaten & 0.61 & 13 & 28 \\
\hline OECD-Durchschnitt & 0.72 & 10 & 32 \\
\hline
\end{tabular}


Tabelle 8.5

Effekte schul- und schülerbezogener Faktoren auf die Leistungen auf der Gesamtskala Lesekompetenz sowie den Skalen für mathematische und für naturwissenschaftliche Grundbildung für alle OECD-Länder zusammen

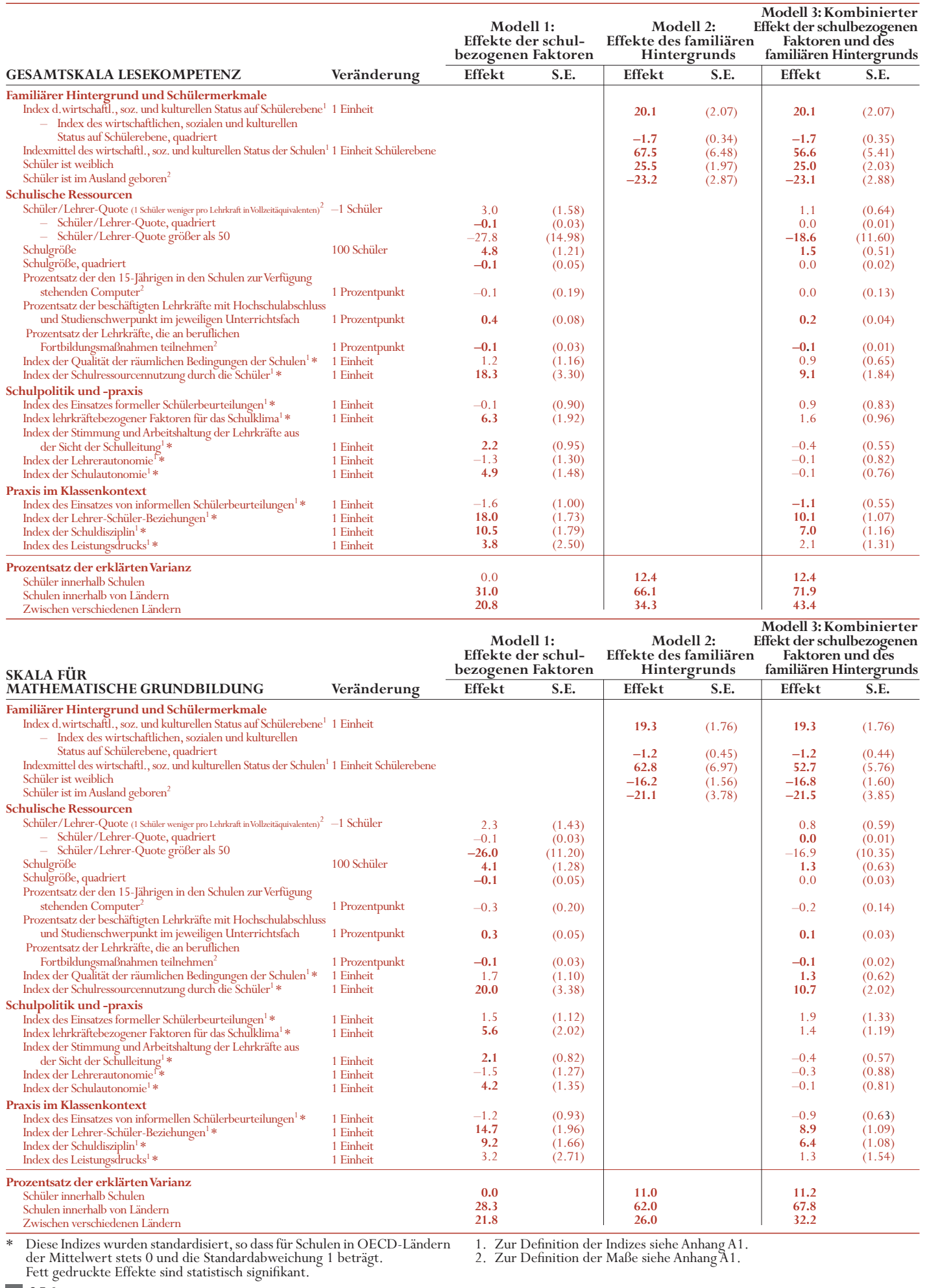

356 
Tabelle 8.5 (Fortsetzung)

Effekte schul- und schülerbezogener Faktoren auf die Leistungen auf der Gesamtskala Lesekompetenz sowie den Skalen für mathematische und für naturwissenschaftliche Grundbildung für alle OECD-Länder zusammen

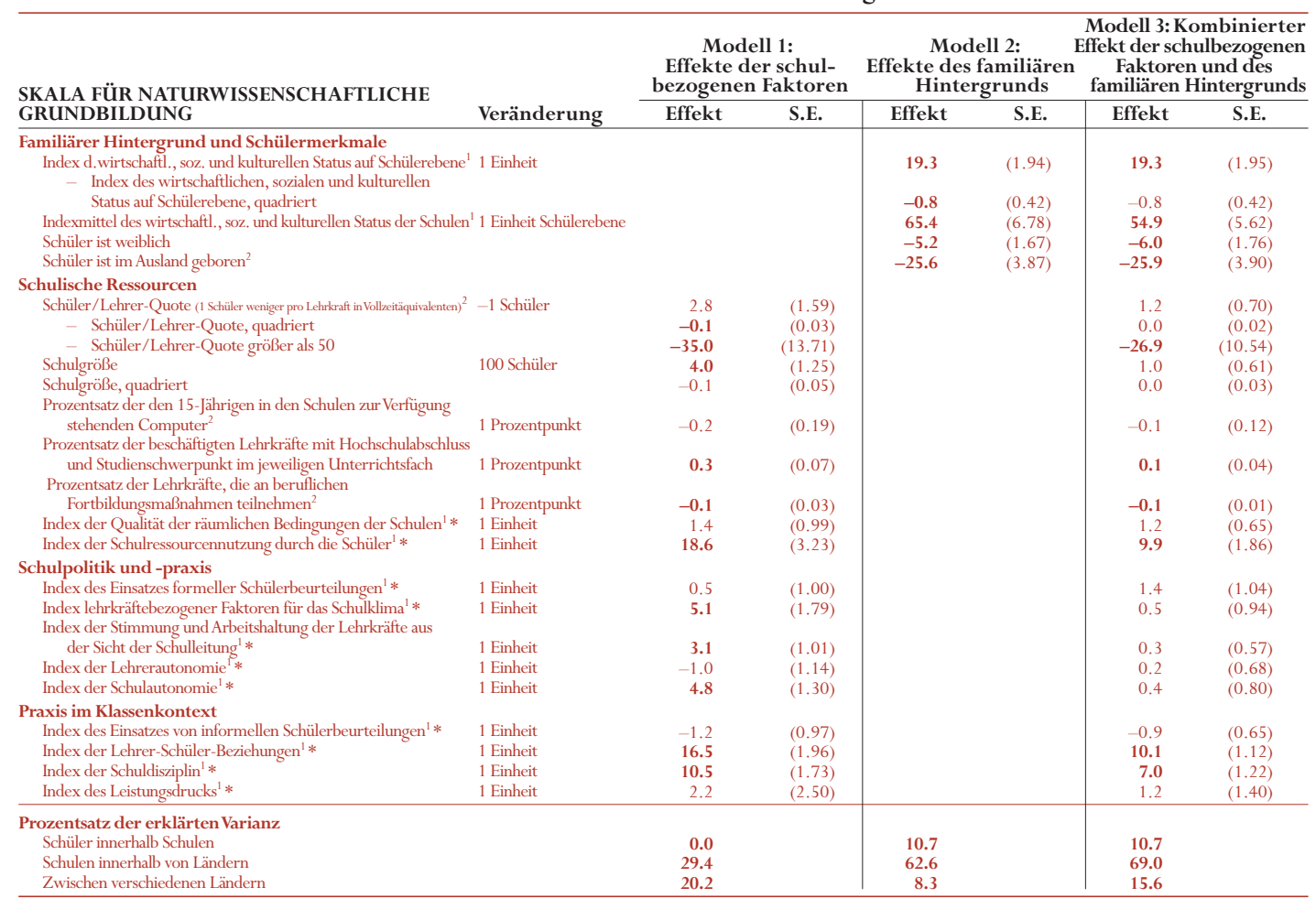

* Diese Indizes wurden standardisiert, so dass für Schulen in OECD-Ländern der Mittelwert stets 0 und die Standardabweichung 1 beträgt.

Fett gedruckte Effekte sind statistisch signifikant

1. Zur Definition der Indizes siehe Anhang A1. 
Tabelle $8.5 a$

Effekte schüler- und schulbezogener Faktoren auf die Leistungen auf der Gesamtskala Lesekompetenz

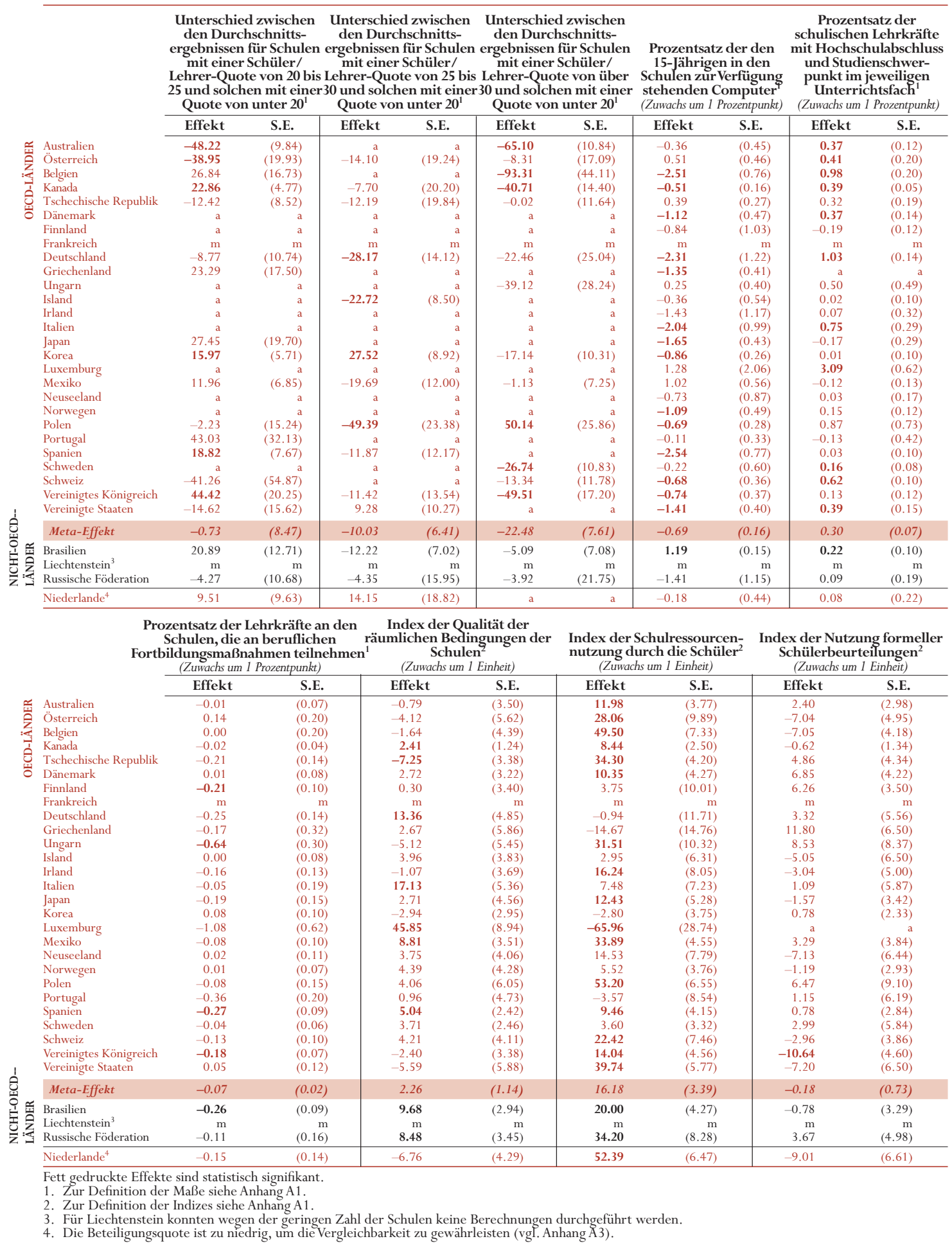


Tabelle $8.5 a$ (Fortsetzung)

Effekte schüler- und schulbezogener Faktoren auf die Leistungen auf der Gesamtskala Lesekompetenz

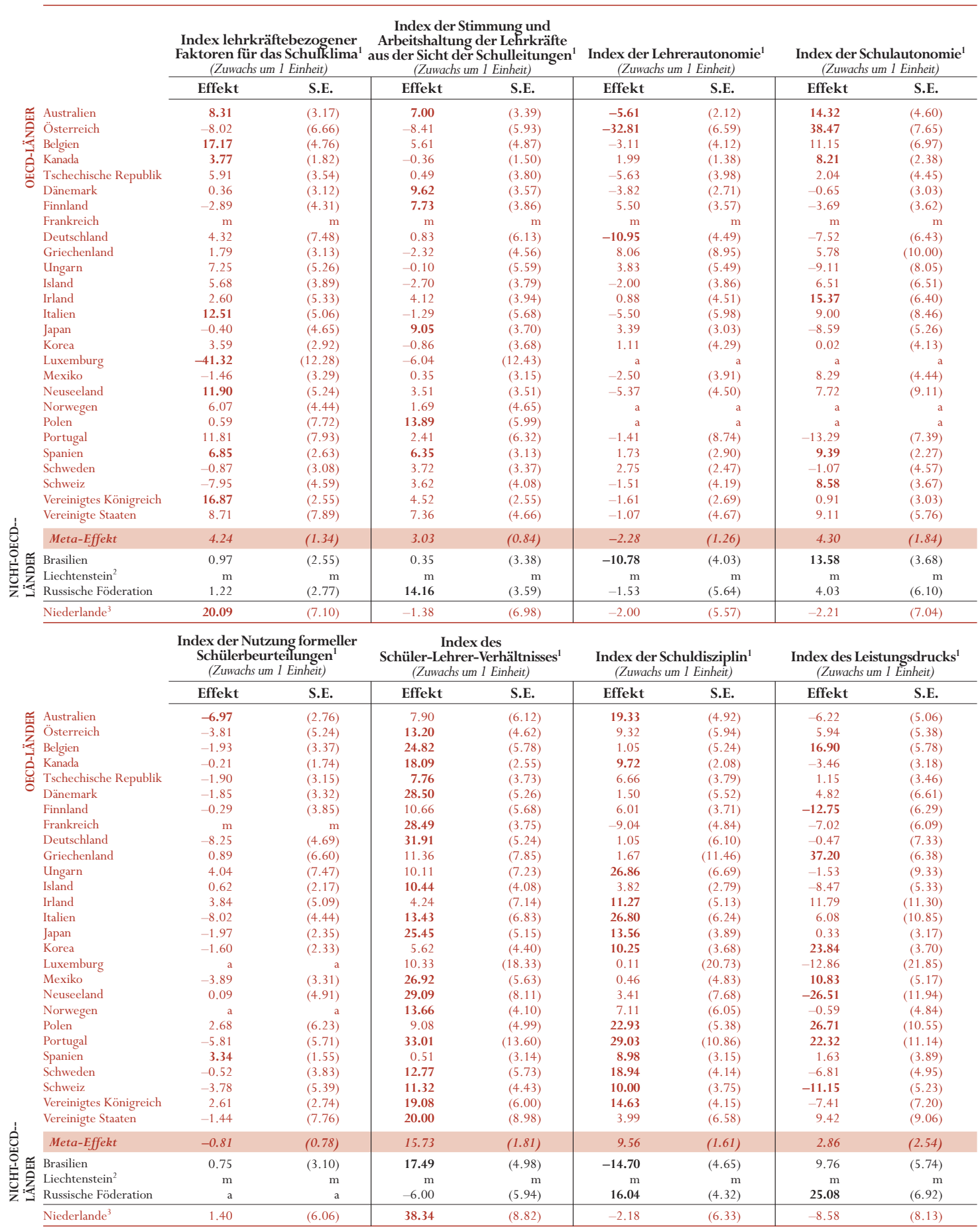

Fett gedruckte Effekte sind statistisch signifikant.

1. Zur Definition der Indizes siehe Anhang A1.

3. Die Beteiligungsquote ist zu niedrig, um die Vergleichbarkeit zu gewährleisten (vgl. Anhang A3). 
Tabelle 8.6

Ländermittel der Indizes ausgewählter schulischer Faktoren und Korrelation mit dem durchschnittlichen wirtschaftlichen, sozialen und kulturellen Status der Schulen (ESCS)

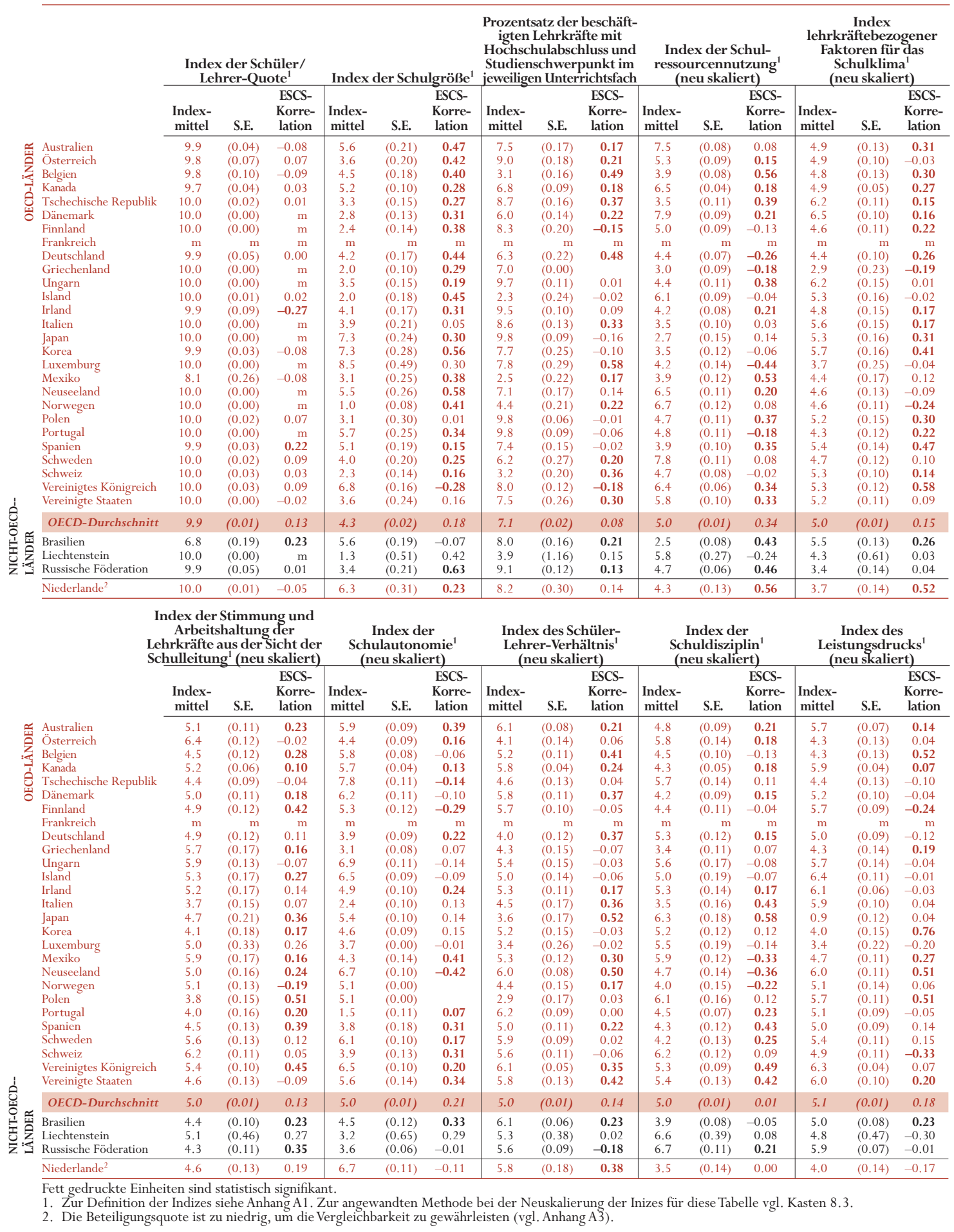




\section{Anhang B2: Leistungsunterschiede zwischen den flämischen und französischen Gemeinschaften in Belgien sowie den Sprachgemeinschaften in der Schweiz}

Für Belgien und die Schweiz konnten die PISA-Ergebnisse dank der Stichprobenkonzeption für die einzelnen subnationalen Einheiten getrennt dargelegt werden. Sie sind in Tabelle B2.1 wiedergegeben.

Tablle B2.1

Leistungsunterschiede zwischen den flämischen und französischen Gemeinschaften Belgiens sowie Sprachgemeinschaften der Schweiz

\begin{tabular}{|c|c|c|c|c|c|c|c|c|c|c|c|c|c|c|c|c|}
\hline \multirow{4}{*}{ Cris } & \multirow{2}{*}{\multicolumn{4}{|c|}{$\begin{array}{l}\text { Standardab- } \\
\text { weichung }\end{array}$}} & \multicolumn{10}{|c|}{ Perzentile } & \multirow{2}{*}{\multicolumn{2}{|c|}{95.}} \\
\hline & & & & & \multicolumn{2}{|c|}{5.} & \multicolumn{2}{|c|}{10.} & \multicolumn{2}{|c|}{25.} & \multicolumn{2}{|c|}{75.} & \multicolumn{2}{|c|}{90.} & & \\
\hline & $\begin{array}{c}\begin{array}{c}\text { Punkt- } \\
\text { zahl }\end{array} \\
\end{array}$ & S.E. & S.D. & S.E. & $\begin{array}{l}\text { Punkt- } \\
\text { zahl }\end{array}$ & S.E. & $\begin{array}{c}\text { Punkt- } \\
\text { zahl }\end{array}$ & S.E. & $\begin{array}{c}\text { Punkt- } \\
\text { zahl }\end{array}$ & S.E. & $\begin{array}{c}\text { Punkt- } \\
\text { zahl }\end{array}$ & S.E. & $\begin{array}{c}\text { Punkt- } \\
\text { zahl }\end{array}$ & S.E. & $\begin{array}{c}\text { Punkt- } \\
\text { zahl }\end{array}$ & S.E. \\
\hline & & & & & & & & & & & & & & & & \\
\hline Belgien (frz.) & 476 & $(7.2)$ & 111 & (3.6) & 283 & $(12.3)$ & 321 & $(11.8)$ & 395 & (10.4) & 561 & (5.6) & 614 & $(4.6)$ & 642 & $(5.8)$ \\
\hline Belgien (fläm.) & 532 & $(4.3)$ & 96 & (3.6) & 348 & $(15.8)$ & 396 & $(9.5)$ & 476 & (7.5) & 601 & $(3.1)$ & 644 & $(3.0)$ & 668 & $(3.2)$ \\
\hline Schweiz (deutsch) & 489 & $(5.2)$ & 105 & $(2,2)$ & 308 & $(6.8)$ & 346 & $(6,2)$ & 417 & (7.1) & 565 & (5.6) & 622 & (6.6) & 653 & $(6.2)$ \\
\hline Schweiz (frz.) & 512 & $(6.0)$ & 89 & (3.8) & 353 & $(10.2)$ & 391 & $(11.3)$ & 452 & $(8.0)$ & 576 & $(8.2)$ & 622 & (8.3) & 647 & $(7.4)$ \\
\hline Schweiz (ital.) & 498 & $(16.4)$ & 83 & (8.7) & 351 & $(16.9)$ & 389 & $(16.0)$ & 446 & $(19.6)$ & 552 & $(24.4)$ & 600 & $(28.6)$ & 630 & $(36.0)$ \\
\hline \multicolumn{17}{|c|}{ Subskala Informationen ermitteln } \\
\hline Belgien (frz.) & 476 & (7.9) & 124 & $(3.7)$ & 260 & (13.9) & 305 & $(12.4)$ & 388 & $(11.1)$ & 572 & $(5.7)$ & 630 & $(6.0)$ & 660 & $(5.5)$ \\
\hline Belgien (fläm.) & 545 & $(4.7)$ & 107 & $(3.8)$ & 340 & $(14.3)$ & 397 & (11.6) & 483 & $(7.4)$ & 621 & (3.2) & 671 & (3.3) & 698 & $(3.2)$ \\
\hline Schweiz (deutsch) & 492 & $(5.4)$ & 116 & (2.4) & 287 & $(7.6)$ & 335 & $(6.9)$ & 415 & $(6.8)$ & 575 & $(5.4)$ & 635 & $(5.8)$ & 668 & (7.1) \\
\hline Schweiz (frz.) & 518 & $(6.4)$ & 101 & (3.9) & 337 & $(12.4)$ & 381 & $(11.3)$ & 452 & $(9.4)$ & 589 & $(6.9)$ & 641 & $(10.0)$ & 670 & $(9.1)$ \\
\hline Schweiz (ital.) & 500 & $(17.4)$ & 103 & $(9,3)$ & 321 & $(30.2)$ & 371 & $(21.7)$ & 437 & $(12.2)$ & 567 & $(25.8)$ & 629 & $(32.4)$ & 660 & (32.9) \\
\hline \multicolumn{17}{|c|}{ Subskala textbezogenes Interpretieren } \\
\hline Belgien (frz.) & 482 & $(5.8)$ & 107 & (2.6) & 300 & $(8.0)$ & 337 & $(9.0)$ & 403 & $(8.9)$ & 563 & (5.5) & 617 & $(4.8)$ & 646 & $(5.3)$ \\
\hline Belgien (fläm.) & 536 & $(4.3)$ & 97 & (3.6) & 356 & $(13.5)$ & 400 & $(10.0)$ & 476 & (6.6) & 606 & $(2.9)$ & 650 & $(3,2)$ & 676 & $(2.8)$ \\
\hline Schweiz (deutsch) & 491 & $(5,0)$ & 103 & $(2.2)$ & 314 & $(5.4)$ & 350 & $(5.8)$ & 420 & $(6.6)$ & 565 & (5.6) & 621 & $(6.4)$ & 652 & $(6.5)$ \\
\hline Schweiz (frz.) & 516 & $(6.2)$ & 92 & (3.8) & 351 & $(14.4)$ & 390 & (10.7) & 456 & (7.9) & 582 & $(8.6)$ & 627 & $(7.8)$ & 658 & (10.3) \\
\hline Schweiz (ital.) & 496 & $(16.6)$ & 85 & $(9.1)$ & 348 & $(21.4)$ & 391 & $(20.5)$ & 444 & $(13.6)$ & 552 & $(22.9)$ & 603 & $(33.0)$ & 629 & $(31.8)$ \\
\hline \multicolumn{17}{|c|}{ Subskala Reflektieren und Bewerten } \\
\hline Belgien (frz.) & 466 & (9.1) & 120 & $(6.8)$ & 247 & $(27.6)$ & 302 & $(18.6)$ & 386 & $(13.2)$ & 556 & $(6.2)$ & 610 & (5.5) & 640 & $(6.5)$ \\
\hline Belgien (fläm.) & 521 & $(4.4)$ & 103 & (3.9) & 324 & $(13.0)$ & 379 & $(11.1)$ & 465 & $(7.2)$ & 593 & $(2.9)$ & 639 & (3.1) & 666 & (3.3) \\
\hline Schweiz (deutsch) & 484 & $(5.8)$ & 118 & $(2.5)$ & 279 & $(9.3)$ & 326 & $(7.2)$ & 404 & (7.9) & 569 & $(6,8)$ & 631 & $(6.9)$ & 667 & $(6.8)$ \\
\hline Schweiz (frz.) & 500 & $(6.1)$ & 95 & (3.9) & 333 & $(14.3)$ & 377 & (11.7) & 439 & (7.7) & 566 & $(8.0)$ & 618 & $(8.8)$ & 651 & $(8.4)$ \\
\hline Schweiz (ital.) & 503 & $(20.4)$ & 99 & $(5.1)$ & 345 & (26.6) & 383 & $(19.9)$ & 441 & (20.6) & 573 & $(21.8)$ & 624 & $(23.5)$ & 661 & $(33.7)$ \\
\hline \multicolumn{17}{|c|}{ Skala für mathematische Grundbildung } \\
\hline Belgien (frz.) & 491 & (7.2) & 109 & $(3.8)$ & 301 & $(13.0)$ & 340 & $(11.0)$ & 415 & $(11.8)$ & 572 & (6.4) & 626 & (5.5) & 653 & (7.5) \\
\hline Belgien (fläm.) & 543 & $(4.6)$ & 98 & $(4.0)$ & 356 & $(18.8)$ & 408 & $(13.8)$ & 487 & $(6.8)$ & 612 & (3.9) & 658 & (4.6) & 684 & $(4.2)$ \\
\hline Schweiz (deutsch) & 525 & $(5.3)$ & 102 & (2.3) & 344 & (10.8) & 386 & (7.9) & 459 & $(6.0)$ & 599 & (6.4) & 651 & (6.7) & 681 & $(5.8)$ \\
\hline Schweiz (frz.) & 547 & $(7.1)$ & 90 & $(4.8)$ & 400 & (14.4) & 431 & $(9.9)$ & 489 & $(9.0)$ & 609 & (10.6) & 663 & $(10.5)$ & 686 & (12.6) \\
\hline Schweiz (ital.) & 525 & $(13.8)$ & 91 & $(10.2)$ & 371 & $(33.0)$ & 422 & $(20.7)$ & 481 & $(15.4)$ & 584 & $(21.4)$ & 628 & $(27.9)$ & 658 & $(34.4)$ \\
\hline \multicolumn{17}{|c|}{ Skala für naturwissenschaftliche Grundbildun } \\
\hline Belgien (frz.) & 467 & $(8.7)$ & 122 & $(5.8)$ & 253 & $(24.8)$ & 299 & $(19.0)$ & 383 & $(11.4)$ & 560 & $(6.2)$ & 620 & (7.7) & 652 & $(5.7)$ \\
\hline Belgien (fläm.) & 519 & $(4.2)$ & 95 & (3.4) & 352 & $(12.0)$ & 392 & $(9.2)$ & 457 & $(6.9)$ & 588 & (3.6) & 634 & $(3.2)$ & 659 & (3.6) \\
\hline Schweiz (deutsch) & 492 & $(5.3)$ & 99 & $(2.7)$ & 329 & $(6.2)$ & 362 & $(6.2)$ & 423 & $(6.8)$ & 562 & $(8.2)$ & 619 & (7.3) & 649 & (6.6) \\
\hline Schweiz (frz.) & 514 & $(7.3)$ & 103 & $(4.8)$ & 344 & $(15.9)$ & 383 & $(11.5)$ & 441 & (11.3) & 587 & (10.9) & 648 & $(12.6)$ & 685 & $(16.0)$ \\
\hline Schweiz (ital.) & 483 & $(16.7)$ & 94 & $(11.5)$ & 316 & $(36.9)$ & 368 & $(32.2)$ & 428 & (17.7) & 539 & (27.4) & 605 & $(24.9)$ & 632 & (29.4) \\
\hline
\end{tabular}





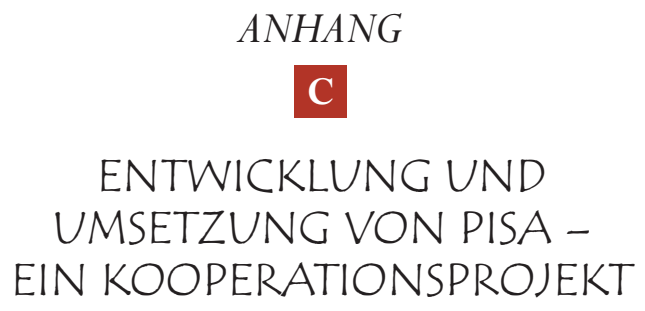




\section{Anhang C: Entwicklung und Umsetzung von PISA - ein Kooperationsprojekt}

\section{Einführung}

PISA ist ein Kooperationsprojekt, das wissenschaftliches Fachwissen der Teilnehmerländer zusammenführt, und in dem Entscheidungen gemeinschaftlich auf der Basis politischer Interessen der teilnehmenden Länder getroffen werden.

Ein Ausschuss der Teilnehmerländer (BPC - Board of Participating Countries), in dem jedes Land vertreten ist, definiert im Rahmen der OECD-Zielsetzung die Politikprioritäten für PISA und überwacht die Einhaltung dieser Prioritäten während der Programmumsetzung. Diese Aufgabe umfasst die Festlegung von Prioritäten für die Ausarbeitung der Indikatoren, die Erstellung der Instrumente zur Leistungsmessung und die Berichterstattung über die Ergebnisse.

Es wurden Arbeitsgruppen mit Experten aus den Teilnehmerstaaten gebildet, die gewährleisten sollen, dass die politischen Zielsetzungen mit der größtmöglichen international verfügbaren verfahrenstechnischen Kompetenz verknüpft werden. Durch ihre Beteiligung an diesen Expertengruppen stellen die Länder sicher, dass die eingesetzten Instrumente international valide sind und zugleich dem kulturellen und curricularen Kontext der OECD-Mitgliedsländer Rechnung tragen, die eingesetzten Beurteilungsinstrumente über sehr gute messtechnische Eigenschaften verfügen und diese Instrumente sowohl authentisch als auch bildungspolitisch relevant sind.

Über die nationalen Projektmanager setzen die Teilnehmer das PISA-Programm gemäß den vereinbarten administrativen Verfahren auf nationaler Ebene um. Die nationalen Projektmanager spielen eine entscheidende Rolle, indem sie gewährleisten, dass die Umsetzung hohen qualitativen Ansprüchen genügt, und indem sie die Ergebnisse, Analysen, Berichte und Veröffentlichungen überprüfen und evaluieren.

Zuständig für Design und Implementierung der Erhebungen innerhalb des vom Ausschuss der teilnehmenden Länder festgelegten Rahmens ist das so genannte PISA-Konsortium, in dem unter der Leitung des Australian Council for Educational Research (ACER) die folgenden Forschungseinrichtungen zusammenarbeiten: Netherlands National Institute for Educational Measurement (Citogroep), The National Institute for Educational Policy Research (NIER), Japan, the Educational Testing Service (ETS), USA, und WESTAT, USA.

Das OECD-Sekretariat hat die übergreifende Managementverantwortung für das Programm, verfolgt dessen praktische Umsetzung, fungiert als Sekretariat für den Ausschuss der teilnehmenden Länder, bemüht sich um Konsensbildung zwischen den Ländern und dient als Ansprechpartner zwischen dem Ausschuss der teilnehmenden Länder und dem internationalen Konsortium, das mit der Implementierung der Aktivitäten beauftragt ist. Das OECD-Sekretariat erstellt auch die Indikatoren, analysiert und arbeitet die internationalen Berichte und Veröffentlichungen in Zusammenarbeit mit dem PISA-Konsortium und in enger Konsultation mit den Mitgliedsländern sowohl auf politischer Ebene (Ausschuss der teilnehmenden Länder) als auch auf Implementierungsebene (nationale Projektmanager) aus.

Nachstehend finden sich Listen mit den Mitgliedern der verschiedenen PISA-Organe wie auch einzelner Fachleute und Consultants, die an der PISA-Erhebung mitgewirkt haben.

\section{Mitglieder des PISA-Ausschusses der teilnehmenden Länder}

Vorsitzender: Eugene Owen

Australien: Wendy Whitham

Belgien: Dominique Barthélémy, Christiane Blondin, Dominique Lafontaine, Liselotte van de Perre

Brasilien: Maria Helena Guimarães de Castro

Dänemark: Birgitte Bovin

Deutschland: Jochen Schweitzer, Helga Hinke, Gudrun Stoltenberg

Finnland: Ritva Jakku-Sihvonen

Frankreich: Gérard Bonnet

Griechenland: Vassilis Koulaidis
Irland: Gerry Shiel

Island: Einar Gudmundsson

Italien: Chiara Croce, Elisabetta Midena, Benedetto

Vertecchi

Japan: Ryo Watanabe

Kanada: Satya Brink, Patrick Bussière, Dianne Pennock

Korea: Kooghyang Ro

Luxemburg: Jean-Paul Reeff

Mexiko: Fernando Córdova Calderón

Neuseeland: Lynne Whitney

Niederlande: Arnold Spee

Norwegen: Alette Schreiner 
Österreich: Friedrich Plank

Polen: Kazimierz Korab

Portugal: Glória Ramalho

Schweden: Anders Auer, Birgitta Fredander, Anita Wester Schweiz: Heinz Gilomen

Spanien: Guillermo Gil

Tschechische Republik: Jan Koucky, Jana Strakova

Ungarn: Péter Vári

Vereinigtes Königreich: Lorna Bertrand, Brian Semple

Vereinigte Staaten: Mariann Lemke

\section{Nationale PISA-Projektmanager}

Australien: Jan Lokan

Belgien: Dominique Lafontaine, Luc van de Poele

Brasilien: Tereza Cristina Cotta, Maria Lucia Guardia, Maria Inês Pestana

Dänemark: Vita Bering Pruzan

Deutschland: Juergen Baumert, Petra Stanat

Finnland: Jouni Välijärvi

Frankreich: Jean-Pierre Jeantheau

Griechenland: Katerina Kassotakis

Irland: Judith Cosgrove

Island: Julius Bjornsson, Ragna Benedikta Garðarsdóttir

Italien: Emma Nardi

Japan: Ryo Watanabe

Kanada: Marc Lachance, Dianne Pennock

Korea: Kooghyang Ro

Lettland: Andris Kangro

Luxemburg: Iris Blanke, Jean-Paul Reeff

Mexiko: Fernando Córdova Calderón

Neuseeland: Steve May

Niederlande: Johan Wijnstra

Norwegen: Svein Lie

Österreich: Günter Haider

Polen: Michal Federowicz

Portugal: Glória Ramalho

Russische Föderation: Galina Kovalyova

Schweden: Bengt-Olov Molander, Astrid Pettersson, Karin

Taube

Schweiz: Huguette McCluskey

Spanien: Guillermo Gil

Tschech.Republik: Jana Straková

Ungarn: Péter Vári

Vereinigtes Königreich: Baljit Gill, Graham Thorpe

Vereinigte Staaten: Ghedam Bairu, Marilyn Binkley

\section{OECD-Sekretariat}

Andreas Schleicher (PISA-Gesamtkoordination und Kontakte zu den Mitgliedsländern)

Claudia Tamassia (Projektmanagement)

Eric Charbonnier (statistische Unterstützung)

Hannah Cocks (statistische Unterstützung)

Juliet Evans (administrative Unterstützung)

\section{PISA-Expertengruppen}

Funktionale Expertengruppe Mathematik

Jan de Lange (Vorsitzender) (Universität Utrecht, Niederlande)

Raimondo Bolletta (Istituto Nazionale di Valutazione, Italien)

Sean Close (St Patrick's College, Irland)

Maria Luisa Moreno (IES “Lope de Vega”, Spanien)

Mogens Niss (IMFUFA, Universität Roskilde, Dänemark)

Kyungmee Park (Universität Hongik, Korea)

Thomas A. Romberg (Vereinigte Staaten)

Peter Schüller (Bundesministerium für Bildung und Kultur, Österreich)

\section{Funktionale Expertengruppe Lesen}

Irwin Kirsch (Vorsitzender) (Educational Testing Service, Vereinigte Staaten)

Marilyn Binkley (National Center for Educational Statistics, Vereinigte Staaten)

Alan Davies (Universität Edinburgh, Vereinigtes Königreich)

Stan Jones (Statistics Canada, Kanada)

John de Jong (Language Testing Services, Niederlande)

Dominique Lafontaine (Université de Liège Sart Tilman, Belgien)

Pirjo Linnakylä (Universität Jyväskylä, Finnland)

Martine Rémond (Institut National de Recherche

Pédagogique, Frankreich)

Wolfgang Schneider (Universität Würzburg, Deutschland)

Ryo Watanabe (National Institute for Educational Research, Japan)

\section{Funktionale Expertengruppe Naturwissenschaften}

Wynne Harlen (Vorsitzender) (University of Bristol, Vereinigtes Königreich)

Peter Fensham (Monash University, Australien)

Raul Gagliardi (Universität Genf, Schweiz)

Svein Lie (Universität Oslo, Norwegen)

Manfred Prenzel (Universität Kiel, Deutschland)

Senta A. Raizen (National Center for Improving Science Education (NCISE), Vereinigte Staaten)

Donghee Shin (Dankook University, Korea)

Elizabeth Stage (University of California, Vereinigte Staaten) 


\section{PISA Technische Beratergruppe}

Ray Adams (ACER, Australien)

Pierre Foy (Statistics Canada, Kanada)

Aletta Grisay (Belgien)

Larry Hedges (The University of Chicago, Vereinigte Staaten)

Eugene Johnson (American Institutes for Research, Vereinigte Staaten)

John de Jong (Language Testing Services, Niederlande)

Geoff Masters (ACER, Australien)

Keith Rust (WESTAT, Vereinigte Staaten)

Norman Verhelst (Citogroep, Niederlande)

J. Douglas Willms (University of New Brunswick, Kanada)

\section{PISA-Konsortium}

Australian Council for Educational Research

Ray Adams (Projektleiter des PISA-Konsortiums)

Alla Berezner (Datenverarbeitung, Datenanalyse)

Claus Carstensen (Datenanalyse)

Lynne Darkin (Lesetestausarbeitung)

Brian Doig (Mathematiktestausarbeitung)

Adrian Harvey-Beavis (Qualitätskontrolle,

Fragebogenausarbeitung)

Kathryn Hill (Lesetestausarbeitung)

John Lindsey (Mathematiktestausarbeitung)

Jan Lokan (Qualitätskontrolle, Ausarbeitung

länderspezifischer Verfahren)

Le Tu Luc (Datenverarbeitung)

Greg Macaskill (Datenverarbeitung)

Joy McQueen (Lesetestausarbeitung und Berichterstattung)

Gary Marks (Fragebogenausarbeitung)

Juliette Mendelovits (Lesetestausarbeitung und Berichterstattung)

Christian Monseur (Leiter des PISA-Konsortiums für Datenverarbeitung, Datenanalyse, Qualitätskontrolle)

Gayl O'Connor (Naturwissenschaftstestausarbeitung)

Alla Routitsky (Datenverarbeitung)

Wolfram Schulz (Datenanalyse)

Ross Turner (Testanalyse und Koordinierung der Berichterstattung)

Nikolai Volodin (Datenverarbeitung)

Craig Williams (Datenverarbeitung, Datenanalyse)

Margaret Wu (stellvertretende Projektleiterin des PISA-

Konsortiums)

\section{Westat}

Nancy Caldwell (Leiterin des PISA-Konsortiums für Feldoperationen und Qualitätskontrolle)

Ming Chen (Stichprobenauswahl und Gewichtung)
Fran Cohen (Stichprobenauswahl und Gewichtung) Susan Fuss (Stichprobenauswahl und Gewichtung) Brice Hart (Stichprobenauswahl und Gewichtung) Sharon Hirabayashi (Stichprobenauswahl und Gewichtung) Sheila Krawchuk (Stichprobenauswahl und Gewichtung) Dward Moore (Feldoperationen und Qualitätskontrolle) Phu Nguyen (Stichprobenauswahl und Gewichtung) Monika Peters (Feldoperationen und Qualitätskontrolle) Merl Robinson (Feldoperationen und Qualitätskontrolle)

Keith Rust (Leiter des PISA-Konsortiums für Stichprobenauswahl und Gewichtung)

Leslie Wallace (Stichprobenauswahl und Gewichtung)

Dianne Walsh (Feldoperationen und Qualitätskontrolle)

Trevor Williams (Fragebogenausarbeitung)

\section{Citogroep}

Steven Bakker (Naturwissenschaftstestausarbeitung)

Bart Bossers (Lesetestausarbeitung)

Truus Decker (Mathematiktestausarbeitung)

Erna van Hest (Lesetestausarbeitung und Qualitätskontrolle)

Kees Lagerwaard (Mathematiktestausarbeitung)

Gerben van Lent (Mathematiktestausarbeitung)

Ico de Roo (Naturwissenschaftstestausarbeitung)

Maria van Toor (logistische Unterstützung und

Qualitätskontrolle)

Norman Verhelst (technische Beratung, Datenanalyse)

\section{Educational Testing Service}

Irwin Kirsch (Lesetestausarbeitung))

\section{Sonstige Experten}

Cordula Altelt (Fragebogenausarbeitung)

Marc Demeuse (Qualitätskontrolle)

Barbara Feuille (Übersetzung)

Harry Ganzeboom (Fragebogenausarbeitung)

Aletta Grisay (technische Beratung, Datenanalyse, Übersetzung, Fragebogenausarbeitung)

Donald Hirsch (redaktionelle Überarbeitung)

Sabine Lutkat (redaktionelle Überarbeitung)

Jules Peschar (Fragebogenausarbeitung)

Erich Ramseier (Fragebogenausarbeitung)

Gundula Schumel (Fragebogenausarbeitung)

Marie-Andrée Somers (Datenanalyse und Berichterstattung) Peter Sutton (redaktionelle Überarbeitung)

Rich Tobin (Fragebogenausarbeitung und Berichterstattung)

J.Douglas Willms (Fragebogenausarbeitung, Datenanalyse und Berichterstattung) 
OECD PUBLICATIONS, 2, rue André-Pascal, 75775 PARIS CEDEX 16 PRINTED IN FRANCE

(96 2001145 P) ISBN 92-64-59671-2 - No. 522802001 